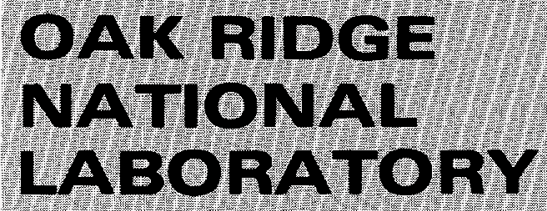

MAGTH MARUETRA

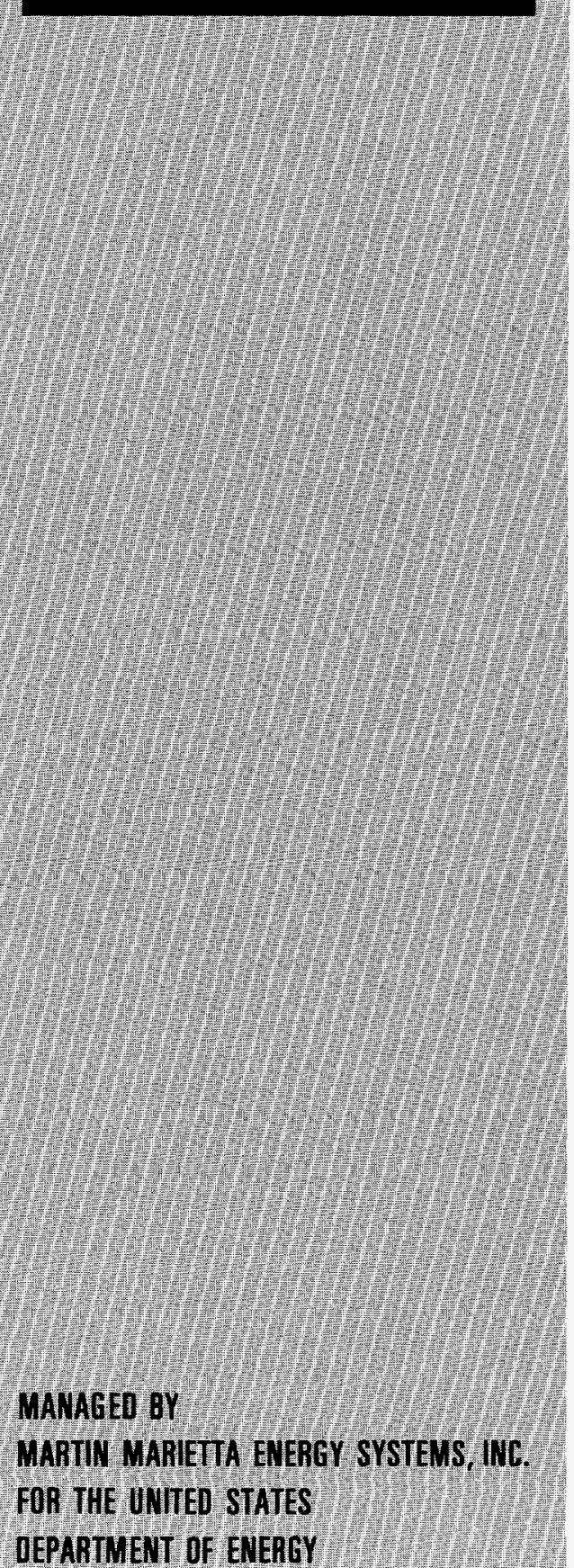

\section{Mathematical Models and Specific Absorbed Fractions of Photon Energy in the Nonpregnant Adult Female and at the End of Each Trimester of Pregnancy}

\author{
M. G. Stabin \\ E. E. Watson \\ M. Cristy \\ J. C. Ryman \\ K. F. Eckerman \\ J. L. Davis \\ D. Marshall \\ M. K. Gehlen \\ DISCLAIMER
}

This report was prepared as an account of work sponsored by an agency of the United States Government. Neither the United States Government nor any agency thereof, nor any of their employees, makes any warranty, express or implied, or assumes any legal liability or responsibility for the accuracy, completeness, or usefulness of any information, apparatus, product, or process disclosed, or represents that its use would not infringe privately owned rights. Reference herein to any specific commercial product, process, or service by trade name, trademark, manufacturer, or otherwise does not necessarily constitute or imply its endorsement, recommendation, or favoring by the United States Government or any agency thereof. The views and opinions of authors expressed herein do not necessarily state or reflect those of the United States Government or any agency thereof.

$$
\begin{aligned}
& \text { RECEIVED } \\
& \text { JUN } 291995 \\
& \text { OSTI }
\end{aligned}
$$


This report has been reproduced directly from the best available copy.

Available to DOE and DOE contractors from the Office of Scientific and Technical Information, P.O. Box 62, Oak Ridge, TN 37831; prices available from (615) 576-8401, FTS 626-8401.

Available to the public from the National Tectnical Information Service, U.S. Department of Commerce, 5285 Port Royal Rd., Springfield, VA 22161.

This report was prepared as an account of work sponsored by an agency of the United States Government. Neither the United States Government nor any agency thereof, nor any of their employees, makes any warranty, express or implied, or assumes any legal liability or responsibility for the accuracy, completeness, or usefulness of any information, apparatus, product, or process disclosed, or represents that its use would not infringe privately owned rights. Reference herein to any specific commercial product, process, or service by trade name, trademark, manufacturer, or otherwise, does not necessarily constitute or imply its endorsement, recommendation, or favoring by the United States Government or any agency thereof. The views and opinions of authors expressed herein do not necessarily state or reflect those of the United States Government or any agency thereot. 


\section{DISCLAIMER}

\section{Portions of this document may be illegible in electronic image products. Images are produced from the best available original document.}


ORNL/TM-12907

\section{MATHEMATICAL MODELS}

AND SPECIFIC ABSORBED FRACTIONS

OF PHOTON ENERGY IN

THE NONPREGNANT ADULT FEMALE AND

AT THE END OF EACH TRIMESTER OF PREGNANCY

M. G. Stabin', E. E. Watson ${ }^{1}$, M. Cristy ${ }^{2}$, J. C. Ryman ${ }^{2}$

K. F. Eckerman ${ }^{2}$, J. L. Davis ${ }^{3}$, D. Marshall ${ }^{4}$, M. K. Gehlen ${ }^{5}$

May 8, 1995

${ }^{1}$ Oak Ridge Institute for Science and Education, Radiation Internal Dose Information Center, P.O. Box 117, Oak Ridge, TN 37831-0117

${ }^{2}$ Oak Ridge National Laboratory, Health Sciences Research Division, 1060 Commerce Park, Oak Ridge, TN 37831

${ }^{3}$ University of Tennessee at Chattanooga, Chattanooga, TN 37403; deceased

${ }^{4}$ University of Florida, Department of Nuclear Engineering, Gainesville, FL 32611

${ }^{5}$ San Diego State University, San Diego, CA 92175

Prepared by the

OAK RIDGE NATIONAL LABORATORY

Oak Ridge, Tennessee 37831

managed by

MARTIN MARIETTA ENERGY SYSTEMS, INC.

for the

U. S. DEPARTMENT OF ENERGY

under contract DE-AC05-84OR21400 


\section{CONTENTS}

ACKNOWLEDGEMENTS $\ldots \ldots \ldots \ldots \ldots \ldots \ldots \ldots \ldots \ldots \ldots \ldots \ldots$ vii

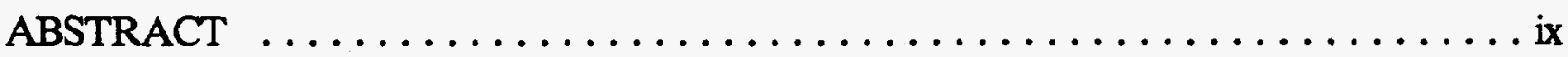

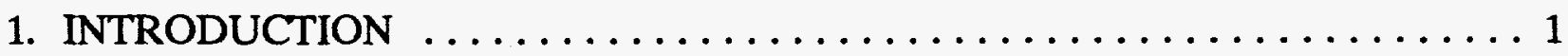

2. NEW NONPREGNANT ADULT FEMALE MODEL $\ldots \ldots \ldots \ldots \ldots \ldots, 3$

3. MODIFICATIONS TO THE ADULT FEMALE PHANTOM $\ldots \ldots \ldots \ldots \ldots 4$

3.1. THE UTERUS AND UTERINE CONTENTS $\ldots \ldots \ldots \ldots \ldots \ldots 6$

3.1.1. Three-Month Pregnant Female . . . . . . . . . . . . 6

3.1.2. Six-Month Pregnant Female . . . . . . . . . . . . . . 9

3.1.3. Nine-Month Pregnant Female . . . . . . . . . . . . 12

3.1.4. Summary - Masses and Volumes $\ldots \ldots \ldots \ldots \ldots \ldots \ldots \ldots$

3.2. FETUS AND FETAL SKELETON $\ldots \ldots \ldots \ldots \ldots \ldots \ldots \ldots$

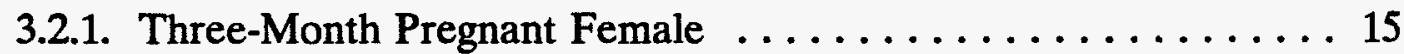

3.2.2. Six-Month Pregnant Female . . . . . . . . . . . . . 15

3.2.3. Nine-Month Pregnant Female . . . . . . . . . . . 17

3.3. FETAL SKELETON COMPOSITION $\ldots \ldots \ldots \ldots \ldots \ldots \ldots$

3.3.1. Summary - Masses and Volumes $\ldots \ldots \ldots \ldots \ldots \ldots \ldots \ldots$

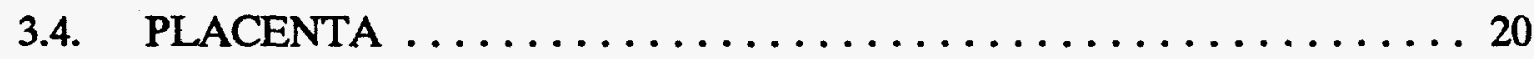

3.4.1. Three-Month Pregnant Female ............... 20

3.4.2. Six-Month Pregnant Female . . . . . . . . . . . . . 20

3.4.3. Nine-Month Pregnant Female . . . . . . . . . . . . 21

3.4.4. Summary - Masses and Volumes $\ldots \ldots \ldots \ldots \ldots \ldots \ldots 22$

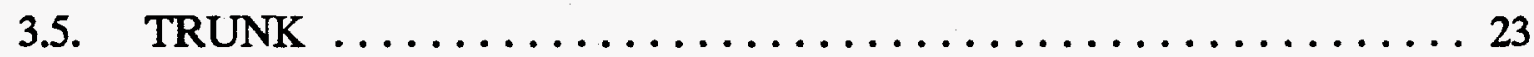

3.5.1. Three-Month Pregnant Female . . . . . . . . . . . 23

3.5.2. Six-Month Pregnant Female . . . . . . . . . . . . . 23

3.5.3. Nine-Month Pregnant Female . ............... 24

3.5.4. Summary - Masses and Volumes ............... 24

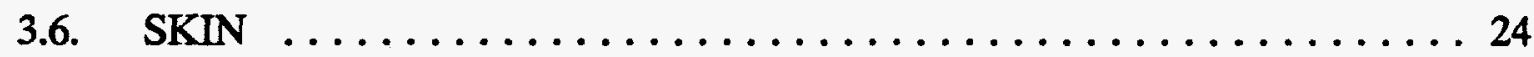

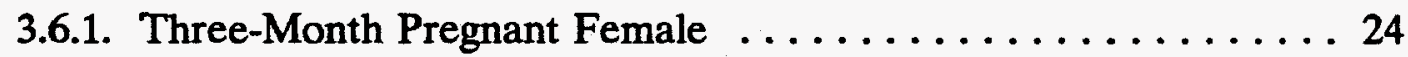

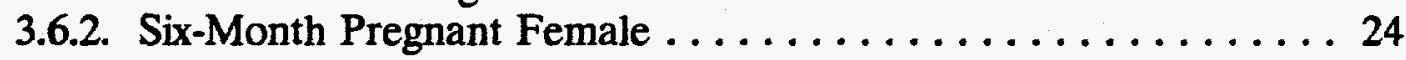

3.6.3. Nine-Month Pregnant Female . ............... 25 
3.7. URINARY BLADDER $\ldots \ldots \ldots \ldots \ldots \ldots \ldots \ldots \ldots \ldots \ldots$

3.7.1. Three-Month Pregnant Female ................ 25

3.7.2. Six-Month Pregnant Female . . . . . . . . . . . . . 26

3.7.3. Nine-Month Pregnant Female . . . . . . . . . . . . 27

3.7.4. Summary - Masses and Volumes $\ldots \ldots \ldots \ldots \ldots \ldots \ldots 28$

3.8. SMALL INTESTINE $\ldots \ldots \ldots \ldots \ldots \ldots \ldots \ldots \ldots \ldots \ldots \ldots$

3.8.1. Three-Month Pregnant Female $\ldots \ldots \ldots \ldots \ldots \ldots \ldots \ldots 28$

3.8.2. Six-Month Pregnant Female . . . . . . . . . . . . . . 29

3.8.3. Nine-Month Pregnant Female . . . . . . . . . . . 30

3.9. UPPER LARGE INTESTINE (ULI) $\ldots \ldots \ldots \ldots \ldots \ldots \ldots \ldots \ldots$

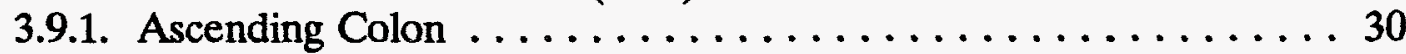

3.9.1.1. Three-month pregnant female ............ 30

3.9.1.2. Six-month pregnant female $\ldots \ldots \ldots \ldots \ldots \ldots \ldots, 32$

3.9.1.3. Nine-month pregnant female $\ldots \ldots \ldots \ldots \ldots \ldots \ldots 33$

3.9.2. Transverse Colon $\ldots \ldots \ldots \ldots \ldots \ldots \ldots \ldots \ldots \ldots \ldots \ldots \ldots$

3.9.2.1. Three-month pregnant female . ........... 33

3.9.2.2. Six-month pregnant female $\ldots \ldots \ldots \ldots \ldots \ldots \ldots 34$

3.9.2.3. Nine-month pregnant female $\ldots \ldots \ldots \ldots \ldots \ldots . . \ldots \ldots$

3.10. LOWER LARGE INTESTINE (LLI) $\ldots \ldots \ldots \ldots \ldots \ldots \ldots \ldots$

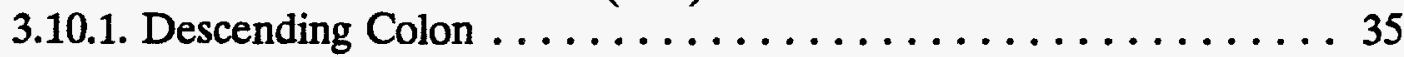

3.10.1.1. Three-month pregnant female $\ldots \ldots \ldots \ldots \ldots \ldots \ldots 35$

3.10.1.2. Six-month pregnant female $\ldots \ldots \ldots \ldots \ldots \ldots \ldots 36$

3.10.1.3. Nine-month pregnant female . . . . . . . . . . 37

3.10.2. Sigmoid Colon ..................... 37

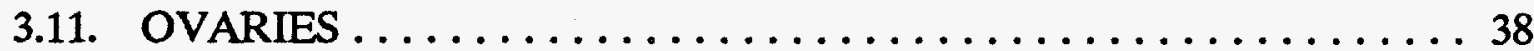

3.11.1. Three-Month Pregnant Female ............... 38

3.11.2. Six- and Nine-Month Pregnant Female . . . . . . . . . 38

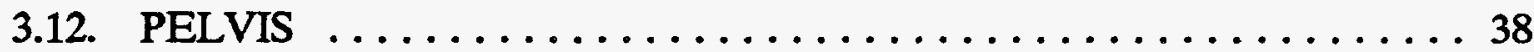

3.12.1. Three-Month Pregnant Female ............... 38

3.12.2. Six- and Nine-Month Pregnant Female . . . . . . . . 38

3.13. LIVER . ............................ 39

3.13.1. Three- and Six-Month Pregnant Female ............ 39

3.13.2. Nine-Month Pregnant Female . . . . . . . . . . . . 39

3.14. OTHER ORGANS $\ldots \ldots \ldots \ldots \ldots \ldots \ldots \ldots \ldots \ldots \ldots \ldots$ 


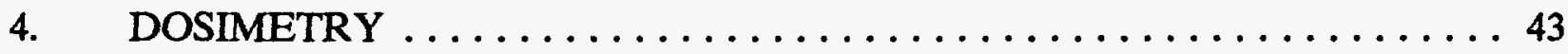

4.1. DOSIMETRIC METHODOLOGY $\ldots \ldots \ldots \ldots \ldots \ldots \ldots \ldots$

4.1.1. Dosimetric Quantities ................... 43

4.2. SPECIFIC ABSORBED FRACTIONS FOR PHOTONS $\ldots \ldots \ldots .45$

4.2.1. Methods ....................... 45

4.2.2. Special Methods for Consistency among the Adult Female Models 48

4.2.3. Examples of Results . . . . . . . . . . . . . . 48

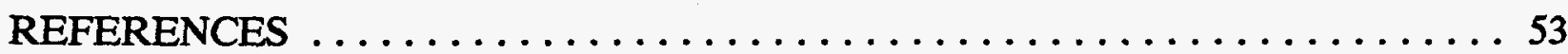

Appendix A: TABLES OF SPECIFIC ABSORBED FRACTIONS $\ldots \ldots \ldots \ldots \ldots 5$

Table A.1. Nonpregnant Female .................. 57

Table A.2. At Three Months Gestation . . . . . . . . . . . . . 75

Table A.3. At Six Months Gestation . . . . . . . . . . . . . . 93

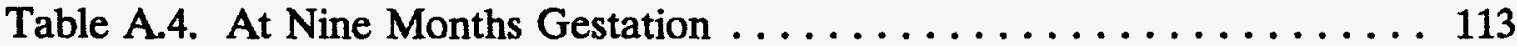




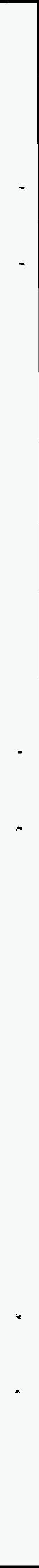




\section{ACKNOWLEDGEMENTS}

The work at Oak Ridge National Laboratory was performed under the auspices of the Department of Energy under contract DE-AC05-84-OR-21400 with Martin Marietta Energy Systems, Inc. Earlier work on the mathematical phantoms was supported jointly by the Department of Energy and the Nuclear Regulatory Commission; this and additional support from the Environmental Protection Agency and the Food and Drug Administration have been critical to the development of our dosimetry research program and is gratefully acknowledged.

The work at Oak Ridge Institute for Science and Education was performed for the Department of Energy under contract DE-AC05-760P00033 and for the Food and Drug Administration under Interagency Agreement No. FDA 224-75-3016, DOE 40-286-71.

The contribution of two of the authors (David Marshall and Kurt Gehlen) to this work was accomplished as part of practicums in the Department of Energy's Applied Health Physics Fellowship Program, which is administered by the Oak Ridge Institute for Science and Education.

The authors further wish to acknowledge the assistance of Barbara Clark, Oak Ridge National Laboratory, for the preparation of the final manuscript for publication and Susan A. Bolcar of the Oak Ridge Institute for Science and Education for graphics development. 


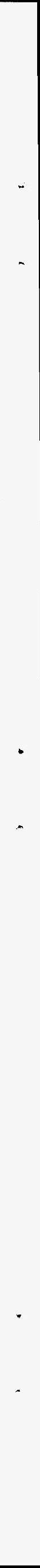




\begin{abstract}
Mathematical phantoms representing the adult female at three, six, and nine months of gestation are described. They are modifications of the 15-year-old male/adult female phantom (15-AF phantom) of Cristy and Eckerman (1987). The model of uterine contents includes the fetus, fetal skeleton, and placenta. The model is suitable for dose calculations for the fetus as a whole; individual organs within the fetus (other than the skeleton) are not modeled. A new model for the nonpregnant adult female is also described, comprising (1) the 15-AF phantom; (2) an adjustment to specific absorbed fractions for organ self-dose from photons to better match Reference Woman masses; and (3) computation of specific absorbed fractions with Reference Woman masses from ICRP Publication 23 for both penetrating and nonpenetrating radiations. Specific absorbed fractions for photons emitted from various source regions are tabulated for the new nonpregnant adult female model and the three pregnancy models.
\end{abstract}




\section{INTRODUCTION}

For a given kind of radiation, the absorbed fraction, $\phi(T-S)$, is the fraction of energy emitted from source organ or region $S$ that is absorbed in target organ or region $T$. When divided by the mass of the target region, $M_{T}$, it is called the specific absorbed fraction, $\Phi(T-S)$. It is one of the quantities used in calculating the S-value $S(T-S)$, used in the schema of the Medical Internal Radiation Dose Committee (MIRD) of the Society of Nuclear Medicine (Loevinger and Berman 1976), or the specific effective energy SEE(T-S), used in the schema of the International Commission on Radiological Protection (ICRP 1979). The S(T-S) and SEE(T-S) quantities represent the absorbed dose rate and equivalent dose rate, respectively, in target tissue $T$ due to a unit activity of a radionuclide in source region $\mathrm{S}$. Whether one is interested in absorbed or equivalent dose the basic physical quantity is the absorbed fraction. Hence there is interest in fundamental tabulations of specific absorbed fractions for radiation types like photons, which travel extensively through the body (except at very low energies) and contribute substantially to $\Phi(T-S)$ even when $S \neq T$. Tables of $\Phi$, for a nonpregnant adult female and for females at the end of each trimester of pregnancy and for a large number of source and target regions, are given in Appendix A.

To obtain $\Phi$ s, one needs a representation of the body (phantom) suitable for application with Monte Carlo techniques in radiation transport calculations. The phantoms described here for nonpregnant and pregnant females are similar in kind to the phantoms developed by Snyder et al. $(1969,1978)$ for a 70-kg adult and by Cristy and Eckerman (1987) for various ages from newborn to adult female and adult male. The Snyder phantom is sometimes also called the MIRD phantom because of its adoption by the MIRD Committee for dose calculations.

A problem of concern in radiation protection is exposure of pregnant women to ionizing radiation, because of the high radiosensitivity of the embryo and fetus. None of the phantoms developed previously adequately represents the female anatomy during the various stages of pregnancy. Cloutier et al. (1973) designed a model for the uterus and urinary bladder to be representative of all nine months of pregnancy and, through Monte Carlo transport techniques, developed absorbed fractions for radiation emitted in the bladder that may be absorbed by the growing uterus. This model has proven useful for many nuclear medicine applications, as the urinary bladder is often a major contributor to absorbed dose within the uterus. It does not, however, completely describe the geometry of the affected abdominal region.

Section 2 describes a new model for the nonpregnant adult female, as given by Cristy and Eckerman (1993). The new model allows dose calculations that better represent the Reference Woman of ICRP Publication 23 (1975). Specific absorbed fractions for this new model, which have not been published previously, are given in Appendix A.1.

Section 3 describes the modifications made to the adult female phantom of Cristy and Eckerman (1987), which completely characterizes, with simple geometric shapes typical of that kind of phantom, the geometry of the internal organs in the female at the end of the first, second, and third trimesters of pregnancy. Only organs and regions changed are described; the reader is referred to Cristy and Eckerman (1987) for a description of the organs and regions not changed. The model at three months has been described previously (Davis et al. 1987). The six-month model is the subject of a master's thesis by Marshall. The model at nine months has been described in part (Watson and 
Stabin 1987), but it did not include the fetus and placenta. Some of the organ modifications given by Marshall and by Watson and Stabin have been further modified here. Specific absorbed fractions for the three models describing the female at each stage of pregnancy are given in Appendices A.2 A.4.

Section 4 describes techniques used in deriving the specific absorbed fractions. It also gives more information on dosimetric terminology and assumptions.

This work does not include a detailed model of the organs and tissues of the developing fetus, as our purpose here is to evaluate the contribution to fetal dose from photons emitted within the organs of the mother. At the third month of pregnancy we offer no explicit model of the fetus, while at six and nine months the model differentiates two fetal tissues (skeleton and soft tissue). These tissues are considered as source regions, because reciprocal $\Phi \mathrm{s}$ were used in deriving the recommended values. Thus the $\Phi \mathbf{s}$ tabulated in Appendix A include photon emitters uniformly distributed within the fetus, within fetal soft tissue, and within the fetal skeleton. The data for source-target pairs comprised of fetal tissues are provided, although the fetal models and the kerma approximation used in the transport calculations might limit their validity (in the kerma approximation the kinetic energy of secondary electrons is assumed to be absorbed at the site of their liberation by the photon interactions). Further evaluation of the dose to fetal tissues from photon emitters incorporated within the fetus awaits the formulation of anatomical models for the developing fetus. The $\Phi \mathbf{s}$ for the near-term fetus might be derived from the newborn in Cristy and Eckerman (1987) and scaled to the earlier stages. 


\section{NEW NONPREGNANT ADULT FEMALE MODEL}

In the series of phantoms developed by Cristy and Eckerman (1987), one phantom was used to represent both a 15-year-old male and an adult female (the "15-AF phantom"). This phantom was originally developed for a 15-year-old male, with organ masses appropriate for a 15-year-old male. Because of the fortuity of the body mass of this phantom being very close to the $58 \mathrm{~kg}$ body mass of Reference Woman, as defined in ICRP Publication 23 (1975), this phantom was used to represent Reference Woman after appropriate changes in masses of breasts, ovaries, and uterus.

Cristy and Eckerman (1993) later improved both the adult male and adult female models, so that the reference organ masses from ICRP Publication 23 could be used for both penetrating and nonpenetrating radiations. First, the 15-AF and adult male phantoms, as described in Cristy and Eckerman (1987), are used as is to generate specific absorbed fractions ( $\Phi$ s) for photons. Second, self-dose values $[\Phi(S-S)]$ are adjusted to be appropriate for the reference masses rather than the phantom masses (see below). Values of $\Phi$ for cross-fire (source and target organs not the same) are not adjusted, because they are not strongly dependent on organ mass. Third, the reference masses are used for non-penetrating radiations as well. The reference masses for the adult female are given in Table 3.7.

Thus the model for adult female comprises three parts: (1) the 15-AF phantom; (2) an adjustment for ongan self-dose from photons; and (3) computation of $\Phi$ with reference masses from ICRP Publication 23 for both penetrating and nonpenetrating radiations.

The correction for self-dose, $\Phi(\mathrm{S} \sim \mathrm{S})$, as applied to $\Phi$ computed from the phantom, is as follows: except at low energies, one would expect theoretically that $\log \Phi$ vs. $\log$ mass would be linear with a slope of $-2 / 3$. Empirically, for a wide variety of organs or regions in the phantoms, Cristy and Eckerman (1993; unpublished data cited therein) found that this holds approximately true for photons with energies from $30 \mathrm{keV}$ to $4 \mathrm{MeV}$. At energies of 10,15 , and $20 \mathrm{keV}$, the slope was approximately $-0.95,-0.90$, and -0.80 . Thus, the correction is made by adjusting the $\Phi$ s computed for the phantom with this relationship, assuming a slope of $-2 / 3$ for energies from $30 \mathrm{keV}$ to $4 \mathrm{MeV}$ and the empirical slopes from 10 to $20 \mathrm{keV}$.

For some organs or regions in the adult female, ICRP Publication 23 does not give reference masses. In those cases masses were scaled from the reference adult male. Masses for cortical and trabecular bone and for endosteal tissue ("bone surface") are $75 \%$ of the male values, instead of the value of $68 \%$ used in ICRP Publication 23, which is too low based on data being assembled for the revision of Reference Man (R. W. Leggett, personal communication cited in Cristy and Eckerman, 1993).

The photon $\Phi \mathrm{s}$ for the nonpregnant adult female based on the new model are given in Table A.1 of Appendix A. They have not been published previously. 


\section{MODIFICATIONS TO THE ADULT FEMALE PHANTOM}

The coordinate system used in the pediatric phantom series of Cristy and Eckerman (1987) was the same as that used in the original MIRD phantom (Snyder et al. 1969). We have retained the same system here, as we have used essentially the same phantom as was proposed by Cristy and Eckerman, with modifications representing pregnancy. In this coordinate system, the origin is at the center of the base of the elliptical cylinder representing the trunk of the phantom. The positive Z-axis is up, the positive $\mathrm{X}$-axis is to the phantom's left, and the positive $\mathrm{Y}$-axis is toward the back of the phantom. Coordinates are given in $\mathrm{cm}$.

The uterus, uterine contents as a whole, fetus, fetal skeleton, and placenta for each phantom are described in a rotated and translated coordinate system. In each case only the $Y$ and $Z$ axes are rotated and translated, which simplifies the rotation-translation equations. For this special case, where the $Y$ and $Z$ axes have been rotated by angle $\theta$ and translated to the origin $\left(0, Y_{0}, Z_{0}\right)$, the new coordinate system $\left(\mathrm{X}^{\prime}, \mathrm{Y}^{\prime}, \mathrm{Z}^{\prime}\right)$ is related to the $(\mathrm{X}, \mathrm{Y}, \mathrm{Z})$ coordinate system by the following equation:

$$
\left[\begin{array}{l}
X^{\prime} \\
Y^{\prime} \\
Z^{\prime}
\end{array}\right]=\left[\begin{array}{ccc}
1 & 0 & 0 \\
0 & \cos \theta & \sin \theta \\
0 & -\sin \theta & \cos \theta
\end{array}\right]\left[\begin{array}{c}
X \\
Y-Y_{0} \\
Z-Z_{0}
\end{array}\right]
$$

To change from the prime system back to the unprimed system, one simply rotates by the angle $-\theta$ :

$$
\left[\begin{array}{c}
X \\
Y-Y_{0} \\
Z-Z_{0}
\end{array}\right]=\left[\begin{array}{ccc}
1 & 0 & 0 \\
0 & \cos (-\theta) & \sin (-\theta) \\
0 & -\sin (-\theta) & \cos (-\theta)
\end{array}\right]\left[\begin{array}{l}
X^{\prime} \\
Y^{\prime} \\
Z^{\prime}
\end{array}\right] \text {. }
$$

Changes in anatomy during pregnancy are illustrated in Figs. 3.1 and 3.2. Note the changes in shape and position of the uterus and the displacement of maternal organs, all of which were taken into account in designing the pregnant phantom series. 

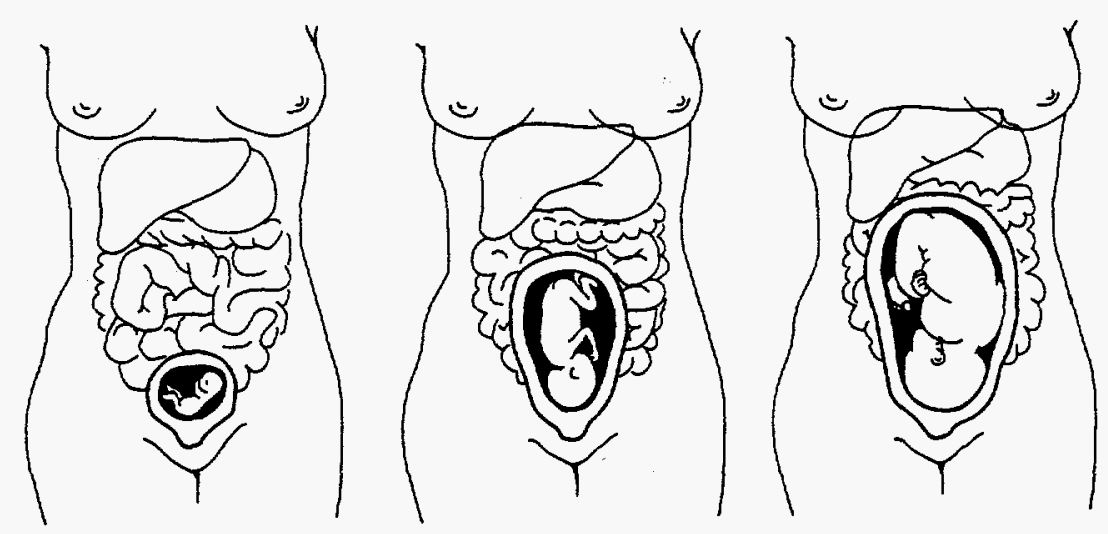

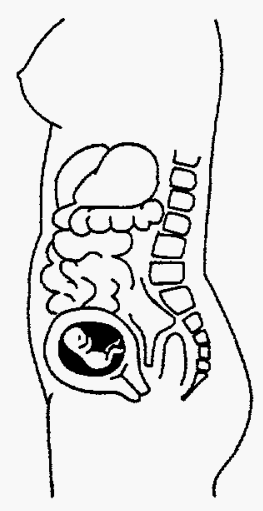

Four Months

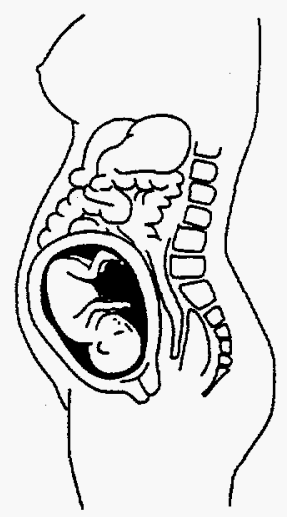

Six Months

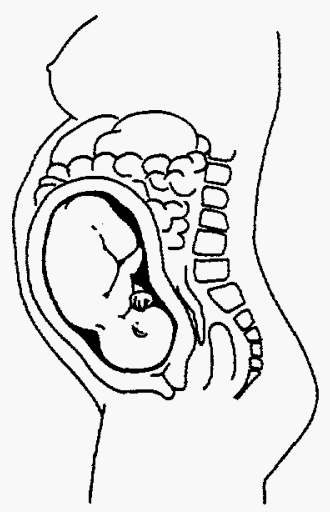

Nine Months

Fig. 3.1. Relative size of the uterus and fetus at four, six, and nine months of gestation. Adapted from Reeder et al. (1983).

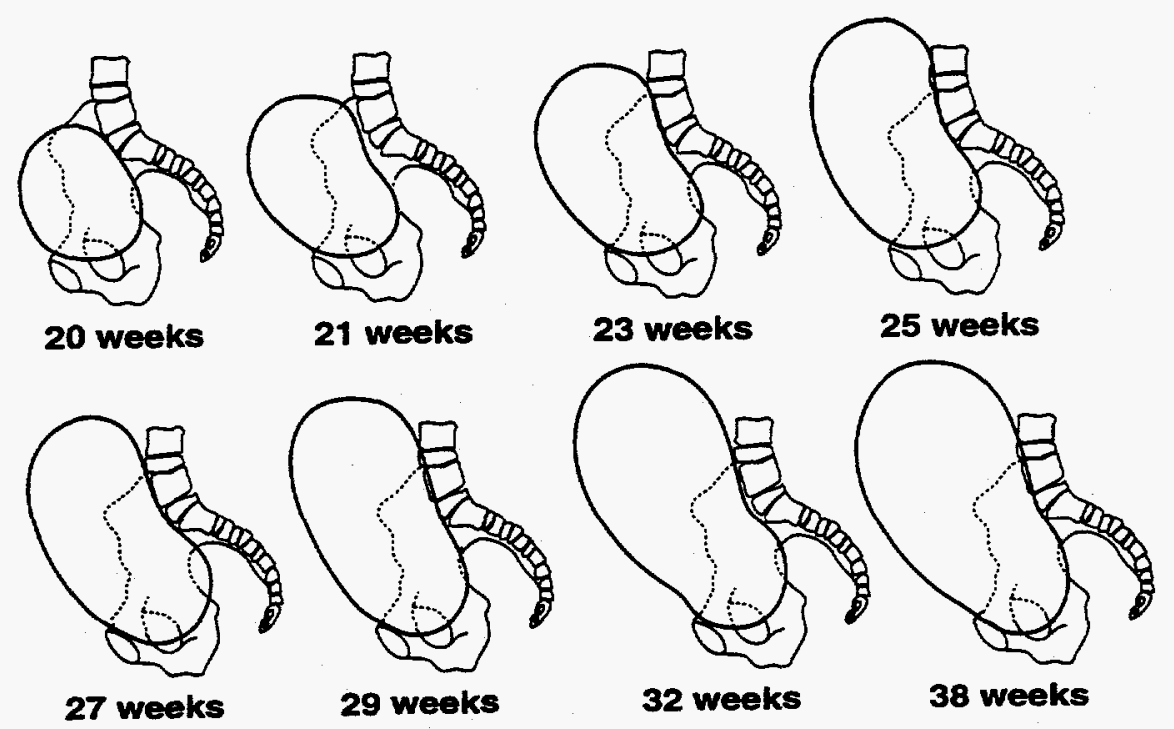

Fig. 3.2. Enlargement of the uterus following the twentieth week of pregnancy. Lateral view, taken from $x$-rays. Note rapid elongation to thirty-second week, then slight increase in anteroposterior diameter thereafter. Adapted from Gillespie (1950). 


\subsection{THE UTERUS AND UTERINE CONTENTS}

\subsubsection{Three-Month Pregnant Female}

The greatest changes to the adult female phantom in modeling pregnancy involve the growth of the uterus and the existence of a compartment representing uterine contents. Our model for the uterus is similar to that described by Cloutier et al. (1973). The uterus plus contents at three months' gestation is represented by a right circular cone with a hemispherical cap. The axis of the cone is along the $\mathrm{Z}^{\prime}$-axis (defined below), which has been rotated $56.9^{\circ}$ from the $\mathrm{Z}$-axis of the phantom (equivalent to a $33.1^{\circ}$ incline to the horizontal plane of the phantom). A cross-section of the model is shown in Fig. 3.3, and an artist's rendition of the organs in the trunk, as viewed from the front (A-P projection), is shown in Fig. 3.4.

The uterus and its contents are described in terms of $\left(X^{\prime}, Y^{\prime}, Z^{\prime}\right)$ coordinates, which are related to the $(X, Y, Z)$ coordinates by the following rotation-translation equation:

$$
\left[\begin{array}{l}
X^{\prime} \\
Y^{\prime} \\
Z^{\prime}
\end{array}\right]=\left[\begin{array}{ccc}
1 & 0 & 0 \\
0 & \cos \theta & \sin \theta \\
0 & -\sin \theta & \cos \theta
\end{array}\right]\left[\begin{array}{c}
X \\
Y-4.51 \\
Z-7.52
\end{array}\right] \text {, where } \theta=56.9^{\circ}
$$

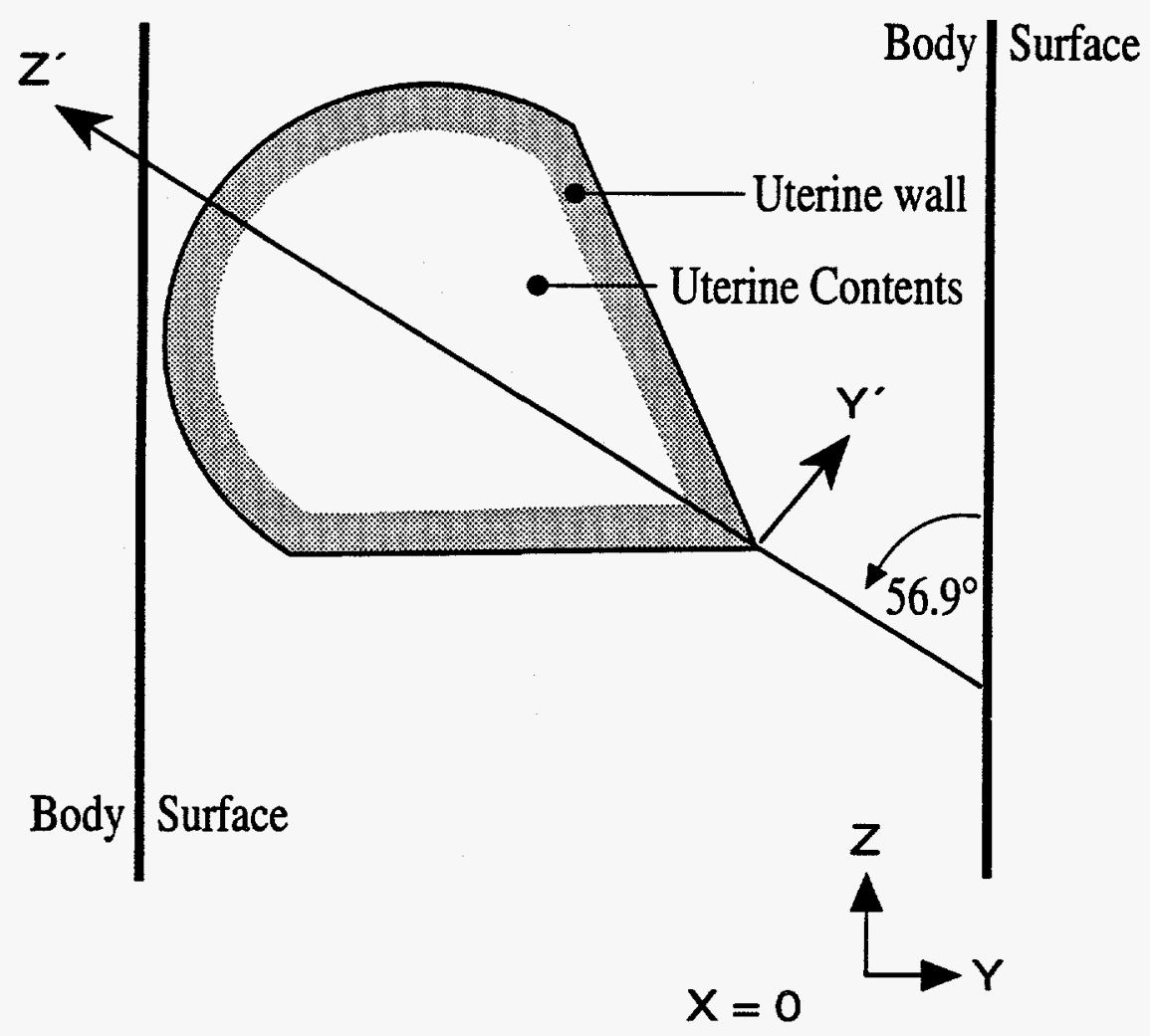

Fig. 3.3. Cross-section of uterine model at 3 months. $Y-Z$ (or $Y^{\prime}-Z^{\prime}$ ) plane; $X=X^{\prime}=0$. 


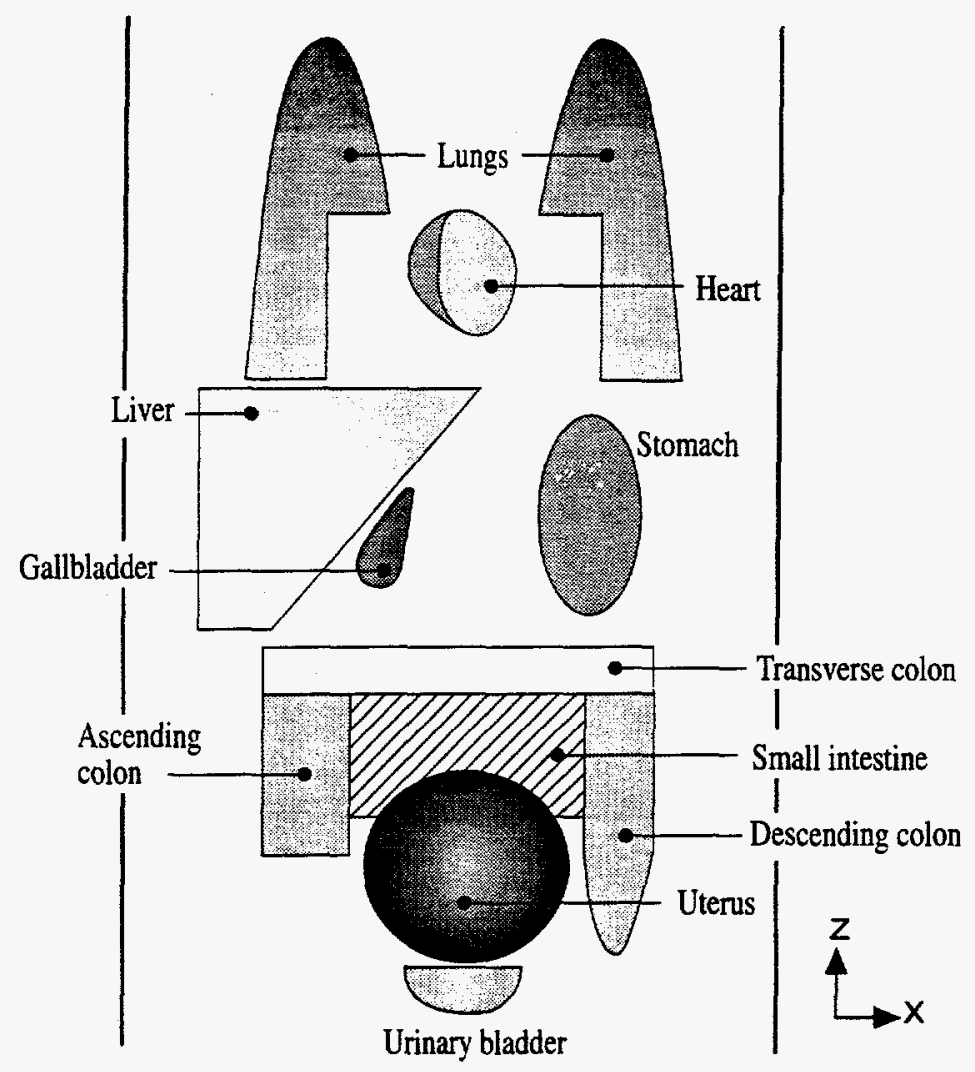

Fig. 3.4. Artist's rendition of organs in the 3-month model, as viewed from the front of the body (A-P projection). Not drawn to scale.

Conical part. The uterine wall in the conical part is represented by the volume between two coaxial right circular cones, and the uterine contents is represented by the volume within the inner cone (Fig. 3.3). Both cones are described in the $\left(\mathrm{X}^{\prime}, \mathrm{Y}^{\prime}, \mathrm{Z}^{\prime}\right)$ coordinate system, with the tip of the outer cone at the origin of this coordinate system.

The uterine wall in the conical part is defined by

$$
\begin{gathered}
X^{\prime 2}+Y^{\prime 2}-\left(\frac{Z^{\prime}}{r}\right)^{2} \leq 0 \\
\text { and } X^{\prime 2}+Y^{\prime 2}-\left(\frac{Z^{\prime}-Z_{0}}{r}\right)^{2}>0 \\
\text { and } Z_{0} \leq Z^{\prime} \leq Z_{2} ;
\end{gathered}
$$

or, for the region of the outer cone that is below the tip of the inner cone,

$$
\begin{aligned}
& X^{\prime 2}+Y^{\prime 2}-\left(\frac{Z^{\prime}}{r}\right)^{2} \leq 0 \\
& \text { and } Z_{1} \leq Z^{\prime} \leq Z_{0} .
\end{aligned}
$$


The uterine contents in the conical part are defined by

$$
\begin{gathered}
X^{\prime 2}+Y^{\prime 2}-\left(\frac{Z^{\prime}-Z_{0}}{r}\right)^{2} \leq 0 \\
\text { and } Z_{0} \leq Z^{\prime} \leq Z_{2}
\end{gathered}
$$

The parameters for the conical part are:

\begin{tabular}{cccc}
$\mathrm{r}$ & $\mathrm{Z}_{0}$ & $\mathrm{Z}_{1}$ & $\mathrm{Z}_{2}$ \\
\hline 1.533 & 1.83 & 0 & 9.2
\end{tabular}

Hemispherical part. The uterine wall in the hemispherical part is represented by the volume between two concentric hemispheres, and the uterine contents is represented by the volume within the inner hemisphere (Fig. 3.3).

The hemispherical part of the uterine wall is defined by

$$
\begin{aligned}
& \left(\frac{X^{\prime}}{A}\right)^{2}+\left(\frac{Y^{\prime}}{A}\right)^{2}+\left(\frac{Z^{\prime}-Z_{0}}{A}\right)^{2} \leq 1 \\
& \text { and }\left(\frac{X^{\prime}}{A-D}\right)^{2}+\left(\frac{Y^{\prime}}{A-D}\right)^{2}+\left(\frac{Z^{\prime}-Z_{0}}{A-D}\right)^{2}>1 \\
& \text { and } Z^{\prime} \geq Z_{0} \text {. }
\end{aligned}
$$

The hemispherical part of the uterine contents is defined by

$$
\begin{gathered}
\left(\frac{X^{\prime}}{A-D}\right)^{2}+\left(\frac{Y^{\prime}}{A-D}\right)^{2}+\left(\frac{Z^{\prime}-Z_{0}}{A-D}\right)^{2} \leq 1 \\
\text { and } Z^{\prime} \geq Z_{0}
\end{gathered}
$$

The parameters for the hemispherical part are:

\begin{tabular}{ccc}
$\mathrm{A}$ & $\mathrm{D}$ & $\mathrm{Z}_{0}$ \\
\hline 6.0 & 1.0 & 9.2
\end{tabular}

Volume. The volume of the entire wall is $359 \mathrm{~cm}^{3}$, and the volume of the entire contents is $440 \mathrm{~cm}^{3}$. 


\subsubsection{Six-Month Pregnant Female}

After the third month of pregnancy, and until about the seventh month, the main area of growth in the uterus occurs along its long axis, although an increase in breadth also occurs, but to a lesser extent (Reynolds 1949; Ivy 1942; Gillespie 1950); see Fig. 3.2. Therefore, in the six-month pregnant phantom, the uterus and its contents are modeled as a right circular cylinder capped at both ends by hemispheres. The long axis of the cylinder is along the $Z^{\prime}$-axis (defined below), which has been rotated $50^{\circ}$ from the $\mathrm{Z}$-axis of the phantom (equivalent to a $40^{\circ}$ incline to the horizontal plane of the phantom). A cross-section of the model is shown in Fig. 3.5, and an artist's rendition of the organs in the trunk, as viewed from the front (A-P projection), is shown in Fig. 3.6. The thickness of the uterine wall in the six-month model was determined from the literature and set to $0.73 \mathrm{~cm}$ (Gillespie 1950). The mass of the uterine wall is $834 \mathrm{~g}$, an increase of $755 \mathrm{~g}$ from the mass of the non-gravid uterus of the adult female model (Cristy and Eckerman 1987). This mass is in good agreement with that found in the literature at this stage of gestation (Ivy 1942; Gillespie 1950; Hytten and Leitch 1964; Moore 1982; ICRP 1975).

The uterus and its contents are described in terms of $\left(X^{\prime}, Y^{\prime}, Z^{\prime}\right)$ coordinates, which are related to the $(X, Y, Z)$ coordinates by the following rotation-translation equation:

$$
\left[\begin{array}{l}
X^{\prime} \\
Y^{\prime} \\
Z^{\prime}
\end{array}\right]=\left[\begin{array}{ccc}
1 & 0 & 0 \\
0 & \cos \theta & \sin \theta \\
0 & -\sin \theta & \cos \theta
\end{array}\right]\left[\begin{array}{c}
X \\
Y+2.1 \\
Z-12.9
\end{array}\right] \text {, where } \theta=50^{\circ}
$$
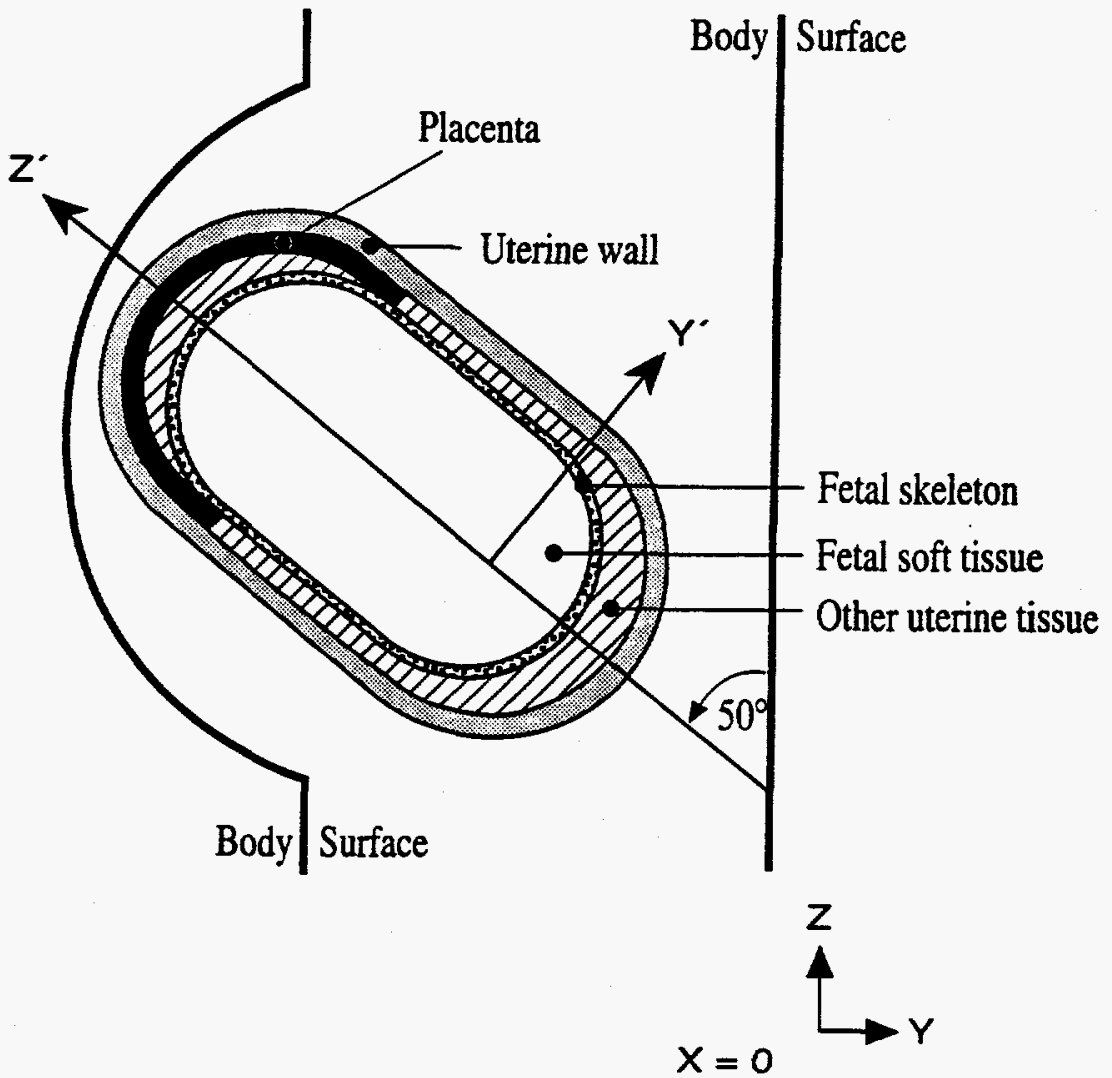

Fig. 3.5. Cross-section of uterine model at 6 months. $Y-Z$ (or $Y^{\prime}-Z^{\prime}$ ) plane; $X=X^{\prime}=0$. 


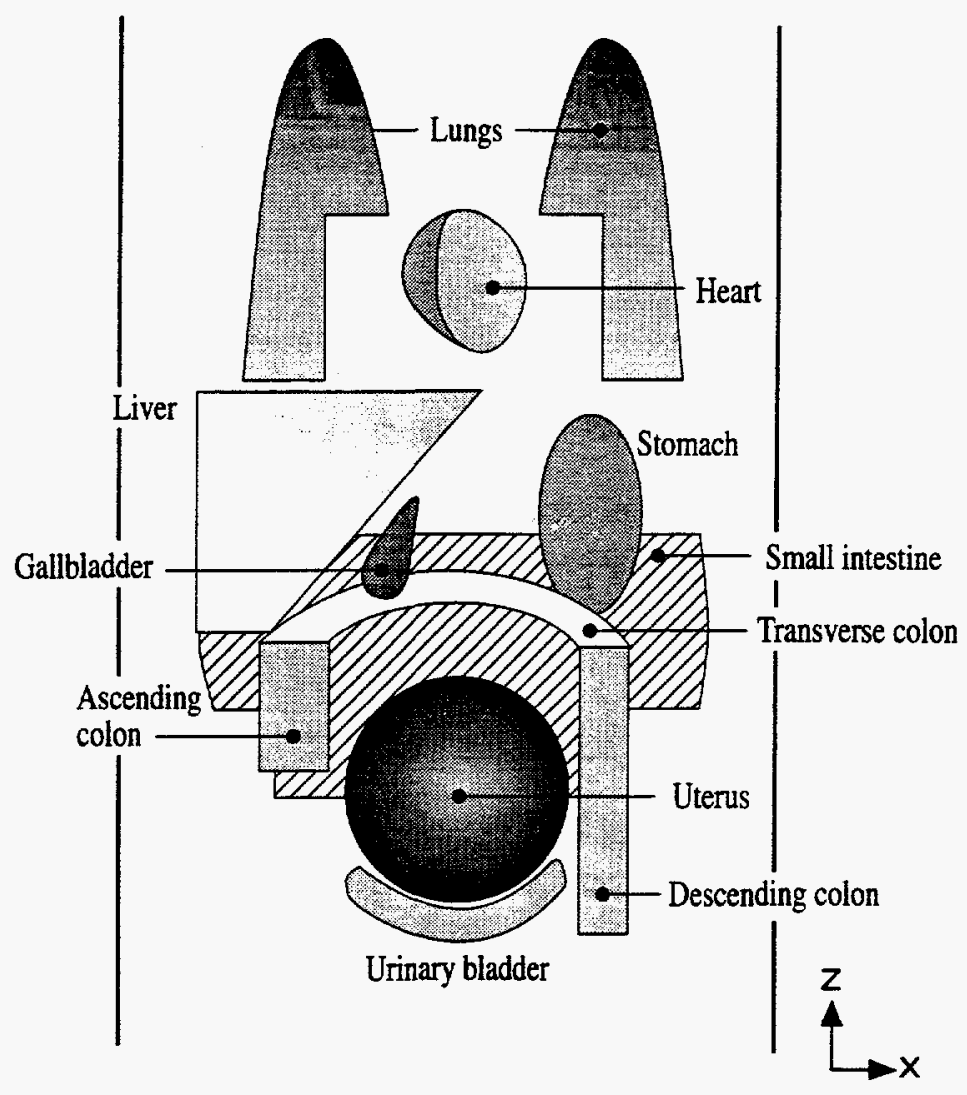

Fig. 3.6. Artist's rendition of organs in the 6-month model, as viewed from the front of the body (A-P projection). Not drawn to scale.

Cylindrical part. The uterine wall in the cylindrical part is represented by the volume between two coaxial right circular cylinders, and the uterine contents is represented by the volume within the inner cylinder (Fig. 3.5). The origin of the $\left(\mathrm{X}^{\prime}, \mathrm{Y}^{\prime}, \mathrm{Z}^{\prime}\right)$ coordinate system is at the base of the cylinders.

The cylindrical part of the uterine wall is defined by

$$
\begin{gathered}
\left(\frac{X^{\prime}}{A}\right)^{2}+\left(\frac{Y^{\prime}}{A}\right)^{2} \leq 1 \\
\text { and }\left(\frac{X^{\prime}}{A-D}\right)^{2}+\left(\frac{Y^{\prime}}{A-D}\right)^{2}>1 \\
\text { and } Z_{1} \leq Z^{\prime} \leq Z_{2}
\end{gathered}
$$

The cylindrical part of the uterine contents is defined by

$$
\begin{aligned}
& \left(\frac{X^{\prime}}{A-D}\right)^{2}+\left(\frac{Y^{\prime}}{A-D}\right)^{2} \leq 1 \\
& \text { and } Z_{1} \leq Z^{\prime} \leq Z_{2}
\end{aligned}
$$


The parameters for the cylindrical part are:

\begin{tabular}{cccc}
$\mathrm{A}$ & $\mathrm{D}$ & $\mathrm{Z}_{1}$ & $\mathrm{Z}_{2}$ \\
\hline 7.25 & 0.73 & 0 & 11.6
\end{tabular}

Hemispherical parts. The uterine wall in each of the two hemispherical parts is represented by the volume between two concentric hemispheres, and the uterine contents is represented by the volume within the inner hemisphere.

The uterine wall in each hemisphere is defined by

$$
\begin{gathered}
\left(\frac{X^{\prime}}{A}\right)^{2}+\left(\frac{Y^{\prime}}{A}\right)^{2}+\left(\frac{Z^{\prime}-Z_{0}}{A}\right)^{2} \leq 1 \\
\text { and }\left(\frac{X^{\prime}}{A-D}\right)^{2}+\left(\frac{Y^{\prime}}{A-D}\right)^{2}+\left(\frac{Z^{\prime}-Z_{0}}{A-D}\right)^{2}>1
\end{gathered}
$$

and $\left[Z^{\prime} \geq Z_{0}\right.$ (upper hemisphere) or $Z^{\prime} \leq Z_{0}$ (lower hemisphere)] .

The uterine contents in each hemisphere are defined by

$$
\left(\frac{X^{\prime}}{A-D}\right)^{2}+\left(\frac{Y^{\prime}}{A-D}\right)^{2}+\left(\frac{Z^{\prime}-Z_{0}}{A-D}\right)^{2} \leq 1
$$

and $\left[Z^{\prime} \geq Z_{0}\right.$ (upper hemisphere) or $Z^{\prime} \leq Z_{0}$ (lower hemisphere) $]$.

The parameters for the hemispherical parts are:

\begin{tabular}{cccc} 
Hemisphere & $\mathrm{A}$ & $\mathrm{D}$ & $\mathrm{Z}_{0}$ \\
\hline Upper & 7.25 & 0.73 & 11.6 \\
Lower & 7.25 & 0.73 & 0
\end{tabular}

Volume. The volume of the entire wall is $802 \mathrm{~cm}^{3}$; the volume of the entire contents is $2710 \mathrm{~cm}^{3}$.

The uterine contents in this model contains the fetus, placenta, and a residual region designated as Other Uterine Contents. As the fetus in this model contains some skeletal material (see Fetus and Fetal Skeleton section below), the average density of the uterine contents is somewhat greater than the value of $1.04 \mathrm{~g} \cdot \mathrm{cm}^{-3}$ assigned to soft tissue. 


\subsubsection{Nine-Month Pregnant Female}

In the nine-month phantom, the uterus and its contents are modeled as the frustrum of a right circular cone capped at both ends by hemispheres. At this stage, the uterus is extended in length as well as breadth; the upper hemisphere is considerably larger than the lower hemisphere, and larger than in the six-month model; see Fig. 3.2. The axis of the cone is along the $Z^{\prime}$-axis (defined below), which has been rotated $50^{\circ}$ from the $Z$-axis of the phantom (equivalent to a $40^{\circ}$ incline to the horizontal plane of the phantom, similar to the axis for the cylinder in the six-month model). A crosssection of the model is given in Fig. 3.7, and an artist's rendition of the organs in the trunk, as viewed from the front (A-P projection), is shown in Fig. 3.8. The thickness of the uterine wall in the ninemonth model was determined from the literature and set equal to $0.65 \mathrm{~cm}$ (Ivy 1942; Gillespie 1950). The mass of the uterine wall in this model is $1095 \mathrm{~g}$, an increase of $1016 \mathrm{~g}$ from the mass of the nongravid uterus of the adult female model (Cristy and Eckerman 1987). This mass is in good agreement with that found in the literature at this stage of gestation (Ivy 1942; Gillespie 1950; Hytten and Leitch 1964; Moore 1982; ICRP 1975).

The uterus and its contents are described in terms of $\left(X^{\prime}, Y^{\prime}, Z^{\prime}\right)$ coordinates, which are related to $(\mathrm{X}, \mathrm{Y}, \mathrm{Z})$ coordinates by the following rotation-translation equation:

$$
\left[\begin{array}{l}
X^{\prime} \\
Y^{\prime} \\
Z^{\prime}
\end{array}\right]=\left[\begin{array}{ccc}
1 & 0 & 0 \\
0 & \cos \theta & \sin \theta \\
0 & -\sin \theta & \cos \theta
\end{array}\right]\left[\begin{array}{c}
X \\
Y-6.75 \\
Z-5.58
\end{array}\right] \text {, where } \theta=50^{\circ}
$$

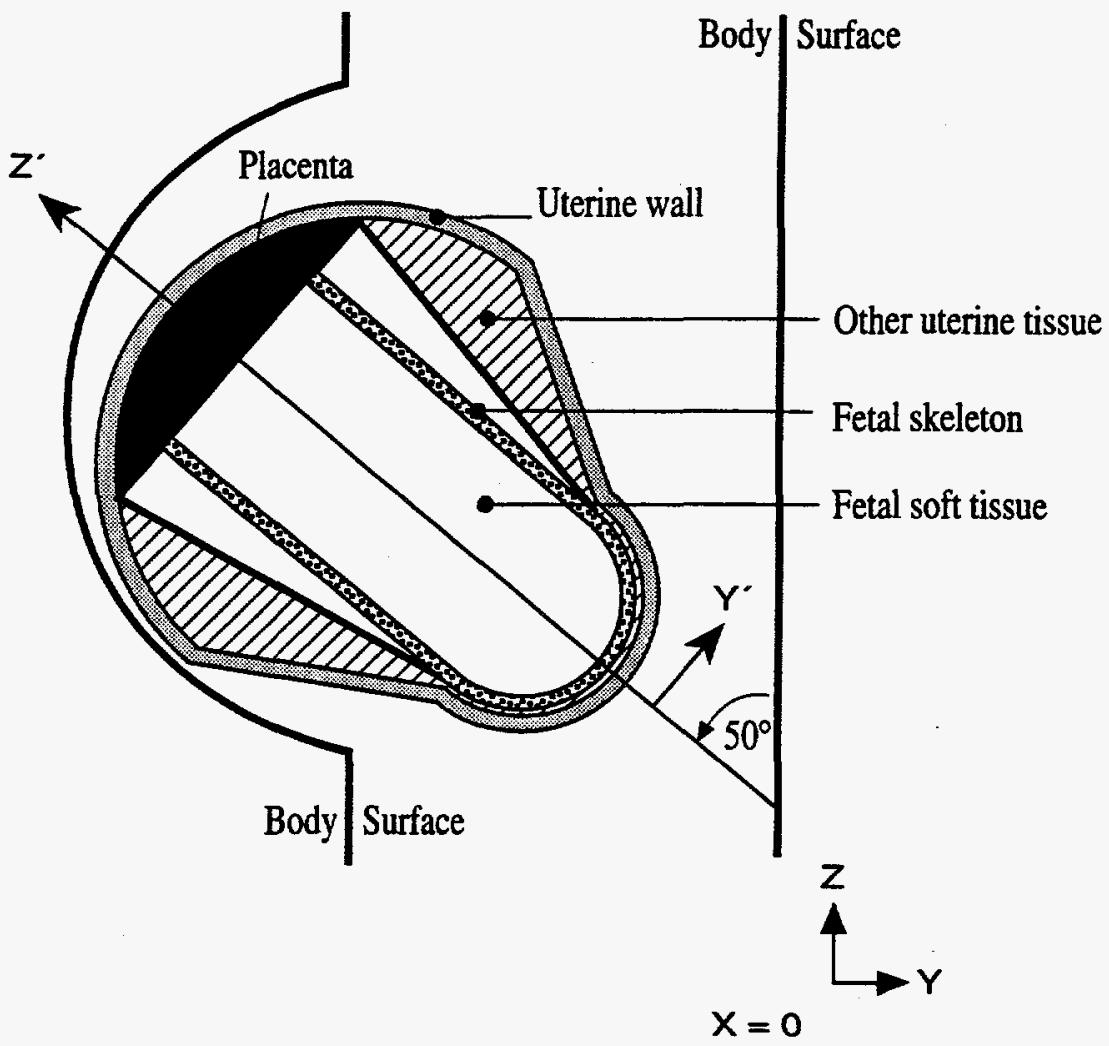

Fig. 3.7. Cross-section of uterine model at 9 months. $Y-Z$ (or $Y^{\prime}-Z^{\prime}$ ) plane; $X=X^{\prime}=0$. 


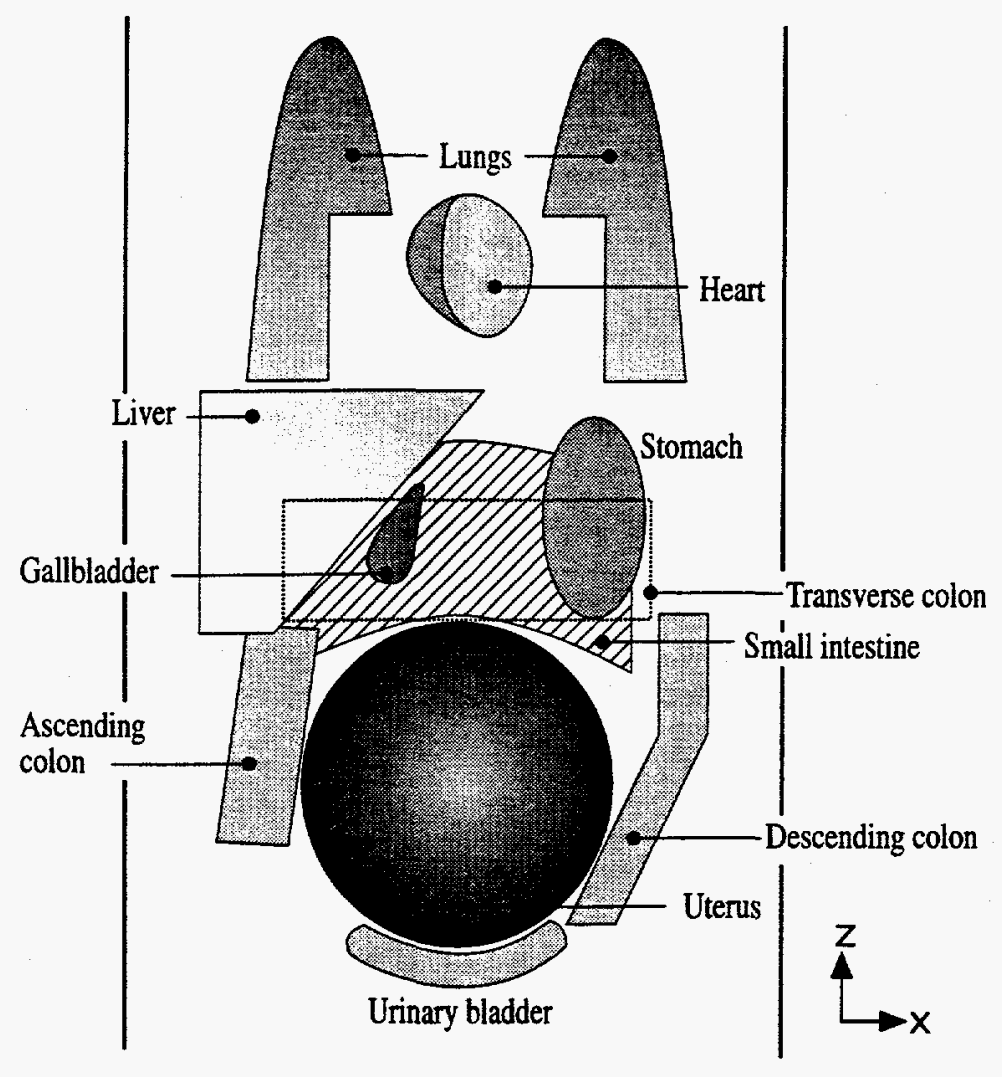

Fig. 3.8. Artist's rendition of organs in the 9-month model, as viewed from the front of the body (A-P projection). Not drawn to scale.

Conical part. The uterine wall in the conical part is represented by the volume between the frustrums of two coaxial right circular cones, and the uterine contents is represented by the volume within the frustrum of the inner cone (Fig. 3.7). The origin of the ( $\left.X^{\prime}, Y^{\prime}, Z^{\prime}\right)$ coordinate system is at the tip of the outer cone (the virtual tip, which is not shown in Fig. 3.7, since it occurs outside of the model).

The conical portion of the uterine wall is defined by

$$
\begin{gathered}
X^{\prime 2}+Y^{\prime 2}-\left(\frac{Z^{\prime}}{r}\right)^{2} \leq 0 \\
\text { and } X^{\prime 2}+Y^{\prime 2}-\left(\frac{Z^{\prime}-Z_{0}}{r}\right)^{2}>0 \\
\text { and } Z_{1} \leq Z^{\prime} \leq Z_{2} .
\end{gathered}
$$


The conical portion of the uterine contents is defined by

$$
\begin{gathered}
X^{\prime 2}+Y^{\prime 2}-\left(\frac{Z^{\prime}-Z_{0}}{r}\right)^{2} \leq 0 \\
\text { and } Z_{1} \leq Z^{\prime} \leq Z_{2} \text {. }
\end{gathered}
$$

The parameters for the conical part are:

\begin{tabular}{cccc}
$\mathrm{r}$ & $\mathrm{Z}_{0}$ & $\mathrm{Z}_{1}$ & $\mathrm{Z}_{2}$ \\
\hline 1.7085 & 1.11 & 10.6 & 20.5
\end{tabular}

Hemispherical parts. The uterine wall in each of the two hemispherical parts is represented by the volume between two concentric hemispheres, and the uterine contents is represented by the volume within the inner hemisphere (Fig. 3.7).

The uterine wall in each hemisphere is defined by

$$
\begin{array}{r}
\left(\frac{X^{\prime}}{A}\right)^{2}+\left(\frac{Y^{\prime}}{A}\right)^{2}+\left(\frac{Z^{\prime}-Z_{0}}{A}\right)^{2} \leq 1 \\
\text { and }\left(\frac{X^{\prime}}{A-D}\right)^{2}+\left(\frac{Y^{\prime}}{A-D}\right)^{2}+\left(\frac{Z^{\prime}-Z_{0}}{A-D}\right)^{2}>1
\end{array}
$$

and $\left[Z^{\prime} \geq Z_{0}\right.$ (upper hemisphere) or $Z^{\prime} \leq Z_{0}$ (lower hemisphere)] .

The uterine contents in each hemisphere are defined by

$$
\left(\frac{X^{\prime}}{A-D}\right)^{2}+\left(\frac{Y^{\prime}}{A-D}\right)^{2}+\left(\frac{Z^{\prime}-Z_{0}}{A-D}\right)^{2} \leq 1
$$

and $\left[Z^{\prime} \geq Z_{0}\right.$ (upper hemisphere) or $Z^{\prime} \leq Z_{0}$ (lower hemisphere)] .

The parameters for the hemispherical parts are:

\begin{tabular}{cccc} 
Hemisphere & $\mathrm{A}$ & $\mathrm{D}$ & $\mathrm{Z}_{0}$ \\
\hline Upper & 12.0 & 0.65 & 20.5 \\
Lower & 6.2 & 0.65 & 10.6
\end{tabular}

Volume. The volume of the entire wall is $1053 \mathrm{~cm}^{3}$; the volume of the entire contents is $5730 \mathrm{~cm}^{3}$. 
The uterine contents compartment in this model contains the fetus, placenta, and a residual region designated as Other Uterine Contents. As the fetus in this model contains some skeletal material (see Fetus and Fetal Skeleton section, below), the average density of the uterine contents is somewhat greater than the value of $1.04 \mathrm{~g} \cdot \mathrm{cm}^{-3}$ assigned to soft tissue.

\subsubsection{Summary - Masses and Volumes}

Table 3.1. Masses and Volumes of the Models of the Uterus and Uterine Contents

\begin{tabular}{|l|c|c|c|c|c|c|c||c||}
\cline { 2 - 8 } \multicolumn{1}{l|}{} & \multicolumn{2}{|c|}{ Nonpregnant } & \multicolumn{2}{c|}{$\begin{array}{c}\text { Three } \\
\text { months }\end{array}$} & \multicolumn{2}{c|}{$\begin{array}{c}\text { Six } \\
\text { months }\end{array}$} & \multicolumn{2}{c|}{$\begin{array}{c}\text { Nine } \\
\text { months }\end{array}$} \\
\cline { 2 - 9 } & $\begin{array}{c}\text { Mass } \\
(\mathrm{g})\end{array}$ & $\begin{array}{c}\text { Vol. } \\
\left(\mathrm{cm}^{3}\right)\end{array}$ & $\begin{array}{c}\text { Mass } \\
(\mathrm{g})\end{array}$ & $\begin{array}{c}\text { Vol. } \\
\left(\mathrm{cm}^{3}\right)\end{array}$ & $\begin{array}{c}\text { Mass } \\
(\mathrm{g})\end{array}$ & $\begin{array}{c}\text { Vol. } \\
\left(\mathrm{cm}^{3}\right)\end{array}$ & $\begin{array}{c}\text { Mass } \\
(\mathrm{g})\end{array}$ & $\begin{array}{c}\text { Vol. } \\
\left(\mathrm{cm}^{3}\right)\end{array}$ \\
\hline \hline Uterine Wall & 79.0 & 76.0 & 374 & 359 & 834 & 802 & 1095 & 1053 \\
\hline Uterine Contents & --- & -- & 458 & 440 & 2840 & 2710 & 6010 & 5730 \\
\hline
\end{tabular}

\subsection{FETUS AND FETAL SKELETON}

\section{Three-Month Pregnant Female}

The uterine contents of the three-month phantom were modeled as a homogeneous mixture of soft tissue. No attempt was made to distinguish the fetus from other tissues within the uterus or to model the presence of fetal skeletal material. The specific absorbed energy in the fetus is taken to be that of the uterine contents.

\subsection{Six-Month Pregnant Female}

In the six- and nine-month phantoms, the fetus, placenta, and other uterine contents (amniotic fluid and membranes) were explicitly modeled as separate regions. The fetal skeleton was also explicitly modeled and given the composition of the skeleton of the newborn phantom of Cristy and Eckerman (1987) (see discussion below). By the sixth month of gestation, the fetus has a partially developed skeletal system, with a significant amount of structure (Ivy 1942; ICRP 1975). The presence of high-Z material (such as calcium) was expected to affect the absorption of low-energy photons differently from a homogeneous mixture of low- and high- $Z$ materials. Therefore, a heterogeneous model was deemed desirable. A simple model of the fetus has been developed for the six-month model, consisting of fetal skeletal tissue and fetal soft tissue contained within a structure inside the uterus. It is not possible to assign a position to the fetus or fetal skeleton at this stage, due to movement of the fetus within the uterus. Therefore, simple models for both the whole fetus and fetal skeleton were adopted which are similar to that of the uterus itself, i.e., (for the fetus) a right circular cylinder capped with hemispheres and (for the fetal skeleton) a cylindrical shell capped with 
hemispherical shells. The fetal skeleton is located inside the fetus under a thin layer of fetal soft tissue. The thickness of the fetal skeleton was determined from the target mass of $115 \mathrm{~g}$ (ICRP 1975) and the designated geometry.

The fetus and fetal skeleton are described in the same rotation-translation $\left(X^{\prime}, Y^{\prime}, Z^{\prime}\right)$ coordinate system used to describe the uterus and its total contents (see Fig. 3.5):

$$
\left[\begin{array}{l}
X^{\prime} \\
Y^{\prime} \\
Z^{\prime}
\end{array}\right]=\left[\begin{array}{ccc}
1 & 0 & 0 \\
0 & \cos \theta & \sin \theta \\
0 & -\sin \theta & \cos \theta
\end{array}\right]\left[\begin{array}{c}
X \\
Y+2.1 \\
Z-12.9
\end{array}\right] \text {, where } \theta=50^{\circ}
$$

Cylindrical part of fetus. The cylindrical part of the fetus is defined by

$$
\begin{aligned}
& \left(\frac{X^{\prime}}{A}\right)^{2}+\left(\frac{Y^{\prime}}{A}\right)^{2} \leq 1 \\
& \text { and } Z_{1} \leq Z^{\prime} \leq Z_{2} \text {. }
\end{aligned}
$$

The parameters for the cylindrical part are:

\begin{tabular}{ccc}
$A$ & $Z_{1}$ & $Z_{2}$ \\
\hline 5.53 & 1.355 & 10.245
\end{tabular}

Hemispherical parts of fetus. The part of the fetus in each hemisphere is defined by

$$
\left(\frac{X^{\prime}}{A}\right)^{2}+\left(\frac{Y^{\prime}}{A}\right)^{2}+\left(\frac{Z^{\prime}-Z_{0}}{A}\right)^{2} \leq 1
$$

and $\left[Z^{\prime} \geq Z_{0}\right.$ (upper hemisphere) or $Z^{\prime} \leq Z_{0}$ (lower hemisphere)]

The parameters for the hemispherical parts are:

\begin{tabular}{ccc} 
Hemisphere & $\mathrm{A}$ & $\mathrm{Z}_{0}$ \\
\hline Upper & 5.53 & 10.245 \\
Lower & 5.53 & 1.355
\end{tabular}


Cylindrical part of fetal skeleton. The cylindrical part of the fetal skeleton is defined by

$$
\begin{aligned}
& \left(\frac{X^{\prime}}{A}\right)^{2}+\left(\frac{Y^{\prime}}{A}\right)^{2} \leq 1 \\
& \text { and }\left(\frac{X^{\prime}}{A-D}\right)^{2}+\left(\frac{Y^{\prime}}{A-D}\right)^{2}>1 \\
& \text { and } Z_{1} \leq Z^{\prime} \leq Z_{2} .
\end{aligned}
$$

The parameters for the cylindrical part are:

\begin{tabular}{cccc}
$\mathrm{A}$ & $\mathrm{D}$ & $\mathrm{Z}_{1}$ & $\mathrm{Z}_{2}$ \\
\hline 5.46 & 0.142 & 1.355 & 10.245
\end{tabular}

Hemispherical parts of fetal skeleton. The hemispherical shells are defined by

$$
\begin{gathered}
\left(\frac{X^{\prime}}{A}\right)^{2}+\left(\frac{Y^{\prime}}{A}\right)^{2}+\left(\frac{Z^{\prime}-Z_{0}}{A}\right)^{2} \leq 1 \\
\text { and }\left(\frac{X^{\prime}}{A-D}\right)^{2}+\left(\frac{Y^{\prime}}{A-D}\right)^{2}+\left(\frac{Z^{\prime}-Z_{0}}{A-D}\right)^{2}>1
\end{gathered}
$$

and $\left[Z^{\prime} \geq Z_{0}\right.$ (upper hemisphere) or $Z^{\prime} \leq Z_{0}$ (lower hemisphere)] .

The parameters for the hemispherical parts are:

\begin{tabular}{cccc} 
Hemisphere & $\mathrm{A}$ & $\mathrm{D}$ & $\mathrm{Z}_{0}$ \\
\hline Upper & 5.46 & 0.142 & 10.245 \\
Lower & 5.46 & 0.142 & 1.355
\end{tabular}

Volume. The volume of the fetus is $1560 \mathrm{~cm}^{3}$, and the volume of the fetal skeleton is $94.6 \mathrm{~cm}^{3}$.

\subsection{Nine-Month Pregnant Female}

The fetus in the nine-month phantom is modeled as a hemisphere (within the lower hemisphere of the uterine contents) attached to the frustrum of a right circular cone that extends throughout the conical section and into the upper hemispherical section of the uterine contents (Fig. 3.7). The fetal skeleton is modeled as a cylindrical shell capped by a hemispherical shell. The fetal skeleton is located inside the fetus under a thin layer of fetal soft tissue in the hemispherical part and deeper within the fetus in the conical part. The thickness of the fetal skeleton was determined from the 
target mass of $350 \mathrm{~g}$ (to match that in the newborn phantom of Cristy and Eckerman, 1987) and the designated geometry.

The fetus and fetal skeleton are described in the same rotation-translation $\left(\mathrm{X}^{\prime}, \mathrm{Y}^{\prime}, \mathrm{Z}^{\prime}\right)$ coordinate system used to describe the uterus and total contents (see Fig. 3.7):

$$
\left[\begin{array}{l}
X^{\prime} \\
Y^{\prime} \\
Z^{\prime}
\end{array}\right]=\left[\begin{array}{ccc}
1 & 0 & 0 \\
0 & \cos \theta & \sin \theta \\
0 & -\sin \theta & \cos \theta
\end{array}\right]\left[\begin{array}{c}
X \\
Y-6.75 \\
Z-5.58
\end{array}\right] \text {, where } \theta=50^{\circ}
$$

Conical part of fetus. The conical part of the fetus is defined by

$$
\begin{gathered}
X^{\prime 2}+Y^{\prime 2}-\left(\frac{Z^{\prime}-Z_{0}}{r}\right)^{2} \leq 0 \\
\text { and } Z_{0} \leq Z^{\prime} \leq Z_{2} .
\end{gathered}
$$

The parameters for the conical part are:

\begin{tabular}{cccc}
$\mathrm{r}$ & $\mathrm{Z}_{0}$ & $\mathrm{Z}_{1}$ & $\mathrm{Z}_{2}$ \\
\hline 5.438 & -17.7 & 10.6 & 27.67
\end{tabular}

Hemispherical part of fetus. The hemispherical part of the fetus is defined by

$$
\begin{gathered}
\left(\frac{X^{\prime}}{A}\right)^{2}+\left(\frac{Y^{\prime}}{A}\right)^{2}+\left(\frac{Z^{\prime}-Z_{0}}{A}\right)^{2} \leq 1 \\
\text { and } Z^{\prime} \leq Z_{0} .
\end{gathered}
$$

The parameters for the hemispherical part are:

\begin{tabular}{cc}
$A$ & $\mathrm{Z}_{0}$ \\
\hline 5.2 & 10.6
\end{tabular}


Cylindrical part of fetal skeleton. The cylindrical shell is defined by

$$
\begin{gathered}
\left(\frac{X^{\prime}}{A}\right)^{2}+\left(\frac{Y^{\prime}}{A}\right)^{2} \leq 1 \\
\text { and }\left(\frac{X^{\prime}}{A-D}\right)^{2}+\left(\frac{Y^{\prime}}{A-D}\right)^{2}>1 \\
\text { and } Z_{1} \leq Z^{\prime} \leq Z_{2} .
\end{gathered}
$$

The parameters for the cylindrical part are:

\begin{tabular}{cccc}
$\mathrm{A}$ & $\mathrm{D}$ & $\mathrm{Z}_{1}$ & $\mathrm{Z}_{2}$ \\
\hline 5.13 & 0.422 & 10.6 & 27.67
\end{tabular}

Hemispherical part of fetal skeleton. The hemispherical shell is defined by

$$
\begin{gathered}
\left(\frac{X^{\prime}}{A}\right)^{2}+\left(\frac{Y^{\prime}}{A}\right)^{2}+\left(\frac{Z^{\prime}-Z_{0}}{A}\right)^{2} \leq 1 \\
\text { and }\left(\frac{X^{\prime}}{A-D}\right)^{2}+\left(\frac{Y^{\prime}}{A-D}\right)^{2}+\left(\frac{Z^{\prime}-Z_{0}}{A-D}\right)^{2}>1 \\
\text { and } Z^{\prime} \leq Z_{0} \text {. }
\end{gathered}
$$

The parameters for the hemispherical part are:

\begin{tabular}{ccc}
$\mathrm{A}$ & $\mathrm{D}$ & $\mathrm{Z}_{0}$ \\
\hline 5.13 & 0.422 & 10.6
\end{tabular}

Volume. The volume of the fetus is $2800 \mathrm{~cm}^{3}$, and the volume of the fetal skeleton is $287 \mathrm{~cm}^{3}$.

\subsection{FETAL SKELETON COMPOSITION}

The composition of the fetal skeleton is different from that of the mother's skeleton. Although the Geigy Scientific Tables (1981) note an increase of approximately $20 \%$ in calcium and phosphorus content in the trabeculae of the femur of the newborn compared with 20-24-week fetuses, the difference was only about $5 \%$ in the cortex of the femur. A decrease of about $10-20 \%$ in water content was noted in both types of samples. A study by Swanson and Iob (1937), however, did not show any significant change in femur or tibia content of calcium or phosphorus in fetuses of masses between 200 and $4000 \mathrm{~g}$, nor did they observe a significant difference in water content in this range. Therefore, the changes in composition of the fetal skeleton between six and nine months were thought to be minor, and the composition of the skeleton in the newborn phantom of Cristy.and 
Eckerman (1987) was used for both the six- and nine-month fetal skeleton compositions. Therefore, the phantoms for the six- and nine-month models have four different media: soft tissue, lung tissue, maternal bone, and fetal bone.

\subsubsection{Summary - Masses and Volumes}

Table 3.2 Masses and volumes for the models of the fetus and fetal tissues.

\begin{tabular}{||l|c|c||c|c||}
\cline { 2 - 5 } \multicolumn{1}{c|}{} & \multicolumn{2}{c|}{ Six months } & \multicolumn{2}{c|}{ Nine months } \\
\cline { 2 - 5 } \multicolumn{1}{c|}{} & $\begin{array}{c}\text { Mass } \\
(\mathrm{g})\end{array}$ & $\begin{array}{c}\text { Volume } \\
\left(\mathrm{cm}^{3}\right)\end{array}$ & $\begin{array}{c}\text { Mass } \\
(\mathrm{g})\end{array}$ & $\begin{array}{c}\text { Volume } \\
\left(\mathrm{cm}^{3}\right)\end{array}$ \\
\hline \hline Fetus & 1640 & 1560 & 2960 & 2800 \\
\hline Fetal Soft Tissue & 1530 & 1470 & 2610 & 2510 \\
\hline Fetal Skeleton & 115 & 94.6 & 350 & 287 \\
\hline
\end{tabular}

For comparison, the body mass of a newborn is $3400 \mathrm{~g}$, and the mass of the newborn skeleton is $350 \mathrm{~g}$ (Cristy and Eckerman 1987).

\subsection{PLACENTA}

\subsubsection{Three-Month Pregnant Female}

No placenta was modeled in the three-month pregnant female.

\subsubsection{Six-Month Pregnant Female}

In the six-month phantom, the placenta was modeled as a short cylindrical shell capped at the upper end by a larger hemispherical shell (see Fig. 3.5). The placenta lies next to the uterine wall in the upper hemisphere of the uterine contents. This model provides a logical progression to the similar but larger placenta in the nine-month model. The mass of the placenta in the six-month model is $310 \mathrm{~g}$, which is consistent with that described in the literature (Hytten and Leitch 1964; ICRP 1975). The thickness of the placenta was determined by developing a model based on this mass that would provide a reasonable representation of the placenta at six months of gestation (Eastman 1956; Carnation Company 1962).

The placenta is also described in the rotation-translation coordinate system that describes the uterus and total contents (see Fig. 3.5):

$$
\left[\begin{array}{l}
X^{\prime} \\
Y^{\prime} \\
Z^{\prime}
\end{array}\right]=\left[\begin{array}{ccc}
1 & 0 & 0 \\
0 & \cos \theta & \sin \theta \\
0 & -\sin \theta & \cos \theta
\end{array}\right]\left[\begin{array}{c}
X \\
Y+2.1 \\
Z-12.9
\end{array}\right] \text {, where } \theta=50^{\circ} .
$$


Cylindrical part. The cylindrical shell is defined by

$$
\begin{gathered}
\left(\frac{X^{\prime}}{A}\right)^{2}+\left(\frac{Y^{\prime}}{A}\right)^{2} \leq 1 \\
\text { and }\left(\frac{X^{\prime}}{A-D}\right)^{2}+\left(\frac{Y^{\prime}}{A-D}\right)^{2}>1 \\
\text { and } Z_{1} \leq Z^{\prime} \leq Z_{2} .
\end{gathered}
$$

The parameters for the cylindrical part are:

\begin{tabular}{cccc}
$\mathrm{A}$ & $\mathrm{D}$ & $\mathrm{Z}_{1}$ & $\mathrm{Z}_{2}$ \\
\hline 6.52 & 0.99 & 9.685 & 11.6
\end{tabular}

Hemispherical part. The hemispherical shell is defined by

$$
\begin{aligned}
& \left(\frac{X^{\prime}}{A}\right)^{2}+\left(\frac{Y^{\prime}}{A}\right)^{2}+\left(\frac{Z^{\prime}-Z_{0}}{A}\right)^{2} \leq 1 \\
& \text { and }\left(\frac{X^{\prime}}{A-D}\right)^{2}+\left(\frac{Y^{\prime}}{A-D}\right)^{2}+\left(\frac{Z^{\prime}-Z_{0}}{A-D}\right)^{2}>1 \\
& \text { and } Z^{\prime} \geq Z_{0} \text {. }
\end{aligned}
$$

The parameters for this part are:

\begin{tabular}{ccc}
$\mathrm{A}$ & $\mathrm{D}$ & $\mathrm{Z}_{0}$ \\
\hline 6.52 & 0.99 & 11.6
\end{tabular}

Volume. The volume of the placenta is $298 \mathrm{~cm}^{3}$.

\subsubsection{Nine-Month Pregnant Female}

In the nine-month phantom, the placenta was modeled as a section of a hemisphere (hemisphere cut by a plane perpendicular to the $Z^{\prime}$-axis). The placenta lies next to the uterine wall in the upper hemisphere of the uterine contents (see Fig. 3.7). This model provides a logical progression from the placenta in the six-month model. The mass of the placenta in the nine-month model is $466 \mathrm{~g}$, which is consistent with that described in the literature (Hytten and Leitch 1964; ICRP 1975). The thickness of the placenta was determined by developing a model based on this mass that would provide a reasonable representation of the placenta at nine months of gestation (Eastman 1956; Carnation Company 1962). 
The placenta is also described in the rotation-translation coordinate system that describes the uterus and total contents (see Fig. 3.7):

$$
\left[\begin{array}{l}
X^{\prime} \\
Y^{\prime} \\
Z^{\prime}
\end{array}\right]=\left[\begin{array}{ccc}
1 & 0 & 0 \\
0 & \cos \theta & \sin \theta \\
0 & -\sin \theta & \cos \theta
\end{array}\right]\left[\begin{array}{c}
X \\
Y-6.75 \\
Z-5.58
\end{array}\right] \text {, where } \theta=50^{\circ} .
$$

The placenta is defined by

$$
\begin{gathered}
\left(\frac{X^{\prime}}{A}\right)^{2}+\left(\frac{Y^{\prime}}{A}\right)^{2}+\left(\frac{Z^{\prime}-Z_{0}}{A}\right)^{2} \leq 1 \\
\text { and } Z_{1} \leq Z^{\prime} \text {. }
\end{gathered}
$$

The parameters are:

\begin{tabular}{ccc}
$A$ & $Z_{0}$ & $Z_{1}$ \\
\hline 11.0 & 20.5 & 27.67
\end{tabular}

Volume. The volume of the placenta is $448 \mathrm{~cm}^{3}$.

\subsubsection{Summary - Masses and Volumes}

Table 3.3. Masses and volumes for the models of the placenta

\begin{tabular}{|c|c|c||c|c|}
\cline { 2 - 5 } \multicolumn{1}{c|}{} & \multicolumn{2}{c|}{ Six months } & \multicolumn{2}{c|}{ Nine months } \\
\cline { 2 - 5 } & $\begin{array}{c}\text { Mass } \\
(\mathrm{g})\end{array}$ & $\begin{array}{c}\text { Volume } \\
\left(\mathrm{cm}^{3}\right)\end{array}$ & $\begin{array}{c}\text { Mass } \\
(\mathrm{g})\end{array}$ & $\begin{array}{c}\text { Volume } \\
\left(\mathrm{cm}^{3}\right)\end{array}$ \\
\hline Placenta & 310 & 298 & 466 & 448 \\
\hline
\end{tabular}




\subsection{TRUNK}

\subsubsection{Three-Month Pregnant Female}

In the three-month phantom, the uterus has not been enlarged enough to cause any changes in the exterior of the adult female phantom.

\subsubsection{Six-Month Pregnant Female}

In the six- and nine-month phantoms, the uterus cannot be contained within the confines of the adult female phantom. The trunk was therefore enlarged to accommodate the enlarged uterus and the modified abdominal organs. The trunk is represented by the elliptical cylinder of the nonpregnant phantom plus the part of an ellipsoid that extends outside the cylinder to the front of the phantom (see Fig. 3.5).

The trunk is defined by the elliptical cylinder of the nonpregnant phantom:

$$
\left(\frac{X}{A}\right)^{2}+\left(\frac{Y}{B}\right)^{2} \leq 1
$$

$$
\text { and } Z_{1} \leq Z \leq Z_{2} \text {; }
$$

plus the trunk extension:

$$
\begin{gathered}
\left(\frac{X}{A_{\alpha x}}\right)^{2}+\left(\frac{Y-Y_{0, \alpha x}}{B_{\alpha x}}\right)^{2}+\left(\frac{Z-Z_{0, \alpha x}}{C_{e x}}\right)^{2} \leq 1 \\
\text { and }\left(\frac{X}{A}\right)^{2}+\left(\frac{Y}{B}\right)^{2}>1 \\
\text { and } Y<0 .
\end{gathered}
$$

The parameters are:

\begin{tabular}{ccccccccc}
$\mathrm{A}$ & $\mathrm{B}$ & $\mathrm{A}_{\mathrm{ex}}$ & $\mathrm{B}_{\mathrm{ex}}$ & $\mathrm{C}_{\mathrm{ex}}$ & $\mathrm{Y}_{0, \mathrm{ex}}$ & $\mathrm{Z}_{0, \mathrm{ex}}$ & $\mathrm{Z}_{1}$ & $\mathrm{Z}_{2}$ \\
\hline 17.25 & 9.8 & 14.75 & 14.0 & 15.0 & -5.8 & 18.5 & 0 & 63.1
\end{tabular}

The trunk extension ranges in $\mathrm{Z}$ from 4.13 to 32.87 . The increase in body mass caused by this addition to the phantom was determined by Monte Carlo techniques to be $4.61 \mathrm{~kg}$. Part of this mass comprises portions of the uterus and other abdominal organs. The mass of soft tissue in the trunk extension not associated with any defined organ space was determined by Monte Carlo techniques to be $1.71 \mathrm{~kg}$. This mass was assigned to "Remaining Tissue" for dosimetric purposes. (Remaining Tissue is that maternal tissue remaining after all defined organs and regions are removed.) 


\subsubsection{Nine-Month Pregnant Female}

The trunk is defined by the same inequalities as for the six-month phantom (above), but with the following parameters:

\begin{tabular}{ccccccccc}
$\mathrm{A}$ & $\mathrm{B}$ & $\mathrm{A}_{\mathrm{ex}}$ & $\mathrm{B}_{\mathrm{ex}}$ & $\mathrm{C}_{\mathrm{ex}}$ & $\mathrm{Y}_{0, \mathrm{ex}}$ & $\mathrm{Z}_{0, \mathrm{ex}}$ & $\mathrm{Z}_{1}$ & $\mathrm{Z}_{2}$ \\
\hline 17.25 & 9.8 & 15.73 & 16.53 & 15.93 & -5.8 & 20.8 & 0 & 63.1
\end{tabular}

The trunk extension ranges in $\mathrm{Z}$ from 5.34 to 36.26 . The increase in body mass caused by this addition to the phantom was determined by Monte Carlo techniques to be $6.73 \mathrm{~kg}$. Part of this mass comprises portions of the uterus and other abdominal organs. The mass of soft tissue in the trunk extension not associated with any defined organ space was determined by Monte Carlo techniques to be compensated by a loss of Remaining Tissue mass inside of the trunk in volume taken up by the enlarged uterus. This resulted in a net loss of Remaining Tissue of $0.51 \mathrm{~kg}$.

\subsubsection{Summary - Masses and Volumes}

Table 3.4. Masses and volumes for the models of the trunk, remaining tissue, and entire phantom

\begin{tabular}{|c|c|c|c|c|c|c|c|c|}
\hline & \multicolumn{2}{|c|}{ Nonpregnant } & \multicolumn{2}{|c|}{ Three months } & \multicolumn{2}{|c|}{ Six months } & \multicolumn{2}{|c|}{ Nine months } \\
\hline & $\begin{array}{c}\text { Mass } \\
\text { (kg) }\end{array}$ & $\begin{array}{c}\text { Volume } \\
\text { (liter) }\end{array}$ & $\begin{array}{c}\text { Mass } \\
(\mathrm{kg})\end{array}$ & $\begin{array}{c}\text { Volume } \\
\text { (liter) }\end{array}$ & $\begin{array}{c}\text { Mass } \\
(\mathrm{kg})\end{array}$ & $\begin{array}{c}\text { Volume } \\
\text { (liter) }\end{array}$ & $\begin{array}{c}\text { Mass } \\
\text { (kg) }\end{array}$ & $\begin{array}{c}\text { Volume } \\
\text { (liter) }\end{array}$ \\
\hline Trunk & 34.5 & 33.5 & 34.5 & 33.5 & 39.6 & 38.4 & 41.3 & 40.1 \\
\hline $\begin{array}{l}\text { Remaining } \\
\text { Tissue }\end{array}$ & 40.0 & 38.5 & 39.3 & 37.8 & 41.7 & 40.1 & 39.5 & 39.0 \\
\hline $\begin{array}{l}\text { Entire } \\
\text { Phantom }\end{array}$ & 56.8 & 53.8 & 56.8 & 53.8 & 61.5 & 58.7 & 63.7 & 60.4 \\
\hline
\end{tabular}

\subsection{SKIN}

\subsubsection{Three-Month Pregnant Female}

Because the trunk did not change in the three-month pregnant female, no changes were needed for the skin.

\subsubsection{Six-Month Pregnant Female}

The skin of the phantom had to be extended to fit around the trunk extension. To maintain the skin mass, the skin thickness around the trunk extension was changed from $0.17 \mathrm{~cm}$ to $0.101 \mathrm{~cm}$. 
The inequalities which define the skin on the trunk extension are

$$
\begin{gathered}
\text { and }\left(\frac{X}{A_{e x}}\right)^{2}+\left(\frac{Y-Y_{0, e x}}{B_{e x}}\right)^{2}+\left(\frac{Z-Z_{0, e x}}{C_{e x}}\right)^{2} \leq 1 \\
\text { and }\left(\frac{Y-Y_{0, e x}}{B_{e x}-D}\right)^{2}+\left(\frac{Z-Z_{0, e x}}{C_{e x}-D}\right)^{2}>1 \\
\text { and } Y<0 .
\end{gathered}
$$

Parameters. The value of $D$ is 0.101 . All other parameters are the same as given above in Section 3.5.2 on the trunk in the six-month pregnant phantom.

\subsubsection{Nine-Month Pregnant Female}

The skin of the phantom had to be extended to fit around the trunk extension. To maintain the skin mass, the skin thickness around the trunk extension was changed from $0.17 \mathrm{~cm}$ to $0.099 \mathrm{~cm}$. The inequalities that define the skin on the trunk extension are as given for the six-month, above, except that the parameters are different.

Parameters. The value of $\mathrm{D}$ is 0.099 . All other parameters are the same as given above in Section 3.5 .3 on the trunk in the nine-month pregnant phantom.

\subsection{URINARY BLADDER}

\subsubsection{Three-Month Pregnant Female}

The enlarged uterus strongly influences the shape and location of the urinary bladder. The bladder is lowered from its normal position and flattened on top by the growth of the uterus (Cloutier et al. 1973). The deformation has been modeled so that both the volume and surface area of the adult female bladder wall have been held approximately constant. With the bladder and contents represented as a hemisphere covered with a cylindrical cap tangent to the lower boundary of the uterus, the bladder contents volume is smaller than that of the adult female. This is consistent with the normal experience of pregnancy. 
The cap part of the bladder wall is defined by a short right circular cylinder (like a coin):

$$
\begin{aligned}
& \left(\frac{X}{A}\right)^{2}+\left(\frac{Y-Y_{0}}{A}\right)^{2} \leq 1 \\
& \text { and } Z_{0} \leq Z \leq Z_{1} .
\end{aligned}
$$

The hemispherical part of the bladder wall is defined by the volume between two concentric hemispheres:

$$
\begin{aligned}
& \text { and }\left(\frac{X}{A}\right)^{2}+\left(\frac{Y-Y_{0}}{A}\right)^{2}+\left(\frac{Z-Z_{0}}{A}\right)^{2} \leq 1 \\
& \text { and } Z \leq Z_{0} \text {. }
\end{aligned}
$$

The bladder contents are defined as the volume within the inner hemisphere:

$$
\begin{gathered}
\left(\frac{X}{A-D}\right)^{2}+\left(\frac{Y-Y_{0}}{A-D}\right)^{2}+\left(\frac{Z-Z_{0}}{A-D}\right)^{2} \leq 1 \\
\text { and } Z \leq Z_{0}
\end{gathered}
$$

The parameters are:

\begin{tabular}{ccccc}
$\mathrm{A}$ & $\mathrm{D}$ & $\mathrm{Y}_{0}$ & $\mathrm{Z}_{0}$ & $\mathrm{Z}_{1}$ \\
\hline 4.12 & 0.23 & -4.41 & 7.29 & 7.52
\end{tabular}

Volume. The volume of the bladder wall is $35.4 \mathrm{~cm}^{3}$; the volume of the bladder contents is $123 \mathrm{~cm}^{3}$.

\subsubsection{Six-Month Pregnant Female}

The urinary bladder in the woman at six months of pregnancy is flattened under the enlarged uterus (Eastman 1956; Carnation Company 1962; Clemente 1981; Cloutier et al. 1973). In the six-month phantom, the bladder is modeled as an ellipsoid curved to fit under the uterus and inside the extended trunk. 
The bladder wall is defined by the volume between two concentric ellipsoids, each with one of the axes curved:

$$
\begin{aligned}
& \qquad\left(\frac{X}{A}\right)^{2}+\left(\frac{Y-Y_{0}}{B}\right)^{2}+\left(\frac{Z-Z_{0}}{C}\right)^{2} \leq 1 \\
& \text { and }\left(\frac{X}{A-D}\right)^{2}+\left(\frac{Y-Y_{0}}{B-D}\right)^{2}+\left(\frac{Z-Z_{0}}{C-D}\right)^{2}>1, \\
& \text { where } Z_{0}=21.8-16 \sqrt{0.9628-\left(\frac{X}{23.8}\right)^{2}} .
\end{aligned}
$$

The bladder contents are defined by the volume within the inner ellipsoid:

$$
\left(\frac{X}{A-D}\right)^{2}+\left(\frac{Y-Y_{0}}{B-D}\right)^{2}+\left(\frac{Z-Z_{0}}{C-D}\right)^{2} \leq 1,
$$

where $Z_{0}$ is as defined above for the bladder wall.

The parameters are:

\begin{tabular}{ccccc}
$\mathrm{A}$ & $\mathrm{B}$ & $\mathrm{C}$ & $\mathrm{D}$ & $\mathrm{Y}_{0}$ \\
\hline 6.5 & 3.5 & 1.425 & 0.23 & -9.0
\end{tabular}

Volume. The volume of the bladder wall is $33.1 \mathrm{~cm}^{3}$; the volume of the bladder contents is $103 \mathrm{~cm}^{3}$.

\subsubsection{Nine-Month Pregnant Female}

The urinary bladder in the woman at nine months of pregnancy is severely flattened under the enlarged uterus and curves up around the sides of the lower hemisphere of the uterus (Eastman 1956; Carnation Company 1962; Clemente 1981; Cloutier et al. 1973). The inequalities defining the bladder wall and contents are identical to those for the six-month model above, except that

$$
Z_{0}=22.2-16 \sqrt{0.9628-\left(\frac{X}{15.8}\right)^{2}}
$$

and the parameters are:

\begin{tabular}{ccccc} 
A & B & C & D & $\mathrm{Y}_{0}$ \\
\hline 5.0 & 3.8 & 0.8 & 0.23 & -8.6
\end{tabular}

Volume. The volume of the bladder wall is $23.0 \mathrm{~cm}^{3}$; the volume of the bladder contents is $40.7 \mathrm{~cm}^{3}$. 


\subsubsection{Summary - Masses and Volumes}

For each phantom the mass and volume of the bladder contents are for a relatively full bladder. In Appendix A two values are given for $\Phi$ (urinary bladder wall - urinary bladder contents): one for the modeled bladder and one which has been adjusted for the bladder in mid-cycle, i.e., half of the volume of contents given here. See Section 4.2.1 for further explanation.

Table 3.5. Masses and volumes for the models of the urinary bladder

\begin{tabular}{||c|c|c|c|c||c|c||c|c||}
\cline { 2 - 9 } \multicolumn{1}{c|}{} & \multicolumn{2}{c|}{ Nonpregnant } & \multicolumn{2}{c|}{ Three months } & \multicolumn{2}{c|}{ Six months } & \multicolumn{2}{c|}{ Nine months } \\
\cline { 2 - 9 } \multicolumn{1}{c|}{} & $\begin{array}{c}\text { Mass } \\
(\mathrm{g})\end{array}$ & $\begin{array}{c}\text { Volume } \\
\left(\mathrm{cm}^{3}\right)\end{array}$ & $\begin{array}{c}\text { Mass } \\
(\mathrm{g})\end{array}$ & $\begin{array}{c}\text { Volume } \\
\left(\mathrm{cm}^{3}\right)\end{array}$ & $\begin{array}{c}\text { Mass } \\
(\mathrm{g})\end{array}$ & $\begin{array}{c}\text { Volume } \\
\left(\mathrm{cm}^{3}\right)\end{array}$ & $\begin{array}{c}\text { Mass } \\
(\mathrm{g})\end{array}$ & $\begin{array}{c}\text { Volume } \\
\left(\mathrm{cm}^{3}\right)\end{array}$ \\
\hline \hline Wall & 35.9 & 34.5 & 36.9 & 35.4 & 34.5 & 33.1 & 23.9 & 23.0 \\
\hline Contents & 160 & 154 & 128 & 123 & 107 & 103 & 42.3 & 40.7 \\
\hline
\end{tabular}

\subsection{SMALL INTESTINE}

\subsection{Three-Month Pregnant Female}

Because the geometry of the small intestine is complicated by the folding of the long cylindrical tube upon itself, the exact geometry is not modeled, and the walls and contents are not distinguished for estimation of photon dose. However, see Section 4.2.1 on dosimetry about corrections to small intestine $\Phi$ estimates. The volume of the small intestine in the three-month model is $806 \mathrm{~cm}^{3}$, in agreement with the value in the nonpregnant adult female phantom.

The small intestine and contents are represented by an elliptical cylinder whose axis is parallel to the $\mathrm{Z}$-axis and which is cut by two vertical planes perpendicular to the $\mathrm{Y}$-axis:

$$
\begin{aligned}
& \left(\frac{X}{9.75}\right)^{2}+\left(\frac{Y+3.72}{11.07}\right)^{2} \leq 1 \\
& \text { and }-5.513 \leq Y \leq 2.16, \\
& \text { and } 15.32 \leq Z \leq 24.34 \text {. }
\end{aligned}
$$

The part of the large intestine within this space is excluded. In addition, the lower part of the small intestine intersects with the spherical portion of the uterus. The intersecting volume is deleted and the front wall of the small intestine has been moved forward to compensate for the lost volume. The additional condition to delete this intersection is

$$
X^{2}-(Y+3.2)^{2}+(Z-12.5457)^{2}>36
$$




\subsubsection{Six-Month Pregnant Female}

In the six-month phantom, the small intestine and contents are represented by two box-like structures stacked one above the other. The upper box is bounded on four sides by planes and on two sides by the inner surface of an ellipsoid. The lower box is bounded on three sides by planes and on the other three sides by the inner surfaces of the same ellipsoid or a right circular cylinder, whichever is most restrictive at a given place. The purpose of the cylinder is to avoid an overlap with the pelvis.

As in other phantoms, no attempt was made to distinguish between wall and contents or to explicitly model the geometry. This structure represents an attempt to model the complex geometry of the small intestine after it has been moved up by the enlarged uterus and pushed against and around some of these organs. The volume of the small intestine, as estimated by Monte Carlo techniques, is $805 \mathrm{~cm}^{3}$, which agrees with the value of $806 \mathrm{~cm}^{3}$ in the nonpregnant adult female.

The upper box is defined by

$$
\begin{aligned}
&-6.0 \leq Y \leq 2.068 \\
& \text { and } 19.83 \leq Z \leq 25.12 \\
& \text { and }\left(\frac{X}{A}\right)^{2}+\left(\frac{Y-Y_{0}}{B}\right)^{2}+\left(\frac{Z-Z_{0}}{B}\right)^{2} \leq 1 .
\end{aligned}
$$

The lower box is defined by

$$
\begin{gathered}
Y \geq-2.82 \\
\text { and } 15.32 \leq Z<19.83 \\
\text { and }\left(\frac{X}{A}\right)^{2}+\left(\frac{Y-Y_{0}}{B}\right)^{2}+\left(\frac{Z-Z_{0}}{B}\right)^{2} \leq 1 \\
\text { and }\left(\frac{X}{10.622}\right)^{2}+\left(\frac{Y-Y_{0}}{10.622}\right)^{2} \leq 1
\end{gathered}
$$

The parts of the large intestine and other organs within these volumes are excluded.

The parameters are:

\begin{tabular}{cccc}
$Y_{0}$ & $Z_{0}$ & $A$ & $B$ \\
\hline-3.572 & 18.75 & 9.75 & 16.0
\end{tabular}




\subsection{Nine-Month Pregnant Female}

In the nine-month phantom, the small intestine and contents are represented by a box-like volume, bounded on the four vertical sides by planes and above and below by cylindrical surfaces. As in other phantoms, no attempt was made to distinguish between wall and contents, or to explicitly model the geometry. This structure represents an attempt to model the complex geometry of the small intestine after it has been moved up by the enlarged uterus and pushed against and around some of these organs. The volume of the small intestine, as estimated by Monte Carlo techniques, is $804 \mathrm{~cm}^{3}$, which agrees with the value of $806 \mathrm{~cm}^{3}$ in the nonpregnant adult female.

The inequalities defining the small intestine are

$$
\begin{aligned}
\text { and }-8.8 & \leq X \leq 8.8 \\
\text { and } 16 \sqrt{0.775-\left(\frac{X}{15.8}\right)^{2}}+11.8 & \leq Z \leq 16 \sqrt{0.775-\left(\frac{X}{15.8}\right)^{2}}+20.8
\end{aligned}
$$

The parts of the large intestine and other organs within this volume are excluded.

\subsection{UPPER LARGE INTESTINE (ULI)}

The ULI comprises the ascending colon and the transverse colon. In all phantoms the volume of the wall and contents were made to match closely those in the nonpregnant phantom.

\subsubsection{Ascending Colon}

\subsubsection{Three-month pregnant female}

The ascending colon comprises an upper part and a lower part. In each part the wall is defined as the volume between two coaxial elliptical cylinders, and the contents are defined as the volume within the inner cylinder. The lower part was modified from that in the nonpregnant female to avoid an overlap with the enlarged uterus. The design criterion for the change in the lower part was to elongate the ellipse of cross-section in the Y-direction while maintaining the perimeter. This produces an ascending colon with a mismatched junction at $\mathrm{Z}=16 \mathrm{~cm}$, but this mathematical anomaly has little effect on dose estimates. 
The wall in the upper part is defined by

$$
\begin{aligned}
& \left(\frac{X-X_{0}}{A_{1}}\right)^{2}+\left(\frac{Y-Y_{0}}{B_{1}}\right)^{2} \leq 1 \\
& \text { and }\left(\frac{X-X_{0}}{A_{1}-D}\right)^{2}+\left(\frac{Y-Y_{0}}{B_{1}-D}\right)^{2}>1 \\
& \text { and } Z_{2} \leq Z \leq Z_{3} .
\end{aligned}
$$

The wall in the lower part is defined by

$$
\begin{aligned}
& \left(\frac{X-X_{0}}{A_{2}}\right)^{2}+\left(\frac{Y-Y_{0}}{B_{2}}\right)^{2} \leq 1 \\
& \text { and }\left(\frac{X-X_{0}}{A_{2}-D}\right)^{2}+\left(\frac{Y-Y_{0}}{B_{2}-D}\right)^{2}>1 \\
& \text { and } Z_{1} \leq Z<Z_{2} .
\end{aligned}
$$

The contents are defined by

$$
\begin{aligned}
& \left(\frac{X-X_{0}}{A_{1}-D}\right)^{2}+\left(\frac{Y-Y_{0}}{B_{1}-D}\right)^{2} \leq 1 \text { and } Z_{2} \leq Z \leq Z_{3} \text { (upper part); } \\
& \text { or }\left(\frac{X-X_{0}}{A_{2}-D}\right)^{2}+\left(\frac{Y-Y_{0}}{B_{2}-D}\right)^{2} \leq 1 \text { and } Z_{1} \leq Z<Z_{2} \text { (lower part). }
\end{aligned}
$$

The parameters are:

\begin{tabular}{cccccccccc}
$\mathrm{A}_{1}$ & $\mathrm{~B}_{1}$ & $\mathrm{~A}_{2}$ & $\mathrm{~B}_{2}$ & $\mathrm{D}$ & $\mathrm{X}_{0}$ & $\mathrm{Y}_{0}$ & $\mathrm{Z}_{1}$ & $\mathrm{Z}_{2}$ & $\mathrm{Z}_{3}$ \\
\hline 2.16 & 2.45 & 1.62 & 2.90 & 0.65 & -7.33 & -2.31 & 13.03 & 16 & 21.63
\end{tabular}




\subsubsection{Six-month pregnant female}

The ascending colon comprises three parts: lower, middle, and upper. In each part the wall is defined as the volume between two coaxial elliptical cylinders, and the contents are defined as the volume within the inner cylinder. The middle part was modified from that in the nonpregnant female to avoid an overlap with the enlarged uterus. The design criterion for the modification was to elongate the ellipse of cross-section in the Y-direction while maintaining the perimeter constant.

The wall in the lower and upper parts is defined by

$$
\begin{aligned}
\left(\frac{X-X_{0}}{A_{1}}\right)^{2}+\left(\frac{Y-Y_{0}}{B_{1}}\right)^{2} \leq 1 \\
\text { and }\left(\frac{X-X_{0}}{A_{1}-D}\right)^{2}+\left(\frac{Y-Y_{0}}{B_{1}-D}\right)^{2}>1
\end{aligned}
$$

and $\left[Z_{1}<Z \leq Z_{2}\right.$ (lower part) or $Z_{3}<Z \leq Z_{4}$ (upper part) $]$.

The wall in the middle part is defined by

$$
\begin{aligned}
& \left(\frac{X-X_{0}}{A_{2}}\right)^{2}+\left(\frac{Y-Y_{0}}{B_{2}}\right)^{2} \leq 1 \\
& \text { and }\left(\frac{X-X_{0}}{A_{2}-D}\right)^{2}+\left(\frac{Y-Y_{0}}{B_{2}-D}\right)^{2}>1 \\
& \text { and } Z_{2}<Z \leq Z_{3} .
\end{aligned}
$$

The contents are defined by

$$
\begin{aligned}
& \left(\frac{X-X_{0}}{A_{1}-D}\right)^{2}+\left(\frac{Y-Y_{0}}{B_{1}-D}\right)^{2} \leq 1 \text { and } Z_{1}<Z \leq Z_{2} \text { (lower part); } \\
& \text { or }\left(\frac{X-X_{0}}{A_{1}-D}\right)^{2}+\left(\frac{Y-Y_{0}}{B_{1}-D}\right)^{2} \leq 1 \text { and } Z_{3}<Z \leq Z_{4} \text { (upper part); } \\
& \text { or }\left(\frac{X-X_{0}}{A_{2}-D}\right)^{2}+\left(\frac{Y-Y_{0}}{B_{2}-D}\right)^{2} \leq 1 \text { and } Z_{2}<Z \leq Z_{3} \text { (middle part). }
\end{aligned}
$$


The parameters are:

\begin{tabular}{ccccccccccc}
$\mathrm{A}_{1}$ & $\mathrm{~B}_{1}$ & $\mathrm{~A}_{2}$ & $\mathrm{~B}_{2}$ & $\mathrm{D}$ & $\mathrm{X}_{0}$ & $\mathrm{Y}_{0}$ & $\mathrm{Z}_{1}$ & $\mathrm{Z}_{2}$ & $\mathrm{Z}_{3}$ & $\mathrm{Z}_{4}$ \\
\hline 2.16 & 2.45 & 1.90 & 2.783 & 0.65 & -9.45 & -6.24 & 15.79 & 17.8 & 19.6 & 24.39
\end{tabular}

\subsubsection{Nine-month pregnant female}

The ascending colon in the nine-month phantom is constricted between the enlarged uterus and the right side of the trunk extension. This constriction causes the cylinder with an ellipsoidal crosssection to be compressed into a cylinder with a triangular cross-section. In addition, the cylinder is slanted at an angle from the vertical. The wall is represented by the volume between this triangular cylinder and a smaller one with the same axis, and the contents are represented by the volume within the inner cylinder.

The wall is defined by

$$
\begin{aligned}
& Y+4.40 X+72.5-4.40 Z_{1} \geq 0 \text { and } Y+4.40 X+70.8-4.40 Z_{1}<0 \\
& \text { and } Y-0.125 X+1.54+0.125 Z_{1} \leq 0 \text { and } Y-0.125 X+1.93+0.125 Z_{1}>0 \\
& \text { and } Y-10.08 X-122.8+10.08 Z_{1} \geq 0 \text { and } Y-10.08 X-126.8+10.08 Z_{1}<0
\end{aligned}
$$

The definition of $Z_{1}$ and a constraint on $Z$ are given by

$$
\begin{gathered}
Z_{1}=(22.407-Z) \tan \left(15^{\circ}\right) \\
13.74 \leq Z \leq 22.41 .
\end{gathered}
$$

The contents are defined by

$$
\begin{aligned}
& Y+4.40 X+70.8-4.40 Z_{1} \geq 0 \\
& \text { and } Y-0.125 X+1.93+0.125 Z_{1} \leq 0 \\
& \text { and } Y-10.08 X-126.8+10.08 Z_{1} \geq 0
\end{aligned}
$$

The constraint on $Z$ and the definition of $Z_{1}$ are as given above.

\subsubsection{Transverse Colon}

\subsubsection{Three-month pregnant female}

No changes were made to the transverse colon in the three-month phantom. 


\subsubsection{Six-month pregnant female}

The transverse colon was modeled as the top half of a vertical torus curving around the top hemisphere of the uterus in the trunk extension. The wall is represented by the volume between this torus and a smaller coaxial torus, and the contents are represented by the volume within the smaller torus.

The wall is defined by

$$
\begin{aligned}
& \left(\frac{Y-Y_{0}}{B}\right)^{2}+\left(\frac{\sqrt{X^{2}+\left(Z-Z_{0}\right)^{2}}-R}{C}\right)^{2} \leq 1 \\
& \text { and }\left(\frac{Y-Y_{0}}{B-D}\right)^{2}+\left(\frac{\sqrt{X^{2}+\left(Z-Z_{0}\right)^{2}}-R}{C-D}\right)^{2}>1 \\
& \text { and } Z \geq Z_{0} .
\end{aligned}
$$

The contents are defined by

$$
\begin{gathered}
\left(\frac{Y-Y_{0}}{B-D}\right)^{2}+\left(\frac{\sqrt{X^{2}+\left(Z-Z_{0}\right)^{2}}-R}{C-D}\right)^{2} \leq 1 \\
\text { and } Z \geq Z_{0} .
\end{gathered}
$$

The parameters are:

\begin{tabular}{cccccc}
$\mathrm{B}$ & $\mathrm{C}$ & $\mathrm{D}$ & $\mathrm{R}$ & $\mathrm{Y}_{0}$ & $\mathrm{Z}_{0}$ \\
\hline 1.8 & 1.146 & 0.3966 & 9.05 & -11.83 & 19.6
\end{tabular}

Because of the curvature of the transverse colon and the positioning of the descending colon, there is a small overlap between the transverse colon wall and the descending colon wall, which is excluded from the transverse colon wall. 


\subsubsection{Nine-month pregnant female}

The transverse colon in the nine-month phantom is triangular in cross section, being flattened on top of the uterus by the top of the trunk extension. As with the other models, the volume of the wall and contents were made to match those of the nonpregnant adult female phantom.

The wall is defined by

$$
\begin{array}{ll}
Z+0.65 Y-26.8+0.65 X_{1} \leq 0 & \text { and } Z+0.65 Y-26.5+0.65 X_{1}>0 \\
\text { and } Z-Y-35.0-X_{1} \geq 0 & \text { and } Z-Y-35.4-X_{1}<0 \\
\text { and } Z-2.14 Y-46.3-2.14 X_{1} \leq 0 & \text { and } Z-2.14 Y-45.7-2.14 X_{1}>0
\end{array}
$$

The definition of $\mathrm{X}_{1}$ and a constraint on $\mathrm{X}$ are given by

$$
\begin{gathered}
X_{1}=\sqrt{105.1-X^{2}} \\
-10.25 \leq X \leq 10.25 .
\end{gathered}
$$

The contents are defined by

$$
\begin{aligned}
& Z+0.65 Y-26.5+0.65 X_{1} \leq 0 \\
& \text { and } Z-Y-35.4-X_{1} \geq 0 \\
& \text { and } Z-2.14 Y-45.7-2.14 X_{1} \leq 0 \text {. }
\end{aligned}
$$

The constraint on $\mathrm{X}$ and the definition of $\mathrm{X}_{1}$ are as given above.

\subsection{LOWER LARGE INTESTINE (LLI)}

The LLI comprises the descending colon and the sigmoid colon. In all phantoms the volumes of the wall and contents were made to match closely those in the nonpregnant phantom.

\subsubsection{Descending Colon}

\subsubsection{Three-month pregnant female}

No changes were made in the descending colon in the three-month phantom. 


\subsubsection{Six-month pregnant female}

The descending colon in the six-month pregnant female is modeled as an elliptical cylinder displaced to fit around the enlarged uterus and slanted slightly towards the phantom's right as it descends. A smaller coaxial elliptical cylinder defines the inner surface of the wall, and the contents are within the inner cylinder.

The descending colon wall is defined by

$$
\begin{aligned}
& \left(\frac{X-X_{0}}{A}\right)^{2}+\left(\frac{Y-Y_{0}}{B}\right)^{2} \leq 1 \\
& \text { and }\left(\frac{X-X_{0}}{A-D}\right)^{2}+\left(\frac{Y-Y_{0}}{B-D}\right)^{2}>1 \\
& \text { and } Z_{1} \leq Z \leq Z_{2} \text {, } \\
& \text { where } X_{0}=X_{1}+\frac{M_{x}\left(Z-Z_{2}\right)}{\left(Z_{2}-Z_{1}\right)} \\
& \text { and where } Y_{0}=Y_{1}+\frac{M_{y}\left(Z_{1}-Z\right)}{\left(Z_{2}-Z_{1}\right)} \text {. }
\end{aligned}
$$

The descending colon contents are defined by

$$
\begin{gathered}
\left(\frac{X-X_{0}}{A-D}\right)^{2}+\left(\frac{Y-Y_{0}}{B-D}\right)^{2} \leq 1 \\
\text { and } Z_{1} \leq Z \leq Z_{2},
\end{gathered}
$$

where $X_{0}$ and $Y_{0}$ are defined as above.

The parameters are:

\begin{tabular}{ccccccccc}
$\mathrm{A}$ & $\mathrm{B}$ & $\mathrm{D}$ & $\mathrm{M}_{\mathrm{z}}$ & $\mathrm{M}_{\mathrm{y}}$ & $\mathrm{X}_{1}$ & $\mathrm{Y}_{1}$ & $\mathrm{Z}_{1}$ & $\mathrm{Z}_{2}$ \\
\hline 1.461 & 1.931 & 0.446 & 0.6728 & 2.45 & 9.06 & -6.24 & 7.86 & 24.39
\end{tabular}




\subsubsection{Nine-month pregnant female}

The descending colon model in the nine-month pregnant female comprises two parts, upper and lower. Each part consists of two coaxial elliptical cylinders, with the wall defined as the volume between the cylinders and the contents defined as the volume within the inner cylinder. The axis of the upper part is oriented vertically, and the axis of the lower part is slanted to avoid the enlarged uterus and goes into the trunk extension.

The wall in the upper part is defined by

$$
\begin{aligned}
& \left(\frac{X-X_{0}}{A}\right)^{2}+\left(\frac{Y-Y_{0}}{B}\right)^{2} \leq 1 \\
& \text { and }\left(\frac{X-X_{0}}{A-D}\right)^{2}+\left(\frac{Y-Y_{0}}{B-D}\right)^{2}>1 \\
& \text { and } Z_{1} \leq Z \leq Z_{2} .
\end{aligned}
$$

The contents in the upper part are defined by

$$
\begin{gathered}
\left(\frac{X-X_{0}}{A-D}\right)^{2}+\left(\frac{Y-Y_{0}}{B-D}\right)^{2} \leq 1 \\
\text { and } Z_{1} \leq Z \leq Z_{2}
\end{gathered}
$$

The parameters are:

\begin{tabular}{ccccccc}
$\mathrm{A}$ & $\mathrm{B}$ & $\mathrm{D}$ & $\mathrm{X}_{0}$ & $\mathrm{Y}_{0}$ & $\mathrm{Z}_{1}$ & $\mathrm{Z}_{2}$ \\
\hline 1.107 & 2.459 & 0.401 & 13.3 & -9.7 & 17.0 & 25.0
\end{tabular}

The wall and contents in the lower part are defined by similar inequalities and parameters, except that $Z_{1}=7.86, Z_{2}=17$, and the axis of the elliptical cylinders is a slanting straight line instead of a vertical straight line. The equations defining the axis, in terms of $X_{0}$ and $Y_{0}$, are

$$
\begin{aligned}
X_{0} & =13.3-(17-Z) \sin \left(35^{\circ}\right) \\
\text { and } Y_{0} & =-9.7-(17-Z) \cos \left(35^{\circ}\right)
\end{aligned}
$$

\subsubsection{Sigmoid Colon}

No changes were made to the sigmoid colon in any of the pregnant phantoms. 


\subsection{OVARIES}

\subsubsection{Three-Month Pregnant Female}

No changes were made to the ovaries in the three-month phantom.

\subsection{Six- and Nine-Month Pregnant Female}

The ovaries were moved outward slightly in the $\mathrm{X}$ direction from their location in the nonpregnant adult female model in order to be outside of the enlarged uterus. They are otherwise unchanged. The inequalities describing the ovaries are

$$
\left(\frac{X-X_{0}}{A}\right)^{2}+\left(\frac{Y}{B}\right)^{2}+\left(\frac{Z-Z_{0}}{C}\right)^{2} \leq 1 .
$$

The parameters are:

\begin{tabular}{lccccc} 
Model & $\mathrm{A}$ & $\mathrm{B}$ & $\mathrm{C}$ & $\mathrm{X}_{0}$ & $\mathrm{Z}_{0}$ \\
\hline Six-month & 1.17 & 0.58 & 1.80 & \pm 8.39 & 13.52 \\
Nine-month & 1.17 & 0.58 & 1.80 & \pm 7.18 & 13.52
\end{tabular}

The positive value of $\mathrm{X}_{0}$ is associated with the left ovary, and the negative value is associated with the right ovary.

\subsection{PELVIS}

\subsection{Three-Month Pregnant Female}

No changes were made in the three-month phantom.

\subsection{Six- and Nine-Month Pregnant Female}

In the six- and nine-month phantoms, the pelvis was expanded to accommodate the enlarged uterus and the surrounding organs. The pelvis has the same mass and bone marrow distribution as in the nonpregnant adult female model. The pelvis is a part of the volume between two nonconcentric circular cylinders with vertical axes; the volume is restricted by two vertical planes, one for the entire cylinder and one for the lower part only. (In the nonpregnant phantom, the cylinders are elliptical.) 
The inequalities defining the pelvis are

$$
\begin{aligned}
& \left(\frac{X}{A}\right)^{2}+\left(\frac{Y-Y_{01}}{A}\right)^{2} \leq 1 \\
& \text { and }\left(\frac{X}{A-D}\right)^{2}+\left(\frac{Y-Y_{02}}{A-D}\right)^{2}>1 \\
& \text { and } Y \geq Y_{02} \\
& \text { and } Z_{1} \leq Z \leq Z_{3} \\
& \text { and } Y \leq Y_{1} \text { if } Z \leq Z_{2} .
\end{aligned}
$$

The parameters are:

\begin{tabular}{cccccccc}
$\mathrm{A}$ & $\mathrm{D}$ & $\mathrm{Y}_{01}$ & $\mathrm{Y}_{02}$ & $\mathrm{Y}_{1}$ & $\mathrm{Z}_{1}$ & $\mathrm{Z}_{2}$ & $\mathrm{Z}_{3}$ \\
\hline 11.28 & 0.658 & -3.572 & -2.82 & 4.7 & 0 & 12.62 & 19.83
\end{tabular}

\subsection{LIVER}

\subsubsection{Three- and Six-Month Pregnant Female}

No changes were made in the three- or six-month phantoms.

\subsubsection{Nine-Month Pregnant Female}

In the nine-month phantom, a portion of the lower tip of the liver intersected with the uterus. This intersection is defined as belonging to the uturus. This intersection reduced the volume of the liver by less than $1.5 \%$, so no attempt was made to make up the difference by extending the liver in other areas. 


\subsection{OTHER ORGANS}

A number of other organs were moved slightly within the six- and nine- month phantoms without altering their geometries otherwise. Most were simply moved up to make room for the enlarged uterus and reduce crowding in the abdomen. Others were moved to allow them to fit around other organs. These changes are summarized in the following table.

Table 3.6 Organs moved in the six- and nine-month models.

\begin{tabular}{|c|c|c|}
\hline Organ & $\begin{array}{c}\text { Six-Month Pregnant } \\
\text { Female }\end{array}$ & $\begin{array}{c}\text { Nine-month } \\
\text { Pregnant Female }\end{array}$ \\
\hline Gallbladder & $\begin{array}{c}\text { Raised } 1.75 \mathrm{~cm}, \\
\text { moved }+3.14 \mathrm{~cm} \text { in } \\
\text { Y direction }\end{array}$ & $\begin{array}{c}\text { Raised } 1.75 \mathrm{~cm}, \\
\text { moved }+3.14 \mathrm{~cm} \text { in } \\
\text { Y direction }\end{array}$ \\
\hline Stomach & Raised $1 \mathrm{~cm}$ & Raised $1 \mathrm{~cm}$ \\
\hline Heart & Raised $1 \mathrm{~cm}$ & Raised $1 \mathrm{~cm}$ \\
\hline Liver & Raised $1 \mathrm{~cm}$ & Raised $1 \mathrm{~cm}$ \\
\hline Lungs & Raised $1 \mathrm{~cm}$ & Raised $1 \mathrm{~cm}$ \\
\hline Thymus & Raised $1 \mathrm{~cm}$ & Raised $1 \mathrm{~cm}$ \\
\hline
\end{tabular}

It should be noted that the brain in the adult female phantom has been raised since the publication of the document describing the pediatric phantom series (Cristy and Eckerman 1987), as a neck region was added to the phantoms. The position of the thyroid has also been changed slightly and has less tissue surrounding it with the introduction of a neck region. The new head-and-neck design is in the six- and nine-month pregnant phantoms but not the nonpregnant or three-month pregnant phantoms. See Section 4.2.2. regarding how these changes are resolved over the series of nonpregnant and pregnant adult female models to reduce inconsistency among the models. 
Table 3.7. Masses of Organs in the Adult Female Modek. Except for organs affected by pregnancy, the masses below are primarily Reference Woman (ICRP 23) values rather than phantom values. Values of for self-dose from the phantoms were adjusted to match these Reference Woman values, as described in Section 2 on the adult female model. Values of for "cross-fire" (source and target regions not the same) were not adjusted, since these is are not strongly dependent on mass. Masses taken from the phantoms instead of ICRP 23 are marked with the superscript "a". See Section 2 for further explanation why Reference Woman masses are used in the models.

\begin{tabular}{|c|c|c|c|c|}
\hline Model: & Nonpregnant & 3-month & 6-month & 9-month \\
\hline Organ or region & \multicolumn{4}{|c|}{ Mass (g) in each model } \\
\hline $\begin{array}{l}\text { Adrenals } \\
\text { Brain } \\
\text { Breasts-excluding skin } \\
\text { Gall bladder contents } \\
\text { Gall bladder wall } \\
\text { GI tract } \\
\text {-Stomach contents } \\
\text {--Stomach wall } \\
\text {-SI contents } \\
\text {--SI wall } \\
\text {-ULI contents } \\
\text {--ULI wall } \\
\text {-LLI contents } \\
\text {-LLI wall } \\
\text { Heart contents } \\
\text { Heart wall } \\
\text { Kidneys } \\
\text { Liver } \\
\text { Lungs } \\
\text { Ovaries } \\
\text { Pancreas } \\
\text { Remaining tissue } \\
\text { Skeleton } \\
\text { - Cortical bone } \\
\text { - Trabecular bone } \\
\text { - Endosteal tissue } \\
\text { - Red marrow } \\
\text { Skin } \\
\text { Spleen } \\
\text { Thymus } \\
\text { Thyroid } \\
\text { Urinary bladder contents } \\
\text { Urinary bladder wall } \\
\text { Uterine wall } \\
\text { Fetus } \\
\text {-Fetal soft tissue } \\
\text {-Fetal skeleton } \\
\text { Placenta } \\
\text { Other uteripe contents }\end{array}$ & $\begin{array}{c}14 \\
1200 \\
360 \\
50 \\
8 \\
\\
230 \\
140 \\
375 \\
600 \\
210 \\
200 \\
135 \\
160 \\
410 \\
240 \\
275 \\
1400 \\
651^{2} \\
11 \\
85 \\
40000^{2} \\
7500 \\
3000 \\
750 \\
90 \\
1300 \\
1790 \\
150 \\
20 \\
17 \\
160^{2, c} \\
35.9^{3} \\
80 \\
- \\
- \\
- \\
- \\
-\end{array}$ & $\begin{array}{r}14 \\
1200 \\
360 \\
50 \\
8 \\
\\
230 \\
140 \\
375 \\
600 \\
210 \\
200 \\
135 \\
160 \\
410 \\
240 \\
275 \\
1400 \\
651^{2} \\
11 \\
85 \\
39300^{2} \\
7500 \\
3000 \\
750 \\
90 \\
1300 \\
1790 \\
150 \\
20 \\
17 \\
128^{2, c} \\
36.9^{2} \\
374^{2} \\
458^{2, d} \\
- \\
- \\
- \\
- \\
\end{array}$ & $\begin{array}{r}14 \\
1200 \\
360 \\
50 \\
8 \\
230 \\
140 \\
375 \\
600 \\
210 \\
200 \\
135 \\
160 \\
410 \\
240 \\
275 \\
1400 \\
651^{2} \\
11 \\
85 \\
41700^{2} \\
7500 \\
3000 \\
750 \\
90 \\
1300 \\
1790 \\
150 \\
20 \\
17 \\
107^{2, c} \\
34.5^{2} \\
834^{2} \\
1640^{2} \\
1530^{2} \\
115^{2} \\
310^{2} \\
886^{2}\end{array}$ & $\begin{array}{c}14 \\
1200 \\
360 \\
50 \\
8 \\
\\
230 \\
140 \\
375 \\
600 \\
210 \\
200 \\
135 \\
160 \\
410 \\
240 \\
275 \\
1400 \\
651^{2} \\
11 \\
85 \\
39500^{2} \\
7500 \\
3000 \\
750 \\
90 \\
1300 \\
1790 \\
150 \\
20 \\
17 \\
42.3^{2, c} \\
23.9^{a} \\
1095^{2} \\
2960^{2} \\
2610^{2} \\
350^{2} \\
466^{2} \\
2580^{2} \\
\end{array}$ \\
\hline $\begin{array}{l}\text { Maternal tissues } \\
\text { Uterine contents } \\
\text { Other Contents } \\
\text { Total }\end{array}$ & $\begin{array}{l}56.8 \mathrm{~kg} \\
0 \\
1.16 \\
58.0\end{array}$ & $\begin{array}{c}56.4 \mathrm{~kg} \\
0.46 \\
1.13 \\
58.0\end{array}$ & $\begin{array}{l}57.5 \mathrm{~kg} \\
2.84 \\
1.11 \\
61.5^{\mathrm{a}}\end{array}$ & $\begin{array}{c}56.6 \mathrm{~kg} \\
6.01 \\
1.04 \\
63.7^{2}\end{array}$ \\
\hline
\end{tabular}

- Mass in phantom.

b "Remaining tissue" is defined as the maternal tissues remaining when all defined organs have been removed. This region of the phantom has been used to model muscle for dosimetric purposes. However, the appropriate mass of muscle to use in such calculations in the adult female is $17,000 \mathrm{~g}$ (ICRP 23). 
These bladder contents are for a relatively full bladder. See Section 4.2.1. regarding how specific absorbed fractions are also adjusted for a half-full bladder.

d Entire uterine contents for 3-month pregnant female.

- The concept of body tissues (here, maternal tissues) is useful in dosimetry because they are the parts "inside the body" from a physiologist's viewpoint - the systemic tissues. They exclude the contents of the GI tract, the urinary bladder, and the gall bladder.

Table 3.8. Centroids of Organs in the Phantoms

The following table lists the centroids of the organs in the phantoms. This information may be useful to the user in studying changes in the phantoms or in certain calculations which employ mean inter-organ distances. Centroids for the nonpregnant adult female phantom are also shown for comparison.

\begin{tabular}{|c|c|c|c|c|}
\hline \multicolumn{5}{|c|}{ Centroid ( $[\mathrm{X}, \mathrm{Y}, \mathrm{Z}]$ coordinates in $\mathrm{cm})$ of organs } \\
\hline & Nonpregnant & 3-Month & 6-Month & 9-Month \\
\hline Adrenals - left & $3.02,4.90,35.87$ & $3.02,4.90,35.87$ & $3.02,4.90,35.87$ & $3.02,4.90,35.87$ \\
\hline Adrenals - right & $-3.02,4.90,35.87$ & $-3.02,4.90,35.87$ & $-3.02,4.90,35.87$ & $-3.02,4.90,35.87$ \\
\hline Brain & $0.00,0.00,79.07$ & $0.00,0.00,79.07$ & $0.00,0.00,83.15$ & $0.00,0.00,83.15$ \\
\hline Breasts - left & $9.23,-9.89,46.86$ & $9.23,-9.89,46.86$ & $9.23,-9.88,46.87$ & $9.23,-9.88,46.87$ \\
\hline Breasts - right & $-9.23,-9.89,46.86$ & $-9.23,-9.89,46.86$ & $-9.23,-9.88,46.87$ & $-9.23,-9.88,46.87$ \\
\hline Gall bladder cont. & $-3.63,-2.89,28.18$ & $-3.63,-2.89,28.18$ & $-3.63,0.25,29.93$ & $-3.63,0.25,29.93$ \\
\hline Gall bladder wall & $-3.51,-2.81,28.55$ & $-3.51,-2.81,28.55$ & $-3.51,0.33,30.30$ & $-3.51,0.33,30.30$ \\
\hline $\begin{array}{l}\text { GI tract } \\
\text {--Stomach contents }\end{array}$ & $6.90,-3.92,31.55$ & $6.90,-3.92,31.55$ & $6.90,-3.92,32.55$ & $6.90,-3.92,32.55$ \\
\hline --Stomach wall & $6.90,-3.92,31.55$ & $6.90,-3.92,31.55$ & $6.90,-3.92,32.55$ & $6.90,-3.92,32.55$ \\
\hline --Small intestine & $0.29,-1.00,19.38$ & $0.30,-1.44,19.92$ & $0.18,-1.10,21.56$ & $0.38,-1.75,29.80$ \\
\hline -ULI contents & $-3.15,-2.31,20.55$ & $-3.45,-2.31,20.54$ & $-0.01,-11.83,25.32$ & $-5.53,-11.39,24.31$ \\
\hline --ULI wall & $-3.15,-2.31,20.55$ & $-3.52,-2.31,20.42$ & $-4.10,-9.43,23.12$ & $-5.46,-12.08,23.99$ \\
\hline --LLI contents & $6.76,-0.91,11.90$ & $6.76,-0.91,11.90$ & $7.56,-5.55,12.92$ & $9.93,-5.74,13.15$ \\
\hline -LLI wall & $6.11,-0.68, \quad 9.83$ & $6.11,-0.68, \quad 9.83$ & $6.71,-4.17,10.60$ & $8.48,-4.30,10.76$ \\
\hline Heart contents & $0.11,-3.48,44.92$ & $0.11,-3.48,44.92$ & $0.11,-3.48,45.92$ & $0.10,-3.48,45.92$ \\
\hline Heart wall & $1.65,-4.03,43.85$ & $1.65,-4.03,43.85$ & $1.65,-4.03,44.85$ & $1.65,-4.03,44.85$ \\
\hline Kidneys - left & $5.43,5.88,29.30$ & $5.43,5.88,29.30$ & $5.43,5.88,29.30$ & $5.43,5.88,29.30$ \\
\hline Kidneys - right & $-5.43,5.88,29.30$ & $-5.43,5.88,29.30$ & $-5.43,5.88,29.30$ & $-5.43,5.88,29.30$ \\
\hline Liver & $-7.68,-1.65,33.51$ & $-7.68,-1.65,33.51$ & $-7.68,-1.65,34.51$ & $-7.68,-1.60,34.62$ \\
\hline Lungs - left & $7.72, \quad 0.46,47.55$ & $7.72, \quad 0.46,47.55$ & $7.72, \quad 0.46,48.55$ & $7.72,0.46,48.55$ \\
\hline Lungs - right & $-7.46, \quad 0.03,47.00$ & $-7.46,0.03,47.00$ & $-7.46,0.03,48.00$ & $-7.46,0.03,48.00$ \\
\hline Ovaries - left & $5.18, \quad 0.00,13.52$ & $5.18,0.00,13.52$ & $8.39,0.00,13.52$ & $7.18,0.00,13.52$ \\
\hline Ovaries - right & $-5.18, \quad 0.00,13.52$ & $-5.18,0.00,13.52$ & $-8.39,0.00,13.52$ & $-7.18,0.00,13.52$ \\
\hline Pancreas & $3.37, \quad 0.00,33.81$ & $3.37,0.00,33.81$ & $3.37,0.00,33.81$ & $3.37,0.00,33.81$ \\
\hline Spleen & $9.49, \quad 2.94,33.35$ & $9.49,2.94,33.35$ & $9.49,2.94,33.35$ & $9.49,2.94,33.35$ \\
\hline Thymus & $0.00,-7.15,52.00$ & $0.00,-7.15,52.00$ & $0.00,-7.15,53.00$ & $0.00,-7.15,53.00$ \\
\hline Thyroid & $0.00,-4.84,64.98$ & $0.00,-4.84,64.98$ & $0.00,-3.48,64.99$ & $0.00,-3.48,64.99$ \\
\hline Urinary bladder cont. & $0.00,-4.41,7.21$ & $0.00,-4.41, \quad 5.83$ & $0.00,-9.00,6.21$ & $0.00,-8.63,6.61$ \\
\hline Urinary bladder wall & $0.00,-4.41,7.21$ & $0.00,-4.41, \quad 6.02$ & $0.00,-9.00,6.25$ & $0.00,-8.67,6.70$ \\
\hline Uterine wall & $0.00,-1.26,12.62$ & $0.00,-4.10,13.13$ & $0.00,-6.54,16.63$ & $0.00,-8.87,18.68$ \\
\hline Placenta & $\ldots, \quad, \cdots$, & $-\cdots,-\cdots$, & $0.00,-12.57,21.69$ & $0.00,-15.45,24.22$ \\
\hline Fetus & $\cdots$, & $0.00,-2.88,12.34$ & $0.00,-6.54,16.63$ & $0.00,-7.95,17.92$ \\
\hline
\end{tabular}




\section{DOSIMETRY}

This section gives general information on dosimetric methodology and specific information on techniques used in generating the specific absorbed fractions tabulated in the appendix. Parts of this section are taken from Cristy and Eckerman (1993). For a more complete discussion of general methodology and techniques, see Cristy and Eckerman $(1987,1993)$.

\subsection{DOSIMETRIC METHODOLOGY}

The dosimetric methodology in which the specific absorbed fractions for sources of photon emitters in the body are used is that of the ICRP and that of MIRD. The methodology considers two sets of anatomical regions within the body. One set specifies the location of radioactivity within the body and is referred to as the source regions. The methodology places no constraints on the nature of the source regions - each might be represented by a point, a surface, or a volume. The second set of regions are the so-called target regions or the tissue over which the radiation dose is of interest. Both the ICRP and MIRD consider the mean absorbed dose to a target region as the fundamental dosimetric quantity. The principal biological effect of interest in radiation protection, cancer induction, is cellular in origin and the mean dose in a target is relevant to the extent that mean dose is representative of the dose to the cells at risk. For lack of information, the cells at risk are assumed to be uniformly distributed in the target region and thus, with this assumption, the mean dose is the relevant quantity.

The assumptions of the dosimetric methodology are:

1. The radioactivity in each source region is assumed to be uniformly distributed. For most regions the distribution is by mass or volume. However, for mineral bone regions it may also be by surface area. For a source in Lungs, the activity may be considered to be either in the volume of air or deposited on surfaces within the lung.

2. For all target regions, the mean energy absorbed in the target volume averaged over the mass of the target is the relevant quantity.

The mean energy absorbed in the target region depends on the nature of the radiations emitted in the source regions, the spatial relationships between the source and target regions, and the nature of the tissues between the regions.

\subsubsection{Dosimetric Quantities}

To see where the specific absorbed fractions for photons are used in dosimetry, let us look at the ICRP methodology. In the ICRP methodology, computation of specific effective energy, SEE, is necessary in the computation of equivalent dose rate in a target region, for occupational or public exposure to radionuclides taken into the body. (In the MIRD schema, the similar S-factor is computed.) Whether one is interested in committed equivalent dose to a region, committed effective dose, or assessment of risk, the basic quantity to be computed first is the equivalent dose rate at various times. 
From ICRP Publication 56, Part 1 (1989), and substituting newer terminology from ICRP Publication 60 (1991) and substituting the word "region" for "organ" or "tissue", we may define equivalent dose rate $\dot{H}$ and specific effective energy $S E E$ as follows:

"The equivalent dose rate in target region $T$ includes contributions from each radionuclide in the body and from each region in which radionuclides are present. The equivalent dose rate at age $t$ in region $T$ of an individual of age $t_{0}$ at the time of intake, $\dot{H}_{T}\left(t, t_{0}\right)$, can be expressed as

$$
\dot{H}_{T}\left(t, t_{0}\right)=c \sum_{S} \sum_{j} q_{S, j}(t) S E E(T-S ; t)_{j},
$$

where $q_{S_{j}}(t)$ is the activity of radionuclide $j$ present in source region $S$ at age $t, S E E(T-S ; t)_{j}$ is the specific effective energy deposited in target region $T$ per nuclear transformation of radionuclide $j$ in source region $S$ at age $t$, and $c$ is any numerical constant required by the units of $q$ and $S E E$. ...

For any radionuclide, the specific effective energy at age $t$ is defined as

$$
S E E(T-S ; t)=\sum_{i} \frac{w_{R, i} Y_{i} E_{i} \phi_{i}(T-S ; t)}{M_{T}(t)}
$$

where $w_{R i}$ is the radiation weighting factor for radiation type $i, Y_{i}$ is the yield of radiations of type $i$ per nuclear transformation, $E_{i}$ is the average or unique energy of radiation type $i, \phi_{i}(T-S ; t)$ is the fraction of energy emitted in source region $S$ that is absorbed within target region $T$ at age $t$, and $M_{T}(t)$ is the mass of target region $T$ at age $t$... The age dependence in $S E E$ arises from the age dependence of the absorbed fraction and the mass of the target region."

The quantity $\phi_{i}(T \sim S ; t)$ is called the absorbed fraction, and when divided by the mass of the target region, $M_{T}$ is called the specific absorbed fraction, $\Phi_{i}(T-S ; t)$. They are also commonly referred to as $A F$ and $S A F$, respectively.

The equation above applies to all kinds of radiations: photons, electrons, alpha particles, etc. For nonpenetrating radiations like electrons and alpha particles, $\Phi(T-S)=0$ when $S \neq T$, except for special cases such as tissues in bone and lung where source and target regions are in close proximity. Thus for most target organs $\mathrm{T}$, the only contribution from cross-fire is that from photons. 


\subsection{SPECIFIC ABSORBED FRACTIONS FOR PHOTONS}

\subsection{Methods}

Four methods are used to calculate the specific absorbed fraction for a given source-target region pair at each of 12 initial photon energies:

$\Phi$ (target - source) is calculated with the Monte Carlo radiation transport program ALGAMP (Ryman et al. 1987);

The converse, $\Phi$ (source - target), is calculated with the Monte Carlo radiation transport program, and this value is used to estimate $\Phi$ (target + source), sometimes after applying an empirical correction factor when the absorbing media of the source and target are of different compositions and densities (Cristy and Eckerman (1987).

$\Phi($ target $\rightarrow$ source) is calculated by numerical integration of the point-source kernel equation, and an empirical correction factor relating Monte Carlo and point-source kernel results is usually applied (Cristy and Eckerman 1987). This was used only in the nonpregnant and 3-month pregnant phantoms.

$\Phi($ target $*$ source) is calculated with the point-source kernel equation and the centroids of each region. This was used only occasionally at low energies $(10-20 \mathrm{keV})$ in the sixand nine-month pregnant phantoms, where method (3) was not used.

The computer program ALGAMP, used to derive the Monte Carlo estimates of $\Phi$, is identical in form to that used by Cristy and Eckerman (1987) in their work on the pediatric and adult phantom series. ALGAMP simulates the transport of monoenergetic photons at any given initial energy (from $10 \mathrm{keV}$ to $4 \mathrm{MeV}$ ) emitted from a given source region. The energy absorbed in the various target regions of the body are scored, the specific absorbed fractions are calculated, and the statistical reliability of each estimate is computed as a coefficient of variation (C.V.).

The program selects phantom descriptive parameters from a file, depending on which phantom from the six-phantom series is chosen. The program used to calculate Monte Carlo estimates of $\Phi$ given in this report was a modified version of ALGAMP, which replaced some parameters describing the adult female with values representing the pregnant adult female at the end of each trimester of pregnancy. In addition, some original programming was necessary to add the additional geometric regions and the changes in the kind of equations describing some organs.

Whenever possible (statistically reliable), the recommended value of $\Phi$ is taken as the weighted average of the two Monte Carlo estimates (with a correction factor sometimes applied to the converse estimate; see above). The weighting is done by the inverse of the variance. A single Monte Carlo estimate is considered statistically reliable if its C.V. is less than 50\%; the weighted average is considered reliable if its C.V. is less than 30\%. The corrected point-source kernel estimate (method 3 or 4 , above) is used if the weighted average is not reliable.

The specific absorbed fractions reported here for the nonpregnant female are those from Cristy and Eckerman (1987), except for the corrections for self-dose for the new adult female model as described in Section 2 of this report. The $\Phi$ s reported here for the 3-month pregnant female were developed by J. L. Davis about the same time and reported in part (Davis et al. 1987). His values for self-dose have also been changed here in a similar manner. In these two phantoms, the number of photon histories in the Monte Carlo simulation was $6 \times 10^{4}$. For the six-and nine-month pregnant females, the use of faster 
and cheaper computers allowed the Monte Carlo program to be run at $10^{7}$ histories, with certain low energy problems studied at even $10^{8}$ histories. With this change, the point-source kernel method (method 3; integrated) was not used since it was believed that the combination of direct and converse Monte Carlo estimates would be adequate. That was true except at low energies of 10-20 keV for widely separated organs, where the dose is so low that even $10^{8}$ histories was not adequate to get reliable estimates. Method 4, the point-source kernel method based on a centroid-to-centroid calculation, was used; or extrapolation on the $\log \Phi$ vs. $\log$ Energy was done; or an extrapolation based on other phantoms in the series was done. Although less accurate, these approximate methods are considered adequate because the self-dose and dose to tissues close to the source region are very large compared to these doses. The $\log \Phi$ vs. $\log$ Enengy curve was then smoothed in the nonpregnant and 3-month phantoms. Smoothing was not necessary in the six- and nine-month phantoms because of the larger number of histories.

The specific absorbed fractions published here have an additional digit beyond the two-digit values published previously in Cristy and Eckerman (1987). The additional digit reduces the potential for "lossof-significance $n$ in the second digit in some computations of SEE or S-factor. Also, previously $\Phi$ s below $10^{-10} \mathrm{~kg}^{-1}$ (at low energies) were listed as zero. Here, the first value below $10^{-10}$, as energy decreases, is kept and the values at lower energies where $\Phi$ is even smaller are given as zero (see Appendix A). The change was made to facilitate interpolation in energy when using the data.

Radiosensitive tissues in the skeleton. The energy deposition within the active bone marrow and within the endosteal cells on bone surfaces was based on the microscopic geometry of bone represented in the pathlength distributions compiled by Beddoe et al. (1976), a significant improvement over previous dosimetry of photons in bone. See Cristy and Eckerman (1987) for details. The hematopoietically active marrow is often called Red Marrow, but we don't like the term. It should be noted that some "red" marrow is redder than others, i.e., that the percentage of active marrow cells (cellularity) within a volume of marrow varies from site to site in the skeleton. Both the distribution of marrow within the body and the relative "redness" at each site have been taken into account in the dosimetry. We use the term "endosteum" rather than the term "bone surfaces" that the ICRP uses, to avoid confusion with sources of radionuclides on the surfaces of mineral bone.

Definition of Body Tissues (Maternal Tissues) as a source or target region. Addressing the use of Whole Body as a source region, Cristy and Eckerman (1993) changed the definition of Whole Body to exclude the contents of the GI tract (stomach, small intestine, upper large intestine, lower large intestine), the urinary bladder, and the gall bladder. We do the same here, but use the term Body Tissues (or Maternal Tissues) to represent this region, and further exclude the contents of the uterus (fetus, placenta, other uterine contents).

In the six- and nine-month pregnant phantoms, $\Phi$ s were calculated directly by the Monte Carlo program using the new definition for either source or target = Body Tissues. In the nonpregnant and threemonth pregnant phantoms, $\Phi$ s were calculated using the old definition of Whole Body, and corrections had to be made there. For Body Tissues as a source, the specific absorbed fractions are calculated by subtracting the contributions from the contents regions: 


$$
\Phi_{k}(T-B T ; t)=\frac{M_{W B} \Phi_{k}(T-W B ; t)-\sum_{\text {cont }} M_{\text {cont }} \Phi_{k}(T-\operatorname{con} ; t)}{M_{W B}-\sum_{\text {cont }} M_{\text {cont }}},
$$

where $B T$ is Body Tissues, $W B$ is the Whole Body with the contents, cont refers to one of the contents, $M$ is mass, $T$ is a particular target region, and the subscript $k$ refers to a particular photon energy in the standard grid of 12 energies.

Whole Body or Body Tissues as a target region is no longer used in the ICRP methodology, because of the use of the effective equivalent dose. However, in nuclear medicine it is still used, and consequently Body Tissues (Maternal Tissues) has been tabulated in the appendix. For the nonpregnant and threemonth pregnant phantoms, the specific absorbed fractions for Body Tissues as a target are calculated by subtracting contributions from the contents regions:

$$
\Phi_{k}(B T-S ; t)=\frac{M_{W B} \Phi_{k}(W B-S ; t)-\sum_{\text {cont }} M_{\text {cont }} \Phi_{k}(\text { cont }-S ; t)}{M_{W B}-\sum_{\text {cont }} M_{\text {cont }}},
$$

where $S$ is a particular source region and the other variables are defined as above.

Special case: small intestine wall and contents. As mentioned in Section 3, the small intestine wall and contents are modelled in the phantoms as one large mixture of wall and contents, i.e., walls and contents are not modelled separately. This results in poor estimates, especially at low energies, for $\Phi$ (SI wall - SI contents), $\Phi$ (Whole Body - SI contents), $\Phi$ (Whole Body - SI wall), and $\Phi$ (SI wall - Whole Body). For these region pairs, $\Phi$ was patterned after the results for LLI contents and wall, with corrections made for the mass differences and constrained to the results for SI wall and contents combined.

$\Phi$ (urinary bladder wall - urinary bladder contents). The bladder modeled in the phantoms is relatively full. For dosimetric purposes, a bladder mid-way in the void cycle, about half full, makes more sense. The error in using the average size rather than considering the whole cycle is only about $10 \%$, and consequently Cristy and Eckerman (1993) adopted the average values to derive $\Phi$ values for the six phantoms (newborn to adult male) of Cristy and Eckerman (1987). Also in deriving $\Phi$ (wall-contents) values, they made use of the fact that $\log \Phi$ vs. $\log$ mass (contents + wall) is linear - meaning that they could derive a power function $\Phi=a \cdot M_{a s s}{ }^{b}-$ and that it is relatively independent of wall thickness, except at the very low photon energies of $10-15 \mathrm{keV}$. Thus at each photon energy from the data for the six phantoms for urinary bladder to wall, they derived the $a$ and $b$ values for the power function and computed $\Phi$ for the desired mass of bladder contents. Here we have used the same power function to modify $\Phi$ (wall-contents) in the pregnant phantom series, again with a half-full bladder. Note that the region masses for urinary bladder contents given in Table 3.7 are for the contents as modeled in the phantoms (the full bladder). Specific absorbed fractions are given in the appendix for both the half-full bladder and the full bladder when the source in the urinary bladder contents and the target is the urinary bladder wall. 


\section{Special Methods for Consistency among the Adult Female Models}

Several problems arose because of differences in methodology used in the nonpregnant ("0-mo.") and three-month pregnant models vs. the six- and nine-month pregnant models. In addition, it was desirable to reduce inconsistencies among the $\Phi$ s for source-target pairs not affected by pregnancy. These differences and their resolutions are as follows:

(1) The number of photon histories in the calculations for the 6-mo. and 9-mo. phantoms was $10^{7}$ to $10^{8}$, compared with $6 \times 10^{4}$ for the 0 -mo. and 3-mo. phantoms. There are a number of organ pairs that are the same in the 0-mo. and 3-mo. phantoms, in the 6-mo. and 9-mo. phantoms, or in all four phantoms. In the former two cases the two values were averaged; in the last case the 6-mo. and 9-mo. values were averaged and used for all four phantoms, because of their better statistical reliability.

(2) The integrated point-source kernel method was used in the 0-mo. and 3-mo. phantoms, but not in the 6-mo. and 9-mo. phantoms. In those cases where the organ pairs are the same in all four phantoms and the $\Phi$ s for the 6-mo. and 9-mo. phantoms were poor at low energies, these values from the 0-mo. and 3-mo. phantoms were substituted.

The 6-mo. and 9-mo. phantoms have a separate neck region that was designed after the calculations of $\Phi$ were done for the 0 -mo. and 3-mo. phantoms. The thyroid is at a slightly different position in the two sets of phantoms, and the amount of tissue around the thyroid is different. In addition, the brain in the two sets is at a different z-position, about $4 \mathrm{~cm}$ higher in the 6-mo. and 9-mo. phantoms. Since the later design is more realistic, $\Phi$ s for thyroid and brain in the 6-mo. and 9-mo. phantoms were used for the 0-mo. and 3-mo. results, whenever possible. There are still some anomalies, especially for the brain.

(4) There were several organs that were considered source organs in the Monte Carlo program in the 3-mo. but not in the 0-mo., namely, skin, stomach wall, ULI wall, LLI wall, urinary bladder wall, and gall bladder wall. Previously tabulated $\Phi$ s for 0 -mo. had been based on the integrated point-source kernel method. Here these Monte Carlo values from the 3-mo. are used for the 0-mo.

\subsection{Examples of Results}

The results are tabulated in Appendix A. Here graphs of the results are shown for selected cases, but most for target $=$ fetus (Figs. 4.1-4.4). These examples were chosen because the main purpose of this work was to get doses to the fetus from maternal sources. For maternal target tissues, two examples are shown: $\Phi$ (urinary bladder wall - urinarybladder contents), where the contents are in mid-cycle (half-full) (Fig. 4.5), and $\Phi$ (SI wall - ULI contents) (Fig. 4.6). Note that $\Phi$ to bladder wall increases with gestation time because of the flattening of the bladder, and that $\Phi$ to SI wall from the ULI contents decreases with gestation time because of the pushing upward of the small intestine by the enlarging uterus. 


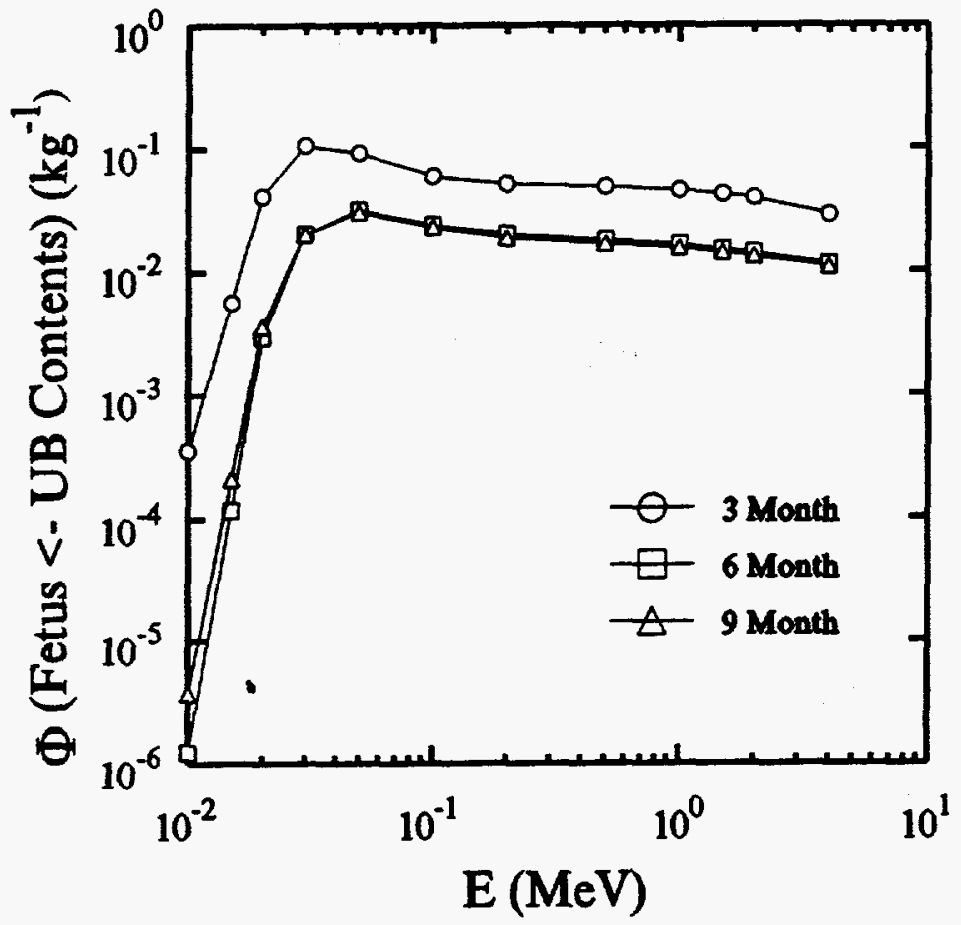

Fig. 4.1. $\Phi$ (fetus - urinary bladder contents) vs. initial photon energy.

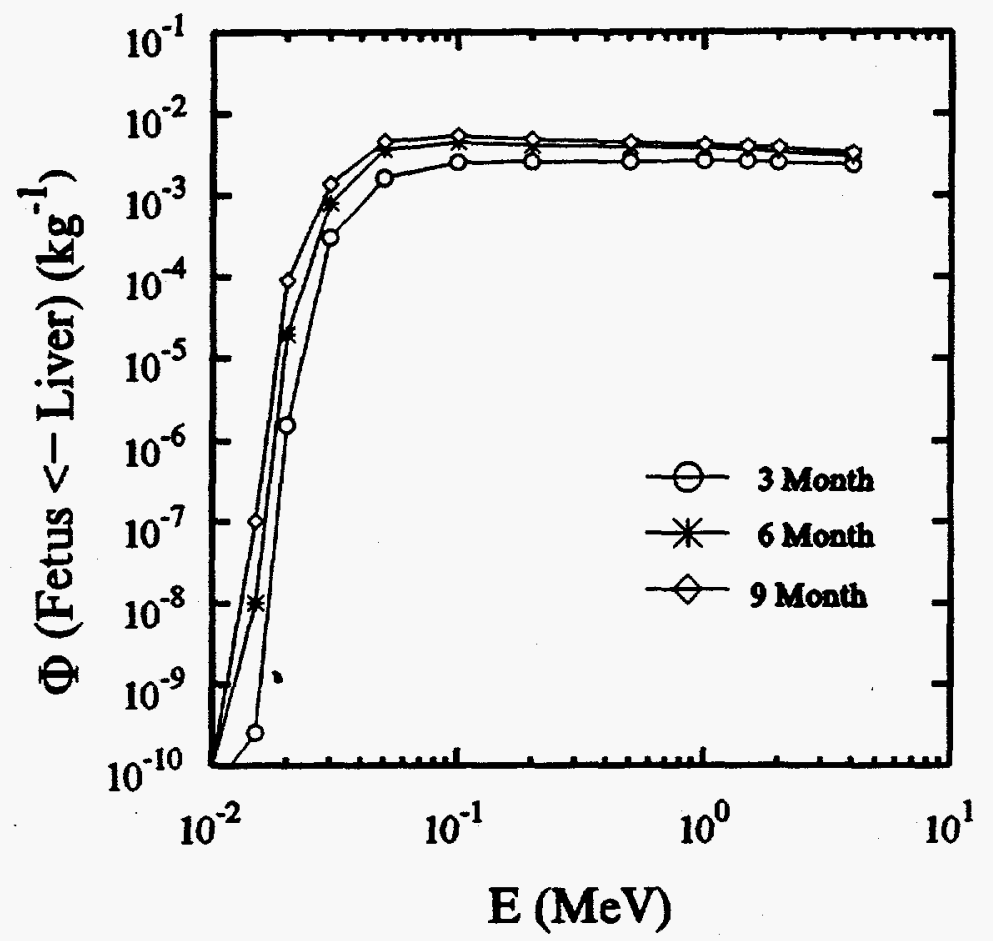

Fig. 4.2. $\Phi$ (fetus - liver) vs. initial photon energy. 


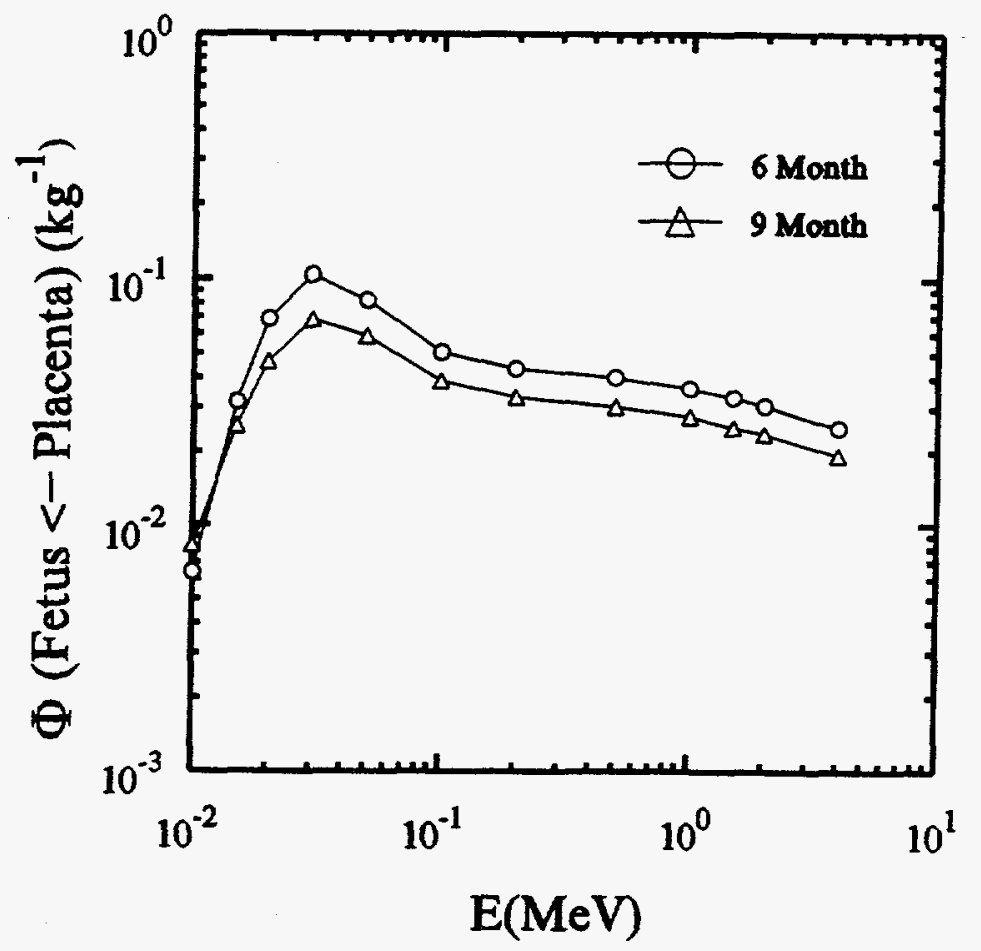

Fig. 4.3. $\Phi$ (fetus - placenta) vs. initial photon energy.

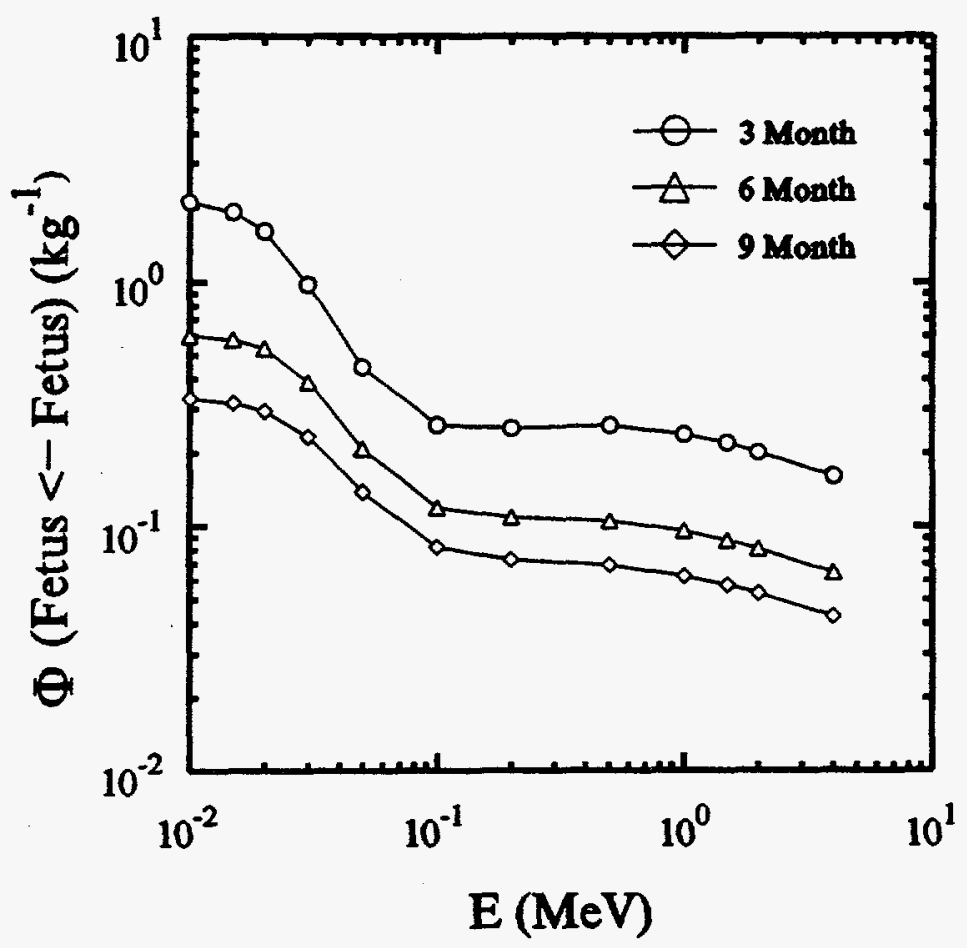

Fig. 4.4. $\Phi$ (fetus - fetus) vs. initial photon energy. 


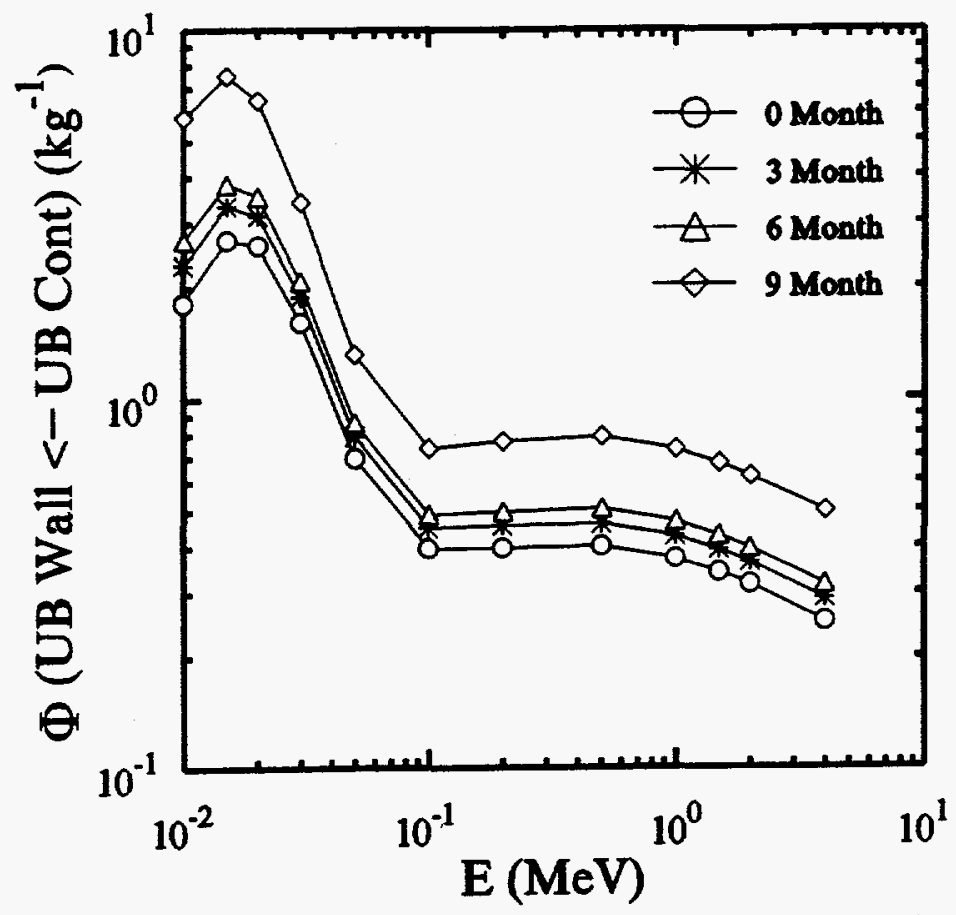

Fig. 4.5. $\Phi$ (urinary bladder wall - urinary bladder contents, mid-cycle) vs. initial photon energy.

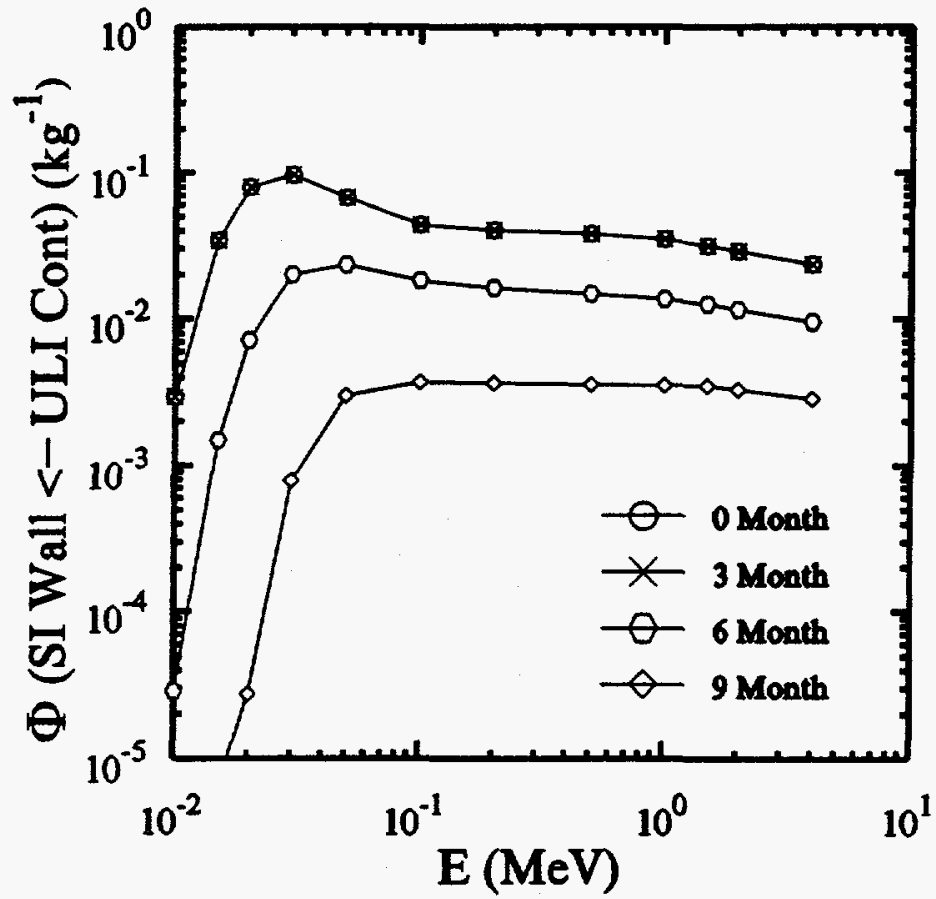

Fig. 4.6. $\Phi($ SI wall - ULI contents) vs. initial photon energy. 



\section{REFERENCES}

Beddoe, A H.; Darley, P.J.; and Spiers, F. W. Measurements of trabecular bone structure in man. Phys. Med. Biol. 21: 589-607, 1976.

Carnation Company. Pregnancy in anatomical transparencies. Los Angeles: Carnation Company, 1962.

Clemente, C. D. Anatomy, a regional atlas of the human body, 2nd ed. Baltimore: Urban and Schwarzenberg, 1981.

Cloutier, R. J.; Smith, S. A.; Watson, E. E.; Snyder, W. S.; and Warner, G. G. Dose to the fetus from radionuclides in the bladder. Health Phys. 25:147-161, 1973.

Cristy, M. and Eckerman, K. F.: Specific absorbed fractions of energy at various ages from internal photon sources. Oak Ridge National Laboratory Report ORNL/TM-8381/V1-V7, 1987.

Cristy, M. and Eckerman, K. F. SEECAL: Program to calculate age-dependent specific effective energies. Oak Ridge National Laboratory Report ORNL/TM-12351, 1993.

Davis, J. S.; Stabin, M.G.; Cristy, M.; and Ryman, J. C. Dosimetry data for the fetus derived from an anatomical model of its mother at the end of the first trimester. In:Age-related factors in radionuclide metabolism and dosimetry, eds. Gerber, G.B.; Métivier, H.; and Smith, H.; pp 389-394. Dordrecht: Martins Nijhoff Publishers, 1987.

Eastman, N. J. Williams' obstetrics, 11th ed. New York: Appleton-Century-Crofts, 1956.

Geigy Scientific Tables. Volume 1, Units of measurement, body fluids, composition of the body, nutrition. Basle, Switzerland:CIBA-Geigy, 1981.

Gillespie, E. C. Principles of uterine growth in pregnancy. Amer. J. Obst. Gynec. 59:940-459, 1950.

Hytten, F. E. and Leitch, I. The physiology of human pregnancy. Philadelphia: F. A. Davis, 1964.

International Commission on Radiological Protection. Report of the task group on Reference Man. ICRP Publication 23, Oxford: Pergamon Press, 1975.

International Commission on Radiological Protection. Limits for intakes of radionuclides by workers. ICRP Publication 30, Annals of the ICRP 2, No. 3/4, 1979.

Ivy, A.C. The functional anatomy of labor, with special emphasis to the human being. Amer. J. Obst. Gynec. 44: 952-972, 1942.

Loevinger, R. and Berman, M. MIRD Pamphlet No. 1, Revised - A revised schema for calculating the absorbed dose from biologically distributed radionuclides. New York: Society of Nuclear Medicine, 1976.

Moore, K. L. The developing human, 3rd ed. Philadelphia; W. B. Saunders, 1982. 
Reeder, S. J.; Mastroianni, Jr., L.; and Martin, L. L. Maternity Nursing, 15th ed. Philadelphia: J. B. Lippincott Company, 1983.

Reynolds, S. R. M. Physiology of the uterus. 2nd ed. New York: Paul B. Hoeber, 1949.

Ryman, J. C. and Eckerman, K. F. ALGAMP - a Monte Carlo radiation transport code for calculating specific absorbed fractions of energy from internal or external photon sources. Oak Ridge National Laboratory Report ORNL/TM-8377, in preparation.

Snyder, W. S.; Ford, M. R.; Warner, G. G.; and Fisher, Jr., H. L. MIRD Pamphlet No. 5 - Estimates of absorbed fractions for monoenergetic photon sources uniformly distributed in various organs of a heterogeneous phantom. J. Nucl. Med. Suppl No. 3, No. 5, 1969.

Snyder, W. S.; Ford, M. R.; and Warner, G. G. MIRD Pamphlet No. 5, Revised - Estimates of specific absorbed fractions for photon sources uniformly distributed in various organs of a heterogeneous phantom. New York: Society of Nuclear Medicine, 1978.

Swanson, W. W. and Iob, L. V. Mineral composition of the bone and cartilage of the human fetus. Am. J. Dis. Child. 54:1025-1029, 1937.

Watson, E. E. and Stabin, M. G. A mathematical model of the nine-month pregnant woman for calculating specific absorbed fractions. In: Age-related factors in radionuclide metabolism and dosimetry, eds. Gerber, G.B.; Métivier, H.; and Smith,H.;pp 389-394. Dordrecht: Martins Nijhoff Publishers, 1987. 
APPENDIX A

TABLES OF SPECIFIC ABSORBED FRACTIONS 

Notes on Table A.1. Nonpregnant Female: Specific Absorbed Fraction of Photon Energy $\left(\mathrm{kg}^{-1}\right)$

Body Tissues. Values of $\Phi$ are given for "Body Tissues" as a source or target region. This region replaces "Whole Body" or "Total Body" given in previous publications (e.g., Cristy and Eckerman 1987) and is defined as the living maternal body tissues, i.e., it excludes the contents of the GI tract, the contents of the urinary and gall bladders, and (for the pregnant woman) the contents of the uterus.

Urinary Bladder. Two rows of $\Phi$ s are given when source $=$ urinary bladder contents and target $=$ urinary bladder wall, labeled "mid" and "full" and designating that the bladder contents are in mid-cycle (half-full) or full.

Muscle. Values of $\Phi$ for muscle are from the Remaining Tissue compartment of each phantom (that part of the maternal tissues remaining after all defined organs are removed).

Cortical and Trabecular Bone. Values of $\Phi$ for source $=$ Cortical Bone, surface or volume distributed, are not given, but source $=$ skeleton may be used for this purpose. Similarly, $\Phi$ s for source $=$ Trabecular Bone, surface or volume distributed, are not given, but source $=$ Red Marrow may be used.

Alphabetical Ordering of Organs. The source and target organs are arranged mostly in alphabetical order. Parts of the GI tract appear together, alphabetized under the heading "GI Tract," but under this heading they appear in natural order from mouth to anus. Active marrow, endosteum (called "bone surface" by the ICRP), and skeleton appear under "Skeletal Tissues"; and (at six and nine months of gestation) fetus, fetal skeleton, fetal soft tissue, placenta, and other uterine contents appear under "Uterine Contents." Body Tissues appears at the end of the tables rather than in alphabetical order. Note that some of these organs appear only as source organs (i.e., skeleton, placenta, and other uterine contents), and endosteum appears only as a target organ. Note also that as target tissues fetal skeleton and fetal soft tissue (at six and nine months of gestation) are indented under "Fetus" and are listed as "Skeleton" and "Soft Tissue" for brevity of space.

Abbreviations. The meanings of the abbreviations used for target regions in Tables A.1 - A.4 are given in the following table. Abbreviations are not used for source regions.

\begin{tabular}{||l|l||}
\hline \multicolumn{2}{|c|}{ Abbreviations used in the Tables for Target Regions } \\
\hline Abbreviation & Meaning \\
\hline Act Marrow & Active Marrow \\
Gall Bl & Gall Bladder \\
GI Tract & Gastrointestinal Tract \\
SI & Small Intestine \\
ULI & Upper Large Intestine \\
LLI & Lower Large Intestine \\
Skeletal Tiss & Skeletal Tissues \\
Urin Bl & Urinary Bladder \\
Uterine Cont & Uterine Contents \\
\hline
\end{tabular}


Table A.1. Nonpregnant Female: Specific Absorbed Fraction of Photon Energy $\left(\mathrm{kg}^{-1}\right)$.

SOURCE $=$ ADRENALS

Energy (MeV)

\begin{tabular}{|c|c|c|c|c|c|c|c|c|c|c|c|c|}
\hline srget & 0.010 & 0.015 & 0.020 & 0.030 & 0.050 & 0.100 & 0.200 & 0.500 & 1.000 & 1.500 & 2.000 & 4.000 \\
\hline $\begin{array}{l}\text { Adrenals } \\
\text { Brain } \\
\text { Breasts } \\
\text { Gall BI Hall } \\
\text { GI tract: } \\
\text { Stonach Wall } \\
\text { SI Hall } \\
\text { ULI Hall } \\
\text { LLI Hall }\end{array}$ & $\begin{array}{l}5.69 E+01 \\
0.0 \\
0.0 \\
1.24 E-14 \\
1.89 E-16 \\
3.04 E-26 \\
3.59 E-28 \\
0.0\end{array}$ & $\begin{array}{l}3.53 E+01 \\
0.0 \\
6.40 E-13 \\
7.21 E-06 \\
\\
2.36 E-06 \\
6.74 E-10 \\
4.20 E-10 \\
5.54 E-13\end{array}$ & $\begin{array}{l}2.03 E+01 \\
1.00 E-14 \\
3.02 E-07 \\
1.15 E-03 \\
6.16 E-04 \\
6.65 E-06 \\
7.77 E-06 \\
1.31 E-07\end{array}$ & $\begin{array}{l}8.87 \mathrm{E} \\
4.33 \mathrm{E} \\
1.43 \mathrm{E}\end{array}$ & $\begin{array}{l}2.0 \\
3.6 \\
4.2 \\
5.5\end{array}$ & $\begin{array}{l}1.471 \\
2.85 \\
2.96 \\
2.518\end{array}$ & $\begin{array}{l}-05 \\
-03 \\
-02\end{array}$ & $\begin{array}{l}00 \\
05 \\
03 \\
02 \\
02 \\
03 \\
03 \\
03\end{array}$ & $\begin{array}{l}1.68 \\
1.39 \\
3.43 \\
1.49 \\
1.14 \\
5.00 \\
5.68 \\
1.48\end{array}$ & $\begin{array}{l}00 \\
.04 \\
.03 \\
.02 \\
.02 \\
.03 \\
.03 \\
.03\end{array}$ & $\begin{array}{l}1.1 \\
4.2\end{array}$ & $\begin{array}{l}2.0 \\
1.0 \\
1.0 \\
3.6 \\
4.1 \\
1.4\end{array}$ \\
\hline $\begin{array}{l}\text { Heart Holl } \\
\text { Kidneys } \\
\text { Liver } \\
\text { Lungs }\end{array}$ & $\begin{array}{l}1.80 E-16 \\
8.65 E-04 \\
5.43 E-05 \\
2.91 E-08\end{array}$ & $\begin{array}{l}1.47 E-06 \\
1.89 E-02 \\
4.26 E-03 \\
7.24 E-04\end{array}$ & $\begin{array}{l}4.50 E-04 \\
5.80 E-02 \\
1.83 E-02 \\
6.90 E-03\end{array}$ & $\begin{array}{l}8.99 E-03 \\
9.13 E-02 \\
3.72 E-02\end{array}$ & & $\begin{array}{l}1.5 \\
4.5\end{array}$ & $\begin{array}{l}2 \\
2 \\
2 \\
2\end{array}$ & & & & & \\
\hline $\begin{array}{l}\text { Muscle } \\
\text { Ovaries } \\
\text { Pancreas } \\
\text { Skeletal Tiss: } \\
\text { Act Marrow } \\
\text { Endosteun } \\
\text { Skin } \\
\text { Spleen }\end{array}$ & $\begin{array}{l}5.39 E-03 \\
0.0 \\
7.75 E-09 \\
= \\
1.94 E-05 \\
6.32 E-05 \\
4.57 E-07 \\
1.06 E-10\end{array}$ & $\begin{array}{l}1.20 E-02 \\
1.72 E-15 \\
1.23 E-03 \\
1.27 E-03 \\
3.82 E-03 \\
1.87 E-05 \\
1.77 E-04\end{array}$ & $\begin{array}{l}1.48 E-02 \\
2.06 E-08 \\
2.66 E-02 \\
4.88 E-03 \\
1.62 E-02 \\
2.60 E-04 \\
7.61 E-03\end{array}$ & $\begin{array}{l}1.30 \\
6.76 \\
9.95\end{array}$ & 9.18 & $\begin{array}{l}03 \\
03 \\
02 \\
32 \\
32\end{array}$ & 2 & $\begin{array}{l}02 \\
03 \\
03\end{array}$ & & $\begin{array}{l}02 \\
03 \\
03 \\
02\end{array}$ & $\begin{array}{l}02 \\
03 \\
03\end{array}$ & $\begin{array}{l}-03 \\
-03 \\
-03 \\
-02\end{array}$ \\
\hline $\begin{array}{l}\text { Thymus } \\
\text { Thyroid } \\
\text { Urin Bl Wall } \\
\text { Uterine Wall } \\
\text { Body Tissues }\end{array}$ & $\begin{array}{l}0.0 \\
0.0 \\
0.0 \\
0.0 \\
1.76 E-02\end{array}$ & $\begin{array}{l}5.70 E-13 \\
0.0 \\
1.00 E-19 \\
1.00 E-14 \\
1.76 E-02\end{array}$ & $\begin{array}{l}07 \\
11 \\
10 \\
88\end{array}$ & $\begin{array}{l}14 \\
16 \\
16 \\
15\end{array}$ & $\begin{array}{l}3 \\
4 \\
14 \\
4\end{array}$ & $\begin{array}{l}.03 \\
.04 \\
.04 \\
.03\end{array}$ & $\begin{array}{l}33 \\
34 \\
34 \\
3\end{array}$ & $\begin{array}{l}03 \\
04 \\
04 \\
03\end{array}$ & $\begin{array}{l}03 \\
04 \\
03 \\
03\end{array}$ & $\begin{array}{l}.03 \\
.04 \\
.03 \\
.03 \\
.03\end{array}$ & $\begin{array}{l}.03 \\
.03 \\
.03 \\
03\end{array}$ & $\begin{array}{l}-03 \\
-04 \\
-04 \\
-03 \\
-03\end{array}$ \\
\hline
\end{tabular}

SOURCE $=$ BRAIN

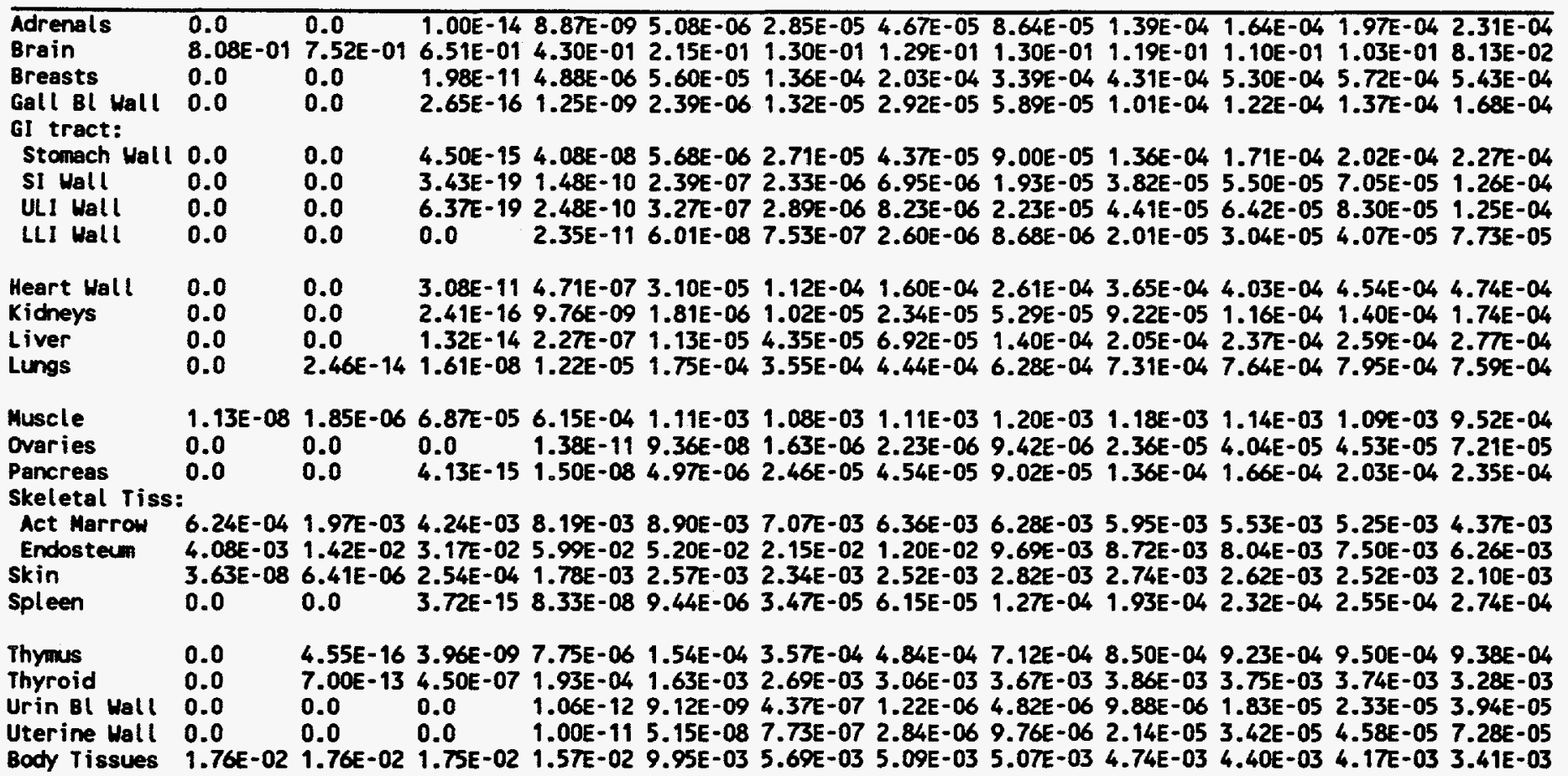


Table A.1 (cont'd). Nonpregnant Female: Specific Absorbed Fraction of Photon Energy $\left(\mathrm{kg}^{-1}\right)$.

SOURCE $=$ BREASTS

Energy (MeV)

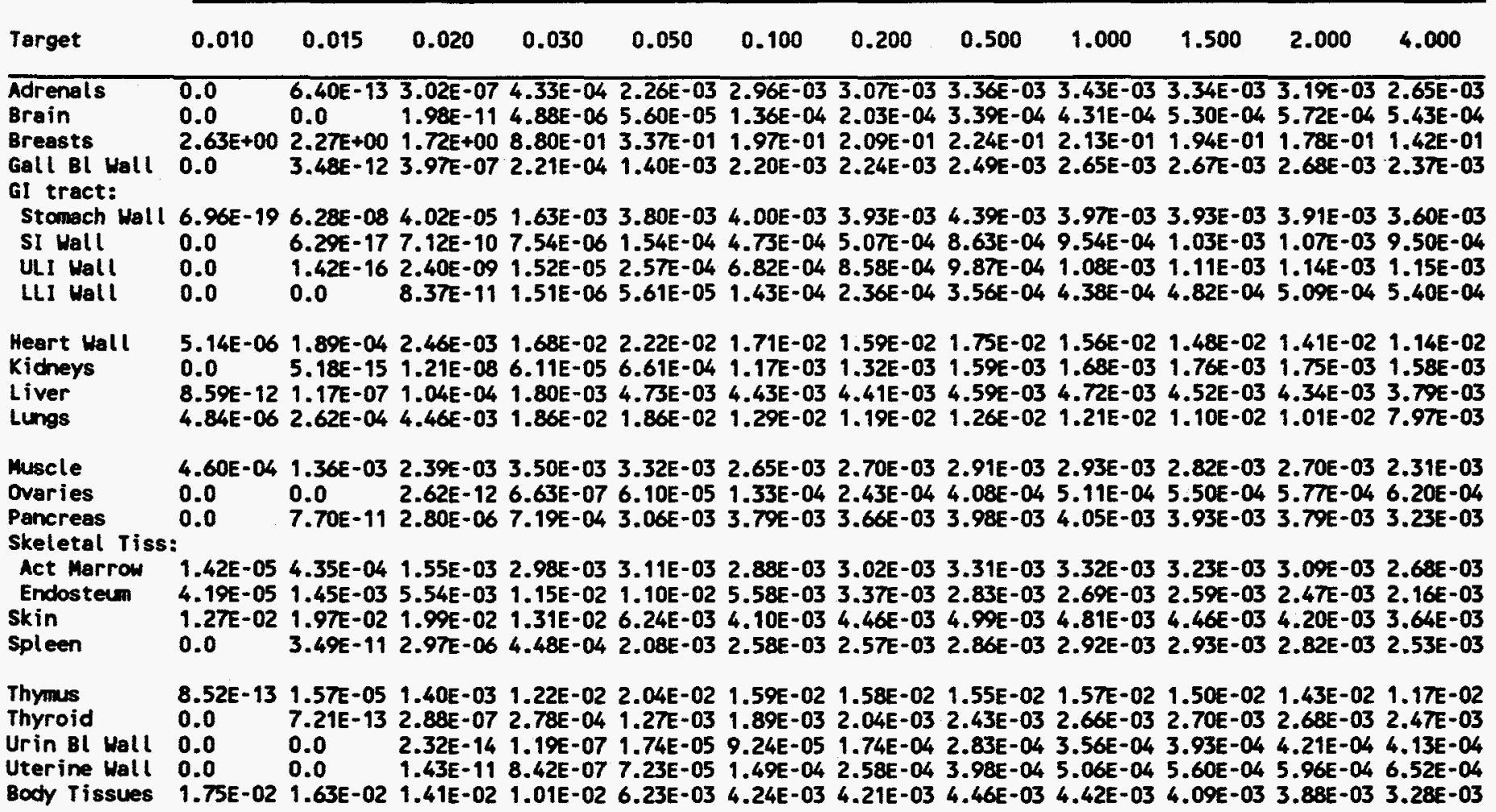

SOURCE = GALL BLADDER CONTENTS

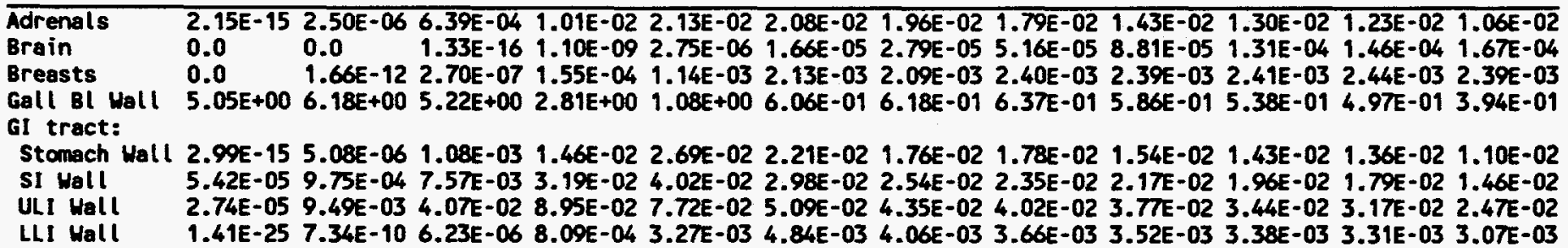

Heart Hall

Kicheys

Liver

Lungs

Muscle

Ovaries

Pancreas

Skeletal Tiss:

Act Marrow

Endosteum

Skin

Spleen

Thyous

Thyroid Urin Bl Wall

Uterine Wall

Body Tissues
7.68E-19 5.94E-08 4.26E-05 2.59E-03 6.39E-03 7.78E-03 7.01E-03 6.55E-03 6.41E-03 6.06E-03 5.73E-03 4.90E-03 7.83E-16 4.01E-06 1.12E-03 1.57E-02 2.89E-02 2.40E-02 2.07E-02 1.90E-02 1.75E-02 1.61E-02 1.50E-02 1.21E-02 5.65E-04 1.43E-02 5.12E-02 9.46E-02 7.96E-02 5.20E-02 4.65E-02 4.36E-02 4.05E-02 3.70E-02 3.44E-02 2.70E-02 1.35E-11 8.77E-08 4.54E-05 1.59E-03 4.95E-03 4.95E-03 4.70E-03 4.41E-03 3.98E-03 3.77E-03 3.66E-03 3.54E-03

9.65E-04 4.78E-03 8.80E-03 1.16E-02 9.73E-03 7.16E-03 6.60E-03 6.47E-03 6.06E-03 5.65E-03 5.36E-03 4.46E-03 3.61E-26 1.43E-09 1.53E-05 2.03E-03 6.98E-03 9.14E-03 7.51E-03 7.68E-03 7.07E-03 6.19E-03 5.68E-03 4.87E-03 2.53E-04 3.38E-03 2.35E-02 7.02E-02 6.66E-02 4.57E-02 3.93E-02 3.51E-02 3.30E-02 3.00E-02 2.76E-02 2.17E-02

3.63E-15 1.66E-06 6.01E-05 1.36E-03 4.55E-03 5.56E-03 5.56E-03 5.46E-03 5.17E-03 4.84E-03 4.60E-03 3.96E-03 6.81E-07 1.63E-05 1.56E-04 3.73E-03 1.12E-02 8.54E-03 4.96E-03 3.22E-03 2.94E-03 2.77E-03 2.64E-03 2.26E-03 4.67E-09 1.61E-06 1.02E-04 9.56E-04 1.92E-03 1.81E-03 1.85E-03 2.08E-03 2.16E-03 2.06E-03 1.97E-03 1.86E-03 2.77E-24 3.58E-09 2.36E-05 2.17E-03 7.90E-03 9.77E-03 8.73E-03 8.01E-03 7.86E-03 7.45E-03 7.09E-03 5.62E-03

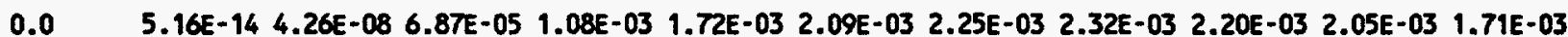
$0.0 \quad 0.0 \quad 1.60 E-12 \quad 5.05 E-07 \quad 4.27 E-05 \quad 1.78 E-04$ 3.28E-04 4.65E-04 5.26E-04 5.62E-04 5.92E-04 5.60E-04 0.0 1.00E-13 2.91E-07 2.09E-04 1.47E-03 3.54E-03 3.67E-03 3.08E-03 2.90E-03 2.85E-03 2.78E-03 $2.36 E-03$ 1.00E-21 3.81E-10 9.77E-06 1.92E-03 7.21E-03 8.67E-03 7.31E-03 6.00E-03 6.28E-03 6.05E-03 5.73E-03 5.00E-03 1.48E-03 4.82E-03 8.64E-03 1.23E-02 1.11E-02 8.10E-03 7.20E-03 6.90E-03 6.47E-03 5.97E-03 5.63E-03 4.68E-03 
Table A.1 (cont'd). Nonpregnant Female: Specific Absorbed Fraction of Photon Energy ( $\left.\mathbf{k g}^{-1}\right)$.

SOURCE = GALL BLADDER WALL

Energy (MeV)

$\begin{array}{lllllllllllll}\text { Target } & 0.010 & 0.015 & 0.020 & 0.030 & 0.050 & 0.100 & 0.200 & 0.500 & 1.000 & 1.500 & 2.000 & 4.000\end{array}$

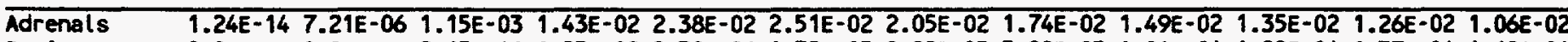

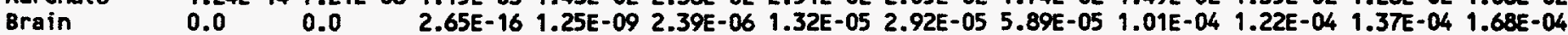

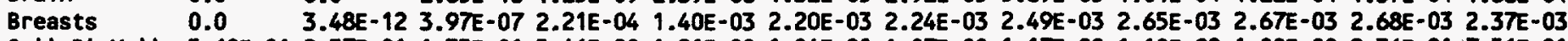

Gall Bl Wall $5.60 E+012.57 E+01 \quad 1.35 E+01 \quad 5.46 E+00 \quad 1.81 E+00 \quad 1.04 E+00 \quad 1.07 E+001.17 E+00 \quad 1.10 E+00 \quad 1.00 E+00 \quad 9.36 E-01 \quad 7.56 E-01$

GI tract:

Stomach Wall 2.01E-14 9.29E-06 1.43E-03 1.61E-02 2.99E-02 2.47E-02 2.10E-02 1.79E-02 1.57E-02 1.47E-02 1.40E-02 1.15E-02 SI Hall 2.07E-04 2.00E-03 9.97E-03 2.82E-02 3.77E-02 2.87E-02 2.48E-02 2.56E-02 $1.94 E-02$ 1.61E-02 $1.46 E-02$ 1.33E-02 ULI Hall 4.44E-04 1.82E-02 5.52E-02 9.52E-02 8.03E-02 5.38E-02 4.66E-02 4.21E-02 3.77E-02 3.42E-02 3.18E-02 2.61E-02

LLI Hall 2.14E-25 1.06E-09 6.86E-06 8.69E-04 2.99E-03 4.15E-03 3.98E-03 3.94E-03 3.79E-03 3.43E-03 3.19E-03 3.01E-03

Heart Wall 3.26E-11 1.89E-07 8.81E-05 3.04E-03 7.71E-03 8.38E-03 7.05E-03 6.69E-03 6.42E-03 6.16E-03 5.98E-03 5.35E-03

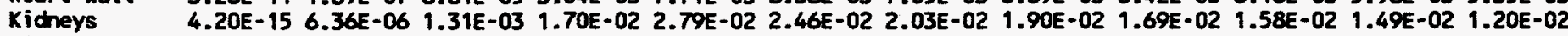
Liver $\quad 2.24 E-03 \quad 2.47 E-02 \quad 6.75 E-02$ 1.13E-01 9.14E-02 5.42E-02 4.56E-02 4.46E-02 4.14E-02 3.80E-02 3.54E-02 2.78E-02 Lungs $\quad 1.94 E-10$ 3.78E-07 8.16E-05 1.99E-03 5.86E-03 5.34E-03 4.86E-03 4.29E-03 3.91E-03 3.79E-03 3.70E-03 3.12E-03

Muscle $\quad 7.25 E-03$ 1.04E-02 1.24E-02 1.25E-02 9.50E-03 7.15E-03 6.54E-03 6.34E-03 6.00E-03 5.58E-03 5.29E-03 4.51E-03 Ovaries $\quad 1.48 E-25$ 2.35E-09 $1.73 E-05$ 1.61E-03 7.07E-03 $1.00 E-02$ 7.91E-03 $6.49 E-03 \quad 5.89 E-03 \quad 5.56 E-03$ 5.31E-03 $4.50 E-03$ Pancreas 4.14E-05 1.25E-02 4.12E-02 9.39E-02 8.32E-02 5.51E-02 4.65E-02 4.16E-02 3.91E-02 3.50E-02 3.20E-02 2.63E-02 Skeletal Tiss:

Act Marrow 1.99E-14 7.00E-07 1.08E-04 2.05E-03 5.55E-03 5.53E-03 4.75E-03 5.64E-03 5.05E-03 4.62E-03 4.36E-03 3.64E-03 Endosteum 1.00E-07 1.25E-05 1.79E-04 3.86E-03 1.15E-02 8.70E-03 4.97E-03 3.22E-03 2.98E-03 2.80E-03 2.67E-03 2.27E-03 Skin

Spleen 8.24E-09 2.24E-06 1.20E-04 1.03E-03 1.87E-03 1.82E-03 1.95E-03 2.06E-03 2.14E-03 2.00E-03 $1.91 E-03$ 1.88E-03

Thymus $\quad 0.0 \quad 1.70 E-13$ 8.31E-08 9.02E-05 9.31E-04 $1.52 E-03$ 1.84E-03 2.10E-03 2.13E-03 2.10E-03 2.09E-03 1.80E-03 Thyroid $\quad 0.0 \quad 0.0 \quad 3.32 E-12$ 6.75E-07 4.94E-05 $1.95 E-04$ 3.22E-04 4.70E-04 5.53E-04 5.90E-04 6.17E-04 5.82E-04 Urin 81 wall $0.0 \quad 1.00 E-13 \quad 3.19 E-07$ 2.23E-04 2.31E-03 4.06E-03 4.05E-03 3.65E-03 3.34E-03 3.11E-03 $2.96 E-03 \quad 2.52 E-03$ Uterine Wall 9.45E-19 5.83E-10 1.07E-05 1.43E-03 6.70E-03 9.10E-03 8.70E-03 7.10E-03 5.63E-03 5.38E-03 5.25E-03 4.40E-03 Body Tissues 1.32E-02 1.24E-02 1.30E-02 1.40E-02 1.17E-02 8.44E-03 7.55E-03 7.24E-03 6.82E-03 6.32E-03 5.99E-03 4.96E-03

\section{SOURCE $=$ GI TRACT: STOMACH CONTENTS}

\begin{tabular}{llllllllllllll}
\hline Adrenals & $5.50 E-18$ & $7.87 E-07$ & $5.41 E-04$ & $7.52 E-03$ & $1.93 E-02$ & $1.77 E-02$ & $1.52 E-02$ & $1.45 E-02$ & $1.35 E-02$ & $1.21 E-02$ & $1.12 E-02$ & $9.80 E-03$ \\
Brain & 0.0 & 0.0 & $2.66 E-15$ & $2.91 E-08$ & $5.57 E-06$ & $2.40 E-05$ & $3.83 E-05$ & $7.76 E-05$ & $1.31 E-04$ & $1.62 E-04$ & $1.89 E-04$ & $2.11 E-04$ \\
Breasts & $1.00 E-12$ & $2.04 E-08$ & $2.31 E-05$ & $1.20 E-03$ & $3.68 E-03$ & $3.59 E-03$ & $3.69 E-03$ & $4.12 E-03$ & $4.29 E-03$ & $4.10 E-03$ & $3.96 E-03$ & $3.71 E-03$ \\
Gall Bl Wall & $6.74 E-16$ & $2.98 E-06$ & $8.25 E-04$ & $1.48 E-02$ & $2.43 E-02$ & $2.26 E-02$ & $2.03 E-02$ & $1.71 E-02$ & $1.48 E-02$ & $1.35 E-02$ & $1.26 E-02$ & $1.10 E-02$ \\
GI tract: & & & & & & & & & & & & & \\
Stomach Wall & $4.20 E-01$ & $9.47 E-01$ & $1.13 E+00$ & $8.50 E-01$ & $4.05 E-01$ & $2.30 E-01$ & $2.21 E-01$ & $2.20 E-01$ & $2.03 E-01$ & $1.84 E-01$ & $1.70 E-01$ & $1.35 E-01$ \\
S1 Wall & $1.77 E-05$ & $2.63 E-04$ & $1.78 E-03$ & $9.55 E-03$ & $1.61 E-02$ & $1.38 E-02$ & $1.23 E-02$ & $1.16 E-02$ & $1.01 E-02$ & $9.48 E-03$ & $9.10 E-03$ & $8.15 E-03$ \\
ULI Wall & $6.93 E-06$ & $1.47 E-03$ & $6.97 E-03$ & $2.17 E-02$ & $2.47 E-02$ & $1.77 E-02$ & $1.54 E-02$ & $1.48 E-02$ & $1.39 E-02$ & $1.28 E-02$ & $1.18 E-02$ & $9.98 E-03$ \\
LLI Wall & $5.86 E-08$ & $9.40 E-06$ & $3.52 E-04$ & $3.29 E-03$ & $6.89 E-03$ & $5.88 E-03$ & $5.37 E-03$ & $5.34 E-03$ & $5.27 E-03$ & $5.08 E-03$ & $4.86 E-03$ & $4.14 E-03$
\end{tabular}

Heart Mall

Kidneys

Liver

Lungs

Muscle

Ovaries

Pancreas

Skeletel Tiss:

Act Merrow

Endosteum

skin

Spleen

Thyous

Thyroid Urin Bl Wall

Uterine Hall

Body Tissues
1.58E-06 1.00E-04 1.90E-03 $1.29 E-02$ 2.06E-02 $1.67 E-02 \quad 1.47 E-02 \quad 1.37 E-02 \quad 1.26 E-02 \quad 1.18 E-02 \quad 1.09 E-02 \quad 8.98 E-03$ 4.27E-17 1.00E-06 3.89E-04 8.64E-03 1.80E-02 1.54E-02 1.38E-02 1.28E-02 1.27E-02 $1.12 E-02$ 9.98E-03 8.18E-03 3.00E-10 1.95E-05 6.66E-04 6.09E-03 1.24E-02 1.14E-02 1.01E-02 9.45E-03 8.86E-03 8.24E-03 7.77E-03 6.49E-03 1.27E-05 2.27E-04 1.75E-03 7.06E-03 1.01E-02 8.25E-03 7.28E-03 6.91E-03 6.30E-03 5.93E-03 5.63E-03 4.71E-03

5.44E-05 1.51E-03 5.06E-03 9.52E-03 8.75E-03 6.38E-03 5.94E-03 5.80E-03 5.55E-03 5.18E-03 4.89E-03 4.07E-03 3.77E-27 2.34E-10 3.24E-06 5.38E-04 2.83E-03 4.64E-03 3.98E-03 3.80E-03 3.67E-03 3.5TE-03 3.50E-03 3.15E-03 1.44E-03 1.35E-02 6.58E-02 1.49E-01 1.19E-01 7.64E-02 6.78E-02 6.35E-02 5.55E-02 5.10E-02 4.80E-02 3.86E-02

1.24E-06 3.37E-05 3.47E-04 1.63E-03 3.54E-03 4.28E-03 4.34E-03 4.29E-03 4.16E-03 4.00E-03 3.81E-03 3.32E-03 3.73E-06 1.12E-04 1.24E-03 6.36E-03 1.18E-02 8.23E-03 4.78E-03 3.32E-03 2.94E-03 2.75E-03 2.62E-03 2.30E-03 9.53E-07 3.57E-05 4.69E-04 1.92E-03 2.34E-03 2.09E-03 2.16E-03 2.47E-03 2.39E-03 2.33E-03 2.27E-03 2.03E-03 5.43E-10 2.54E-04 1.05E-02 5.53E-02 6.67E-02 4.56E-02 3.99E-02 3.45E-02 3.02E-02 2.87E-02 2.74E-02 2.34E-02

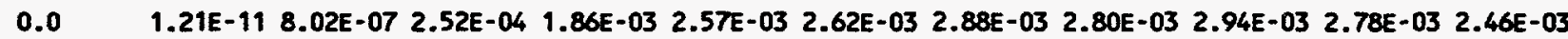

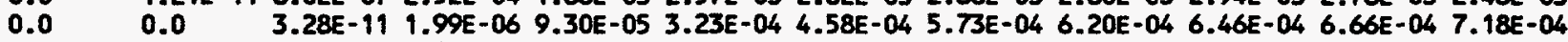

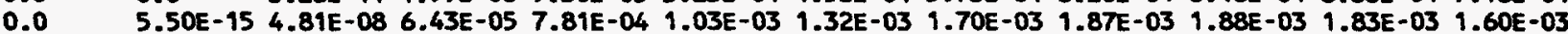
1.01E-22 5.23E-09 1.18E-06 3.95E-04 2.55E-03 3.75E-03 3.83E-03 3.77E-03 3.94E-03 3.78E-03 3.56E-03 3.00E-03 9.11E-04 3.10E-03 6.19E-03 9.88E-03 9.29E-03 6.70E-03 6.03E-03 5.84E-03 5.52E-03 5.12E-03 4.85E-03 4.05E-03 
Table A.1 (cont'd). Nonpregnant Female: Specific Absorbed Fraction of Photon Energy $\left(\mathrm{kg}^{-1}\right)$.

SOURCE = GI TRACT: STOMACH WALL

Energy (MeV)

\begin{tabular}{|c|c|c|c|c|c|c|c|c|c|c|c|c|}
\hline Target & 0.010 & 0.015 & 0.020 & 0.030 & 0.050 & 0.100 & 0.200 & 0.500 & 1.000 & 1.500 & 2.000 & 4.000 \\
\hline $\begin{array}{l}\text { Adrenals } \\
\text { Brain } \\
\text { Breasts } \\
\text { Gall Bl Wall } \\
\text { GI tract: } \\
\text { Stomach Wall } \\
\text { SI Wall } \\
\text { ULI Mall } \\
\text { LLI Wall }\end{array}$ & $\begin{array}{l}1.89 E-16 \\
0.0 \\
6.96 E-19 \\
2.01 E-14 \\
5.80 E+00 \\
4.00 E-05 \\
3.50 E-04 \\
1.86 E-10\end{array}$ & $\begin{array}{l}2.36 E-06 \\
0.0 \\
6.28 E-08 \\
9.29 E-06 \\
3.77 E+00 \\
1.09 E-03 \\
6.59 E-03 \\
3.91 E-05\end{array}$ & $\begin{array}{l}6.16 E-04 \\
4.50 E-15 \\
4.02 E-05 \\
1.43 E-03 \\
2.44 E+00 \\
3.42 E-03 \\
1.94 E-02 \\
6.25 E-04\end{array}$ & $\begin{array}{l}8.67 E-03 \\
4.08 E-08 \\
1.63 E-03 \\
1.61 E-02 \\
1.15 E+00 \\
1.11 E-02 \\
3.22 E-02 \\
4.67 E-03\end{array}$ & $\begin{array}{l}2.04 E-02 \\
5.68 E-06 \\
3.80 E-03 \\
2.99 E-02 \\
4.69 E-01 \\
1.74 E-02 \\
3.02 E-02 \\
7.83 E-03\end{array}$ & $\begin{array}{l}1.77 E-02 \\
2.71 E-05 \\
4.00 E-03 \\
2.47 E-02 \\
2.73 E-01 \\
1.46 E-02 \\
2.29 E-02 \\
6.08 E-03\end{array}$ & $\begin{array}{l}1.64 E-02 \\
4.37 E-05 \\
3.93 E-03 \\
2.10 E-02 \\
2.70 E-01 \\
1.27 E-02 \\
2.01 E-02 \\
6.34 E-03\end{array}$ & $\begin{array}{l}1.42 E-02 \\
9.00 E-05 \\
4.39 E-03 \\
1.79 E-02 \\
\\
2.89 E-01 \\
1.16 E-02 \\
1.80 E-02 \\
5.60 E-03\end{array}$ & $\begin{array}{l}1.14 E-02 \\
1.36 E-04 \\
3.97 E-03 \\
1.57 E-02 \\
2.68 E-01 \\
1.09 E-02 \\
1.61 E-02 \\
5.44 E-03\end{array}$ & $\begin{array}{l}1.14 E-02 \\
1.71 E-04 \\
3.93 E-03 \\
1.47 E-02 \\
2.43 E-01 \\
9.88 E-03 \\
1.46 E-02 \\
5.22 E-03\end{array}$ & $\begin{array}{l}1.15 E-02 \\
2.02 E-04 \\
3.91 E-03 \\
1.40 E-02 \\
2.26 E-01 \\
9.19 E-03 \\
1.37 E-02 \\
5.07 E-03\end{array}$ & $\begin{array}{l}1.08 E-02 \\
2.27 E-04 \\
3.60 E-03 \\
1.15 E-02 \\
1.78 E-01 \\
8.16 E-03 \\
1.12 E-02 \\
4.31 E-03\end{array}$ \\
\hline $\begin{array}{l}\text { Heart Wall } \\
\text { Kidneys } \\
\text { Liver } \\
\text { Lungs }\end{array}$ & $\begin{array}{l}1.00 E-05 \\
1.40 E-15 \\
1.78 E-06 \\
1.33 E-05\end{array}$ & $\begin{array}{l}3.15 E-04 \\
2.80 E-06 \\
7.76 E-05 \\
9.31 E-04\end{array}$ & $\begin{array}{l}4.22 E-03 \\
6.54 E-04 \\
1.13 E-03 \\
3.79 E-03\end{array}$ & $\begin{array}{l}1.80 E-02 \\
8.07 E-03 \\
7.06 E-03 \\
9.34 E-03\end{array}$ & $\begin{array}{l}2 . \\
1 . \\
1 . \\
1 .\end{array}$ & & $\begin{array}{l}1.64 \\
1.32 \\
1.02 \\
7.71\end{array}$ & & $\begin{array}{l}-02 \\
-02 \\
-03 \\
-03\end{array}$ & $\begin{array}{l}1.38 \\
1.0 \\
8.26\end{array}$ & $\begin{array}{l}1.25 E-02 \\
9.55 E-03 \\
7.78 E-03 \\
6.00 E-03\end{array}$ & $\begin{array}{l}1.01 E-02 \\
8.55 E-03 \\
6.53 E-03 \\
4.96 E-03\end{array}$ \\
\hline $\begin{array}{l}\text { Muscle } \\
\text { Ovaries } \\
\text { Pancreas } \\
\text { Skeletal Tiss }\end{array}$ & $\begin{array}{l}2.85 E-03 \\
8.31 E-26 \\
2.62 E-03\end{array}$ & $\begin{array}{l}6.5 \\
8.5 \\
4.5\end{array}$ & $\begin{array}{l}1.00 E \\
6.06 E \\
1.19 E\end{array}$ & $\begin{array}{l}02 \\
04 \\
01\end{array}$ & & & & & $\begin{array}{l}5.9 \\
4.1\end{array}$ & & & $\begin{array}{l}4.28 \\
3.22 \\
3.9\end{array}$ \\
\hline $\begin{array}{l}\text { Act Marrow } \\
\text { Endosteum } \\
\text { skin } \\
\text { Spleen }\end{array}$ & $\begin{array}{l}1.50 E-05 \\
2.67 E-06 \\
5.00 E-06 \\
2.16 E-08\end{array}$ & $\begin{array}{l}1.26 E-04 \\
4.18 E-04 \\
1.11 E-04 \\
1.11 E-03\end{array}$ & $\begin{array}{l}6.06 E-04 \\
2.17 E-03 \\
6.74 E-04 \\
1.47 E-02\end{array}$ & $\begin{array}{l}1.94 E-03 \\
7.54 E-03 \\
2.11 E-03 \\
6.14 E-02\end{array}$ & $\begin{array}{l}3.72 E-03 \\
1.25 E-02 \\
2.34 E-03 \\
6.68 E-02\end{array}$ & $\begin{array}{l}4.38 \\
8.35 \\
2.12 \\
4.48\end{array}$ & $\begin{array}{l}4.41 E-03 \\
4.83 E-03 \\
2.14 E-03 \\
3.83 E-02\end{array}$ & $\begin{array}{l}4.43 E-03 \\
3.42 E-03 \\
2.43 E-03 \\
3.72 E-02\end{array}$ & $\begin{array}{l}4.29 E-03 \\
.3 .04 E-03 \\
2.62 E-03 \\
3.40 E-02\end{array}$ & $\begin{array}{l}4.08 E-03 \\
2.83 E-03 \\
2.51 E-03 \\
2.98 E-02\end{array}$ & $\begin{array}{l}3.91 E-03 \\
2.69 E-03 \\
2.43 E-03 \\
2.70 E-02\end{array}$ & $\begin{array}{l}3.36 E-03 \\
2.34 E-03 \\
2.06 E-03 \\
2.25 E-02\end{array}$ \\
\hline $\begin{array}{l}\text { Thymus } \\
\text { Thyroid } \\
\text { Urin Bl Well } \\
\text { Uterine Wall } \\
\text { Body Tissues }\end{array}$ & $\begin{array}{l}0.0 \\
0.0 \\
0.0 \\
1.00 E-23\end{array}$ & $\begin{array}{l}3.81 E-11 \\
0.0 \\
2.06 E-14 \\
1.00 E-10 \\
1\end{array}$ & $\begin{array}{l}1.11 E-06 \\
5.77 E-11 \\
3.25 E-08 \\
2.13 E-06\end{array}$ & $\begin{array}{l}3.52 E-04 \\
2.42 E-06 \\
5.16 E-05 \\
6.59 E-04\end{array}$ & $\begin{array}{l}1.91 E-03 \\
1.00 E-04 \\
7.93 E-04 \\
2.94 E-03 \\
1.03 E-02\end{array}$ & $\begin{array}{l}2.98 E-03 \\
3.47 E-04 \\
1.01 E-03 \\
4.34 E-03\end{array}$ & $\begin{array}{l}3.01 E-03 \\
5.32 E-04 \\
1.13 E-03 \\
3.45 E-03\end{array}$ & $\begin{array}{l}2.85 E \\
6.51 E \\
1.22 E \\
3.35 E\end{array}$ & $\begin{array}{l}=-03 \\
=-04 \\
=-03 \\
=-03\end{array}$ & $\begin{array}{l}-03 \\
-04 \\
-04 \\
-03 \\
-03\end{array}$ & $\begin{array}{l}-03 \\
-04 \\
-03 \\
-03\end{array}$ & $\begin{array}{l}2.60 E-03 \\
7.24 E-04 \\
1.61 E-03 \\
3.08 E-03 \\
4.49 E-03\end{array}$ \\
\hline
\end{tabular}

SOURCE = GI TRACT: SMALL INTESTINE CONTENTS

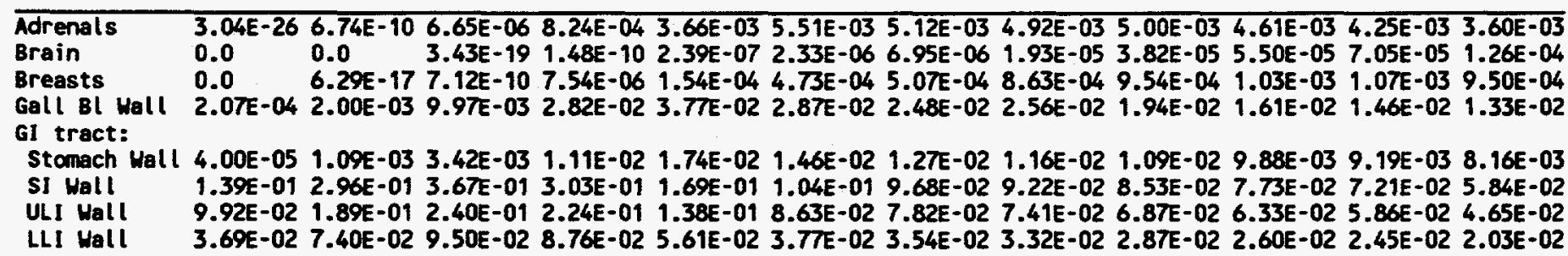

Heart Wall $0.0 \quad 1.28 E-13 \quad 5.46 E-08$ 5.02E-05 $7.82 E-04 \quad 1.47 E-03 \quad 1.54 E-03 \quad 1.67 E-03 \quad 1.80 E-03 \quad 1.74 E-03 \quad 1.65 E-03 \quad 1.44 E-03$ Kicheys Liver

Lungs 1.11E-07 2.29E-05 1.01E-03 8.01E-03 1.52E-02 1.41E-02 1.31E-02 1.24E-02 1.08E-02 1.07E-02 1.04E-02 7.99E-03 1.65E-05 2.62E-04 9.33E-04 4.19E-03 8.29E-03 7.89E-03 6.97E-03 6.62E-03 6.52E-03 6.20E-03 5.88E-03 5.09E-03 0.0 3.11E-13 8.20E-08 7.17E-05 7.50E-04 1.14E-03 1.24E-03 1.22E-03 $1.35 E-03 \quad 1.46 E-03 \quad 1.50 E-03 \quad 1.39 E-03$

Muscle Ovaries Pancreas Skeletal Tiss:

Act Marrow Endosteum

9.40E-04 2.80E-03 5.16E-03 8.6TE-03 9.09E-03 7.16E-03 6.54E-03 6.33E-03 5.97E-03 5.67E-03 5.38E-03 4.45E-03 4.19E-03 3.04E-02 7.67E-02 1.17E-01 9.03E-02 5.90E-02 5.27E-02 4.83E-02 4.45E-02 4.10E-02 3.80E-02 3.05E-02 1.46E-17 1.71E-07 8.55E-05 3.33E-03 9.84E-03 1.03E-02 8.96E-03 7.86E-03 7.68E-03 7.17E-03 6.68E-03 5.59E-03

Skin

Spleen

3.90E-04 1.15E-03 2.62E-03 6.89E-03 1.12E-02 1.09E-02 9.99E-03 1.00E-02 9.22E-03 8.37E-03 7.73E-03 6.32E-03 4.99E-04 1.64E-03 4.13E-03 1.22E-02 1.87E-02 1.13E-02 6.20E-03 4.15E-03 3.85E-03 3.58E-03 3.35E-03 2.83E-03 2.59E-09 1.33E-06 1.11E-04 8.02E-04 1.81E-03 1.84E-03 1.90E-03 2.10E-03 2.19E-03 2.21E-03 2.18E-03 1.91E-03 6.02E-10 6.59E-07 1.07E-04 2.23E-03 6.62E-03 7.17E-03 6.47E-03 6.48E-03 6.11E-03 5.58E-03 5.15E-03 4.12E-03

Thymus $\quad 0.0 \quad 0.0 \quad 6.84 E-11$ 2.37E-06 1.47E-04 3.22E-04 4.57E-04 5.70E-04 6.83E-04 7.45E-04 7.79E-04 8.23E-04 Thyroid $\quad 0.0 \quad 0.0 \quad 3.16 E-15$ 2.13E-08 $6.12 E-06$ 5.80E-05 7.88E-05 $1.18 E-04 \quad 1.61 E-04$ 1.93E-04 2.19E-04 2.97E-04

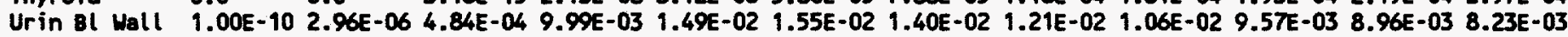
Uterine Hall 2.78E-04 4.50E-03 3.25E-02 8.73E-02 7.86E-02 5.39E-02 4.62E-02 4.29E-02 3.86E-02 3.51E-02 3.24E-02 2.63E-02 Body Tissues 3.12E-03 7.16E-03 1.00E-02 1.23E-02 1.10E-02 7.98E-03 7.08E-03 6.79E-03 6.37E-03 5.91E-03 5.61E-03 4.62E-03 
Table A.1 (cont'd). Nonpregnant Female: Specific Absorbed Fraction of Photon Energy $\left(\mathrm{kg}^{-1}\right)$.

SOURCE = GI TRACT: SMALL INTESTINE WALL_ Energy (MeV)

$\begin{array}{llllllllllllll}\text { Target } & 0.010 & 0.015 & 0.020 & 0.030 & 0.050 & 0.100 & 0.200 & 0.500 & 1.000 & 1.500 & 2.000 & 4.000\end{array}$

Adrenals $\quad 3.04 E-26 \quad 6.74 E-10 \quad 6.65 E-06 \quad 8.24 E-04 \quad 3.66 E-03 \quad 5.51 E-03 \quad 5.12 E-03 \quad 4.92 E-03 \quad 5.00 E-03 \quad 4.61 E-034.25 E-03 \quad 3.60 E-03$

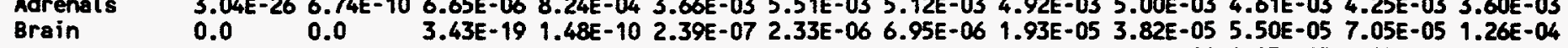

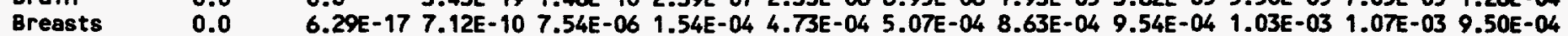
Gall BL Wall 2.07E-04 2.00E-03 9.97E-03 2.82E-02 3.77E-02 2.87E-02 2.48E-02 2.56E-02 1.94E-02 1.61E-02 1.46E-02 1.33E-02 GI tract:

Stomach Wall 4.00E-05 1.09E-03 3.42E-03 1.11E-02 1.74E-02 1.46E-02 1.27E-02 1.16E-02 1.09E-02 9.88E-03 9.19E-03 8.16E-03 SI Wall 1.52E+00 1.26E+00 9.78E-01 5.74E-01 2.66E-01 1.62E-01 1.57E-01 1.57E-01 1.41E-01 1.28E-01 $1.20 E-019.40 E-02$ ULI Wall 9.92E-02 1.89E-01 2.40E-01 2.24E-01 1.38E-01 8.63E-02 7.82E-02 7.41E-02 6.87E-02 6.33E-02 5.86E-02 4.65E-02 LLI Wall 3.69E-02 7.40E-02 9.50E-02 8.76E-02 5.61E-02 3.77E-02 3.54E-02 3.32E-02 2.87E-02 2.60E-02 2.45E-02 2.03E-02

Heart Wall $\quad 0.0 \quad 1.28 E-13 \quad 5.46 E-08$ 5.02E-05 $7.82 E-04 \quad 1.47 E-03 \quad 1.54 E-03 \quad 1.67 E-03 \quad 1.80 E-03 \quad 1.74 E-03 \quad 1.65 E-03 \quad 1.44 E-03$

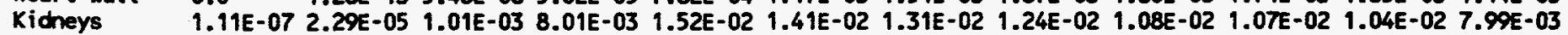
Liver $\quad 1.65 E-05$ 2.62E-04 9.33E-04 4.19E-03 8.29E-03 7.89E-03 6.97E-03 6.62E-03 6.52E-03 6.20E-03 5.88E-03 5.09E-03

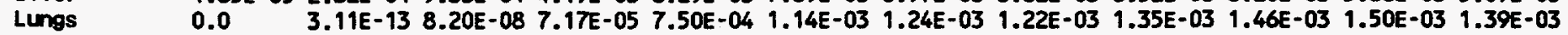

Muscle $\quad 9.40 E-04 \quad 2.80 E-03$ 5.16E-03 8.67E-03 9.09E-03 7.16E-03 6.54E-03 6.33E-03 5.97E-03 5.67E-03 5.38E-03 4.45E-03 Ovaries $\quad 4.19 E-03$ 3.04E-02 7.67E-02 1.17E-01 9.03E-02 5.90E-02 5.27E-02 4.83E-02 4.45E-02 4.10E-02 3.80E-02 3.05E-02 Pancress 1.46E-17 1.71E-07 8.55E-05 3.33E-03 9.84E-03 1.03E-02 8.96E-03 7.86E-03 7.68E-03 7.17E-03 6.68E-03 5.59E-03 Skeletal Tiss:

Act Marrow 3.90E-04 1.15E-03 2.62E-03 6.89E-03 1.12E-02 1.09E-02 9.99E-03 1.00E-02 9.22E-03 8.37E-03 7.73E-03 6.32E-03 Endosteum 4.99E-04 1.64E-03 4.13E-03 1.22E-02 1.87E-02 1.13E-02 6.20E-03 4.15E-03 3.85E-03 3.58E-03 3.35E-03 2.83E-03 Skin $\quad 2.59 E-09$ 1.33E-06 $1.11 E-04$ 8.02E-04 $1.81 E-03 \quad 1.84 E-03 \quad 1.90 E-03 \quad 2.10 E-03 \quad 2.19 E-03 \quad 2.21 E-03 \quad 2.18 E-03 \quad 1.91 E-03$ Spleen $\quad 6.02 E-106.59 E-07$ 1.07E-04 2.23E-03 6.62E-03 7.17E-03 6.47E-03 6.48E-03 6.11E-03 5.58E-03 5.15E-03 4.12E-03

Thymus $\quad 0.0 \quad 0.0 \quad 6.84 E-11$ 2.37E-06 1.47E-04 3.22E-04 4.57E-04 5.70E-04 6.83E-04 7.45E-04 7.79E-04 8.23E-04

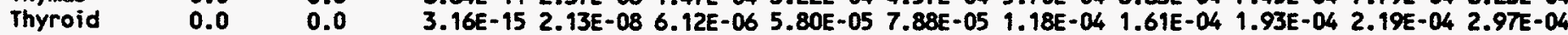
Urin Bl Wall 1.00E-10 2.96E-06 4.84E-04 9.99E-03 1.49E-02 1.55E-02 1.40E-02 1.21E-02 1.06E-02 9.57E-03 8.96E-03 8.23E-03 Uterine Mall 2.78E-04 4.50E-03 3.25E-02 8.73E-02 7.86E-02 5.39E-02 4.62E-02 4.29E-02 3.86E-02 3.51E-02 3.24E-02 2.63E-02 Body Tissues 1.66E-02 1.54E-02 1.45E-02 1.39E-02 1.15E-02 8.31E-03 7.43E-03 7.17E-03 6.69E-03 6.23E-03 5.91E-03 4.85E-03

\section{SOURCE = GI TRACT: UPPER LARGE INTESTINE CONTENTS}

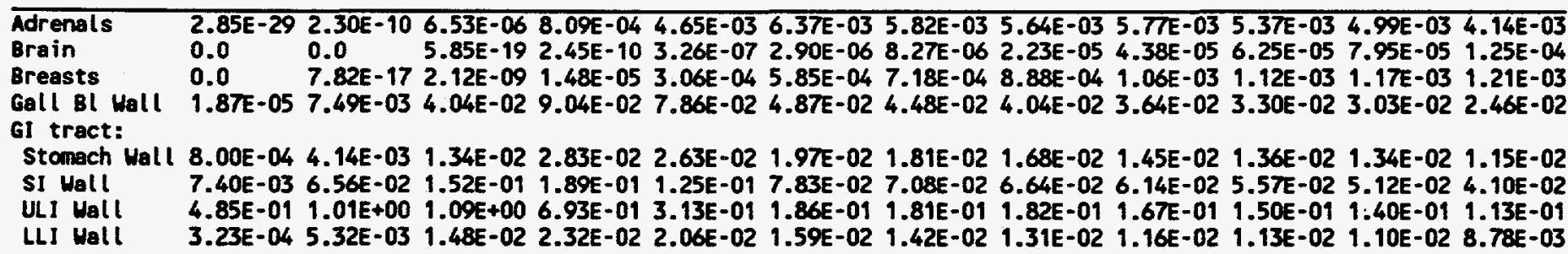

Heart Wall $\quad 0.0 \quad 2.37 E-13$ 1.38E-07 1.02E-04 1.15E-03 2.02E-03 2.06E-03 2.16E-03 2.38E-03 2.18E-03 2.07E-03 2.02E-03 Kidneys $\quad 9.48 E-17$ 9.44E-07 3.35E-04 6.33E-03 $1.44 E-02$ 1.34E-02 $1.18 E-02$ 1.11E-02 $1.08 E-02$ 9.51E-03 8.68E-03 7.65E-03

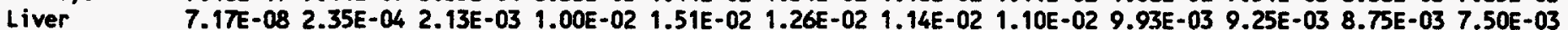
Lungs $\quad 0.0 \quad 4.31 E-13 \quad 1.63 E-07 \quad 1.16 E-04 \quad 8.43 E-04 \quad 1.49 E-03 \quad 1.48 E-03 \quad 1.66 E-03 \quad 1.76 E-03 \quad 1.80 E-03 \quad 1.78 E-03 \quad 1.59 E-03$

Muscle $\quad 9.11 E-05$ 1.15E-03 3.93E-03 8.42E-03 8.78E-03 6.83E-03 6.28E-03 6.10E-03 5.71E-03 5.38E-03 5.10E-03 $4.26 E-03$ ovaries $\quad 4.42 E-04$ 2.68E-02 8.34E-02 $1.05 E-01$ 7.07E-02 $4.87 E-02$ 4.37E-02 4.10E-02 3.96E-02 3.50E-02 3.15E-02 2.63E-02 Pancreas 9.22E-19 2.30E-07 1.63E-04 4.46E-03 1.26E-02 1.27E-02 1.04E-02 9.46E-03 8.93E-03 8.28E-03 7.70E-03 6.14E-03 Skeletal Tiss:

Act Marrow 6.66E-06 4.48E-04 2.05E-03 5.99E-03 8.89E-03 8.63E-03 8.15E-03 8.14E-03 7.57E-03 6.90E-03 6.41E-03 5.39E-03

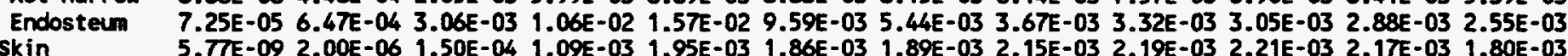
skin Spleen 9.64E-18 2.22E-07 9.85E-05 2.22E-03 6.97E-03 7.21E-03 6.33E-03 5.88E-03 5.76E-03 5.34E-03 5.01E-03 4.58E-03

Thymus $\quad 0.0 \quad 1.71 E-191.37 E-10 \quad 4.35 E-06$ 1.75E-04 3.37E-04 4.81E-04 6.26E-04 6.99E-04 7.38E-04 7.69E-04 8.47E-04

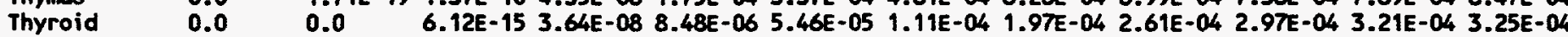
Urin BL Wall 1.00E-09 2.26E-06 3.13E-04 4.50E-03 1.02E-02 1.17E-02 9.63E-03 9.37E-03 9.40E-03 8.7TE-03 8.06E-03 6.01E-03 Uterine Mall 1.00E-07 4. 10E-05 2.61E-03 2.23E-02 3.51E-02 2.83E-02 2.35E-02 2.10E-02 $1.88 E-02$ 1.80E-02 $1.72 E-02$ 1.37E-02 Body Tissues 1.55E-03 4.49E-03 7.62E-03 1.09E-02 1.01E-02 7.44E-03 6.63E-03 6.35E-03 5.94E-03 5.51E-03 5.23E-03 4.35E-03 
Table A.1 (cont'd). Nonpregnant Female: Specific Absorbed Fraction of Photon Energy $\left(\mathbf{k g}^{-1}\right)$.

SOURCE $=$ GI TRACT: UPPER LARGE INTESTINE WALL

Energy (MeV)

\begin{tabular}{|c|c|c|c|c|c|c|c|c|c|c|c|c|}
\hline rget & 0.010 & 0.015 & 0.020 & 0.030 & 0.050 & 0.100 & 0.200 & 0.500 & 1.000 & 1.500 & 2.000 & 4.000 \\
\hline $\begin{array}{l}\text { Irenals } \\
\text { ain } \\
\text { easts } \\
\text { ll Bl Wall } \\
\text { tract: }\end{array}$ & $\begin{array}{l}3.59 E-28 \\
0.0 \\
0.0 \\
4.44 E-04\end{array}$ & $\begin{array}{l}4.20 E-10 \\
0.0 \\
1.42 E-16 \\
1.82 E-02\end{array}$ & $\begin{array}{l}6.37 \\
2.40 \\
5.52\end{array}$ & $\begin{array}{l}2.4 \\
1.5 \\
9.5\end{array}$ & & & & & & & & \\
\hline $\begin{array}{l}\text { Stomach Wall } \\
\text { SI Wall } \\
\text { ULI Hall } \\
\text { LLI Wall }\end{array}$ & $\begin{array}{l}3.50 \\
9.921 \\
4.021 \\
3.56\end{array}$ & & $\begin{array}{l}2.40 E \\
1.74 E \\
2.01 E\end{array}$ & & & & & $\begin{array}{l}2.18 E-01 \\
1.37 E-02\end{array}$ & & & & $\begin{array}{l}1.12 E-02 \\
4.65 E-02 \\
1.34 E-01 \\
7.98 E-03\end{array}$ \\
\hline
\end{tabular}

Heart Mall $0.0 \quad 4.01 E-13 \quad 1.58 E-07 \quad 1.18 E-04 \quad 1.25 E-03 \quad 1.90 E-03 \quad 1.95 E-03 \quad 2.07 E-03 \quad 2.10 E-03 \quad 2.10 E-03 \quad 2.05 E-03 \quad 2.00 E-03$ Kidneys Liver

Lungs 2.02E-15 2.14E-06 3.76E-04 6.98E-03 1.46E-02 1.36E-02 1.21E-02 1.13E-02 1.02E-02 9.57E-03 9.04E-03 7.03E-03 1.27E-05 2.70E-04 2.36E-03 1.04E-02 1.46E-02 $1.23 E-02 \quad 1.12 E-02 \quad 1.04 E-02$ 9.76E-03 9.10E-03 8.60E-03 7.81E-03 0.0 7.30E-13 1.88E-07 1.16E-04 1.08E-03 1.36E-03 $1.43 E-03$ 1.49E-03 1.56E-03 $1.54 E-03 \quad 1.49 E-03 \quad 1.32 E-03$

Muscle

Ovaries

Pancreas

Skeletal Tiss:

Act Marrow

Endosteum

Skin

Spleien

6.39E-04 2.17E-03 4.46E-03 7.96E-03 8.15E-03 6.34E-03 5.82E-03 5.68E-03 5.40E-03 5.10E-03 4.82E-03 4.03E-03 3.50E-02 1.08E-01 1.46E-01 1.29E-01 7.92E-02 5.33E-02 5.03E-02 4.57E-02 4.31E-02 3.96E-02 3.67E-02 3.04E-02 1.52E-17 3.88E-07 1.89E-04 4.87E-03 1.25E-02 1.24E-02 1.04E-02 8.91E-03 8.77E-03 8.02E-03 7.45E-03 6.45E-03

6.66E-06 3.97E-04 2.01E-03 5.90E-03 8.88E-03 8.63E-03 8.06E-03 8.20E-03 7.65E-03 6.90E-03 6.35E-03 5.30E-03 6.23E-05 5.92E-04 2.92E-03 1.05E-02 1.57E-02 9.57E-03 5.41E-03 3.58E-03 3.38E-03 3.11E-03 2.89E-03 2.54E-03 3.56E-08 5.56E-06 2.00E-04 1.09E-03 2.02E-03 1.87E-03 2.02E-03 2.11E-03 2.18E-03 2.17E-03 2.17E-03 $1.78 E-03$ 1.27E-10 3.90E-07 1.16E-04 2.91E-03 6.22E-03 7.11E-03 6.30E-03 6.08E-03 5.79E-03 5.60E-03 5.44E-03 4.98E-03

Thymus $\quad 0.0 \quad 2.91 E-191.57 E-104.43 E-06 \quad 1.49 E-04 \quad 4.15 E-04 \quad 6.38 E-04 \quad 7.35 E-04 \quad 7.72 E-04 \quad 8.10 E-04 \quad 8.32 E-04 \quad 8.43 E-04$

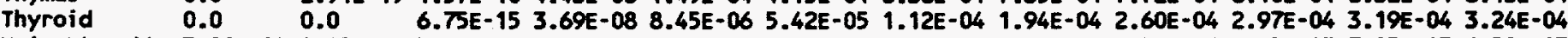
Urin Bl Wall 3.88E-09 2.95E-06 3.27E-04 5.22E-03 1.37E-02 1.41E-02 1.23E-02 $1.05 E-02$ 9.31E-03 8.4TE-03 7.93E-03 6.58E-03 Uterine Hall 8.95E-10 1.21E-04 3.68E-03 2.40E-02 3.62E-02 2.96E-02 2.52E-02 2.21E-02 1.99E-02 $1.85 E-02$ 1.72E-02 $1.31 E-02$ Body Tissues 1.54E-02 1.32E-02 1.26E-02 1.26E-02 1.06E-02 7.77E-03 7.02E-03 6.78E-03 6.33E-03 5.85E-03 5.54E-03 4.63E-03

SOURCE = GI TRACT: LOWER LARGE INTESTINE CONTENTS

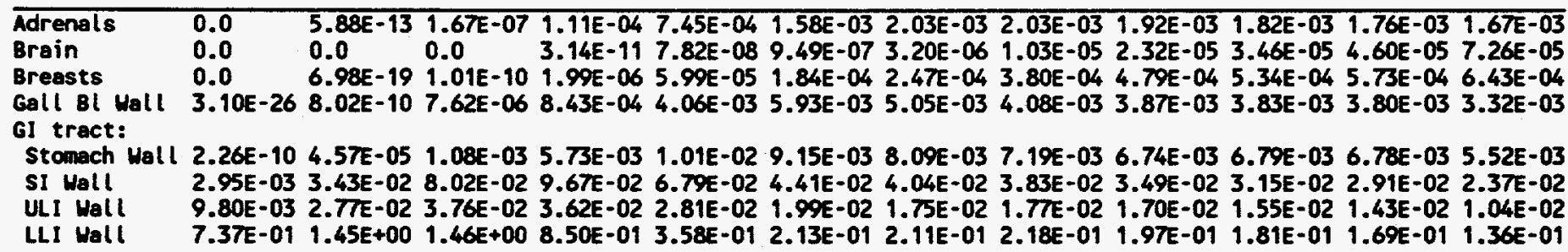

Heart Wall $\quad 0.0 \quad 1.02 E-15 \quad 4.83 E-09$ 1.48E-05 3.71E-04 5.00E-04 6.34E-04 7.83E-04 8.72E-04 9.14E-04 9.44E-04 1.01E-03

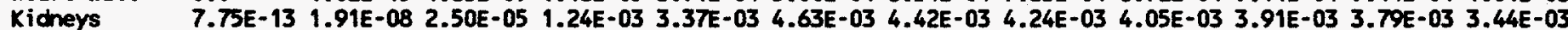

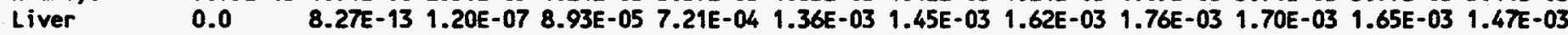
lungs $\quad 0.0 \quad 3.42 E-15$ 6.50E-09 $1.33 E-05$ 2.34E-04 4.11E-04 4.46E-04 5.36E-04 6.72E-04 7.12E-04 7.23E-04 7.35E-04

Muscle $\quad 9.67 E-05$ 1.82E-03 5.31E-03 9.90E-03 1.00E-02 7.72E-03 7.11E-03 6.97E-03 6.49E-03 6.07E-03 5.72E-03 4.80E-03 Ovaries $\quad 3.73 E-03$ 9.30E-02 2.25E-01 2.35E-01 1.32E-01 8.11E-02 7.82E-02 8.01E-02 6.90E-02 6.11E-02 5.58E-02 4.60E-02 Pancreas $\quad 1.52 E-27$ 2.49E-10 3.93E-06 5.16E-04 2.71E-03 4.24E-03 3.62E-03 3.37E-03 3.16E-03 2.96E-03 2.87E-03 2.85E-03 Skeletal Tiss:

Act Marrow 3.07E-05 1.75E-03 7.62E-03 1.54E-02 1.61E-02 1.34E-02 1.24E-02 1.22E-02 1.10E-02 1.01E-02 9.49E-03 7.67E-03 Endosteum 3.93E-05 2.52E-03 1.17E-02 2.60E-02 2.68E-02 1.36E-02 7.37E-03 5.36E-03 $4.64 E-03$ 4.32E-03 4.09E-03 3.42E-03 skin Spleen 1.85E-04 3.90E-04 6.77E-04 1.35E-03 2.16E-03 2.21E-03 2.20E-03 2.31E-03 2.49E-03 2.41E-03 2.33E-03 1.95E-03 1.58E-21 9.26E-09 1.73E-05 9.34E-04 3.28E-03 4.54E-03 3.58E-03 3.42E-03 3.39E-03 3.31E-03 3.26E-03 3.22E-03

Thymus $\quad 0.0 \quad 0.0 \quad 3.50 E-12 \quad 4.74 E-07 \quad 3.24 E-05 \quad 1.31 E-04 \quad 2.21 E-04 \quad 3.29 E-04 \quad 4.09 E-04 \quad 4.45 E-04 \quad 4.67 E-04 \quad 4.55 E-04$

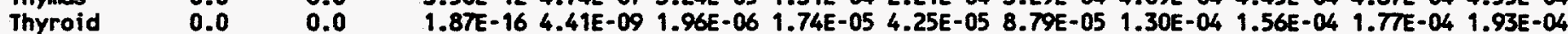
Urin Bl Wall 7.41E-09 4.47E-04 9.43E-03 3.59E-02 4.68E-02 3.30E-02 2.63E-02 2.32E-02 2.10E-02 $1.97 \mathrm{TE}-02$ 1.87E-02 $1.65 E-02$ Uterine Wall 1.00E-07 4.98E-05 4.61E-03 3.33E-02 5.14E-02 3.66E-02 3.18E-02 2.82E-02 2.54E-02 2.39E-02 2.22E-02 1.59E-02 Body Tissues 1.76E-03 5.25E-03 8.84E-03 1.22E-02 $1.10 E-02 \quad 7.74 E-03 \quad 6.83 E-03 \quad 6.59 E-03 \quad 6.12 E-03 \quad 5.68 E-03$ 5.39E-03 $4.50 E-03$ 
Table A.1 (cont'd). Nonpregnant Female: Specific Absorbed Fraction of Photon Energy (kg-1).

SOURCE $=$ GI TRACT: LOWER LARGE INTESTINE WALL Energy (MeV)

$\begin{array}{llllllllllllllll}\text { Terget } & 0.010 & 0.015 & 0.020 & 0.030 & 0.050 & 0.100 & 0.200 & 0.500 & 1.000 & 1.500 & 2.000 & 4.000\end{array}$

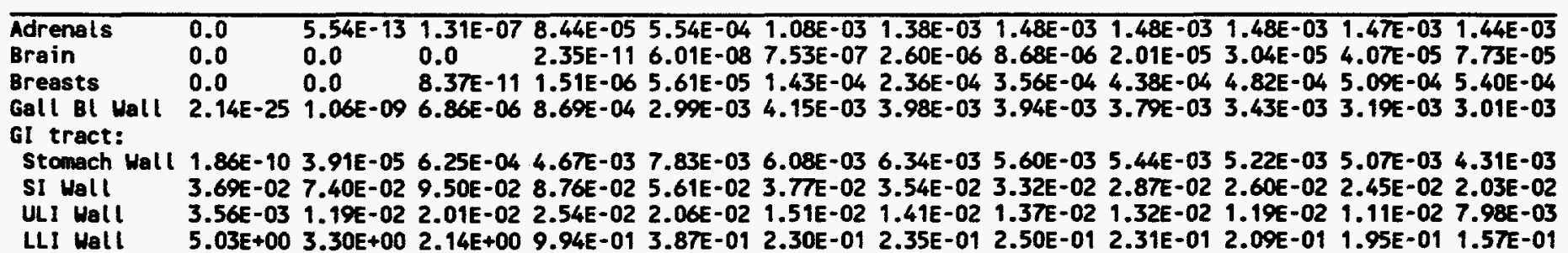

Heart wall $\quad 0.0 \quad 8.10 E-16 \quad 3.74 E-09$ 1.13E-05 2.35E-04 3.60E-04 5.24E-04 7.29E-04 8.23E-04 8.44E-04 8.50E-04 8.38E-04

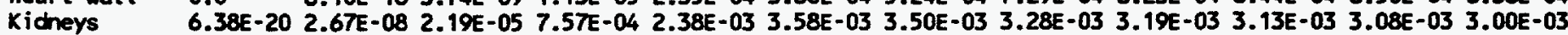

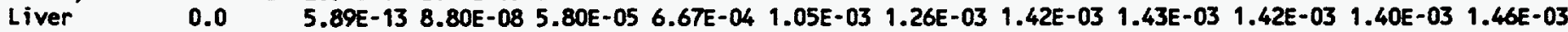
lungs $\quad 0.0 \quad 2.13 E-15$ 4.43E-09 9.80E-06 $1.78 E-04$ 3.33E-04 4.60E-04 5.24E-04 5.49E-04 5.77E-04 5.97E-04 6.46E-04

Muscle $\quad 2.50 E-03 \quad 5.30 E-03 \quad 7.40 E-03$ 1.01E-02 $1.09 E-02 \quad 8.83 E-03 \quad 7.24 E-03 \quad 6.50 E-03 \quad 6.20 E-03$ 5.70E-03 5.40E-03 4.56E-03 Overies $\quad 8.04 E-021.85 E-01$ 2.44E-01 2.04E-01 1.15E-01 7.32E-02 6.85E-02 6.85E-02 6.15E-02 5.58E-02 5.18E-02 4.03E-02 Pancreas $\quad 2.39 E-16 \quad 1.85 E-10$ 2.80E-06 6.17E-04 2.18E-03 3.17E-03 2.87E-03 3.02E-03 2.98E-03 2.88E-03 2.77E-03 2.38E-03 Skeletal Tiss:

Act Marrow 2.45E-04 2.67E-03 8.40E-03 1.54E-02 1.58E-02 1.32E-02 1.24E-02 1.22E-02 1.10E-02 1.01E-02 9.47E-03 7.62E-03 Endosteum 3.13E-04 3.81E-03 1.29E-02 2.63E-02 2.68E-02 $1.37 E-02$ 7.60E-03 5.49E-03 $4.75 E-03$ 4.41E-03 $4.17 E-03$ 3.47E-03

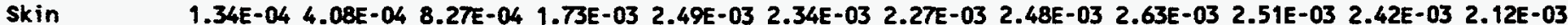
Spleen $\quad 2.43 E-13$ 8.07E-09 1.30E-05 9.20E-04 2.38E-03 3.34E-03 3.36E-03 3.17E-03 2.82E-03 2.68E-03 2.60E-03 2.67E-03

Thymus $\quad 0.0 \quad 0.0 \quad 2.70 E-12 \quad 3.60 E-07$ 2.50E-05 1.05E-04 1.80E-04 2.83E-04 3.51E-04 3.85E-04 4.05E-04 4.01E-04

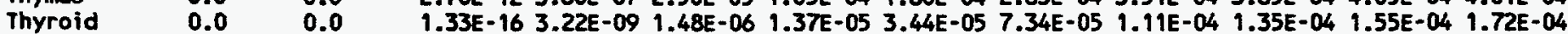
Urin Bl Wall 1.77E-07 1.26E-03 1.49E-02 5.28E-02 5.95E-02 4.28E-02 3.63E-02 3.19E-02 2.83E-02 2.56E-02 2.39E-02 1.96E-02 Uterine Wall 2.00E-07 7.97E-05 4.66E-03 2.96E-02 4.58E-02 3.48E-02 2.84E-02 2.40E-02 2.20E-02 2.17E-02 2.13E-02 $1.92 E-02$ Bocty Tissues 1.59E-02 1.43E-02 1.41E-02 1.43E-02 1.17E-02 8.14E-03 7.29E-03 7.07E-03 6.59E-03 6.11E-03 5.79E-03 4.79E-03

\section{SOURCE $=$ HEART CONTENTS}

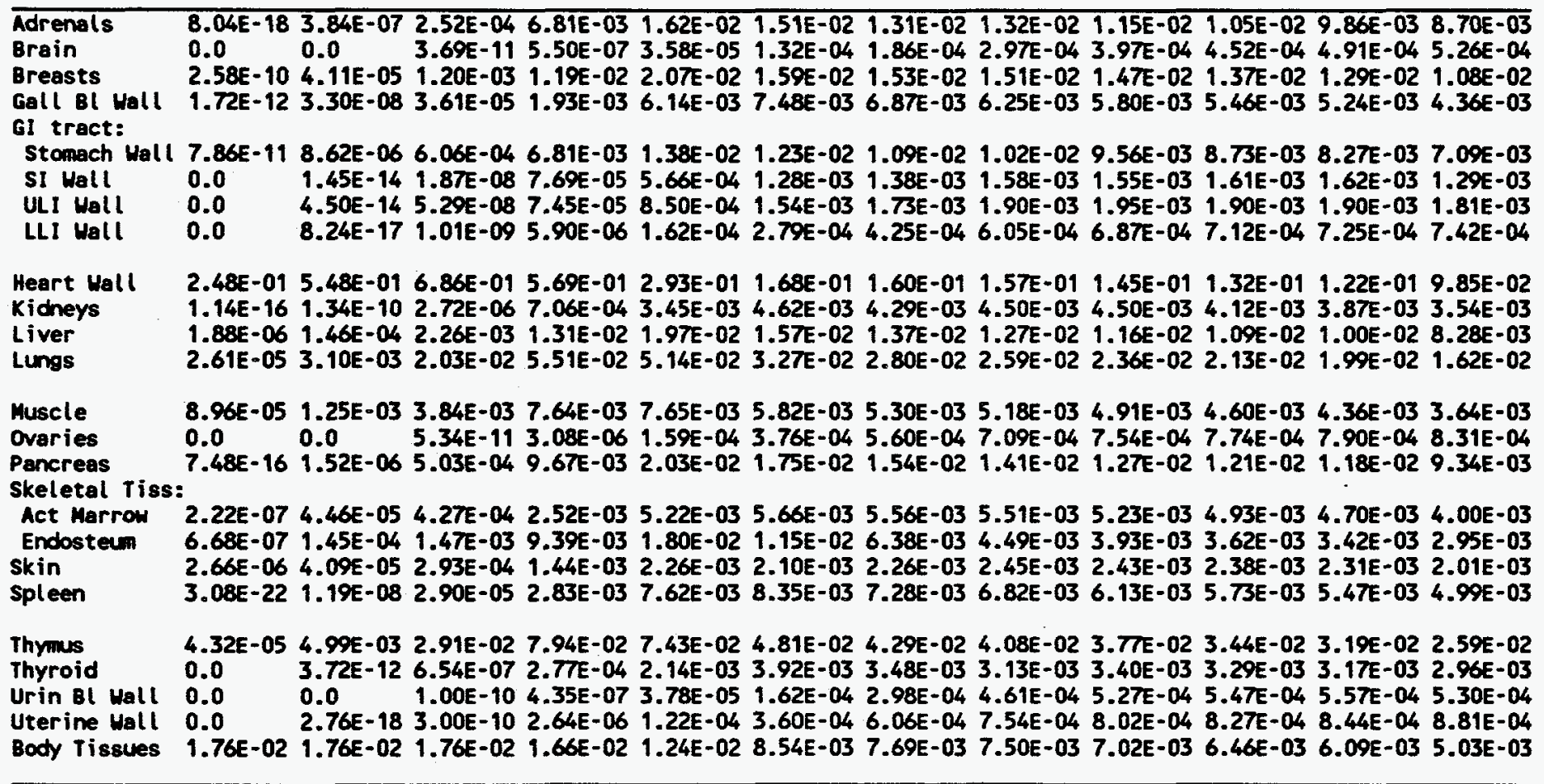


Table A.1 (cont'd). Nonpregnant Female: Specific Absorbed Fraction of Photon Energy $\left(\mathrm{kg}^{-1}\right)$.

SOURCE = HEART WALL

Energy (MeV)

\begin{tabular}{|c|c|c|c|c|c|c|c|c|c|c|c|c|}
\hline Target & 0.010 & 0.015 & 0.020 & 0.030 & 0.050 & 0.100 & 0.200 & 0.500 & 1.000 & 1.500 & 2.000 & 4.000 \\
\hline $\begin{array}{l}\text { Adrenals } \\
\text { Brain } \\
\text { Breasts } \\
\text { Gall BL Wall } \\
\text { GI tract: } \\
\text { Stomach Wall } \\
\text { SI Wall } \\
\text { ULI Wall } \\
\text { LLI Wall }\end{array}$ & $\begin{array}{l}1.80 E-16 \\
0.0 \\
5.14 E-06 \\
3.26 E-11 \\
\\
1.00 E-05 \\
0.0 \\
0.0 \\
0.0\end{array}$ & $\begin{array}{l}1.47 E-06 \\
0.0 \\
1.89 E-04 \\
1.89 E-07 \\
\\
3.15 E-04 \\
1.28 E-13 \\
4.01 E-13 \\
8.10 E-16\end{array}$ & $\begin{array}{l}4.50 E-04 \\
3.08 E-11 \\
2.46 E-03 \\
8.81 E-05\end{array}$ & $\begin{array}{l}8.99 E-03 \\
4.71 E-07 \\
1.68 E-02 \\
3.04 E-03\end{array}$ & $\begin{array}{l}1.90 E-02 \\
3.10 E-05 \\
2.22 E-02 \\
7.71 E-03\end{array}$ & $\begin{array}{l}1.66 E-02 \\
1.12 E-04 \\
1.71 E-02 \\
8.38 E-03 \\
1.86 E-02 \\
1.47 E-03 \\
1.90 E-03 \\
3.60 E-04\end{array}$ & $\begin{array}{l}1.41 E-02 \\
1.60 E-04 \\
1.59 E-02 \\
7.05 E-03\end{array}$ & $\begin{array}{l}1.35 E-02 \\
2.61 E-04 \\
1.75 E-02 \\
6.69 E-03\end{array}$ & $\begin{array}{l}1.33 E-02 \\
3.65 E-04 \\
1.56 E-02 \\
6.42 E-03\end{array}$ & $\begin{array}{l}1.17 E-02 \\
4.03 E-04 \\
1.48 E-02 \\
6.16 E-03\end{array}$ & $\begin{array}{l}1.06 E-02 \\
4.54 E-04 \\
1.41 E-02 \\
5.98 E-03\end{array}$ & $\begin{array}{l}8.59 E-03 \\
4.74 E-04 \\
1.14 E-02 \\
5.35 E-03 \\
1.01 E-02 \\
1.44 E-03 \\
2.00 E-03 \\
8.38 E-04\end{array}$ \\
\hline
\end{tabular}

Heart Hall 3.49E+00 2.54E+00 1.79E+00 9.29E-01 4.02E-01 2.35E-01 2.36E-01 2.46E-01 2.25E-01 2.03E-01 1.87E-01 1.52E-01 Kidneys $\quad 6.80 E-26 \quad 6.76 E-10 \quad 6.27 E-06 \quad 8.52 E-04$ 3.73E-03 5.12E-03 4.79E-03 4.73E-03 4.69E-03 4.37E-03 4.15E-03 3.56E-03 Liver $\quad 1.17 E-05$ 1.08E-03 5.73E-03 1.75E-02 2.14E-02 1.64E-02 1.46E-02 1.37 E-02 $1.26 E-02$ 1.16E-02 1.08E-02 9.07E-03 Lungs $\quad 2.10 E-04$ 7.13E-03 2.72E-02 5.56E-02 4.83E-02 3.06E-02 2.63E-02 2.48E-02 2.24E-02 2.05E-02 1.90E-02 1.54E-02

Muscle $\quad 1.95 E-03$ 4.54E-03 6.94E-03 9.17E-03 8.03E-03 5.95E-03 5.55E-03 5.46E-03 5.18E-03 4.82E-03 4.55E-03 3.78E-03

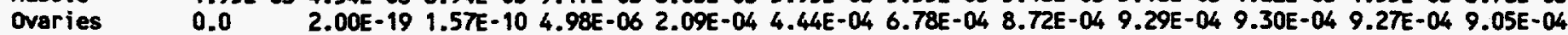
Pencreas 5.67E-13 1.76E-05 2.04E-03 1.61E-02 2.90E-02 2.25E-02 1.93E-02 1.80E-02 1.55E-02 1.41E-02 1.32E-02 1.13E-02 Skeletal Tiss:

Act Marrow 4.11E-06 1.44E-04 7.16E-04 2.7TE-03 5.07E-03 5.36E-03 5.29E-03 5.30E-03 5.05E-03 4.81E-03 4.56E-03 3.90E-03 Endosteum 1.24E-05 4.76E-04 2.51E-03 1.05E-02 1.75E-02 1.09E-02 6.07E-03 4.33E-03 3.82E-03 3.55E-03 3.34E-03 2.87E-03 skin

Spleen

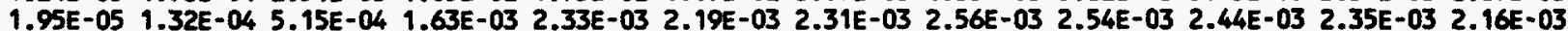
2.77E-19 1.13E-07 1.23E-04 4.39E-03 1.24E-02 1.06E-02 9.46E-03 8.80E-03 8.35E-03 8.14E-03 7.95E-03 6.73E-03

Thymus Thyroid 4.56E-04 9.39E-03 3.11E-02 6.71E-02 6.12E-02 4.03E-02 3.66E-02 3.48E-02 3.19E-02 2.96E-02 2.72E-02 2.25E-02

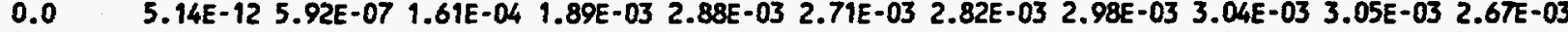
Urin BL Wall $0.0 \quad 0.0 \quad 1.00 E-11$ 6.96E-07 4.91E-05 1.92E-04 3.17E-04 4.62E-04 5.44E-04 5.81E-04 6.08E-04 5.74E-04 Uterine Hall $0.0 \quad 2.00 E-18$ 1.09E-10 4.20E-06 2.00E-04 5.20E-04 7.00E-04 7.70E-04 7.70E-04 7.60E-04 7.50E-04 7.40E-04 Body Tissues $1.76 E-02$ 1.76E-02 1.76E-02 1.62E-02 1.21E-02 8.23E-03 7.49E-03 7.35E-03 6.83E-03 6.29E-03 5.94E-03 4.92E-03

\section{SOURCE $=$ KIDNEYS}

\begin{tabular}{lllllllllllll}
\hline Adrenals & $8.65 E-04$ & $1.89 E-02$ & $5.80 E-02$ & $9.13 E-02$ & $7.05 E-02$ & $4.90 E-02$ & $4.52 E-02$ & $4.42 E-02$ & $4.03 E-02$ & $3.68 E-02$ & $3.44 E-02$ & $2.83 E-02$ \\
Brain & 0.0 & 0.0 & $2.41 E-16$ & $9.76 E-09$ & $1.81 E-06$ & $1.02 E-05$ & $2.34 E-05$ & $5.29 E-05$ & $9.22 E-05$ & $1.16 E-04$ & $1.40 E-04$ & $1.74 E-04$ \\
Breasts & 0.0 & $5.18 E-15$ & $1.21 E-08$ & $6.11 E-05$ & $6.61 E-04$ & $1.17 E-03$ & $1.32 E-03$ & $1.59 E-03$ & $1.68 E-03$ & $1.76 E-03$ & $1.75 E-03$ & $1.58 E-03$ \\
Gall Bl Wall & $4.20 E-15$ & $6.36 E-06$ & $1.31 E-03$ & $1.70 E-02$ & $2.79 E-02$ & $2.46 E-02$ & $2.03 E-02$ & $1.90 E-02$ & $1.69 E-02$ & $1.58 E-02$ & $1.49 E-02$ & $1.20 E-02$ \\
GI tract: & & & & & & & & & & & & \\
Stomach Wall & $1.40 E-15$ & $2.80 E-06$ & $6.54 E-04$ & $8.07 E-03$ & $1.83 E-02$ & $1.51 E-02$ & $1.32 E-02$ & $1.19 E-02$ & $1.18 E-02$ & $1.04 E-02$ & $9.55 E-03$ & $8.55 E-03$ \\
SI Wall & $1.11 E-07$ & $2.29 E-05$ & $1.01 E-03$ & $8.01 E-03$ & $1.52 E-02$ & $1.41 E-02$ & $1.31 E-02$ & $1.24 E-02$ & $1.08 E-02$ & $1.07 E-02$ & $1.04 E-02$ & $7.99 E-03$ \\
ULI Wall & $2.02 E-15$ & $2.14 E-06$ & $3.76 E-04$ & $6.98 E-03$ & $1.46 E-02$ & $1.36 E-02$ & $1.21 E-02$ & $1.13 E-02$ & $1.02 E-02$ & $9.57 E-03$ & $9.04 E-03$ & $7.03 E-03$ \\
LLI Wall & $6.38 E-20$ & $2.67 E-08$ & $2.19 E-05$ & $7.57 E-04$ & $2.38 E-03$ & $3.58 E-03$ & $3.50 E-03$ & $3.28 E-03$ & $3.19 E-03$ & $3.13 E-03$ & $3.08 E-03$ & $3.00 E-03$
\end{tabular}

Heart Wall

Kidneys

Liver

6.80E-26 6.76E-10 6.27E-06 8.52E-04 3.75E-03 5.12E-03 4.79E-03 4.73E-03 4.69E-03 4.37E-03 4.15E-03 3.56E-03

Lungs 3.42E+00 2.88E+00 2.15E+00 1.09E+00 4.27E-01 2.48E-01 2.59E-01 2.67E-01 2.46E-01 2.27E-01 2.11E-01 1.72E-01 6.82E-05 8.58E-04 5.20E-03 1.85E-02 2.29E-02 1.80E-02 1.64E-02 $1.55 E-02$ 1.42E-02 $1.34 E-02 \quad 1.26 E-02$ 1.00E-02 3.36E-10 5.80E-07 1.15E-04 2.22E-03 4.66E-03 4.95E-03 4.72E-03 4.58E-03 4.43E-03 4.21E-03 4.05E-03 3.65E-03

Muscle

Overies

Pancreas

Skeletal Tiss:

Act Marrow 4.36E-06 3.43E-04 1.88E-03 6.33E-03 9.91E-03 9.94E-03 9.95E-03 9.87E-03 9.29E-03 8.72E-03 8.22E-03 6.94E-03 Endosteun 8.52E-06 7.72E-04 4.62E-03 1.63E-02 2.25E-02 1.25E-02 7.18E-03 5.34E-03 4.77E-03 4.41E-03 4.17E-03 3.59E-03

Skin

Spleen

1.72E-03 5.13E-03 8.69E-03 1.03E-02 8.05E-03 6.06E-03 5.82E-03 5.82E-03 5.56E-03 5.20E-03 4.92E-03 4.13E-03 1.40E-27 3.29E-10 5.40E-06 7.33E-04 3.48E-03 5.08E-03 4.69E-03 4.89E-03 4.80E-03 4.44E-03 4.19E-03 3.87E-03 4.09E-12 6.31E-05 4.00E-03 3.00E-02 4.17E-02 3.19E-02 2.77E-02 2.63E-02 2.39E-02 2.21E-02 2.05E-02 1.66E-02

Thymus

Thyinus $\quad 0.0$

Thyroid $\quad 0.0$ 1.84E-05 2.25E-04 1.35E-03 3.05E-03 2.63E-03 2.20E-03 2.44E-03 2.64E-03 2.74E-03 2.62E-03 $2.52 E-03$ 2.19E-03 1.41E-04 1.02E-02 4.37E-02 8.44E-02 6.53E-02 4.22E-02 3.81E-02 3.66E-02 3.35E-02 3.07E-02 2.85E-02 2.30E-02

$0.0 \quad 3.30 E-16 \quad 5.08 E-092.66 E-05 \quad 5.31 E-04 \quad 1.09 E-03 \quad 1.18 E-03 \quad 1.42 E-03 \quad 1.52 E-03 \quad 1.53 E-03 \quad 1.52 E-03 \quad 1.49 E-03$

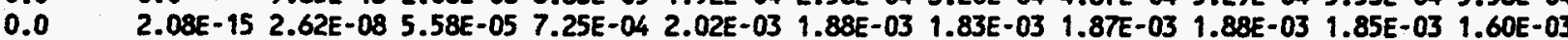
$1.78 E-25$ 6.82E-10 1.94E-06 6.33E-04 2.81E-03 4.45E-03 3.86E-03 3.83E-03 4.03E-03 3.82E-03 $3.68 E-03 \quad 3.76 E-03$ Body Tissues 1.76E-02 1.76E-02 1.72E-02 1.49E-02 1.07E-02 7.54E-03 6.97E-03 6.82E-03 6.48E-03 6.00E-03 5.69E-03 4.73E-03 
Table A.1 (cont'd). Nonpregnant Female: Specific Absorbed Fraction of Photon Energy (kg').

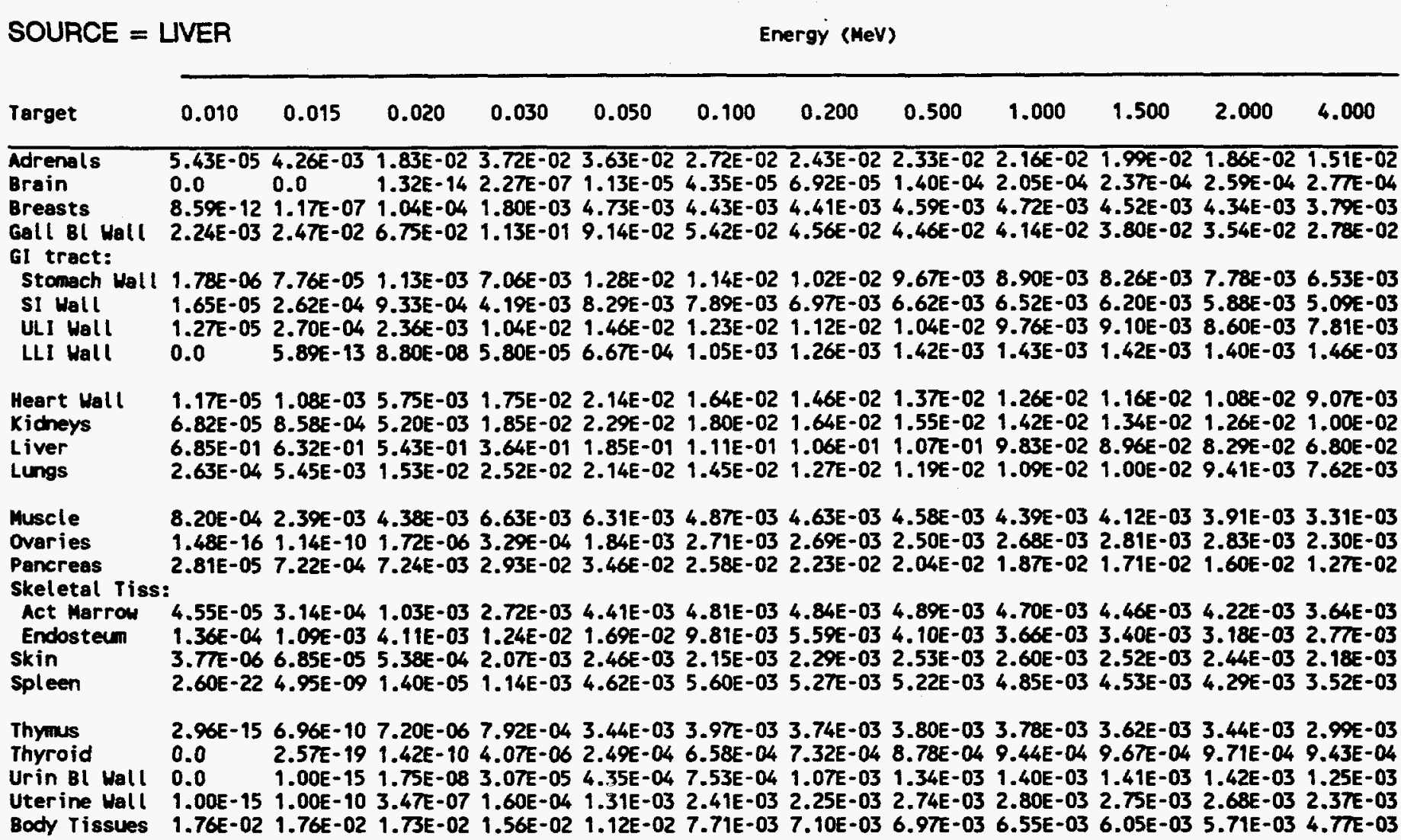

SOURCE $=$ LUNGS

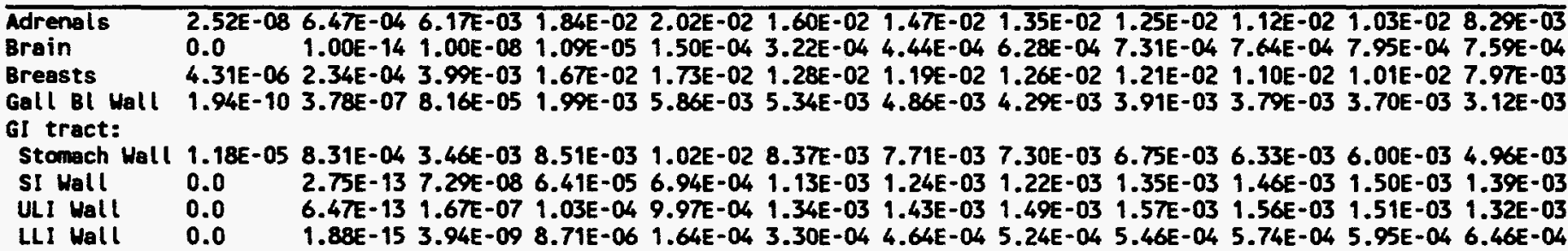

Heart Hall

Kidneys

Liver

Lungs

Muscle

Ovaries

Pancreas

Skeletal Tiss:

Act Marrow

Endosteun

Skin

Spleen

Thymus

Thyroid

Urin Bl Wal

Uterine Hell

Body Tissues
1.88E-04 6.23E-03 2.45E-02 5.00E-02 4.42E-02 2.94E-02 2.63E-02 2.48E-02 2.24E-02 2.05E-02 1.90E-02 1.54E-02 2.96E-10 5.14E-07 1.02E-04 1.99E-03 4.31E-03 4.89E-03 4.73E-03 4.58E-03 4.43E-03 4.21E-03 4.05E-03 3.65E-03 2.36E-04 4.85E-03 1.37E-02 2.27E-02 1.97E-02 1.39E-02 1.27E-02 1.19E-02 1.09E-02 1.00E-02 9.41E-03 7.62E-03 1.38E+00 1.05E+00 7.02E-01 3.21E-01 1.23E-01 6.92E-02 6.74E-02 6.98E-02 6.40E-02 5.83E-02 5.37E-02 4.27E-02

2.64E-03 7.39E-03 1.15E-02 1.25E-02 8.76E-03 6.13E-03 5.82E-03 5.70E-03 5.36E-03 4.94E-03 4.63E-03 3.78E-03 $0.07 .51 E-19$ 2.45E-10 5.10E-06 1.66E-04 3.38E-04 4.48E-04 6.13E-04 7.05E-04 7.29E-04 7.43E-04 7.74E-04 1.37E-07 3.19E-05 1.53E-03 1.04E-02 1.56E-02 1.27E-02 1.10E-02 1.02E-02 9.82E-03 8.88E-03 8.13E-03 6.31E-03

2.40E-06 2.12E-04 1.42E-03 4.89E-03 6.76E-03 6.37E-03 6.27E-03 6.25E-03 5.92E-03 5.49E-03 5.21E-03 4.38E-03 7.17E-06 7.14E-04 5.27E-03 2.03E-02 2.52E-02 1.29E-02 7.16E-03 5.40E-03 4.81E-03 4.42E-03 4.15E-03 3.50E-03 2.43E-06 5.79E-05 5.49E-04 2.22E-03 2.63E-03 2.24E-03 2.33E-03 2.82E-03 2.58E-03 2.41E-03 2.29E-03 1.96E-03 1.45E-04 1.30E-03 6.22E-03 1.55E-02 1.52E-02 1.13E-02 1.02E-02 9.93E-03 9.15E-03 8.28E-03 7.65E-03 6.44E-03

1.42E-12 2.19E-05 1.87E-03 1.75E-02 2.45E-02 1.83E-02 $1.64 E-02$ 1.55E-02 1.43E-02 1.31 E-02 $1.22 E-02 \quad 1.02 E-02$ 3.10E-20 5.57E-08 5.88E-05 2.13E-03 6.13E-03 6.01E-03 5.44E-03 5.23E-03 5.05E-03 4.65E-03 4.37E-03 4.07E-03

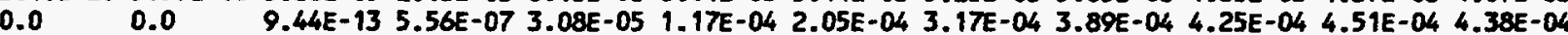
$0.0 \quad 1.00 E-18$ 1.01E-10 3.64E-06 1.14E-04 3.29E-04 4.74E-04 5.38E-04 6.12E-04 6.43E-04 6.63E-04 7.30E-04 1.76E-02 1.76E-02 1.75E-02 1.58E-02 1.10E-02 7.19E-03 6.47E-03 6.27E-03 5.86E-03 5.38E-03 5.06E-03 4.14E-03 
Table A.1 (cont'd). Nonpregnant Female: Specific Absorbed Fraction of Photon Energy $\left(\mathrm{kg}^{-1}\right)$.

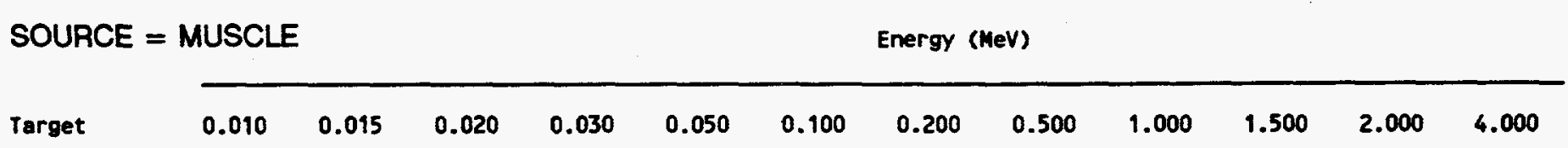

Adrenals

Brain

Breasts

Gall Bl Wall

GI tract:

stomech Wall

SI Hall

ULI Hall

LLI Wall

Heart Wall

Kicheys

Liver

Lungs

Muscle

Ovaries

Pancreas

Skeletal Tiss:

Act Marrow

Endosteum

Skin

spleen

Thymus

Thyroid

Urin Bt Wall

Uterine Wall

Body Tissues
5.39E-03 $1.20 E-02 \quad 1.48 E-02 \quad 1.30 E-02 \quad 9.18 E-03 \quad 6.98 E-03 \quad 6.75 E-03 \quad 6.77 E-03 \quad 6.39 E-03 \quad 5.92 E-03 \quad 5.58 E-03 \quad 4.61 E-03$ 1.13E-08 1.85E-06 6.87E-05 6.15E-04 1.11E-03 1.08E-03 1.11E-03 1.20E-03 1.18E-03 $1.14 E-03$ 1.09E-03 9.52E-04

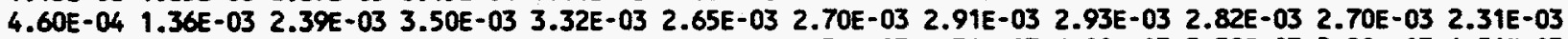
7.25E-03 1.04E-02 1.24E-02 1.25E-02 9.50E-03 7.15E-03 6.54E-03 6.34E-03 6.00E-03 5.58E-03 5.29E-03 4.51E-03 2.85E-03 6.93E-03 1.00E-02 1.18E-02 9.42E-03 6.73E-03 6.30E-03 6.26E-03 5.95E-03 5.51E-03 5.22E-03 4.28E-03 9.40E-04 2.80E-03 5.16E-03 8.67E-03 9.09E-03 7.16E-03 6.54E-03 6.33E-03 5.97E-03 5.67E-03 5.38E-03 4.45E-03 6.39E-04 2.17E-03 4.46E-03 7.96E-03 8.15E-03 6.34E-03 5.82E-03 5.68E-03 5.40E-03 5.10E-03 $4.82 E-03$ 4.03E-03 2.50E-03 5.30E-03 7.40E-03 1.01E-02 1.09E-02 8.83E-03 7.24E-03 6.50E-03 6.20E-03 5.70E-03 5.40E-03 4.56E-03

1.95E-03 4.54E-03 6.94E-03 9.17E-03 8.03E-03 5.95E-03 5.55E-03 5.46E-03 5.18E-03 4.82E-03 4.55E-03 3.78E-03 1.72E-03 5.13E-03 8.69E-03 1.03E-02 8.05E-03 6.06E-03 5.82E-03 5.82E-03 5.56E-03 5.20E-03 4.92E-03 4.13E-03 8.20E-04 2.39E-03 4.38E-03 6.63E-03 6.31E-03 4.87E-03 4.63E-03 4.58E-03 4.39E-03 4.12E-03 3.91E-03 3.31E-03 2.95E-03 8.28E-03 1.29E-02 1.39E-02 9.46E-03 6.19E-03 5.82E-03 5.70E-03 5.36E-03 4.94E-03 4.63E-03 3.78E-03

2.28E-02 2.12E-02 1.88E-02 1.39E-02 8.69E-03 6.16E-03 6.07E-03 6.22E-03 5.89E-03 5.53E-03 5.21E-03 4.35E-03 3.98E-03 9.43E-03 1.28E-02 $1.39 E-02$ 1.17E-02 8.80E-03 $8.15 E-03 \quad 7.90 E-03 \quad 7.36 E-03 \quad 6.91 E-03 \quad 6.50 E-03 \quad 5.28 E-03$ 2.65E-03 7.28E-03 1.16E-02 1.40E-02 1.11E-02 8.02E-03 7.38E-03 7.20E-03 6.75E-03 6.28E-03 5.91E-03 4.86E-03

6.30E-04 1.78E-03 3.25E-03 5.44E-03 6.26E-03 5.55E-03 5.4TE-03 5.56E-03 5.32E-03 4.94E-03 4.65E-03 3.93E-03 2.09E-03 6.95E-03 1.39E-02 2.55E-02 2.60E-02 1.30E-02 7.69E-03 5.63E-03 5.38E-03 5.07E-03 4.76E-03 3.95E-03 5.48E-03 8.05E-03 8.51E-03 6.83E-03 4.34E-03 3.25E-03 3.41E-03 3.79E-03 3.75E-03 3.54E-03 3.40E-03 2.87E-03 1.57E-03 4.79E-03 8.57E-03 1.16E-02 9.32E-03 6.65E-03 6.26E-03 6.20E-03 5.87E-03 5.47E-03 5.17E-03 4.32E-03

2.79E-03 8.03E-03 1.23E-02 1.32E-02 9.57E-03 6.66E-03 6.30E-03 6.30E-03 5.96E-03 5.56E-03 5.25E-03 4.34E-03 4.32E-03 1.03E-02 1.34E-02 1.26E-02 8.21E-03 5.56E-03 $5.47 \mathrm{TE}-03$ 5.62E-03 5.34E-03 $4.96 E-03 \quad 4.66 E-03 \quad 3.86 E-03$ 6.00E-03 8.83E-03 1.04E-02 1.27E-02 1.31E-02 1.07E-02 8.56E-03 6.80E-03 6.30E-03 6.00E-03 5.60E-03 4.76E-03 1.90E-03 5.67E-03 9.99E-03 1.36E-02 1.20E-02 8.87E-03 8.11E-03 7.88E-03 7.40E-03 6.87E-03 6.42E-03 5.32E-03 1.73E-02 1.64E-02 1.50E-02 1.18E-02 7.72E-03 5.67E-03 5.64E-03 5.74E-03 5.45E-03 5.07E-03 4.83E-03 4.01E-03

\section{SOURCE $=$ OVARIES}

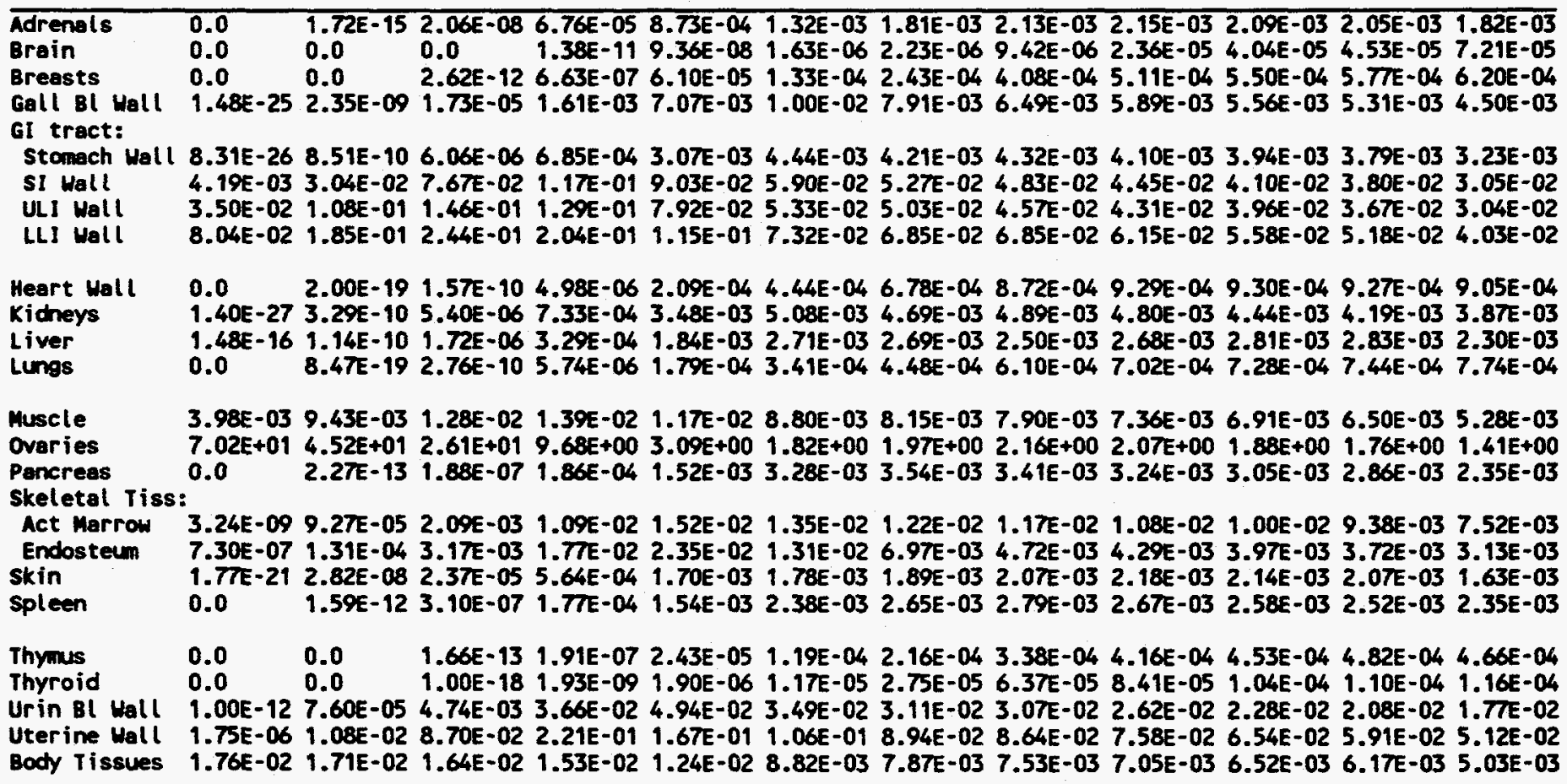


Table A.1 (cont'd). Nonpregnant Female: Specific Absorbed Fraction of Photon Energy ( $\left.\mathrm{kg}^{-1}\right)$.

SOURCE $=$ PANCREAS

Energy (MeV)

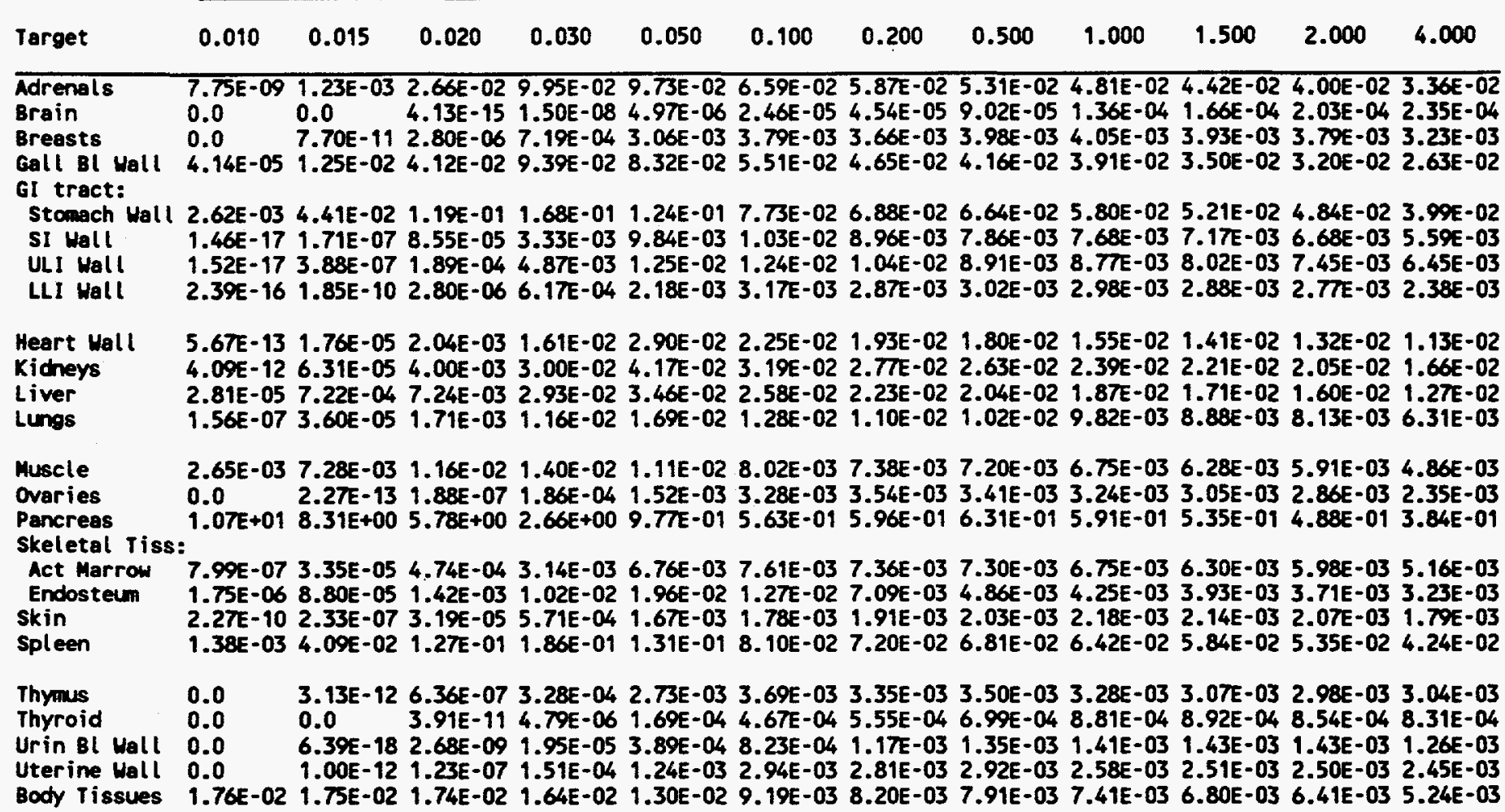

SOURCE = SKELETAL TISSUES: ACTIVE MARROW

\begin{tabular}{|c|c|}
\hline $\begin{array}{l}\text { Adrenals } \\
\text { Brain } \\
\text { Breasts } \\
\text { Gall BI Wall } \\
\text { GI tract: } \\
\text { Stcnach Wall } \\
\text { SI Wall } \\
\text { ULI Wall } \\
\text { LLI Wall }\end{array}$ & 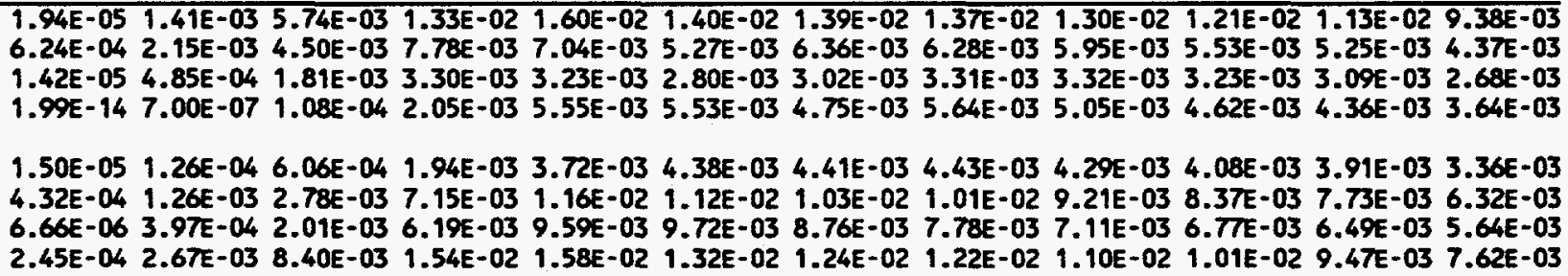 \\
\hline $\begin{array}{l}\text { Heart Wall } \\
\text { Kidneys } \\
\text { Liver } \\
\text { Lungs }\end{array}$ & $\begin{array}{llll}-03 & 4.81 E-03 & 4.56 E-03 & 3.90 E-03 \\
-03 & 8.72 E-03 & 8.22 E-03 & 6.94 E-03 \\
-03 & 4.46 E-03 & 4.22 E-03 & 3.64 E-03 \\
-03 & 5.49 E-03 & 5.21 E-03 & 4.38 E-03\end{array}$ \\
\hline $\begin{array}{l}\text { Muscle } \\
\text { Ovaries } \\
\text { Pencreas } \\
\text { Skeletal Tis }\end{array}$ & 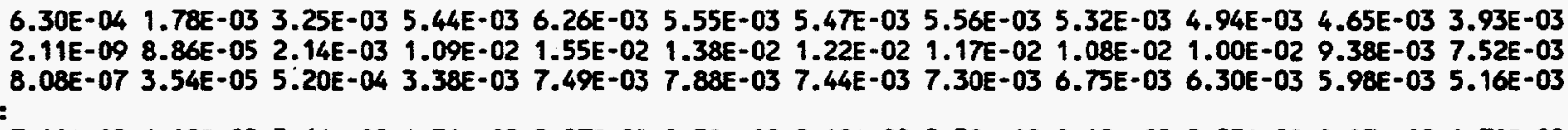 \\
\hline $\begin{array}{l}\text { Act Marrow } \\
\text { Endosteum } \\
\text { Skin } \\
\text { Spleen }\end{array}$ & $\begin{array}{llllllllllll}41 E-02 & 6.48 E-02 & 5.66 E-02 & 4.31 E-02 & 2.97 E-02 & 2.39 E-02 & 2.49 E-02 & 2.59 E-02 & 2.42 E-02 & 2.25 E-02 & 2.07 E-02 & 1.71 E-02 \\
55 E-01 & 1.59 E-01 & 1.50 E-01 & 1.23 E-01 & 7.26 E-02 & 2.78 E-02 & 1.72 E-02 & 1.50 E-02 & 1.37 E-02 & 1.25 E-02 & 1.16 E-02 & 9.62 E-03 \\
54 E-04 & 1.18 E-03 & 1.86 E-03 & 2.49 E-03 & 2.70 E-03 & 2.55 E-03 & 2.76 E-03 & 3.02 E-03 & 3.14 E-03 & 2.99 E-03 & 2.88 E-03 & 2.50 E-03 \\
57 E-06 & 1.39 E-04 & 9.30 E-04 & 3.06 E-03 & 5.46 E-03 & 5.48 E-03 & 5.17 E-03 & 5.20 E-03 & 5.05 E-03 & 4.80 E-03 & 4.58 E-03 & 3.96 E-03\end{array}$ \\
\hline $\begin{array}{l}\text { Thyous } \\
\text { Thyroid } \\
\text { Urin Bl Wall } \\
\text { Uterine Wall } \\
\text { Body Tissues }\end{array}$ & $\begin{array}{l}3.80 E-06 \\
1.00 E-14 \\
2.67 E-09\end{array}$ \\
\hline
\end{tabular}


Table A.1 (cont'd). Nonpregnant Female: Specific Absorbed Fraction of Photon Energy $\left(\mathrm{kg}^{-1}\right)$.

SOURCE = SKELETAL TISSUES: SKELETON

Energy (MeV)

Target $\quad \begin{array}{llllllllllll}0.010 & 0.015 & 0.020 & 0.030 & 0.050 & 0.100 & 0.200 & 0.500 & 1.000 & 1.500 & 2.000 & 4.000\end{array}$

Adrenals $\quad 1.38 E-05 \quad 7.83 E-04 \quad 3.25 E-03 \quad 6.42 E-03 \quad 8.01 E-03 \quad 6.74 E-036.69 E-037.28 E-03$ 6.66E-03 6.01E-03 5.59E-03 4.83E-03

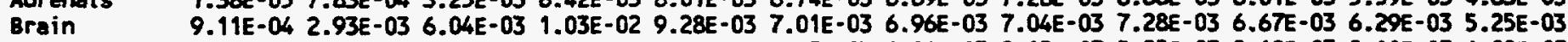

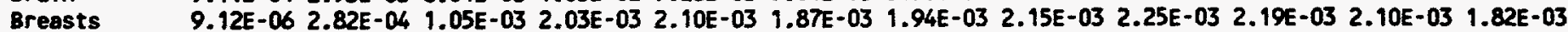
Gall Bl Wall 3.27E-10 3.08E-07 4.06E-05 7.48E-04 2.33E-03 2.73E-03 2.70E-03 2.68E-03 2.52E-03 2.34E-03 2.21E-03 1.90E-03 GI tract:

Stomach Wall 5.81E-07 8.19E-05 4.13E-04 1.42E-03 2.57E-03 2.74E-03 2.61E-03 2.59E-03 2.51E-03 2.38E-03 2.27E-03 1.98E-03 SI Wall $9.89 E-05$ 3.17E-04 7.32E-04 1.96E-03 3.46E-03 3.65E-03 3.46E-03 3.44E-03 3.22E-03 2.98E-03 2.79E-03 2.36E-03 UL1 Wall 6.02E-05 4.02E-04 8.90E-04 1.89E-03 3.04E-03 3.08E-03 2.91E-03 2.93E-03 2.75E-03 2.62E-03 2.55E-03 2.27E-03 LLI Wall 1.28E-04 1.00E-03 2.22E-03 4.03E-03 5.05E-03 4.60E-03 4.33E-03 4.51E-03 4.17E-03 3.87E-03 3.65E-03 3.07E-03

Heart Hall 2.71E-06 9.31E-05 4.93E-04 1.96E-03 3.62E-03 3.53E-03 3.37E-03 3.35E-03 3.17E-03 2.99E-03 2.83E-03 2.43E-03 Kidneys Liver Lungs 1.85E-06 1.51E-04 8.86E-04 3.00E-03 4.53E-03 4.32E-03 4.11E-03 4.26E-03 3.98E-03 3.72E-03 3.54E-03 3.04E-03 3.22E-05 2.25E-04 7.17E-04 2.04E-03 3.20E-03 3.12E-03 3.06E-03 3.21E-03 3.01E-03 2.80E-03 2.65E-03 2.31E-03 1.08E-05 1.64E-04 1.14E-03 3.89E-03 5.22E-03 4.31E-03 4.13E-03 4.41E-03 3.93E-03 3.66E-03 3.48E-03 2.93E-03

Muscle $\quad 4.68 E-04$ 1.29E-03 2.39E-03 4.15E-03 4.92E-03 4.48E-03 4.52E-03 4.66E-03 4.47E-03 4.18E-03 3.97E-03 3.29E-03 Ovaries $\quad 2.45 E-10 \quad 2.33 E-05 \quad 5.41 E-04 \quad 2.78 E-03 \quad 4.35 E-03 \quad 4.23 E-03 \quad 3.86 E-03 \quad 3.86 E-03 \quad 3.56 E-03 \quad 3.31 E-03 \quad 3.11 E-03 \quad 2.63 E-03$ Pancreas 3.94E-07 1.72E-05 2.55E-06 1.70E-03 3.89E-03 4.17E-03 3.91E-03 3.86E-03 3.53E-03 3.29E-03 3.13E-03 2.73E-03 Skeletal Tiss:

Act Marrow 3.24E-02 2.84E-02 2.49E-02 1.95E-02 1.42E-02 1.17E-02 1.21E-02 1.26E-02 1.15E-02 1.08E-02 9.92E-03 8.25E-03 Endosteun 1.66E-01 1.62E-01 1.56E-01 1.32E-01 7.94E-02 3.00E-02 1.86E-02 $1.65 E-02$ 1.50E-02 $1.38 E-02$ 1.27E-02 $1.05 E-02$ Skin Spleen 4.23E-04 1.25E-03 2.07E-03 2.94E-03 3.12E-03 2.83E-03 3.13E-03 3.55E-03 3.44E-03 3.29E-03 3.17E-03 2.76E-03 9.82E-07 8.17E-05 5.31E-04 2.08E-03 3.44E-03 3.35E-03 3.15E-03 3.18E-03 3.09E-03 2.91E-03 2.79E-03 2.41E-03

Thyous

Thyroid

2.68E-07 1.96E-04 1.05E-03 2.35E-03 3.09E-03 2.96E-03 2.93E-03 3.19E-03 3.02E-03 2.80E-03 2.63E-03 2.25E-03 Urin Bl Wall 1.92E-18 5.20E-08 1.87E-05 6.17E-04 1.88E-03 2.73E-03 2.64E-03 2.41E-03 2.23E-03 2.16E-03 2.12E-03 1.82E-03 Body Tissues 1.76E-02 1.75E-02 1.71E-02 1.53E-02 1.08E-02 6.48E-03 5.68E-03 5.73E-03 5.41E-03 5.01E-03 4.76E-03 3.90E-03

SOURCE $=$ SKIN

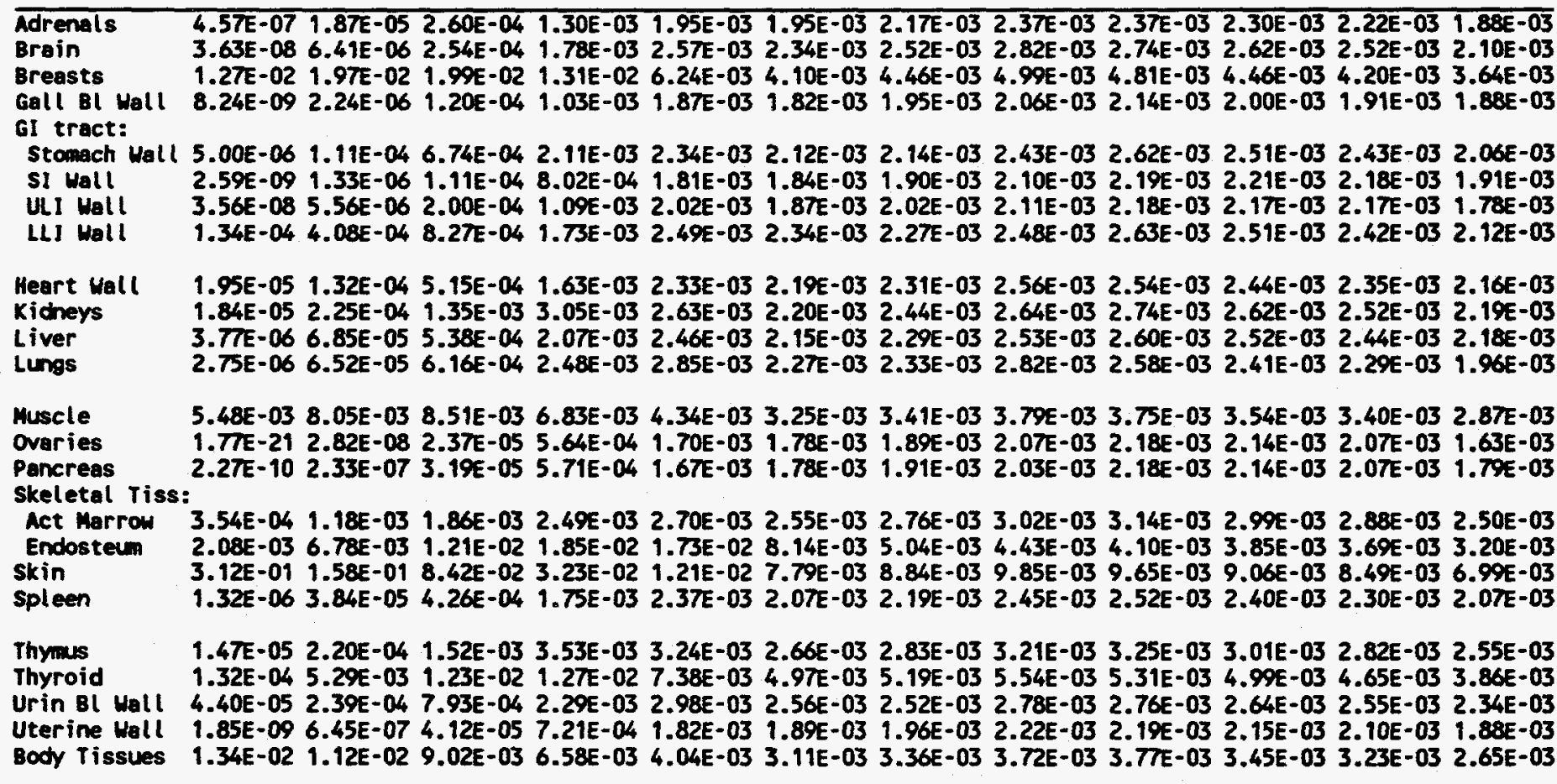


Table A.1 (cont'd). Nonpregnant Female: Specific Absorbed Fraction of Photon Energy $\left(\mathrm{kg}^{-1}\right)$.

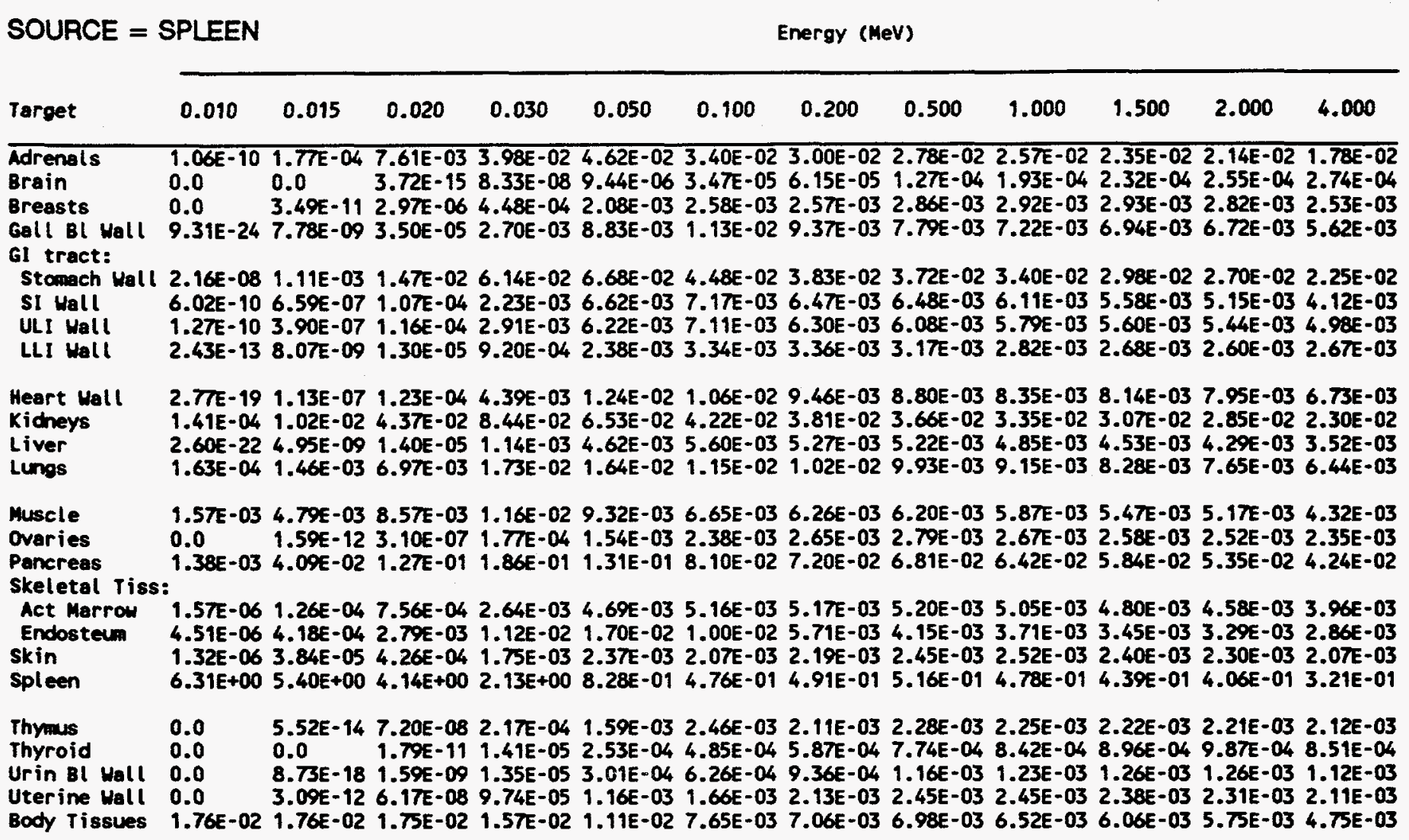

\section{SOURCE $=$ THYMUS}

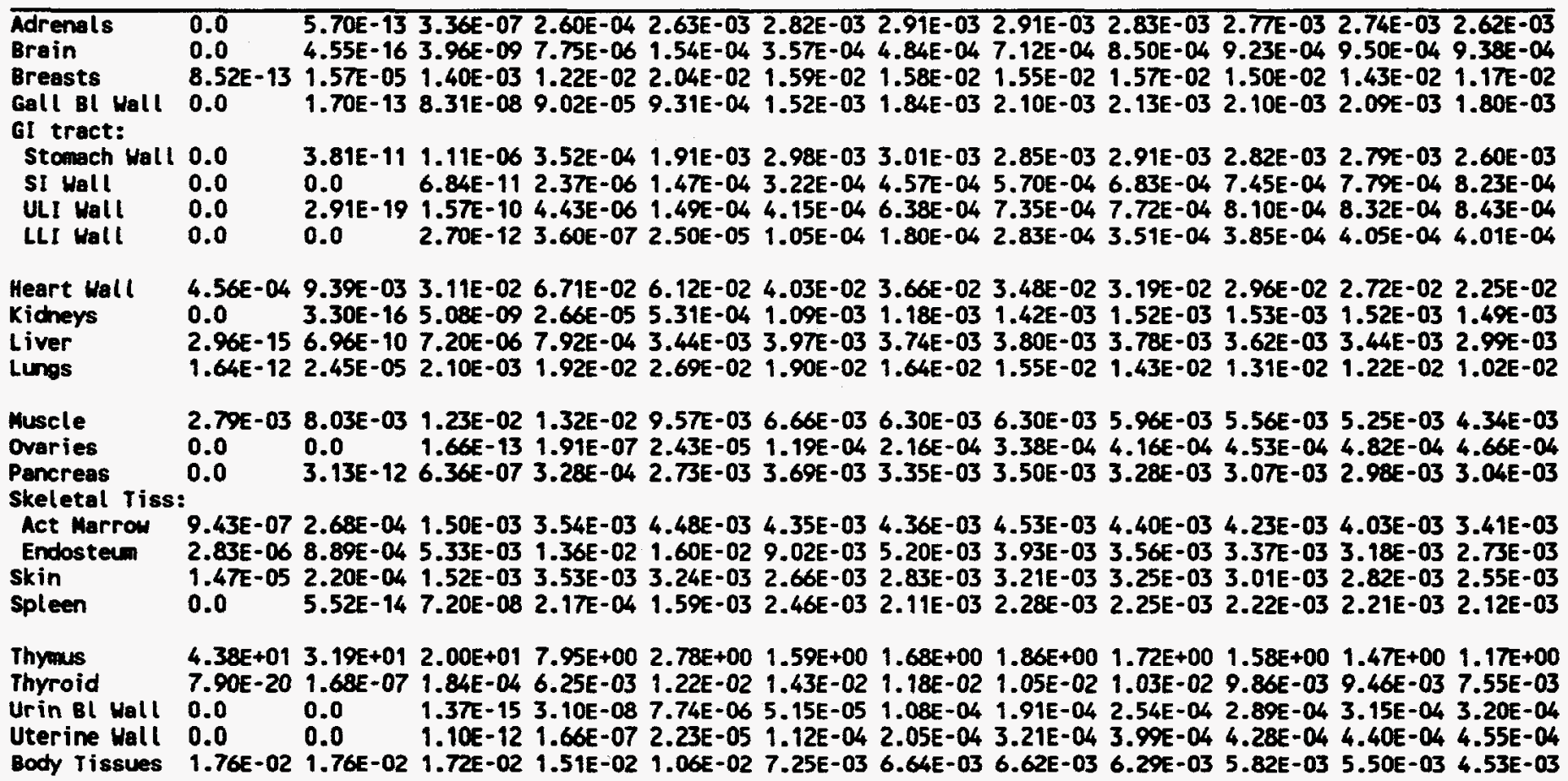


Table A.1 (cont'd). Nonpregnant Female: Specific Absorbed Fraction of Photon Energy $\left(\mathrm{kg}^{-1}\right)$.

SOUACE $=$ THYROID

Energy (MeV)

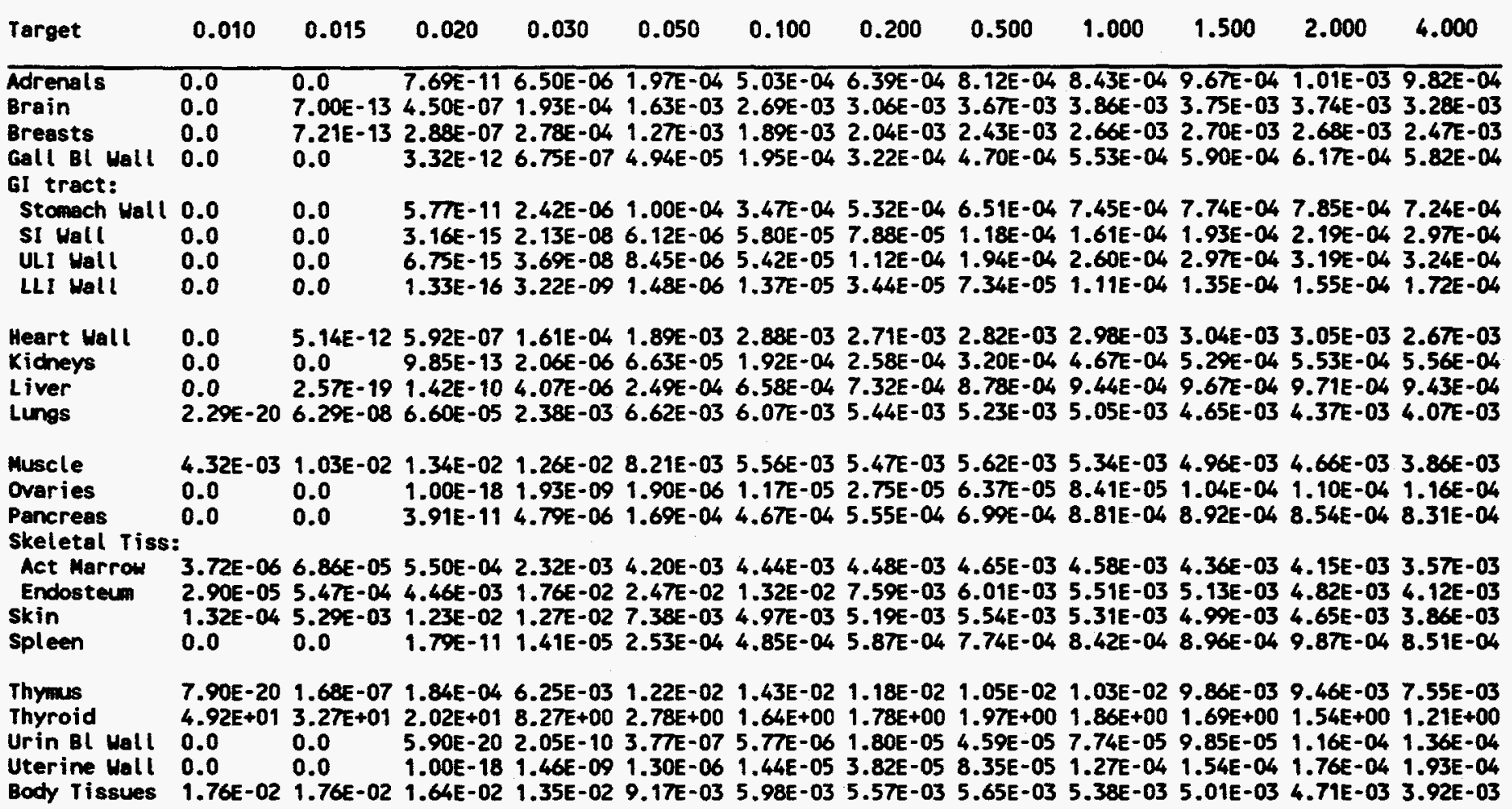

SOURCE = URINARY BLADDER CONTENTS

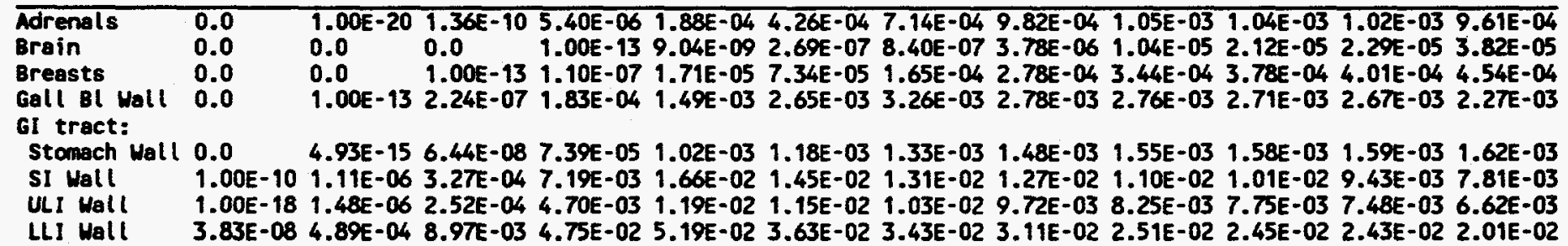

Heart wall $0.0 \quad 0.0 \quad 7.57 E-13 \quad 6.40 E-07$ 4.79E-05 $1.86 E-04 \quad 2.36 E-04 \quad 3.17 E-04 \quad 3.91 E-04 \quad 4.39 E-04 \quad 4.73 E-04 \quad 5.74 E-04$

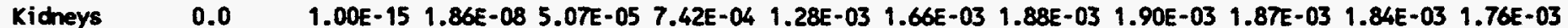

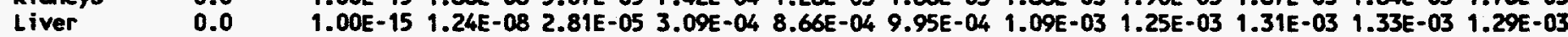

Lungs $\quad 0.0 \quad 0.0 \quad 1.00 E-12$ 5.82E-07 3.28E-05 1.05E-04 1.85E-04 3.04E-04 4.23E-04 4.57E-04 4.67E-04 4.84E-04

muscle

Ovaries

Pancreas

Skeletal Tiss:

Act Marrow

Endosteum

Skin

Spleen

2.72E-04 2.61E-03 6.78E-03 1.19E-02 1.10E-02 8.11E-03 7.47E-03 7.2TE-03 6.84E-03 6.39E-03 6.00E-03 4.96E-03 1.00E-14 3.02E-05 3.56E-03 3.20E-02 4.95E-02 3.66E-02 3.11E-02 2.95E-02 2.52E-02 2.22E-02 2.05E-02 1.81E-02 0.0 1.00E-17 1.96E-09 1.80E-05 5.76E-04 7.84E-04 9.69E-04 1.14E-03 $1.23 E-03$ 1.28E-03 $1.31 E-031.39 E-03$

1.00E-17 2.31E-07 3.06E-05 1.35E-03 4.60E-03 5.37E-03 5.02E-03 5.28E-03 4.63E-03 4.24E-03 3.99E-03 3.35E-03 6.00E-08 3.00E-06 5.39E-05 2.82E-03 9.73E-03 7.95E-03 4.55E-03 2.96E-03 2.70E-03 2.53E-03 2.40E-03 2.14E-03 7.54E-07 3.26E-05 4.72E-04 2.12E-03 2.75E-03 2.41E-03 2.42E-03 2.52E-03 2.57E-03 2.61E-03 2.59E-03 2.25E-03

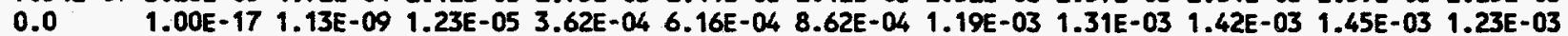

Thymus $\quad 0.0 \quad 0.0 \quad 1.00 E-15$ 2.81E-08 $\quad 0.44 E-06 \quad 5.04 E-05 \quad 1.06 E-04 \quad 1.89 E-04 \quad 2.52 E-04 \quad 2.70 E-04 \quad 2.84 E-04 \quad 3.19 E-04$

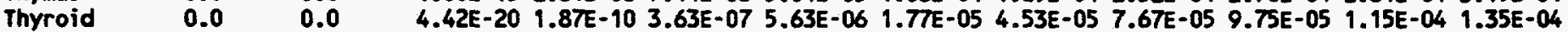
Us Wall-mid $1.82 E+00 \quad 2.70 E+00$ 2.62E+00 1.62E+00 6.95E-01 3.96E-01 3.99E-01 4.03E-01 3.72E-01 3.41E-01 3.16E-01 2.51E-01 UB Mall-full 1.07E+00 1.72E+00 1.78E+00 1.17E+00 5.30E-01 3.05E-01 2.92E-01 3.04E-01 2.72E-01 2.49E-01 2.32E-01 1.84E-01 Uterine Wall 1.29E-05 7.07E-03 5.68E-02 1.44E-01 1.22E-01 7.71E-02 6.78E-02 6.06E-02 5.48E-02 5.25E-02 4.98E-02 3.75E-02 Body Tissues 8.47E-04 2.98E-03 6.04E-03 9.89E-03 9.53E-03 7.02E-03 6.33E-03 6.08E-03 5.70E-03 5.29E-03 5.02E-03 4.18E-03 
Table A.1 (cont'd). Nonpregnant Female: Specific Absorbed Fraction of Photon Energy (kg-1).

SOURCE = URINARY BLADDER WALL

Energy (MeV)

\begin{tabular}{|c|c|c|c|c|c|c|c|c|c|c|c|c|}
\hline arget & 0.010 & 0.015 & 0.020 & 0.030 & 0.050 & 0.100 & 0.200 & 0.500 & 1.000 & 1.500 & 2.000 & 4.000 \\
\hline $\begin{array}{l}\text { Adrenals } \\
\text { Brain } \\
\text { Breasts } \\
\text { Gell BI Wall } \\
\text { GI tract: } \\
\text { Stcmach Wall } \\
\text { SI Wall } \\
\text { ULI Wall } \\
\text { LLI Wall }\end{array}$ & $\begin{array}{l}0.0 \\
0.0 \\
0.0 \\
0.0 \\
0.0 \\
1.00 E-10 \\
3.88 E-09 \\
1.77 E-07\end{array}$ & $\begin{array}{l}1.00 E-19 \\
0.0 \\
0.0 \\
1.00 E-13 \\
2.06 E-14 \\
2.96 E-06 \\
2.95 E-06 \\
1.26 E-03\end{array}$ & $\begin{array}{l}1.85 E-10 \\
0.0 \\
2.32 E-14 \\
3.19 E-07 \\
3.25 E-08 \\
4.84 E-04 \\
3.27 E-04 \\
1.49 E-02\end{array}$ & $\begin{array}{l}5.84 E-06 \\
1.06 E-12 \\
1.19 E-07 \\
2.23 E-04 \\
5.16 E-05 \\
9.99 E-03 \\
5.22 E-03 \\
5.28 E-02\end{array}$ & $\begin{array}{l}1.91 E-04 \\
9.12 E-09 \\
1.74 E-05 \\
2.31 E-03 \\
7.93 E-04 \\
1.49 E-02 \\
1.37 E-02 \\
5.95 E-02\end{array}$ & $\begin{array}{l}6.06 E-04 \\
4.37 E-07 \\
9.24 E-05 \\
4.06 E-03 \\
1.01 E-03 \\
1.55 E-02 \\
1.41 E-02 \\
4.28 E-02\end{array}$ & $\begin{array}{l}7.38 E-04 \\
1.22 E-06 \\
1.74 E-04 \\
4.05 E-03 \\
1.13 E-03 \\
1.40 E-02 \\
1.23 E-02 \\
3.63 E-02\end{array}$ & $\begin{array}{l}9.32 E-04 \\
4.82 E-06 \\
2.83 E-04 \\
3.65 E-03 \\
1.22 E-03 \\
1.21 E-02 \\
1.05 E-02 \\
3.19 E-02\end{array}$ & $\begin{array}{l}1.02 E-03 \\
9.88 E-06 \\
3.56 E-04 \\
3.34 E-03 \\
1.32 E-03 \\
1.06 E-02 \\
9.31 E-03 \\
2.83 E-02\end{array}$ & $\begin{array}{l}1.06 E-03 \\
1.83 E-05 \\
3.93 E-04 \\
3.11 E-03 \\
1.39 E-03 \\
9.57 E-03 \\
8.47 E-03 \\
2.56 E-02\end{array}$ & $\begin{array}{l}1.07 E-03 \\
2.33 E-05 \\
4.21 E-04 \\
2.96 E-03 \\
1.45 E-03 \\
8.96 E-03 \\
7.93 E-03 \\
2.39 E-02\end{array}$ & $\begin{array}{l}9.62 E-04 \\
3.94 E-05 \\
4.13 E-04 \\
2.52 E=03\end{array}$ \\
\hline
\end{tabular}

Heart Wall $\quad 0.0 \quad 0.0 \quad 1.00 E-11$ 6.96E-07 4.91E-05 1.92E-04 3.17E-04 4.62E-04 5.44E-04 5.81E-04 6.08E-04 5.74E-04

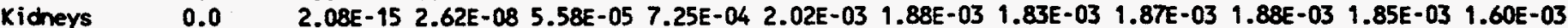

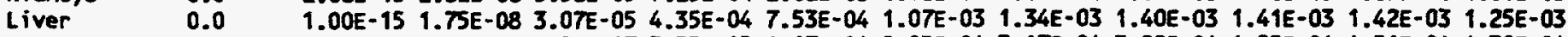
Lungs $\quad 0.0 \quad 0.0 \quad 1.00 E-11$ 6.26E-07 3.33E-05 1.17E-04 2.05E-04 3.17E-04 3.89E-04 4.25E-04 4.51E-04 4.38E-04

Muscle $\quad 6.00 E-03 \quad 8.83 E-03 \quad 1.04 E-02 \quad 1.27 E-02 \quad 1.31 E-02 \quad 1.07 E-02$ 8.56E-03 6.80E-03 6.30E-03 6.00E-03 5.60E-03 4.76E-03 Ovaries $\quad 1.00 E-12$ 7.60E-05 4.74E-03 3.66E-02 4.94E-02 3.49E-02 3.11E-02 3.07E-02 2.62E-02 2.28E-02 2.08E-02 1.77E-02

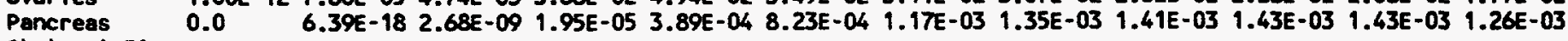
Skeletal Tiss:

Act Marrow 1.00E-14 2.31E-07 3.06E-05 1.35E-03 4.60E-03 5.37E-03 5.02E-03 5.28E-03 4.63E-03 4.24E-03 3.99E-03 3.35E-03 Endosteum $0.0 \quad 5.82 E-08$ 5.39E-05 2.82E-03 9.73E-03 7.95E-03 4.55E-03 2.96E-03 2.70E-03 2.53E-03 2.40E-03 2.14E-03

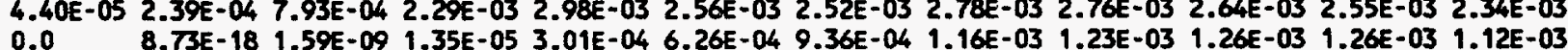
skin Spleen

Thymus $\quad 0.0 \quad 0.0 \quad 1.37 E-15$ 3.10E-08 7.74E-06 5.15E-05 1.08E-04 1.91E-04 2.54E-04 2.89E-04 3.15E-04 3.20E-04

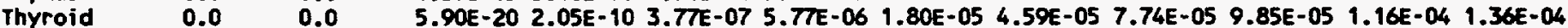
Ur BI Wall 1.72E+01 9.11E+00 5.22E+00 2.15E+00 8.10E-01 4.69E-01 4.81E-01 5.24E-01 4.92E-01 4.48E-01 4.13E-01 3.28E-01 Uterine Hall 3.09E-04 2.03E-02 8.72E-02 1.63E-01 1.30E-01 7.79E-02 6.36E-02 6.53E-02 5.71E-02 5.40E-02 5.19E-02 4.39E-02 Body Tissues 1.47E-02 1.29E-02 1.24E-02 1.21E-02 9.99E-03 7.28E-03 6.62E-03 6.39E-03 6.02E-03 5.57E-03 5.28E-03 4.38E-03

SOURCE = UTERINE WALL

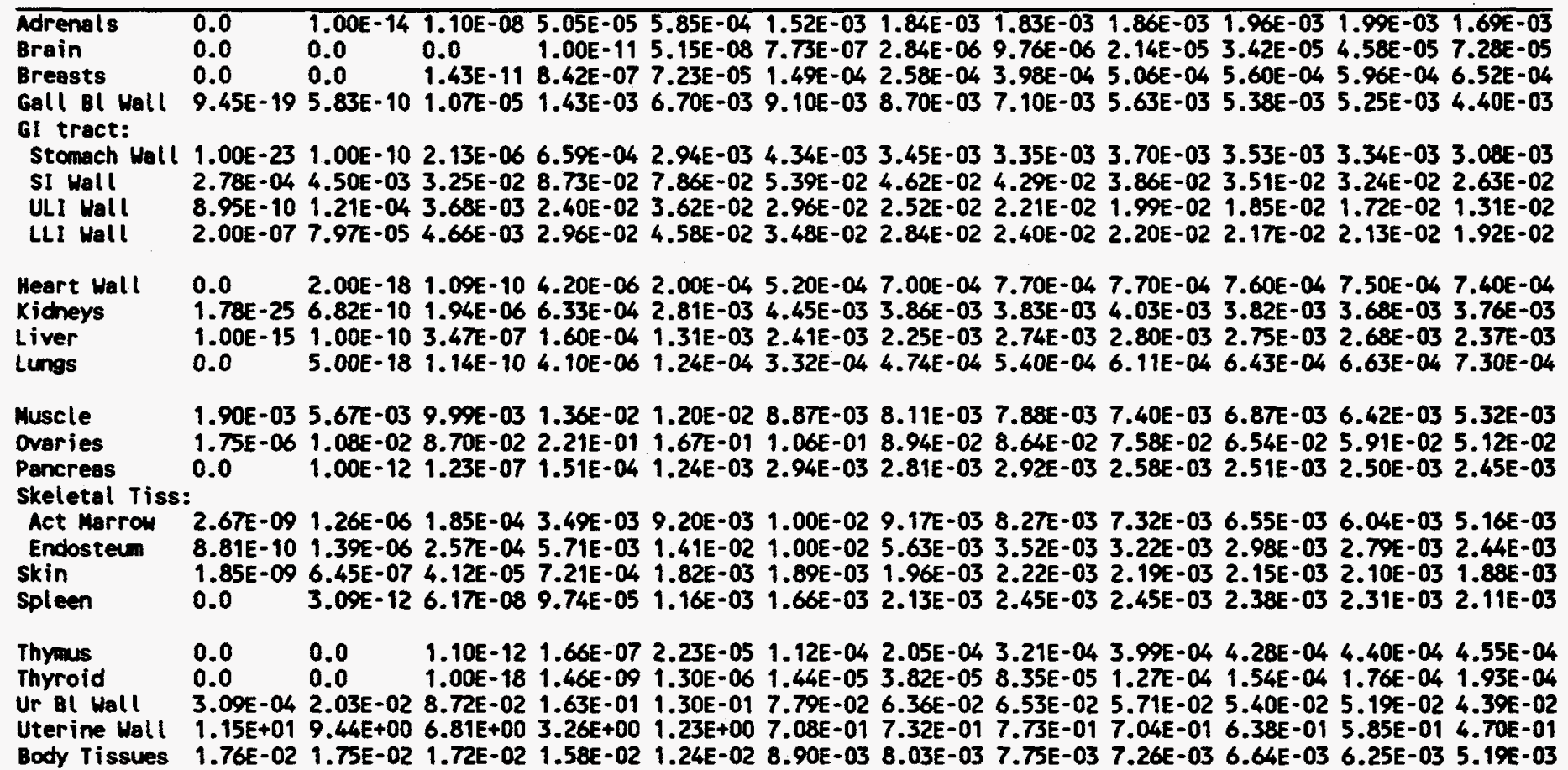


Table A.1 (cont'd). Nonpregnant Female: Specific Absorbed Fraction of Photon Energy $\left(\mathrm{kg}^{-1}\right)$.

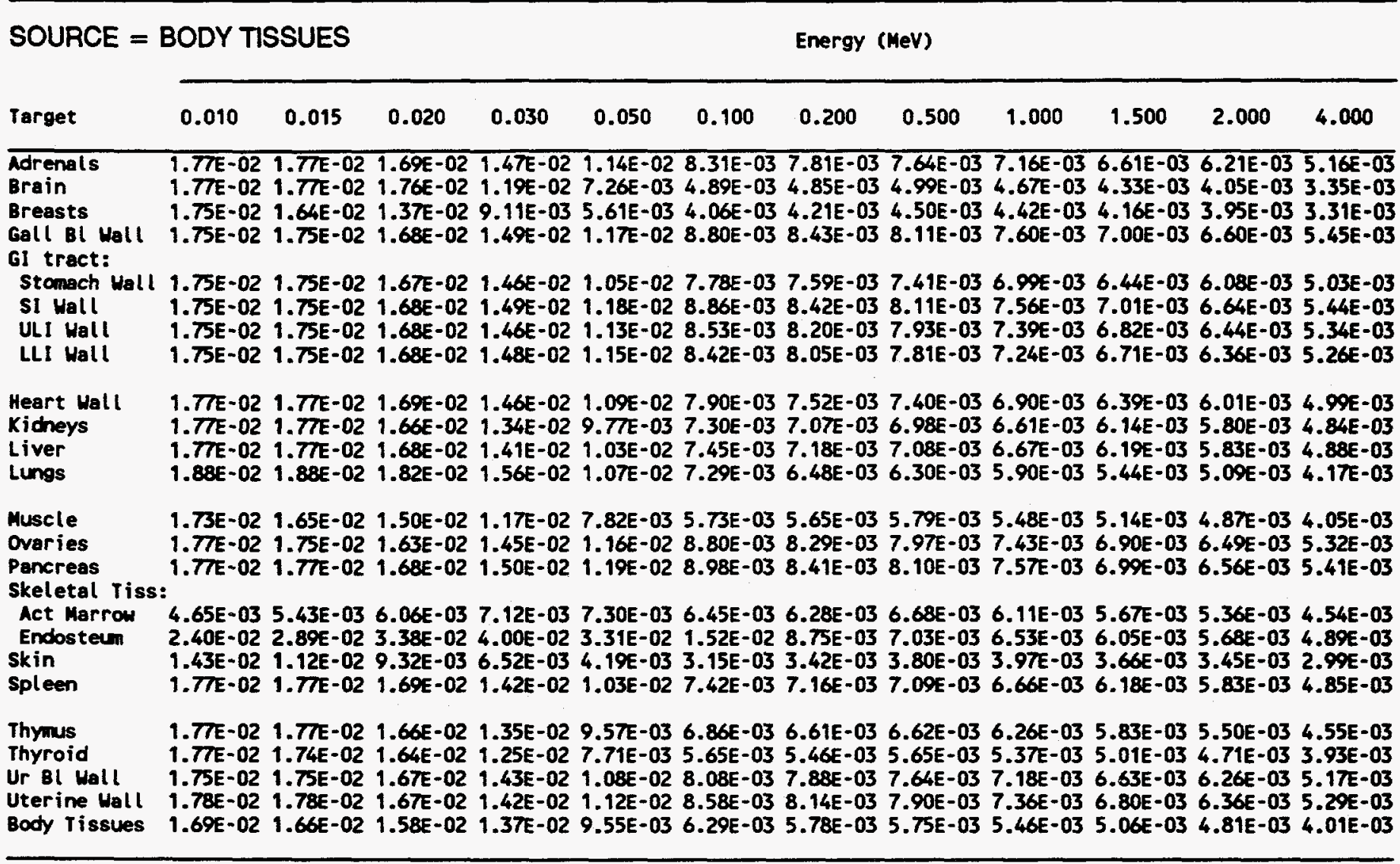



Notes on Table A.2. At Three Months Gestation: Specific Absorbed Fraction of Photon Energy $\left(\mathrm{kg}^{-1}\right)$

Body Tissues. Values of $\Phi$ are given for "Body Tissues" as a source or target region. This region replaces "Whole Body" or "Total Body" given in previous publications (e.g., Cristy and Eckerman 1987) and is defined as the living maternal body tissues, i.e., it excludes the contents of the GI tract, the contents of the urinary and gall bladders, and (for the pregnant woman) the contents of the uterus.

Urinary Bladder. Two rows of $\Phi$ s are given when source $=$ urinary bladder contents and target $=$ urinary bladder wall, labeled "mid" and "full" and designating that the bladder contents are in mid-cycle (half-full) or full.

Muscle. Values of $\Phi$ for muscle are from the Remaining Tissue compartment of each phantom (that part of the maternal tissues remaining after all defined organs are removed).

Cortical and Trabecular Bone. Values of $\Phi$ for source $=$ Cortical Bone, surface or volume distributed, are not given, but source $=$ skeleton may be used for this purpose. Similarly, $\Phi$ s for source $=$ Trabecular Bone, surface or volume distributed, are not given, but source $=$ Red Marrow may be used.

Alphabetical Ordering of Organs. The source and target organs are arranged mostly in alphabetical order. Parts of the GI tract appear together, alphabetized under the heading "GI Tract," but under this heading they appear in natural order from mouth to anus. Active marrow, endosteum (called "bone surface" by the ICRP), and skeleton appear under "Skeletal Tissues"; and (at six and nine months of gestation) fetus, fetal skeleton, fetal soft tissue, placenta, and other uterine contents appear under "Uterine Contents." Body Tissues appears at the end of the tables rather than in alphabetical order. Note that some of these organs appear only as source organs (i.e., skeleton, placenta, and other uterine contents), and endosteum appears only as a target organ. Note also that as target tissues fetal skeleton and fetal soft tissue (at six and nine months of gestation) are indented under "Fetus" and are listed as "Skeleton" and "Soft Tissue" for brevity of space.

Abbreviations. The meanings of the abbreviations used for target regions in Tables A.1 - A.4 are given in the following table. Abbreviations are not used for source regions.

\begin{tabular}{||l|l|}
\hline \multicolumn{2}{|c|}{ Abbreviations used in the Tables for Target Regions } \\
\hline Abbreviation & Meaning \\
\hline Act Marrow & Active Marrow \\
Gall Bl & Gall Bladder \\
GI Tract & Gastrointestinal Tract \\
SI & Small Intestine \\
ULI & Upper Large Intestine \\
LLI & Lower Large Intestine \\
Skeletal Tiss & Skeletal Tissues \\
Urin Bl & Urinary Bladder \\
Uterine Cont & Uterine Contents \\
\hline
\end{tabular}


Table A.2. At Three Months Gestation: Specific Absorbed Fraction of Photon Energy $\left(\mathrm{kg}^{-1}\right)$.

SOURCE $=$ ADRENALS

Energy (MeV)

\begin{tabular}{|c|c|c|c|c|c|c|c|c|c|c|c|c|}
\hline rget & 0.010 & 0.015 & 0.020 & .030 & .050 & .100 & . 200 & .500 & .000 & 1.500 & 2.000 & 4.000 \\
\hline $\begin{array}{l}\text { renals } \\
\text { ain } \\
\text { easts } \\
\text { ll Bl Wall } \\
\text { tract: }\end{array}$ & $\begin{array}{l}5.69 E+01 \\
0.0 \\
0.0 \\
1.24 E-14\end{array}$ & $\begin{array}{l}3.53 E+0 \\
0.0 \\
6.40 E-1 \\
7.21 E-0\end{array}$ & & & & & & & & & & \\
\hline $\begin{array}{l}\text { Stomech Hall } \\
\text { SI Hall } \\
\text { ULI Wall } \\
\text { LLI Hall }\end{array}$ & $\begin{array}{l}1.89 E \\
3.04 E \\
3.59 E \\
0.0\end{array}$ & $\begin{array}{l}7 \\
4 \\
5\end{array}$ & & & & & & & & & & \\
\hline
\end{tabular}

Heart Wall $\quad 1.80 E-16 \quad 1.47 E-06 \quad 4.50 E-04$ 8.99E-03 $1.90 E-02 \quad 1.66 E-02 \quad 1.41 E-02 \quad 1.35 E-02 \quad 1.33 E-02 \quad 1.17 E-02$ 1.06E-02 8.59E-03 Kidneys $\quad 8.65 E-04$ 1.89E-02 5.80E-02 9.13E-02 7.05E-02 4.90E-02 4.52E-02 4.42E-02 4.03E-02 3.68E-02 3.44E-02 2.83E-02

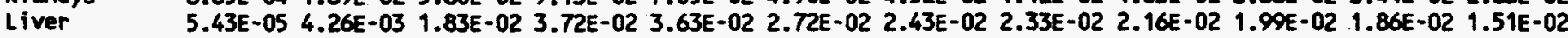
Lungs $\quad 2.91 E-08$ 7.24E-04 6.90E-03 2.06E-02 2.18E-02 1.62E-02 1.47E-02 $1.35 E-02$ 1.25E-02 $1.12 E-02$ 1.03E-02 8.29E-03

Muscle $\quad 5.39 E-03$ 1.20E-02 $1.48 E-02$ 1.30E-02 9.18E-03 6.98E-03 6.75E-03 6.77E-03 6.39E-03 5.92E-03 5.58E-03 4.61E-03

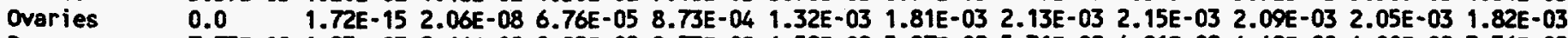
Pancreas 7.75E-09 1.23E-03 2.66E-02 9.95E-02 9.73E-02 6.59E-02 5.87E-02 5.31E-02 4.81E-02 4.42E-02 4.00E-02 3.36E-02 Skeletal Tiss:

$\begin{array}{lllllllllll}\text { Act Marrow 1.94E-05 1.27E-03 4.88E-03 } 1.15 E-02 & 1.47 E-02 & 1.39 E-02 & 1.39 E-02 & 1.37 E-02 & 1.30 E-02 & 1.21 E-02 & 1.13 E-02 & 9.38 E-03\end{array}$ Endosteum 6.32E-05 3.82E-03 1.62E-02 3.92E-02 4.21E-02 2.04E-02 1.15E-02 8.76E-03 7.87E-03 7.14E-03 6.64E-03 5.68E-03

skin

Spleen 4.57E-07 1.87E-05 2.60E-04 $1.30 E-03$ 1.95E-03 $1.95 E-03$ 2.17E-03 $2.37 E-03$ 2.37E-03 $2.30 E-03 \quad 2.22 E-03 \quad 1.88 E-03$ 1.06E-10 1.77E-04 7.61E-03 3.98E-02 4.62E-02 3.40E-02 3.00E-02 2.78E-02 2.57E-02 2.35E-02 2.14E-02 1.78E-02

Thymus $\quad 0.0 \quad 5.70 E-13 \quad 3.36 E-07$ 2.60E-04 2.63E-03 2.82E-03 2.91E-03 2.91E-03 2.83E-03 2.7TE-03 2.74E-03 2.62E-03

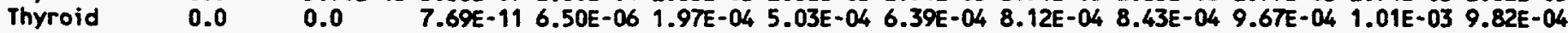
Urin Bl Well $0.0 \quad 0.0 \quad 7.14 E-11$ 3.84E-06 $1.50 E-04 \quad 4.40 E-04$ 5.29E-04 6.74E-04 8.10E-04 9.00E-04 9.73E-04 8.82E-04

Uterine Cont:

Fetus 0.0

Uterine Wall 0.0

6.43E- 15 2.24E-08 5.47E-05 7.08E-04 1.33E-03 1.69E-03 1.78E-03 1.84E-03 1.90E-03 1.84E-03 1.78E-03

2.72E-14 4.52E-08 6.88E-05 6.24E-04 1.4 EE-03 $1.74 E-03 \quad 1.90 E-03 \quad 2.02 E-03 \quad 1.90 E-03 \quad 1.89 E-03 \quad 1.80 E-03$ Body Tissues 1.77E-02 1.77E-02 1.76E-02 1.65E-02 1.26E-02 8.68E-03 7.76E-03 7.56E-03 7.13E-03 6.50E-03 6.12E-03 5.14E-03

\section{SOURCE = BRAIN}

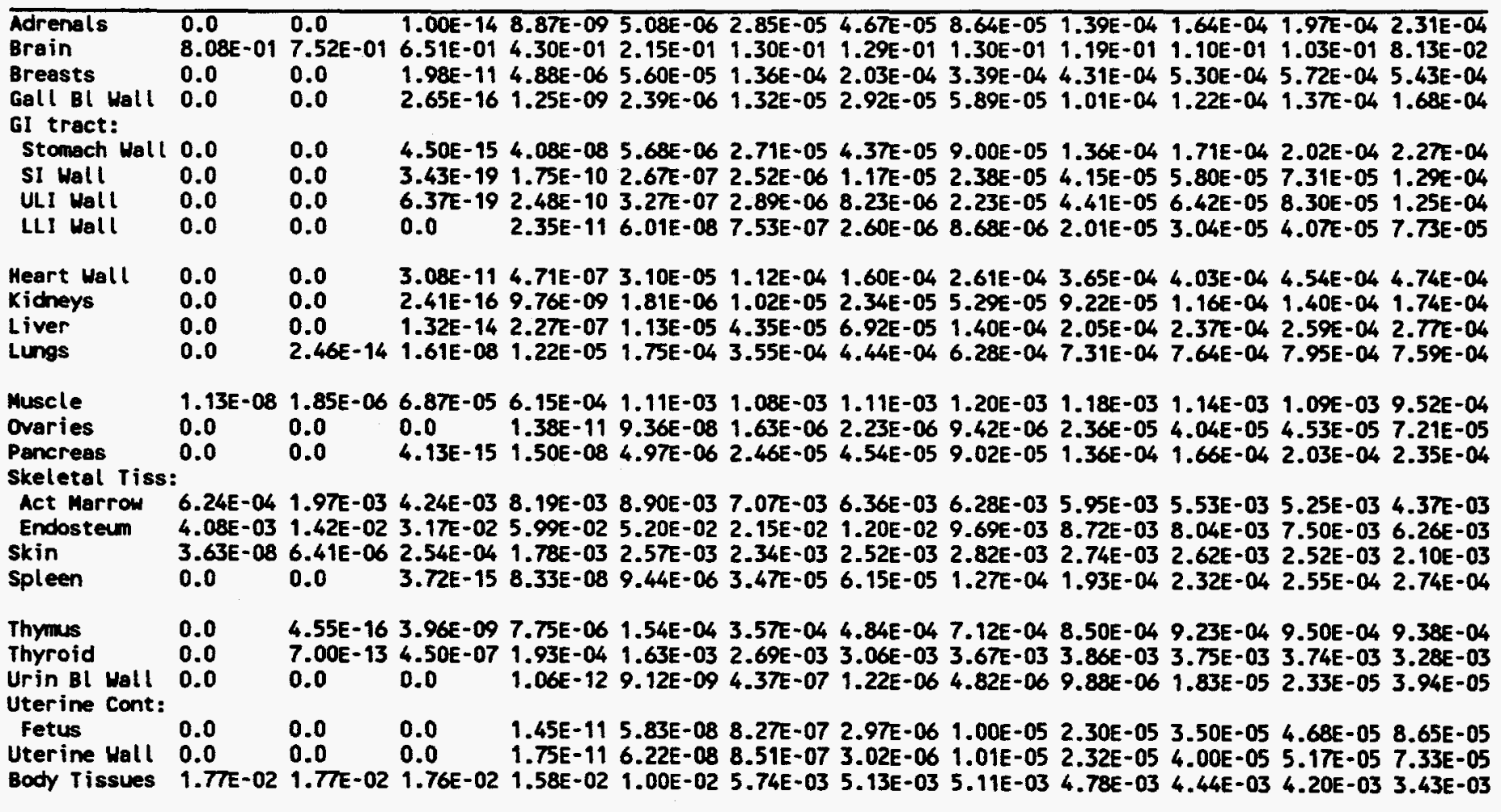


Table A.2 (cont'd). At Three Months Gestation: Specific Absorbed Fraction of Photon Energy (kg-1).

SOURCE $=$ BREASTS

Energy (MeV)

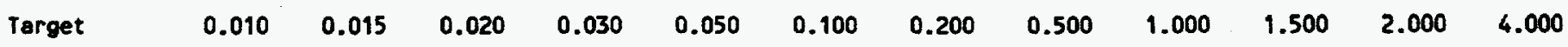

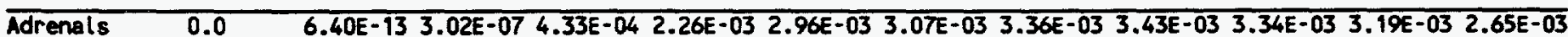

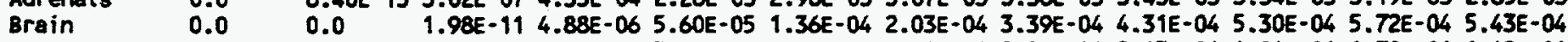

Breasts $\quad 2.63 E+002.27 E+001.72 E+00$ 8.80E-01 3.37E-01 1.97E-01 2.09E-01 2.24E-01 2.13E-01 1.94E-01 1.78E-01 1.42E-01

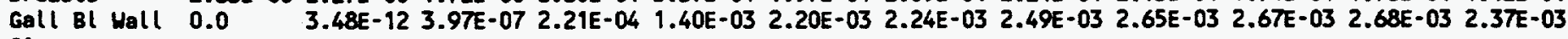

G1 tract:

stomach Wall 6.96E-19 6.28E-08 4.02E-05 1.63E-03 3.80E-03 4.00E-03 3.93E-03 4.39E-03 3.97E-03 3.93E-03 3.91E-03 3.60E-03

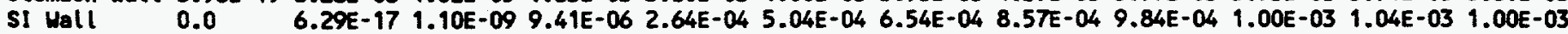
ULI Wall $\quad 0.0 \quad 1.42 E-16 \quad 2.40 E-09$ 1.52E-05 2.57E-04 6.82E-04 8.58E-04 9.87E-04 $1.08 E-03 \quad 1.11 E-03 \quad 1.14 E-03 \quad 1.15 E-03$

LLI Wall $\quad 0.0 \quad 0.0 \quad 8.37 E-11$ 1.51E-06 5.61E-05 $1.43 E-04 \quad 2.36 E-04 \quad 3.56 E-04 \quad 4.38 E-04 \quad 4.82 E-04 \quad 5.09 E-04 \quad 5.40 E-04$

Heart Wall 5.14E-06 1.89E-04 2.46E-03 1.68E-02 2.22E-02 1.71E-02 1.59E-02 $1.75 E-02 \quad 1.56 E-02 \quad 1.48 E-02 \quad 1.41 E-02 \quad 1.14 E-02$

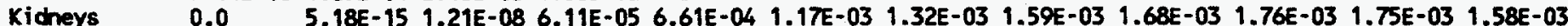
Liver $\quad 8.59 E-12$ 1.17E-07 1.04E-04 1.80E-03 4.73E-03 4.43E-03 4.41E-03 4.59E-03 4.72E-03 4.52E-03 4.34E-03 3.79E-03 lungs $\quad 4.84 E-06$ 2.62E-04 4.46E-03 1.86E-02 $1.86 E-02 \quad 1.29 E-02 \quad 1.19 E-02 \quad 1.26 E-02 \quad 1.21 E-02 \quad 1.10 E-02 \quad 1.01 E-02 \quad 7.97 E-03$

Muscle $\quad 4.60 E-04 \quad 1.36 E-03 \quad 2.39 E-03 \quad 3.50 E-03 \quad 3.32 E-03 \quad 2.65 E-03 \quad 2.70 E-03 \quad 2.91 E-03 \quad 2.93 E-03 \quad 2.82 E-03 \quad 2.70 E-03 \quad 2.31 E-03$

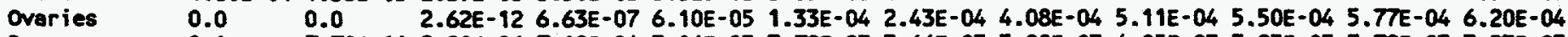
Pancreas $\quad 0.0 \quad 7.70 E-11$ 2.80E-06 7.19E-04 3.06E-03 3.79E-03 3.66E-03 3.98E-03 4.05E-03 3.93E-03 3.79E-03 3.23E-03 Skeletal Tiss:

Act Marrow 1.42E-05 4.35E-04 1.55E-03 2.98E-03 3.11E-03 2.88E-03 3.02E-03 3.31E-03 3.32E-03 3.23E-03 3.09E-03 2.68E-03 Endosteum 4.19E-05 1.45E-03 5.54E-03 1.15E-02 $1.10 E-02$ 5.58E-03 3.37E-03 2.83E-03 2.69E-03 2.59E-03 2.47E-03 2.16E-03

Skin

Spleen 1.27E-02 1.97E-02 1.99E-02 1.31E-02 6.24E-03 4.10E-03 4.46E-03 4.99E-03 4.81E-03 4.46E-03 4.20E-03 3.64E-03

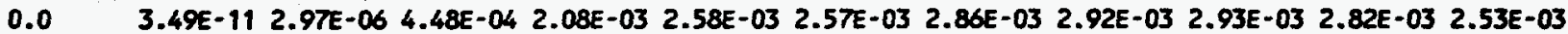

Thymus $\quad 8.52 E-13 \quad 1.57 E-05 \quad 1.40 E-03 \quad 1.22 E-02 \quad 2.04 E-02 \quad 1.59 E-02 \quad 1.58 E-02 \quad 1.55 E-02 \quad 1.57 E-02 \quad 1.50 E-02 \quad 1.43 E-02 \quad 1.17 E-02$

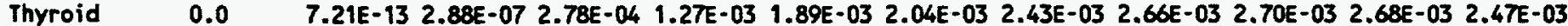

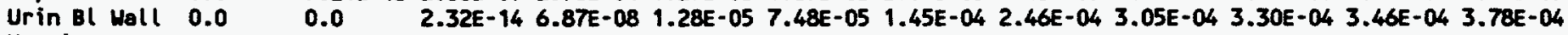
Uterine cont:

Fetus 0.0

Uterine Hall $0.0 \quad 0.0 \quad 1.43 E-11$ 8.42E-07 7.23E-05 1.49E-04 2.58E-04 3.98E-04 5.06E-04 5.60E-04 5.96E-04 6.52E-04 Body Tissues 1.76E-02 1.64E-02 1.42E-02 1.02E-02 6.28E-03 4.28E-03 4.24E-03 4.49E-03 4.45E-03 4.12E-03 3.91E-03 3.30E-03

\section{SOURCE $=$ GALL BLADDER CONTENTS}

Adrenals $2.15 E-15$ 2.50E-06 6.39E-04 1.01E-02 2.13E-02 2.08E-02 1.96E-02 $1.79 E-02$ 1.43E-02 $1.30 E-02 \quad 1.23 E-02$ 1.06E-02

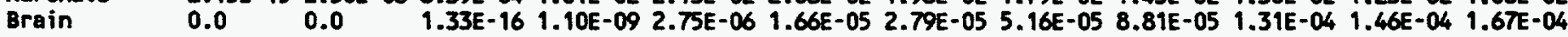

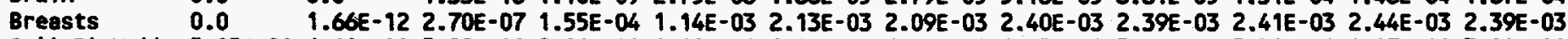

Gall Bl Wall 5.05E+00 6.18E+00 5.22E+00 2.81E+00 1.08E+00 6.06E-01 6.18E-01 6.37E-01 5.86E-01 5.38E-01 4.97E-01 3.94E-01 G1 tract:

Stomach Hall 2.99E-15 5.08E-06 1.08E-03 1.46E-02 2.69E-02 2.21E-02 1.76E-02 $1.78 E-02 \quad 1.54 E-02 \quad 1.43 E-02 \quad 1.36 E-02 \quad 1.10 E-02$ S1 Wall 3.14E-06 $1.40 E-03 \quad 1.05 E-02 \quad 3.87 E-02$ 4.48E-02 $3.23 E-02 \quad 2.81 E-02 \quad 2.63 E-02 \quad 2.39 E-02 \quad 2.20 E-02 \quad 2.00 E-02 \quad 1.62 E-02$ ULI Wall 2.74E-05 9.49E-03 4.07E-02 8.95E-02 7.72E-02 5.09E-02 4.35E-02 4.02E-02 3.77E-02 3.44E-02 3.17E-02 2.47E-02 LLI Woll 1.41E-25 7.34E-10 6.23E-06 8.09E-04 3.27E-03 4.84E-03 4.06E-03 3.66E-03 3.52E-03 3.38E-03 3.31E-03 3.07E-03

Heart Wall 7.68E-19 5.94E-08 4.26E-05 2.32E-03 6.69E-03 6.97E-03 6.80E-03 6.58E-03 6.58E-03 5.85E-03 5.38E-03 5.02E-03

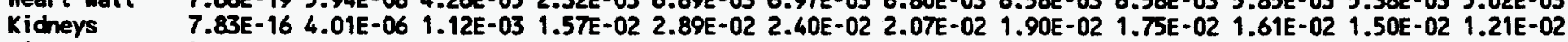
Liver $\quad 5.65 E-04 \quad 1.43 E-02$ 5.12E-02 9.46E-02 7.96E-02 5.20E-02 4.65E-02 $4.36 E-02 \quad 4.05 E-02 \quad 3.70 E-02 \quad 3.44 E-02 \quad 2.70 E-02$ Lungs $\quad 1.35 E-11$ 8.77E-08 4.44E-05 1.49E-03 5.23E-03 5.03E-03 4.57E-03 4.26E-03 4.18E-03 4.05E-03 3.96E-03 3.30E-03

Muscle $\quad 9.65 E-04 \quad 4.78 E-03 \quad 8.80 E-03$ 1.16E-02 $9.73 E-03 \quad 7.16 E-03 \quad 6.60 E-03 \quad 6.47 E-03 \quad 6.06 E-03 \quad 5.65 E-03$ 5.36E-03 4.46E-03 Ovaries $\quad 3.61 E-26$ 1.43E-09 1.53E-05 2.03E-03 6.98E-03 9.14E-03 7.51E-03 7.68E-03 7.07E-03 6.19E-03 5.68E-03 4.87E-03 Pancreas 2.53E-04 3.38E-03 2.35E-02 7.02E-02 6.66E-02 4.57E-02 3.93E-02 3.51E-02 3.30E-02 3.00E-02 2.76E-02 2.17E-02 Skeletal Tiss:

Act Marrow 3.63E-15 1.66E-06 6.01E-05 1.36E-03 4.55E-03 5.56E-03 5.56E-03 5.46E-03 5.17E-03 4.84E-03 4.60E-03 3.96E-03 Endosteum 6.81E-07 1.63E-05 $1.56 E-04$ 3.73E-03 1.12E-02 8.54E-03 4.96E-03 3.22E-03 2.94E-03 2.77E-03 2.64E-03 2.26E-03

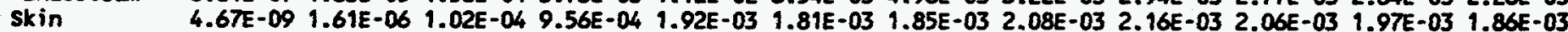
Spleen $\quad 2.77 E-24$ 3.58E-09 2.36E-05 2.17E-03 7.90E-03 9.77E-03 8.73E-03 8.01E-03 7.86E-03 7.45E-03 7.09E-03 5.62E-03

Thymus $\quad 0.0 \quad 5.16 E-14 \quad 4.26 E-08$ 6.87E-05 8.91E-04 1.47E-03 2.01E-03 2.34E-03 2.24E-03 2.08E-03 1.98E-03 1.71E-03 Thyroid $\quad 0.0 \quad 0.0 \quad 1.60 E-12$ 5.05E-07 4.27E-05 $1.78 E-04$ 3.28E-04 4.65E-04 5.26E-04 5.62E-04 5.92E-04 5.60E-04

$\begin{array}{lllllllllllllll}\begin{array}{l}\text { Urin Bl Wall } \\ \text { Uterine Cont: }\end{array} & 0.0 & 2.16 E-14 & 6.46 E-08 & 1.10 E-04 & 1.12 E-03 & 2.18 E-03 & 2.60 E-03 & 2.49 E-03 & 2.32 E-03 & 2.20 E-03 & 2.18 E-03 & 2.02 E-03 \\ \text { fetus } & 5.76 E-13 & 2.36 E-08 & 4.43 E-05 & 2.06 E-03 & 7.77 E-03 & 9.27 E-03 & 7.78 E-03 & 7.19 E-03 & 7.13 E-03 & 7.00 E-03 & 6.65 E-03 & 5.24 E-03\end{array}$

Uterine Cont:

Fetus

Uterine Wall 1.38E-19 1.03E-07 8.86E-05 3.13E-03 8.10E-03 9.44E-03 7.75E-03 7.68E-03 6.56E-03 6.30E-03 6.21E-03 6.09E-03

Body Tissues 1.49E-03 4.86E-03 8.69E-03 1.23E-02 1.11E-02 8.08E-03 7.18E-03 6.87E-03 6.45E-03 5.95E-03 5.61E-03 4.66E-03 
Table A.2 (cont'd). At Three Months Gestation: Specific Absorbed Fraction of Photon Energy (kg $\left.{ }^{-1}\right)$.

SOURCE = GALL BLADDER WAL

Energy (MeV)

\begin{tabular}{|c|c|c|c|c|c|c|c|c|c|c|c|c|}
\hline rget & 0.010 & 0.015 & 0.020 & 0.030 & 0.050 & 0.100 & 0.200 & 0.500 & 1.000 & 1.500 & 2.000 & 4.000 \\
\hline $\begin{array}{l}\text { Adrenals } \\
\text { Brain } \\
\text { Breasts } \\
\text { Gall Bl Wall } \\
\text { GI tract: } \\
\text { Stomach Hall } \\
\text { SI Wall } \\
\text { ULI Wall } \\
\text { LLI Wall }\end{array}$ & $\begin{array}{l}1.24 E-14 \\
0.0 \\
0.0 \\
5.60 E+01 \\
2.01 E-14 \\
7.60 E-05 \\
2.78 E-04 \\
2.14 E-25\end{array}$ & $\begin{array}{l}7.21 E-06 \\
0.0 \\
3.48 E-12 \\
2.57 E+01 \\
9.29 E-06 \\
2.59 E-03 \\
1.71 E-02 \\
1.06 E-09\end{array}$ & $\begin{array}{l}1.15 E-03 \\
2.65 E-16 \\
3.97 E-07 \\
1.35 E+01 \\
1.43 E-03 \\
1.26 E-02 \\
4.89 E-02 \\
6.86 E-06\end{array}$ & $\begin{array}{l}1.43 E \\
1.25 E \\
2.21 E \\
5.46 \\
1.611 \\
3.91 E \\
9.121 \\
8.69\end{array}$ & $\begin{array}{l}2.38 E-02 \\
2.39 E-06 \\
1.40 E-03 \\
1.81 E+00 \\
. \\
2.99 E-02 \\
4.25 E-02 \\
7.54 E-02 \\
2.99 E-03\end{array}$ & $\begin{array}{l}2.5 \\
1.3 \\
2.2 \\
1.0 \\
2.4 \\
3.1 \\
4.8 \\
4.1\end{array}$ & $\begin{array}{l}-02 \\
-05 \\
-03 \\
+00 \\
-02 \\
-02 \\
-02 \\
-03\end{array}$ & $\begin{array}{l}1.7 \\
5.8 \\
2.4 \\
1.1 \\
1.7 \\
2.5 \\
4.0 \\
3.9\end{array}$ & $\begin{array}{l}1.49 E-02 \\
1.01 E-04 \\
2.65 E-03 \\
1.10 E+00 \\
1.57 E-02 \\
2.28 E-02 \\
3.58 E-02 \\
3.79 E-03\end{array}$ & $\begin{array}{l}1.2 \\
2.6 \\
1.0 \\
1.4 \\
2.0 \\
3.1 \\
3.4\end{array}$ & $\begin{array}{l}1.26 E-02 \\
1.37 E-04 \\
2.68 E-03 \\
9.36 E-01\end{array}$ & $\begin{array}{l}1.06 E-02 \\
1.68 E-04 \\
2.37 E-03 \\
7.56 E-01 \\
1.15 E-02 \\
1.62 E-02 \\
2.58 E-02 \\
3.01 E-03\end{array}$ \\
\hline $\begin{array}{l}\text { Heart Wall } \\
\text { Kidneys } \\
\text { Liver } \\
\text { Lungs }\end{array}$ & $\begin{array}{l}3.26 E-11 \\
4.20 E-15 \\
2.24 E-03 \\
1.97 E-10\end{array}$ & $\begin{array}{l}1.89 \\
6.36 \\
2.47 \\
3.78\end{array}$ & $\begin{array}{l}8.81 E \\
1.31 E \\
6.75 E \\
8.08 E\end{array}$ & $\begin{array}{l}3.0 \\
1.7 \\
1.1 \\
1.9\end{array}$ & $\begin{array}{l}7.71 \\
2.79 \\
9.14 \\
5.76\end{array}$ & $\begin{array}{l}8.38 \\
2.46 \\
5.42 \\
5.59\end{array}$ & $\begin{array}{l}3 \\
12 \\
12 \\
13\end{array}$ & $\begin{array}{l}6 . \\
1 . \\
4 . \\
4 .\end{array}$ & $\begin{array}{l}6.4 \\
1.6 \\
4.1\end{array}$ & $\begin{array}{l}6 . \\
1.5 \\
3 .\end{array}$ & & $\begin{array}{l}5.3 \\
1.2 \\
2.7\end{array}$ \\
\hline $\begin{array}{l}\text { Muscle } \\
\text { Ovaries } \\
\text { Pancreas } \\
\text { Skeletal Tiss: }\end{array}$ & $\begin{array}{l}7.25 E-03 \\
1.48 E-25 \\
4.14 E-05 \\
:\end{array}$ & $\begin{array}{l}1.04 \\
2.35 \\
1.25\end{array}$ & $\begin{array}{l}02 \\
05 \\
02\end{array}$ & $\begin{array}{l}1.25 \mathrm{E} \\
1.61 \mathrm{E} \\
9.39 \mathrm{E}\end{array}$ & $\begin{array}{l}9.50 \\
7.07 \\
8.32\end{array}$ & $\begin{array}{l}3 \\
2 \\
2\end{array}$ & $\begin{array}{l}6.54 \mathrm{E} \\
7.91 \mathrm{E} \\
4.65 \mathrm{E}\end{array}$ & & $\begin{array}{l}6.00 \\
5.85\end{array}$ & 5. & $\begin{array}{l}03 \\
03\end{array}$ & $\begin{array}{l}E-03 \\
E-03 \\
E-02\end{array}$ \\
\hline $\begin{array}{l}\text { Act Marrow } \\
\text { Endosteum } \\
\text { skin } \\
\text { Spleen }\end{array}$ & $\begin{array}{l}1.99 E-14 \\
1.00 E-07 \\
8.24 E-09 \\
9.31 E-24\end{array}$ & $\begin{array}{l}2.64 E-06 \\
1.25 E-05 \\
2.24 E-06 \\
7.78 E-09\end{array}$ & $\begin{array}{l}8.01 E-05 \\
1.79 E-04 \\
1.20 E-04 \\
3.50 E-05\end{array}$ & $\begin{array}{l}1.42 E-03 \\
3.86 E-03 \\
1.03 E-03 \\
2.70 E-03\end{array}$ & $\begin{array}{l}4.76 E-03 \\
1.15 E-02 \\
1.87 E-03 \\
8.83 E-03\end{array}$ & $\begin{array}{l}5.74 E-03 \\
8.70 E-03 \\
1.82 E-03 \\
1.13 E-02\end{array}$ & $\begin{array}{l}5.54 \mathrm{E}-03 \\
4.97 \mathrm{E}-03 \\
1.95 \mathrm{E}-03 \\
9.37 \mathrm{E}-03\end{array}$ & $\begin{array}{l}5.33 E-03 \\
3.22 E-03 \\
2.06 E-03 \\
7.79 E-03\end{array}$ & $\begin{array}{l}5.35 E-03 \\
2.98 E-03 \\
2.14 E-03 \\
7.22 E-03\end{array}$ & $\begin{array}{l}5.05 E-03 \\
2.80 E-03 \\
2.00 E-03\end{array}$ & $\begin{array}{l}4.84 E-03 \\
2.67 E-03 \\
1.91 E-03\end{array}$ & $\begin{array}{l}4.06 E-03 \\
2.27 E-03 \\
1.88 E-03 \\
5.62 E-03\end{array}$ \\
\hline $\begin{array}{l}\text { Thyowus } \\
\text { Thyroid } \\
\text { Urin Bl Wall } \\
\text { Uterine Cont: }\end{array}$ & $\begin{array}{l}0.0 \\
0.0 \\
0.0\end{array}$ & $\begin{array}{l}1.70 E-13 \\
0.0 \\
3.45 E-14\end{array}$ & $\begin{array}{l}8.31 E-08 \\
3.32 E-12 \\
7.26 E-08\end{array}$ & $\begin{array}{l}32 E-05 \\
75 E-07 \\
38 E-04\end{array}$ & $\begin{array}{l}4 E-03 \\
4 E-05 \\
5 E-03\end{array}$ & $\begin{array}{l}1.20 \\
1.95 \\
1.84\end{array}$ & $\begin{array}{l}37 E-03 \\
22 E-04 \\
99 E-03\end{array}$ & & $\begin{array}{l}1.85 E-03 \\
5.53 E-04 \\
2.10 E-03\end{array}$ & $\begin{array}{l}1.99 \\
5.90 \\
2.07\end{array}$ & $\begin{array}{l}2.09 E-03 \\
6.17 E-04 \\
2.05 E-03\end{array}$ & $\begin{array}{l}1.80 E-03 \\
5.82 E-04 \\
1.97 E-03\end{array}$ \\
\hline $\begin{array}{l}\text { Fetus } \\
\text { Uterine Uall } \\
\text { Body Tissues }\end{array}$ & $\begin{array}{l}1.90 E-20 \\
9.45 E-19 \\
1.33 E-02\end{array}$ & $\begin{array}{l}4.39 E-08 \\
2.04 E-07 \\
1.25 E-02\end{array}$ & $\begin{array}{l}5.30 E-05 \\
1.09 E-04 \\
1.31 E-02\end{array}$ & $\begin{array}{l}2.44 E-03 \\
3.34 E-03 \\
1.40 E-02\end{array}$ & $\begin{array}{l}8.01 E-03 \\
7.92 E-03 \\
1.17 E-02\end{array}$ & $\begin{array}{l}8.54 E-03 \\
8.87 E-03 \\
8.42 E-03\end{array}$ & $\begin{array}{l}7.58 E-03 \\
7.48 E-03 \\
7.55 E-03\end{array}$ & $\begin{array}{l}7.17 E-03 \\
7.31 E-03 \\
7.26 E-03\end{array}$ & $6.80 E-03$ & $6.28 E-03$ & 5.95E-03 & $\begin{array}{l}5.00 E-03 \\
5.32 E-03 \\
4.94 E-03\end{array}$ \\
\hline
\end{tabular}

\section{SOURCE = GI TRACT: STOMACH CONTENTS}

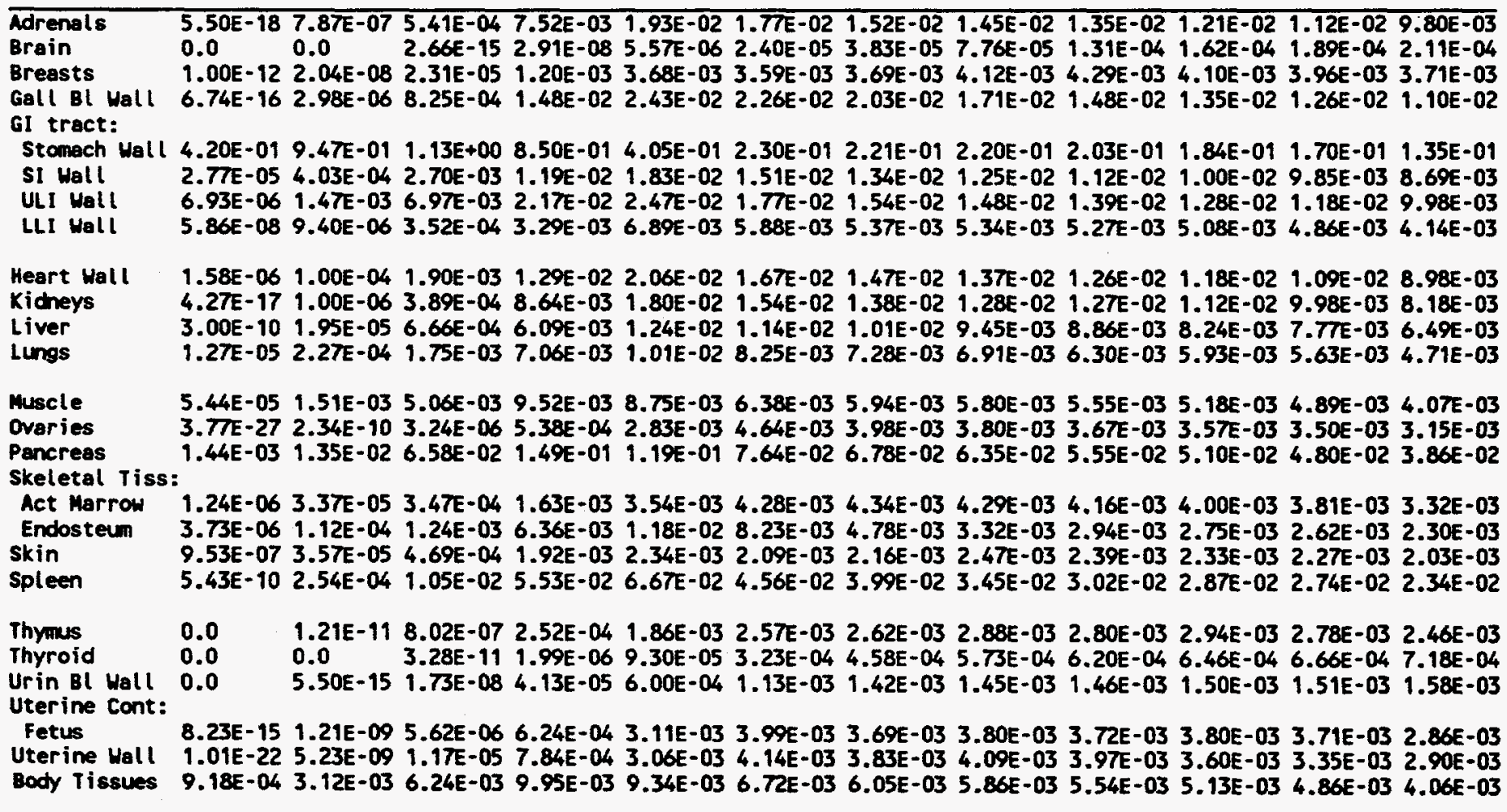


Table A.2 (cont'd). At Three Months Gestation: Specific Absorbed Fraction of Photon Energy (kg-1).

SOURCE = GI TRACT: STOMACH WALL

Energy (MeV)

$\begin{array}{lllllllllllll}\text { Target } & 0.010 & 0.015 & 0.020 & 0.030 & 0.050 & 0.100 & 0.200 & 0.500 & 1.000 & 1.500 & 2.000 & 4.000\end{array}$

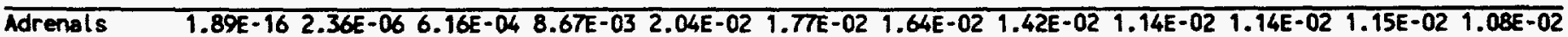

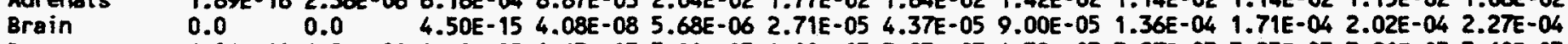

Breasts $\quad 6.96 E-19$ 6.28E-08 4.02E-05 1.63E-03 3.80E-03 4.00E-03 3.93E-03 4.39E-03 3.97E-03 3.93E-03 3.91E-03 3.60E-03

Gall BI Hall 2.01E-14 9.29E-06 1.43E-03 1.61E-02 2.99E-02 2.47E-02 2.10E-02 1.79E-02 1.57E-02 1.47E-02 1.40E-02 1.15E-02

Gi tract:

Stomach Wall 5.80E+00 3.77E+00 2.44E+00 1.15E+00 4.69E-01 2.73E-01 2.70E-01 2.89E-01 2.68E-01 2.43E-01 2.26E-01 1.78E-01 SI Hall $9.00 E-05$ 2.26E-03 5.77E-03 $1.45 E-02$ 2.05E-02 $1.66 E-02$ 1.41E-02 $1.37 \mathrm{E}-02$ 1.29E-02 $1.20 \mathrm{E}-02$ 1.11E-02 8.81E-03 ULI Wall 3.50E-04 7.82E-03 2.15E-02 3.06E-02 2.75E-02 $1.93 E-02 \quad 1.81 E-02$ 1.57E-02 $1.54 E-02 \quad 1.38 E-02$ 1.27E-02 $1.15 E-02$ LLI Wall $\quad 1.86 E-10$ 3.91E-05 6.25E-04 4.67E-03 7.83E-03 6.08E-03 6.34E-03 5.60E-03 5.44E-03 5.22E-03 5.07E-03 4.31E-03

Heart Wall 1.00E-05 3.15E-04 4.22E-03 1.80E-02 2.41E-02 1.86E-02 1.64E-02 1.56E-02 $1.40 E-02 \quad 1.32 E-02$ 1.25E-02 $1.01 E-02$ Kicheys $\quad 1.40 E-15$ 2.80E-06 $6.54 E-04$ 8.07E-03 $1.83 E-02 \quad 1.51 E-02 \quad 1.32 E-02 \quad 1.19 E-02 \quad 1.18 E-02 \quad 1.04 E-02$ 9.55E-03 8.55E-03

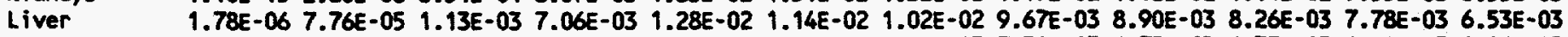
Lungs $\quad 1.33 E-05$ 9.31E-04 3.79E-03 9.34E-03 1.11E-02 8.60E-03 7.71E-03 7.30E-03 6.75E-03 6.33E-03 6.00E-03 4.96E-03

Muscle $\quad 2.85 E-03 \quad 6.93 E-03$ 1.00E-02 1.18E-02 9.42E-03 6.73E-03 6.30E-03 6.26E-03 5.95E-03 5.51E-03 5.22E-03 4.28E-03 Ovaries $\quad 8.31 E-26 \quad 8.51 E-10$ 6.06E-06 6.85E-04 3.07E-03 4.44E-03 4.21E-03 4.32E-03 4.10E-03 3.94E-03 3.79E-03 3.23E-03 Pancreas 2.62E-03 4.41E-02 1.19E-01 1.68E-01 1.24E-01 7.73E-02 6.88E-02 6.64E-02 5.80E-02 5.21E-02 4.84E-02 3.99E-02 Skeletal Tiss:

Act Marrow 1.50E-05 1.26E-04 6.06E-04 1.94E-03 3.72E-03 4.38E-03 4.41E-03 4.43E-03 4.29E-03 4.08E-03 3.91E-03 3.36E-03 Endosteun 2.67E-06 4.18E-04 2.17E-03 7.54E-03 9.25E-02 8.35E-03 4.83E-03 3.42E-03 3.04E-03 2.83E-03 2.69E-03 2.34E-03

Skin

Spleen 5.00E-06 1.11E-04 6.74E-04 2.11E-03 2.34E-03 2.12E-03 2.14E-03 2.43E-03 2.62E-03 2.51E-03 2.43E-03 2.06E-03 2.16E-08 1.11E-03 1.47E-02 6.14E-02 6.68E-02 4.48E-02 3.83E-02 3.72E-02 3.40E-02 2.98E-02 2.70E-02 2.25E-02

Thymus $\quad 0.0 \quad 3.81 E-11$ 1.11E-06 3.52E-04 $1.91 E-03 \quad 2.98 E-03 \quad 3.01 E-03 \quad 2.85 E-03 \quad 2.91 E-03 \quad 2.82 E-03 \quad 2.79 E-03 \quad 2.60 E-03$

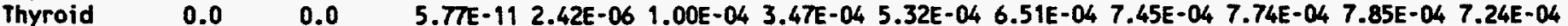
Urin Bl Wall $0.0 \quad 2.06 E-14$ 3.25E-08 5.16E-05 7.93E-04 $1.01 E-03 \quad 1.13 E-03 \quad 1.22 E-03 \quad 1.32 E-03 \quad 1.39 E-03 \quad 1.45 E-03 \quad 1.61 E-03$ Uterine Cont:

Fetus

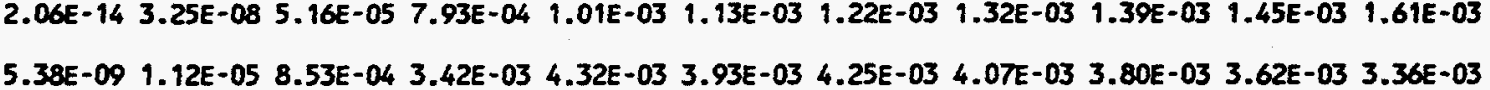
Uterine Hall 3.52E-21 $1.65 E-08$ 1.99E-05 $1.01 E-03 \quad 3.69 E-03 \quad 4.16 E-03 \quad 3.91 E-03 \quad 4.17 E-03 \quad 4.26 E-03 \quad 4.00 E-03 \quad 3.72 E-03 \quad 3.39 E-03$ Body Tissues 1.62E-02 1.44E-02 1.37E-02 1.31E-02 1.03E-02 7.35E-03 6.73E-03 6.55E-03 6.20E-03 5.71E-03 5.41E-03 4.50E-03

\section{SOURCE = GI TRACT: SMAL INTESTINE CONTENTS}

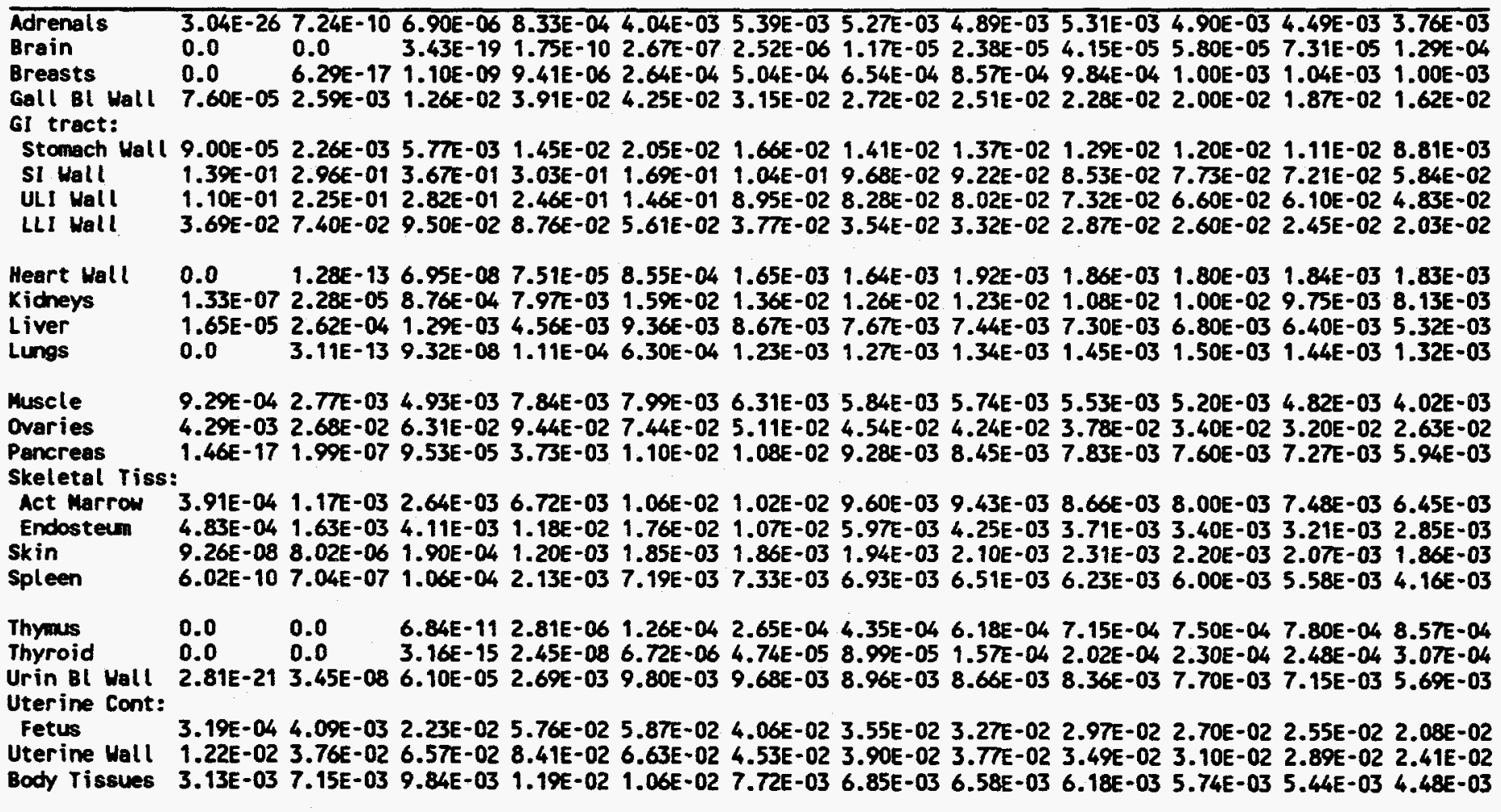


Table A.2 (cont'd). At Three Months Gestation: Specific Absorbed Fraction of Photon Energy (kg').

SOURCE = GI TRACT: SMALL INTESTINE WALL

Energy (MeV)

\begin{tabular}{|c|c|c|c|c|c|c|c|c|c|c|c|c|}
\hline Target & 0.010 & 0.015 & 0.020 & 0.030 & 0.050 & 0.100 & 0.200 & 0.500 & 1.000 & 1.500 & 2.000 & 4.000 \\
\hline $\begin{array}{l}\text { Adrenals } \\
\text { Brain } \\
\text { Breasts } \\
\text { Gall BI Mall } \\
\text { GI tract: } \\
\text { Stomach Hall } \\
\text { SI Hall } \\
\text { ULI Wall } \\
\text { LLI Hall }\end{array}$ & $\begin{array}{l}3.04 E-26 \\
0.0 \\
0.0 \\
7.60 E-05 \\
9.00 E-05 \\
1.52 E+00 \\
1.10 E-01 \\
3.69 E-02\end{array}$ & $\begin{array}{l}7.24 E-10 \\
0.0 \\
6.29 E-17 \\
2.59 E-03 \\
2.26 E-03 \\
1.26 E+00 \\
2.25 E-01 \\
7.40 E-02\end{array}$ & $\begin{array}{l}6.90 E-06 \\
3.43 E-19 \\
1.10 E-09 \\
1.26 E-02 \\
5.77 E-03 \\
9.78 E-01 \\
2.82 E-01 \\
9.50 E-02\end{array}$ & $\begin{array}{l}8.33 E-04 \\
1.75 E-10 \\
9.41 E-06 \\
3.91 E-02 \\
1.45 E-02 \\
5.74 E-01 \\
2.46 E-01 \\
8.76 E-02\end{array}$ & $\begin{array}{l}4.04 E-03 \\
2.67 E-07 \\
2.64 E-04 \\
4.25 E-02 \\
2.05 E-02 \\
2.66 E-01 \\
1.46 E-01 \\
5.61 E-02\end{array}$ & $\begin{array}{l}5.39 E-03 \\
2.52 E-06 \\
5.04 E-04 \\
3.15 E-02 \\
1.66 E-02 \\
1.62 E-01 \\
8.95 E-02 \\
3.77 E-02\end{array}$ & $\begin{array}{l}5.27 E-03 \\
1.17 E-05 \\
6.54 E-04 \\
2.72 E-02 \\
1.41 E-02 \\
1.57 E-01 \\
8.28 E-02 \\
3.54 E-02\end{array}$ & $\begin{array}{l}4.89 E-03 \\
2.38 E-05 \\
8.57 E-04 \\
2.51 E-02 \\
1.37 E-02 \\
1.57 E-01 \\
8.02 E-02\end{array}$ & $\begin{array}{l}4.15 E-05 \\
9.84 E-04 \\
2.28 E-02 \\
1.29 E-02 \\
1.41 E-01 \\
7.32 E-02 \\
2.87 E-02\end{array}$ & $\begin{array}{l}4.90 E-03 \\
5.80 E-05 \\
1.00 E-03 \\
2.00 E-02\end{array}$ & $\begin{array}{l}4.49 E-03 \\
7.31 E-05 \\
1.04 E-03 \\
1.87 E-02\end{array}$ & $\begin{array}{l}3.76 E-03 \\
1.29 E-04 \\
1.00 E-03 \\
1.62 E-02\end{array}$ \\
\hline
\end{tabular}

Heart Wall $\quad 0.0 \quad 1.28 E-13 \quad 6.95 E-08 \quad 7.51 E-05 \quad 8.55 E-04 \quad 1.65 E-03 \quad 1.64 E-03 \quad 1.92 E-03 \quad 1.86 E-03 \quad 1.80 E-03 \quad 1.84 E-03 \quad 1.83 E-03$

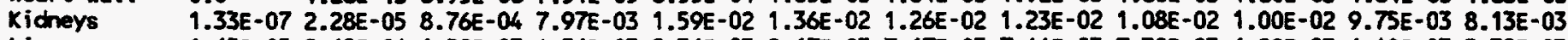

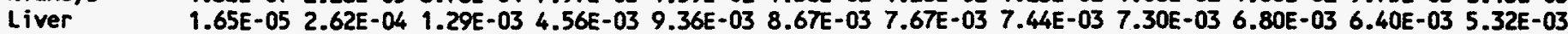
Lungs $\quad 0.0 \quad 3.11 E-139.32 E-08$ 1.11E-04 $6.30 E-04 \quad 1.23 E-03 \quad 1.27 E-03 \quad 1.34 E-03 \quad 1.45 E-03 \quad 1.50 E-03 \quad 1.44 E-03 \quad 1.32 E-03$

Muscle $\quad 9.29 E-04 \quad 2.77 E-03 \quad 4.93 E-03 \quad 7.84 E-03 \quad 7.99 E-03 \quad 6.31 E-03 \quad 5.84 E-03 \quad 5.74 E-03 \quad 5.53 E-03 \quad 5.20 E-03$ 4.82E-03 4.02E-03 ovaries 4.29E-03 2.68E-02 6.31E-02 $9.44 E-02$ 7.44E-02 5.11E-02 4.54E-02 4.24E-02 3.78E-02 3.40E-02 3.20E-02 2.63E-02 Pancreas 1.46E-17 1.99E-07 9.53E-05 3.73E-03 1.10E-02 1.08E-02 9.28E-03 8.45E-03 7.83E-03 7.60E-03 7.27E-03 5.94E-03 Skeletal Tiss:

Act Marrow 3.91E-04 1.17E-03 2.64E-03 6.72E-03 1.06E-02 1.02E-02 9.60E-03 9.43E-03 8.66E-03 8.00E-03 7.48E-03 6.45E-03 Endosteum 4.83E-04 1.63E-03 4.11E-03 1.18E-02 1.76E-02 1.07E-02 5.97E-03 4.25E-03 3.71E-03 3.40E-03 3.21E-03 2.85E-03 Skin

Spleen 9.26E-08 8.02E-06 1.90E-04 $1.20 E-03 \quad 1.85 E-03$ 1.86E-03 $1.94 E-03$ 2.10E-03 2.31E-03 2.20E-03 2.07E-03 1.86E-03 6.02E-10 7.04E-07 1.06E-04 2.13E-03 7.19E-03 7.33E-03 6.93E-03 6.51E-03 6.23E-03 6.00E-03 5.58E-03 4.16E-03

Thymus $\quad 0.0 \quad 0.0 \quad 6.84 E-11 \quad 2.81 E-06 \quad 1.26 E-04 \quad 2.65 E-04 \quad 4.35 E-04 \quad 6.18 E-04 \quad 7.15 E-04 \quad 7.50 E-04 \quad 7.80 E-04 \quad 8.57 E-04$

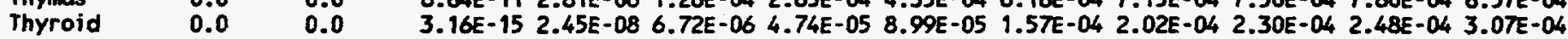
Urin Bl Wall 2.81E-21 3.45E-08 6.10E-05 2.69E-03 9.80E-03 9.68E-03 8.96E-03 8.66E-03 8.36E-03 7.70E-03 7.15E-03 5.69E-03 Uterine Cont:

Fetus

3.19E-04 4.09E-03 2.23E-02 5.76E-02 5.87E-02 4.06E-02 3.55E-02 3.27E-02 2.97E-02 2.70E-02 2.55E-02 2.08E-02 Body Tissues 1.68E-02 1.54E-02 $1.44 E-02$ 1.35E-02 $1.11 E-02$ 8.05E-03 7.20E-03 6.96E-03 6.51E-03 6.06E-03 5.75E-03 $4.72 E-03$

\section{SOURCE $=$ GI TRACT: UPPER LARGE INTESTINE CONTENTS}

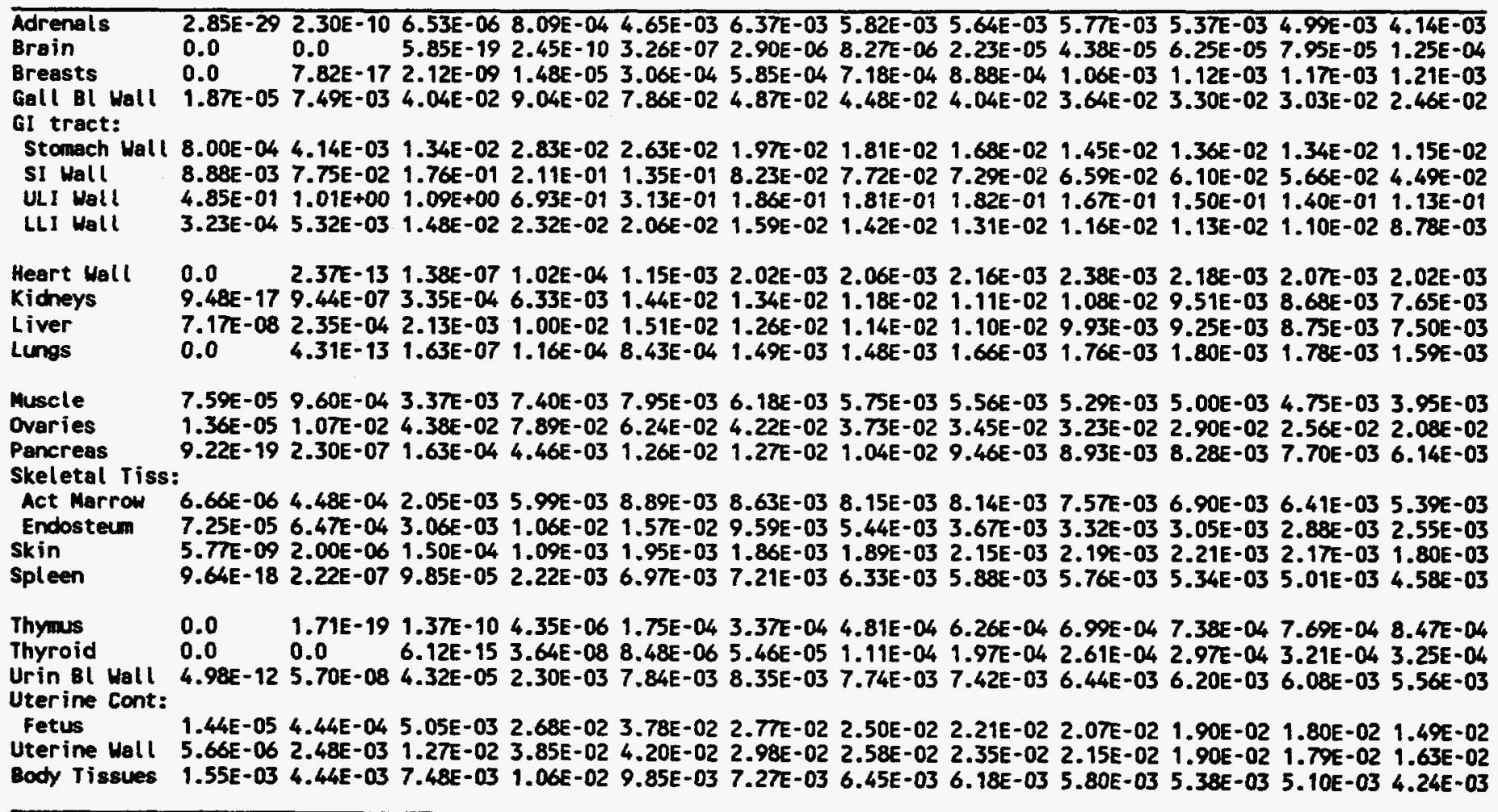


Table A.2 (cont'd). At Three Months Gestation: Specific Absorbed Fraction of Photon Energy (kg-1).

SOURCE = GI TRACT: UPPER LARGE INTESTINE WALL

Energy (MeV)

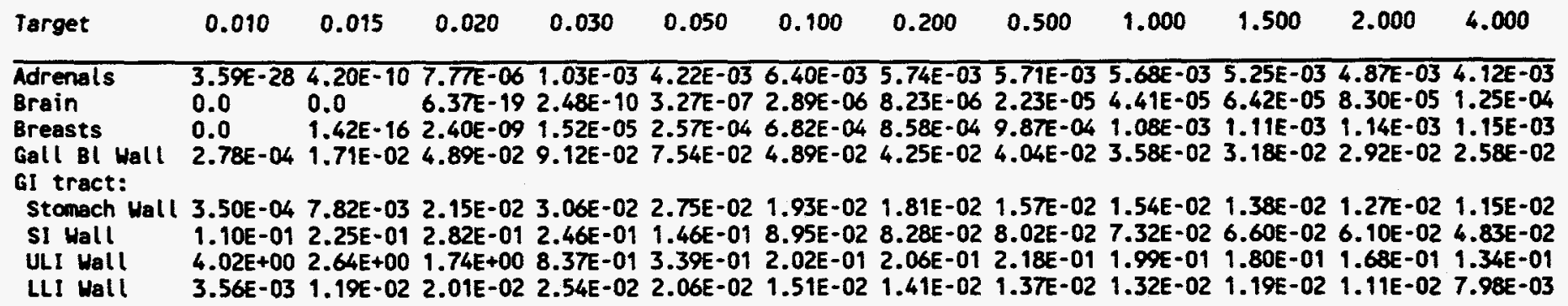

Heart Hall $0.0 \quad 4.01 E-13 \quad 1.58 E-07$ 1.18E-04 $1.25 E-03 \quad 1.90 E-03 \quad 1.95 E-03 \quad 2.07 E-03 \quad 2.10 E-03 \quad 2.10 E-03 \quad 2.05 E-03 \quad 2.00 E-03$ $2.02 E-152.14 E-06$ 3.76E-04 6.98E-03 1.46E-02 1.36E-02 1.21E-02 1.13E-02 1.02E-02 9.57E-03 9.04E-03 7.03E-03

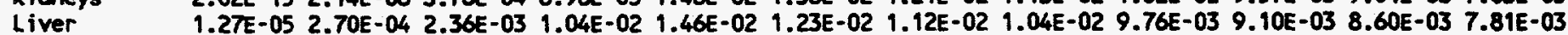
Lungs $\quad 0.0 \quad 7.30 E-131.89 E-07$ 1.66E-04 8.85E-04 $1.38 E-03 \quad 1.48 E-03 \quad 1.59 E-03 \quad 1.62 E-03 \quad 1.63 E-03 \quad 1.63 E-03 \quad 1.36 E-03$

Muscle $\quad 6.39 E-04 \quad 2.17 E-03 \quad 4.46 E-03$ 7.96E-03 8.15E-03 6.34E-03 5.82E-03 5.68E-03 5.40E-03 5.10E-03 4.82E-03 4.03E-03 Ovaries $\quad 3.78 E-04$ 2.91E-02 7.55E-02 9.03E-02 6.80E-02 4.49E-02 4.14E-02 3.82E-02 3.68E-02 3.30E-02 3.01E-02 2.41E-02 Pancreas $\quad 1.52 E-17$ 3.88E-07 1.89E-04 4.87E-03 1.25E-02 1.24E-02 1.04E-02 8.91E-03 8.77E-03 8.02E-03 7.45E-03 6.45E-03 Skeletal Tiss:

Act Marrow 2.15E-04 1.48E-03 3.61E-03 7.11E-03 9.41E-03 8.95E-03 8.54E-03 8.18E-03 7.40E-03 7.10E-03 6.88E-03 5.66E-03 Endosteum 2.75E-04 2.08E-03 5.55E-03 1.24E-02 $1.63 E-02$ 9.74E-03 5.52E-03 3.83E-03 3.29E-03 3.13E-03 3.02E-03 2.54E-03 Skin Spleen 3.56E-08 5.56E-06 2.00E-04 1.09E-03 2.02E-03 1.87E-03 2.02E-03 2.11E-03 2.18E-03 2.17E-03 2.17E-03 1.78E-03 1.27E-10 3.90E-07 1.16E-04 2.91E-03 6.22E-03 7.11E-03 6.30E-03 6.08E-03 5.79E-03 5.60E-03 5.44E-03 4.98E-03

Thymus $\quad 0.0 \quad 2.91 E-19 \quad 1.57 E-10 \quad 4.43 E-06 \quad 1.49 E-04 \quad 4.15 E-04 \quad 6.38 E-04 \quad 7.35 E-04 \quad 7.72 E-04 \quad 8.10 E-04 \quad 8.32 E-04 \quad 8.43 E-04$

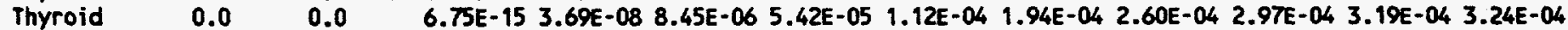
Urin Bl Woll 7.89E-12 7.80E-08 5.33E-05 2.57E-03 7.31E-03 8.14E-03 7.19E-03 7.33E-03 6.76E-03 6.26E-03 5.93E-03 5.19E-03 Uterine Cont:

Fetus

Uterine Hall

Body Tissues

2.94E-05 6.84E-04 6.39E-03 3.02E-02 3.83E-02 2.91E-02 2.57E-02 2.24E-02 2.05E-02 1.90E-02 1.83E-02 1.49E-02 1.70E-04 6.11E-03 2.04E-02 4.18E-02 4.14E-02 3.07E-02 2.66E-02 2.47E-02 2.20E-02 2.10E-02 2.05E-02 1.65E-02 1.55E-02 1.30E-02 1.23E-02 1.23E-02 1.04E-02 7.59E-03 6.84E-03 6.62E-03 6.19E-03 5.73E-03 5.43E-03 4.53E-03

\section{SOURCE = GI TRACT: LOWER LARGE INTESTINE CONTENTS}

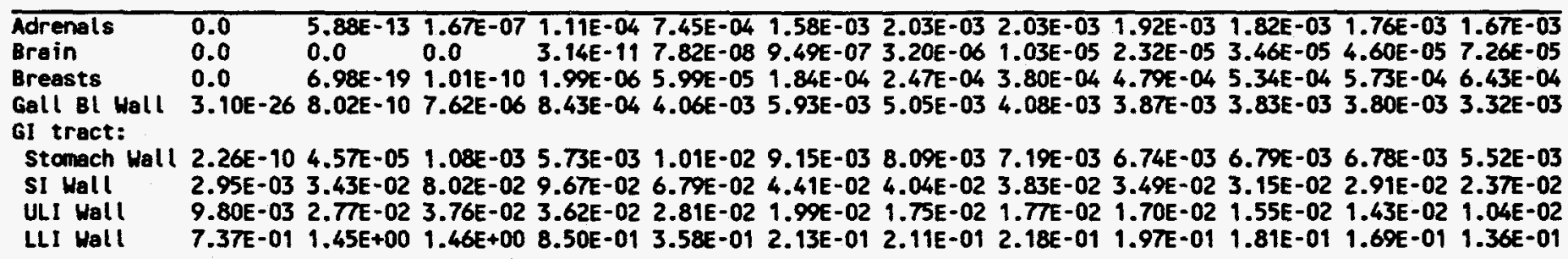

Heart Hall $\quad 0.0 \quad 1.02 E-15 \quad 4.83 E-09$ 1.48E-05 3.71E-04 5.00E-04 6.34E-04 7.83E-04 8.72E-04 9.14E-04 9.44E-04 1.01E-03

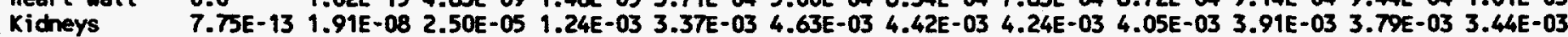

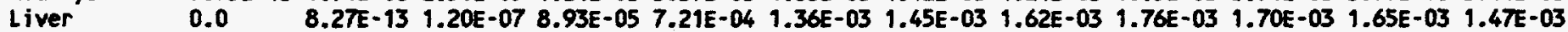
Lungs $\quad 0.0 \quad 3.42 E-15 \quad 6.50 E-09$ 1.33E-05 2.34E-04 4.11E-04 4.46E-04 5.36E-04 6.72E-04 7.12E-04 7.23E-04 7.35E-04

muscle $\quad 1.00 E-04$ 1.76E-03 5.05E-03 9.11E-03 9.17E-03 7.15E-03 6.64E-03 6.52E-03 6.12E-03 5.66E-03 5.35E-03 4.46E-03 Ovaries $\quad 3.73 E-03$ 9.30E-02 2.25E-01 2.35E-01 1.32E-01 8.11E-02 7.82E-02 8.01E-02 6.90E-02 6.11E-02 5.58E-02 4.60E-02 Pancreas $\quad 1.52 E-27$ 2.49E-10 3.93E-06 5.16E-04 2.71E-03 4.24E-03 3.62E-03 3.37E-03 3.16E-03 2.96E-03 2.87E-03 2.85E-03 Skeletal Tiss:

Act Marrow 3.07E-05 1.75E-03 7.62E-03 1.54E-02 1.61E-02 1.34E-02 1.24E-02 $1.22 E-02 \quad 1.10 E-02 \quad 1.01 E-02$ 9.49E-03 7.67E-03

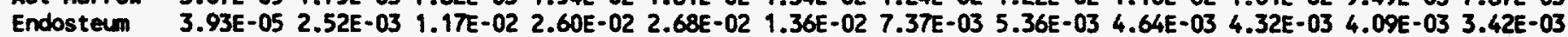

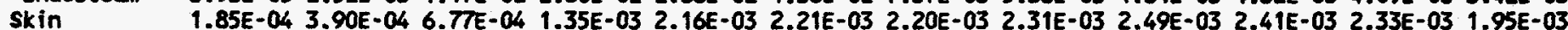
Spleen $\quad 1.58 E-219.26 E-09$ 1.73E-05 9.34E-04 3.28E-03 4.54E-03 3.58E-03 3.42E-03 3.39E-03 3.31E-03 3.26E-03 3.22E-03

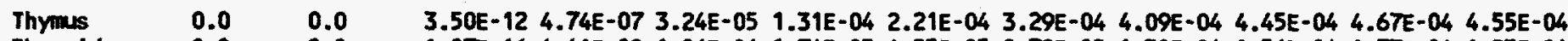

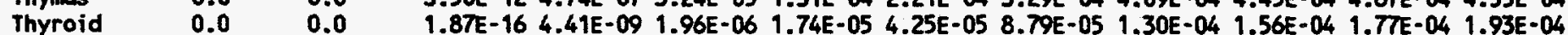
Urin Bl Hall 3.73E-07 5.54E-04 1.41E-02 5.42E-02 5.32E-02 3.65E-02 3.16E-02 2.95E-02 2.62E-02 2.40E-02 2.20E-02 1.64E-02 Uterine Cont:

Fetus

9.28E-08 3.96E-04 8.04E-03 4.04E-02 4.77E-02 3.57E-02 3.06E-02 2.80E-02 2.42E-02 2.20E-02 2.09E-02 1.79E-02 Uterine Hall 2.92E-06 3.59E-03 1.84E-02 5.39E-02 5.27E-02 3.71E-02 3.32E-02 3.00E-02 $2.73 E-02$ 2.40E-02 $2.23 E-02 \quad 1.98 E-02$ Body Tissues 1.7TE-03 5.28E-03 8.81E-03 1.20E-02 1.07E-02 7.53E-03 6.66E-03 6.43E-03 5.98E-03 5.56E-03 5.28E-03 4.39E-03 
Table A.2 (cont'd). At Three Months Gestation: Specific Absorbed Fraction of Photon Energy (kg-1).

SOURCE = GI TRACT: LOWER LARGE INTESTINE WALL

Energy (MeV)

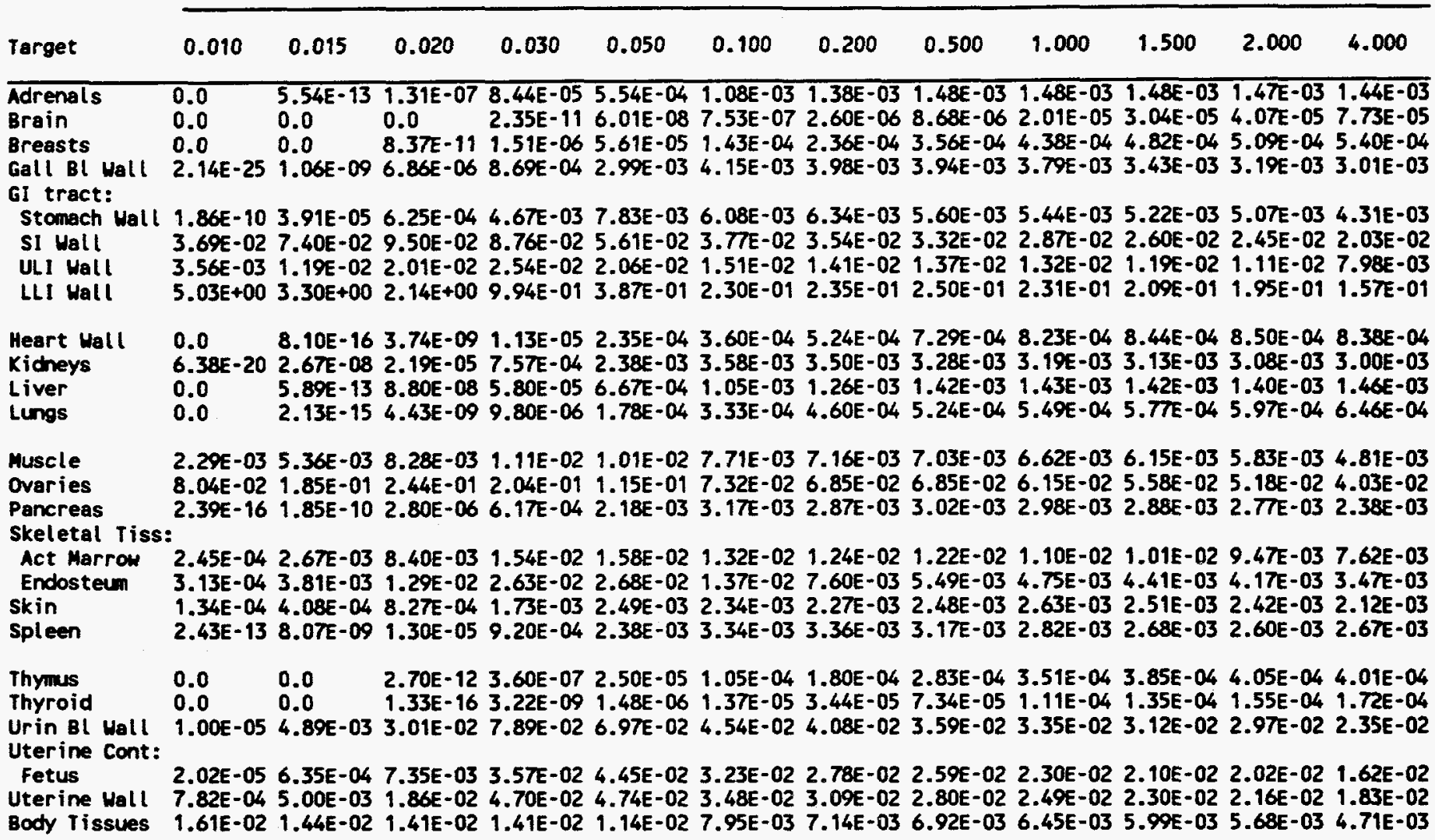

SOURCE $=$ HEART CONTENTS

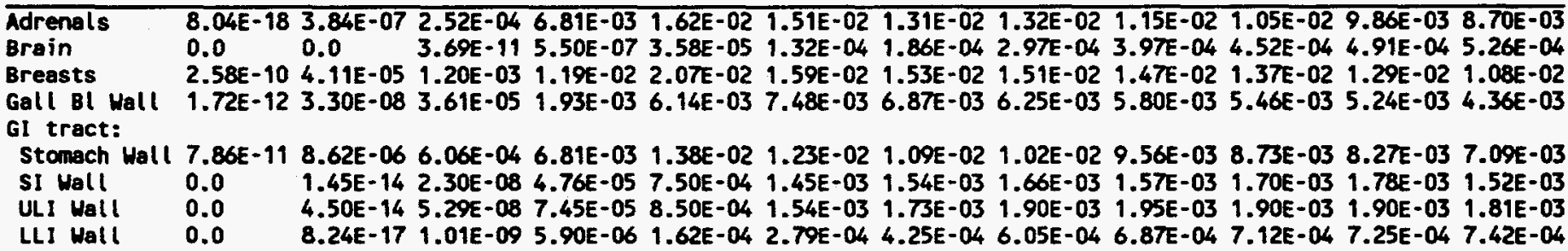

Heart Hall

Kidneys

2.48E-01 5.48E-01 6.86E-01 5.69E-01 2.93E-01 1.68E-01 1.60E-01 1.57E-01 1.45E-01 1.32E-01 1.22E-01 9.85E-02

Liver

$1.14 E-16 \quad 1.34 E-10$ 2.72E-06 7.06E-04 3.45E-03 4.62E-03 $4.29 E-03 \quad 4.50 E-03$ 4.50E-03 $4.12 E-03$ 3.87E-03 3.54E-03 1.88E-06 1.46E-04 2.26E-03 1.31E-02 1.97E-02 1.57E-02 $1.37 \mathrm{FE}-02$ 1.27E-02 $1.16 \mathrm{E}-02$ 1.09E-02 1.00 E-02 8.28E-03

Lungs

2.61E-05 3.10E-03 2.03E-02 5.51E-02 5.14E-02 3.27E-02 2.80E-02 2.59E-02 2.36E-02 2.13E-02 1.99E-02 1.62E-02

Muscle

Ovaries

Pancreas

Skeletal Tiss

Act Marrow

Endosteum

Skin

8.96E-05 1.25E-03 3.84E-03 7.64E-03 7.65E-03 5.82E-03 5.30E-03 5.18E-03 4.91E-03 4.60E-03 4.36E-03 3.64E-03

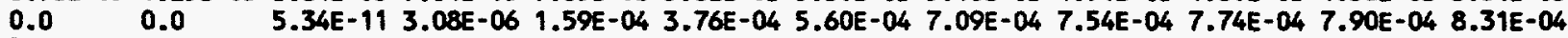
7.48E- 16 1.52E-06 5.03E-04 9.67E-03 2.03E-02 1.75E-02 $1.54 E-02$ 1.41E-02 $1.27 E-02$ 1.21E-02 $1.18 E-02$ 9.34E-03

Spleen

2.22E-07 4.46E-05 4.27E-04 2.52E-03 5.22E-03 5.66E-03 5.56E-03 5.51E-03 5.23E-03 4.93E-03 4.70E-03 4.00E-03 6.68E-07 1.45E-04 1.4TE-03 9.39E-03 1.80E-02 1.15E-02 $6.38 E-03 \quad 4.49 E-03 \quad 3.93 E-03 \quad 3.62 E-03 \quad 3.42 E-03 \quad 2.95 E-03$ 2.66E-06 4.09E-05 2.93E-04 1.44E-03 2.26E-03 2.10E-03 2.26E-03 2.45E-03 2.43E-03 2.38E-03 2.31E-03 2.01E-03 3.08E-22 1.19E-08 2.90E-05 2.83E-03 7.62E-03 8.35E-03 7.28E-03 6.82E-03 6.13E-03 5.73E-03 5.47E-03 4.99E-03

Thymus

Thyroid

4.32E-05 4.99E-03 2.91E-02 7.94E-02 7.43E-02 4.81E-02 4.29E-02 4.08E-02 3.77E-02 3.44E-02 3.19E-02 2.59E-02

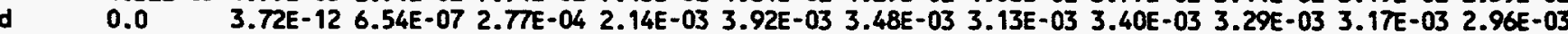

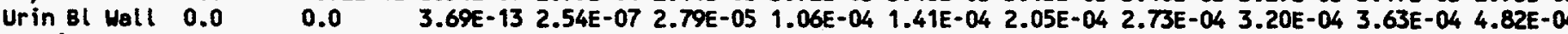
uterine cont:

Fetus

0.0

Uterine Hall 0.0

3.69E-13 2.54E-07 2.79E-05 1.06E-04 1.41E-04 2.05E-04 2.73E-04 3.20E-04 3.63E-04 4.82E-04

Body Tissues

7.46E-19 1.68E-10 4.08E-06 1.64E-04 3.08E-04 4.99E-04 7.34E-04 8.48E-04 8.90E-04 9.02E-04 9.16E-04 2.76E-18 3.00E-10 4.83E-06 $1.28 E-04$ 3.78E-04 6.00E-04 7.17E-04 6.99E-04 7.30E-04 7.67E-04 $9.11 E-04$

1.77E-02 1.77E-02 1.7TE-02 1.67E-02 1.25E-02 8.60E-03 7.74E-03 7.56E-03 7.07E-03 6.51E-03 6.13E-03 5.07E-03 
Table A.2 (cont'd). At Three Months Gestation: Specific Absorbed Fraction of Photon Energy ( $\left.\mathrm{kg}^{-1}\right)$.

SOURCE $=$ HEART WALL

Energy (MeV)

\begin{tabular}{|c|c|c|c|c|c|c|c|c|c|c|c|c|}
\hline irs & 010 & 15 & 020 & 130 & 0.050 & 100 & .200 & .500 & 1.000 & 1.500 & .000 & 4.000 \\
\hline $\begin{array}{l}\text { Brain } \\
\text { Breasts } \\
\text { Gall Bi Wall } \\
\text { GI tract: }\end{array}$ & $\begin{array}{l}1.80 E-16 \\
0.0 \\
5.14 E-06 \\
3.26 E-11\end{array}$ & $\begin{array}{l}1.47 \mathrm{E}-06 \\
0.0 \\
1.89 E-04 \\
1.89 E-07\end{array}$ & $\begin{array}{l}4.50 \\
3.08 \\
2.46 \\
8.8\end{array}$ & $\begin{array}{l}3 \\
7 \\
2 \\
3\end{array}$ & $\begin{array}{l}1.9 \\
3.1 \\
2.2 \\
7.7\end{array}$ & $\begin{array}{l}14 \\
12\end{array}$ & & & & & & $\begin{array}{l}04 \\
02 \\
03\end{array}$ \\
\hline $\begin{array}{l}\text { Stomach Wall } \\
\text { SI Wall } \\
\text { ULI Wall } \\
\text { LLI Wall }\end{array}$ & $\begin{array}{l}1.00 E-05 \\
0.0 \\
0.0 \\
0.0\end{array}$ & $\begin{array}{l}3.15 E-04 \\
1.28 E-13 \\
4.01 E-13 \\
8.10 E-16\end{array}$ & 8 & $\begin{array}{l}7 . \\
1 . \\
1 .\end{array}$ & $\begin{array}{l}8 \\
1 \\
2\end{array}$ & & & & & & $\begin{array}{l}E-02 \\
E-03 \\
E-03 \\
E-04\end{array}$ & \\
\hline
\end{tabular}

Heart Well 3.49E+00 2.54E+00 1.79E+00 9.29E-01 4.02E-01 2.35E-01 2.36E-01 2.46E-01 2.25E-01 2.03E-01 1.87E-01 1.52E-01 Kioneys 6.80E-26 6.76E-10 6.27E-06 8.52E-04 3.75E-03 5.12E-03 4.79E-03 4.73E-03 4.69E-03 $4.37 E-03$ 4.15E-03 3.56E-03

Liver

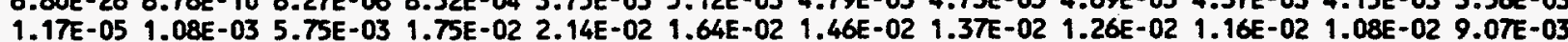

Lungs 2.10E-04 7.13E-03 2.72E-02 5.56E-02 4.83E-02 3.06E-02 2.63E-02 2.48E-02 2.24E-02 2.05E-02 1.90E-02 1.54E-02

Muscle

Overies

Pancreas

Skeletal Tiss:

Act Marrow

Endosteum

1.95E-03 4.54E-03 6.94E-03 9.17E-03 8.03E-03 5.95E-03 5.55E-03 5.46E-03 5.18E-03 4.82E-03 4.55E-03 3.78E-03

skin

Spleen $0.0 \quad 2.00 E-19$ 1.57E-10 4.98E-06 2.09E-04 4.44E-04 6.78E-04 8.72E-04 9.29E-04 9.30E-04 9.27E-04 9.05E-04 5.67E-13 1.76E-05 2.04E-03 1.61E-02 2.90E-02 2.25E-02 $1.93 E-02 \quad 1.80 E-02 \quad 1.55 E-02 \quad 1.41 E-02 \quad 1.32 E-02 \quad 1.13 E-02$

4.11E-06 1.44E-04 7.16E-04 2.77E-03 5.07E-03 5.36E-03 5.29E-03 5.30E-03 5.05E-03 4.81E-03 4.56E-03 3.90E-03 1.24E-05 4.76E-04 2.51E-03 1.05E-02 1.75E-02 1.09E-02 6.07E-03 4.33E-03 3.82E-03 3.55E-03 3.34E-03 2.87E-03 1.95E-05 1.32E-04 5.15E-04 1.03E-03 2.33E-03 2.19E-03 2.31E-03 2.56E-03 2.54E-03 2.44E-03 2.35E-03 2.16E-03 2.77E-19 1.13E-07 1.23E-04 4.39E-03 1.24E-02 1.06E-02 9.46E-03 8.80E-03 8.35E-03 8.14E-03 7.95E-03 6.73E-03

Thymus 4.56E-04 9.39E-03 3.11E-02 6.71E-02 6.12E-02 4.03E-02 3.66E-02 3.48E-02 3.19E-02 2.96E-02 2.72E-02 2.25E-02

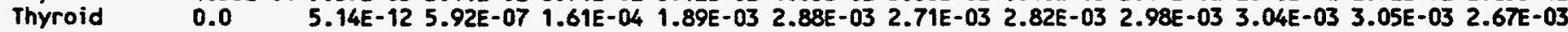
Urin Bl Wall $0.0 \quad 0.0 \quad 1.08 E-124.16 E-073.69 E-05$ 1.66E-04 2.10E-04 2.81E-04 3.47E-04 3.90E-04 4.27E-04 5.24E-04 Uterine Cont:

Fetus

0.0

5.04E-18 4.74E-10 6.64E-06 1.40E-04 3.85E-04 6.34E-04 7.94E-04 8.71E-04 9.20E-04 9.46E-04 1.01E-03

Uterine Hall 0.0

2.20E-17 8.85E-10 7.97E-06 1.95E-04 4.27E-04 6.45E-04 8.01E-04 8.95E-04 9.50E-04 9.78E-04 1.00E-03 Body Tissues 1.7TE-02 1.7TE-02 1.77E-02 1.64E-02 1.22E-02 8.30E-03 7.55E-03 7.40E-03 6.88E-03 6.34E-03 5.97E-03 4.95E-03

\section{SOURCE $=$ KIDNEYS}

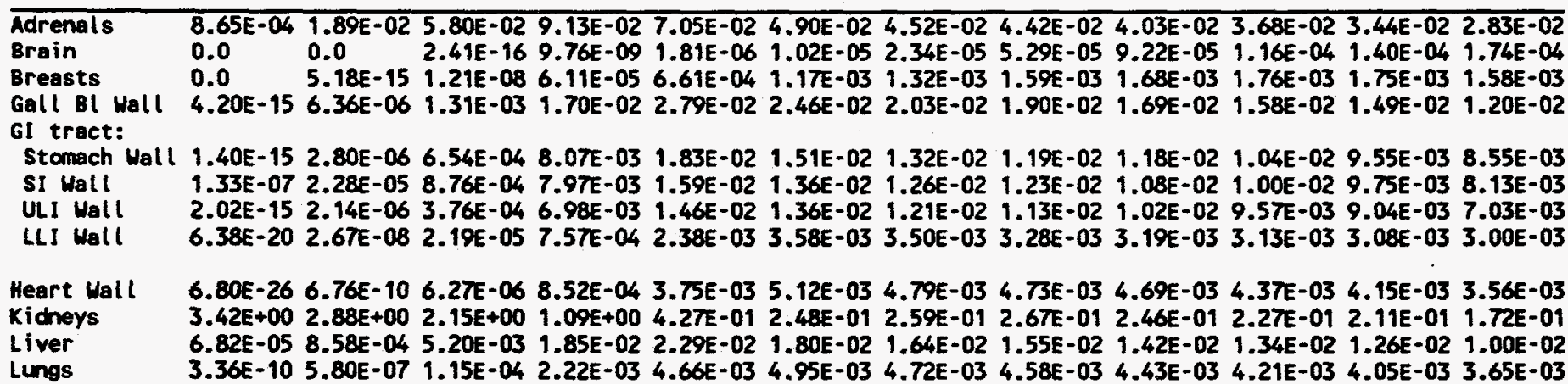

Muscle $\quad 1.72 E-03$ 5.13E-03 8.69E-03 1.03E-02 8.05E-03 6.06E-03 5.82E-03 5.82E-03 5.56E-03 5.20E-03 4.92E-03 4.13E-03 Ovaries $\quad 1.40 E-27$ 3.29E-10 5.40E-06 7.33E-04 3.48E-03 5.08E-03 4.69E-03 4.89E-03 4.80E-03 4.44E-03 4.19E-03 3.87E-03 Pancreas 4.09E-12 6.31E-05 4.00E-03 3.00E-02 4.17E-02 3.19E-02 2.77E-02 2.63E-02 2.39E-02 2.21E-02 2.05E-02 1.66E-02 Skeletal Tiss:

Act Marrow 4.36E-06 3.43E-04 1.88E-03 6.33E-03 9.91E-03 9.94E-03 9.95E-03 9.87E-03 9.29E-03 8.72E-03 8.22E-03 6.94E-03 Endosteun 8.52E-06 7.72E-04 4.62E-03 1.63E-02 2.25E-02 1.25E-02 7.18E-03 5.34E-03 4.77E-03 4.41E-03 4.17E-03 3.59E-03 skin

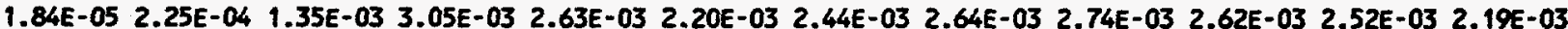
Spleen $\quad 1.41 E-04$ 1.02E-02 4.37E-02 8.44E-02 6.53E-02 4.22E-02 3.81E-02 3.66E-02 3.35E-02 3.07E-02 2.85E-02 2.30E-02

Thymus $\quad 0.0 \quad 3.30 E-16 \quad 5.08 E-09$ 2.66E-05 5.31E-04 $1.09 E-03$ 1.18E-03 $1.42 E-03 \quad 1.52 E-03 \quad 1.53 E-03 \quad 1.52 E-03 \quad 1.49 E-03$ Thyroid $\quad 0.0 \quad 0.0 \quad 9.85 E-13$ 2.06E-06 6.63E-05 $1.92 E-04 \quad 2.58 E-04 \quad 3.20 E-04 \quad 4.67 E-04 \quad 5.29 E-04$ 5.53E-04 5.56E-04 Urin Bl Wall $0.0 \quad 2.08 E-15$ 1.26E-08 3.97E-05 $4.57 E-04 \quad 1.04 E-03 \quad 1.51 E-03 \quad 1.86 E-03 \quad 1.93 E-03 \quad 1.90 E-03 \quad 1.82 E-03 \quad 1.62 E-03$ Uterine Cont:

Fetus

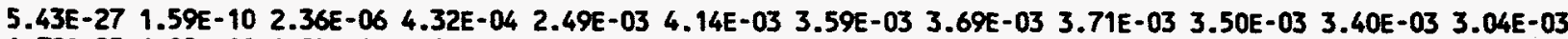
Uterine Wall $1.78 E-25 \quad 6.82 E-10 \quad 4.92 E-06 \quad 4.07 E-04 \quad 2.60 E-03 \quad 3.94 E-03 \quad 3.82 E-03 \quad 3.69 E-03 \quad 3.83 E-03 \quad 3.60 E-03 \quad 3.38 E-03 \quad 3.09 E-03$ Body Tissues 1.77E-02 1.77E-02 1.73E-02 1.50E-02 1.08E-02 7.57E-03 7.00E-03 6.84E-03 6.50E-03 6.02E-03 5.71E-03 4.75E-03 
Table A.2 (cont'd). At Three Months Gestation: Specific Absorbed Fraction of Photon Energy ( $\left.\mathrm{kg}^{-1}\right)$.

SOURCE = LIVER

Energy (MeV)

\begin{tabular}{|c|c|c|c|c|c|c|c|c|c|c|c|c|}
\hline Target & 0.010 & 0.015 & 0.020 & 0.030 & 0.050 & 0.100 & 0.200 & 0.500 & 1.000 & 1.500 & 2.000 & 4.000 \\
\hline $\begin{array}{l}\text { Adrenals } \\
\text { Brain } \\
\text { Breasts } \\
\text { Gall BI Hall } \\
\text { GI tract: } \\
\text { stomach Wall } \\
\text { SI Wall } \\
\text { ULI Mall } \\
\text { LLI Wall }\end{array}$ & $\begin{array}{l}5.43 E-05 \\
0.0 \\
8.59 E-12 \\
2.24 E-03 \\
1.78 E-06 \\
1.65 E-05 \\
1.27 E-05 \\
0.0\end{array}$ & $\begin{array}{l}4.26 E-03 \\
0.0 \\
1.17 E-07 \\
2.47 E-02 \\
7.76 E-05 \\
2.62 E-04 \\
2.70 E-04 \\
5.89 E-13\end{array}$ & $\begin{array}{l}1.83 E-02 \\
1.32 E-14 \\
1.04 E-04 \\
6.75 E-02\end{array}$ & $\begin{array}{l}3.72 E-02 \\
2.27 E-07 \\
1.80 E-03 \\
1.13 E-01 \\
7.06 E-03 \\
4.56 E-03 \\
1.04 E-02 \\
5.80 E-05\end{array}$ & $\begin{array}{l}3.63 E-02 \\
1.13 E-05 \\
4.73 E-03 \\
9.14 E-02 \\
1.28 E-02 \\
9.36 E-03 \\
1.46 E-02 \\
6.67 E-04\end{array}$ & $\begin{array}{l}2.72 E-02 \\
4.35 E-05 \\
4.43 E-03 \\
5.42 E-02 \\
1.14 E-02 \\
8.67 E-03 \\
1.23 E-02 \\
1.05 E-03\end{array}$ & $\begin{array}{l}2.43 E-02 \\
6.92 E-05 \\
4.41 E-03 \\
4.56 E-02 \\
1.02 E-02 \\
7.67 E-03 \\
1.12 E-02 \\
1.26 E-03\end{array}$ & $\begin{array}{l}2.33 E-02 \\
1.40 E-04 \\
4.59 E-03 \\
4.46 E-02 \\
9.67 E-03 \\
7.44 E-03 \\
1.04 E-02 \\
1.42 E-03\end{array}$ & $\begin{array}{l}2.16 E-02 \\
2.05 E-04 \\
4.72 E-03 \\
4.14 E-02 \\
8.90 E-03 \\
7.30 E-03 \\
9.76 E-03 \\
1.43 E-03\end{array}$ & $\begin{array}{l}1.99 E-02 \\
2.37 E-04 \\
4.52 E-03 \\
3.80 E-02 \\
8.26 E-03 \\
6.80 E-03 \\
9.10 E-03 \\
1.42 E-03\end{array}$ & $\begin{array}{l}1.86 E-02 \\
2.59 E-04 \\
4.34 E-03 \\
3.54 E-02\end{array}$ & $\begin{array}{l}1.51 E-02 \\
2.77 E-04 \\
3.79 E-03 \\
2.78 E-02 \\
6.53 E-03 \\
5.32 E-03 \\
7.81 E-03 \\
1.46 E-03\end{array}$ \\
\hline
\end{tabular}

Heart Wall 1.17E-05 $1.08 E-03 \quad 5.75 E-03 \quad 1.75 E-02 \quad 2.14 E-02 \quad 1.64 E-02 \quad 1.46 E-02 \quad 1.37 E-02 \quad 1.26 E-02 \quad 1.16 E-02 \quad 1.08 E-02 \quad 9.07 E-03$

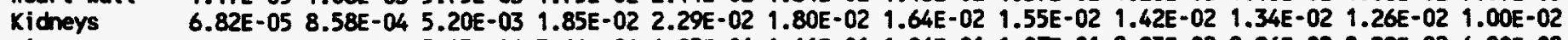
Liver $\quad 6.85 E-01 \quad 6.32 E-01$ 5.43E-01 3.64E-01 1.85E-01 1.11E-01 1.06E-01 1.07E-01 9.83E-02 8.96E-02 8.29E-02 6.80E-02 Lungs $\quad 2.63 E-04$ 5.45E-03 $1.53 E-02$ 2.52E-02 2.14E-02 $1.45 E-02 \quad 1.27 E-02 \quad 1.19 E-02 \quad 1.09 E-02 \quad 1.00 E-02$ 9.41E-03 $7.62 E-03$

Muscle $\quad 8.20 E-04 \quad 2.39 E-03 \quad 4.38 E-03 \quad 6.63 E-03 \quad 6.31 E-03 \quad 4.87 E-03 \quad 4.63 E-03 \quad 4.58 E-03 \quad 4.39 E-03 \quad 4.12 E-03 \quad 3.91 E-03 \quad 3.31 E-03$

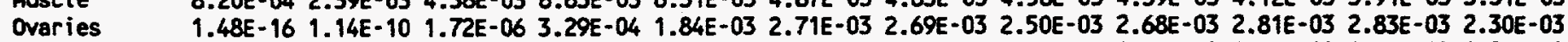
Pancreas 2.81E-05 7.22E-04 7.24E-03 2.93E-02 3.46E-02 2.58E-02 2.23E-02 2.04E-02 1.87E-02 1.71E-02 1.60E-02 1.27E-02 Skeletal Tiss:

Act Marrow 4.55E-05 3.14E-04 1.03E-03 2.72E-03 4.41E-03 4.81E-03 4.84E-03 4.89E-03 4.70E-03 4.46E-03 4.22E-03 3.64E-03 Endosteum 1.36E-04 1.09E-03 4.11E-03 1.24E-02 1.69E-02 9.81E-03 5.59E-03 4.10E-03 3.66E-03 3.40E-03 3.18E-03 2.77E-03

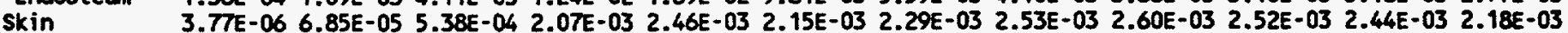
Spleen $\quad 2.60 E-22 \quad 4.95 E-09$ 1.40E-05 1.14E-03 4.62E-03 5.60E-03 5.27E-03 5.22E-03 4.85E-03 4.53E-03 4.29E-03 3.52E-03

Thymus $\quad 2.96 E-15$ 6.96E-10 7.20E-06 7.92E-04 3.44E-03 3.97E-03 3.74E-03 3.80E-03 3.78E-03 3.62E-03 3.44E-03 2.99E-03 Thyroid $\quad 0.0 \quad 2.57 E-19 \quad 1.42 E-104.07 E-06 \quad 2.49 E-04 \quad 6.58 E-04 \quad 7.32 E-04 \quad 8.78 E-04 \quad 9.44 E-04 \quad 9.67 E-04 \quad 9.71 E-04 \quad 9.43 E-04$ Urin Bl Wall $0.0 \quad 4.81 E-16 \quad 3.67 E-09$ 1.64E-05 2.50E-04 7.40E-04 8.45E-04 9.73E-04 $1.11 \mathrm{E}-03 \quad 1.10 \mathrm{E}-03$ 1.15E-03 $1.14 \mathrm{E}-03$ Uterine Cont:

Fetus

Uterine Hall

$\begin{array}{llllllllllll}2.54 E-25 & 2.53 E-10 & 1.55 E-06 & 2.99 E-04 & 1.61 E-03 & 2.52 E-03 & 2.54 E-03 & 2.54 E-03 & 2.62 E-03 & 2.60 E-03 & 2.52 E-03 & 2.33 E-03\end{array}$ Body Tissues 1.77E-02 1.77E-02 1.75E-02 1.57E-02 1.13E-02 7.75E-03 7.13E-03 7.01E-03 6.58E-03 6.07E-03 5.74E-03 4.79E-03

SOURCE = LUNGS

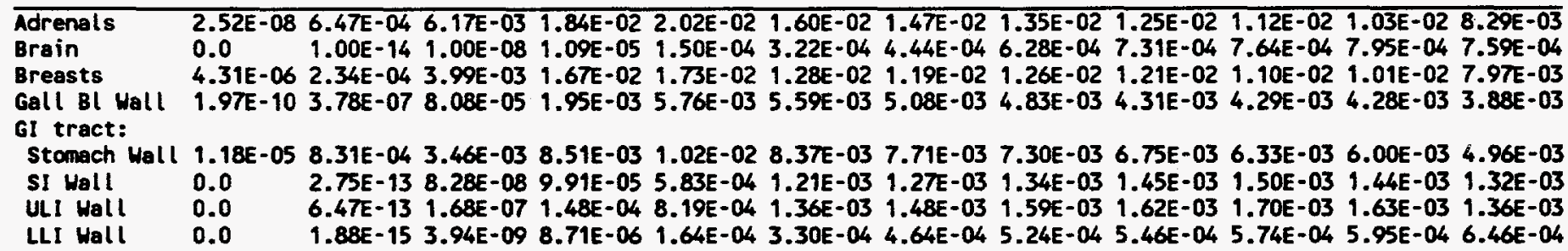

Heart Wall 1.88E-04 6.23E-03 2.45E-02 5.00E-02 4.42E-02 2.94E-02 2.63E-02 2.48E-02 2.24E-02 2.05E-02 1.90E-02 1.54E-02 Kidneys $\quad 2.96 E-10$ 5.14E-07 1.02E-04 1.99E-03 4.31E-03 4.89E-03 4.73E-03 $4.58 E-03$ 4.43E-03 4.21E-03 4.05E-03 3.65E-03

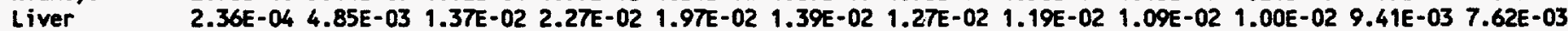
lungs $\quad 1.37 E+001.05 E+00$ 7.07E-01 3.23E-01 1.23E-01 6.83E-02 6.67E-02 6.83E-02 6.37E-02 5.73E-02 5.31E-02 4.33E-02

Muscle 2.64E-03 7.39E-03 1.15E-02 1.25E-02 8.76E-03 6.13E-03 5.82E-03 5.70E-03 5.36E-03 4.94E-03 4.63E-03 3.78E-03 Ovaries $\quad 0.0 \quad 7.51 E-19$ 2.45E-10 5.10E-06 1.66E-04 3.38E-04 4.48E-04 6.13E-04 7.05E-04 7.29E-04 7.43E-04 7.74E-04 Pancreas 1.37E-07 3.19E-05 1.53E-03 1.04E-02 1.56E-02 1.27E-02 1.10E-02 1.02E-02 9.82E-03 8.88E-03 8.13E-03 6.31E-03 Skeletal Tiss:

Act Marrow 2.40E-06 2.12E-04 1.42E-03 4.89E-03 6.76E-03 6.37E-03 6.27E-03 6.25E-03 5.92E-03 5.49E-03 5.21E-03 4.38E-03 Endosteum 7.17E-06 7.14E-04 5.27E-03 2.03E-02 2.52E-02 1.29E-02 7.16E-03 5.40E-03 4.81E-03 4.42E-03 4.15E-03 3.50E-03

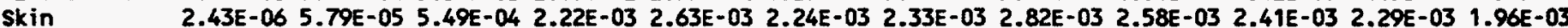
spleen $\quad 1.45 E-04 \quad 1.30 E-03$ 6.22E-03 1.55E-02 1.52E-02 1.13E-02 1.02E-02 9.93E-03 9.15E-03 8.28E-03 7.65E-03 6.44E-03

Thymus $\quad 1.42 E-12 \quad 2.19 E-05 \quad 1.87 E-03 \quad 1.75 E-02 \quad 2.45 E-02 \quad 1.83 E-02 \quad 1.64 E-02 \quad 1.55 E-02 \quad 1.43 E-02 \quad 1.31 E-02 \quad 1.22 E-02 \quad 1.02 E-02$ Thyroid $\quad 3.10 E-20 \quad 5.57 E-08$ 5.88E-05 2.13E-03 6.13E-03 6.01E-03 5.44E-03 5.23E-03 5.05E-03 4.65E-03 4.37E-03 4.07E-03

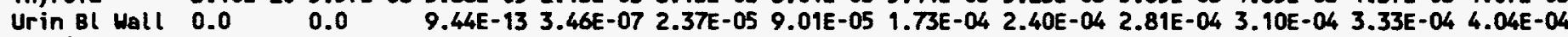
Uterine Cont:

Fetus

0.0

Uterine Hall 0.0

3.08E-18 3.31E-10 4.96E-06 1.35E-04 3.24E-04 4.68E-04 5.90E-04 7.01E-04 7.50E-04 7.65E-04 7.41E-04 $1.43 E-17$ 6.47E-10 5.99E-06 1.47E-04 3.34E-04 5.11E-04 6.46E-04 6.94E-04 7.10E-04 7.25E-04 7.41E-04 1.77E-02 1.77E-02 1.76E-02 1.59E-02 1.11E-02 7.25E-03 6.52E-03 6.32E-03 5.90E-03 5.41E-03 5.09E-03 4.16E-03 
Table A.2 (cont'd). At Three Months Gestation: Specific Absorbed Fraction of Photon Energy ( $\left.\mathrm{kg}^{-1}\right)$.

SOURCE $=$ MUSCLE

Energy (MeV)

\begin{tabular}{|c|}
\hline Target \\
\hline
\end{tabular}

Adrenals

Brain

Breasts

Gall Bl Wal

G1 tract: Stomach Wall 2.85E-03 6.93E-03 1.00E-02 1.18E-02 9.42E-03 6.73E-03 6.30E-03 6.26E-03 5.95E-03 5.51E-03 5.22E-03 4.28E-03 SI Wall

ULI Nall

LLI Mall

Heart Wall

Kidneys

Liver

lungs

Muscle

Ovaries

Pancreas

Skeletal Tiss:

Act Marrow

Endosteum

Skin

Spleen

Thymus

Thyroid

Urin B1 Wall

Uterine Cont:

Fetus

Uterine Wall

Body Tissues

5.39E-03 1.20E-02 1.48E-02 $1.30 E-02$ 9.18E-03 6.98E-03 6.75E-03 6.7TE-03 6.39E-03 5.92E-03 5.58E-03 4.61E-03

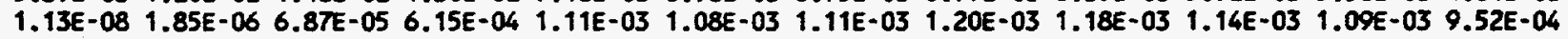

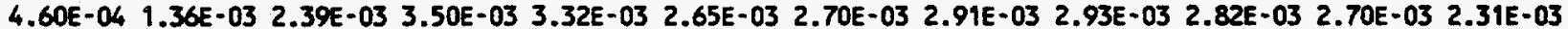
7.25E-03 1.04E-02 1.24E-02 1.25E-02 9.50E-03 7.15E-03 6.54E-03 6.34E-03 6.00E-03 5.58E-03 5.29E-03 4.51E-03 9.29E-04 2.77E-03 4.93E-03 7.84E-03 7.99E-03 6.31E-03 5.84E-03 5.74E-03 5.53E-03 5.20E-03 4.82E-03 4.02E-03 6.39E-04 2.17E-03 4.46E-03 7.96E-03 8.15E-03 6.34E-03 5.82E-03 5.68E-03 5.40E-03 5.10E-03 4.82E-03 4.03E-03 2.29E-03 5.36E-03 8.28E-03 1.11E-02 1.01E-02 7.71E-03 7.16E-03 7.03E-03 6.62E-03 6.15E-03 5.83E-03 4.81E-03

1.95E-03 4.54E-03 6.94E-03 9.17E-03 8.03E-03 5.95E-03 5.55E-03 5.46E-03 5.18E-03 4.82E-03 4.55E-03 3.78E-03 1.72E-03 5.13E-03 8.69E-03 1.03E-02 8.05E-03 6.06E-03 5.82E-03 5.82E-03 5.56E-03 5.20E-03 4.92E-03 4.13E-03 8.20E-04 2.39E-03 4.38E-03 6.63E-03 6.31E-03 4.87E-03 4.63E-03 4.58E-03 4.39E-03 4.12E-03 3.91E-03 3.31E-03 2.95E-03 8.28E-03 1.29E-02 1.39E-02 9.46E-03 6.19E-03 5.82E-03 5.70E-03 5.36E-03 4.94E-03 4.63E-03 3.78E-03

2.28E-02 2.12E-02 1.88E-02 1.39E-02 8.69E-03 6.16E-03 6.07E-03 6.22E-03 5.89E-03 5.53E-03 5.21E-03 4.35E-03 4.30E-03 9.24E-03 1.12E-02 1.13E-02 9.82E-03 7.56E-03 7.12E-03 6.94E-03 6.50E-03 6.07E-03 5.79E-03 4.76E-03 2.65E-03 7.28E-03 1.16E-02 1.40E-02 1.11E-02 8.02E-03 7.38E-03 7.20E-03 6.75E-03 6.28E-03 5.91E-03 4.86E-03

6.30E-04 1.78E-03 3.25E-03 5.44E-03 6.26E-03 5.55E-03 5.47E-03 5.56E-03 5.32E-03 4.94E-03 4.65E-03 3.93E-03 2.09E-03 6.95E-03 1.39E-02 2.55E-02 2.60E-02 $1.30 E-02 \quad 7.69 E-03 \quad 5.63 E-03 \quad 5.38 E-03 \quad 5.07 E-03 \quad 4.76 E-03 \quad 3.95 E-03$ 5.48E-03 8.05E-03 8.51E-03 6.83E-03 4.34E-03 3.25E-03 3.41E-03 3.79E-03 3.75E-03 3.54E-03 3.40E-03 2.87E-03 1.57E-03 4.79E-03 8.57E-03 1.16E-02 9.32E-03 6.65E-03 6.26E-03 6.20E-03 5.87E-03 5.47E-03 5.17E-03 4.32E-03

2.79E-03 8.03E-03 1.23E-02 1.32E-02 9.57E-03 6.66E-03 6.30E-03 6.30E-03 5.96E-03 5.56E-03 5.25E-03 4.34E-03 4.32E-03 1.03E-02 1.34E-02 1.26E-02 8.21E-03 5.56E-03 5.47E-03 5.62E-03 5.34E-03 4.96E-03 4.66E-03 3.86E-03 4.83E-03 8.25E-03 1.07E-02 1.23E-02 1.04E-02 7.67E-03 7.11E-03 6.95E-03 6.63E-03 6.20E-03 5.79E-03 4.89E-03 1.60E-06 3.48E-04 1.92E-03 5.64E-03 7.12E-03 5.87E-03 5.40E-03 5.21E-03 4.96E-03 4.70E-03 4.43E-03 3.73E-03 1.35E-03 3.64E-03 5.95E-03 8.29E-03 8.13E-03 6.36E-03 5.91E-03 5.76E-03 5.47E-03 5.20E-03 4.87E-03 4.08E-03 1.74E-02 1.66E-02 1.51E-02 1.19E-02 7.74E-03 5.68E-03 5.65E-03 5.76E-03 5.46E-03 5.08E-03 4.84E-03 4.02E-03

SOURCE $=$ OVARIES

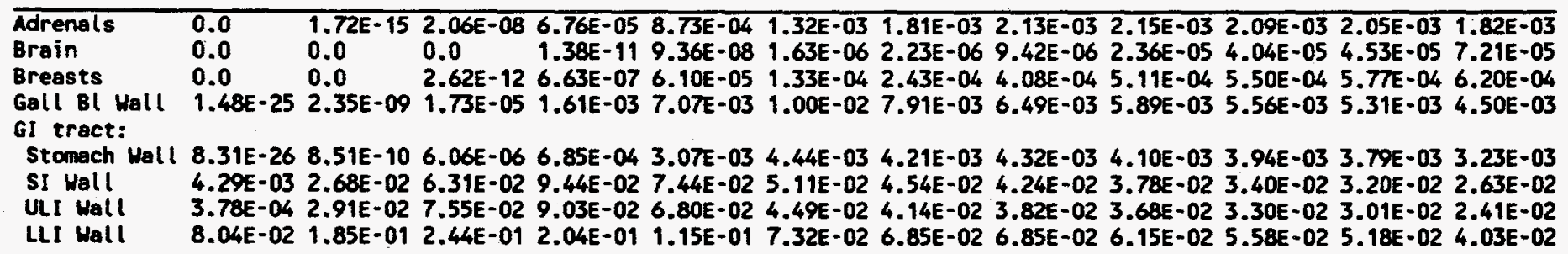

Heart Wall $0.0 \quad 2.00 E-19$ 1.57E-10 4.98E-06 2.09E-04 4.44E-04 6.78E-04 8.72E-04 9.29E-04 9.30E-04 9.27E-04 $9.05 E-04$ Kidneys $\quad 1.40 E-27$ 3.29E-10 5.40E-06 7.33E-04 3.48E-03 5.08E-03 4.69E-03 4.89E-03 $4.80 E-03$ 4.44E-03 4.19E-03 3.87E-03 Liver $\quad 1.48 E-16 \quad 1.14 E-101.72 E-06 \quad 3.29 E-04$ 1.84E-03 2.71E-03 2.69E-03 2.50E-03 2.68E-03 2.81E-03 2.83E-03 2.30E-03 Lungs $\quad 0.0 \quad 8.47 E-19$ 2.76E-10 5.74E-06 $1.79 E-04 \quad 3.41 E-04 \quad 4.48 E-04 \quad 6.10 E-04 \quad 7.02 E-04 \quad 7.28 E-04 \quad 7.44 E-04 \quad 7.74 E-04$

Muscle 4.30E-03 9.24E-03 1.12E-02 1.13E-02 9.82E-03 7.56E-03 7.12E-03 6.94E-03 6.50E-03 6.07E-03 5.79E-03 4.76E-03

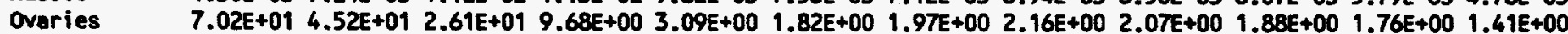
Pancreas $\quad 0.0 \quad 2.27 E-13$ 1.88E-07 1.86E-04 1.52E-03 3.28E-03 3.54E-03 3.41E-03 3.24E-03 3.05E-03 2.86E-03 2.35E-03 Skeletal Tiss:

Act Marrow 3.24E-09 9.27E-05 2.09E-03 1.09E-02 1.52E-02 $1.35 E-02 \quad 1.22 E-02 \quad 1.17 E-02 \quad 1.08 E-02 \quad 1.00 E-02$ 9.38E-03 7.52E-03 Endosteun 7.30E-07 1.31E-04 3.17E-03 1.77E-02 2.35E-02 $1.31 E-02$ 6.97E-03 $4.72 E-03 \quad 4.29 E-03$ 3.97E-03 3.72E-03 3.13E-03 skin

Spleen 1.7TE-21 2.82E-08 2.37E-05 5.64E-04 1.70E-03 1.78E-03 1.89E-03 2.07E-03 2.18E-03 2.14E-03 2.07E-03 $1.63 E-03$

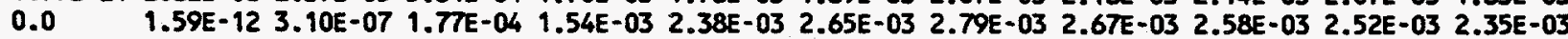

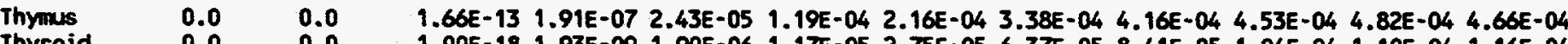

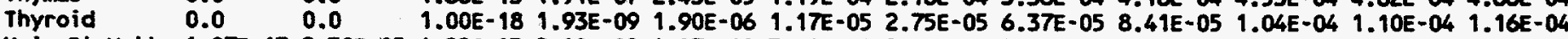
Urin Bl Wall 1.07E-13 2.30E-05 4.22E-03 2.10E-02 4.07E-02 3.00E-02 2.28E-02 2.20E-02 1.97E-02 1.80E-02 1.72E-02 1.55E-02 Uterine Cont:

Fetus Uterine Wall

3.07E-05 1.41E-02 7.93E-02 1.67E-01 1.29E-01 8.12E-02 7.24E-02 6.70E-02 6.04E-02 5.50E-02 5.10E-02 4.04E-02 Body Tissues 1.T7E-02 1.72E-02 1.61E-02 1.44E-02 1.17E-02 8.34E-03 7.45E-03 7.14E-03 $6.72 E-03$ 6.21E-03 5.88E-03 4.81E-03 
Table A.2 (cont'd). At Three Months Gestation: Specific Absorbed Fraction of Photon Energy (kg.').

SOURCE = PANCREAS

Energy (MeV)

\begin{tabular}{|c|c|c|c|c|c|c|c|c|c|c|c|c|}
\hline Target & 0.010 & 0.015 & 0.020 & 0.030 & 0.050 & 0.100 & 0.200 & 0.500 & 1.000 & 1.500 & 2.000 & 4.000 \\
\hline $\begin{array}{l}\text { Adrenals } \\
\text { Brain } \\
\text { Breasts } \\
\text { Gall BI Hall } \\
\text { GI tract: } \\
\text { Stomach Hall } \\
\text { SI Hall } \\
\text { ULI Hall } \\
\text { LLI Hall }\end{array}$ & $\begin{array}{l}0.0 \\
0.0 \\
4.14 E-05 \\
2.62 E-03 \\
1.46 E-17 \\
1.52 E-17 \\
2.39 E-16\end{array}$ & $\begin{array}{l}1.23 E-03 \\
0.0 \\
7.70 E-11 \\
1.25 E-02\end{array}$ & $\begin{array}{l}2.66 E-02 \\
4.13 E-15 \\
2.80 E-06 \\
4.12 E-02\end{array}$ & $\begin{array}{l}\text { 3.73E-03 } \\
4.87 \mathrm{E}-03 \\
6.17 \mathrm{E}-04\end{array}$ & $\begin{array}{l}1.10 E-02 \\
1.25 E-02 \\
2.18 E-03\end{array}$ & $\begin{array}{l}6.59 E-02 \\
2.46 E-05 \\
3.79 E-03 \\
5.51 E-02\end{array}$ & $\begin{array}{l}1.04 E-02 \\
2.87 E-03\end{array}$ & $\begin{array}{l}8.45 E-03 \\
8.91 E-03 \\
3.02 E-03\end{array}$ & $\begin{array}{l}8.77 E-03 \\
2.98 E-03\end{array}$ & $\begin{array}{l}8.02 E-03 \\
2.88 E-03\end{array}$ & $2.77 E-03$ & $\begin{array}{l}3.30 E-0 L \\
2.35 E-04 \\
3.23 E-03 \\
2.63 E-02 \\
3.99 E-02 \\
5.94 E-03 \\
6.45 E-03 \\
2.38 E-03\end{array}$ \\
\hline
\end{tabular}

Heart Wall

Kidneys

Liver

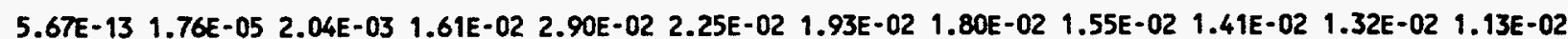

Lungs 4.09E-12 6.31E-05 4.00E-03 3.00E-02 4.17E-02 3.19E-02 2.77E-02 2.63E-02 2.39E-02 2.21E-02 2.05E-02 1.66E-02 2.81E-05 7.22E-04 7.24E-03 2.93E-02 3.46E-02 2.58E-02 2.23E-02 2.04E-02 1.87E-02 1.71E-02 1.60E-02 1.27E-02 1.56E-07 3.60E-05 1.71E-03 1.16E-02 1.69E-02 1.28E-02 1.10E-02 1.02E-02 9.82E-03 8.88E-03 8.13E-03 6.31E-03

Muscle

Ovaries

Pancreas

2.65E-03 7.28E-03 1.16E-02 1.40E-02 1.11E-02 8.02E-03 7.38E-03 7.20E-03 6.75E-03 6.28E-03 5.91E-03 4.86E-03

Skeletal Tiss:

Act Marrow

Endosteum

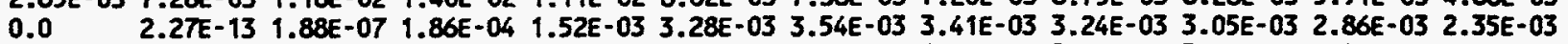
$1.07 E+01$ 8.31E+00 5.78E+00 2.66E+00 9.77E-01 5.63E-01 5.96E-01 6.31E-01 5.91E-01 5.35E-01 4.88E-01 3.84E-01

skin

Spleen

7.99E-07 3.35E-05 4.74E-04 3.14E-03 6.76E-03 7.61E-03 7.36E-03 7.30E-03 6.75E-03 6.30E-03 5.98E-03 5.16E-03 1.75E-06 8.80E-05 1.42E-03 1.02E-02 $1.96 E-02$ 1.27E-02 7.09E-03 4.86E-03 4.25E-03 3.93E-03 3.71E-03 3.23E-03 2.27E-10 2.33E-07 3.19E-05 5.71E-04 1.67E-03 1.78E-03 1.91E-03 2.03E-03 2.18E-03 2.14E-03 2.07E-03 1.79E-03 1.38E-03 4.09E-02 1.27E-01 1.86E-01 1.31E-01 8.10E-02 7.20E-02 6.81E-02 6.42E-02 5.84E-02 5.35E-02 4.24E-02

Thyous $\quad 0.0 \quad 3.13 E-12 \quad 6.36 E-07 \quad 3.28 E-04 \quad 2.73 E-03 \quad 3.69 E-03 \quad 3.35 E-03 \quad 3.50 E-03 \quad 3.28 E-03 \quad 3.07 E-03 \quad 2.98 E-03 \quad 3.04 E-03$ Thyroid $\quad 0.0 \quad 0.0 \quad: 3.91 E-11$ 4.79E-06 $1.69 E-04$ 4.67E-04 5.55E-04 6.99E-04 8.81E-04 8.92E-04 8.54E-04 8.31E-04 Urin Bl Wall 0.0 Uterine Cont: Fetus 0.0 Uterine Wall 0.0 6.39E-18 8.88E-10 $1.23 E-05$ 4.38E-04 7.96E-04 $1.06 E-03$ 1.20E-03 $1.28 E-03$ 1.30E-03 $1.29 E-03$ 1.14E-03 Body Tissues 1.77E-02 1.77E-02 $1.75 E-02$ 1.65E-02 1.31E-02 9.24E-03 8.24E-03 7.94E-03 7.45E-03 6.84E-03 6.44E-03 5.27E-03

\section{SOURCE $=$ SKELETAL TISSUES: ACTIVE MARROW}

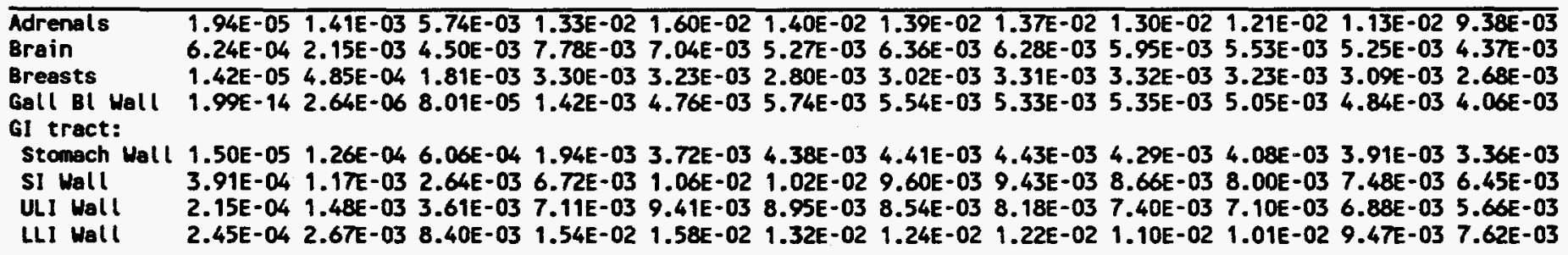

Heart Wall

Kicheys

4.11E-06 1.61E-04 8.43E-04 3.22E-03 5.85E-03 5.67E-03 5.29E-03 5.30E-03 5.05E-03 4.81E-03 4.56E-03 3.90E-03

Liver 4.36E-06 3.81E-04 2.21E-03 7.44E-03 1.10E-02 1.03E-02 9.95E-03 9.8TE-03 9.29E-03 8.72E-03 8.22E-03 6.94E-03

Lungs 4.55E-05 3.50E-04 1.20E-03 3.17E-03 5.06E-03 5.13E-03 4.84E-03 4.89E-03 4.70E-03 4.46E-03 4.22E-03 3.64E-03 2.69E-06 2.65E-04 1.85E-03 6.31E-03 8.22E-03 6.78E-03 6.27E-03 6.25E-03 5.92E-03 5.49E-03 5.21E-03 4.38E-03

Muscle

Ovaries

Pancreas

Skeletal Tiss: $\begin{array}{llllllllllllll}\text { Act Marrow } & 7.41 E-02 & 6.48 E-02 & 5.66 E-02 & 4.31 E-02 & 2.97 E-02 & 2.39 E-02 & 2.49 E-02 & 2.59 E-02 & 2.42 E-02 & 2.25 E-02 & 2.07 E-02 & 1.71 E-02 \\ \text { Endosteum } & 1.65 E-01 & 1.59 E-01 & 1.50 E-01 & 1.23 E-01 & 7.26 E-02 & 2.78 E-02 & 1.72 E-02 & 1.50 E-02 & 1.37 E-02 & 1.25 E-02 & 1.16 E-02 & 9.62 E-03\end{array}$ Skin

Spleen

6.30E-04 1.78E-03 3.25E-03 5.44E-03 6.26E-03 5.55E-03 5.47E-03 5.56E-03 5.32E-03 4.94E-03 4.65E-03 3.93E-03 2.11E-09 8.86E-05 2.14E-03 1.09E-02 1.55E-02 1.38E-02 1.22E-02 1.17E-02 1.08E-02 1.00E-02 9.38E-03 7.52E-03 8.08E-07 3.54E-05 5.20E-04 3.38E-03 7.49E-03 7.88E-03 7.44E-03 7.30E-03 6.75E-03 6.30E-03 5.98E-03 5.16E-03

Thyous

Thyroid

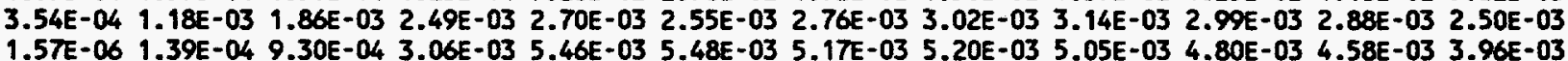
Urin Bl Wall 5.40E-17 2.16E-07 5.97E-05 1.66E-03 5.09E-03 5.69E-03 5.28E-03 4.85E-03 4.57E-03 4.40E-03 4.21E-03 3.33E-03 Uterine Cont:

Fetus

Uterine Wall

1.73E-10 3.94E-07 9.48E-05 2.21E-03 6.50E-03 7.19E-03 6.47E-03 6.46E-03 5.60E-03 5.30E-03 5.12E-03 4.30E-03 Body Tissues

2.67E-09 2.08E-06 2.34E-04 2.91E-03 7.21E-03 7.29E-03 6.88E-03 6.65E-03 6.14E-03 5.70E-03 5.41E-03 4.54E-03 1.77E-02 1.76E-02 1.73E-02 1.56E-02 1.13E-02 7.23E-03 6.41E-03 6.37E-03 6.07E-03 5.57E-03 5.24E-03 4.40E-03 
Table A.2 (cont'd). At Three Months Gestation: Specific Absorbed Fraction of Photon Energy (kg-1).

SOURCE $=$ SPLEEN

Energy (MeV)

\begin{tabular}{|c|c|c|c|c|c|c|c|c|c|c|c|c|}
\hline Target & 0.010 & 0.015 & 0.020 & 0.030 & 0.050 & 0.100 & 0.200 & 0.500 & 1.000 & 1.500 & 2.000 & 4.000 \\
\hline $\begin{array}{l}\text { Adrenals } \\
\text { Brain } \\
\text { Breasts } \\
\text { Gall BL Wall } \\
\text { GI tract: }\end{array}$ & $\begin{array}{l}1.06 E-10 \\
0.0 \\
0.0 \\
9.31 E-24\end{array}$ & $\begin{array}{l}1.77 E-04 \\
0.0 \\
3.49 E-11 \\
7.78 E-09\end{array}$ & $\begin{array}{l}7.61 E-03 \\
3.72 E-15 \\
2.97 E-06 \\
3.50 E-05\end{array}$ & $\begin{array}{l}3.98 E-02 \\
8.33 E-08 \\
4.48 E-04 \\
2.70 E-03\end{array}$ & $\begin{array}{l}4.62 E-02 \\
9.44 E-06 \\
2.08 E-03 \\
8.83 E-03\end{array}$ & $\begin{array}{l}3.40 E-02 \\
3.47 E-05 \\
2.58 E-03 \\
1.13 E-02\end{array}$ & $\begin{array}{l}3.00 E-02 \\
6.15 E-05 \\
2.57 E-03 \\
9.37 E-03\end{array}$ & $\begin{array}{l}2.78 E-02 \\
1.27 E-04 \\
2.86 E-03 \\
7.79 E-03\end{array}$ & $\begin{array}{l}1.93 E-04 \\
2.92 E-03 \\
7.22 E-03\end{array}$ & $\begin{array}{l}2.32 E-04 \\
2.93 E-03 \\
6.94 E-03\end{array}$ & $\begin{array}{l}2.14 E-02 \\
2.55 E-04 \\
2.82 E-03 \\
6.72 E-03\end{array}$ & $\begin{array}{l}1.78 E-02 \\
2.74 E-04 \\
2.53 E-03 \\
5.62 E-03\end{array}$ \\
\hline $\begin{array}{l}\text { stomech Wall } \\
\text { SI Wall } \\
\text { ULI Wall } \\
\text { LLI Wall }\end{array}$ & $\begin{array}{l}2.16 E-08 \\
6.02 E-10 \\
1.27 E-10 \\
2.43 E-13\end{array}$ & $\begin{array}{l}1.11 E-03 \\
7.04 E-07 \\
3.90 E-07 \\
8.07 E-09\end{array}$ & $\begin{array}{l}1.47 E-02 \\
1.06 E-04 \\
1.16 E-04 \\
1.30 E-05\end{array}$ & $\begin{array}{l}6.14 E-02 \\
2.13 E-03 \\
2.91 E-03 \\
9.20 E-04\end{array}$ & $\begin{array}{l}6.68 E-02 \\
7.19 E-03 \\
6.22 E-03 \\
2.38 E-03\end{array}$ & $\begin{array}{l}4.48 E-02 \\
7.33 E-03 \\
7.11 E-03 \\
3.34 E-03\end{array}$ & $\begin{array}{l}3.83 E-02 \\
6.93 E-03 \\
6.30 E-03 \\
3.36 E-03\end{array}$ & $\begin{array}{l}3.72 E-02 \\
6.51 E-03 \\
6.08 E-03 \\
3.17 E-03\end{array}$ & $\begin{array}{l}-02 \\
-03 \\
-03 \\
-03\end{array}$ & $\begin{array}{l}2.98 E-02 \\
6.00 E-03 \\
5.60 E-03 \\
2.68 E-03\end{array}$ & $\begin{array}{l}2.70 E-02 \\
5.58 E-03 \\
5.44 E-03 \\
2.60 E-03\end{array}$ & .03 \\
\hline
\end{tabular}

Heart Hall 2.77E-19 $1.13 E-07$ 1.23E-04 4.39E-03 1.24E-02 1.06E-02 9.46E-03 8.80E-03 8.35E-03 8.14E-03 7.95E-03 6.73E-03 Kidneys

Liver

Lungs 1.41E-04 1.02E-02 4.37E-02 8.44E-02 6.53E-02 4.22E-02 3.81E-02 3.66E-02 3.35E-02 3.07E-02 2.85E-02 2.30E-02 2.60E-22 4.95E-09 1.40E-05 1.14E-03 4.62E-03 5.60E-03 5.27E-03 5.22E-03 4.85E-03 4.53E-03 4.29E-03 3.52E-03 1.63E-04 1.46E-03 6.97E-03 1.73E-02 1.64E-02 1.15E-02 1.02E-02 9.93E-03 9.15E-03 8.28E-03 7.65E-03 6.44E-03

Muscle

Ovaries

Pancreas

1.57E-03 4.79E-03 8.57E-03 1.16E-02 9.32E-03 6.65E-03 6.26E-03 6.20E-03 5.87E-03 5.47E-03 5.17E-03 4.32E-03

Skeletal Tiss:

Act Marrow

Endosteum

skin

Spleen

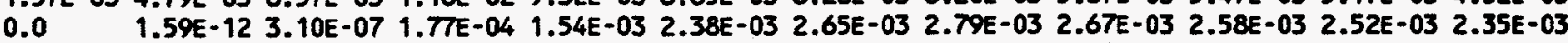
1.38E-03 4.09E-02 1.27E-01 1.86E-01 1.31E-01 8.10E-02 7.20E-02 6.81E-02 6.42E-02 5.84E-02 5.35E-02 4.24E-02

1.57E-06 1.26E-04 7.56E-04 2.64E-03 4.69E-03 5.16E-03 5.17E-03 5.20E-03 5.05E-03 4.80E-03 4.58E-03 3.96E-03 4.51E-06 4.18E-04 2.79E-03 1.12E-02 1.70E-02 1.00E-02 5.71E-03 4.15E-03 3.71E-03 3.45E-03 3.29E-03 2.86E-03 1.32E-06 3.84E-05 4.26E-04 1.75E-03 2.37E-03 2.07E-03 2.19E-03 2.45E-03 2.52E-03 2.40E-03 2.30E-03 2.07E-03 $6.31 E+00$ 5.40E+00 4.14E+00 2.13E+00 8.28E-01 4.76E-01 4.91E-01 5.16E-01 4.78E-01 4.39E-01 4.06E-01 3.21E-01

Thymus $\quad 0.0 \quad 5.52 E-14 \quad 7.20 E-08$ 2.17E-04 $1.59 E-03 \quad 2.46 E-03 \quad 2.11 E-03 \quad 2.28 E-03 \quad 2.25 E-03 \quad 2.22 E-03 \quad 2.21 E-03 \quad 2.12 E-03$ Thyroid $\quad 0.0 \quad 0.0 \quad 1.79 E-11 \quad 1.41 E-05 \quad 2.53 E-04 \quad 4.85 E-04 \quad 5.87 E-04 \quad 7.74 E-04 \quad 8.42 E-04 \quad 8.96 E-049.87 E-04 \quad 8.51 E-04$ Urin Bl Hall $0.0 \quad 8.73 E-18$ 7.99E-10 9.92E-06 2.43E-04 6.25E-04 9.17E-04 $1.09 E-03 \quad 1.14 E-03 \quad 1.10 E-03 \quad 1.12 E-03 \quad 1.04 E-03$ Uterine Cont:

Fetus 0.0

Uterine Mall 0.0

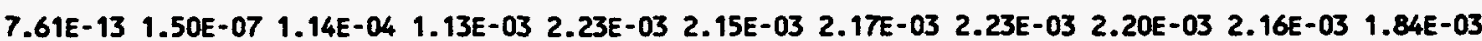
3.09E-12 2.99E-07 1.27E-04 1.06E-03 2.17E-03 2.05E-03 2.34E-03 2.12E-03 2.10E-03 2.21E-03 2.35E-03 Body Tissues 1.77E-02 1.77E-02 1.76E-02 1.58E-02 1.12E-02 7.69E-03 7.10E-03 7.02E-03 6.56E-03 6.09E-03 5.77E-03 4.78E-03

SOURCE $=$ THYMUS

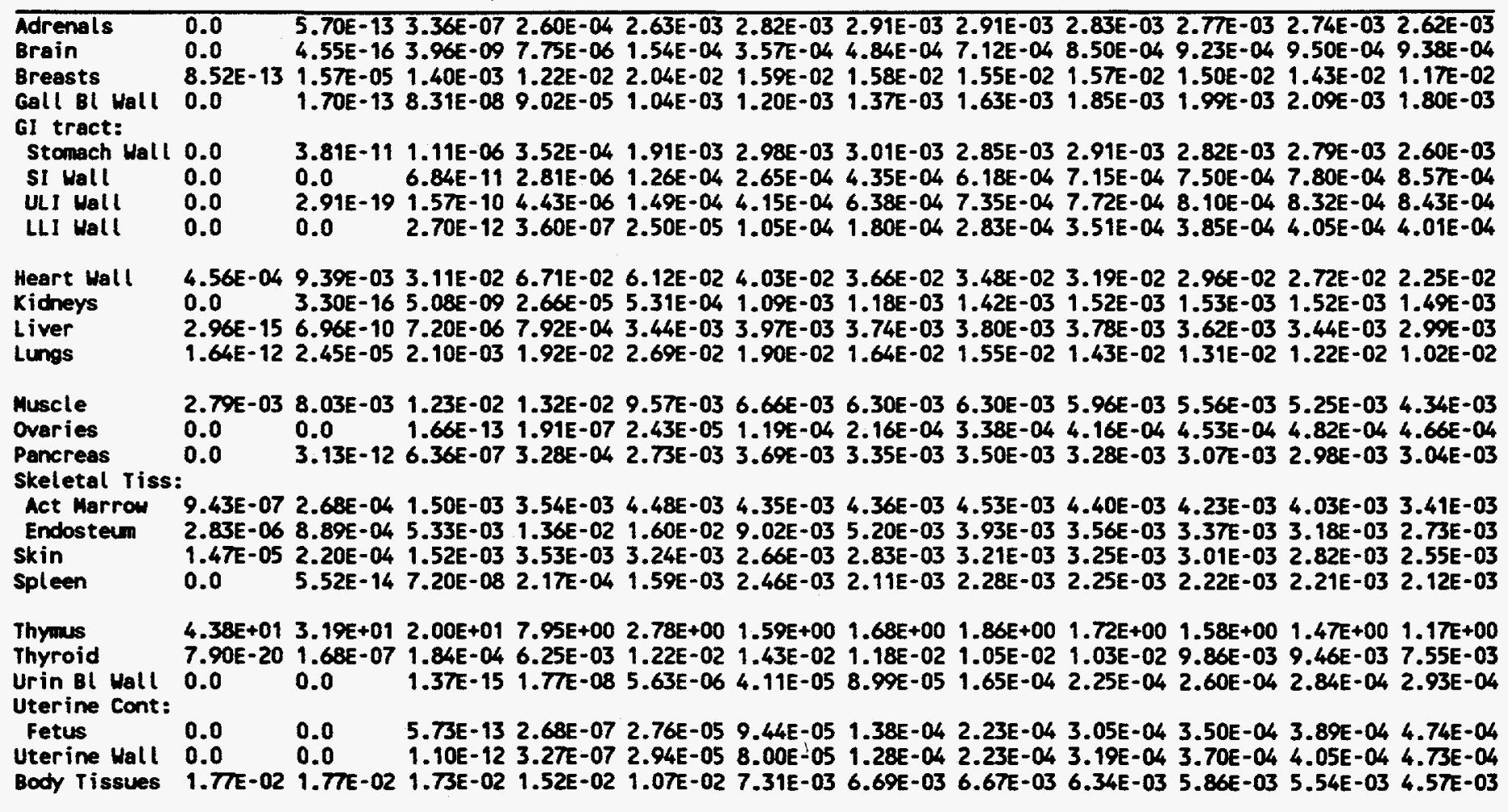


Table A.2 (cont'd). At Three Months Gestation: Specific Absorbed Fraction of Photon Energy ( $\left.\mathrm{kg}^{-1}\right)$.

SOURCE $=$ THYROID

Energy (MeV)

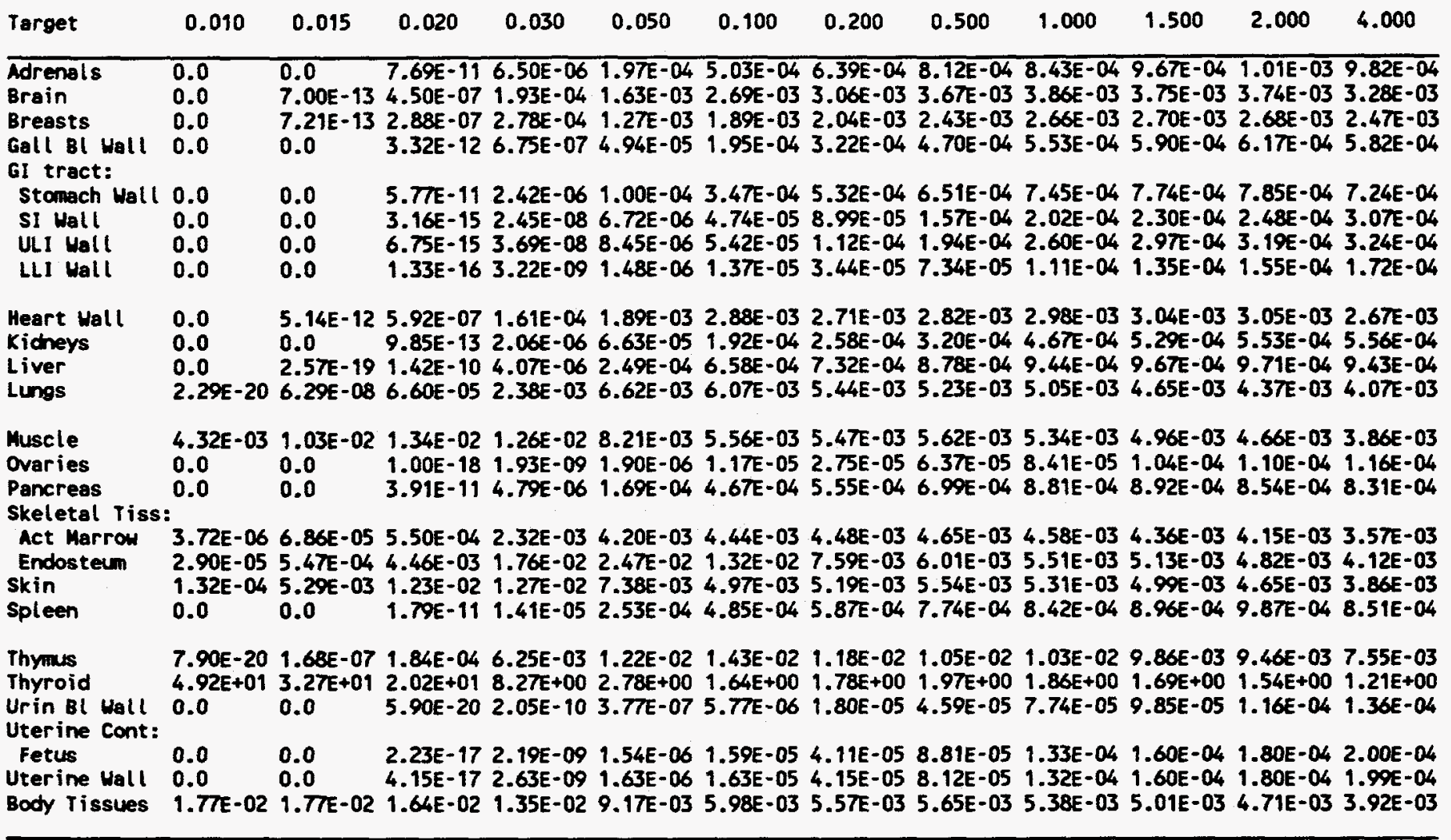

SOURCE = URINARY BLADDER CONTENTS

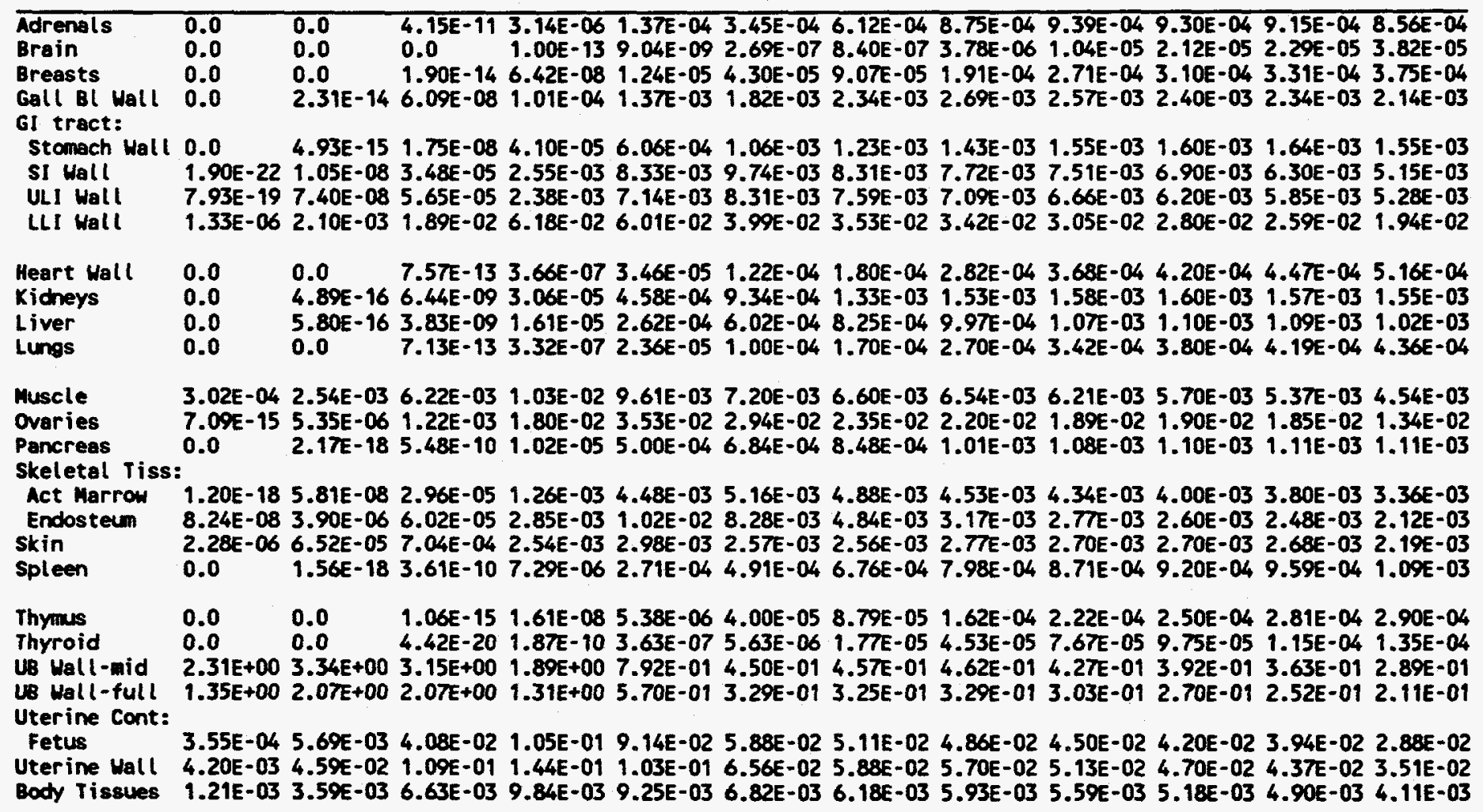


Table A.2 (cont'd). At Three Months Gestation: Specific Absorbed Fraction of Photon Energy $\left(\mathrm{kg}^{-1}\right)$.

SOURCE = URINARY BLADDER WAU

Energy (MeV)

\begin{tabular}{|c|c|c|c|c|c|c|c|c|c|c|c|c|}
\hline Target & 0.010 & 0.015 & 0.020 & 0.030 & 0.050 & 0.100 & 0.200 & 0.500 & 1.000 & 1.500 & 2.000 & 4.000 \\
\hline $\begin{array}{l}\text { Adrensls } \\
\text { Brain } \\
\text { Breasts } \\
\text { Gall Bl Wall } \\
\text { GI tract: } \\
\text { Stomach Hall } \\
\text { SI Hall } \\
\text { ULI Wall } \\
\text { LLI Hall }\end{array}$ & $\begin{array}{l}0.0 \\
0.0 \\
0.0 \\
0.0 \\
0.0 \\
2.81 E-21 \\
7.89 E-12 \\
1.00 E-05\end{array}$ & $\begin{array}{l}0.0 \\
0.0 \\
0.0 \\
3.45 E-14 \\
2.06 E-14 \\
3.45 E-08 \\
7.80 E-08 \\
4.89 E-03\end{array}$ & $\begin{array}{l}7.14 E-11 \\
0.0 \\
2.32 E-14 \\
7.26 E-08 \\
3.25 E-08 \\
6.10 E-05 \\
5.33 E-05 \\
3.01 E-02\end{array}$ & $\begin{array}{l}3.84 E-06 \\
1.06 E-12 \\
6.87 E-08 \\
1.08 E-04 \\
5.16 E-05 \\
2.69 E-03 \\
2.57 E-03 \\
7.89 E-02\end{array}$ & $\begin{array}{l}1.50 E-04 \\
9.12 E-09 \\
1.28 E-05 \\
1.65 E-03 \\
7.93 E-04 \\
9.80 E-03 \\
7.31 E-03 \\
6.97 E-02\end{array}$ & $\begin{array}{l}4.40 E-04 \\
4.37 E-07 \\
7.48 E-05 \\
1.84 E-03 \\
1.01 E-03 \\
9.68 E-03 \\
8.14 E-03 \\
4.54 E-02\end{array}$ & $\begin{array}{l}5.29 E-04 \\
1.22 E-06 \\
1.45 E-04 \\
1.99 E-03\end{array}$ & $\begin{array}{l}6.74 E-04 \\
4.82 E-06 \\
2.46 E-04 \\
2.10 E-03\end{array}$ & $\begin{array}{l}8.10 E-04 \\
9.88 E-06 \\
3.05 E-04 \\
2.10 E-03\end{array}$ & $\begin{array}{l}9.00 E-04 \\
1.83 E-05 \\
3.30 E-04 \\
2.07 E-03\end{array}$ & $\begin{array}{l}9.73 E-04 \\
2.33 E-05 \\
3.46 E-0.4 \\
2.05 E-03\end{array}$ & $\begin{array}{l}8.82 E-04 \\
3.94 E-05 \\
3.78 E-04 \\
1.97 E-03\end{array}$ \\
\hline
\end{tabular}

$1.08 E-12 \quad 4.16 E-07$ 3.69E-05 1.66E-04 2.10E-04 2.81E-04 3.4TE-04 3.90E-04 4.2TE-04 5.24

0.0

Liver $\quad 0.0$

Lungs $\quad 0.0$

2.08E-15 $1.26 E-08$ 3.97E-05 4.57E-04 1.04E-03 1.51E-03 $9.86 E-03$ 1.93E-03 1.90E-03 $1.82 E-03$ 1.62E-03

4.81E-16 3.67E-09 1.64E-05 2.50E-04 7.40E-04 8.45E-04 9.73E-04 1.11E-03 1.10E-03 $1.15 E-03 \quad 1.14 E-03$

0.0 1.06E-12 3.89E-07 2.57E-05 9.20E-05 1.73E-04 2.41E-04 2.82E-04 3.11E-04 3.34E-04 4.04E-04

Muscle $\quad 4.83 E-03 \quad 8.25 E-03$ 1.07E-02 1.23E-02 1.04E-02 7.67E-03 7.11E-03 6.95E-03 6.63E-03 6.20E-03 5.79E-03 4.89E-03 Ovaries $\quad 1.07 E-13$ 2.30E-05 4.22E-03 2.10E-02 4.07E-02 3.00E-02 2.28E-02 2.20E-02 1.97 E-02 $1.80 E-02$ 1.72E-02 $1.55 E-02$

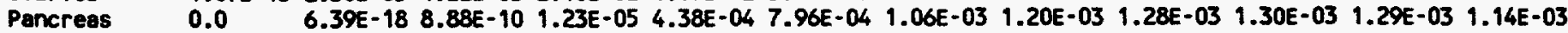

Skeletal Tiss:

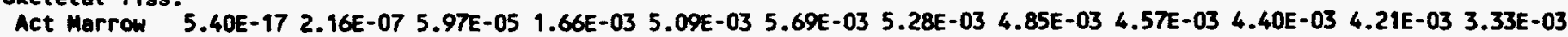
Endosteum $\quad 0.0 \quad 1.00 E-06 \quad 1.05 E-04 \quad 3.57 E-03 \quad 1.11 E-02 \quad 8.62 E-03 \quad 5.03 E-03 \quad 3.24 E-03 \quad 2.82 E-03 \quad 2.68 E-03$ 2.58E-03 2.19E-03

Skin

Spleen 4.40E-05 2.39E-04 7.93E-04 2.29E-03 2.98E-03 2.56E-03 2.52E-03 2.78E-03 2.76E-03 2.64E-03 2.55E-03 2.34E-03 $0.0 \quad 8.73 E-18 \quad 7.99 E-10 \quad 9.92 E-06 \quad 2.43 E-04 \quad 6.25 E-04 \quad 9.17 E-04 \quad 1.09 E-03 \quad 1.14 E-03 \quad 1.10 E-03 \quad 1.12 E-03 \quad 1.04 E-03$

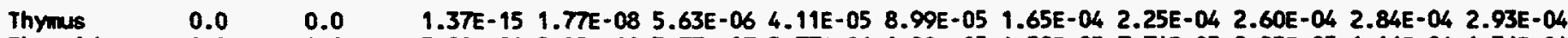

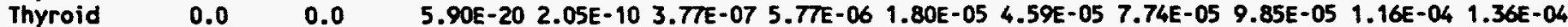
Ur Bl Mall 1.68E+01 9.09E+00 5.20E+00 2.16E+00 8.26E-01 4.79E-01 4.91E-01 5.37E-01 4.87E-01 4.32E-01 3.97E-01 3.07E-01 Uterine Cont:

Fetus

Uterine Hall $4.86 E-02$ 1.34E-01 $1.85 E-01$ 1.78E-01 $9.99 E-02$ 6.29E-02 5.61E-02 5.27E-02 4.87 -02 $4.40 E-02$ 4.02E-02 $3.20 E-02$ Body Tissues 1.47E-02 1.29E-02 1.24E-02 1.21E-02 9.96E-03 7.25E-03 6.64E-03 6.42E-03 6.06E-03 5.62E-03 5.33E-03 4.41E-03

\section{SOURCE = UTERINE CONTENTS: FETUS}

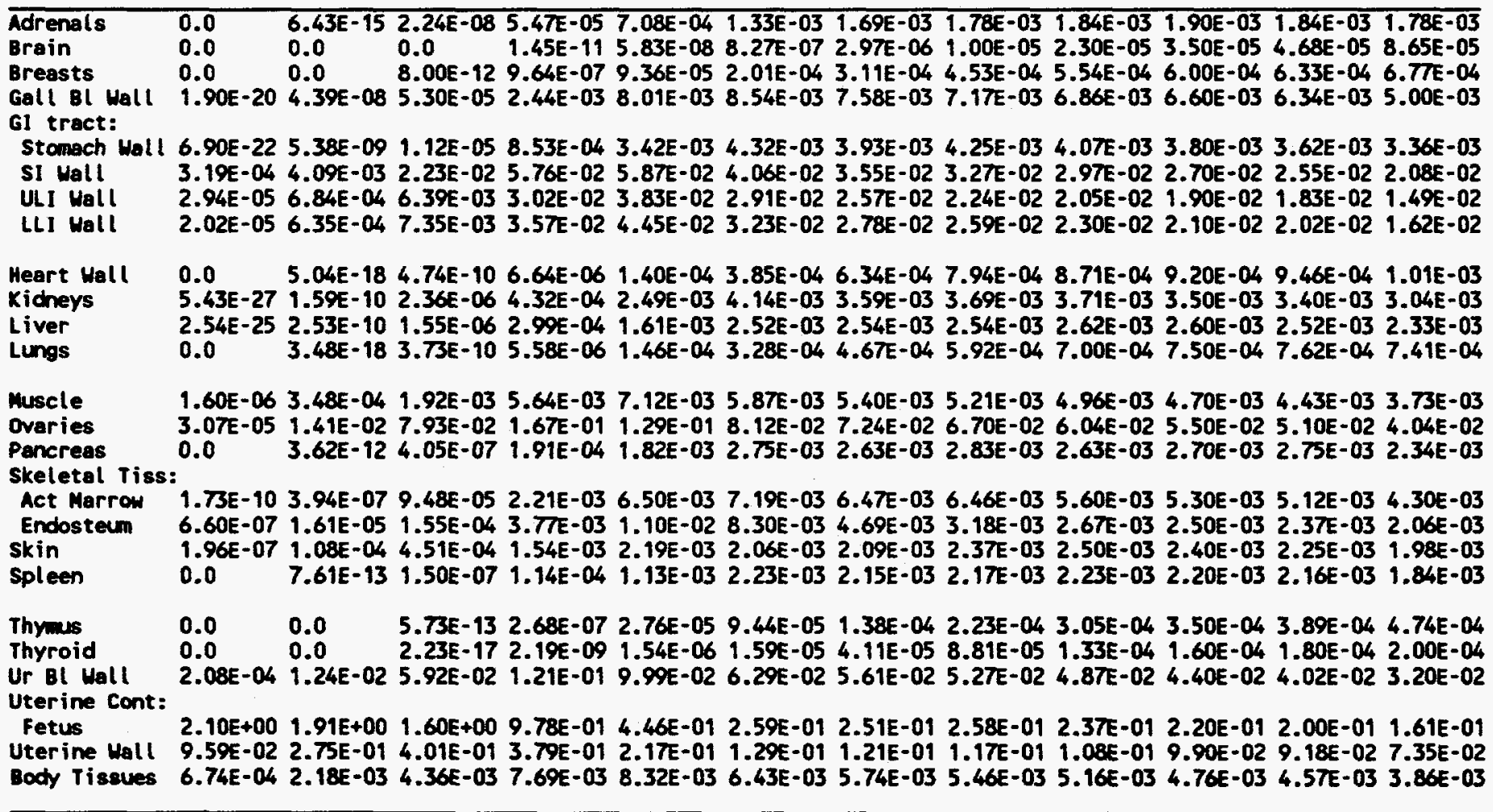


Table A.2 (cont'd). At Three Months Gestation: Specific Absorbed Fraction of Photon Energy (kg').

SOURCE = UTERINE WALL

Energy (MeV)

\begin{tabular}{|c|c|c|c|c|c|c|c|c|c|c|c|c|}
\hline$t$ & .010 & 0.015 & .020 & .030 & 0.050 & 0.100 & 0.200 & 0.500 & 1.000 & 1.500 & 2.000 & 4.000 \\
\hline $\begin{array}{l}\text { Adrenals } \\
\text { Brain } \\
\text { Breasts } \\
\text { Gall Bl Wall } \\
\text { GI tract: } \\
\text { Stcmach Nall } \\
\text { SI Wall } \\
\text { ULI Wall } \\
\text { LLI Wall }\end{array}$ & $\begin{array}{l}3.52 E-21 \\
1.22 E-02 \\
1.70 E-04 \\
7.82 E-04\end{array}$ & $\begin{array}{l}2.72 E-14 \\
0.0 \\
0.0 \\
2.04 E-07 \\
1.65 E-08 \\
3.76 E-02 \\
6.11 E-03 \\
5.00 E-03\end{array}$ & $\begin{array}{l}0.0 \\
1.43 E-11 \\
1.09 E-04 \\
1.99 E-05 \\
6.57 E-02 \\
2.04 E-02 \\
1.86 E-02\end{array}$ & $\begin{array}{l}1.75 \\
8.42 \\
3.34 \\
1.01 \\
8.41 \\
4.18 \\
4.70\end{array}$ & $\begin{array}{l}4 \\
18 \\
13 \\
3 \\
2 \\
2 \\
2\end{array}$ & $\begin{array}{l}1.4 \\
8.5 \\
1.4 \\
8.8 \\
4.1 \\
4.5 \\
3.0 \\
3.4\end{array}$ & $\begin{array}{l}3.5 \\
2.5 \\
7.4 \\
3.5 \\
3.5 \\
2.5 \\
3.0\end{array}$ & $\begin{array}{l}1.01 E-05 \\
3.98 E-04 \\
7.31 E-03 \\
4.17 E-03 \\
3.77 E-02 \\
2.47 E-02 \\
2.80 E-02\end{array}$ & $\begin{array}{l}E-03 \\
E-02 \\
E-02 \\
-02\end{array}$ & $\begin{array}{l}O E-02 \\
O E-02 \\
O E-02\end{array}$ & $\begin{array}{l}-02 \\
-02\end{array}$ & $\begin{array}{l}7 . \\
6 .\end{array}$ \\
\hline
\end{tabular}

Heart Wall $\quad 0.0 \quad 2.20 E-17$ 8.85E-10 7.97E-06 1.95E-04 4.27E-04 6.45E-04 8.01E-04 8.95E-04 9.50E-04 9.78E-04 $1.00 E-03$ Kidneys $\quad 1.78 E-25 \quad 6.82 E-10 \quad 4.92 E-06 \quad 4.07 E-04 \quad 2.60 E-03 \quad 3.94 E-03 \quad 3.82 E-03 \quad 3.69 E-03 \quad 3.83 E-03 \quad 3.60 E-03$ 3.38E-03 3.09E-03

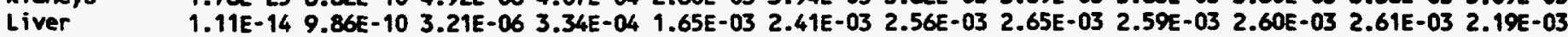
Lungs $\quad 0.0 \quad 1.62 E-17$ 7.28E-10 6.74E-06 1.59E-04 3.38E-04 5.08E-04 6.45E-04 6.95E-04 7.20E-04 7.26E-04 7.41E-04

Muscle $\quad 1.35 E-03$ 3.64E-03 5.95E-03 8.29E-03 8.13E-03 6.36E-03 5.91E-03 5.76E-03 5.47E-03 5.20E-03 4.87E-03 4.08E-03 Ovaries $\quad 9.44 E-03$ 1.22E-01 2.33E-01 2.35E-01 1.44E-01 9.07E-02 8.21E-02 7.86E-02 7.45E-02 6.90E-02 6.34E-02 4.86E-02

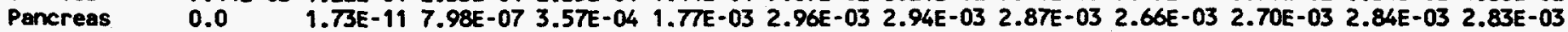
Skeletal riss:

Act Marrow 2.67E-09 2.08E-06 2.34E-04 2.91E-03 7.21E-03 7.29E-03 6.88E-03 6.65E-03 6.14E-03 5.70E-03 5.41E-03 4.54E-03 Endosteun 3.55E-06 5.12E-05 3.41E-04 4.92E-03 1.21E-02 8.40E-03 4.72E-03 3.25E-03 2.80E-03 2.60E-03 2.52E-03 2.17E-03 Skin

Spleen

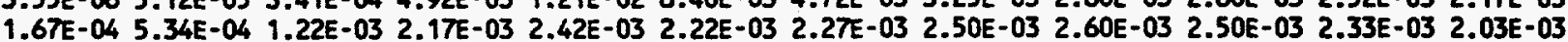
0.0 3.09E-12 2.99E-07 1.27E-04 1.06E-03 2.17E-03 2.05E-03 2.34E-03 2.12E-03 2.10E-03 2.21E-03 2.35E-03

Thymus $\quad 0.0 \quad 0.0 \quad 1.10 E-12 \quad 3.27 E-07 \quad 2.94 E-05 \quad 8.00 E-05 \quad 1.28 E-04 \quad 2.23 E-04 \quad 3.19 E-04 \quad 3.70 E-04 \quad 4.05 E-04 \quad 4.73 E-04$

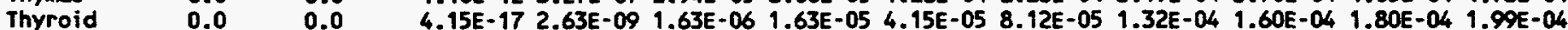
Ur BI Wall 4.86E-02 1.34E-01 1.85E-01 1.78E-01 1.15E-01 7.13E-02 6.46E-02 6.60E-02 5.85E-02 5.30E-02 4.90E-02 4.06E-02 Uterine Cont:

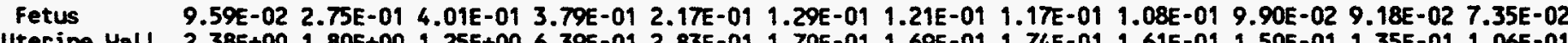

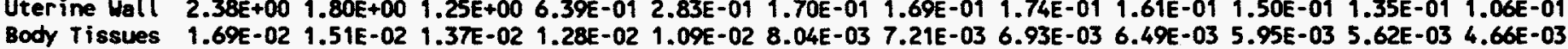

\section{SOURCE $=$ BODY TISSUES}

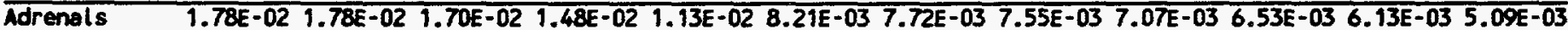

Brain

Breasts 1.7TE-02 1.77E-02 1.76E-02 1.19E-02 7.26E-03 4.89E-03 4.85E-03 4.99E-03 4.67E-03 4.33E-03 4.05E-03 3.35E-03

1.76E-02 1.65E-02 1.38E-02 9.17E-03 5.63E-03 4.07E-03 4.22E-03 4.50E-03 4.42E-03 4.16E-03 3.94E-03 3.30E-03

Gall BL Wall 1.11E-02 9.80E-03 9.64E-03 9.79E-03 9.11E-03 8.02E-03 7.27E-03 6.47E-03 5.89E-03 5.50E-03 5.24E-03 4.49E-03 61 tract:

Stomach Wall 1.60E-02 1.38E-02 1.22E-02 1.06E-02 8.56E-03 6.62E-03 6.39E-03 6.30E-03 5.94E-03 5.50E-03 5.21E-03 4.30E-03 S1 Wall $\quad 1.65 E-02 \quad 1.48 E-02 \quad 1.33 E-02 \quad 1.14 E-02 \quad 9.35 E-03 \quad 7.33 E-03 \quad 7.00 E-03 \quad 6.81 E-03 \quad 6.46 E-03 \quad 6.01 E-03 \quad 5.58 E-03 \quad 4.64 E-03$ ULI Wall $\quad 1.51 E-02$ 1.25E-02 1.09E-02 1.01E-02 8.74E-03 6.90E-03 6.62E-03 6.41E-03 6.00E-03 5.69E-03 5.31E-03 4.43E-03 LLI Wall 1.57E-02 1.38E-02 1.27E-02 1.19E-02 9.79E-03 7.42E-03 7.06E-03 6.84E-03 6.42E-03 5.94E-03 5.62E-03 4.67E-03

Heart Wall 1.78E-02 1.78E-02 1.70E-02 1.47E-02 1.09E-02 7.86E-03 7.49E-03 7.37E-03 6.87E-03 6.36E-03 5.98E-03 4.96E-03 Kicheys $\quad 1.78 E-02$ 1.78E-02 $1.67 E-02$ 1.34E-02 $9.56 E-03 \quad 7.09 E-03 \quad 6.88 E-03 \quad 6.80 E-03 \quad 6.44 E-03$ 5.99E-03 5.66E-03 4.72E-03

liver 1.78E-02 1.78E-02 1.69E-02 1.40E-02 1.01E-02 7.29E-03 7.04E-03 6.94E-03 6.54E-03 6.07E-03 5.71E-03 4.78E-03

Lungs 1.88E-02 1.88E-02 1.82E-02 1.57E-02 1.07E-02 7.29E-03 6.48E-03 6.29E-03 5.89E-03 5.43E-03 5.08E-03 4.16E-03

Muscle

Ovaries

Pancreas Skeletal Tiss:

Act Marrow

Endosteum

skin

1.74E-02 1.66E-02 1.50E-02 1.16E-02 7.66E-03 5.60E-03 5.53E-03 5.68E-03 5.37E-03 5.04E-03 4.77E-03 3.97E-03 1.78E-02 1.71E-02 1.47E-02 1.18E-02 9.54E-03 7.45E-03 7.09E-03 6.84E-03 6.42E-03 5.98E-03 5.64E-03 4.64E-03 1.78E-02 1.78E-02 1.66E-02 1.44E-02 1.13E-02 8.55E-03 8.04E-03 7.75E-03 7.26E-03 6.70E-03 6.29E-03 5.19E-03

Spleen

4.68E-03 5.46E-03 6.06E-03 7.04E-03 7.14E-03 6.28E-03 6.12E-03 6.53E-03 5.97E-03 5.54E-03 5.24E-03 4.44E-03 2.38E-02 2.86E-02 3.35E-02 3.96E-02 3.26E-02 1.50E-02 8.63E-03 7.06E-03 6.38E-03 5.87E-03 5.53E-03 4.62E-03

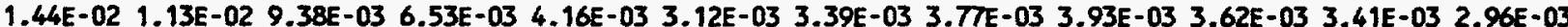
1.78E-02 1.78E-02 1.70E-02 1.41E-02 1.00E-02 7.18E-03 6.95E-03 6.91E-03 6.49E-03 6.02E-03 5.68E-03 4.72E-03

Thymus

Thyroid

1.78E-02 1.78E-02 1.67E-02 1.36E-02 9.63E-03 6.90E-03 6.64E-03 6.65E-03 6.28E-03 5.85E-03 5.52E-03 4.56E-03 Ur Bl Wall Uterine Cont:

Fetus 1.78E-02 1.73E-02 1.65E-02 1.26E-02 7.77E-03 5.69E-03 5.50E-03 5.69E-03 5.40E-03 5.04E-03 4.74E-03 3.95E-03 Uterine Wall 1.46E-02 1.29E-02 1.17E-02 1.03E-02 8.70E-03 6.81E-03 6.56E-03 6.40E-03 6.03E-03 5.56E-03 5.26E-03 4.45E-03 Body Tissues

9.21E-04 2.39E-03 4.53E-03 7.74E-03 8.29E-03 6.39E-03 5.72E-03 5.44E-03 5.14E-03 4.75E-03 4.55E-03 3.84E-03 1.68E-02 1.51E-02 1.27E-02 1.01E-02 8.30E-03 6.63E-03 6.42E-03 6.27E-03 5.92E-03 5.51E-03 5.20E-03 4.34E-03 1.68E-02 1.65E-02 1.57E-02 1.36E-02 9.49E-03 6.24E-03 5.73E-03 5.70E-03 5.42E-03 5.03E-03 4.7TE-03 3.98E-03 
Notes on Table A.3. At Six Month Gestation: Specific Absorbed Fraction of Photon Energy (kg $\left.{ }^{-1}\right)$

Body Tissues. Values of $\Phi$ are given for "Body Tissues" as a source or target region. This region replaces "Whole Body" or "Total Body" given in previous publications (e.g., Cristy and Eckerman 1987) and is defined as the living maternal body tissues, i.e., it excludes the contents of the GI tract, the contents of the urinary and gall bladders, and (for the pregnant woman) the contents of the uterus.

Urinary Bladder. Two rows of $\Phi$ s are given when source $=$ urinary bladder contents and target $=$ urinary bladder wall, labeled "mid" and "full" and designating that the bladder contents are in mid-cycle (half-full) or full.

Muscle. Values of $\Phi$ for muscle are from the Remaining Tissue compartment of each phantom (that part of the maternal tissues remaining after all defined organs are removed).

Cortical and Trabecular Bone. Values of $\Phi$ for source $=$ Cortical Bone, surface or volume distributed, are not given, but source $=$ skeleton may be used for this purpose. Similarly, $\Phi$ s for source $=$ Trabecular Bone, surface or volume distributed, are not given, but source $=$ Red Marrow may be used.

Alphabetical Ordering of Organs. The source and target organs are arranged mostly in alphabetical order. Parts of the GI tract appear together, alphabetized under the heading "GI Tract," but under this heading they appear in natural order from mouth to anus. Active marrow, endosteum (called "bone surface" by the ICRP), and skeleton appear under "Skeletal Tissues"; and (at six and nine months of gestation) fetus, fetal skeleton, fetal soft tissue, placenta, and other uterine contents appear under "Uterine Contents." Body Tissues appears at the end of the tables rather than in alphabetical order. Note that some of these organs appear only as source organs (i.e., skeleton, placenta, and other uterine contents), and endosteum appears only as a target organ. Note also that as target tissues fetal skeleton and fetal soft tissue (at six and nine months of gestation) are indented under "Fetus" and are listed as "Skeleton" and "Soft Tissue" for brevity of space.

Abbreviations. The meanings of the abbreviations used for target regions in Tables A.1 - A.4 are given in the following table. Abbreviations are not used for source regions.

\begin{tabular}{||l|l||}
\hline \multicolumn{2}{|c|}{ Abbreviations used in the Tables for Target Regions } \\
\hline Abbreviation & Meaning \\
\hline Act Marrow & Active Marrow \\
Gall Bl & Gall Bladder \\
GI Tract & Gastrointestinal Tract \\
SI & Small Intestine \\
ULI & Upper Large Intestine \\
LLI & Lower Large Intestine \\
Skeletal Tiss & Skeletal Tissues \\
Urin Bl & Urinary Bladder \\
Uterine Cont & Uterine Contents \\
\hline
\end{tabular}


Table A.3. At Six Months Gestation: Specific Absorbed Fraction of Photon Energy $\left(\mathrm{kg}^{-1}\right)$.

SOURCE $=$ ADRENALS

Energy (MeV)

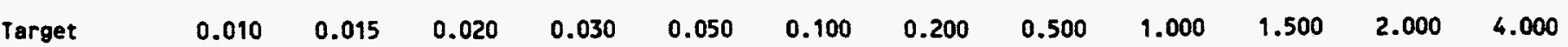

\begin{tabular}{lllllllllllllll}
\hline Adrenals & $5.69 E+01$ & $3.53 E+01$ & $2.03 E+01$ & $7.54 E+00$ & $2.42 E+00$ & $1.47 E+00$ & $1.59 E+00$ & $1.75 E+00$ & $1.68 E+00$ & $1.54 E+00$ & $1.44 E+00$ & $1.18 E+00$
\end{tabular}

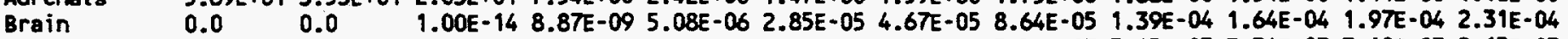

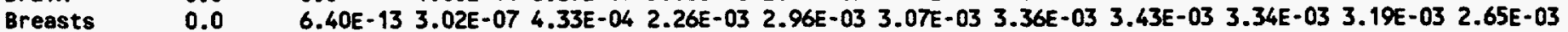

Gall 8i Wall 1.00E-05 3.97E-03 2.99E-02 7.67E-02 7.33E-02 5.18E-02 4.73E-02 4.45E-02 3.91E-02 3.70E-02 3.27E-02 2.50E-02

GI tract:

stomach Wall 1.00E-17 4.92E-08 6.56E-04 1.06E-02 2.18E-02 1.94E-02 1.70E-02 1.55E-02 1.37E-02 1.33E-02 1.23E-02 1.02E-02 S1 Wall $1.00 E-12$ 1.00E-07 $1.24 E-05$ 1.24E-03 5.63E-03 7.29E-03 6.80E-03 6.52E-03 6.09E-03 5.72E-03 5.45E-03 $4.60 E-03$ ULI Wall $\quad 0.0 \quad 1.00 E-11$ 5.00E-07 1.98E-04 1.82E-03 2.83E-03 2.91E-03 3.08E-03 2.98E-03 2.93E-03 2.76E-03 2.55E-03 LLI Wall $0.0 \quad 0.0 \quad 5.14 E-10 \quad 4.27 E-05 \quad 5.63 E-04 \quad 1.11 E-03 \quad 1.31 E-03 \quad 1.41 E-03 \quad 1.57 E-03 \quad 1.58 E-03 \quad 1.53 E-03 \quad 1.50 E-03$

Heart Wall 1.00E-17 1.00E-07 2.34E-04 6.21E-03 1.51E-02 1.44E-02 1.30E-02 $1.19 E-02 \quad 1.08 E-02 \quad 1.00 E-02$ 9.68E-03 7.89E-03

Kidneys

Liver 8.65E-04 1.89E-02 5.80E-02 9.13E-02 7.05E-02 4.90E-02 4.52E-02 4.42E-02 4.03E-02 3.68E-02 3.44E-02 2.83E-02 4.10E-06 1.70E-03 1.14E-02 3.22E-02 3.60E-02 2.75E-02 2.44E-02 2.27E-02 2.08E-02 1.91E-02 $1.77 E-02 \quad 1.44 E-02$

Lungs

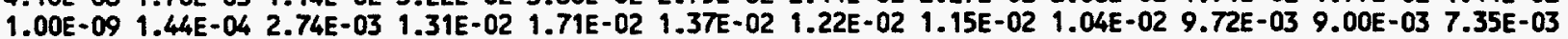

Muscle

Ovaries

Pancreas

5.13E-03 1.17E-02 1.46E-02 1.32E-02 9.34E-03 7.03E-03 6.76E-03 6.71E-03 6.30E-03 5.88E-03 5.52E-03 4.59E-03

Skeletal Tiss:

Act Marrow

Endosteum

Skin

Spleen

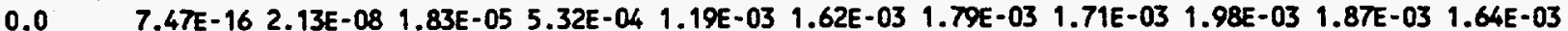
7.75E-09 1.23E-03 2.66E-02 9.95E-02 9.73E-02 6.59E-02 5.87E-02 5.31E-02 4.81E-02 4.42E-02 4.00E-02 3.36E-02

Thymus

0.0

Urin Bl Wall

Fetus

Skeleton

1.94E-05 1.27E-03 4.88E-03 1.15E-02 1.47E-02 1.39E-02 1.39E-02 1.37E-02 1.30E-02 1.21E-02 1.13E-02 9.38E-03 6.32E-05 3.82E-03 1.62E-02 3.92E-02 4.21E-02 2.04E-02 1.45E-02 8.76E-03 7.87E-03 7.14E-03 6.64E-03 5.68E-03 3.72E-08 6.02E-06 2.22E-04 1.23E-03 1.86E-03 1.90E-03 2.03E-03 2.24E-03 2.26E-03 2.23E-03 2.16E-03 1.94E-03 1.06E-10 1.7TE-04 7.61E-03 3.98E-02 4.62E-02 3.40E-02 3.00E-02 2.78E-02 2.57E-02 2.35E-02 2.14E-02 1.78E-02

6.75E-13 1.14E-09 2.22E-07 3.75E-04 3.83E-03 5.41E-03 4.05E-03 2.83E-03 2.68E-03 2.38E-03 2.32E-03 2.04E-03 $\begin{array}{lllllllll} & \end{array}$ Uterine Wall 3.86E-12 2.56E-09 2.56E-07 1.70E-04 1.37E-03 2.33E-03 2.40E-03 2.48E-03 2.51E-03 2.43E-03 2.35E-03 2.15E-03 Body Tissues 1.74E-02 1.74E-02 1.73E-02 1.62E-02 1.26E-02 8.69E-03 7.76E-03 7.48E-03 6.98E-03 6.49E-03 6.09E-03 5.07E-03

SOURCE = BRAIN

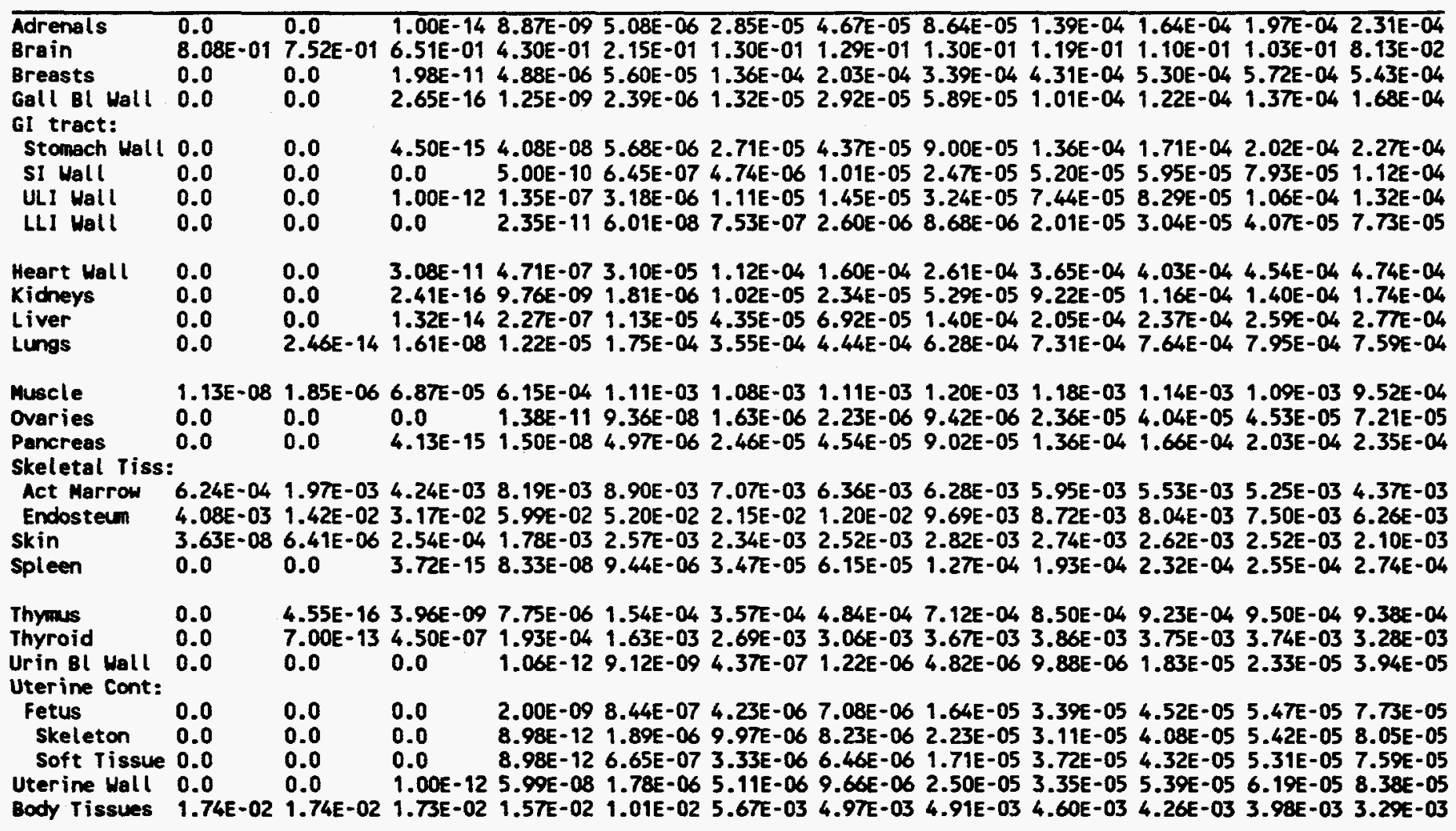


Table A.3 (cont'd). At Six Months Gestation: Specific Absorbed Fraction of Photon Energy (kg-1).

SOURCE $=$ BREASTS

Energy (MeV)

\begin{tabular}{|c|c|c|c|c|c|c|c|c|c|c|c|c|}
\hline rget & 0.010 & 0.015 & .020 & 0.030 & 0.050 & 0.100 & 0.200 & 0.500 & 1.000 & 1.500 & 2.000 & 4.000 \\
\hline $\begin{array}{l}\text { Adrenals } \\
\text { Brain } \\
\text { Breasts } \\
\text { Gall BI Wall } \\
\text { GI tract: } \\
\text { Stomach Wall } \\
\text { SI Wall } \\
\text { ULI Wall } \\
\text { LLI Wall }\end{array}$ & $\begin{array}{l}0.0 \\
0.0 \\
2.63 E+00 \\
0.0 \\
\\
1.00 E-11 \\
0.0 \\
1.07 E-06 \\
0.0\end{array}$ & $\begin{array}{l}6.40 E-13 \\
0.0 \\
2.27 E+00 \\
3.48 E-12 \\
1.00 E-06 \\
1.00 E-11 \\
5.12 E-05 \\
2.87 E-06\end{array}$ & $\begin{array}{l}3.02 E-07 \\
1.98 E-11 \\
1.72 E+00 \\
5.96 E-07 \\
1.13 E-04 \\
5.00 E-07 \\
7.96 E-04 \\
1.82 E-05\end{array}$ & $\begin{array}{l}4.33 E-04 \\
4.88 E-06 \\
8.80 E-01 \\
2.44 E-04 \\
2.15 E-03 \\
5.59 E-05 \\
2.97 E-03 \\
2.13 E-04\end{array}$ & $\begin{array}{l}2.26 E-03 \\
5.60 E-05 \\
3.37 E-01 \\
1.71 E-03 \\
5.15 E-03 \\
4.81 E-04 \\
3.15 E-03 \\
4.71 E-04\end{array}$ & $\begin{array}{l}2.96 E-03 \\
1.36 E-04 \\
1.97 E-01 \\
2.40 E-03 \\
5.05 E-03 \\
8.21 E-04 \\
2.50 E-03 \\
5.09 E-04\end{array}$ & $\begin{array}{l}3.07 E-03 \\
2.03 E-04 \\
2.09 E-01 \\
2.49 E-03\end{array}$ & $\begin{array}{l}3.36 E-03 \\
3.39 E-04 \\
2.24 E-01 \\
2.62 E-03 \\
5.16 E-03 \\
1.12 E-03 \\
2.72 E-03 \\
7.25 E-04\end{array}$ & $\begin{array}{l}3.43 \\
4.31 \\
2.13 \\
2.78 \\
5.18 \\
1.24 \\
2.64 \\
8.07\end{array}$ & $\begin{array}{l}3.34 \\
5.30 \\
1.94 \\
2.62 \\
5.03 \\
1.31 \\
2.42 \\
7.66\end{array}$ & $\begin{array}{l}1.78 E-01 \\
2.55 E-03 \\
4.70 E-03 \\
1.22 E-03 \\
2.48 E-03 \\
8.02 E-04\end{array}$ & $\begin{array}{l}2 . \\
5 . \\
1 . \\
2 . \\
4 . \\
1 . \\
1 . \\
7 .\end{array}$ \\
\hline $\begin{array}{l}\text { leart Wall } \\
\text { fidneys } \\
\text { iver } \\
\text { ungs }\end{array}$ & $\begin{array}{l}2.38 E-06 \\
0.0 \\
1.00 E-09 \\
5.75 E-06\end{array}$ & $\begin{array}{l}1.52 E-04 \\
5.18 E-15 \\
1.00 E-06 \\
2.80 E-04\end{array}$ & $\begin{array}{l}2.91 \\
1.21 \\
1.16 \\
4.41\end{array}$ & $\begin{array}{l}1.82 \\
6.11 \\
2.34 \\
1.86\end{array}$ & $\begin{array}{l}2.48 E-02 \\
6.61 E-04 \\
5.63 E-03 \\
1.95 E-02\end{array}$ & $\begin{array}{l}1.87 \\
1.17 \\
5.33 \\
1.33\end{array}$ & $\begin{array}{l}1.7 \\
1.3 \\
5.1 \\
1.2\end{array}$ & $\begin{array}{l}1.8 \\
1 . \\
5 . \\
1 .\end{array}$ & $\begin{array}{l}1.7 \\
1.6 \\
5.2 \\
1.1\end{array}$ & $\begin{array}{l}1.60 \\
1.76 \\
5.07 \\
1.08\end{array}$ & $\begin{array}{l}.02 \\
-03 \\
.03 \\
.02\end{array}$ & $\begin{array}{l}1.2 \\
1.5 \\
4.1 \\
8.3\end{array}$ \\
\hline $\begin{array}{l}\text { luscte } \\
\text { Pvaries } \\
\text { ancreas }\end{array}$ & $\begin{array}{l}4.33 E-04 \\
0.0 \\
0.0\end{array}$ & $\begin{array}{l}1.32 E-03 \\
0.0 \\
7.70 E-11\end{array}$ & $\begin{array}{l}2.38 E-03 \\
2.62 E-12 \\
2.80 E-06\end{array}$ & & $\begin{array}{l}-03 \\
-05 \\
-03\end{array}$ & $\begin{array}{l}2.68 \\
1.84 \\
3.79\end{array}$ & $\begin{array}{l}03 \\
03 \\
03\end{array}$ & $\begin{array}{l}03 \\
04 \\
03\end{array}$ & & & & $\begin{array}{l}E-03 \\
E-04 \\
E-03\end{array}$ \\
\hline $\begin{array}{l}\text { Skeletal Tiss: } \\
\text { Act Marrow } \\
\text { Endosteum } \\
\text { Skin } \\
\text { Spleen }\end{array}$ & $\begin{array}{l}1.42 E-05 \\
4.19 E-05 \\
1.26 E-02 \\
0.0\end{array}$ & $\begin{array}{l}4.35 E-04 \\
1.45 E-03 \\
2.03 E-02 \\
3.49 E-11\end{array}$ & $\begin{array}{l}1.55 E-03 \\
5.54 E-03 \\
2.08 E-02 \\
2.97 E-06\end{array}$ & $\begin{array}{l}2.98 \\
1.15 \\
1.411 \\
4.481\end{array}$ & $\begin{array}{l}3.11 \\
1.10 \\
6.51 \\
2.08\end{array}$ & $\begin{array}{l}2.88 \\
5.58 \\
4.24 \\
2.58\end{array}$ & $\begin{array}{l}3 . \\
3 . \\
4 . \\
2 .\end{array}$ & $\begin{array}{l}2 . \\
5 . \\
2 .\end{array}$ & $\begin{array}{l}3.3 \\
2.6 \\
4.9 \\
2.9\end{array}$ & $\begin{array}{l}3.23 \\
2.59 \\
4.59 \\
2.93\end{array}$ & $\begin{array}{l}03 \\
03 \\
03 \\
03\end{array}$ & $\begin{array}{l}2.68 E-03 \\
2.16 E-03 \\
3.57 E-03 \\
2.53 E-03\end{array}$ \\
\hline $\begin{array}{l}\text { Thymus } \\
\text { Thyroid } \\
\text { Urin Bl Wall } \\
\text { Uterine Cont: }\end{array}$ & $\begin{array}{l}1.00 E-14 \\
0.0 \\
0.0\end{array}$ & $\begin{array}{l}6.62 E-06 \\
7.21 E-13 \\
0.0\end{array}$ & $\begin{array}{l}7.12 E-04 \\
2.88 E-07 \\
2.32 E-14\end{array}$ & $\begin{array}{l}9.70 E-03 \\
2.78 E-04 \\
1.12 E-06\end{array}$ & $\begin{array}{l}1.71 E-02 \\
1.27 E-03 \\
4.67 E-05\end{array}$ & $\begin{array}{l}1.41 E-02 \\
1.89 E-03 \\
1.18 E-04\end{array}$ & $\begin{array}{l}1.39 E-02 \\
2.04 E-03 \\
1.74 E-04\end{array}$ & $\begin{array}{l}1.47 E-02 \\
2.43 E-03 \\
2.62 E-04\end{array}$ & $\begin{array}{l}1.44 E-02 \\
2.66 E-03 \\
4.06 E-04\end{array}$ & $\begin{array}{l}1.37 E-02 \\
2.70 E-03 \\
4.55 E-04\end{array}$ & $\begin{array}{l}1.31 E-02 \\
2.68 E-03 \\
4.61 E-04\end{array}$ & $\begin{array}{l}1.13 E-02 \\
2.47 E-03 \\
5.14 E-04\end{array}$ \\
\hline $\begin{array}{l}\text { Fetus } \\
\text { Skeleton } \\
\text { Soft Tissue } \\
\text { Uterine Wall } \\
\text { Body Tissues }\end{array}$ & $\begin{array}{l}0.0 \\
0.0 \\
0.0 \\
1.00 E-13 \\
1.72 E-02\end{array}$ & $\begin{array}{l}1.30 E-09 \\
1.00 E-09 \\
1.00 E-11 \\
1.00 E-08 \\
1.62 E-02\end{array}$ & $\begin{array}{l}0 E-06 \\
0 E-05 \\
7 E-06 \\
B E-05\end{array}$ & $\begin{array}{l}14 \\
14 \\
14\end{array}$ & $\begin{array}{l}24 \\
33 \\
24\end{array}$ & $\begin{array}{l}9.02 E \\
1.72 E \\
7.91 E \\
0.07 E\end{array}$ & $\begin{array}{l}9 . \\
1 . \\
8 . \\
1 .\end{array}$ & 33 & $\begin{array}{l}03 \\
03 \\
03 \\
03\end{array}$ & $\begin{array}{l}03 \\
03 \\
03 \\
03\end{array}$ & $\begin{array}{l}03 \\
03 \\
03 \\
03 \\
03\end{array}$ & $\begin{array}{l}1.08 E-03 \\
1.09 E-03 \\
1.08 E-03 \\
1.13 E-03 \\
3\end{array}$ \\
\hline
\end{tabular}

SOURCE = GALL BLADDER CONTENTS

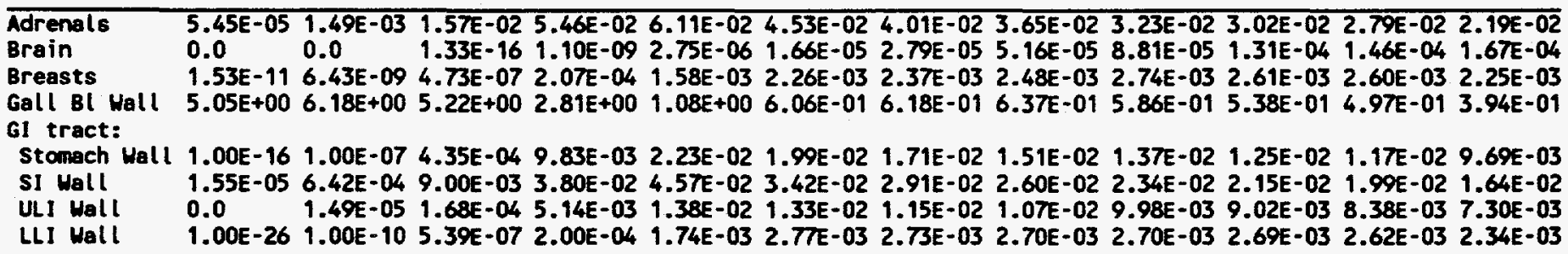

Heart Wall 1.00E-18 1.00E-07 3.52E-05 1.97E-03 7.35E-03 8.12E-03 7.33E-03 6.87E-03 6.41E-03 6.08E-03 5.74E-03 5.04E-03 Kidneys $\quad 8.26 E-06$ 6.66E-04 1.50E-02 6.25E-02 6.64E-02 4.69E-02 4.12E-02 3.82E-02 3.45E-02 3.14E-02 2.92E-02 2.39E-02

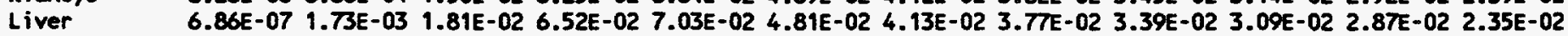
Lungs $\quad 1.00 E-101.00 E-06$ 7.76E-05 2.30E-03 6.34E-03 6.28E-03 5.66E-03 5.35E-03 5.04E-03 $4.80 E-03$ 4.46E-03 $3.79 E-03$

Muscle $\quad 8.96 E-04 \quad 4.86 E-03 \quad 9.40 E-03$ 1.23E-02 1.02E-02 7.61E-03 7.00E-03 6.76E-03 6.30E-03 5.86E-03 5.50E-03 4.59E-03 Ovaries $\quad 3.00 E-26 \quad 1.43 E-092.93 E-06$ 5.65E-04 3.74E-03 5.43E-03 5.13E-03 4.67E-03 5.11E-03 4.48E-03 4.09E-03 3.44E-03 Pancreas 1.00E-03 1.50E-02 7.45E-02 1.44E-01 1.13E-01 7.19E-02 6.34E-02 5.93E-02 5.39E-02 4.79E-02 4.43E-02 3.67E-02 Skeletal Tiss:

Act Merrow 1.00E-11 1.77E-05 4.23E-04 3.12E-03 7.26E-03 8.28E-03 8.11E-03 7.81E-03 7.34E-03 6.87E-03 6.50E-03 5.52E-03 Endosteum 6.81E-07 4.27E-05 1.02E-03 8.17E-03 1.76E-02 1.20E-02 6.81E-03 4.66E-03 4.05E-03 3.73E-03 3.51E-03 3.04E-03

Skin

Spleen 1.00E-09 8.82E-07 1.14E-05 4.17E-04 1.29E-03 $1.52 E-03 \quad 1.61 E-03 \quad 1.80 E-03 \quad 1.86 E-03 \quad 1.83 E-03 \quad 1.83 E-031.63 E-03$

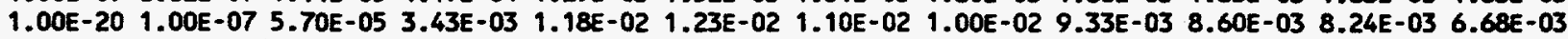

Thymus $\quad 0.0 \quad 5.00 E-14 \quad 6.01 E-096.98 E-05 \quad 9.38 E-04 \quad 1.70 E-03 \quad 1.89 E-03 \quad 1.88 E-03 \quad 2.06 E-03 \quad 2.11 E-03 \quad 1.90 E-03 \quad 1.61 E-03$ Thyroid $\quad 0.0 \quad 0.0 \quad 1.00 E-11 \quad 1.77 E-06 \quad 1.02 E-04 \quad 3.37 E-04 \quad 3.44 E-04 \quad 4.66 E-04 \quad 5.07 E-04 \quad 6.31 E-04 \quad 5.95 E-04 \quad 6.91 E-04$ Urin BL Wall $0.0 \quad 1.00 E-15$ 2.40E-09 2.14E-05 5.78E-04 $1.39 E-03$ 1.40E-03 $1.60 E-03 \quad 1.75 E-03 \quad 1.67 E-03 \quad 1.49 E-03 \quad 1.68 E-03$

Uterine Cont:

Fetus

1.00E-12 1.00E-07 5.88E-05 2.79E-03 1.00E-02 1.08E-02 9.17E-03 8.11E-03 7.33E-03 6.71E-03 6.31E-03 5.34E-03

Skeleton $0.0 \quad 2.50 E-05$ 2.93E-04 9.42E-03 2.79E-02 $2.24 E-02$ 1.34E-02 $9.21 E-03 \quad 7.72 E-03 \quad 6.97 E-03$ 6.84E-03 $5.67 E-03$

Soft Tissue 0.0 5.00E-08 4.10E-05 2.29E-03 8.69E-03 9.92E-03 8.85E-03 8.00E-03 7.30E-03 6.70E-03 6.31E-03 5.33E-03 Uterine Wall 1.00E-07 3.88E-05 2.73E-04 4.27E-03 9.89E-03 9.76E-03 8.72E-03 8.04E-03 7.25E-03 6.92E-03 6.48E-03 5.45E-03 Body Tissues 1.45E-03 4.53E-03 8.31E-03 1.25E-02 1.19E-02 8.79E-03 7.69E-03 7.22E-03 6.68E-03 6.20E-03 5.82E-03 4.85E-03 
Table A.3 (cont'd). At Six Months Gestation: Specific Absorbed Fraction of Photon Energy (kg-1).

SOURCE = GALL BLADDER WALL

Energy (MeV)

$\begin{array}{lllllllllllll}\text { Target } & 0.010 & 0.015 & 0.020 & 0.030 & 0.050 & 0.100 & 0.200 & 0.500 & 1.000 & 1.500 & 2.000 & 4.000\end{array}$

Adrenals $\quad 1.00 E-05$ 3.97E-03 2.99E-02 7.67E-02 7.33E-02 5.18E-02 4.73E-02 4.45E-02 3.91E-02 3.70E-02 3.27E-02 2.50E-02

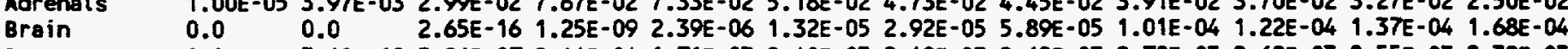

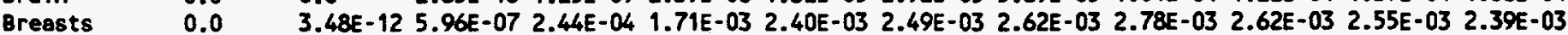

Gall 8l Wall $5.60 E+012.57 E+01 \quad 1.35 E+01 \quad 5.46 E+001.81 E+001.04 E+001.07 E+001.17 E+001.10 E+001.00 E+00 \quad 9.36 E-01 \quad 7.56 E-01$

GI tract:

Stonach Wall 1.00E-15 1.00E-06 5.35E-04 1.06E-02 2.31E-02 2.03E-02 1.75E-02 $1.55 E-02 \quad 1.39 E-02 \quad 1.28 E-02 \quad 1.21 E-02$ 1.01E-02

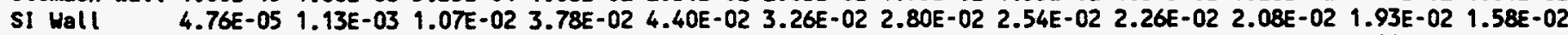
UL.I Wall 7.56E-07 1.95E-05 1.96E-04 5.06E-03 1.32E-02 $1.26 E-02 \quad 1.12 E-02 \quad 1.01 E-02 \quad 9.41 E-03 \quad 8.75 E-03$ 8.44E-03 6.87E-03 LLI Hall 1.00E-26 1.00E-10 7.77E-07 2.04E-04 1.67E-03 2.67E-03 2.70E-03 2.68E-03 2.67E-03 2.52E-03 2.50E-03 2.29E-03

Heart Wall 1.00E-10 5.00E-07 7.07E-05 2.68E-03 8.60E-03 9.08E-03 8.03E-03 7.48E-03 6.86E-03 6.54E-03 6.32E-03 5.26E-03

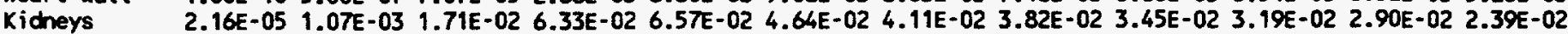
4.11E-06 3. 19E-03 2.32E-02 7.07E-02 7.25E-02 4.92E-02 4.24E-02 3.88E-02 3.51E-02 3.20E-02 2.96E-02 2.43E-02

Lungs 1.00E-09 1.00E-06 1.34E-04 2.70E-03 6.77E-03 6.54E-03 6.11E-03 5.76E-03 5.35E-03 4.97E-03 4.86E-03 4.18E-03

Muscle

Ovaries

Pancreas

Skeletal Tiss:

Act Marrow

Endosteum

Skin

Spleen

7.29E-03 1.15E-02 1.38E-02 1.39E-02 1.06E-02 7.83E-03 7.28E-03 7.07E-03 6.59E-03 6.13E-03 5.77E-03 4.78E-03 1.00E-26 1.00E-10 1.33E-06 5.35E-04 3.55E-03 5.57E-03 5.02E-03 4.86E-03 3.98E-03 3.81E-03 3.95E-03 3.60E-03 9.00E-05 3.20E-02 1.13E-01 1.78E-01 1.28E-01 8.01E-02 7.19E-02 6.66E-02 6.00E-02 5.45E-02 5.05E-02 4.14E-02

1.00E-11 4.44E-05 6.62E-04 4.23E-03 9.01E-03 9.01E-03 8.36E-03 8.03E-03 7.61E-03 7.05E-03 6.70E-03 5.64E-03 1.67E-07 9.81E-05 1.41E-03 9.20E-03 1.85E-02 1.24E-02 7.03E-03 4.84E-03 4.21E-03 3.86E-03 3.66E-03 3.13E-03 6.00E-09 1.11E-06 1.32E-05 4.31E-04 1.31E-03 1.53E-03 1.63E-03 1.81E-03 1.89E-03 1.87E-03 1.80E-03 1.59E-03 1.00E-23 1.00E-08 7.87E-05 3.99E-03 1.25E-02 1.30E-02 1.16E-02 1.04E-02 9.77E-03 9.24E-03 8.45E-03 7.35E-03

Thymus $\quad 0.0 \quad 1.70 E-13 \quad 7.84 E-09$ 9.12E-05 $1.09 E-03 \quad 1.77 E-03 \quad 1.99 E-03 \quad 2.12 E-03 \quad 1.70 E-03 \quad 1.86 E-03 \quad 2.08 E-03 \quad 1.95 E-03$ Thyroid $\quad 0.0 \quad 0.0 \quad 1.00 E-11$ 4.06E-06 $1.02 E-04 \quad 3.45 E-04$ 4.03E-04 7.20E-04 6.97E-04 7.20E-04 7.38E-04 7.33E-04 Urin Bl Mall $0.0 \quad 1.00 E-15$ 1.82E-09 2.28E-05 5.50E-04 $1.29 E-03 \quad 1.44 E-03 \quad 1.43 E-03 \quad 1.52 E-03 \quad 1.81 E-03 \quad 1.67 E-03 \quad 1.32 E-03$ Uterine Cont:

Fetus

1.00E-15 1.00E-07 6.63E-05 2.74E-03 9.60E-03 1.03E-02 8.88E-03 7.83E-03 7.06E-03 6.57E-03 6.18E-03 5.26E-03

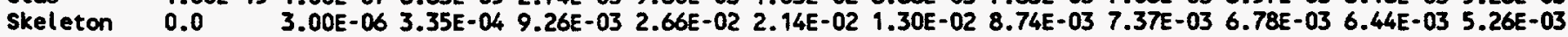

Soft Tissue $0.0 \quad 5.00 E-08 \quad 4.60 E-05$ 2.25E-03 8.31E-03 9.44E-03 8.55E-03 7.76E-03 7.04E-03 6.55E-03 6.17E-03 5.27E-03 Uterine Mall 1.00E-07 4.92E-05 3.12E-04 4.21E-03 9.50E-03 9.33E-03 8.40E-03 7.71E-03 7.08E-03 6.60E-03 6.16E-03 5.36E-03 Body Tissues 1.31E-02 1.21E-02 $1.29 E-02$ 1.42E-02 1.25E-02 9.08E-03 8.03E-03 7.58E-03 7.01E-03 6.50E-03 6.11E-03 5.07E-03

\section{SOURCE = GI TRACT: STOMACH CONTENTS}

\begin{tabular}{lllllllllllll}
\hline Adrenals & $1.00 E-18$ & $4.35 E-08$ & $4.27 E-04$ & $9.62 E-03$ & $2.14 E-02$ & $1.92 E-02$ & $1.69 E-02$ & $1.54 E-02$ & $1.37 E-02$ & $1.28 E-02$ & $1.22 E-02$ & $1.00 E-02$ \\
Brain & 0.0 & 0.0 & $2.66 E-15$ & $2.91 E-08$ & $5.57 E-06$ & $2.40 E-05$ & $3.83 E-05$ & $7.76 E-05$ & $1.31 E-04$ & $1.62 E-04$ & $1.89 E-04$ & $2.11 E-04$ \\
Breasts & $1.00 E-11$ & $1.00 E-07$ & $5.51 E-05$ & $1.63 E-03$ & $4.66 E-03$ & $4.75 E-03$ & $4.58 E-03$ & $4.72 E-03$ & $4.96 E-03$ & $4.72 E-03$ & $4.46 E-03$ & $3.98 E-03$ \\
Gall BI Wall & $1.00 E-16$ & $1.00 E-06$ & $3.53 E-04$ & $9.62 E-03$ & $2.28 E-02$ & $2.04 E-02$ & $1.75 E-02$ & $1.53 E-02$ & $1.38 E-02$ & $1.28 E-02$ & $1.23 E-02$ & $9.85 E-03$ \\
GI tract: & & & & & & & & & & & & \\
Stomach Wall & $4.20 E-01$ & $9.47 E-01$ & $1.13 E+00$ & $8.50 E-01$ & $4.05 E-01$ & $2.30 E-01$ & $2.21 E-01$ & $2.20 E-01$ & $2.03 E-01$ & $1.84 E-01$ & $1.70 E-01$ & $1.35 E-01$ \\
SI Wall & $8.54 E-06$ & $5.68 E-04$ & $4.16 E-03$ & $1.63 E-02$ & $2.29 E-02$ & $1.86 E-02$ & $1.62 E-02$ & $1.46 E-02$ & $1.34 E-02$ & $1.25 E-02$ & $1.16 E-02$ & $9.49 E-03$ \\
ULI Wall & 0.0 & $2.00 E-05$ & $3.53 E-04$ & $5.07 E-03$ & $1.04 E-02$ & $9.61 E-03$ & $8.79 E-03$ & $8.43 E-03$ & $7.72 E-03$ & $7.34 E-03$ & $7.12 E-03$ & $5.84 E-03$ \\
LLI Wall & $5.00 E-08$ & $9.40 E-06$ & $3.49 E-04$ & $3.50 E-03$ & $6.79 E-03$ & $6.30 E-03$ & $5.61 E-03$ & $5.45 E-03$ & $5.17 E-03$ & $5.00 E-03$ & $4.63 E-03$ & $4.05 E-03$
\end{tabular}

Heart Wall 1.58E-06 $1.00 E-04 \quad 1.90 E-03 \quad 1.29 E-02 \quad 2.06 E-02 \quad 1.67 E-02 \quad 1.47 E-02 \quad 1.37 E-02 \quad 1.26 E-02 \quad 1.18 E-02 \quad 1.09 E-02 \quad 8.98 E-03$ Kidneys Liver

Lungs 1.00E-17 5.00E-07 3.27E-04 7.35E-03 1.66E-02 1.51E-02 1.33E-02 1.22E-02 1.13E-02 1.05E-02 9.74E-03 8.35E-03 3.00E-10 1.95E-05 6.66E-04 6.09E-03 1.24E-02 1.14E-02 1.01E-02 9.45E-03 8.86E-03 8.24E-03 7.77E-03 6.49E-03 1.27E-05 2.27E-04 1.73E-03 7.06E-03 1.01E-02 8.25E-03 7.28E-03 6.91E-03 6.30E-03 5.93E-03 5.63E-03 4.71E-03

Muscle $\quad 4.42 E-05$ 1.35E-03 4.63E-03 9.34E-03 9.03E-03 6.66E-03 6.10E-03 5.92E-03 5.54E-03 5.19E-03 4.90E-03 4.10E-03 Ovaries $\quad 1.00 E-27 \quad 1.00 E-101.15 E-06 \quad 3.05 E-04 \quad 2.09 E-03 \quad 3.28 E-03 \quad 3.10 E-03 \quad 3.19 E-03 \quad 3.18 E-03 \quad 3.02 E-03 \quad 2.86 E-03 \quad 2.64 E-03$ Pancreas 1.50E-03 1.47E-02 7.65E-02 1.66E-01 1.34E-01 8.38E-02 7.35E-02 6.76E-02 6.10E-02 5.61E-02 5.16E-02 4.13E-02 Skeletal Tiss:

$\begin{array}{llllllllllll}\text { Act Marrow 1.24E-06 3.37E-05 3.4TE-04 } 1.63 E-03 & 3.54 E-03 & 4.28 E-03 & 4.34 E-03 & 4.29 E-03 & 4.16 E-03 & 4.00 E-03 & 3.81 E-03 & 3.32 E-03\end{array}$ Endosteum 3.73E-06 1.12E-04 1.24E-03 6.36E-03 1.18E-02 8.23E-03 4.78E-03 3.32E-03 2.94E-03 2.73E-03 2.62E-03 2.30E-03 Skin

Spleen 2.10E-07 1.38E-05 2.68E-04 1.38E-03 2.05E-03 $1.91 E-03 \quad 1.93 E-03 \quad 2.16 E-03 \quad 2.25 E-03 \quad 2.17 E-03 \quad 2.10 E-03$ 1.86E-03 5.50E-10 2.20E-04 9.36E-03 5.80E-02 7.00E-02 4.81E-02 4.10E-02 3.77E-02 3.38E-02 3.08E-02 2.85E-02 2.32E-02

Thyous $\quad 0.0 \quad 1.21 E-11 \quad 8.02 E-07 \quad 2.52 E-04 \quad 1.86 E-03 \quad 2.57 E-03 \quad 2.62 E-03 \quad 2.88 E-03 \quad 2.80 E-03 \quad 2.94 E-03 \quad 2.78 E-03 \quad 2.46 E-03$ Thyroid $\quad 0.0 \quad 0.0 \quad 1.00 E-10$ 7.22E-06 $1.77 E-04 \quad 4.24 E-04 \quad 4.75 E-04 \quad 5.74 E-04 \quad 7.66 E-047.29 E-04 \quad 7.74 E-04$ 8.16E-04 Urin Bl Wall 0.0 1.00E-15 5.19E-10 $1.57 E-05$ 4.09E-04 9.27E-04 1.09E-03 $1.21 E-03 \quad 1.33 E-03 \quad 1.40 E-03 \quad 1.32 E-03 \quad 1.29 E-03$

Uterine Cont:

Fetus

1.00E-10 5.00E-07 5.67E-05 2.00E-03 7.14E-03 7.71E-03 6.77E-03 6.18E-03 5.78E-03 5.44E-03 5.09E-03 4.34E-03 Skeleton 1.30E-06 3.10E-05 2.93E-04 6.98E-03 2.02E-02 1.62E-02 1.02E-02 6.91E-03 6.04E-03 5.50E-03 5.35E-03 4.67E-03

Soft Tissue 5.00E-10 5.00E-07 3.88E-05 1.62E-03 6.19E-03 7.09E-03 6.51E-03 6.12E-03 5.78E-03 5.37E-03 5.06E-03 4.32E-03 Uterine Hall 6.02E-08 9.23E-06 3.28E-04 3.50E-03 7.61E-03 7.39E-03 6.66E-03 6.31E-03 5.92E-03 5.53E-03 5.28E-03 4.53E-03 Body Tissues 8.88E-04 2.93E-03 5.92E-03 9.89E-03 9.67E-03 7.11E-03 6.31E-03 6.02E-03 5.64E-03 5.27E-03 4.97E-03 4.16E-03 
Table A.3 (cont'd). At Six Months Gestation: Specific Absorbed Fraction of Photon Energy ( $\left.\mathrm{kg}^{-1}\right)$.

SOURCE = GI TRACT: STOMACH WALL

Energy (MeV)

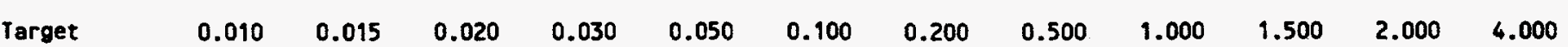

\begin{tabular}{lllllllllllll}
\hline Adrenals & $1.00 E-17$ & $4.92 E-08$ & $6.56 E-04$ & $1.06 E-02$ & $2.18 E-02$ & $1.94 E-02$ & $1.70 E-02$ & $1.55 E-02$ & $1.37 E-02$ & $1.33 E-02$ & $1.23 E-02$ & $1.02 E-02$ \\
Brain & 0.0 & 0.0 & $4.50 E-15$ & $4.08 E-08$ & $5.68 E-06$ & $2.71 E-05$ & $4.37 E-05$ & $9.00 E-05$ & $1.36 E-04$ & $1.71 E-04$ & $2.02 E-04$ & $2.27 E-04$ \\
Breasts & $1.00 E-11$ & $1.00 E-06$ & $1.13 E-04$ & $2.15 E-03$ & $5.15 E-03$ & $5.05 E-03$ & $4.91 E-03$ & $5.16 E-03$ & $5.18 E-03$ & $5.03 E-03$ & $4.70 E-03$ & $4.29 E-03$ \\
Gall BI Hall & $1.00 E-15$ & $1.00 E-06$ & $5.35 E-04$ & $1.06 E-02$ & $2.31 E-02$ & $2.03 E-02$ & $1.75 E-02$ & $1.55 E-02$ & $1.39 E-02$ & $1.28 E-02$ & $1.21 E-02$ & $1.01 E-02$ \\
GI tract: & & & & & & & & & & & & \\
Stomach Hall & $5.80 E+00$ & $3.77 E+00$ & $2.44 E+00$ & $1.15 E+00$ & $4.69 E-01$ & $2.73 E-01$ & $2.70 E-01$ & $2.89 E-01$ & $2.68 E-01$ & $2.43 E-01$ & $2.26 E-01$ & $1.78 E-01$ \\
SI Hall & $1.16 E-04$ & $2.77 E-03$ & $9.60 E-03$ & $2.26 E-02$ & $2.59 E-02$ & $2.02 E-02$ & $1.75 E-02$ & $1.61 E-02$ & $1.47 E-02$ & $1.35 E-02$ & $1.27 E-02$ & $1.04 E-02$ \\
ULI Hall & $5.00 E-05$ & $5.00 E-04$ & $2.00 E-03$ & $5.80 E-03$ & $1.08 E-02$ & $9.59 E-03$ & $8.80 E-03$ & $8.41 E-03$ & $7.90 E-03$ & $7.35 E-03$ & $7.03 E-03$ & $5.80 E-03$ \\
LLI Wall & 0.0 & $3.07 E-05$ & $6.81 E-04$ & $4.53 E-03$ & $7.55 E-03$ & $6.72 E-03$ & $6.05 E-03$ & $5.77 E-03$ & $5.46 E-03$ & $5.26 E-03$ & $4.79 E-03$ & $4.19 E-03$
\end{tabular}

Heart Hall 1.00E-05 3.15E-04 $4.22 E-03 \quad 1.80 E-02 \quad 2.41 E-02 \quad 1.86 E-02 \quad 1.64 E-02 \quad 1.56 E-02 \quad 1.40 E-02 \quad 1.32 E-02 \quad 1.25 E-02 \quad 1.01 E-02$

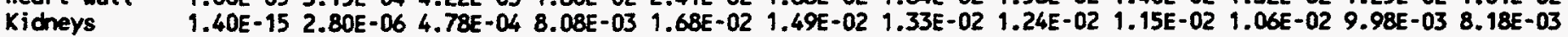
$1.78 E-067.76 E-051.13 E-037.06 E-031.28 E-02$ 1.14E-02 1.02E-02 $9.67 E-03$ 8.90E-03 8.26E-03 7.78E-03 $6.53 E-03$ Lungs $\quad 1.33 E-05$ 9.31E-04 3.79E-03 9.34E-03 1.11E-02 8.60E-03 7.71E-03 7.30E-03 6.73E-03 6.33E-03 6.00E-03 4.96E-03

Muscle $\quad 2.67 E-03 \quad 6.62 E-03 \quad 9.67 E-03 \quad 1.18 E-02 \quad 9.74 E-03 \quad 7.02 E-03 \quad 6.51 E-03 \quad 6.39 E-03 \quad 5.99 E-03 \quad 5.61 E-03 \quad 5.25 E-03 \quad 4.39 E-03$ Ovaries $\quad 1.00 E-26 \quad 1.00 E-10 \quad 1.93 E-06$ 3.76E-04 2.29E-03 3.31E-03 3.18E-03 3.23E-03 3.20E-03 3.11E-03 3.00E-03 2.57E-03 Pancreas 6.05E-03 6.00E-02 1.37E-01 1.94E-01 1.39E-01 8.55E-02 7.69E-02 7.19E-02 6.54E-02 6.01E-02 5.42E-02 4.37E-02 Skeletal Tiss:

$\begin{array}{lllllllllllll}\text { Act Marrow } 1.50 E-05 & 1.26 E-04 & 6.06 E-04 & 1.94 E-03 & 3.72 E-03 & 4.38 E-03 & 4.41 E-03 & 4.43 E-03 & 4.29 E-03 & 4.08 E-03 & 3.91 E-03 & 3.36 E-03\end{array}$ Endosteum 2.67E-06 4.18E-04 2.17E-03 7.54E-03 1.25E-02 8.35E-03 4.83E-03 3.42E-03 3.04E-03 2.83E-03 2.69E-03 2.34E-03 skin $\begin{array}{llllllllllll}2.71 E-06 & 5.26 E-05 & 4.31 E-04 & 1.57 E-03 & 2.12 E-03 & 1.97 E-03 & 2.02 E-03 & 2.24 E-03 & 2.28 E-03 & 2.24 E-03 & 2.15 E-03 & 1.88 E-03\end{array}$ Spleen $\quad 2.16 E-08$ 7.72E-04 $1.48 E-02$ 6.45E-02 7.12E-02 4.84E-02 4.13E-02 3.84E-02 3.45E-02 3.14E-02 2.90E-02 2.37E-02

Thymus $\quad 0.0 \quad 3.81 E-11 \quad 1.11 E-06 \quad 3.52 E-04 \quad 1.91 E-03 \quad 2.98 E-03 \quad 3.01 E-03 \quad 2.85 E-03 \quad 2.91 E-03 \quad 2.82 E-03 \quad 2.79 E-03 \quad 2.60 E-03$ Thyroid $\quad 0.0 \quad 0.0 \quad 1.00 E-10$ 1.07E-05 2.01E-04 4.10E-04 5.02E-04 6.83E-04 7.64E-04 7.41E-04 8.27E-04 8.67E-04 Urin Bl Hell 0.0 1.00E-14 5.19E-10 1.97E-05 4.47E-04 9.50E-04 1.18E-03 1.23E-03 1.53E-03 1.40E-03 1.48E-03 1.20E-03 Uterine Cont:

Fetus

Soft Tissue 1.00E-09 5.00E-07 7.16E-05 2.02E-03 6.69E-03 7.48E-03 6.80E-03 6.30E-03 5.85E-03 5.49E-03 5.25E-03 4.51E-03 Uterine Wall 1.45E-07 1.90E-05 6.04E-04 4.37E-03 8.22E-03 7.74E-03 6.97E-03 6.55E-03 6.30E-03 5.89E-03 5.53E-03 4.76E-03 Body Tissues 1.60E-02 1.42E-02 1.34E-02 1.30E-02 1.06E-02 7.57E-03 6.84E-03 6.62E-03 6.21E-03 5.79E-03 5.43E-03 4.53E-03

SOURCE = GI TRACT: SMALL INTESTINE CONTENTS

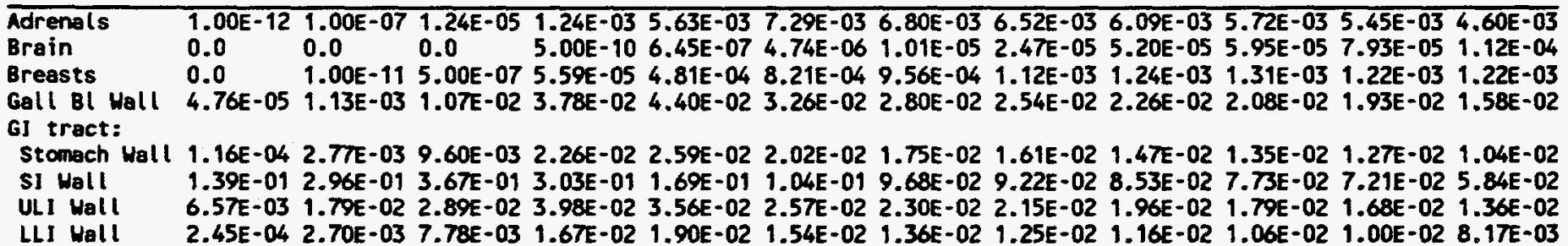

Heart Hall

Kidneys

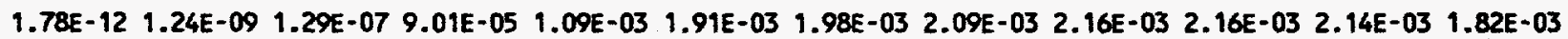

Liver

Lungs 1.57E-07 3.16E-05 1.36E-03 1.19E-02 2.06E-02 1.81E-02 1.60E-02 1.47E-02 1.34E-02 $1.24 E-02 \quad 1.16 E-02 \quad 9.64 E-03$ 1.65E-05 2.62E-04 1.36E-03 6.44E-03 1.15E-02 1.07E-02 9.39E-03 8.74E-03 8.18E-03 7.53E-03 7.02E-03 6.03E-03 1.75E-12 $1.20 E-09$ 1.24E-07 8.49E-05 8.26E-04 1.37E-03 $1.45 E-03$ 1.59E-03 $1.64 E-03$ 1.67E-03 $1.66 E-031.44 E-03$

Muscle

Ovaries

Pancreas

Skeletal Tiss:

Act Marrow

Endosteum

Skin

spleen

7.99E-04 2.38E-03 4.43E-03 7.39E-03 7.88E-03 6.41E-03 5.91E-03 5.68E-03 5.34E-03 5.00E-03 4.70E-03 3.96E-03 3.78E-03 2.59E-02 5.53E-02 6.91E-02 5.16E-02 3.62E-02 3.27E-02 3.06E-02 2.77F-02 2.51E-02 2.36E-02 1.93E-02 1.00E-15 1.00E-06 2.51E-04 6.06E-03 1.53E-02 1.47E-02 $1.28 E-02$ 1.15E-02 1.04E-02 9.63E-03 9.08E-03 $7.45 E-03$

6.14E-05 5.59E-04 2.03E-03 6.06E-03 9.53E-03 9.64E-03 9.36E-03 8.87E-03 8.28E-03 7.75E-03 7.24E-03 6.06E-03 7.52E-05 8.31E-04 3.31E-03 1.12E-02 1.71E-02 1.07E-02 6.17E-03 4.23E-03 3.69E-03 3.40E-03 3.20E-03 2.77E-03 9.71E-08 2.17E-06 $1.96 E-05 \quad 4.38 E-04 \quad 1.25 E-03 \quad 1.51 E-03 \quad 1.61 E-03 \quad 1.72 E-03 \quad 1.79 E-03 \quad 1.78 E-03 \quad 1.69 E-03 \quad 1.53 E-03$ 5.00E-09 3.00E-06 2.38E-04 4.24E-03 1.05E-02 1.02E-02 9.14E-03 8.58E-03 7.84E-03 7.51E-03 7.09E-03 5.86E-03

Thymus $\quad 0.0 \quad 0.0 \quad 6.84 E-11 \quad 3.75 E-06 \quad 1.47 E-04 \quad 3.97 E-04 \quad 5.12 E-04 \quad 6.57 E-04 \quad 7.79 E-04 \quad 8.22 E-04 \quad 8.32 E-04 \quad 7.93 E-04$

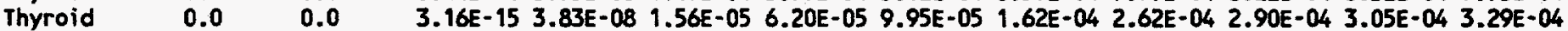
Urin Bl Wall 1.00E-21 1.00E-09 2.57E-06 5.29E-04 3.63E-03 5.15E-03 4.87E-03 4.78E-03 4.56E-03 4.26E-03 4.14E-03 3.54E-03 Uterine Cont:

Fetus

2.01E-05 6.18E-04 7.03E-03 3.19E-02 4.34E-02 3.24E-02 2.67E-02 2.33E-02 2.09E-02 1.91 E-02 $1.78 E-02 \quad 1.45 E-02$

Skeleton $2.71 E-04 \quad 4.79 E-03 \quad 3.68 E-02$ 1.11E-01 $1.20 E-01 \quad 6.24 E-02$ 3.59E-02 2.60E-02 $2.18 E-02$ 2.02E-02 $1.87 E-02 \quad 1.53 E-02$

Soft Tissue 6.04E-06 3.00E-04 4.79E-03 2.59E-02 3.77E-02 3.01E-02 2.59E-02 2.30E-02 2.08E-02 $1.91 E-02$ 1.76E-02 $1.44 E-02$ Uterine Hall 1.28E-02 3.52E-02 5.16E-02 5.76E-02 4.43E-02 3.14E-02 2.81E-02 2.63E-02 2.38E-02 2.19E-02 2.02E-02 1.66E-02 Body Tissues 3.07E-03 7.01E-03 9.65E-03 1.17E-02 1.04E-02 7.57E-03 6.72E-03 6.45E-03 6.06E-03 5.63E-03 5.34E-03 4.39E-03 
Table A.3 (cont'd). At Six Months Gestation: Specific Absorbed Fraction of Photon Energy (kg-1).

SOURCE = GI TRACT: SMALL INTESTINE WALL

Energy (MeV)

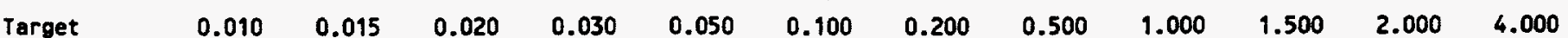

Adrenals $\quad 1.00 E-12$ 1.00E-07 $1.24 E-05$ 1.24E-03 5.63E-03 7.29E-03 6.80E-03 6.52E-03 6.09E-03 5.72E-03 5.45E-03 4.60E-03

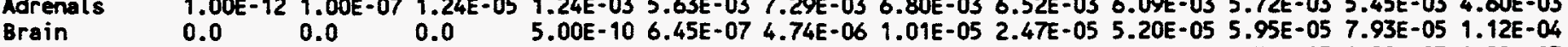

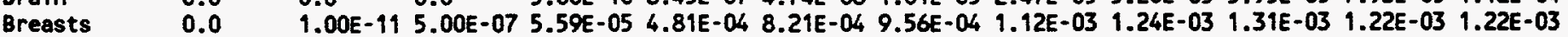

Gall Bl Hall 4.76E-05 1.13E-03 1.07E-02 3.78E-02 4.40E-02 3.26E-02 2.80E-02 2.54E-02 2.26E-02 2.08E-02 1.93E-02 1.58E-02

Gi tract:

Stomach Hall 1.16E-04 2.77E-03 9.60E-03 2.26E-02 2.59E-02 2.02E-02 1.75E-02 1.61E-02 1.47E-02 1.35E-02 1.27E-02 1.04E-02 SI Wall $1.52 E+001.26 E+00$ 9.78E-01 5.74E-01 2.66E-01 1.62E-01 1.57E-01 1.57E-01 1.41E-01 1.28E-01 1.20E-01 9.40E-02 UL1 Nall 6.57E-03 1.79E-02 2.89E-02 3.98E-02 3.56E-02 2.57E-02 2.30E-02 2.15E-02 1.96E-02 1.79E-02 1.68E-02 1.36E-02 LLI Wall 2.45E-04 2.70E-03 7.78E-03 1.67E-02 $1.90 \mathrm{E}-02$ 1.54E-02 $1.36 \mathrm{E}-02$ 1.25E-02 $1.16 \mathrm{E}-02$ 1.06E-02 $1.00 \mathrm{E}-02$ 8.17E-03

Heart Hall 1.78E-12 1.24E-09 1.29E-07 9.01E-05 1.09E-03 1.91E-03 1.98E-03 2.09E-03 2.16E-03 2.16E-03 2.14E-03 1.82E-03

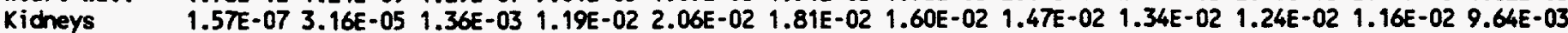

Liver 1.65E-05 2.62E-04 1.36E-03 6.44E-03 1.15E-02 1.07E-02 9.39E-03 8.74E-03 8.18E-03 7.53E-03 7.02E-03 6.03E-03

Lungs 1.75E-12 1.20E-09 1.24E-07 8.49E-05 8.26E-04 $1.37 \mathrm{E}-03$ 1.45E-03 $1.59 \mathrm{E}-03$ 1.64E-03 $1.67 \mathrm{E}-03$ 1.66E-03 $1.44 \mathrm{E}-03$

Muscle

Ovaries

Pancreas

Skeletal Tiss:

Act Marrow

Endosteum

skin

Spleen

7.99E-04 2.38E-03 4.43E-03 7.39E-03 7.88E-03 6.41E-03 5.91E-03 5.68E-03 5.34E-03 5.00E-03 4.70E-03 3.96E-03 3.78E-03 2.59E-02 5.53E-02 6.91E-02 5.16E-02 3.62E-02 3.27E-02 3.06E-02 2.77E-02 2.51E-02 2.36E-02 1.93E-02 1.00E-15 1.00E-06 2.51E-04 6.06E-03 1.53E-02 1.47E-02 1.28E-02 1.15E-02 1.04E-02 9.63E-03 9.08E-03 7.45E-03

Thymus

Thyroid

6.14E-05 5.59E-04 2.03E-03 6.06E-03 9.53E-03 9.64E-03 9.36E-03 8.87E-03 8.28E-03 7.75E-03 7.24E-03 6.06E-03 7.52E-05 8.31E-04 3.31E-03 $1.12 E-02$ 1.71E-02 $1.07 E-02 \quad 6.17 E-03 \quad 4.23 E-03 \quad 3.69 E-03 \quad 3.40 E-03 \quad 3.20 E-03 \quad 2.77 E-03$ 1.D0E-08 1.00E-06 1.96E-05 4.38E-04 1.25E-03 1.51E-03 1.61E-03 1.72E-03 1.79E-03 $1.78 E-03 \quad 1.69 E-03 \quad 1.53 E-03$ 5.00E-09 3.00E-06 2.38E-04 4.24E-03 1.05E-02 1.02E-02 9.14E-03 8.58E-03 7.84E-03 7.51E-03 7.09E-03 5.86E-03

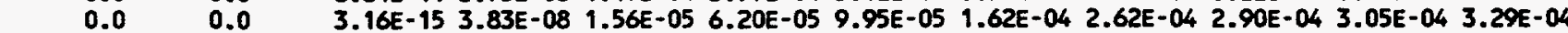
Urin BI Mall 1.00E-21 1.00E-09 2.57E-06 5.29E-04 3.63E-03 5.15E-03 4.87E-03 4.78E-03 4.56E-03 4.26E-03 4.14E-03 3.54E-03 Uterine Cont:

Fetus

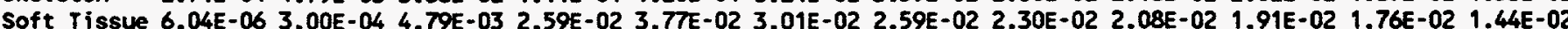
Uterine Hall 1.28E-02 3.52E-02 5.16E-02 5.76E-02 4.43E-02 3.14E-02 2.81E-02 2.63E-02 2.38E-02 2.19E-02 2.02E-02 1.66E-02 Body Tissues 1.65E-02 1.51E-02 1.41E-02 1.32E-02 1.09E-02 7.90E-03 7.06E-03 6.83E-03 6.39E-03 5.94E-03 5.64E-03 4.63E-03

\section{SOURCE = GI TRACT: UPPER LARGE INTESTINE CONTENTS}

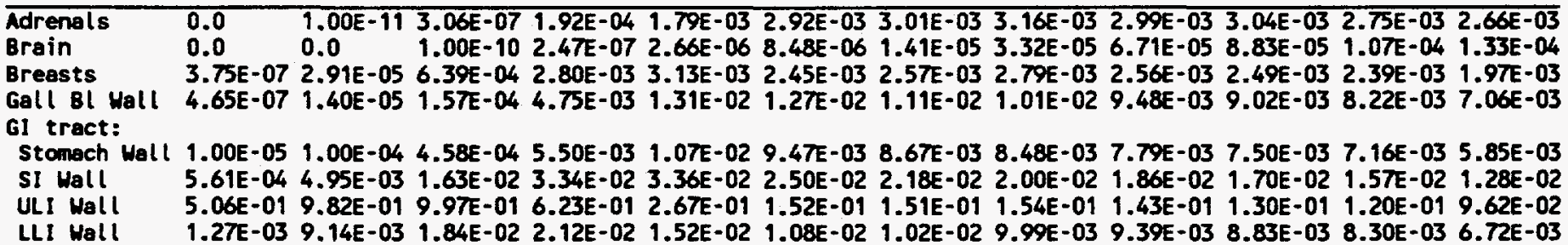

Heart Hall

Kidneys

Liver

Lungs

1.18E-09 1.05E-07 2.56E-06 2.28E-04 1.36E-03 1.95E-03 1.98E-03 2.19E-03 2.16E-03 2.29E-03 2.11E-03 2.03E-03 $0.0 \quad 1.00 E-11 \quad 3.02 E-06 \quad 5.60 E-04 \quad 3.24 E-03 \quad 4.28 E-03 \quad 4.25 E-03 \quad 4.35 E-03$ 4.02E-03 $3.88 E-03 \quad 3.65 E-03 \quad 3.30 E-03$ 4.00E-08 2.95E-04 1.78E-03 7.42E-03 1.18E-02 1.02E-02 9.23E-03 9.00E-03 8.38E-03 8.06E-03 7.57E-03 6.45E-03 0.0 1.00E-12 9.69E-07 1.07E-04 7.75E-04 1.13E-03 $1.23 E-03 \quad 1.37 E-03 \quad 1.47 E-03 \quad 1.49 E-03 \quad 1.44 E-03 \quad 1.40 E-03$

Muscle $\quad 3.03 E-04 \quad 2.66 E-03 \quad 6.17 E-03 \quad 8.59 E-03 \quad 7.03 E-03 \quad 5.20 E-03 \quad 4.94 E-03 \quad 4.97 E-03 \quad 4.73 E-03 \quad 4.47 E-03 \quad 4.21 E-03 \quad 3.56 E-03$ Overies $\quad 1.00 E-104.92 E-06$ 1.00E-03 8.26E-03 1.33E-02 1.13E-02 1.02E-02 9.53E-03 8.96E-03 8.46E-03 7.48E-03 6.44E-03 Pancreas Skeletal Tiss:

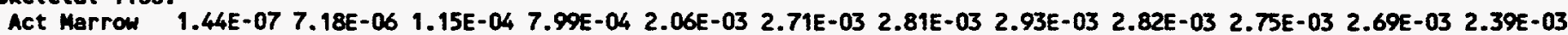
Endosteum 1.59E-07 1.11E-05 2.26E-04 1.98E-03 4.85E-03 4.03E-03 2.52E-03 1.85E-03 1.68E-03 $1.62 E-03 \quad 1.58 E-03 \quad 1.43 E-03$

Skin

Spleen 6.57E-06 1.15E-04 8.79E-04 2.27E-03 2.19E-03 1.78E-03 1.89E-03 2.07E-03 2.10E-03 2.07E-03 2.01E-03 1.73E-03 $\begin{array}{llllllllllll}1.00 E-19 & 1.00 E-09 & 7.52 E-07 & 2.57 E-04 & 1.88 E-03 & 2.75 E-03 & 2.74 E-03 & 2.88 E-03 & 2.95 E-03 & 2.76 E-03 & 2.70 E-03 & 2.47 E-03\end{array}$

Thymus $\quad 0.0 \quad 1.00 E-12 \quad 1.07 E-06 \quad 6.90 E-05 \quad 4.36 E-04 \quad 7.01 E-04 \quad 7.59 E-04 \quad 9.14 E-04 \quad 1.07 E-03 \quad 1.07 E-03 \quad 1.16 E-03 \quad 1.02 E-03$

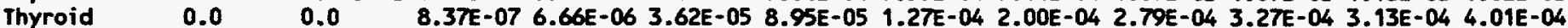
Urin Bl Wall 1.00E-13 1.00E-08 7.05E-06 7.09E-04 3.60E-03 4.38E-03 4.26E-03 4.19E-03 4.30E-03 4.16E-03 4.09E-03 3.47E-03 Uterine Cont:

Fetus

7.07E-07 9.93E-05 3.32E-03 2.21E-02 3.33E-02 2.51E-02 2.08E-02 1.86E-02 1.68E-02 1.54E-02 1.43E-02 1.18E-02 Skeleton 1.08E-05 8.12E-04 $1.74 E-02$ 7.91E-02 9.49E-02 $4.91 E-02 \quad 2.80 E-02 \quad 2.08 E-02 \quad 1.81 E-02 \quad 1.59 E-02 \quad 1.52 E-02 \quad 1.25 E-02$ Soft Tissue 2.68E-07 5.30E-05 2.25E-03 1.77E-02 2.86E-02 2.32E-02 2.02E-02 1.84E-02 $1.67 E-02$ 1.53E-02 1.42E-02 $1.18 E-02$ Uterine Hall 4.43E-04 1.34E-02 3.61E-02 5.16E-02 4.02E-02 2.75E-02 2.49E-02 2.39E-02 2.20E-02 2.00E-02 1.88E-02 1.52E-02 Body Tissues 1.71E-03 5.05E-03 8.12E-03 9.52E-03 7.58E-03 5.56E-03 5.18E-03 5.14E-03 4.86E-03 4.58E-03 4.32E-03 3.65E-03 
Table A.3 (cont'd). At Six Months Gestation: Specific Absorbed Fraction of Photon Energy (kg-1).

SOURCE = GI TRACT: UPPER LARGE INTESTINE WALL

Energy (MeV)

$\begin{array}{lllllllllllll}\text { Target } & 0.010 & 0.015 & 0.020 & 0.030 & 0.050 & 0.100 & 0.200 & 0.500 & 1.000 & 1.500 & 2.000 & 4.000\end{array}$

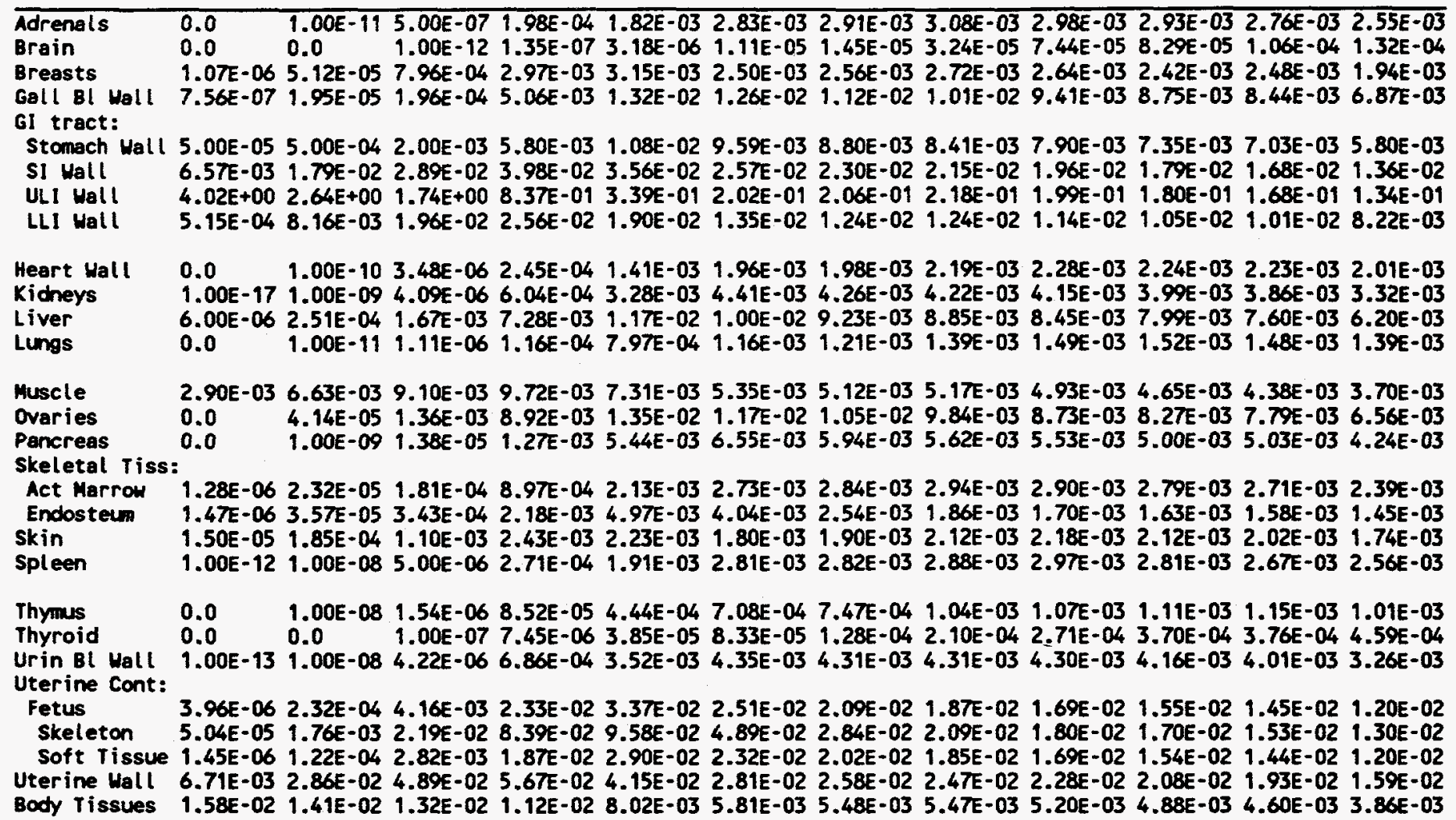

SOURCE = GI TRACT: LOWER LARGE INTESTINE CONTENTS

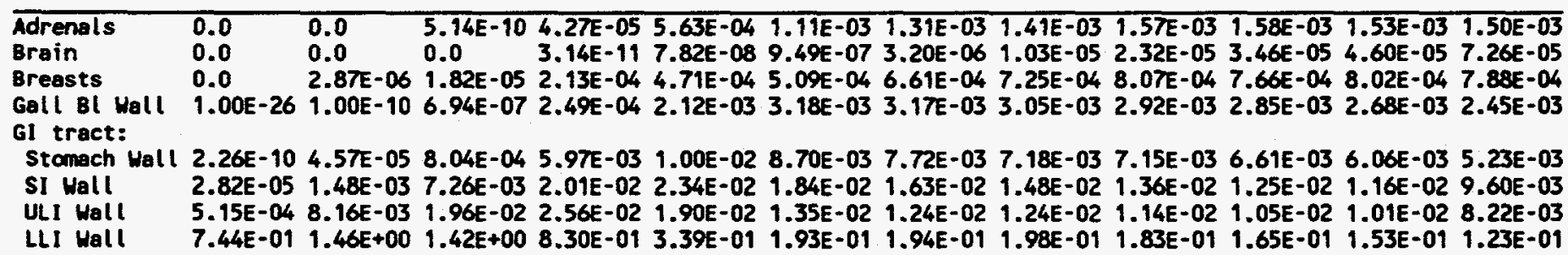

Heart wall $\quad 0.0 \quad 1.00 E-16 \quad 1.00 E-09$ 1.58E-05 2.49E-04 4.99E-04 6.11E-04 7.40E-04 8.45E-04 8.61E-04 8.81E-04 8.77E-04 Kidneys $\quad 1.00 E-14$ 1.00E-09 6.84E-07 2.20E-04 $1.65 E-03 \quad 2.52 E-03 \quad 2.62 E-03 \quad 2.69 E-03 \quad 2.74 E-03 \quad 2.63 E-03$ 2.63E-03 2.27E-03

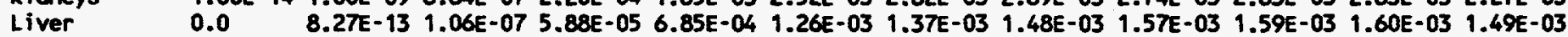
lungs $\quad 0.0 \quad 3.42 E-15$ 2.13E-08 $1.14 E-05$ 1.66E-04 3.49E-04 4.21E-04 5.42E-04 6.47E-04 7.02E-04 6.89E-04 7.32E-04

Muscle $\quad 2.82 E-04$ 2.97E-03 7.33E-03 1.08E-02 9.08E-03 6.67E-03 6.24E-03 6.16E-03 5.81E-03 5.45E-03 5.12E-03 4.30E-03 Overies $\quad 1.37 E-07$ 4.35E-05 2.59E-03 1.97E-02 2.81E-02 2.21E-02 1.95E-02 $1.83 E-02 \quad 1.62 E-02 \quad 1.45 E-02$ 1.38E-02 $1.13 E-02$ Pencreas 1.00E-28 1.00E-10 1.38E-06 4.47E-04 2.53E-03 3.38E-03 3.39E-03 3.27E-03 3.28E-03 3.07E-03 2.93E-03 2.65E-03 Skeletal Tiss:

Act Marrow 1.72E-06 5.00E-05 5.46E-04 2.61E-03 4.72E-03 5.05E-03 4.93E-03 4.81E-03 4.60E-03 4.27E-03 4.05E-03 3.49E-03 Endosteum 2.19E-06 7.60E-05 9.40E-04 5.44E-03 1.03E-02 7.23E-03 4.24E-03 2.97E-03 2.63E-03 2.45E-03 $2.31 E-03 \quad 2.08 E-03$ Skin spleen 1.63E-04 3.90E-04 8.05E-04 1.84E-03 2.17E-03 1.89E-03 1.93E-03 2.16E-03 2.16E-03 2.13E-03 2.06E-03 $1.78 E-03$ 1.00E-22 1.00E-10 1.28E-06 4.56E-04 2.49E-03 3.19E-03 3.15E-03 3.09E-03 3.02E-03 2.98E-03 2.86E-03 2.63E-03

Thyous $\quad 0.0 \quad 0.0 \quad 1.00 E-12 \quad 1.00 E-07 \quad 4.50 E-05 \quad 1.39 E-04 \quad 1.69 E-04 \quad 2.53 E-04 \quad 3.46 E-04 \quad 3.98 E-04 \quad 4.30 E-04 \quad 4.39 E-04$

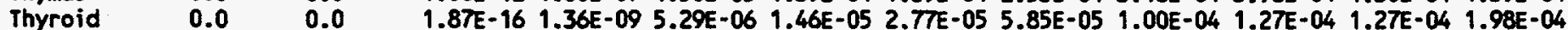
Urin Bl Wall 1.00E-09 1.61E-04 3.08E-03 1.67E-02 2.41E-02 1.87 E-02 $1.67 E-02 \quad 1.55 E-02 \quad 1.45 E-02 \quad 1.40 E-02 \quad 1.25 E-02 \quad 1.05 E-02$ Uterine Cont:

Fetus

$\begin{array}{llllllllll} & \end{array}$ 2.13E-05 1.18E-03 2.04E-02 8.27E-02 9.77E-02 5.15E-02 2.94E-02 2.16E-02 1.84E-02 1.70 -02 $1.57 E-02 \quad 1.29 E-02$ Soft Tissue 6.56E-07 8.58E-05 2.73E-03 $1.93 E-02$ 3.05E-02 2.46E-02 2.13E-02 $1.90 E-02$ 1.75E-02 $1.58 E-02 \quad 1.48 E-02 \quad 1.23 E-02$ Uterine Hell 1.14E-04 7.10E-03 2.47E-02 4.24E-02 3.70E-02 2.63E-02 2.33E-02 2.17E-02 2.01E-02 $1.83 E-02$ 1.70E-02 $1.41 E-02$ Body Tissues 1.86E-03 5.49E-03 8.95E-03 1.10E-02 9.04E-03 6.56E-03 5.97E-03 5.81E-03 5.47E-03 5.12E-03 4.81E-03 4.05E-03 
Table A.3 (cont'd). At Six Months Gestation: Specific Absorbed Fraction of Photon Energy (kg').

SOURCE = GI TRACT: LOWER LARGE INTESTINE WALL

Energy (MeV)

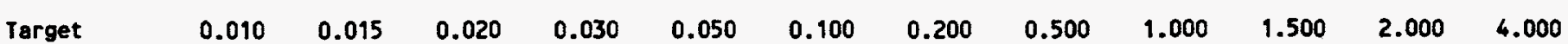

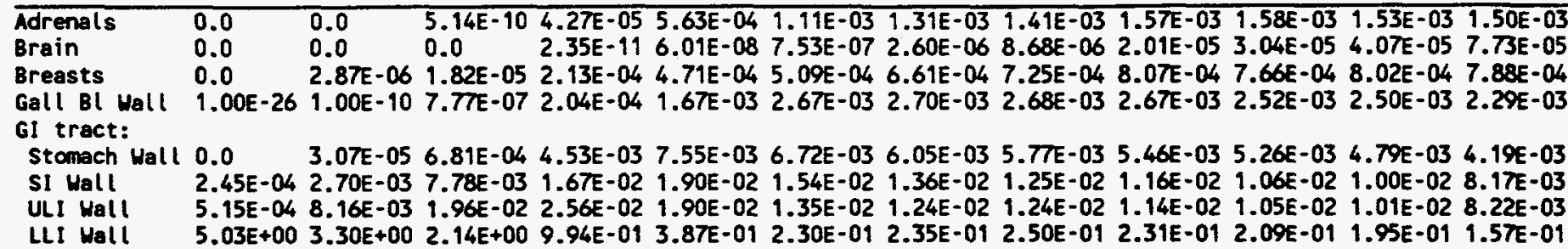

Heart Wall $\quad 0.0 \quad 8.10 E-16 \quad 3.74 E-09$ 1.58E-05 2.49E-04 $4.99 E-04 \quad 6.11 E-04 \quad 7.40 E-04 \quad 8.43 E-04$ 8.61E-04 8.81E-04 8.77E-04

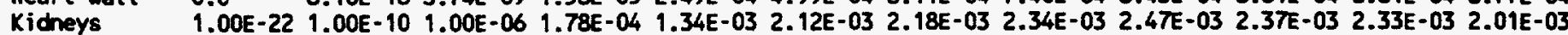
Liver $\begin{array}{llllllllllll}0.0 & 1.00 E-13 & 8.88 E-08 & 4.57 E-05 & 5.39 E-04 & 1.05 E-03 & 1.15 E-03 & 1.28 E-03 & 1.31 E-03 & 1.37 E-03 & 1.37 E-03 & 1.28 E-03\end{array}$ Lungs

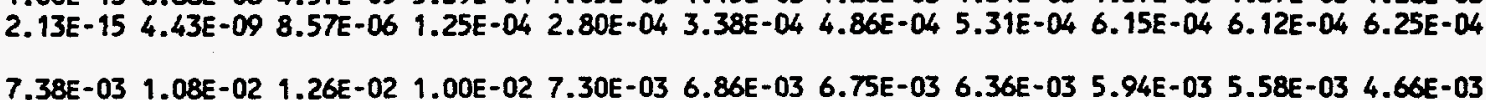

Muscle

Ovaries

Pancreas

Skeletal Tiss:

Act Marrow

Endosteum

Skin

Spleen

0.0

$\begin{array}{lllllllllllll}3.05 E-03 & 7.38 E-03 & 1.08 E-02 & 1.26 E-02 & 1.00 E-02 & 7.30 E-03 & 6.86 E-03 & 6.75 E-03 & 6.36 E-03 & 5.94 E-03 & 5.58 E-03 & 4.66 E-03 \\ 2.70 E-07 & 6.12 E-05 & 2.88 E-03 & 1.92 E-02 & 2.69 E-02 & 2.17 E-02 & 1.88 E-02 & 1.69 E-02 & 1.59 E-02 & 1.45 E-02 & 1.36 E-02 & 1.07 E-02\end{array}$

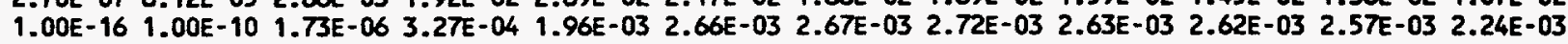

6.00E-06 1.56E-04 1.07E-03 3.84E-03 5.97E-03 6.03E-03 5.87E-03 5.68E-03 5.34E-03 4.97E-03 4.67E-03 4.00E-03 1.34E-06 2.35E-04 1.84E-03 8.08E-03 1.33E-02 8.79E-03 5.11E-03 3.58E-03 3.15E-03 2.92E-03 2.73E-03 2.40E-03 1.36E-04 4.88E-04 9.76E-04 2.02E-03 2.36E-03 2.04E-03 2.05E-03 2.31E-03 2.26E-03 2.24E-03 2.16E-03 1.83E-03 1.00E-16 1.00E-10 9.09E-07 3.52E-04 1.91E-03 2.50E-03 2.49E-03 2.69E-03 2.53E-03 2.61E-03 2.41E-03 2.32E-03

Thymus $\quad 0.0 \quad 0.0 \quad 2.70 E-12 \quad 4.54 E-08 \quad 4.50 E-05 \quad 1.39 E-04 \quad 1.69 E-04 \quad 2.53 E-04 \quad 3.46 E-04 \quad 3.98 E-04 \quad 4.30 E-04 \quad 4.39 E-04$ Thyroid $\quad 0.0 \quad 0.0 \quad 1.33 E-16 \quad 6.39 E-104.26 E-06 \quad 1.56 E-05$ 2.28E-05 $5.46 E-05$ 7.70E-05 $1.10 E-04$ 1.33E-04 $1.60 E-04$ Urin Bl Wall 1.00E-08 2.38E-04 3.09E-03 $1.75 E-02 \quad 2.57 E-02 \quad 2.03 E-02 \quad 1.78 E-02 \quad 1.65 E-02 \quad 1.56 E-02 \quad 1.47 E-02 \quad 1.33 E-02 \quad 1.14 E-02$ Uterine Cont:

Fetus

6.37E-06 2.67E-04 3.78E-03 2.04E-02 3.05E-02 2.33E-02 $1.91 E-02 \quad 1.70 E-02 \quad 1.55 E-02 \quad 1.43 E-02 \quad 1.32 E-02 \quad 1.09 E-02$

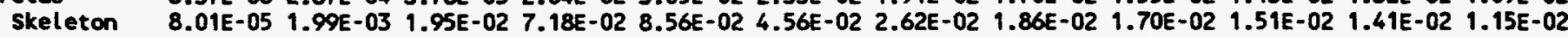

Soft Tissue 2.24E-06 1.39E-04 2.59E-03 1.65E-02 2.64E-02 $2.16 E-02 \quad 1.86 E-02 \quad 1.68 E-02 \quad 1.54 E-02 \quad 1.42 E-02 \quad 1.32 E-02 \quad 1.08 E-02$ Uterine Wall 1.82E-03 1.24E-02 2.67E-02 3.95E-02 3.40E-02 2.42E-02 2.17E-02 2.03E-02 $1.87 \mathrm{E}-02$ 1.70E-02 1.60E-02 $1.31 \mathrm{E}-02$ Body Tissues 1.60E-02 1.45E-02 1.41E-02 1.32E-02 1.01E-02 7.19E-03 6.57E-03 6.40E-03 6.01E-03 5.61E-03 5.27E-03 4.41E-03

\section{SOURCE $=$ HEART CONTENTS}

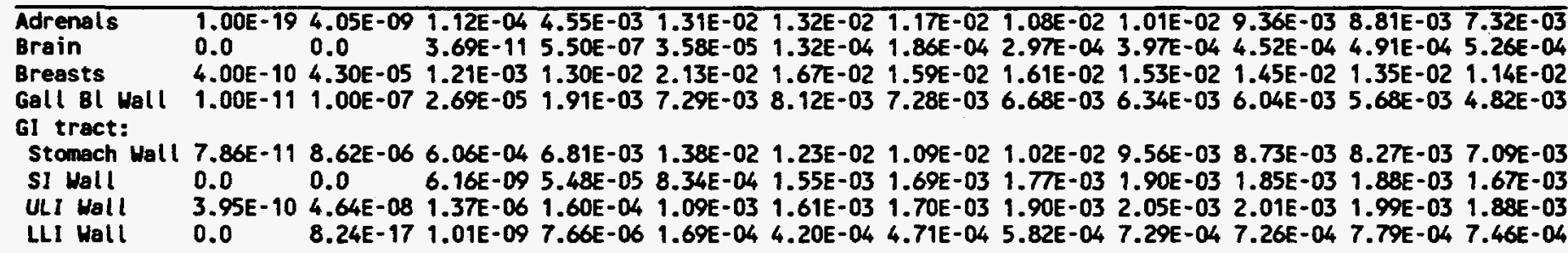

Meart Wall 2.48E-01 5.48E-01 6.86E-01 5.69E-01 2.93E-01 1.68E-01 1.60E-01 1.57E-01 1.45E-01 1.32E-01 1.22E-01 9.85E-02 Kicheys Liver

Lungs $1.00 E-17$ 1.00E-10 1.40E-06 4.03E-04 2.63E-03 3.69E-03 3.71E-03 3.74E-03 3.60E-03 3.66E-03 3.45E-03 2.96E-03 1.88E-06 1.46E-04 2.26E-03 1.31E-02 1.97E-02 1.57 E-02 $1.37 E-02 \quad 1.27 E-02 \quad 1.16 E-02 \quad 1.09 E-02 \quad 1.00 E-02$ 8.2BE-03 2.61E-05 3.10E-03 2.03E-02 5.51E-02 5.14E-02 3.27E-02 2.80E-02 2.59E-02 2.36E-02 2.13E-02 1.99E-02 1.62E-02

Muscle Ovaries Pancreas Skeletal Tiss: Endosteun Skin

Spleen

8.42E-05 1.16E-03 3.49E-03 7.31E-03 7.60E-03 5.72E-03 5.22E-03 5.08E-03 4.76E-03 4.44E-03 4.19E-03 3.53E-03

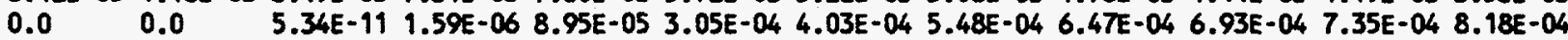
1.00E-16 1.00E-07 1.78E-04 5.80E-03 1.55E-02 1.45E-02 1.26E-02 1.15E-02 1.05E-02 9.79E-03 9.30E-03 $7.77 E-03$

2.22E-07 4.46E-05 4.27E-04 2.52E-03 5.22E-03 5.66E-03 5.56E-03 5.51E-03 5.23E-03 4.93E-03 4.70E-03 4.00E-03 6.68E-07 1.45E-04 1.4TE-03 9.39E-03 1.80E-02 1.15E-02 6.38E-03 4.49E-03 3.93E-03 3.62E-03 3.42E-03 2.95E-03

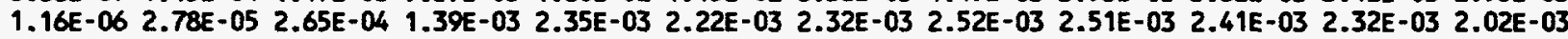
1.00E-23 1.00E-09 2.46E-05 1.69E-03 6.54E-03 7.16E-03 6.47E-03 6.20E-03 5.83E-03 5.61E-03 5.27E-03 4.49E-03

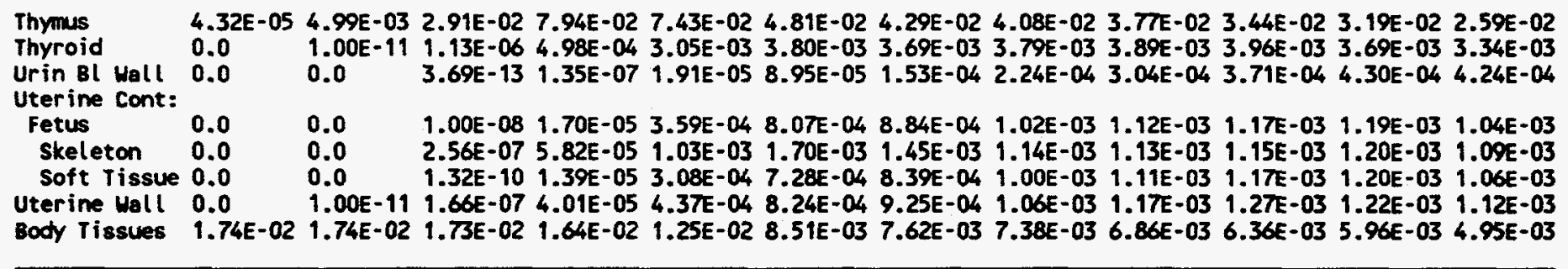


Table A.3 (cont'd). At Six Months Gestation: Specific Absorbed Fraction of Photon Energy $\left(\mathrm{kg}^{-1}\right)$.

SOURCE = HEART WAL

Energy (MeV)

$\begin{array}{lllllllllllll}\text { Target } & 0.010 & 0.015 & 0.020 & 0.030 & 0.050 & 0.100 & 0.200 & 0.500 & 1.000 & 1.500 & 2.000 & 4.000\end{array}$

\begin{tabular}{lllllllllllll}
\hline Adrenals & $1.00 E-17$ & $1.00 E-07$ & $2.34 E-04$ & $6.21 E-03$ & $1.51 E-02$ & $1.44 E-02$ & $1.30 E-02$ & $1.19 E-02$ & $1.08 E-02$ & $1.00 E-02$ & $9.68 E-03$ & $7.89 E-03$ \\
Brain & 0.0 & 0.0 & $3.08 E-11$ & $4.71 E-07$ & $3.10 E-05$ & $1.12 E-04$ & $1.60 E-04$ & $2.61 E-04$ & $3.65 E-04$ & $4.03 E-04$ & $4.54 E-04$ & $4.74 E-04$ \\
Breasts & $2.38 E-06$ & $1.52 E-04$ & $2.91 E-03$ & $1.82 E-02$ & $2.48 E-02$ & $1.87 E-02$ & $1.79 E-02$ & $1.81 E-02$ & $1.74 E-02$ & $1.60 E-02$ & $1.52 E-02$ & $1.28 E-02$ \\
Gall Bl Hall & $1.00 E-10$ & $5.00 E-07$ & $7.07 E-05$ & $2.68 E-03$ & $8.60 E-03$ & $9.08 E-03$ & $8.03 E-03$ & $7.48 E-03$ & $6.86 E-03$ & $6.54 E-03$ & $6.32 E-03$ & $5.26 E-03$ \\
GI tract: & & & & & & & & & & & & \\
Stomach Wall & $1.00 E-05$ & $3.15 E-04$ & $4.22 E-03$ & $1.80 E-02$ & $2.41 E-02$ & $1.86 E-02$ & $1.64 E-02$ & $1.56 E-02$ & $1.40 E-02$ & $1.32 E-02$ & $1.25 E-02$ & $1.01 E-02$ \\
SI Wall & $1.78 E-12$ & $1.24 E-09$ & $1.29 E-07$ & $9.01 E-05$ & $1.09 E-03$ & $1.91 E-03$ & $1.98 E-03$ & $2.09 E-03$ & $2.16 E-03$ & $2.16 E-03$ & $2.14 E-03$ & $1.82 E-03$ \\
ULI Hall & 0.0 & $1.00 E-10$ & $3.48 E-06$ & $2.45 E-04$ & $1.41 E-03$ & $1.96 E-03$ & $1.98 E-03$ & $2.19 E-03$ & $2.28 E-03$ & $2.24 E-03$ & $2.23 E-03$ & $2.01 E-03$ \\
LLI Wall & 0.0 & $8.10 E-16$ & $3.74 E-09$ & $1.58 E-05$ & $2.49 E-04$ & $4.99 E-04$ & $6.11 E-04$ & $7.40 E-04$ & $8.43 E-04$ & $8.61 E-04$ & $8.81 E-04$ & $8.77 E-04$
\end{tabular}

Heart Hall 3.49E+00 2.54E+00 1.79E+00 9.29E-01 4.02E-01 2.35E-01 2.36E-01 2.46E-01 2.25E-01 2.03E-01 1.87E-01 1.52E-01 Kidneys $\quad 2.00 E-26 \quad 2.00 E-102.80 E-06$ 5.59E-04 3.18E-03 4.32E-03 4.10E-03 $4.13 E-03 \quad 4.00$ E-03 $3.93 E-03 \quad 3.80 E-03 \quad 3.35 E-03$

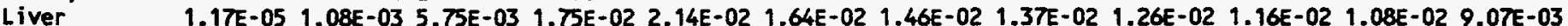
Lungs $\quad 2.10 E-04 \quad 7.13 E-03$ 2.72E-02 5.56E-02 4.83E-02 3.06E-02 2.63E-02 2.48E-02 2.24E-02 2.05E-02 1.90E-02 1.54E-02

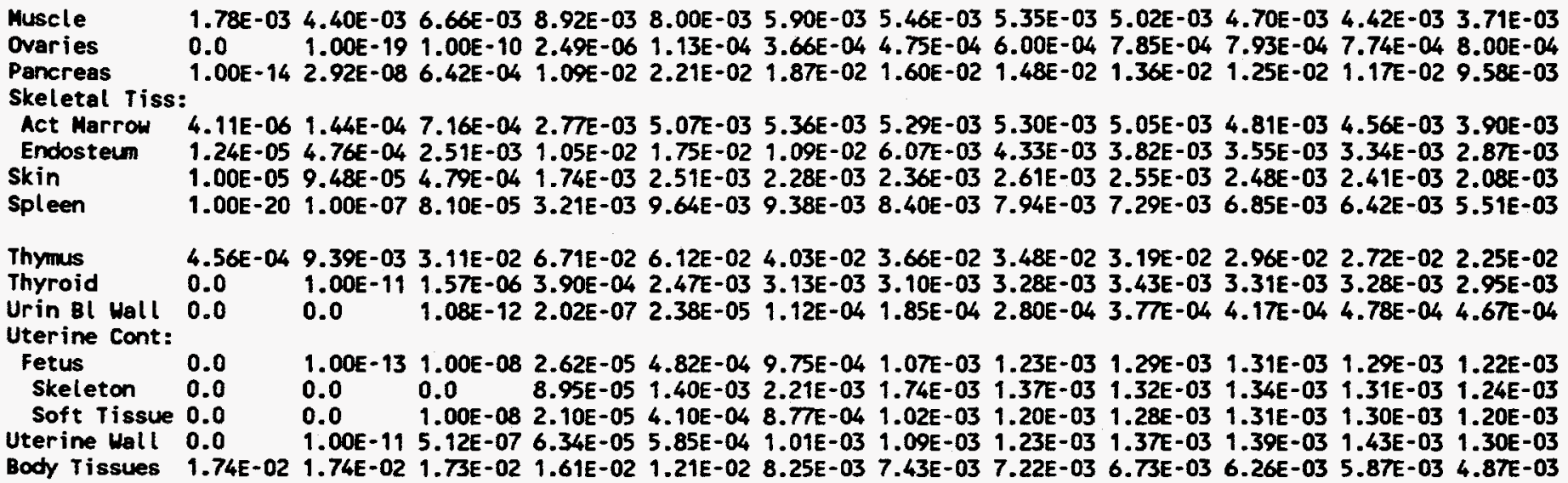

SOURCE $=$ KIDNEYS

\begin{tabular}{lllllllllllll}
\hline Adrenals & $8.65 E-04$ & $1.89 E-02$ & $5.80 E-02$ & $9.13 E-02$ & $7.05 E-02$ & $4.90 E-02$ & $4.52 E-02$ & $4.42 E-02$ & $4.03 E-02$ & $3.68 E-02$ & $3.44 E-02$ & $2.83 E-02$ \\
Brain & 0.0 & 0.0 & $2.41 E-16$ & $9.76 E-09$ & $1.81 E-06$ & $1.02 E-05$ & $2.34 E-05$ & $5.29 E-05$ & $9.22 E-05$ & $1.16 E-04$ & $1.40 E-04$ & $1.74 E-04$ \\
Breasts & 0.0 & $5.18 E-15$ & $1.21 E-08$ & $6.11 E-05$ & $6.61 E-04$ & $1.17 E-03$ & $1.32 E-03$ & $1.59 E-03$ & $1.68 E-03$ & $1.76 E-03$ & $1.75 E-03$ & $1.58 E-03$ \\
Gall BI Hall & $2.16 E-05$ & $1.07 E-03$ & $1.71 E-02$ & $6.33 E-02$ & $6.57 E-02$ & $4.64 E-02$ & $4.11 E-02$ & $3.82 E-02$ & $3.45 E-02$ & $3.19 E-02$ & $2.90 E-02$ & $2.39 E-02$ \\
GI tract: & & & & & & & & & & & & \\
Stomach Wall & $1.40 E-15$ & $2.80 E-06$ & $4.78 E-04$ & $8.08 E-03$ & $1.68 E-02$ & $1.49 E-02$ & $1.33 E-02$ & $1.24 E-02$ & $1.15 E-02$ & $1.06 E-02$ & $9.98 E-03$ & $8.18 E-03$ \\
SI Wall & $1.57 E-07$ & $3.16 E-05$ & $1.36 E-03$ & $1.19 E-02$ & $2.06 E-02$ & $1.81 E-02$ & $1.60 E-02$ & $1.47 E-02$ & $1.34 E-02$ & $1.24 E-02$ & $1.16 E-02$ & $9.64 E-03$ \\
ULI Hall & $1.00 E-17$ & $1.00 E-09$ & $4.09 E-06$ & $6.04 E-04$ & $3.28 E-03$ & $4.41 E-03$ & $4.26 E-03$ & $4.22 E-03$ & $4.15 E-03$ & $3.99 E-03$ & $3.86 E-03$ & $3.32 E-03$ \\
LLI Wall & $1.00 E-22$ & $1.00 E-10$ & $1.00 E-06$ & $1.78 E-04$ & $1.34 E-03$ & $2.12 E-03$ & $2.18 E-03$ & $2.34 E-03$ & $2.47 E-03$ & $2.37 E-03$ & $2.33 E-03$ & $2.01 E-03$
\end{tabular}

Heart Wall 2.00E-26 2.00E-10 2.80E-06 5.59E-04 3.18E-03 4.32E-03 4.10E-03 $4.13 E-03 \quad 4.00 E-03 \quad 3.93 E-03 \quad 3.80 E-03 \quad 3.35 E-03$

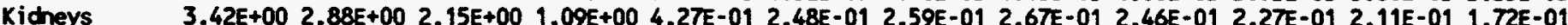

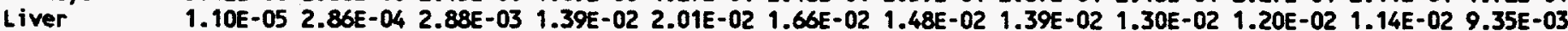
Lungs 1.00E-11 1.00E-07 4.59E-05 1.34E-03 3.83E-03 4.09E-03 3.91E-03 3.96E-03 3.93E-03 3.75E-03 3.59E-03 3.06E-03

Muscle

Ovaries

Pancreas

Skeletal Tiss:

Act Marrow

Endosteun

Skin

Spleen

1.58E-03 4.87E-03 8.34E-03 1.02E-02 7.97E-03 5.97E-03 5.71E-03 5.74E-03 5.44E-03 5.111E-03 4.83E-03 4.05E-03 0.0 1.00E-11 3.56E-06 4.78E-04 2.81E-03 4.00E-03 4.12E-03 4.19E-03 4.35E-03 4.06E-03 3.80E-03 3.31E-03 4.09E-12 6.31E-05 4.00E-03 3.00E-02 4.17E-02 3.19E-02 2.77E-02 2.63E-02 2.39E-02 2.21E-02 2.05E-02 1.66E-02 4.36E-06 3.43E-04 1.88E-03 6.33E-03 9.91E-03 9.94E-03 9.95E-03 9.87E-03 9.29E-03 8.72E-03 8.22E-03 6.94E-03 8.52E-06 7.72E-04 4.62E-03 1.63E-02 2.25E-02 $1.25 E-02 \quad 7.18 E-03 \quad 5.34 E-03 \quad 4.77 E-03 \quad 4.41 E-03 \quad 4.17 E-03 \quad 3.59 E-03$ 1.58E-05 2.09E-04 1.31E-03 2.84E-03 2.56E-03 2.12E-03 2.23E-03 2.51E-03 2.53E-03 2.48E-03 2.37E-03 2.10E-03 1.41E-04 1.02E-02 4.37E-02 8.44E-02 6.53E-02 4.22E-02 3.81E-02 3.66E-02 3.35E-02 3.07E-02 2.85E-02 2.30E-02

Thymus $\quad 0.0 \quad 1.00 E-16 \quad 8.34 E-10 \quad 2.31 E-05 \quad 4.38 E-04 \quad 8.78 E-04 \quad 9.88 E-04 \quad 1.21 E-03 \quad 1.39 E-03 \quad 1.38 E-03 \quad 1.46 E-03 \quad 1.32 E-03$ Thyroid $\quad 0.0 \quad 0.0 \quad 9.85 E-13$ 2.06E-06 6.63E-05 $1.92 E-04 \quad 2.58 E-04 \quad 3.20 E-04 \quad 4.67 E-04 \quad 5.29 E-04 \quad 5.53 E-04 \quad 5.56 E-04$ Urin Bl Wall 0.0 Uterine cont:

Fetus 0.0

Skeleton $\quad 0.0$

Soft Tissue 0.0

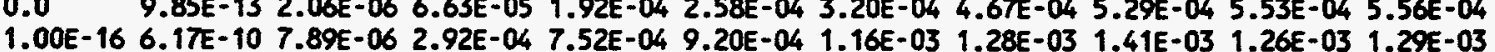

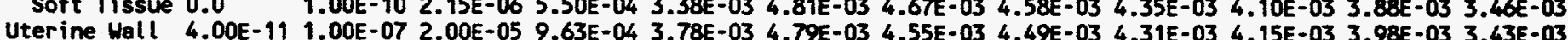
Body Tissues 1.74E-02 1.74E-02 1.70E-02 1.49E-02 1.08E-02 7.52E-03 6.91E-03 6.81E-03 6.42E-03 5.99E-03 5.64E-03 4.70E-03 
Table A.3 (cont'd). At Six Months Gestation: Specific Absorbed Fraction of Photon Energy (kg $\left.{ }^{-1}\right)$.

SOURCE $=$ LIVER

Energy (MeV)

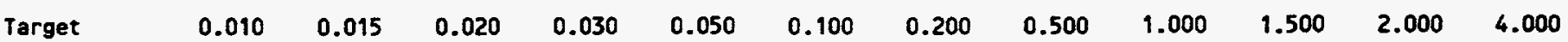

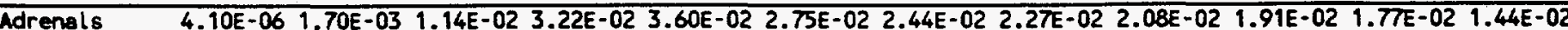

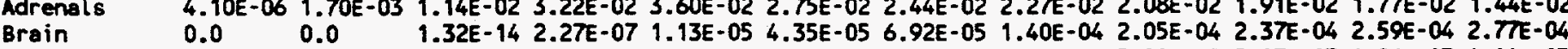

Breasts $\quad 1.00 E-09$ 1.00E-06 1.16E-04 2.34E-03 5.63E-03 5.33E-03 5.13E-03 5.43E-03 5.28E-03 5.07E-03 4.86E-03 4.14E-03

Gall Bl Wall 4.11E-06 3.19E-03 2.32E-02 7.07E-02 7.25E-02 4.92E-02 4.24E-02 3.88E-02 3.51E-02 3.20E-02 2.96E-02 2.43E-02

GI tract:

Stomach Wall 1.78E-06 7.76E-05 $1.13 E-03$ 7.06E-03 1.28E-02 1.14E-02 1.02E-02 9.67E-03 8.90E-03 8.26E-03 7.78E-03 6.53E-03 SI Wall $1.65 E-05$ 2.62E-04 $1.36 E-03 \quad 6.44 E-03$ 1.15E-02 $1.07 E-02$ 9.39E-03 8.74E-03 $8.18 E-03$ 7.53E-03 $7.02 E-03$ 6.03E-03 UL1 Wall 6.00E-06 2.51E-04 1.67E-03 7.28E-03 1.17E-02 1.00E-02 9.23E-03 8.85E-03 8.45E-03 7.99E-03 7.60E-03 6.20E-03 LLI Wall $0.0 \quad 1.00 E-13 \quad 8.88 E-08$ 4.57E-05 $5.39 E-04 \quad 1.05 E-03 \quad 1.15 E-03 \quad 1.28 E-03 \quad 1.31 E-03 \quad 1.37 E-03 \quad 1.37 E-03 \quad 1.28 E-03$

Heart Wall 1.17E-05 1.08E-03 5.73E-03 1.75E-02 2.14E-02 1.64 E-02 $1.46 E-02 \quad 1.37 E-02 \quad 1.26 E-02 \quad 1.16 E-02$ 1.08E-02 9.07E-03

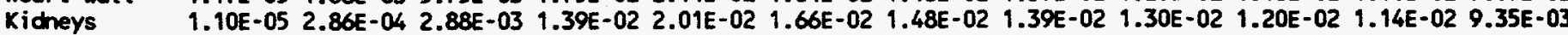
Liver $\quad 6.85 E-01$ 6.32E-01 5.43E-01 3.64E-01 1.85E-01 1.11E-01 1.06E-01 1.07E-01 9.83E-02 8.96E-02 8.29E-02 6.80E-02 Lungs $\quad 2.63 E-04$ 5.45E-03 $1.53 E-02$ 2.52E-02 2.14E-02 $1.45 E-02 \quad 1.27 E-02 \quad 1.19 E-02 \quad 1.09 E-02$ 1.00E-02 9.41E-03 $7.62 E-03$

Muscle $\quad 7.63 E-04$ 2.29E-03 4.21E-03 6.65E-03 6.48E-03 5.02E-03 4.69E-03 4.65E-03 4.42E-03 4.15E-03 3.93E-03 3.31E-03 Ovaries $\quad 1.00 E-16$ 1.00E-10 9.34E-07 1.80E-04 1.37E-03 2.18E-03 2.24E-03 2.35E-03 2.41E-03 2.34E-03 2.31E-03 2.08E-03 Pancreas

Skeletal Tiss:

Act Marrow

Endosteum

Skin

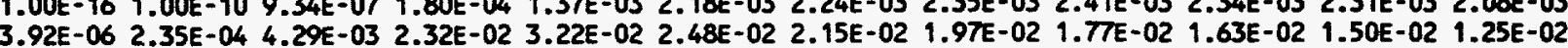

Spleen

4.55E-05 3.14E-04 1.03E-03 2.72E-03 4.41E-03 4.81E-03 4.84E-03 4.89E-03 4.70E-03 4.46E-03 4.22E-03 3.64E-03 1.36E-04 1.09E-03 4.11E-03 1.24E-02 1.69E-02 9.81E-03 5.59E-03 4.10E-03 3.66E-03 3.40E-03 3.18E-03 2.77T-03

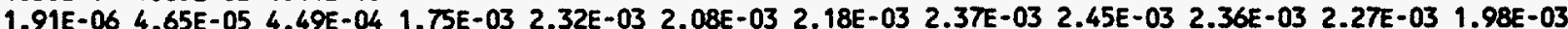
1.00E-22 1.00E-09 9.44E-06 9.31E-04 4.44E-03 5.56E-03 5.21E-03 5.07E-03 4.89E-03 4.59E-03 4.37E-03 3.81E-03

Thymus $\quad 2.96 E-15$ 6.96E-10 7.20E-06 7.92E-04 3.44E-03 3.97E-03 3.74E-03 3.80E-03 3.78E-03 3.62E-03 3.44E-03 2.99E-03 Thyroid $\quad 0.0 \quad 1.00 E-13$ 3.55E-08 3.75E-05 4.02E-04 7.13E-04 8.05E-04 9.72E-04 1.08E-03 1.11E-03 1.11E-03 1.00E-03 Urin Bl wall 0.0 1.00E-16 1.00E-09 7.72E-06 2.24E-04 5.92E-04 6.93E-04 8.86E-04 1.00E-03 1.05E-03 1.06E-03 9.91E-04 Uterine Cont:

Fetus

1.00E-17 1.00E-08 1.93E-05 8.00E-04 3.58E-03 4.45E-03 4.05E-03 3.92E-03 3.70E-03 3.57E-03 3.37E-03 2.95E-03 Skeleton $\quad 2.91 E-07$ 8.55E-06 9.41E-05 2.76E-03 1.01E-02 9.46E-03 6.13E-03 4.32E-03 3.76E-03 3.54E-03 3.37E-03 3.08E-03 Soft Tissue 0.0 $\quad 8.66 E-07$ 1.35E-05 6.51E-04 3.08E-03 4.08E-03 3.87E-03 3.86E-03 3.70E-03 3.56E-03 3.39E-03 2.97E-03 Uterine Hall 7.51E-08 5.97E-06 1.33E-04 1.44E-03 3.84E-03 4.26E-03 4.00E-03 3.95E-03 3.85E-03 3.71E-03 3.55E-03 3.07E-03 Body Tissues 1.74E-02 1.74E-02 1.72E-02 1.58E-02 1.16E-02 7.94E-03 7.23E-03 7.06E-03 6.63E-03 6.16E-03 5.77E-03 4.80E-03

SOURCE $=$ LUNGS

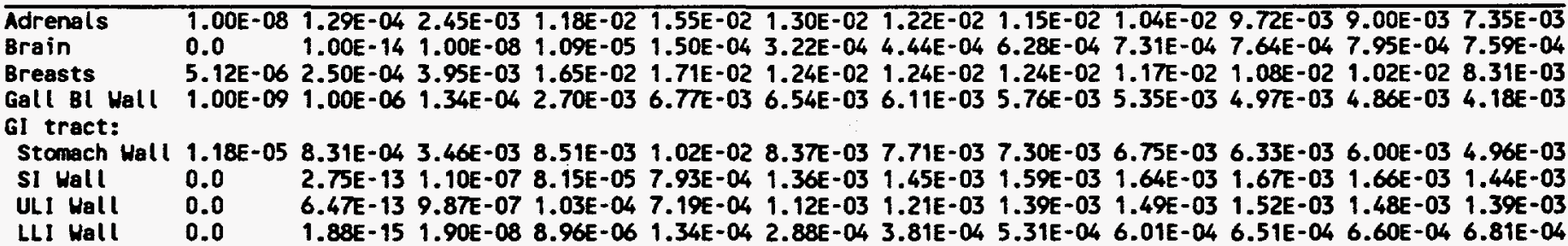

Heart Wall 1.88E-04 6.23E-03 2.45E-02 5.00E-02 4.42E-02 2.94E-02 2.63E-02 2.48E-02 2.24E-02 2.05E-02 1.90E-02 $1.54 E-02$ Kidneys 1.00E-10 1.00E-07 4.10E-05 1.24E-03 3.50E-03 3.91E-03 3.91E-03 3.96E-03 3.93E-03 3.75E-03 3.59E-03 3.06E-03 2.36E-04 4.85E-03 1.37E-02 2.27E-02 1.97E-02 $1.39 E-02$ 1.27E-02 $1.19 E-02$ 1.09E-02 1.00E-02 9.41E-03 7.62E-03 Liver

Lungs 1.38E+00 1.07E+00 7.29E-01 3.36E-01 1.26E-01 6.94E-02 6.85E-02 6.90E-02 6.32E-02 5.78E-02 5.27E-02 4.24E-02

Muscle

Ovaries

Pancreas

Skeletal Tiss:

Act Marrow

Endosteum

skin

Spleen

2.49E-03 7.09E-03 1.11E-02 1.22E-02 8.57E-03 5.86E-03 5.54E-03 5.45E-03 5.07E-03 4.70E-03 4.41E-03 3.65E-03 $0.0 \quad 1.00 E-19 \quad 1.00 E-10 \quad 2.25 E-06 \quad 8.78 E-05 \quad 2.72 E-04 \quad 3.60 E-04 \quad 5.11 E-04 \quad 6.24 E-04 \quad 6.80 E-04 \quad 6.98 E-04 \quad 7.06 E-04$ 5.00E-08 1.00E-05 5.67E-04 6.87E-03 1.24E-02 1.06E-02 9.56E-03 8.74E-03 7.99E-03 7.41E-03 6.98E-03 5.80E-03

2.40E-06 2.12E-04 1.42E-03 4.89E-03 6.76E-03 6.37E-03 6.27E-03 6.25E-03 5.92E-03 5.49E-03 5.21E-03 4.38E-03 7.17E-06 7.14E-04 5.27E-03 2.03E-02 2.52E-02 1.29E-02 7.16E-03 5.40E-03 4.81E-03 4.42E-03 4.15E-03 3.50E-03 9.21E-07 3.79E-05 5.29E-04 2.30E-03 2.84E-03 2.46E-03 2.58E-03 2.84E-03 2.72E-03 2.68E-03 2.53E-03 2.13E-03 5.16E-06 1.82E-04 2.28E-03 9.27E-03 1.14E-02 8.98E-03 8.32E-03 7.84E-03 7.33E-03 6.83E-03 6.26E-03 5.29E-03

Thyous $\quad 1.42 E-12 \quad 2.19 E-05 \quad 1.87 E-03 \quad 1.75 E-02 \quad 2.45 E-02 \quad 1.83 E-02 \quad 1.64 E-02 \quad 1.55 E-02 \quad 1.43 E-02 \quad 1.31 E-02 \quad 1.22 E-02 \quad 1.02 E-02$ Thyroid 1.00E-18 1.00E-07 1.29E-04 3.10E-03 6.74E-03 6.28E-03 6.09E-03 6.17E-03 5.89E-03 5.70E-03 5.35E-03 4.42E-03

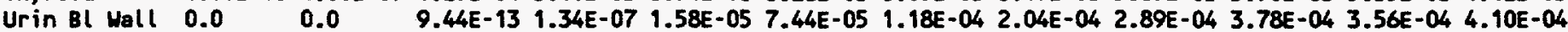
Uterine Cont:

Fetus 0.0

Skeleton 0.0

Soft Tissue 0.0

1.30E-17 1.30E-09 1.45E-05 2.82E-04 6.04E-04 7.16E-04 8.33E-04 9.20E-04 9.57E-04 9.31E-04 8.95E-04 0.0 4.55E-08 5.18E-05 8.05E-04 1.16E-03 1.02E-03 9.90E-04 8.94E-04 9.30E-04 9.51E-04 8.96E-04 $0.0 \quad 1.00 E-08$ 1.11E-05 2.42E-04 5.51E-04 6.81E-04 8.04E-04 9.05E-04 9.60E-04 9.22E-04 9.10E-04 $1.00 E-13$ 5.00E-08 2.93E-05 3.27E-04 6.08E-04 6.91E-04 8.38E-04 9.41E-04 9.60E-04 9.97E-04 9.29E-04 Body Tissues $1.74 E-02$ 1.74E-02 $1.73 E-02$ 1.56E-02 $1.09 E-02$ 7.02E-03 6.29E-03 6.10E-03 $5.65 E-03$ 5.25E-03 4.91E-03 4.05E-03 
Table A.3 (cont'd). At Six Months Gestation: Specific Absorbed Fraction of Photon Energy (kg').

SOURCE $=$ MUSCLE

Energy (MeV)

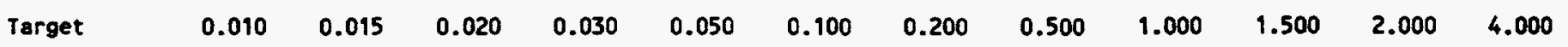

Adrenals 5.13E-03 $1.17 \mathrm{E}-02$ 1.46E-02 $1.32 E-02$ 9.34E-03 7.03E-03 6.76E-03 6.71E-03 6.30E-03 5.88E-03 5.52E-03 4.59E-03 Brain $\quad 1.13 E-08$ 1.85E-06 6.87E-05 6.15E-04 1.11E-03 1.08E-03 1.11E-03 $1.20 E-03 \quad 1.18 E-03 \quad 1.14 E-03$ 1.09E-03 9.52E-04 Breasts $\quad 4.33 E-04$ 1.32E-03 2.38E-03 3.58E-03 3.40E-03 2.68E-03 2.71E-03 2.90E-03 2.87E-03 2.75E-03 2.62E-03 2.24E-03 Goll Bl Wall 7.29E-03 1.15E-02 1.38E-02 $1.39 E-02$ 1.06E-02 $7.83 E-03$ 7.28E-03 7.07E-03 6.59E-03 6.13E-03 5.77E-03 $4.78 E-03$ GI tract:

Stomach Wall 2.67E-03 6.62E-03 9.67E-03 1.18E-02 9.74E-03 7.02E-03 6.51E-03 6.39E-03 5.99E-03 5.61E-03 5.25E-03 4.39E-03 SI Hall 7.99E-04 2.38E-03 4.43E-03 7.39E-03 7.88E-03 6.41E-03 5.91E-03 5.68E-03 5.34E-03 5.00E-03 4.70E-03 3.96E-03 ULI Wall 2.90E-03 6.63E-03 9.10E-03 9.72E-03 7.31E-03 5.35E-03 5.12E-03 5.17E-03 $4.93 E-03$ 4.65E-03 4.38E-03 3.70E-03 LLI Wall 3.05E-03 7.38E-03 1.08E-02 1.26E-02 1.00E-02 7.30E-03 6.86E-03 6.73E-03 6.36E-03 5.94E-03 5.58E-03 4.66E-03

Heart Wall $\quad 1.78 E-03 \quad 4.40 E-03 \quad 6.66 E-03$ 8.92E-03 8.00E-03 5.90E-03 5.46E-03 5.35E-03 5.02E-03 4.70E-03 4.42E-03 3.71E-03 Kidneys $\quad 1.58 E-03$ 4.87E-03 8.34E-03 1.02E-02 7.97E-03 5.97E-03 5.71E-03 5.74E-03 5.44E-03 5.11E-03 4.83E-03 4.05E-03 Liver $\quad 7.63 E-042.29 E-03 \quad 4.21 E-03 \quad 6.65 E-03 \quad 6.48 E-03 \quad 5.02 E-03 \quad 4.69 E-03 \quad 4.65 E-03 \quad 4.42 E-03 \quad 4.15 E-03$ 3.93E-03 3.31E-03 Lungs $\quad 2.67 E-03$ 7.75E-03 1.22E-02 1.35E-02 9.31E-03 6.08E-03 5.54E-03 5.45E-03 5.07E-03 4.70E-03 4.41E-03 3.65E-03

Muscle $\quad 2.35 E-02$ 2.17E-02 $1.91 E-02 \quad 1.39$ E-02 8.38E-03 5.86E-03 5.84E-03 6.01E-03 5.71E-03 5.33E-03 5.01E-03 4.18E-03 Ovaries $\quad 4.08 E-03$ 9.39E-03 $1.12 E-02$ 1.07E-02 $9.20 E-03$ 7.30E-03 6.95E-03 6.80E-03 6.39E-03 5.97E-03 5.60E-03 $4.67 E-03$ Pancreas 2.44E-03 6.90E-03 1.11E-02 $1.37 E-02$ 1.12E-02 8.08E-03 7.44E-03 7.17E-03 6.68E-03 6.20E-03 5.82E-03 4.83E-03 Skeletal Tiss:

Aet Marrow 6.04E-04 1.52E-03 2.82E-03 4.77E-03 5.61E-03 5.29E-03 5.18E-03 5.27E-03 4.99E-03 4.70E-03 4.41E-03 3.72E-03 Endosteum 2.12E-03 6.59E-03 1.31E-02 2.41E-02 2.51E-02 1.25E-02 7.31E-03 5.7TE-03 5.24E-03 4.84E-03 4.53E-03 3.88E-03 skin

Spleen 5.26E-03 7.95E-03 8.37E-03 6.86E-03 4.32E-03 3.18E-03 3.37E-03 3.69E-03 3.62E-03 3.46E-03 3.26E-03 2.T7E-03 1.45E-03 4.53E-03 8.31E-03 1.15E-02 9.37E-03 6.64E-03 6.22E-03 6.13E-03 5.78E-03 5.39E-03 5.07E-03 4.23E-03

Thymus Thyroid

2.64E-03 7.65E-03 1.18E-02 1.30E-02 9.27E-03 6.26E-03 5.95E-03 5.97E-03 5.63E-03 5.24E-03 4.92E-03 4.10E-03 4.32E-03 1.03E-02 1.34E-02 1.26E-02 8.21E-03 5.56E-03 5.47E-03 5.62E-03 5.34E-03 4.96E-03 4.66E-03 3.86E-03 Uterine Cont:

Fetus

Skeleton 5.03E-03 8.37E-03 1.01E-02 1.06E-02 8.19E-03 6.01E-03 5.79E-03 5.89E-03 5.62E-03 5.28E-03 4.99E-03 4.19E-03

4.56E-07 2.91E-05 5.54E-04 3.36E-03 6.13E-03 5.40E-03 4.65E-03 4.39E-03 4.12E-03 3.91E-03 3.69E-03 3.17E-03 Soft Tissue 2.45E-07 1.80E-05 $3.81 E-04$ 2.76E-03 5.28E-03 4 -94E-03 $4.54 E-03 \quad 4.33 E-03 \quad 4.12 E-03 \quad 3.88 E-03$ 3.68E-03 3.14E-03 Uterine Hall 1.60E-03 4.25E-03 6.40E-03 8.04E-03 7.39E-03 5.83E-03 5.46E-03 5.37E-03 5.06E-03 4.75E-03 4.51E-03 3.78E-03 Body Tissues 1.73E-02 1.68E-02 1.58E-02 1.31E-02 8.96E-03 5.95E-03 5.53E-03 5.55E-03 5.23E-03 4.90E-03 4.59E-03 3.87E-03

SOURCE = OVARIES

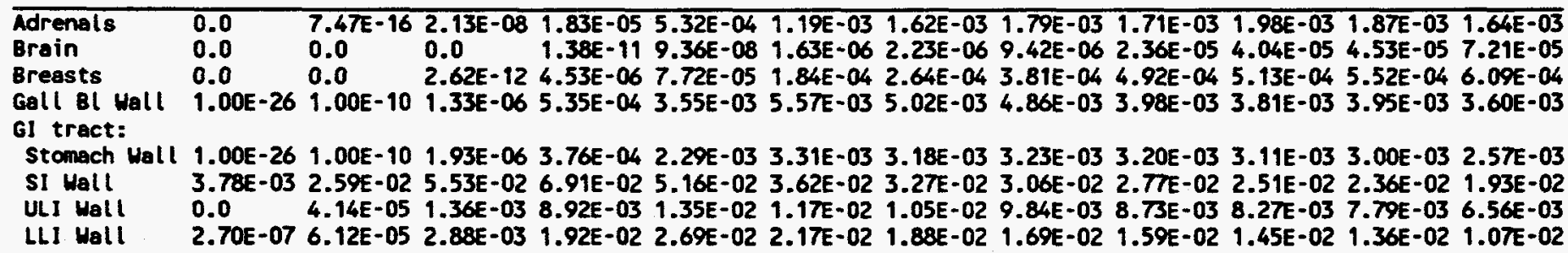

Heart Hall $0.0 \quad 1.00 E-191.00 E-102.49 E-06 \quad 1.13 E-04 \quad 3.66 E-04 \quad 4.75 E-04 \quad 6.00 E-04 \quad 7.85 E-04 \quad 7.93 E-04 \quad 7.74 E-04 \quad 8.00 E-04$ Kicheys $\quad 0.0 \quad 1.00 E-11 \quad 3.56 E-06 \quad 4.78 E-04 \quad 2.81 E-03 \quad 4.00 E-03 \quad 4.12 E-03 \quad 4.19 E-03 \quad 4.35 E-03 \quad 4.06 E-03 \quad 3.80 E-03 \quad 3.31 E-03$ Liver $\quad 1.00 E-16$ 1.00E-10 9.34E-07 1.80E-04 1.37E-03 2.18E-03 2.24E-03 2.35E-03 2.41E-03 2.34E-03 2.31E-03 2.08E-03 lungs $\quad 0.0 \quad 1.00 E-19$ 1.00E-10 2.52E-06 9.59E-05 2.86E-04 3.60E-04 5.11E-04 6.24E-04 6.80E-04 6.98E-04 7.06E-04

Muscle $\quad 4.08 E-03$ 9.39E-03 1.12E-02 1.07E-02 9.20E-03 7.30E-03 6.95E-03 6.80E-03 6.39E-03 5.97E-03 5.60E-03 4.67E-03

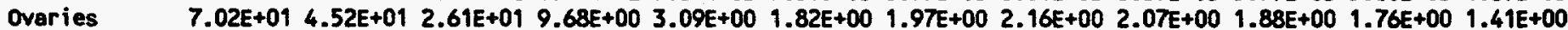

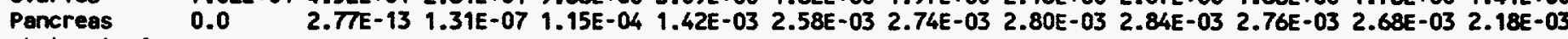
Skeletal Tiss:

Act Marrow 8.93E-05 3.31E-03 1.22E-02 2.42E-02 2.33E-02 1.89E-02 1.80E-02 $1.75 E-02 \quad 1.61$ E-02 $1.49 E-02$ 1.38E-02 $1.13 E-02$ Endosteum 1.18E-04 4.83E-03 1.92E-02 4.05E-02 3.57E-02 1.63E-02 9.10E-03 6.77E-03 5.96E-03 5.46E-03 5.07E-03 4.28E-03 Skin Spleen 1.00E-21 1.00E-08 7.41E-06 4.02E-04 1.37E-03 1.59E-03 1.70E-03 1.84E-03 1.91E-03 1.90E-03 $1.82 E-03$ 1.63E-03

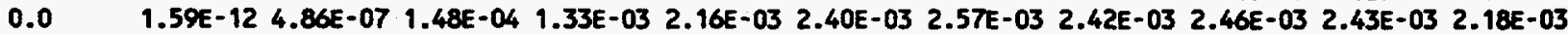

Thymes $\quad 0.0 \quad 0.0 \quad 1.00 E-14$ 1.31E-07 $1.18 E-05 \quad 6.80 E-05 \quad 1.23 E-04 \quad 2.83 E-04 \quad 3.20 E-04 \quad 3.50 E-04 \quad 3.90 E-04 \quad 4.38 E-04$

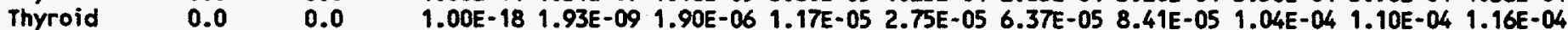
Urin Bl Wall 1.00E-15 1.00E-07 5.71E-05 3.24E-03 1.08E-02 1.13E-02 1.03E-02 9.01E-03 8.68E-03 8.23E-03 8.39E-03 6.77E-03 Uterine Cont:

Fetus

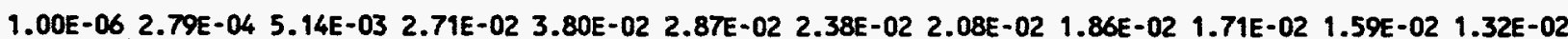
Skeleton 1.00E-06 1.87E-03 2.75E-02 9.93E-02 $1.10 E-01$ 5.69E-02 3.36E-02 $2.39 E-02$ 2.04E-02 $1.84 E-02$ 1.66E-02 $1.44 E-02$ Soft Tissue 2.06E-06 1.58E-04 3.44E-03 2.16E-02 $3.26 E-02 \quad 2.66 E-02 \quad 2.30 E-02 \quad 2.06 E-02 \quad 1.85 E-02 \quad 1.70 E-02 \quad 1.58 E-02 \quad 1.31 E-02$ Uterine Hall 1.18E-03 2.20E-02 5.10E-02 6.33E-02 4.66E-02 3.25E-02 2.93E-02 2.75E-02 2.49E-02 2.28E-02 2.13E-02 $1.73 E-02$ Bocty Tissues 1.74E-02 1.72E-02 1.65E-02 1.49E-02 1.15E-02 8.05E-03 7.22E-03 6.94E-03 6.49E-03 6.04E-03 5.66E-03 4.72E-03 
Table A.3 (cont'd). At Six Months Gestation: Specific Absorbed Fraction of Photon Energy (kg $\left.{ }^{-1}\right)$.

SOURCE $=$ PANCREAS

Energy (MeV)

$\begin{array}{lllllllllllll}\text { Target } & 0.010 & 0.015 & 0.020 & 0.030 & 0.050 & 0.100 & 0.200 & 0.500 & 1.000 & 1.500 & 2.000 & 4.000\end{array}$

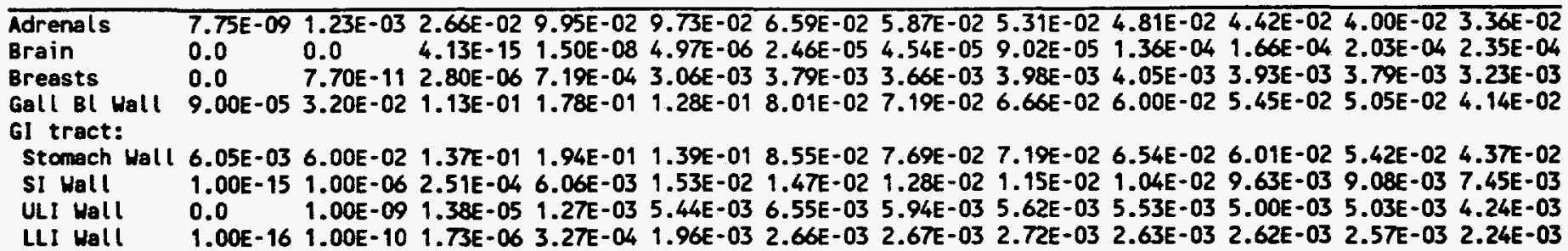

Heart Hall 1.00E-14 2.92E-08 6.42E-04 1.09E-02 2.21E-02 $1.87 E-02 \quad 1.60 E-02 \quad 1.48 E-02 \quad 1.36 E-02 \quad 1.25 E-02 \quad 1.17 E-02 \quad 9.58 E-03$

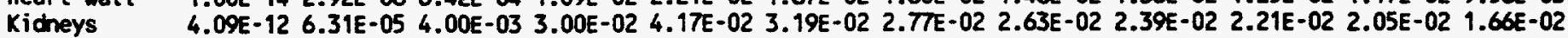

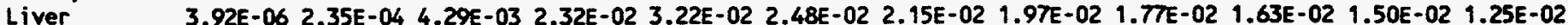
Lungs $\quad 0.0 \quad 4.00 E-06 \quad 6.35 E-04$ 7.38E-03 $1.33 E-02 \quad 1.10 E-02$ 9.56E-03 8.74E-03 7.99E-03 7.41E-03 6.98E-03 5.80E-03

Muscle $\quad 2.44 E-03 \quad 6.90 E-03 \quad 1.11 E-02 \quad 1.37 E-02 \quad 1.12 E-02 \quad 8.08 E-03 \quad 7.44 E-03 \quad 7.17 E-03 \quad 6.68 E-03 \quad 6.20 E-03 \quad 5.82 E-03 \quad 4.83 E-03$

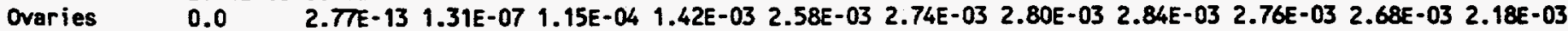
Pancreas 1.07E+01 8.31E+00 5.78E+00 2.66E+00 9.77E-01 5.63E-01 5.96E-01 6.31E-01 5.91E-01 5.35E-01 4.88E-01 3.84E-01 Skeletal Tiss:

Act Marrow 7.99E-07 3.35E-05 4.74E-04 3.14E-03 6.76E-03 7.61E-03 7.36E-03 7.30E-03 6.75E-03 6.30E-03 5.98E-03 5.16E-03

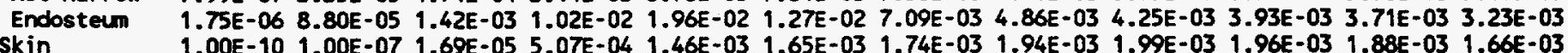

Skin Spleen $\quad 1.38 E-03$ 4.09E-02 1.27E-01 1.86E-01 1.31E-01 8.10E-02 7.20E-02 6.81E-02 6.42E-02 5.84E-02 5.35E-02 4.24E-02

Thymus $\quad 0.0 \quad 1.00 E-12 \quad 8.36 E-08$ 2.40E-04 2.09E-03 3.07E-03 2.99E-03 2.95E-03 2.94E-03 3.97E-03 3.23E-03 2.71E-03 Thyroid $\quad 0.0 \quad 0.0 \quad 3.91 E-11 \quad 4.79 E-06 \quad 1.69 E-04 \quad 4.67 E-04$ 5.55E-04 6.99E-04 8.81E-04 8.92E-04 8.54E-04 8.31E-04 Urin Bl Wall $0.0 \quad 1.00 E-18$ 1.60E-10 6.44E-06 2.42E-04 6.83E-04 8.24E-04 9.59E-04 9.81E-04 1.09E-03 1.07E-03 1.13E-03 Uterine Cont: Fetus $\quad 0.0$ Soft Tissue $0.0 \quad 5.62 E-07$ 1.07E-05 8.08E-04 4.11E-03 5.54E-03 5.20E-03 4.82E-03 4.56E-03 4.30E-03 4.10E-03 3.50E-03 Uterine Wall 5.00E-11 1.00E-07 2.17E-05 1.12E-03 4.35E-03 5.20E-03 4.83E-03 4.54E-03 4.44E-03 4.16E-03 4.03E-03 3.41E-03 Body Tissues 1.74E-02 1.73E-02 1.71E-02 1.62E-02 1.31E-02 9.34E-03 8.31E-03 7.90E-03 7.33E-03 6.79E-03 6.35E-03 5.27E-03

SOURCE = SKELETAL TISSUES: ACTIVE MARROW

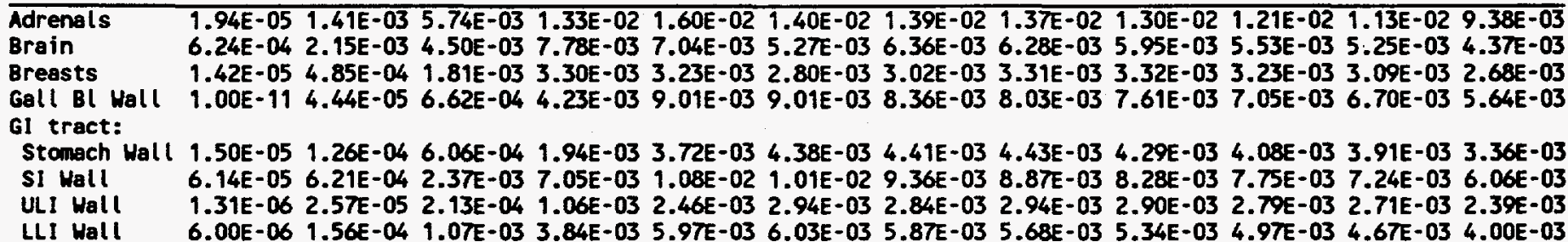

Heart Hall 4.11E-06 1.61E-04 8.43E-04 3.22E-03 5.85E-03 5.67E-03 5.29E-03 5.30E-03 5.05E-03 4.81E-03 4.56E-03 3.90E-03 Kidneys

Liver $4.36 E-06 \quad 3.81 E-04$ 2.21E-03 7.44E-03 1.10E-02 1.03E-02 9.95E-03 9.87E-03 9.29E-03 8.72E-03 8.22E-03 6.94E-03

Lungs 4.55E-05 3.50E-04 1.20E-03 3.17E-03 5.06E-03 5.13E-03 4.84E-03 4.89E-03 4.70E-03 4.46E-03 4.22E-03 3.64E-03 2.69E-06 2.65E-04 1.85E-03 6.31E-03 8.22E-03 6.78E-03 6.27E-03 6.25E-03 5.92E-03 5.49E-03 5.21E-03 4.38E-03

Muscle $\quad 6.04 E-04 \quad 1.74 E-03 \quad 3.19 E-03 \quad 5.31 E-03 \quad 5.92 E-03 \quad 5.21 E-03 \quad 5.18 E-03 \quad 5.27 E-03 \quad 4.99 E-03 \quad 4.70 E-03 \quad 4.41 E-03 \quad 3.72 E-03$ Ovaries $\quad 8.93 E-05 \quad 3.68 E-03 \quad 1.39 E-02$ 2.73E-02 2.50E-02 $1.96 E-02 \quad 1.80 E-02 \quad 1.75 E-02 \quad 1.61 E-02 \quad 1.49 E-02 \quad 1.38 E-02 \quad 1.13 E-02$ Pancreas 8.08E-07 3.54E-05 5.20E-04 3.38E-03 7.49E-03 7.88E-03 7.44E-03 7.30E-03 6.75E-03 6.30E-03 5.98E-03 5.16E-03 Skeletal Tiss:

Act Marrow 7.41E-02 6.48E-02 5.66E-02 4.31E-02 2.97E-02 2.39E-02 2.49E-02 2.59E-02 2.42E-02 2.25E-02 2.07E-02 1.71E-02

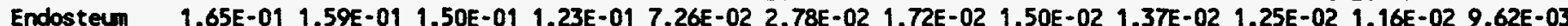

Skin

Spleen

3.43E-04 1.19E-03 1.96E-03 2.65E-03 2.71E-03 2.52E-03 2.90E-03 3.19E-03 3.19E-03 3.05E-03 2.93E-03 2.47E-03

1.57E-06 9.39E-04 9.30E-04 3.06E-03 5.46E-03 5.48E-03 5.17E-03 5.20E-03 5.05E-03 4.80E-03 4.58E-03 3.96E-03

Thymus $\quad 9.43 E-07 \quad 2.97 E-04 \quad 1.76 E-03 \quad 3.96 E-03 \quad 4.93 E-03 \quad 4.53 E-03 \quad 4.36 E-03 \quad 4.53 E-03 \quad 4.40 E-03 \quad 4.23 E-03 \quad 4.03 E-03 \quad 3.41 E-03$ Thyroid $\quad 3.80 E-06$ 7.61E-05 $6.47 E-04$ 2.70E-03 $4.26 E-03 \quad 4.62 E-03 \quad 4.48 E-03 \quad 4.65 E-03 \quad 4.58 E-03 \quad 4.36 E-03 \quad 4.15 E-03 \quad 3.57 E-03$ Urin Bl Wall 1.00E-18 1.00E-09 4.63E-06 3.42E-04 1.74E-03 2.57E-03 2.64E-03 2.70E-03 2.65E-03 2.54E-03 2.48E-03 2.17E-03 Uterine Cont:

Fetus

1.00E-10 3.00E-07 6.23E-05 1.56E-03 5.21E-03 5.83E-03 5.14E-03 4.71E-03 4.36E-03 4.12E-03 3.89E-03 3.33E-03 Skeleton $\quad 1.03 E-091.64 E-06 \quad 3.05 E-04 \quad 5.48 E-03 \quad 1.48 E-02 \quad 1.18 E-02 \quad 7.59 E-03 \quad 5.32 E-03 \quad 4.54 E-03 \quad 4.32 E-03 \quad 4.11 E-03 \quad 3.47 E-03$ Soft Tissue 1.00E-08 1.00E-06 3.98E-05 1.26E-03 4.48E-03 5.38E-03 4.87E-03 4.60E-03 4.37E-03 4.09E-03 3.85E-03 3.32E-03 Uterine Hall 1.00E-08 2.77E-05 5.24E-04 3.35E-03 6.21E-03 6.17E-03 5.69E-03 5.44E-03 5.20E-03 4.77E-03 4.50E-03 $3.86 E-03$ Body Tissues 1.74E-02 1.73E-02 1.69E-02 1.54E-02 1.12E-02 7.04E-03 6.26E-03 6.19E-03 5.83E-03 5.46E-03 5.13E-03 4.31E-03 
Table A.3 (cont'd). At Six Months Gestation: Specific Absorbed Fraction of Photon Energy (kg-1).

SOURCE $=$ SKELETAL TISSUES: SKELETON

Energy (MeV)

$\begin{array}{lllllllllllll}\text { Target } & 0.010 & 0.015 & 0.020 & 0.030 & 0.050 & 0.100 & 0.200 & 0.500 & 1.000 & 1.500 & 2.000 & 4.000\end{array}$

Adrenals $\quad 1.10 E-05 \quad 7.14 E-042.87 E-03 \quad 6.81 E-03 \quad 8.04 E-03 \quad 6.73 E-03 \quad 6.32 E-03 \quad 6.77 E-03 \quad 6.50 E-03 \quad 5.99 E-03 \quad 5.63 E-03 \quad 4.73 E-03$ Brain $\quad 9.11 E-04$ 2.93E-03 6.04E-03 1.03E-02 9.28E-03 7.01E-03 6.96E-03 7.04E-03 7.28E-03 6.67E-03 6.29E-03 5.25E-03 Breasts $\quad 9.12 E-06 \quad 2.82 E-04 \quad 1.05 E-03 \quad 2.03 E-03 \quad 2.10 E-03 \quad 1.87 E-03 \quad 1.94 E-03 \quad 2.15 E-03 \quad 2.25 E-03 \quad 2.19 E-03 \quad 2.10 E-03 \quad 1.82 E-03$ Gall Bl Wall 4.68E-07 1.93E-05 2.69E-04 1.71E-03 3.96E-03 4.06E-03 3.75E-03 3.68E-03 3.50E-03 3.24E-03 3.09E-03 2.64E-03 Gl tract:

Stomach Wall 5.81E-07 8.19E-05 4.13E-04 1.42E-03 2.57E-03 2.74E-03 2.61E-03 2.59E-03 2.51E-03 2.38E-03 2.27E-03 1.98E-03 S1 Wall $\quad 1.66 E-05 \quad 1.63 E-04 \quad 6.38 E-04 \quad 2.06 E-03 \quad 3.53 E-03 \quad 3.51 E-03 \quad 3.33 E-03 \quad 3.26 E-03 \quad 3.07 E-03 \quad 2.86 E-03 \quad 2.71 E-03 \quad 2.34 E-03$ ULI Wall 2.94E-07 6.93E-06 $6.53 E-05 \quad 4.06 E-04 \quad 1.04 E-03 \quad 1.32 E-03 \quad 1.33 E-03 \quad 1.40 E-03 \quad 1.41 E-03 \quad 1.37 E-03 \quad 1.34 E-03 \quad 1.23 E-03$ LLI Wall 2.91E-07 4.60E-05 3.51E-04 1.50E-03 2.78E-03 2.89E-03 2.67E-03 2.74E-03 2.60E-03 2.44E-03 2.30E-03 2.03E-03

Heart Hall 2.71E-06 9.31E-05 4.93E-04 1.96E-03 3.62E-03 3.53E-03 3.37E-03 3.35E-03 3.17E-03 2.99E-03 2.83E-03 2.43E-03 Kicheys $\quad 1.85 E-06 \quad 1.51 E-04$ 8.86E-04 3.00E-03 4.53E-03 4.32E-03 4.11E-03 4.26E-03 3.98E-03 3.72E-03 3.54E-03 3.04E-03

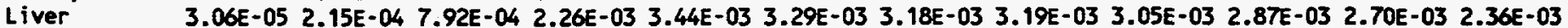
Lungs $\quad 1.03 E-05$ 1.62E-04 1.13E-03 4.09E-03 5.54E-03 4.59E-03 4.29E-03 4.30E-03 4.01E-03 3.73E-03 3.52E-03 2.97E-03

Muscle $\quad 4.63 E-041.29 E-03 \quad 2.36 E-03 \quad 4.09 E-03 \quad 4.75 E-03 \quad 4.25 E-03 \quad 4.30 E-03 \quad 4.45 E-03 \quad 4.26 E-03 \quad 4.02 E-03 \quad 3.78 E-03 \quad 3.20 E-03$ Ovaries Pancreas Skeletal Tiss:

Act Marrow 3.24E-02 2.84E-02 2.49E-02 1.95E-02 1.42E-02 1 1.17E-02 $1.21 E-02 \quad 1.26 E-02 \quad 1.15 E-02 \quad 1.08 E-02 \quad 9.92 E-03 \quad 8.25 E-03$

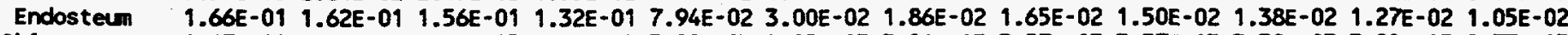
Skin Spleen 4.67E-04 1.36E-03 2.17E-03 3.09E-03 3.22E-03 2.95E-03 3.24E-03 3.57E-03 3.57E-03 3.39E-03 3.22E-03 2.77E-03 9.82E-07 8.17E-05 5.31E-04 2.08E-03 3.44E-03 3.35E-03 3.15E-03 3.18E-03 3.09E-03 2.91E-03 2.79E-03 2.41E-03

Thymus $\quad 6.17 E-07 \quad 1.73 E-04 \quad 1.01 E-03 \quad 2.44 E-03 \quad 3.19 E-03 \quad 2.97 E-03 \quad 2.82 E-03 \quad 3.02 E-03 \quad 2.96 E-03 \quad 2.83 E-03 \quad 2.70 E-03 \quad 2.30 E-03$ Thyroid $\quad 3.52 E-101.07 E-04 \quad 8.48 E-04$ 3.28E-03 $4.68 E-03 \quad 4.39 E-03 \quad 4.20 E-03 \quad 4.67 E-03 \quad 4.60 E-03 \quad 4.34 E-03 \quad 4.10 E-03$ 3.49E-03 Urin BL Wall $1.00 E-18$ 1.00E-09 $1.71 E-06 \quad 1.58 E-04$ 1.00E-03 $1.52 E-03 \quad 1.55 E-03 \quad 1.60 E-03 \quad 1.58 E-03 \quad 1.52 E-03 \quad 1.48 E-03 \quad 1.34 E-03$ Uterine Cont:

Fetus

$\begin{array}{llllllllllllll}1.00 E-17 & 3.54 E-08 & 1.56 E-05 & 4.41 E-04 & 1.68 E-03 & 2.06 E-03 & 1.95 E-03 & 1.84 E-03 & 1.80 E-03 & 1.71 E-03 & 1.63 E-03 & 1.47 E-03\end{array}$ Skeleton $\quad 3.88 E-10 \quad 5.44 E-07 \quad 9.30 E-05 \quad 1.64 E-03 \quad 4.74 E-03 \quad 4.11 E-03 \quad 2.69 E-03 \quad 2.02 E-03 \quad 1.84 E-03 \quad 1.78 E-03 \quad 1.71 E-03 \quad 1.51 E-03$

Soft Tissue $1.67 E-10 \quad 5.31 E-08 \quad 1.03 E-05 \quad 3.56 E-04 \quad 1.44 E-03 \quad 1.89 E-03 \quad 1.88 E-03 \quad 1.83 E-03 \quad 1.80 E-03 \quad 1.71 E-03 \quad 1.62 E-03 \quad 1.45 E-03$ Uterine Wall 1.14E-07 7.39E-06 1.43E-04 9.30E-04 1.98E-03 2.20E-03 2.13E-03 2.10E-03 2.06E-03 1.93E-03 1.85E-03 1.62E-03 Body Tissues 1.74E-02 1.73E-02 1.69E-02 1.53E-02 1.07E-02 6.35E-03 5.60E-03 5.60E-03 5.29E-03 4.96E-03 4.65E-03 3.90E-03

SOURCE $=$ SKIN

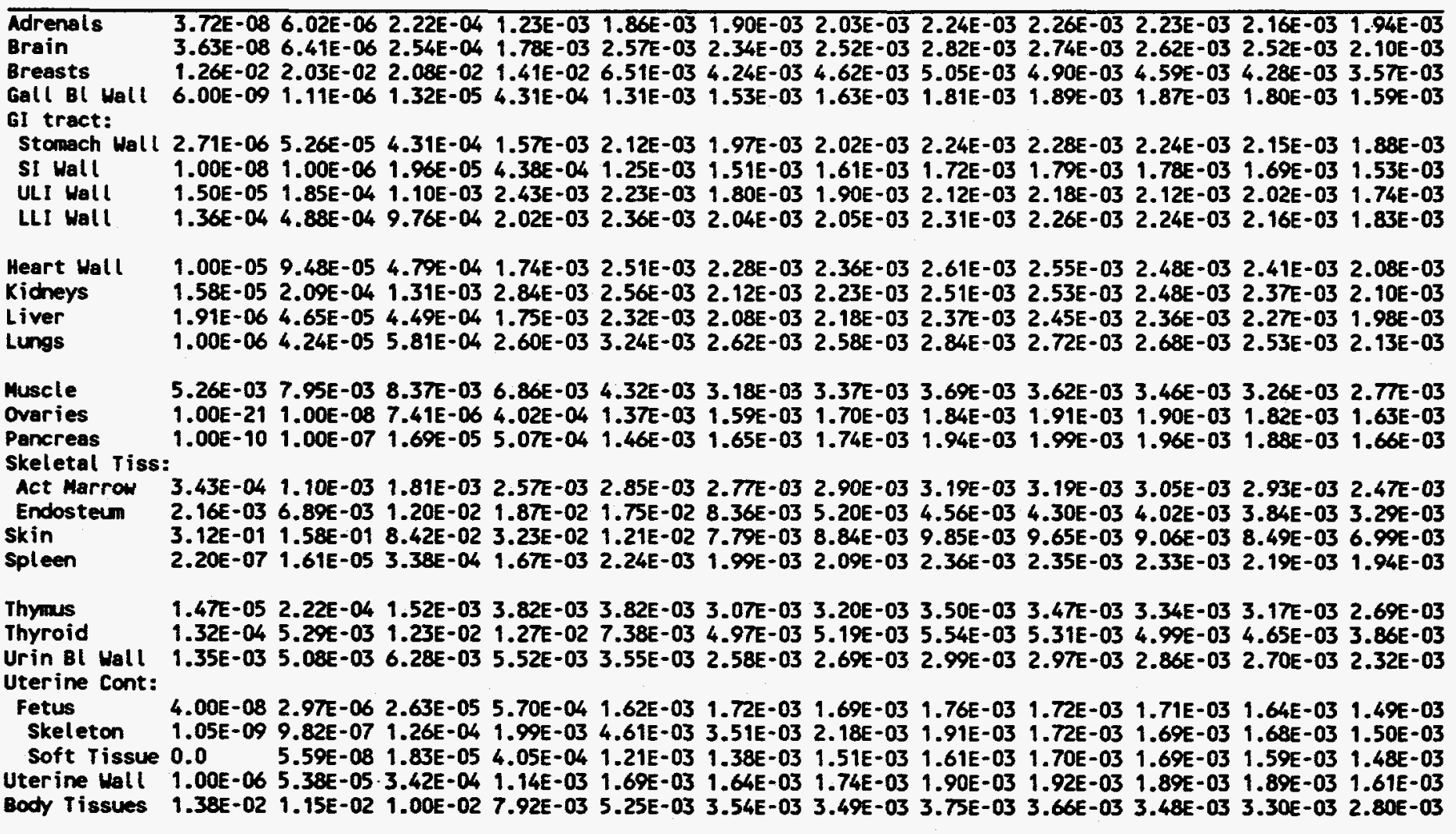


Table A.3 (cont'd). At Six Months Gestation: Specific Absorbed Fraction of Photon Energy (kg').

SOURCE $=$ SPLEEN

Energy (MeV)

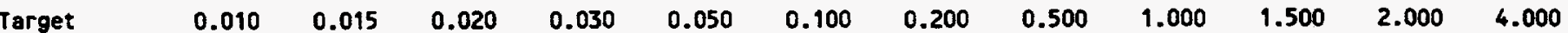

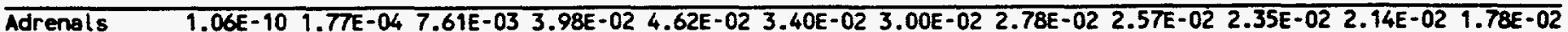

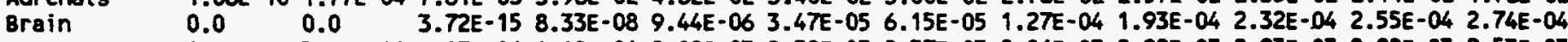

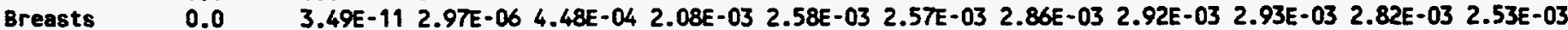

Gall BI Hall 1.00E-23 1.00E-08 7.87E-05 3.99E-03 1.25E-02 1.30E-02 1.16E-02 1.04E-02 9.77E-03 9.24E-03 8.45E-03 7.35E-03

GI tract:

Stonach Mall 2.16E-08 7.72E-04 1.48E-02 6.45E-02 7.12E-02 4.84E-02 4.13E-02 3.84E-02 3.45E-02 3.14E-02 2.90E-02 2.37E-02 SI Wall 5.00E-09 3.00E-06 2.38E-04 4.24E-03 1.05E-02 $1.02 E-02 \quad 9.14 E-03 \quad 8.58 E-03 \quad 7.84 E-03 \quad 7.51 E-03$ 7.09E-03 5.86E-03 ULI Wall 1.00E-12 1.00E-08 5.00E-06 2.71E-04 1.91E-03 2.81E-03 2.82E-03 2.88E-03 2.97E-03 2.81E-03 2.67E-03 2.56E-03 LLI Wall 1.00E-16 1.00E-10 9.09E-07 3.52E-04 1.91E-03 2.50E-03 2.49E-03 2.69E-03 2.53E-03 2.61E-03 2.41E-03 2.32E-03

Heart Wall 1.00E-20 1.00E-07 8.10E-05 3.21E-03 9.64E-03 9.38E-03 8.40E-03 7.94E-03 7.29E-03 6.85E-03 6.42E-03 5.51E-03 Kicheys $\quad 1.41 E-04$ 1.02E-02 $4.37 E-02$ 8.44E-02 6.53E-02 4.22E-02 3.81E-02 3.66E-02 3.35E-02 3.07E-02 2.85E-02 2.30E-02 Liver $\quad 1.00 E-22$ 1.00E-09 9.44E-06 9.31E-04 4.44E-03 5.56E-03 5.21E-03 5.07E-03 4.89E-03 4.59E-03 4.37E-03 3.81E-03 lungs $\quad 5.74 E-06$ 2.04E-04 2.57E-03 1.02E-02 $1.23 E-02$ 9.22E-03 8.32E-03 7.84E-03 7.33E-03 6.83E-03 6.26E-03 5.29E-03

Muscle $\quad 1.45 E-03 \quad 4.53 E-03 \quad 8.31 E-03 \quad 1.15 E-02 \quad 9.37 E-03 \quad 6.64 E-03 \quad 6.22 E-03 \quad 6.13 E-03 \quad 5.78 E-03 \quad 5.39 E-03$ 5.07E-03 $4.23 E-03$ $\begin{array}{lllllllllllll}\text { Overies } & 0.0 & 1.59 E-12 & 4.86 E-07 & 1.48 E-04 & 1.33 E-03 & 2.16 E-03 & 2.40 E-03 & 2.57 E-03 & 2.42 E-03 & 2.46 E-03 & 2.43 E-03 & 2.18 E-03\end{array}$ Pancreas $\quad 1.38 E-03$ 4.09E-02 1.27E-01 1.86E-01 1.31E-01 8.10E-02 7.20E-02 6.81E-02 6.42E-02 5.84E-02 5.35E-02 4.24E-02

Skeletal Tiss:

Act Merrow 1.5TE-06 1.26E-04 7.56E-04 2.64E-03 4.69E-03 5.16E-03 5.17E-03 5.20E-03 5.05E-03 4.80E-03 4.58E-03 3.96E-03 Endosteum 4.51E-06 4.18E-04 2.79E-03 1.12E-02 1.70E-02 $1.00 E-02$ 5.71E-03 $4.15 E-03$ 3.71E-03 3.45E-03 3.29E-03 2.86E-03 Skin $\quad 2.20 E-07$ 1.61E-05 3.38E-04 1.67E-03 2.24E-03 1.99E-03 2.09E-03 2.36E-03 2.35E-03 2.33E-03 2.19E-03 1.94E-03 Spleen $\quad 6.31 E+005.40 E+00 \quad 4.14 E+00$ 2.13E+00 8.28E-01 4.76E-01 4.91E-01 5.16E-01 4.78E-01 4.39E-01 4.06E-01 3.21E-01

Thymus $\quad 0.0 \quad 5.50 E-15 \quad 5.00 E-08 \quad 1.24 E-04 \quad 1.26 E-03 \quad 1.94 E-03 \quad 1.97 E-03 \quad 2.02 E-03 \quad 2.15 E-03 \quad 2.13 E-03 \quad 2.05 E-03 \quad 1.78 E-03$ Thyroid $\quad 0.0 \quad 0.0 \quad 1.79 E-11$ 1.41E-05 2.53E-04 4.85E-04 5.87E-04 7.74E-04 8.42E-04 8.96E-04 9.87E-04 8.51E-04 Urin Bl Wall 0.0 1.00E-19 1.00E-10 4.05E-06 1.57E-04 4.65E-04 5.92E-04 7.74E-04 8.99E-04 9.88E-04 9.68E-04 1.03E-03 Uterine Cont:

Fetus $\quad 0.0$

Skeleton 0.0

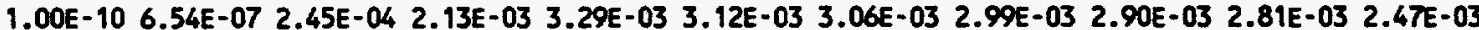
5.51E-08 2.98E-06 8.24E-04 5.93E-03 7.13E-03 4.83E-03 3.52E-03 3.13E-03 2.92E-03 2.80E-03 2.56E-03 4.97E-09 4.06E-07 2.01E-04 1.84E-03 2.99E-03 2.99E-03 3.01E-03 2.96E-03 2.89E-03 2.80E-03 2.46E-03 Uterine Hall $8.00 E-10$ 8.69E-08 2.77E-06 3.65E-04 2.12E-03 3.00E-03 2.94E-03 3.01E-03 2.98E-03 2.86E-03 2.74E-03 2.47E-03 Body Tissues 1.74E-02 1.74E-02 1.73E-02 1.57E-02 1.13E-02 7.73E-03 7.06E-03 6.92E-03 6.49E-03 6.04E-03 5.67E-03 4.71E-03

SOURCE $=$ THYMUS

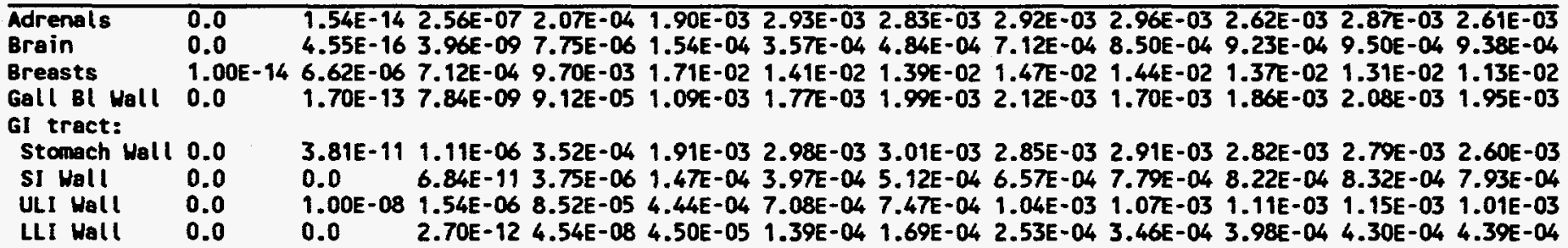

Heart Wall

Kidneys

Liver

Lungs

4.56E-04 9.39E-03 3.11E-02 6.71E-02 6.12E-02 4.03E-02 3.66E-02 3.48E-02 3.19E-02 2.96E-02 2.72E-02 2.25E-02

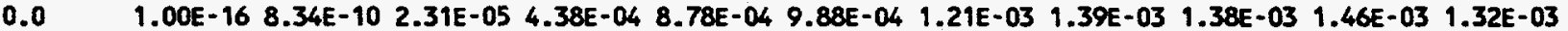
2.96E-15 6.96E-10 7.20E-06 7.92E-04 3.44E-03 3.97E-03 3.74E-03 3.80E-03 3.78E-03 3.62E-03 3.44E-03 2.99E-03 1.64E-12 2.45E-05 2.10E-03 1.92E-02 2.69E-02 1.90E-02 1.64E-02 1.55E-02 1.43E-02 1.31E-02 1.22E-02 1.02E-02

Muscle

Ovaries

Pancreas

2.64E-03 7.65E-03 1.18E-02 1.30E-02 9.27E-03 6.26E-03 5.95E-03 5.97E-03 5.63E-03 5.24E-03 4.92E-03 4.10E-03

Skeletal Tiss:

Act Marrow

Endosteum

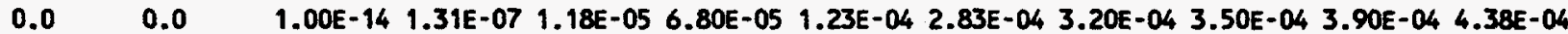
0.0 1.00E-12 8.36E-08 2.40E-04 2.09E-03 3.07E-03 2.99E-03 2.95E-03 2.94E-03 3.17E-03 3.23E-03 2.71E-03

Skin

Spleen

9.43E-07 2.6BE-04 1.50E-03 3.54E-03 4.48E-03 4.35E-03 4.36E-03 4.53E-03 4.40E-03 4.23E-03 4.03E-03 3.41E-03 2.83E-06 8.89E-04 5.33E-03 1.36E-02 1.60E-02 9.02E-03 5.20E-03 3.93E-03 3.56E-03 3.37E-03 3.18E-03 2.73E-03 1.47E-05 2.22E-04 1.52E-03 3.82E-03 3.82E-03 3.07E-03 3.20E-03 3.50E-03 3.47E-03 3.34E-03 3.17E-03 2.69E-03

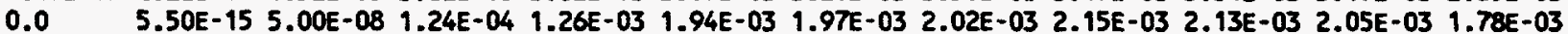

Thymus

Thyroid

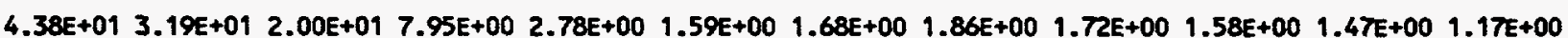
Uterine Cont:

Fetus

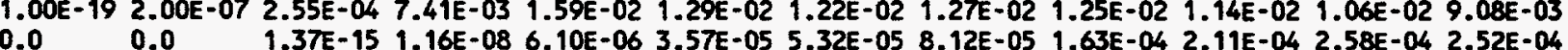

0.0

Soft Tissue 0.0

Uterine Hall 0.0

1.00E-12 3.65E-09 4.98E-06 1.07E-04 2.67E-04 3.28E-04 4.31E-04 5.17E-04 5.59E-04 5.80E-04 6.17E-04 1.00E-10 2.33E-08 2.00E-05 3.17E-04 6.37E-04 4.90E-04 5.43E-04 5.87E-04 5.23E-04 5.60E-04 6.62E-04 1.07E-11 2.17E-09 3.85E-06 9.04E-05 2.38E-04 3.11E-04 4.22E-04 5.15E-04 5.58E-04 5.85E-04 6.16E-04 1.00E-13 1.00E-07 2.47E-05 1.73E-04 3.13E-04 3.91E-04 5.15E-04 6.14E-04 6.53E-04 6.70E-04 6.62E-04 Body Tissues 1.74E-02 1.74E-02 1.70E-02 1.49E-02 1.04E-02 6.87E-03 6.35E-03 6.33E-03 5.96E-03 5.56E-03 5.22E-03 4.34E-03 
Table A.3 (cont'd). At Six Months Gestation: Specific Absorbed Fraction of Photon Energy (kg').

SOURCE $=$ THYROID

Energy (MeV)

\begin{tabular}{|c|c|c|c|c|c|c|c|c|c|c|c|c|}
\hline rget & 0.010 & 0.015 & 0.020 & 0.030 & 0.050 & 0.100 & 0.200 & 0.500 & 1.000 & 1.500 & 2.000 & 4.000 \\
\hline $\begin{array}{l}\text { Adrenals } \\
\text { Brain } \\
\text { Breasts } \\
\text { Gall Bl Wall } \\
\text { GI tract: } \\
\text { Stomach Wall } \\
\text { SI Wall } \\
\text { ULI Wall } \\
\text { LLI Wall }\end{array}$ & $\begin{array}{l}0.0 \\
0.0 \\
0.0 \\
0.0 \\
0.0 \\
0.0 \\
0.0 \\
0.0\end{array}$ & $\begin{array}{l}0.0 \\
7.00 E-13 \\
7.21 E-13 \\
0.0 \\
0.0 \\
0.0 \\
0.0 \\
0.0\end{array}$ & $\begin{array}{l}7.69 E-11 \\
4.50 E-07 \\
2.88 E-07 \\
1.00 E-11 \\
1.00 E-10 \\
3.16 E-15 \\
1.00 E-07 \\
1.33 E-16\end{array}$ & $\begin{array}{l}6.50 E-06 \\
1.93 E-04 \\
2.78 E-04 \\
4.06 E-06 \\
1.07 E-05 \\
3.83 E-08 \\
7.45 E-06 \\
6.39 E-10\end{array}$ & $\begin{array}{l}1.6 \\
1.2 \\
1.0\end{array}$ & $\begin{array}{l}5.03 \\
2.69 \\
1.89 \\
3.45 \\
4.10 \\
6.20 \\
8.33 \\
1.56\end{array}$ & $\begin{array}{l}4 \\
3 \\
3 \\
4\end{array}$ & $\begin{array}{l}6 . \\
1 . \\
2 . \\
5 .\end{array}$ & $\begin{array}{l}04 \\
04 \\
04 \\
05\end{array}$ & $\begin{array}{l}34 \\
34 \\
34\end{array}$ & $\begin{array}{l}04 \\
04 \\
04 \\
04\end{array}$ & $\begin{array}{l}2.4 \\
7.3 \\
.\end{array}$ \\
\hline $\begin{array}{l}\text { Heart Hall } \\
\text { Kicheys } \\
\text { Liver } \\
\text { Lungs }\end{array}$ & $\begin{array}{l}0.0 \\
0.0 \\
0.0 \\
1.00 E-17\end{array}$ & $\begin{array}{l}1.00 E-11 \\
0.0 \\
1.00 E-13 \\
1.00 E-07\end{array}$ & $\begin{array}{l}1.5 \\
9.8 \\
3.5 \\
1.4\end{array}$ & $\begin{array}{l}4 \\
6 \\
5\end{array}$ & & & & & & & & $\begin{array}{l}03 \\
.04 \\
.03 \\
.03\end{array}$ \\
\hline $\begin{array}{l}\text { Muscle } \\
\text { Ovaries } \\
\text { Pancreas } \\
\text { Skeletal Tiss: } \\
\text { Act Marrow } \\
\text { Endosteun } \\
\text { Skin } \\
\text { Spleen }\end{array}$ & $\begin{array}{l}4.32 E-03 \\
0.0 \\
0.0 \\
\\
3.72 E-06 \\
2.90 E-05 \\
1.32 E-04 \\
0.0\end{array}$ & $\begin{array}{l}1.03 E-02 \\
0.0 \\
0.0 \\
\\
6.86 E-05 \\
5.47 E-04 \\
5.29 E-03 \\
0.0\end{array}$ & $\begin{array}{l}1.34 E-02 \\
1.00 E-18 \\
3.91 E-11 \\
5.50 E-04 \\
4.46 E-03 \\
1.23 E-02 \\
1.79 E-11\end{array}$ & $\begin{array}{l}2.32 E-03 \\
1.76 E-02 \\
1.27 E-02 \\
1.41 E-05\end{array}$ & $\begin{array}{l}4.20 \\
2.47 \\
7.38 \\
2.53\end{array}$ & $\begin{array}{l}4.44 \\
1.32 \\
4.97 \\
4.85\end{array}$ & $\begin{array}{l}3 \\
3 \\
3 \\
4\end{array}$ & $\begin{array}{l}4 . \\
6 . \\
5 . \\
7 .\end{array}$ & $\begin{array}{l}3 \\
3 \\
3 \\
4\end{array}$ & $\begin{array}{l}03 \\
04 \\
04\end{array}$ & $\begin{array}{l}03 \\
04 \\
04\end{array}$ & $\begin{array}{l}-03 \\
-03 \\
-03 \\
-04\end{array}$ \\
\hline $\begin{array}{l}\text { Thymus } \\
\text { Thyroid } \\
\text { Urin Bl Wall } \\
\text { Uterine Cont: } \\
\text { Fetus } \\
\text { Skeleton } \\
\text { Soft Tissue } \\
\text { Uterine Wall } \\
\text { Body Tissues }\end{array}$ & $\begin{array}{l}1.00 E-19 \\
4.92 E+01 \\
0.0 \\
0.0 \\
0.0 \\
0.0 \\
3.00 E-10 \\
1.74 E-02\end{array}$ & $\begin{array}{l}2.00 E-07 \\
3.27 E+0 \\
0.0 \\
0.0 \\
0.0 \\
0.0 \\
1.00 E-08 \\
1.71 E-02\end{array}$ & $\begin{array}{l}2.55 E-04 \\
2.02 E+01 \\
5.90 E-20 \\
\\
1.65 E-08 \\
8.87 E-08 \\
1.10 E-08 \\
1.01 E-07\end{array}$ & $\begin{array}{l}4.37 E-07 \\
1.28 E-06 \\
3.22 E-07 \\
2.20 E-06\end{array}$ & $\begin{array}{l}06 \\
05 \\
06 \\
05\end{array}$ & $\begin{array}{l}3.79 \\
9.12 \\
3.35 \\
4.53 \\
5.89\end{array}$ & $\begin{array}{l}-05 \\
-04 \\
-05 \\
-05\end{array}$ & $\begin{array}{l}9.51 \\
1.200 \\
9.35 \\
1.20\end{array}$ & $\begin{array}{l}1.46 E-04 \\
1.19 E-04 \\
1.48 E-04 \\
1.77 E-04 \\
5.28 E-03\end{array}$ & $\begin{array}{l}1.75 \\
1.58 \\
1.75 \\
2.30\end{array}$ & $\begin{array}{l}1.5 \\
1.1\end{array}$ & $\begin{array}{l}.04 \\
.04 \\
.04 \\
.04 \\
.03\end{array}$ \\
\hline
\end{tabular}

SOURCE = URINARY BLADDER CONTENTS

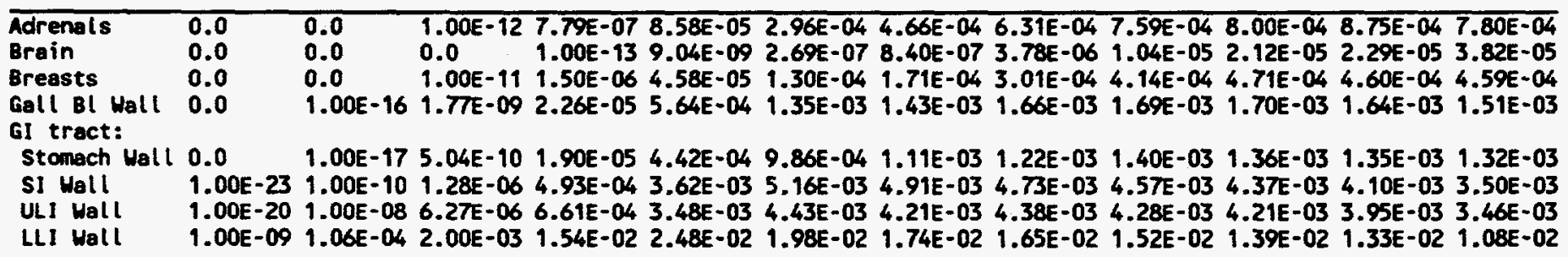

Heart Wall $\quad 0.0 \quad 0.0 \quad 7.57 E-13$ 2.02E-07 2.38E-05 1.25E-04 1.85E-04 2.80E-04 3.77E-04 4.17E-04 4.78E-04 4.67E-04 Kioneys $\quad 0.0 \quad 1.00 E-17 \quad 6.02 E-10 \quad 8.30 E-06 \quad 2.96 E-04 \quad 7.83 E-04 \quad 9.24 E-04 \quad 1.13 E-03 \quad 1.26 E-03 \quad 1.30 E-03 \quad 1.38 E-03 \quad 1.15 E-03$ Liver $\quad 0.0$ Lungs $\quad 0.0$

$1.00 E-16$ 1.00E-08 7.53E-06 2.22E-04 5.82E-04 7.06E-04 8.79E-04 9.76E-04 1.07E-03 $1.08 E-03$ 1.04E-03 $0.0 \quad 1.00 E-13 \quad 4.51 E-08 \quad 1.76 E-05$ 8.70E-05 1.22E-04 $1.95 E-04$ 2.93E-04 3.18E-04 3.67E-04 3.73E-04

Muscle

Ovaries

Pancreas

Skeletal Tiss:

Act Marrow

Endosteum

Skin

Spleen

4.27E-04 3.12E-03 6.48E-03 9.18E-03 7.80E-03 5.81E-03 5.53E-03 5.60E-03 5.35E-03 5.03E-03 4.75E-03 4.01E-03 1.00E- 15 1.00E-07 4.10E-05 2.93E-03 1.05E-02 1.11E-02 1.01E-02 9.53E-03 8.86E-03 8.24E-03 7.51E-03 6.75E-03

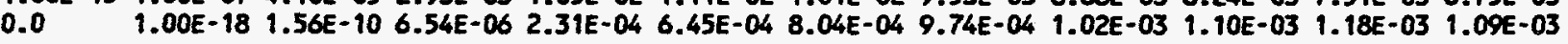

Thyous

Thyroid

9.99E-22 1.00E-09 3.17E-06 3.03E-04 1.69E-03 2.53E-03 2.61E-03 2.65E-03 2.60E-03 2.54E-03 2.41E-03 2.10E-03 2.10E-09 2.39E-07 6.89E-06 7.85E-04 4.45E-03 4.59E-03 2.93E-03 2.10E-03 1.90E-03 $1.82 E-03 \quad 1.74 E-03 \quad 1.55 E-03$

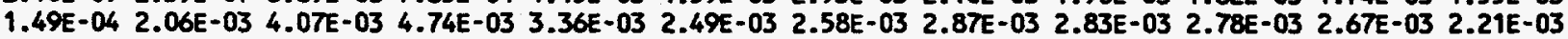
$0.0 \quad 1.00 E-18 \quad 1.00 E-10 \quad 3.40 E-06 \quad 1.52 E-04 \quad 4.53 E-04 \quad 5.53 E-04 \quad 7.51 E-04 \quad 8.67 E-04 \quad 9.13 E-049.87 E-049.93 E-04$

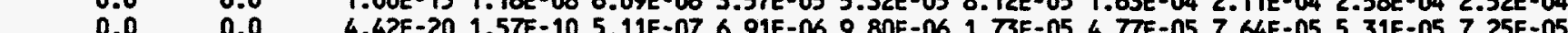
U8 Wall-mid 2.70E+00 3.83E+00 3.56E+00 2.09E+00 8.63E-01 4.89E-01 4.98E-01 5.06E-01 4.67E-01 4.29E-01 3.97E-01 3.16E-01 UB Wall-full 1.71E+00 2.59E+00 2.44E+00 1.45E+00 5.91E-01 3.33E-01 3.40E-01 3.51E-01 3.25E-01 2.95E-01 2.73E-01 2.19E-01 Uterine Cont:

Fetus

Skeleton

1.23E-06 1.17E-04 2.95E-03 2.03E-02 3.17E-02 2.40E-02 2.01E-02 1.81E-02 1.65E-02 $1.51 E-02 \quad 1.41 E-02 \quad 1.15 E-02$

1.59E-05 8.88E-04 1.54E-02 7.19E-02 8.92E-02 4.65E-02 2.76E-02 2.03E-02 1.75E-02 1.59E-02 1.47E-02 1.23E-02

Soft Tissue 5.14E-07 6.48E-05 2.01E-03 1.64E-02 2.73E-02 2.23E-02 1.95E-02 $1.79 E-02 \quad 1.64 E-02$ 1.51E-02 $1.41 E-02 \quad 1.15 E-02$ Uterine Hall 4.71E-04 7.54E-03 2.23E-02 3.87E-02 3.41E-02 2.39E-02 2.17E-02 2.07E-02 1.91 E-02 $1.76 E-02$ 1.66E-02 $1.35 E-02$ Body Tissues 1.34E-03 3.96E-03 6.56E-03 8.26E-03 7.01E-03 5.30E-03 4.96E-03 4.96E-03 4.73E-03 4.46E-03 4.21E-03 3.55E-03 
Table A.3 (cont'd). At Six Months Gestation: Specific Absorbed Fraction of Photon Energy (kg-1).

SOURCE = URINARY BLADDER WALL

Energy (MeV)

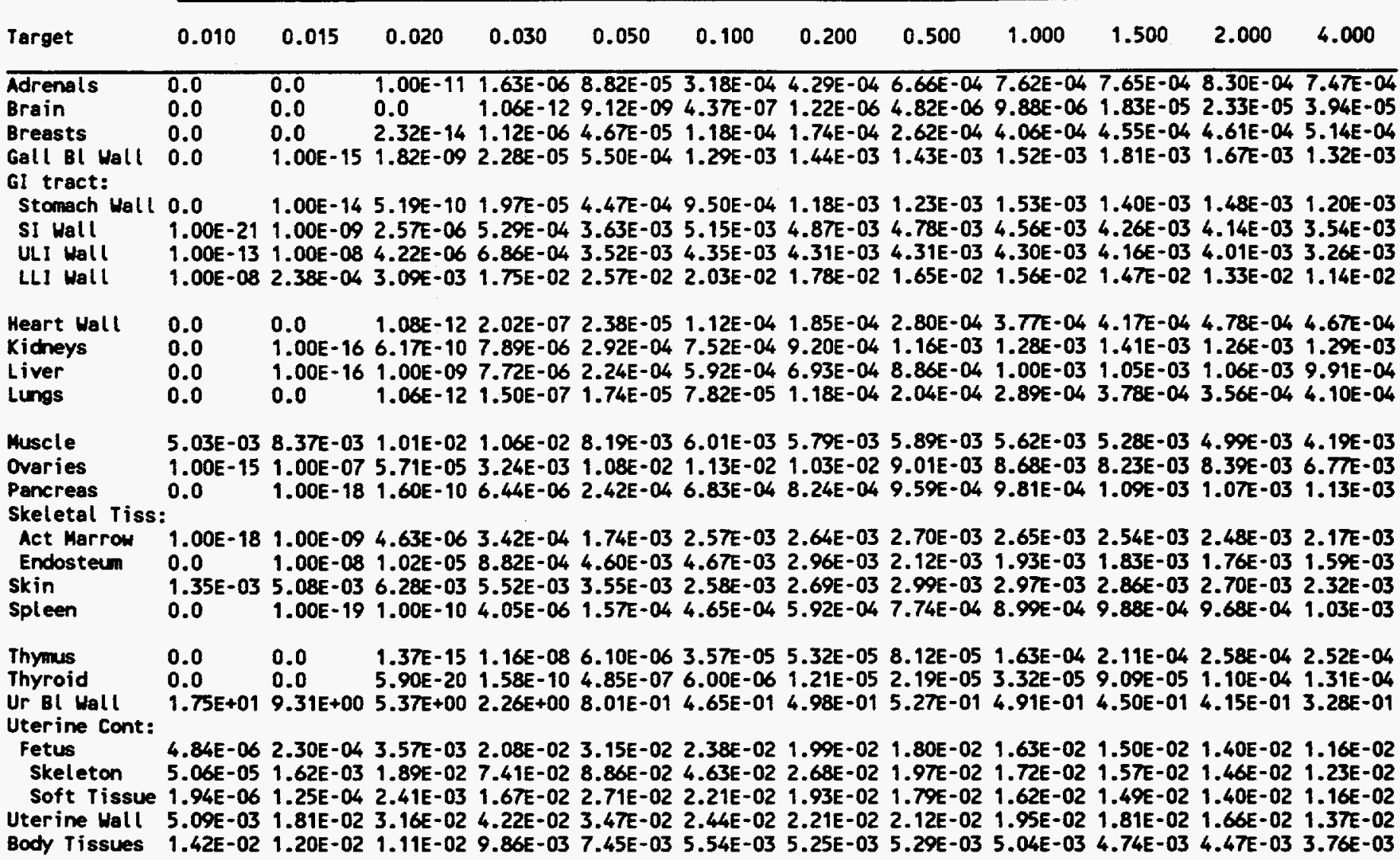

SOURCE = UTERINE CONTENTS: FETUS

\begin{tabular}{llllllllllllll}
\hline Adrenals & $3.50 E-13$ & $4.40 E-10$ & $6.97 E-08$ & $9.10 E-05$ & $1.14 E-03$ & $2.25 E-03$ & $2.42 E-03$ & $2.47 E-03$ & $2.55 E-03$ & $2.52 E-03$ & $2.35 E-03$ & $2.09 E-03$ \\
Brain & 0.0 & 0.0 & 0.0 & $2.00 E-09$ & $8.44 E-07$ & $4.23 E-06$ & $7.08 E-06$ & $1.64 E-05$ & $3.39 E-05$ & $4.52 E-05$ & $5.47 E-05$ & $7.73 E-05$ \\
Breasts & 0.0 & $1.00 E-09$ & $2.09 E-06$ & $1.46 E-04$ & $5.60 E-04$ & $7.97 E-04$ & $8.09 E-04$ & $1.00 E-03$ & $1.12 E-03$ & $1.17 E-03$ & $1.15 E-03$ & $1.08 E-03$ \\
Gall BI Wall & $7.70 E-16$ & $7.70 E-08$ & $5.14 E-05$ & $2.21 E-03$ & $8.77 E-03$ & $9.18 E-03$ & $8.41 E-03$ & $7.67 E-03$ & $7.06 E-03$ & $6.57 E-03$ & $6.18 E-03$ & $5.26 E-03$ \\
GI tract: & & & & & & & & & & & & & \\
Stomach Wall & $7.70 E-11$ & $7.70 E-07$ & $7.89 E-05$ & $2.07 E-03$ & $6.75 E-03$ & $7.46 E-03$ & $6.86 E-03$ & $6.54 E-03$ & $5.87 E-03$ & $5.48 E-03$ & $5.27 E-03$ & $4.50 E-03$ \\
SI Wall & $1.69 E-05$ & $4.83 E-04$ & $5.21 E-03$ & $2.65 E-02$ & $3.80 E-02$ & $3.01 E-02$ & $2.59 E-02$ & $2.32 E-02$ & $2.09 E-02$ & $1.91 E-02$ & $1.78 E-02$ & $1.45 E-02$ \\
ULI Wall & $3.62 E-06$ & $1.87 E-04$ & $3.07 E-03$ & $1.90 E-02$ & $2.90 E-02$ & $2.29 E-02$ & $1.96 E-02$ & $1.03 E-02$ & $1.69 E-02$ & $1.55 E-02$ & $1.45 E-02$ & $1.20 E-02$ \\
LLI Wall & $5.72 E-06$ & $2.15 E-04$ & $2.82 E-03$ & $1.70 E-02$ & $2.67 E-02$ & $2.15 E-02$ & $1.89 E-02$ & $1.68 E-02$ & $1.55 E-02$ & $1.43 E-02$ & $1.32 E-02$ & $1.09 E-02$
\end{tabular}

Heart Wall $\quad 0.0 \quad 7.70 E-14 \quad 7.70 E-09$ 2.11E-05 $4.03 E-04 \quad 8.73 E-04 \quad 1.01 E-03 \quad 1.20 E-03 \quad 1.29 E-03 \quad 1.31 E-03 \quad 1.29 E-03 \quad 1.22 E-03$

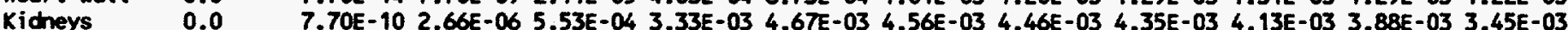

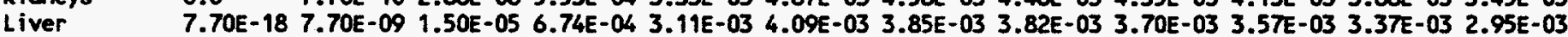
lungs $\quad 0.0 \quad 1.00 E-17$ 1.00E-09 $1.31 E-05$ 2.49E-04 5.55E-04 6.40E-04 7.73E-04 9.20E-04 9.57E-04 9.31E-04 8.95E-04

Muscle $\quad 3.82 E-07 \quad 2.28 E-05 \quad 4.15 E-04 \quad 2.82 E-03 \quad 5.31 E-03 \quad 4.97 E-03 \quad 4.55 E-03 \quad 4.36 E-03 \quad 4.12 E-03 \quad 3.91 E-03 \quad 3.69 E-03 \quad 3.17 E-03$ Ovaries $\quad 7.70 E-07$ 2.25E-04 3.98E-03 2.21E-02 3.25E-02 2.66E-02 2.24E-02 2.04E-02 $1.86 E-02$ 1.71E-02 $1.59 E-02 \quad 1.32 E-02$

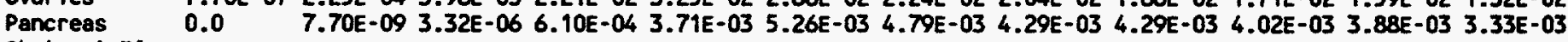
Skeletal Tiss:

Act Marrow 5.70E- 11 1.70E-07 3.66E-05 1.05E-03 3.80E-03 4.93E-03 4.82E-03 $4.59 E-03 \quad 4.36 E-03 \quad 4.12 E-03 \quad 3.89 E-03 \quad 3.33 E-03$ Endosteum 1.00E-07 5.11E-06 5.93E-05 1.8BE-03 6.84E-03 6.19E-03 3.75E-03 2.49E-03 2.18E-03 2.05E-03 $1.94 \mathrm{E}-03$ 1.74E-03 Skin

Spleen 3.10E-08 2.30E-06 2.04E-05 3.90E-04 1.16E-03 1.35E-03 $1.42 E-03 \quad 1.58 E-03 \quad 1.72 E-03 \quad 1.71 E-03 \quad 1.64 E-03 \quad 1.49 E-03$

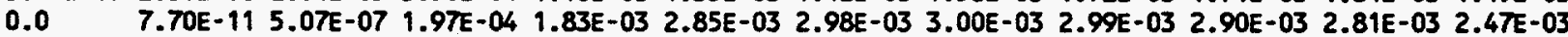

Thymus $\quad 0.0 \quad 7.70 E-13 \quad 3.65 E-09 \quad 4.98 E-06 \quad 1.07 E-04 \quad 2.67 E-04 \quad 3.28 E-04 \quad 4.31 E-04 \quad 5.17 E-04 \quad 5.59 E-04$ 5.80E-04 6.17E-04

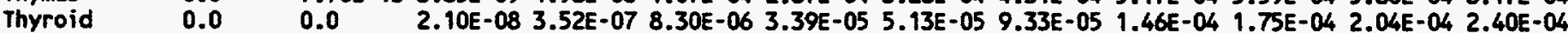
Ur BI Mall 3.70E-06 1.86E-04 2.59E-03 1.72E-02 2.71E-02 2.21E-02 1.87E-02 $1.80 E-02$ 1.63E-02 1.50 E-02 $1.40 E-02$ 1.16E-02 Uterine Cont:

Fetus

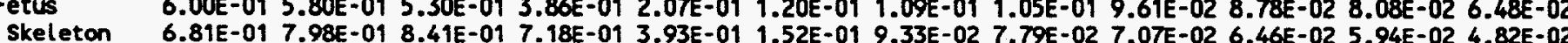

Soft Tissue 5.94E-01 5.64E-01 5.07E-01 3.61E-01 1.93E-01 1.17E-01 1.10E-01 1.08E-01 9.79E-02 8.95E-02 8.24E-02 6.60E-02 Uterine Wall 3.41E-05 4.07E-03 2.32E-02 5.97E-02 6.01E-02 4.24E-02 3.72E-02 3.43E-02 3.08E-02 2.83E-02 2.62E-02 2.13E-02 Body Tissues 3.98E-07 7.96E-05 7.02E-04 3.43E-03 5.90E-03 5.31E-03 4.70E-03 4.40E-03 4.13E-03 3.90E-03 3.69E-03 3.15E-03 
Table A.3 (cont'd). At Six Months Gestation: Specific Absorbed Fraction of Photon Energy (kg-1).

SOURCE = UTERINE CONTENTS: FETAL SKELETON

Energy (MeV)

$\begin{array}{lllllllllllll}\text { Target } & 0.010 & 0.015 & 0.020 & 0.030 & 0.050 & 0.100 & 0.200 & 0.500 & 1.000 & 1.500 & 2.000 & 4.000\end{array}$

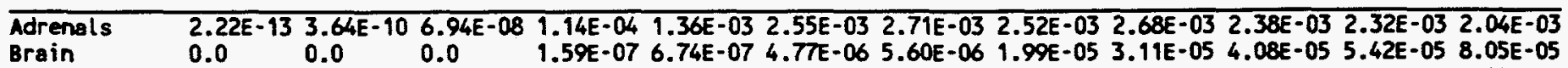

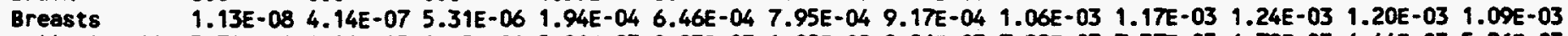

Gall Bl Wall 3.78E-07 1.01E-05 1.05E-04 2.81E-03 8.93E-03 1.02E-02 8.86E-03 7.82E-03 7.37E-03 6.78E-03 6.44E-03 5.26E-03

GI tract:

Stomach Wall 1.20E-06 2.06E-05 1.55E-04 2.66E-03 7.25E-03 7.83E-03 6.89E-03 6.53E-03 6.15E-03 5.51E-03 5.52E-03 4.61E-03 SI Wall 9.17E-05 1.54E-03 $1.13 E-02$ 3.37E-02 3.99E-02 3.07E-02 2.66E-02 2.42E-02 2.18E-02 2.02E-02 1.87E-02 1.53E-02 ULI Wall $1.71 E-05$ 5.68E-04 6.80E-03 2.53E-02 $3.16 E-02$ 2.39E-02 $2.12 E-02 \quad 1.99 E-02 \quad 1.80 E-02 \quad 1.70 E-02 \quad 1.53 E-02 \quad 1.30 E-02$ LL1 Wall 2.70E-05 6.43E-04 6.09E-03 2.19E-02 2.85E-02 2.22E-02 1.96E-02 $1.79 E-02 \quad 1.70 E-02 \quad 1.51 E-02 \quad 1.41 E-02 \quad 1.15 E-02$

Heart Wall 5.87E-08 5.49E-07 2.68E-06 2.71E-05 4.60E-04 9.39E-04 1.00E-03 1.17E-03 1.32E-03 1.34E-03 1.31E-03 1.24E-03

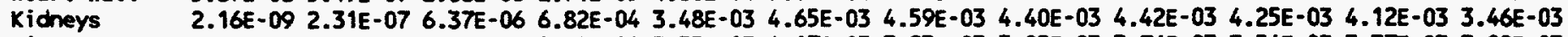
Liver $\quad 9.50 E-08$ 2.72E-06 2.94E-05 8.42E-04 3.35E-03 4.17E-03 3.93E-03 3.85E-03 3.76E-03 3.54E-03 3.37E-03 3.08E-03

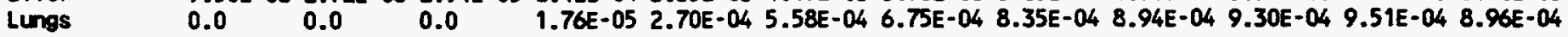

Muscle $\quad 4.01 E-06$ 9.60E-05 9.14E-04 3.72E-03 5.79E-03 5.19E-03 4.78E-03 4.61E-03 4.37E-03 4.12E-03 3.90E-03 3.31E-03 ovaries $\quad 1.43 E-05$ 6.04E-04 8.61E-03 2.99E-02 3.60E-02 2.79E-02 2.29E-02 $2.32 E-02$ 2.04E-02 $1.84 E-02 \quad 1.66 E-02 \quad 1.44 E-02$ Pancreas 1.99E-09 2.24E-07 6.42E-06 7.24E-04 3.95E-03 5.14E-03 5.23E-03 4.61E-03 4.29E-03 4.08E-03 3.99E-03 3.44E-03 Skeletal Tiss:

Act Marrow 3.41E-10 4.75E-07 8.07E-05 1.39E-03 4.20E-03 5.19E-03 5.04E-03 4.86E-03 4.54E-03 4.32E-03 4.11E-03 3.47E-03 Endosteum 4.95E-10 7.34E-07 $1.30 E-04$ 2.47E-03 7.52E-03 $6.38 E-03 \quad 3.83 E-03 \quad 2.58 E-03 \quad 2.24 E-03 \quad 2.12 E-03 \quad 2.03 E-03 \quad 1.77 E-03$ skin

Spleen 3.70E-10 3.17E-07 3.81E-05 5.08E-04 $1.26 E-03 \quad 1.42 E-03 \quad 1.48 E-03 \quad 1.69 E-03 \quad 1.72 E-03 \quad 1.69 E-03 \quad 1.68 E-03 \quad 1.50 E-03$ 6.58E-11 1.76E-08 9.30E-07 2.49E-04 1.95E-03 3.00E-03 3.01E-03 3.12E-03 3.13E-03 2.92E-03 2.80E-03 2.56E-03

Thymus $\quad 0.0 \quad 6.15 E-11 \quad 7.27 E-09$ 6.07E-06 $1.12 E-04 \quad 3.05 E-04 \quad 3.33 E-04 \quad 4.85 E-04 \quad 5.87 E-04 \quad 5.23 E-04 \quad 5.60 E-04 \quad 6.62 E-04$

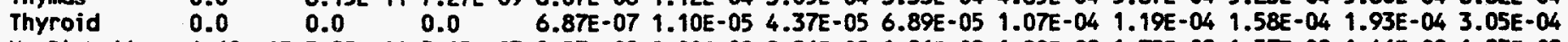
Ur Bi Wall 1.69E-05 5.22E-04 5.95E-03 2.27E-02 2.90E-02 2.26E-02 1.96E-02 1.98E-02 1.72E-02 $1.57 E-02$ 1.46E-02 $1.23 E-02$ Uterine Cont:

Fetus

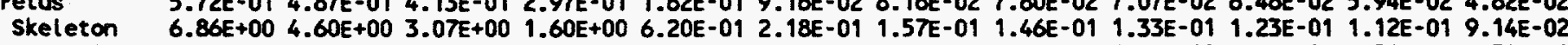
Soft Tissue 9.64E-02 1.76E-01 2.13E-01 1.99E-01 1.27E-01 8.21E-02 7.58E-02 7.28E-02 6.59E-02 6.02E-02 5.54E-02 4.50E-02 Uterine Wall 1.47E-04 1.64E-02 5.37E-02 8.24E-02 6.73E-02 4.60E-02 4.14E-02 3.93E-02 3.54E-02 3.23E-02 2.99E-02 2.43E-02 Body $T$ issues 2.21E-06 3.23E-04 1.58E-03 4.55E-03 6.45E-03 5.55E-03 4.95E-03 4.69E-03 4.40E-03 4.14E-03 3.92E-03 $3.32 E-03$

\section{SOURCE = UTERINE CONTENTS: FETAL SOFT TISSUE}

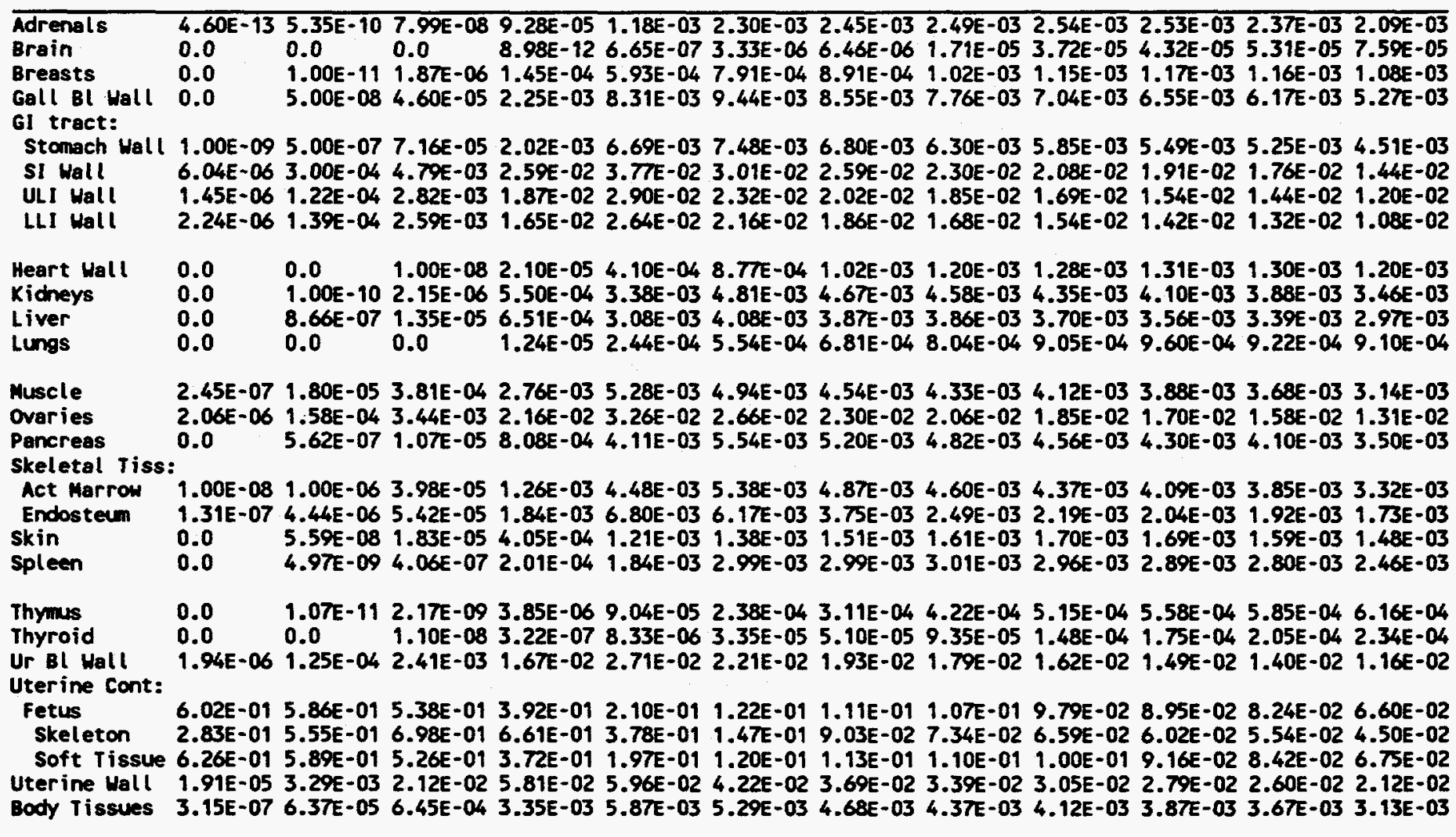


Table A.3 (cont'd). At Six Months Gestation: Specific Absorbed Fraction of Photon Energy (kg-1).

SOURCE $=$ UTERINE CONTENTS: PLACENTA

Energy (MeV)

$\begin{array}{lllllllllllll}\text { Target } & 0.010 & 0.015 & 0.020 & 0.030 & 0.050 & 0.100 & 0.200 & 0.500 & 1.000 & 1.500 & 2.000 & 4.000\end{array}$

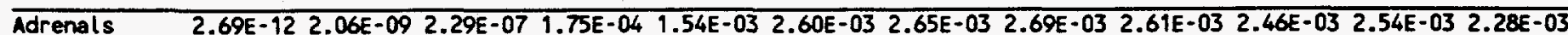

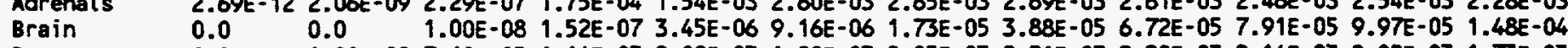

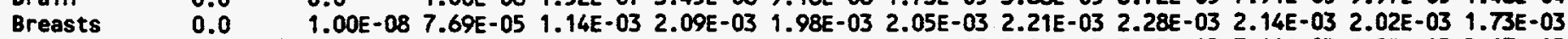

Gall BL Wall 0.0 2.05E-05 1.81E-04 3.87E-03 1.02E-02 1.02E-02 9.15E-03 8.15E-03 7.63E-03 7.14E-03 6.55E-03 5.67E-03

Gl tract:

Stomach Wall 5.88E-08 1.38E-05 6.62E-04 6.24E-03 1.21E-02 1.10E-02 9.69E-03 9.21E-03 8.53E-03 8.23E-03 7.64E-03 6.31E-03 SI Wall $1.67 E-051.11 E-03 \quad 5.06 E-03 \quad 1.53 E-02 \quad 2.17 E-02 \quad 1.83 E-02 \quad 1.60 E-02 \quad 1.47 E-02 \quad 1.32 E-02 \quad 1.21 E-02 \quad 1.14 E-02 \quad 9.51 E-03$ ULI Hall 1.76E-04 1.28E-02 4.77E-02 8.21E-02 6.49E-02 4.23E-02 3.84E-02 3.66E-02 3.33E-02 3.08E-02 2.86E-02 2.37E-02

LLI Mall 1.03E-04 1.13E-03 6.17E-03 1.58E-02 $1.77 E-02 \quad 1.39 E-02 \quad 1.23 E-02 \quad 1.16 E-02 \quad 1.08 E-02 \quad 1.02 E-02$ 9.64E-03 $7.77 E-03$

Heart Wall $\quad 0.0 \quad 0.0 \quad 3.17 E-07 \quad 1.17 E-04 \quad 1.04 E-03 \quad 1.64 E-03 \quad 1.71 E-03 \quad 1.89 E-03 \quad 1.97 E-03 \quad 1.94 E-03 \quad 1.89 E-03 \quad 1.84 E-03$ Kicheys $\quad 7.30 E-11 \quad 2.19 E-08$ 1.26E-06 3.78E-04 2.48E-03 3.54E-03 3.56E-03 3.52E-03 3.54E-03 3.30E-03 3.19E-03 2.76E-03

Liver 1.11E-08 2.70E-06 1.74E-04 2.19E-03 5.64E-03 5.86E-03 5.43E-03 5.31E-03 5.18E-03 4.86E-03 4.64E-03 4.00E-03

Lungs

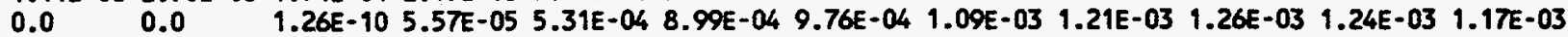

Muscle

Ovaries

Pancreas

Skeletal Tiss:

Act Marrow

Endosteum

Skin

1.57E-05 8.67E-04 2.95E-03 5.52E-03 5.53E-03 4.40E-03 4.16E-03 4.16E-03 3.98E-03 3.77E-03 3.57E-03 3.05E-03 2.13E-09 2.57E-07 7.70E-06 9.29E-04 4.90E-03 6.32E-03 6.00E-03 5.67E-03 5.27E-03 5.10E-03 4.90E-03 4.08E-03 1.68E-08 1.11E-06 2.17E-05 1.44E-03 5.68E-03 6.51E-03 5.91E-03 5.57E-03 5.33E-03 5.13E-03 4.64E-03 4.31E-03

Spleen

3.26E-09 1.69E-07 2.77E-06 1.43E-04 9.80E-04 1.76E-03 1.93E-03 2.05E-03 2.05E-03 2.06E-03 1.96E-03 1.80E-03 1.30E-08 5.93E-07 8.90E-06 4.05E-04 2.35E-03 2.80E-03 $1.90 E-03 \quad 1.38 E-03 \quad 1.26 E-03 \quad 1.25 E-03 \quad 1.21 E-031.14 E-03$ 2.00E-06 4.66E-05 4.35E-04 1.52E-03 1.79E-03 1.57E-03 1.63E-03 1.88E-03 1.88E-03 1.85E-03 1.76E-03 1.55E-03

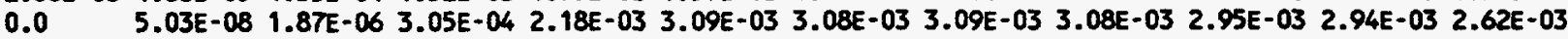

Thymus $\quad 0.0 \quad 3.13 E-09$ 1.68E-07 4.61E-05 3.57E-04 5.68E-04 6.56E-04 8.53E-04 9.99E-04 1.01E-03 1.03E-03 9.36E-04 Thyroid $\quad 0.0 \quad 0.0 \quad 1.00 E-07 \quad 4.31 E-06 \quad 3.44 E-05$ 9.33E-05 $1.21 E-04 \quad 1.65 E-04 \quad 2.66 E-04 \quad 3.22 E-04 \quad 3.46 E-04 \quad 3.32 E-04$ Ur Bl Wall 5.99E-08 2.67E-06 3.96E-05 1.77E-03 6.24E-03 6.82E-03 6.48E-03 6.45E-03 6.24E-03 5.94E-03 5.65E-03 4.66E-03 Uterine Cont:

Fetus

6.42E-03 3.14E-02 6.85E-02 1.03E-01 8.19E-02 5.07E-02 4.33E-02 4.02E-02 3.60E-02 3.30E-02 3.07E-02 2.50E-02 Skeleton 5.18E-02 2.52E-01 4.01E-01 4.19E-01 2.51E-01 1.00E-01 6.14E-02 5.11E-02 4.60E-02 4.17E-02 3.80E-02 3.08E-02

Soft Tissue 2.99E-03 1.48E-02 4.34E-02 7.94E-02 6.90E-02 4.69E-02 4.19E-02 3.94E-02 3.55E-02 3.24E-02 3.02E-02 2.44E-02 Uterine Hall 7.28E-02 1.73E-01 1.98E-01 1.49E-01 8.17E-02 5.15E-02 4.88E-02 4.77E-02 4.41E-02 4.03E-02 3.74E-02 3.01E-02 Body Tissues 1.07E-03 3.18E-03 5.19E-03 6.67E-03 6.03E-03 4.74E-03 4.42E-03 $4.35 E-03 \quad 4.15 E-03$ 3.92E-03 3.70E-03 3.15E-03

SOURCE = UTERINE CONTENTS: OTHER UTERINE CONTENTS

\begin{tabular}{lllllllllllll}
\hline Adrenals & $5.25 E-12$ & $2.62 E-09$ & $2.15 E-07$ & $1.07 E-04$ & $1.07 E-03$ & $2.01 E-03$ & $2.15 E-03$ & $2.21 E-03$ & $2.27 E-03$ & $2.27 E-03$ & $2.18 E-03$ & $1.89 E-03$ \\
Brain & 0.0 & 0.0 & 0.0 & $3.94 E-12$ & $5.10 E-08$ & $2.82 E-06$ & $3.87 E-06$ & $1.67 E-05$ & $2.76 E-05$ & $3.68 E-05$ & $4.61 E-05$ & $7.42 E-05$ \\
Breasts & 0.0 & $1.00 E-10$ & $7.75 E-06$ & $1.93 E-04$ & $5.09 E-04$ & $5.94 E-04$ & $6.13 E-04$ & $8.07 E-04$ & $8.90 E-04$ & $8.93 E-04$ & $8.83 E-04$ & $8.64 E-04$ \\
Gall BI Wall & $7.30 E-07$ & $1.50 E-05$ & $1.27 E-04$ & $2.61 E-03$ & $7.39 E-03$ & $8.11 E-03$ & $7.40 E-03$ & $6.78 E-03$ & $6.16 E-03$ & $5.91 E-03$ & $5.54 E-03$ & $4.78 E-03$ \\
GI tract: & & & & & & & & & & & & \\
Stomach Hall & $2.76 E-08$ & $4.21 E-06$ & $1.49 E-04$ & $2.03 E-03$ & $5.45 E-03$ & $5.97 E-03$ & $5.46 E-03$ & $5.19 E-03$ & $4.91 E-03$ & $4.56 E-03$ & $4.46 E-03$ & $3.68 E-03$ \\
SI Wall & $1.24 E-04$ & $8.40 E-03$ & $2.69 E-02$ & $4.82 E-02$ & $4.40 E-02$ & $3.34 E-02$ & $2.84 E-02$ & $2.57 E-02$ & $2.40 E-02$ & $2.20 E-02$ & $1.95 E-02$ & $1.56 E-02$ \\
ULI Wall & $2.00 E-05$ & $1.78 E-03$ & $9.31 E-03$ & $2.27 E-02$ & $2.47 E-02$ & $1.92 E-02$ & $1.71 E-02$ & $1.50 E-02$ & $1.45 E-02$ & $1.35 E-02$ & $1.26 E-02$ & $1.01 E-02$ \\
LLI Wall & $1.86 E-05$ & $2.44 E-03$ & $1.25 E-02$ & $3.06 E-02$ & $3.35 E-02$ & $2.00 E-02$ & $2.21 E-02$ & $2.05 E-02$ & $1.84 E-02$ & $1.71 E-02$ & $1.57 E-02$ & $1.28 E-02$
\end{tabular}

Heart Wall $\quad 0.0 \quad 0.0 \quad 5.00 E-08 \quad 2.43 E-05 \quad 3.43 E-04 \quad 7.36 E-04 \quad 8.48 E-04 \quad 9.93 E-04 \quad 1.06 E-03 \quad 1.15 E-03 \quad 1.14 E-03 \quad 1.08 E-03$ Kidneys Liver

Lungs

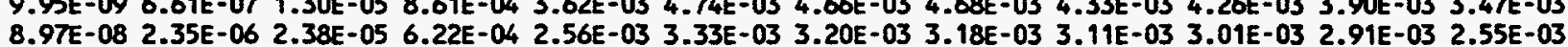
$\begin{array}{lllllll}0.0 & 0.0 & 0.0 & 1.32 E-05 & 1.91 E-04 & 4.51 E-04 & 5.44 E-04 \\ 6.84 E-04 & 8.05 E-04 & 8.60 E-04 & 8.67 E-04 & 7.91 E-04\end{array}$

Muscle $\quad 1.19 E-05$ 7.07E-04 2.60E-03 5.83E-03 7.09E-03 5.99E-03 5.51E-03 5.32E-03 4.98E-03 4.68E-03 4.43E-03 3.74E-03 Ovaries $\quad 6.00 E-05$ 5.31E-03 2.82E-02 5.78E-02 5.22E-02 3.72E-02 3.29E-02 3.02E-02 2.73E-02 2.49E-02 2.31E-02 1.88E-02 Pancreas 3.62E-09 3.08E-07 7.19E-06 6.12E-04 3.14E-03 4.30E-03 4.08E-03 3.88E-03 3.74E-03 3.56E-03 3.39E-03 2.99E-03 Skeletal Tiss:

Act Marrow 8.94E-08 1.16E-05 3.65E-04 3.21E-03 6.89E-03 7.44E-03 6.91E-03 6.59E-03 6.17E-03 5.67E-03 5.33E-03 4.46E-03 Endosteum 1.18E-07 1.71E-05 5.84E-04 5.60E-03 1.19E-02 8.58E-03 4.98E-03 3.39E-03 2.91E-03 2.69E-03 2.56E-03 2.23E-03 skin Spleen 9.71E-10 5.26E-07 4.58E-05 5.46E-04 1.40E-03 1.55E-03 1.61E-03 1.81E-03 1.83E-03 1.74E-03 $1.76 E-031.54 E-03$

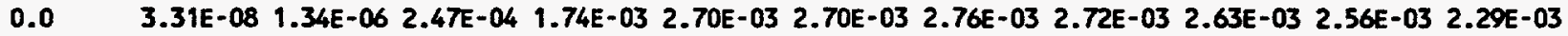

Thymus $\quad 0.0 \quad 4.68 E-10 \quad 2.72 E-08$ 8.34E-06 8.99E-05 $1.98 E-04 \quad 2.76 E-04 \quad 4.03 E-04 \quad 4.68 E-04 \quad 5.05 E-04 \quad 5.42 E-04 \quad 5.37 E-04$

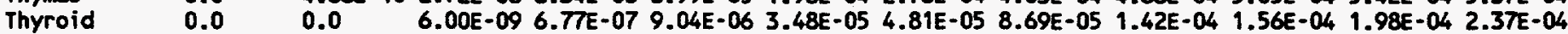
Ur BI Wall 4.77E-05 3.91E-03 1.62E-02 3.69E-02 3.73E-02 2.70E-02 2.44E-02 2.26E-02 2.08E-02 $1.89 E-02$ 1.76E-02 $1.46 E-02$ Uterine Cont:

Fetus

1.80E-02 5.99E-02 1.05E-01 1.38E-01 1.04E-01 6.42E-02 5.48E-02 5.03E-02 4.53E-02 4.13E-02 3.82E-02 3.08E-02 skeleton 1.41E-01 4.79E-01 6.21E-01 5.53E-01 3.11E-01 1.24E-01 7.76E-02 6.44E-02 5.91E-02 5.38E-02 4.99E-02 4.00E-02

Soft Tissue 9.46E-03 3.06E-02 6.75E-02 1.08E-01 8.78E-02 5.92E-02 5.29E-02 4.92E-02 4.44E-02 4.04E-02 3.72E-02 3.03E-02 Uterine Hall 6.05E-02 1.51E-01 1.77E-01 1.39E-01 8.22E-02 5.19E-02 4.89E-02 4.78E-02 4.29E-02 4.01E-02 3.66E-02 2.92E-02 Body Tissues 1.04E-03 3.06E-03 5.07E-03 7.50E-03 8.06E-03 6.46E-03 5.73E-03 5.43E-03 5.05E-03 4.73E-03 4.47E-03 3.76E-03 
Table A.3 (cont'd). At Six Months Gestation: Specific Absorbed Fraction of Photon Energy (kg $\left.{ }^{-1}\right)$.

SOURCE = UTERINE WALL

Energy (MeV)

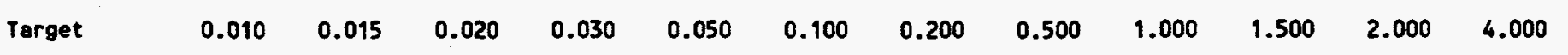

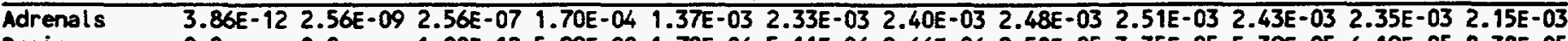

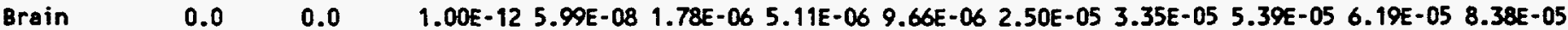
Breasts $\quad 1.00 E-13 \quad 1.00 E-08$ 5.68E-05 5.33E-04 9.63E-04 9.97E-04 $1.04 E-03 \quad 1.22 E-03 \quad 1.33 E-03 \quad 1.30 E-03 \quad 1.28 E-03 \quad 1.13 E-03$ Gall Bl Wall 1.00E-07 4.92E-05 3.12E-04 4.21E-03 9.50E-03 9.33E-03 8.40E-03 7.71E-03 7.08E-03 6.60E-03 6.16E-03 5.36E-03 G1 tract:

stomach Wall 1.45E-07 1.90E-05 6.04E-04 4.37E-03 8.22E-03 7.74E-03 6.97E-03 6.55E-03 6.30E-03 5.89E-03 5.53E-03 4.76E-03 S1 Wall 1.28E-02 3.52E-02 5.16E-02 5.76E-02 4.43E-02 3.14E-02 2.81E-02 2.63E-02 2.38E-02 2.19E-02 2.02E-02 1.66E-02 ULI Wall 6.71E-03 2.86E-02 4.89E-02 5.67E-02 4.15E-02 2.81E-02 2.58E-02 2.47E-02 2.28E-02 2.08E-02 $1.93 E-02$ 1.59E-02 LLI Wall 1.82E-03 1.24E-02 2.67E-02 3.95E-02 3.40E-02 2.42E-02 2.17E-02 2.03E-02 1.87E-02 1.70E-02 1.60E-02 1.31E-02

Heart Hall $\quad 0.0 \quad 1.00 E-11 \quad 5.12 E-07 \quad 6.34 E-05 \quad 5.85 E-04 \quad 1.01 E-03 \quad 1.09 E-03 \quad 1.23 E-03 \quad 1.37 E-03 \quad 1.39 E-03 \quad 1.43 E-03 \quad 1.30 E-03$ Kidneys $\quad 4.00 E-11$ 1.00E-07 2.00E-05 9.63E-04 3.78E-03 4.79E-03 4.55E-03 $4.49 E-03$ 4.31E-03 4.15E-03 3.98E-03 3.43E-03 Liver $\quad 7.51 E-08$ 5.97E-06 $1.33 E-04$ 1.44E-03 3.84E-03 4.26E-03 4.00E-03 3.95E-03 3.85E-03 3.71E-03 3.55E-03 3.07E-03 Lungs $\quad 0.0 \quad 1.12 E-13$ 5.60E-08 3.28E-05 3.35E-04 6.12E-04 6.91E-04 8.38E-04 9.41E-04 9.60E-04 9.97E-04 9.29E-04

Muscle $\quad 1.60 E-03 \quad 4.25 E-03$ 6.40E-03 8.04E-03 7.39 -03 5.83E-03 5.46E-03 5.37E-03 5.06E-03 4.75E-03 4.51E-03 3.78E-03 Overies $\quad 1.18 E-03$ 2.20E-02 5.10E-02 6.33E-02 4.66E-02 $3.25 E-02$ 2.93E-02 2.75E-02 2.49E-02 2.28E-02 2.13E-02 1.73E-02 Pancreas $\quad 5.00 E-11$ 1.00E-07 2.17E-05 1.12E-03 4.35E-03 5.20E-03 4.83E-03 4.54E-03 4.44E-03 4.16E-03 4.03E-03 3.41E-03 Skeletal Tiss:

Act Marrow 1.00E-08 2.77E-05 5.24E-04 3.35E-03 6.21E-03 6.17E-03 5.69E-03 5.44E-03 5.20E-03 4.77F-03 4.50E-03 3.86E-03 Endosteum 5.83E-07 3.80E-05 7.35E-04 5.01E-03 9.54E-03 6.83E-03 4.05E-03 2.79E-03 2.49E-03 2.31E-03 2.20E-03 1.90E-03 Skin

Spleen 1.00E-06 5.38E-05 3.42E-04 $1.14 E-03 \quad 1.69 E-03 \quad 1.64 E-03 \quad 1.74 E-03 \quad 1.90 E-03 \quad 1.92 E-03 \quad 1.89 E-03 \quad 1.89 E-03 \quad 1.61 E-03$ 8.00E-10 8.69E-08 2.77E-06 3.65E-04 2.12E-03 3.00E-03 2.94E-03 3.01E-03 2.98E-03 2.86E-03 2.74E-03 2.47E-03

Thymus $\quad 0.0 \quad 1.00 E-13$ 1.00E-07 2.47E-05 1.73E-04 3.13E-04 3.91E-04 5.15E-04 6.14E-04 6.53E-04 6.70E-04 6.62E-04 Thyroid 3.00E-10 1.00E-08 1.01E-07 2.20E-06 1.61E-05 4.53E-05 6.92E-05 1.20E-04 1.77E-04 2.29E-04 2.22E-04 2.74E-04 Ur bl Wall 5.09E-03 1.81E-02 3.16E-02 4.22E-02 3.47E-02 2.44E-02 2.21E-02 2.12E-02 1.95E-02 1.81E-02 1.66E-02 1.37E-02 Uterine Cont:

Fetus

Skeleton

Soft Tissue

Uterine Wall

Body Tissues

3.96E-05 6.76E-03 3.19E-02 7.31E-02 6.96E-02 4.53E-02 3.80E-02 3.44E-02 3.08E-02 2.83E-02 2.62E-02 2.13E-02 4.11E-04 5.24E-02 1.75E-01 2.73E-01 2.01E-01 8.67E-02 5.17E-02 4.04E-02 3.54E-02 3.23E-02 2.99E-02 2.43E-02 1.91E-05 3.29E-03 2.12E-02 5.81E-02 5.96E-02 4.22E-02 3.69E-02 3.39E-02 3.05E-02 2.79E-02 2.60E-02 2.12E-02 1.03E+00 7.30E-01 4.85E-01 2.41E-01 1.08E-01 6.71E-02 6.66E-02 6.71E-02 6.22E-02 5.68E-02 5.26E-02 4.23E-02 1.62E-02 1.41E-02 1.24E-02 1.06E-02 8.47E-03 6.39E-03 5.87E-03 5.71E-03 5.36E-03 5.01E-03 4.74E-03 3.96E-03

SOURCE $=$ BODY TISSUES

\begin{tabular}{|c|c|}
\hline $\begin{array}{l}\text { Adrenals } \\
\text { Brain } \\
\text { Breasts } \\
\text { Gall Bl Woll } \\
\text { GI tract: } \\
\text { Stomach Wall } \\
\text { SI Wall } \\
\text { ULI Wall } \\
\text { LLI Wall }\end{array}$ & 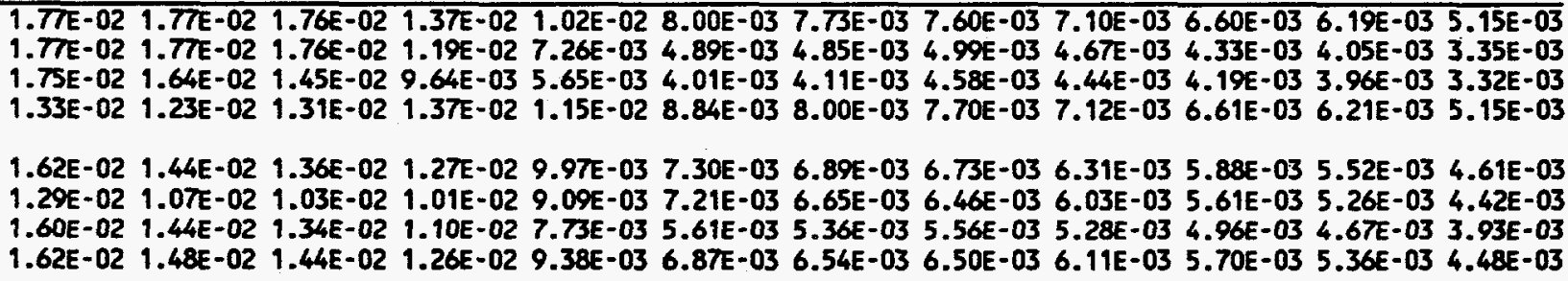 \\
\hline $\begin{array}{l}\text { Heart Wall } \\
\text { Kidneys } \\
\text { Liver } \\
\text { Lungs }\end{array}$ & 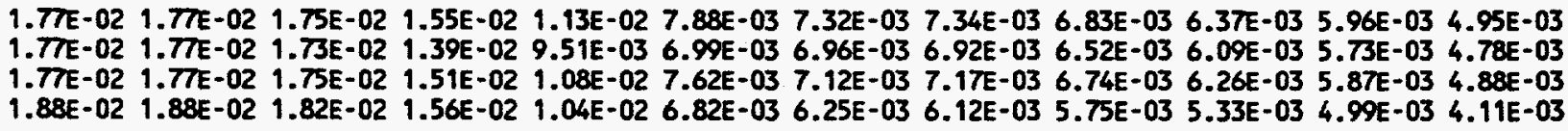 \\
\hline $\begin{array}{l}\text { Muscle } \\
\text { Ovaries } \\
\text { Pancreas } \\
\text { Skeletal Tiss: } \\
\text { Act Marrow } \\
\text { Endosteum } \\
\text { Skin } \\
\text { Spleen }\end{array}$ & 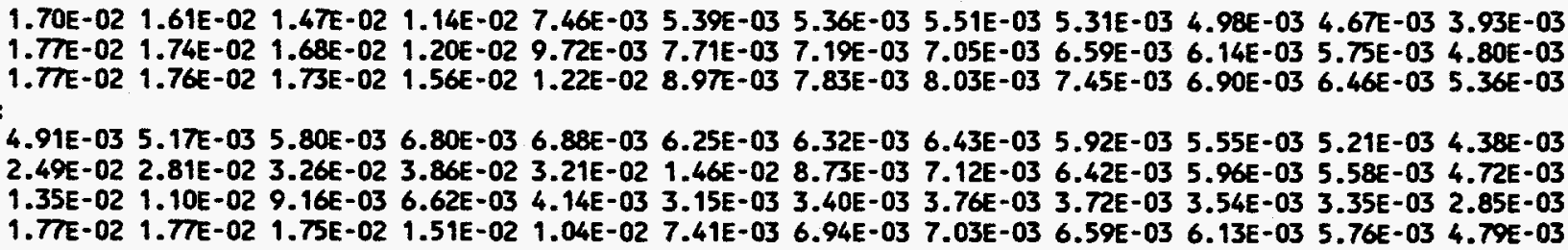 \\
\hline $\begin{array}{l}\text { Thymus } \\
\text { Thyroid } \\
\text { Ur Bl Hall } \\
\text { Uterine Cont: } \\
\text { Fetus } \\
\text { Skeleton } \\
\text { Soft Tissue } \\
\text { Uterine Wall } \\
\text { Body Tissues }\end{array}$ & 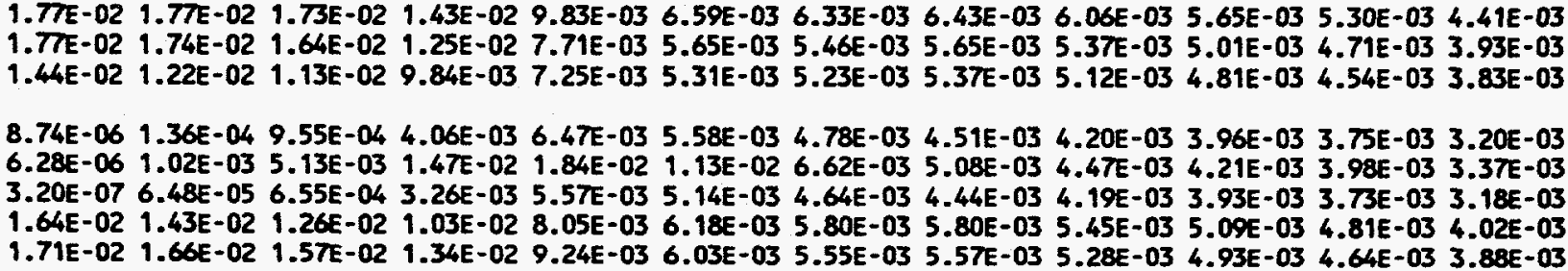 \\
\hline
\end{tabular}



Notes on Table A.4. At Nine Months Gestation: Specific Absorbed Fraction of Photon Energy ( $\left.\mathrm{kg}^{-1}\right)$

Body Tissues. Values of $\Phi$ are given for "Body Tissues" as a source or target region. This region replaces "Whole Body" or "Total Body" given in previous publications (e.g., Cristy and Eckerman 1987) and is defined as the living maternal body tissues, i.e., it excludes the contents of the GI tract, the contents of the urinary and gall bladders, and (for the pregnant woman) the contents of the uterus.

Urinary Bladder. Two rows of $\Phi$ s are given when source $=$ urinary bladder contents and target $=$ urinary bladder wall, labeled "mid" and "full" and designating that the bladder contents are in mid-cycle (half-full) or full.

Muscle. Values of $\Phi$ for muscle are from the Remaining Tissue compartment of each phantom (that part of the maternal tissues remaining after all defined organs are removed).

Cortical and Trabecular Bone. Values of $\Phi$ for source $=$ Cortical Bone, surface or volume distributed, are not given, but source $=$ skeleton may be used for this purpose. Similarly, $\Phi$ s for source $=$ Trabecular Bone, surface or volume distributed, are not given, but source $=$ Red Marrow may be used.

Alphabetical Ordering of Organs. The source and target organs are arranged mostly in alphabetical order. Parts of the GI tract appear together, alphabetized under the heading "GI Tract," but under this heading they appear in natural order from mouth to anus. Active marrow, endosteum (called "bone surface" by the ICRP), and skeleton appear under "Skeletal Tissues"; and (at six and nine months of gestation) fetus, fetal skeleton, fetal soft tissue, placenta, and other uterine contents appear under "Uterine Contents." Body Tissues appears at the end of the tables rather than in alphabetical order. Note that some of these organs appear only as source organs (i.e., skeleton, placenta, and other uterine contents), and endosteum appears only as a target organ. Note also that as target tissues fetal skeleton and fetal soft tissue (at six and nine months of gestation) are indented under "Fetus" and are listed as "Skeleton" and "Soft Tissue" for brevity of space.

Abbreviations. The meanings of the abbreviations used for target regions in Tables A.1 - A.4 are given in the following table. Abbreviations are not used for source regions.

\begin{tabular}{||l|l|}
\hline \multicolumn{2}{|c|}{ Abbreviations used in the Tables for Target Regions } \\
\hline Abbreviation & Meaning \\
\hline Act Marrow & Active Marrow \\
Gall Bl & Gall Bladder \\
GI Tract & Gastrointestinal Tract \\
SI & Small Intestine \\
ULI & Upper Large Intestine \\
LLI & Lower Large Intestine \\
Skeletal Tiss & Skeletal Tissues \\
Urin Bl & Urinary Bladder \\
Uterine Cont & Uterine Contents \\
\hline
\end{tabular}


Table A.4. At Nine Months Gestation: Specific Absorbed Fraction of Photon Energy $\left(\mathrm{kg}^{-1}\right)$.

SOURCE $=$ ADRENALS

Energy (MeV)

$\begin{array}{lllllllllllll}\text { Target } & 0.010 & 0.015 & 0.020 & 0.030 & 0.050 & 0.100 & 0.200 & 0.500 & 1.000 & 1.500 & 2.000 & 4.000\end{array}$

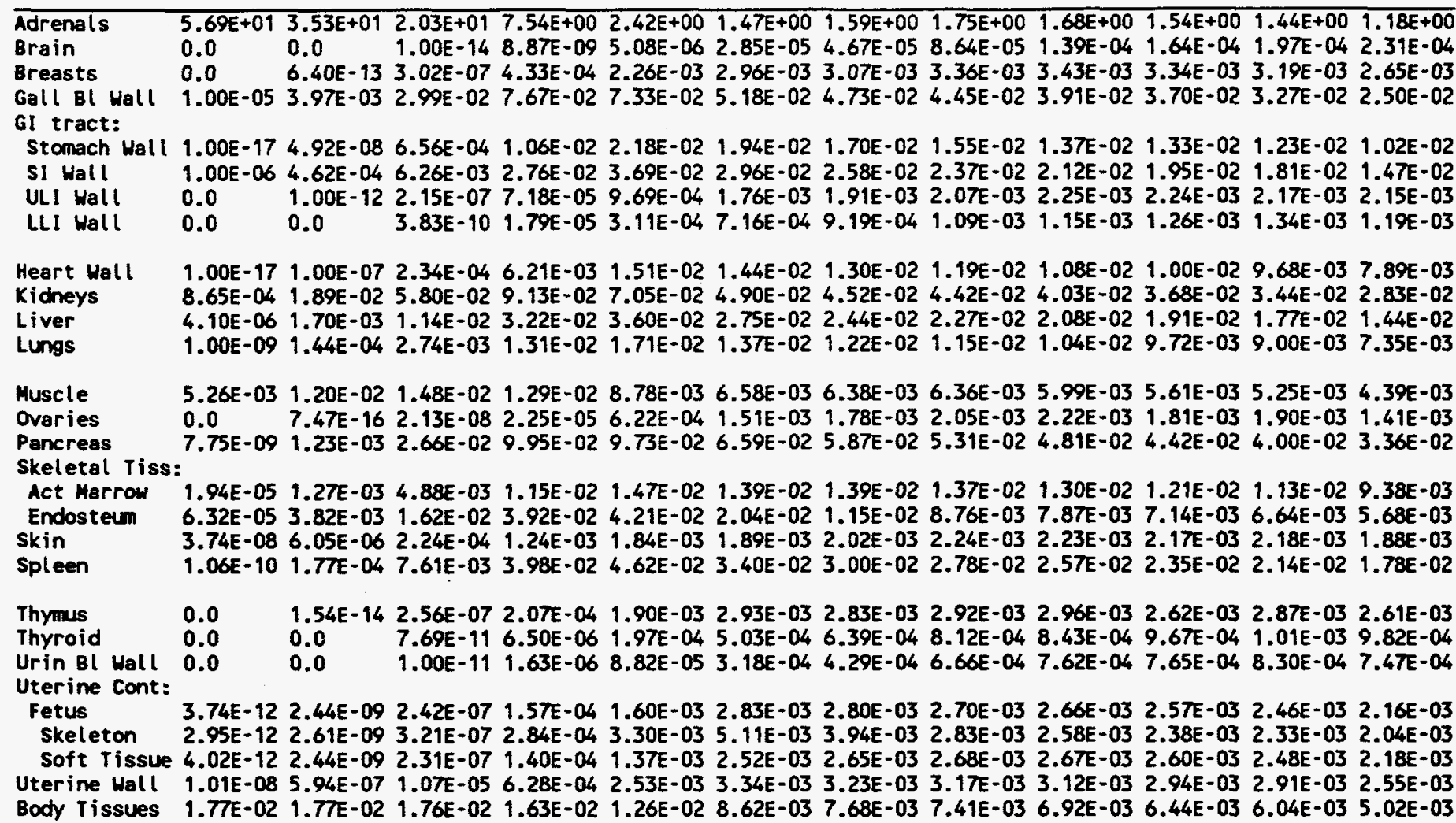

SOURCE = BRAIN

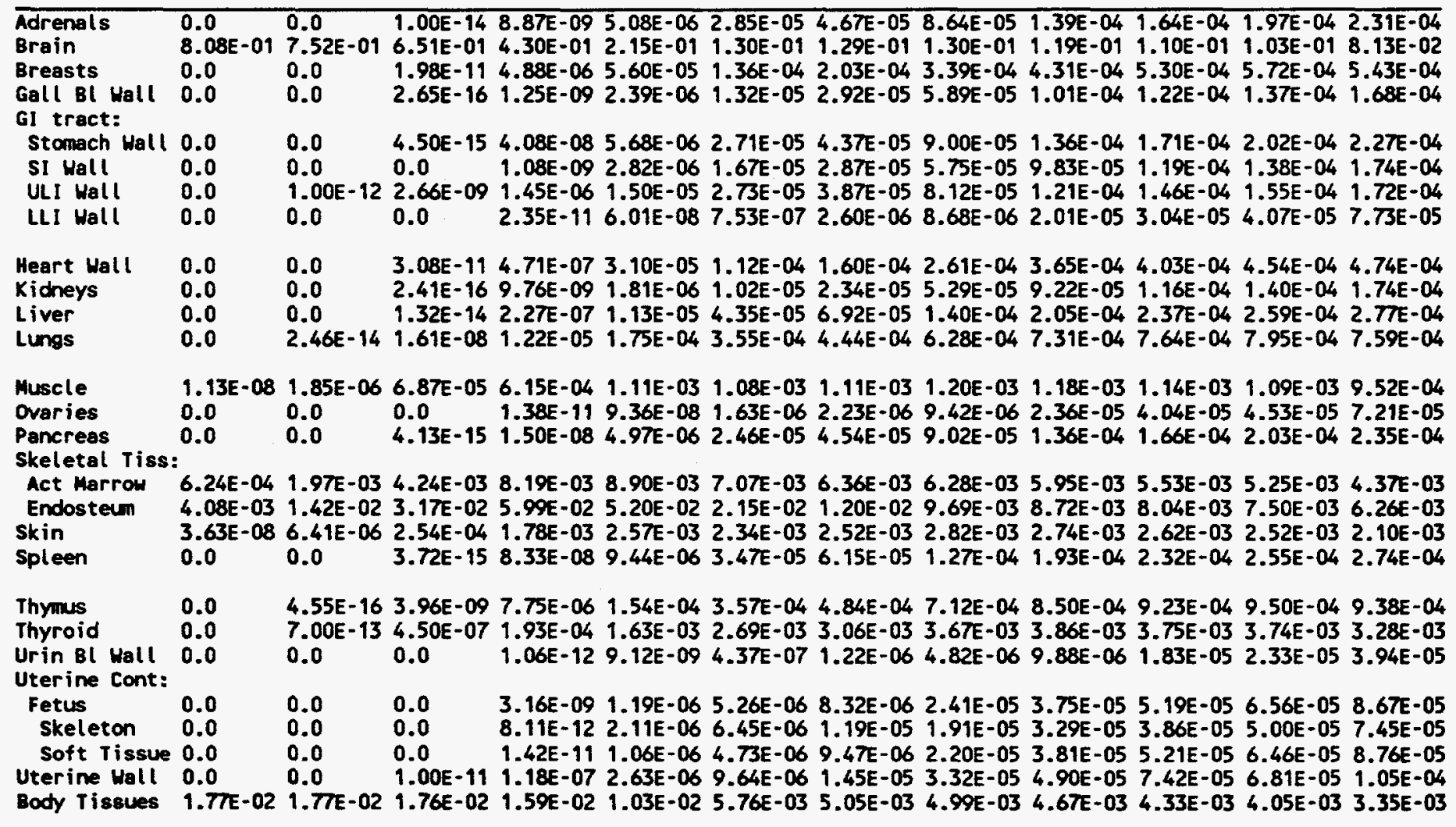


Table A.4 (cont'd). At Nine Months Gestation: Specific Absorbed Fraction of Photon Energy ( $\left.\mathbf{k g}^{-1}\right)$.

SOURCE $=$ BREASTS

Energy (MeV)

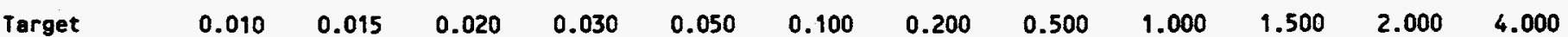

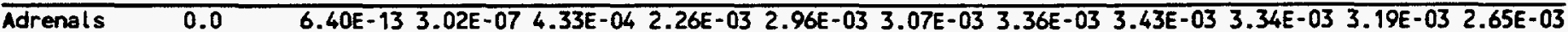

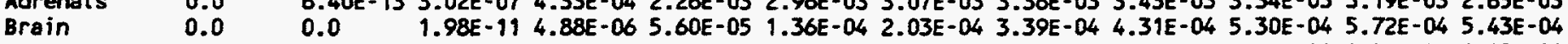

Breasts $\quad 2.63 E+00 \quad 2.27 E+001.72 E+00$ 8.80E-01 3.37E-01 1.97E-01 2.09E-01 2.24E-01 2.13E-01 $1.94 E-01$ 1.78E-01 $1.42 E-01$

Gall Bl Hall $0.0 \quad 3.48 E-12$ 5.96E-07 2.44E-04 1.71E-03 2.40E-03 2.49E-03 2.62E-03 2.78E-03 2.62E-03 2.55E-03 2.39E-03

GI tract:

Stomach Wall 1.00E-11 1.00E-06 1.13E-04 2.15E-03 5.15E-03 5.05E-03 4.91E-03 5.16E-03 5.18E-03 5.03E-03 4.70E-03 4.29E-03 SI Wall 4.27E-09 5.89E-07 1.94E-05 5.19E-04 2.34E-03 2.94E-03 2.92E-03 3.10E-03 3.07E-03 3.05E-03 2.96E-03 2.59E-03 ULI Hall 7.46E-06 1.36E-04 1.06E-03 3.61E-03 3.70E-03 2.82E-03 2.84E-03 3.10E-03 3.01E-03 2.86E-03 2.54E-03 2.21E-03 LLI Wall $\quad 0.0 \quad 1.06 E-05$ 3.63E-05 2.05E-04 3.47E-04 4.03E-04 4.65E-04 6.21E-04 7.59E-04 7.25E-04 7.43E-04 7.08E-04

Heart Wall 2.38E-06 $1.52 E-04 \quad 2.91 E-03 \quad 1.82 E-02 \quad 2.48 E-02 \quad 1.87 E-02 \quad 1.79 E-02 \quad 1.81 E-02 \quad 1.74 E-02 \quad 1.60 E-02 \quad 1.52 E-02 \quad 1.28 E-02$

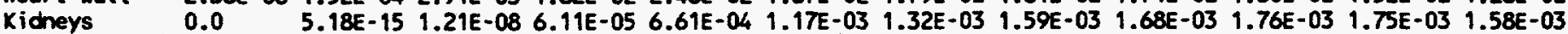

Liver 1.00E-09 1.00E-06 1.16E-04 2.34E-03 5.63E-03 5.33E-03 5.13E-03 5.43E-03 5.28E-03 5.07E-03 4.86E-03 4.14E-03

Lungs 5.73E-06 2.80E-04 4.41E-03 1.86E-02 1.95E-02 1.33E-02 1.24E-02 1.24E-02 1.17E-02 1.08E-02 1.02E-02 8.31E-03

Muscle

Ovaries

Pancreas

4.46E-04 1.39E-03 2.52E-03 3.80E-03 3.55E-03 2.76E-03 2.78E-03 2.98E-03 2.92E-03 2.79E-03 2.66E-03 2.27E-03 Skeletal Tiss:

Act Marrow

Endosteum

Skin

Spleen

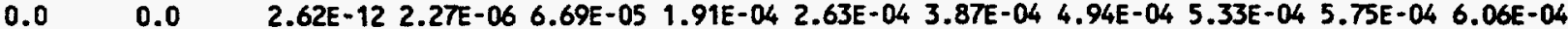
$0.0 \quad 7.70 E-11$ 2.80E-06 7.19E-04 3.06E-03 3.79E-03 3.66E-03 3.98E-03 4.05E-03 3.93E-03 3.79E-03 3.23E-03

Thymus

Thyroid

1.42E-05 4.35E-04 1.55E-03 2.98E-03 3.11E-03 2.88E-03 3.02E-03 3.31E-03 3.32E-03 3.23E-03 3.09E-03 2.68E-03

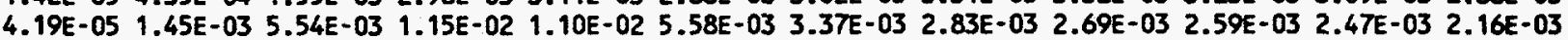
1.28E-02 2.05E-02 2.12E-02 1.44E-02 6.67E-03 4.33E-03 4.70E-03 5.18E-03 4.99E-03 4.67E-03 4.33E-03 3.59E-03

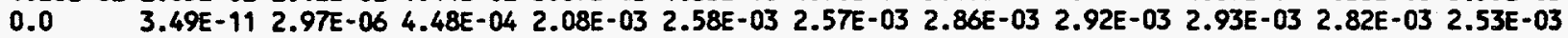

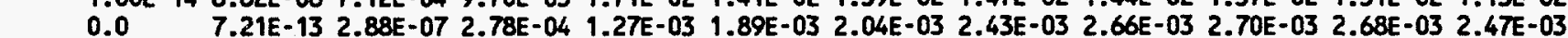

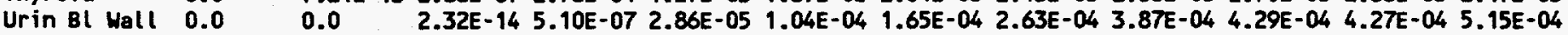
Uterine Cont:

Fetus $\quad 0.0$

Skeleton 0.0

Soft Tissue 0.0

1.60E-09 3.16E-06 1.77E-04 8.13E-04 1.10E-03 1.15E-03 1.31E-03 1.34E-03 1.32E-03 1.33E-03 1.20E-03 1.00E-11 2.59E-06 2.58E-04 $1.41 E-03 \quad 1.63 E-03 \quad 1.30 E-03 \quad 1.14 E-03$ 1.09E-03 $1.13 E-03 \quad 1.14 E-03 \quad 9.88 E-04$ 1.00E-10 3.26E-06 1.66E-04 7.22E-04 1.02E-03 1.12E-03 $1.32 E-03 \quad 1.38 E-03 \quad 1.34 E-03 \quad 1.35 E-03 \quad 1.21 E-03$ Uterine Wall 1.00E-13 1.00E-08 4.06E-05 5.71E-04 1.21E-03 1.28E-03 1.35E-03 $1.53 E-03$ 1.61E-03 $1.55 E-03$ 1.52E-03 $1.42 E-03$ Body Tissues 1.75E-02 1.64E-02 1.45E-02 1.06E-02 6.60E-03 4.46E-03 4.39E-03 4.61E-03 4.46E-03 4.21E-03 3.96E-03 3.32E-03

SOURCE = GALL BLADDER CONTENTS

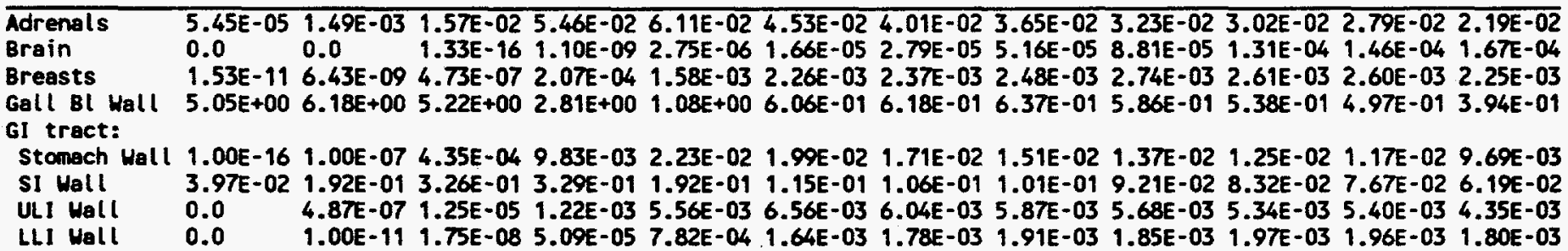

Heart Wall

Kidneys

Liver

Lungs

Muscle

Ovaries

Pancreas

Skeletal Tiss:

Act Marrow

Endosteum

Skin

Spleen

Thyous $\quad 0.0$

Thyroid $\quad 0.0$

Urin BL Wall 0.0

Uterine Cont :

Fetus

Skeleton

Soft Tissue 0.0

Uterine Hall

Body Tissues
1.00E-18 1.00E-07 3.51E-05 1.98E-03 7.42E-03 8.15E-03 7.40E-03 7.10E-03 6.22E-03 5.87E-03 5.70E-03 4.82E-03 8.26E-06 6.66E-04 1.50E-02 6.25E-02 6.64E-02 4.69E-02 4.12E-02 3.82E-02 3.45E-02 3.14E-02 2.92E-02 2.39E-02 6.86E-07 1.73E-03 1.81E-02 6.52E-02 7.03E-02 4.81E-02 4.13E-02 3.77E-02 3.39E-02 3.09E-02 2.87E-02 2.35E-02 1.00E-10 1.00E-06 7.76E-05 2.30E-03 6.36E-03 6.36E-03 5.70E-03 5.29E-03 5.07E-03 4.81E-03 4.48E-03 3.72E-03

7.88E-05 8.09E-04 2.34E-03 4.90E-03 5.91E-03 5.09E-03 4.71E-03 4.54E-03 4.30E-03 4.04E-03 3.82E-03 3.25E-03 3.00E-26 1.43E-09 2.29E-06 6.99E-04 4.25E-03 5.62E-03 5.67E-03 5.23E-03 5.06E-03 4.58E-03 4.42E-03 3.69E-03 1.00E-03 1.50E-02 7.45E-02 1.44E-01 1.13E-01 7.19E-02 6.34E-02 5.93E-02 5.39E-02 4.79E-02 4.43E-02 3.67E-02

1.00E-11 1.77E-05 4.23E-04 3.12E-03 7.26E-03 8.28E-03 8.11E-03 7.81E-03 7.34E-03 6.87E-03 6.50E-03 5.52E-03 6.81E-07 4.27E-05 1.02E-03 8.17E-03 1.76E-02 1.20E-02 6.81E-03 4.66E-03 4.05E-03 3.73E-03 3.51E-03 3.04E-03 1.00E-09 7.68E-07 9.89E-06 3.63E-04 $1.19 E-03$ 1.46E-03 $1.53 E-03 \quad 1.72 E-03$ 1.78E-03 $1.79 E-03 \quad 1.73 E-031.56 E-03$ 1.00E-20 1.00E-07 5.70E-05 3.43E-03 1.18E-02 1.23E-02 1.10E-02 1.00E-02 9.33E-03 8.60E-03 8.24E-03 6.68E-03

- 5.00E-14 6.01E-09 7.20E-05 9.34E-04 1.79E-03 1.83E-03 1.85E-03 1.94E-03 1.91E-03 1.96E-03 $1.92 E-03$ $0.0 \quad 1.00 E-11$ 1.77E-06 1.02E-04 3.37E-04 3.44E-04 4.66E-04 5.07E-04 6.31E-04 5.95E-04 6.91E-04 1.00E-15 3.63E-09 2.15E-05 6.12E-04 1.43E-03 1.70E-03 1.74E-03 1.81E-03 2.04E-03 $1.95 E-03$ 1.67E-03

1.00E-11 1.00E-06 1.50E-04 3.76E-03 1.13E-02 1.17E-02 9.88E-03 8.66E-03 7.84E-03 7.20E-03 6.79E-03 5.72E-03 $0.0 \quad 1.19 E-05$ 1.68E-04 7.03E-03 2.34E-02 2.05E-02 1.28E-02 8.75E-03 7.20E-03 6.68E-03 6.34E-03 5.38E-03 1.50E-07 1.48E-04 3.33E-03 9.72E-03 1.06E-02 9.50E-03 8.65E-03 7.92E-03 7.26E-03 6.86E-03 5.75E-03 1.00E-04 4.35E-03 1.24E-02 2.15E-02 2.03E-02 1.53E-02 $1.39 E-02 \quad 1.29 E-02 \quad 1.16 E-02 \quad 1.08 E-02 \quad 1.02 E-02 \quad 8.29 E-03$ 1.25E-03 3.48E-03 6.40E-03 1.02E-02 1.04E-02 7.89E-03 6.89E-03 6.43E-03 5.98E-03 5.56E-03 5.22E-03 4.38E-03 
Table A.4 (cont'd). At Nine Months Gestation: Specific Absorbed Fraction of Photon Energy (kg').

SOURCE = GALL BLADDER WALL $\quad$ Energy (MeV)

$\begin{array}{lllllllllllll}\text { Target } & 0.010 & 0.015 & 0.020 & 0.030 & 0.050 & 0.100 & 0.200 & 0.500 & 1.000 & 1.500 & 2.000 & 4.000\end{array}$

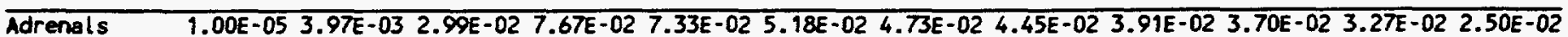

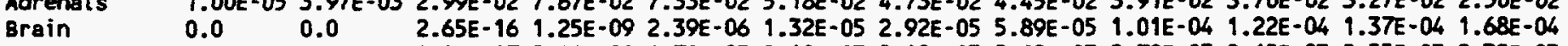

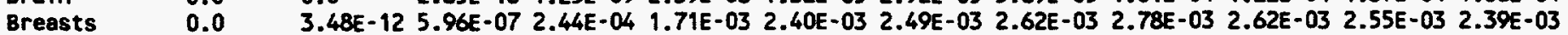
Gall BI Wall 5.60E+01 2.57E+01 1.35E+01 5.46E+00 1.81E+00 1.04E+00 1.07E+00 1.17E+00 $1.10 E+00 \quad 1.00 E+00 \quad 9.36 E-01 \quad 7.56 E-01$ GI tract:

Stomech Wall 1.00E-15 1.00E-06 5.35E-04 1.06E-02 2.31E-02 2.03E-02 1.75E-02 1.55E-02 1.39E-02 1.28E-02 1.21E-02 1.01E-02 SI Nall 3.24E-01 4.56E-01 4.78E-01 3.72E-01 2.01E-01 1.20E-01 1.12E-01 1.09E-01 9.93E-02 9.05E-02 8.35E-02 6.73E-02 ULI Wall 5.45E-09 5.23E-07 1.34E-05 1.28E-03 5.59E-03 6.67E-03 6.22E-03 5.99E-03 5.68E-03 5.37E-03 5.15E-03 4.44E-03 LLI Wall $\quad 0.0 \quad 1.00 E-911$ 1.00E-07 4.82E-05 7.88E-04 1.59E-03 1.71E-03 $1.89 E-03 \quad 1.81 E-03 \quad 1.86 E-03 \quad 2.01 E-03 \quad 1.73 E-03$

Heart wall 1.00E-10 5.00E-07 7.07E-05 2.68E-03 8.60E-03 9.08E-03 8.03E-03 7.48E-03 6.86E-03 6.54E-03 6.32E-03 5.26E-03

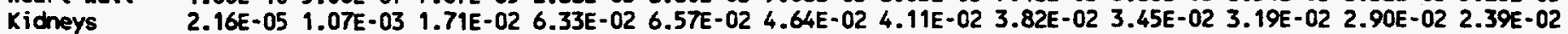
Liver $\quad 4.11 E-06 \quad 3.19 E-03 \quad 2.32 E-02 \quad 7.07 E-02 \quad 7.25 E-02 \quad 4.92 E-02 \quad 4.24 E-02 \quad 3.88 E-02 \quad 3.51 E-02 \quad 3.20 E-02 \quad 2.96 E-02 \quad 2.43 E-02$ Lungs $\quad 1.00 E-09$ 1.00E-06 1.34E-04 2.70E-03 6.82E-03 6.58E-03 6.17E-03 5.76E-03 5.34E-03 5.10E-03 4.84E-03 4.02E-03

Muscle $\quad 6.27 E-04 \quad 1.98 E-03 \quad 3.54 E-03 \quad 5.60 E-03 \quad 6.17 E-03 \quad 5.22 E-03 \quad 4.85 E-03 \quad 4.71 E-03 \quad 4.44 E-03 \quad 4.17 E-03 \quad 3.94 E-03 \quad 3.34 E-03$ Ovaries $\quad 1.00 E-26 \quad 1.00 E-10$ 2.08E-06 6.81E-04 4.02E-03 5.56E-03 5.63E-03 5.70E-03 4.62E-03 4.69E-03 4.29E-03 3.72E-03 Pencreas 9.00E-05 3.20E-02 1.13E-01 1.78E-01 1.28E-01 8.01E-02 7.19E-02 6.66E-02 6.00E-02 5.45E-02 5.05E-02 4.14E-02 Skeletal Tiss:

Act Marrow 1.00E-11 4.44E-05 6.62E-04 4.29E-03 8.84E-03 8.89E-03 8.35E-03 8.03E-03 7.57E-03 7.08E-03 6.70E-03 5.66E-03 Endosteum 1.67E-07 9.81E-05 1.41E-03 9.20E-03 $1.85 E-02 \quad 1.24 E-02$ 7.03E-03 $4.84 E-03 \quad 4.21 E-03 \quad 3.86 E-03$ 3.66E-03 3.13E-03 Skin $\quad 6.00 E-099.53 E-07$ 1.14E-05 3.78E-04 $1.21 E-03 \quad 1.47 E-03 \quad 1.54 E-03 \quad 1.77 E-03 \quad 1.85 E-03 \quad 1.79 E-03 \quad 1.76 E-03 \quad 1.57 E-03$ Spleen $\quad 1.00 E-23$ 1.00E-08 7.87E-05 3.99E-03 1.25E-02 1.30E-02 1.16E-02 1.04E-02 9.77E-03 9.24E-03 8.45E-03 7.35E-03

Thymus $\quad 0.0 \quad 1.70 E-13 \quad 7.84 E-09$ 8.35E-05 1.09E-03 1.94E-03 1.92E-03 2.21E-03 1.93E-03 2.08E-03 $1.81 E-03 \quad 2.18 E-03$ Thyroid $\quad 0.0 \quad 0.0 \quad 1.00 E-11 \quad 4.06 E-06 \quad 1.02 E-04 \quad 3.45 E-04 \quad 4.03 E-04 \quad 7.20 E-04 \quad 6.97 E-04 \quad 7.20 E-04 \quad 7.38 E-04 \quad 7.33 E-04$ Urin Bl Wall $0.0 \quad 1.00 E-15$ 2.76E-09 $1.91 E-05$ 5.74E-04 $1.38 E-03 \quad 1.72 E-03 \quad 1.65 E-03 \quad 1.93 E-03 \quad 1.78 E-03 \quad 1.96 E-03 \quad 1.51 E-03$ Uterine Cont:

Fetus

Skeleton

Soft Tissue 0.0 Uterine Wall 1.82E-03 8.66E-03 1.61E-02 2.21E-02 1.98E-02 $1.49 E-02$ 1.35E-02 $1.27 E-02$ 1.15E-02 $1.07 E-02$ 1.01E-02 8.16E-03 Body Tissues 1.15E-02 9.69E-03 1.01E-02 1.17E-02 1.10E-02 8.17E-03 7.19E-03 6.76E-03 6.28E-03 5.84E-03 5.48E-03 4.58E-03

\section{SOURCE = GI TRACT: STOMACH CONTENTS}

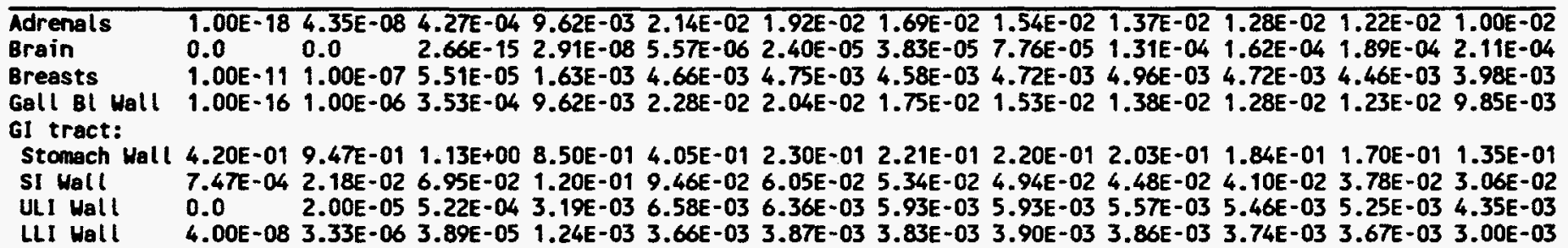

Heart Wall

Kidneys

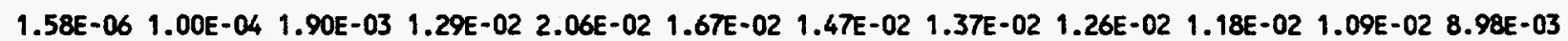

Liver 1.00E-17 5.00E-07 3.27E-04 7.35E-03 1.66E-02 $1.51 E-02 \quad 1.33 E-02 \quad 1.22 E-02 \quad 1.13 E-02 \quad 1.05 E-02 \quad 9.74 E-03 \quad 8.35 E-03$

Lungs 3.00E-10 1.95E-05 6.66E-04 6.09E-03 1.24E-02 $1.14 E-02 \quad 1.01 E-02 \quad 9.45 E-03 \quad 8.86 E-03 \quad 8.24 E-03$ 7.77E-03 6.49E-03

Muscle

Ovaries

Pancreas

Skeletal Tiss:

Act Marrow

Endosteum

1.27E-05 2.27E-04 1.75E-03 7.06E-03 1.01E-02 8.25E-03 7.28E-03 6.91E-03 6.30E-03 5.93E-03 5.63E-03 4.71E-03

Skin

Spleen

2.57E-05 8.14E-04 2.92E-03 6.51E-03 6.99E-03 5.44E-03 5.01E-03 4.90E-03 4.62E-03 4.34E-03 4.09E-03 3.45E-03 1.00E-27 1.00E-10 8.78E-07 2.73E-04 2.05E-03 3.34E-03 3.25E-03 3.25E-03 3.13E-03 3.15E-03 2.97E-03 2.51E-03 1.50E-03 1.47E-02 7.65E-02 1.66E-01 1.34E-01 8.38E-02 7.35E-02 6.76E-02 6.10E-02 5.61E-02 5.16E-02 4.13E-02

1.24E-06 3.37E-05 3.47E-04 1.63E-03 3.54E-03 4.28E-03 4.34E-03 4.29E-03 4.16E-03 4.00E-03 3.81E-03 3.32E-03 3.73E-06 1.12E-04 1.24E-03 6.36E-03 1.18E-02 8.23E-03 4.78E-03 3.32E-03 2.94E-03 2.75E-03 2.62E-03 2.30E-03

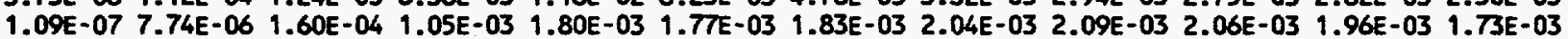
5.50E-10 2.20E-04 9.36E-03 5.80E-02 7.00E-02 4.81E-02 4.10E-02 3.77E-02 3.38E-02 3.08E-02 2.85E-02 2.32E-02

Thyous $\quad 0.0 \quad 1.21 E-11 \quad 8.02 E-07 \quad 2.52 E-04 \quad 1.86 E-03 \quad 2.57 E-03 \quad 2.62 E-03 \quad 2.88 E-03 \quad 2.80 E-03 \quad 2.94 E-03 \quad 2.78 E-03 \quad 2.46 E-03$

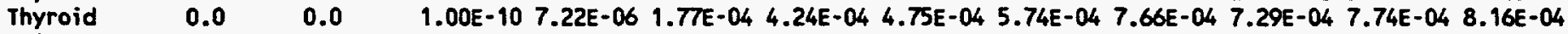
Urin Bl Wall $0.0 \quad 1.00 E-15$ 5.19E-10 $1.08 E-05 \quad 3.92 E-04 \quad 9.78 E-04 \quad 1.16 E-03 \quad 1.28 E-03 \quad 1.50 E-03 \quad 1.37 E-03 \quad 1.49 E-03 \quad 1.31 E-03$

Uterine Cont:

Fetus

3.98E-09 2.35E-06 2.18E-04 3.32E-03 9.04E-03 9.32E-03 8.00E-03 7.16E-03 6.61E-03 6.09E-03 5.69E-03 4.82E-03

Skeleton 3.65E-07 1.23E-05 1.49E-04 5.00E-03 1.67E-02 1.50E-02 9.52E-03 6.66E-03 5.59E-03 5.25E-03 4.89E-03 4.09E-03

Soft Tissue 5.08E-09 2.67E-06 2.27E-04 3.10E-03 8.03E-03 8.58E-03 7.79E-03 7.22E-03 6.72E-03 6.21E-03 5.80E-03 4.93E-03 Uterine Wall 2.92E-03 8.37E-03 1.36E-02 1.85E-02 1.68E-02 1.28E-02 1.15E-02 $1.10 E-02$ 1.01E-02 9.28E-03 8.87E-03 7.26E-03 Body Tissues 9.08E-04 2.83E-03 5.47E-03 9.05E-03 9.07E-03 6.76E-03 5.99E-03 5.70E-03 5.33E-03 4.99E-03 4.70E-03 3.94E-03 
Table A.4 (cont'd). At Nine Months Gestation: Specific Absorbed Fraction of Photon Energy (kg').

SOURCE = GI TRACT: STOMACH WALL

Energy (MeV)

$\begin{array}{lllllllllllll}\text { Target } & 0.010 & 0.015 & 0.020 & 0.030 & 0.050 & 0.100 & 0.200 & 0.500 & 1.000 & 1.500 & 2.000 & 4.000\end{array}$

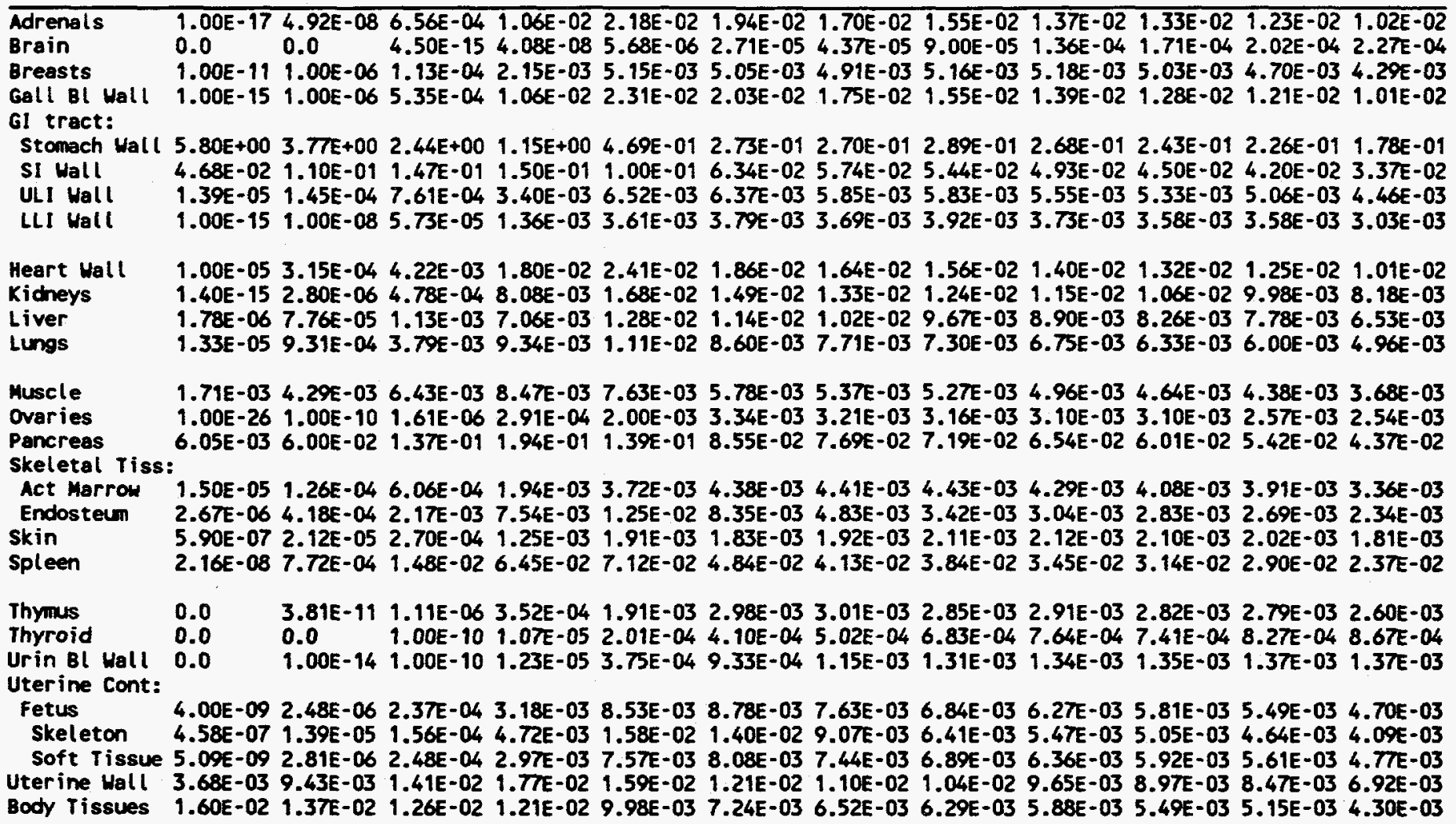

SOURCE = GI TRACT: SMALL INTESTINE CONTENTS

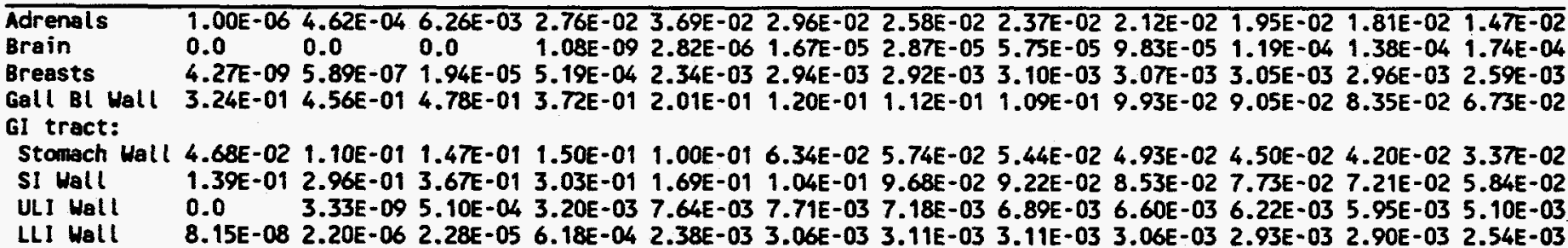

Heart Wall Kidneys

Liver

Lungs

4.71E-07 1.25E-05 1.29E-04 3.43E-03 9.55E-03 9.57E-03 8.64E-03 7.96E-03 7.45E-03 6.83E-03 6.56E-03 5.50E-03 6.97E-06 4.53E-04 8.75E-03 3.71E-02 4.33E-02 3.22E-02 2.83E-02 2.63E-02 2.37E-02 2.18E-02 2.01E-02 1.66E-02 6.01E-03 1.83E-02 3.47E-02 5.49E-02 4.91E-02 3.42E-02 3.02E-02 2.81E-02 2.54E-02 2.32E-02 2.16E-02 1.77E-02 1.82E-07 5.60E-06 6.38E-05 1.97E-03 5.63E-03 5.66E-03 5.09E-03 4.89E-03 4.56E-03 4.33E-03 4.12E-03 3.57E-03

Muscle

Ovaries

Pancreas

Skeletal Tiss:

Act Marrow 2.42E-05 1.61E-04 6.04E-04 2.55E-03 5.70E-03 6.67E-03 6.57E-03 6.44E-03 6.03E-03 5.68E-03 5.41E-03 4.51E-03 $\begin{array}{lllllllllllll}\text { Endosteum } & 7.28 E-05 & 4.76 E-04 & 1.73 E-03 & 7.29 E-03 & 1.45 E-02 & 1.02 E-02 & 5.83 E-03 & 4.04 E-03 & 3.50 E-03 & 3.23 E-03 & 3.06 E-03 & 2.61 E-03 \\ \text { Skin } & 1.16 E-08 & 1.60 E-06 & 5.29 E-05 & 5.68 E-04 & 1.38 E-03 & 1.54 E-03 & 1.61 E-03 & 1.79 E-03 & 1.83 E-03 & 1.82 E-03 & 1.77 E-03 & 1.56 E-03\end{array}$

Skin

Spleen

7.39E-04 2.10E-03 3.70E-03 6.01E-03 6.47E-03 5.31E-03 4.93E-03 4.78E-03 4.51E-03 $4.24 E-03 \quad 4.01 E-03 \quad 3.39 E-03$ 1.71E-08 1.01E-06 1.82E-05 1.08E-03 4.70E-03 6.06E-03 5.73E-03 5.38E-03 4.97E-03 4.88E-03 4.53E-03 3.76E-03 5.70E-02 1.63E-01 2.49E-01 2.54E-01 1.56E-01 9.47E-02 8.63E-02 8.23E-02 7.49E-02 6.79E-02 6.27E-02 5.03E-02 3.22E-03 1.29E-02 2.91E-02 5.07E-02 4.69E-02 3.29E-02 2.89E-02 2.70E-02 2.43E-02 2.23E-02 2.08E-02 1.71E-02

Thymus

Thyroid

7.61E-13 7.47E-10 9.91E-08 9.72E-05 1.07E-03 1.79E-03 1.83E-03 1.99E-03 2.12E-03 2.17E-03 2.05E-03 1.91E-03 Uterine cont Fetus

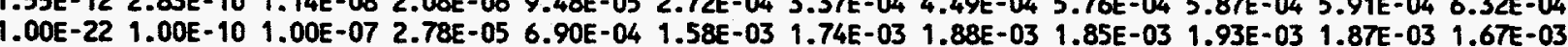

1.64E-07 1.93E-05 5.69E-04 5.92E-03 1.39E-02 1.33E-02 1.12E-02 9.72E-03 8.77E-03 8.12E-03 7.64E-03 6.37E-03 Skeleton $\quad 1.88 E-06 \quad 4.44 E-05 \quad 4.19 E-04 \quad 9.89 E-03 \quad 2.75 E-02 \quad 2.21 E-02 \quad 1.38 E-02 \quad 9.34 E-03 \quad 7.65 E-03 \quad 7.03 E-03 \quad 6.65 E-03 \quad 5.56 E-03$ Soft Tissue 1.97E-07 2.12E-05 5.86E-04 5.39E-03 1.21E-02 1.21E-02 1.09E-02 9.73E-03 8.89E-03 8.28E-03 7.73E-03 6.49E-03 Uterine Hall 8.17E-03 2.06E-02 2.93E-02 3.23E-02 2.52E-02 $1.81 E-02$ 1.66E-02 $1.56 E-02 \quad 1.41 E-02 \quad 1.31 E-02 \quad 1.21 E-02 \quad 1.00 E-02$ Body Tissues 3.12E-03 7.12E-03 9.81E-03 1.19E-02 1.06E-02 7.69E-03 6.83E-03 6.56E-03 6.16E-03 5.72E-03 5.42E-03 4.46E-03 
Table A.4 (cont'd). At Nine Months Gestation: Specific Absorbed Fraction of Photon Energy (kg').

SOURCE = GI TRACT: SMALL INTESTINE WALL

Energy (MeV)

$\begin{array}{lllllllllllll}\text { Target } & 0.010 & 0.015 & 0.020 & 0.030 & 0.050 & 0.100 & 0.200 & 0.500 & 1.000 & 1.500 & 2.000 & 4.000\end{array}$

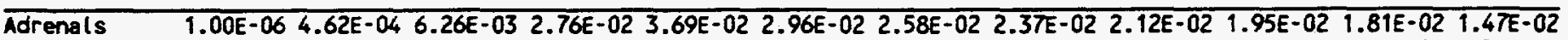

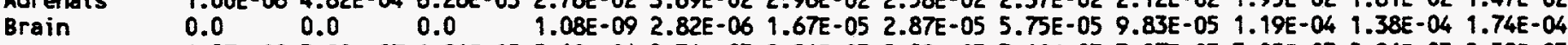
Breasts $\quad 4.27 E-09$ 5.89E-07 1.94E-05 5.19E-04 2.34E-03 2.94E-03 2.92E-03 3.10E-03 3.07E-03 3.05E-03 2.96E-03 2.59E-03 Gall Bl Wall 3.24E-01 4.56E-01 4.78E-01 3.72E-01 2.01E-01 1.20E-01 1.12E-01 1.09E-01 9.93E-02 9.05E-02 8.35E-02 6.73E-02 GI tract:

Stomach Wall 4.68E-02 1.10E-01 1.47E-01 1.50E-01 1.00E-01 6.34E-02 5.74E-02 5.44E-02 4.93E-02 4.50E-02 4.20E-02 3.37E-02 SI Wall $\quad 1.52 E+001.26 E+00$ 9.78E-01 5.74E-01 2.66E-01 1.62E-01 1.57E-01 1.57E-01 1.41E-01 $1.28 E-011.20 E-019.40 E-02$ ULI Hall $\quad 0.0 \quad 3.33 E-09$ 5.10E-04 3.20E-03 7.64E-03 7.71E-03 7.18E-03 6.89E-03 6.60E-03 6.22E-03 5.95E-03 5.10E-03 LLJ Wall 8.15E-08 2.20E-06 2.28E-05 6.18E-04 2.38E-03 3.06E-03 3.11E-03 3.11E-03 3.06E-03 2.93E-03 2.90E-03 2.54E-03

Heart Wall 4.71E-07 1.25E-05 1.29E-04 3.43E-03 9.55E-03 9.57E-03 8.64E-03 7.96E-03 7.45E-03 6.83E-03 6.56E-03 5.50E-03

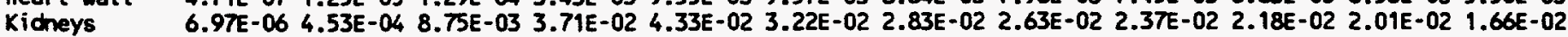
6.01E-03 1.83E-02 3.47E-02 5.49E-02 4.91E-02 3.42E-02 3.02E-02 2.81E-02 2.54E-02 2.32E-02 2.16E-02 1.77E-02

Lungs 1.82E-07 5.60E-06 6.38E-05 1.97E-03 5.63E-03 5.66E-03 5.09E-03 4.89E-03 4.56E-03 4.33E-03 4.12E-03 3.57E-03

Muscle

Ovaries

Pancreas

7.39E-04 2.10E-03 3.70E-03 6.01E-03 6.4TE-03 5.31E-03 4.93E-03 4.78E-03 4.51E-03 4.24E-03 4.01E-03 3.39E-03

Skeletal Tiss:

Act Marrow

Endosteum

Skin

Spleen

1.71E-08 1.01E-06 1.82E-05 1.08E-03 4.70E-03 6.06E-03 5.73E-03 5.38E-03 4.97E-03 4.88E-03 4.53E-03 3.76E-03 5.70E-02 1.63E-01 2.49E-01 2.54E-01 1.56E-01 9.47E-02 8.63E-02 8.23E-02 7.49E-02 6.79E-02 6.27E-02 5.03E-02

2.42E-05 1.61E-04 6.04E-04 2.55E-03 5.70E-03 6.67E-03 6.57E-03 6.44E-03 6.03E-03 5.68E-03 5.41E-03 4.51E-03 7.28E-05 4.76E-04 1.73E-03 7.29E-03 $1.45 E-02$ 1.02E-02 5.83E-03 4.04E-03 3.50E-03 3.23E-03 3.06E-03 2.61E-03 1.16E-08 1.60E-06 5.29E-05 5.68E-04 $1.38 E-03$ 1.54E-03 1.61E-03 1.79E-03 1.83E-03 $1.82 E-03 \quad 1.77 E-03 \quad 1.56 E-03$ 3.22E-03 1.29E-02 2.91E-02 5.07E-02 4.69E-02 3.29E-02 2.89E-02 2.70E-02 2.43E-02 2.23E-02 2.08E-02 1.71E-02

Thymus $\quad 7.61 E-13 \quad 7.47 E-10$ 9.91E-08 9.72E-05 1.07E-03 1.79E-03 1.83E-03 1.99E-03 2.12E-03 2.17E-03 2.05E-03 1.91E-03 Thyroid $\quad 1.55 E-12$ 2.83E-10 1.14E-08 2.08E-06 9.48E-05 2.72E-04 3.37E-04 4.49E-04 5.76E-04 5.87E-04 5.91E-04 6.32E-04 Urin Bl Wall 1.00E-22 1.00E-10 1.00E-07 2.78E-05 6.90E-04 1.58E-03 1.74E-03 1.88E-03 $1.85 E-03$ 1.93E-03 1.87E-03 $1.67 E-03$ Uterine Cont:

Fetus

Skeleton 1.97E-07 2.12E-05 5.86E-04 5.39E-03 1.21E-02 1.21E-02 1.09E-02 9.73E-03 8.89E-03 8.28E-03 7.73E-03 6.49E-03 Uterine Wall 8.17E-03 2.06E-02 2.93E-02 3.23E-02 2.52E-02 1.81E-02 1.66E-02 $1.56 E-02 \quad 1.41 E-02$ 1.31E-02 1.21E-02 1.00E-02 Body Tissues 1.67E-02 1.53E-02 1.43E-02 1.35E-02 1.11E-02 8.02E-03 7.17E-03 6.94E-03 6.49E-03 6.04E-03 5.73E-03 4.70E-03

\section{SOURCE = GI TRACT: UPPER LARGE INTESTINE CONTENTS}

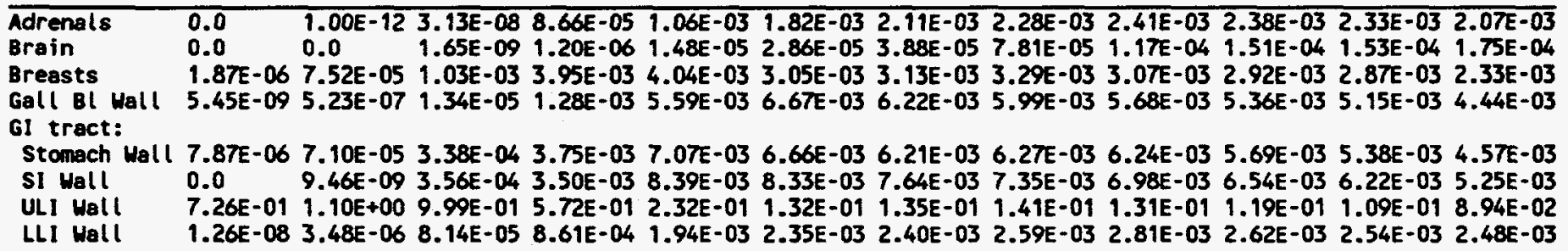

Heart Holl Kidneys Liver Lungs

Muscle Ovaries Pancreas Skeletal Tiss:

Act Marrow Endosteum

skin

Spleen

Thymus

Thyroid

Urin Bl Wall

Uterine Cont:

Fetus

Skeleton

Soft Tissue

Uterine Hall

Body Tissues

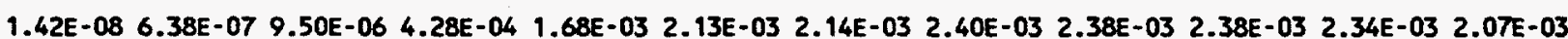
$\begin{array}{llllllllllll}0.0 & 1.00 E-12 & 7.07 E-07 & 2.29 E-04 & 1.68 E-03 & 2.57 E-03 & 2.67 E-03 & 2.82 E-03 & 2.88 E-03 & 2.89 E-03 & 2.70 E-03 & 2.35 E-03\end{array}$ 5.00E-09 5.00E-06 1.93E-04 2.69E-03 6.55E-03 6.44E-03 6.12E-03 6.13E-03 6.00E-03 5.68E-03 5.48E-03 4.61E-03

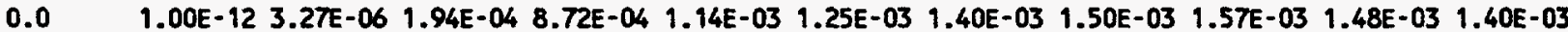

6.35E-04 4.23E-03 7.49E-03 8.17E-03 5.62E-03 4.03E-03 3.99E-03 4.14E-03 4.00E-03 3.80E-03 3.58E-03 3.06E-03 5.00E-11 2.00E-06 6.47E-04 6.18E-03 1.03E-02 9.00E-03 8.26E-03 7.87E-03 7.20E-03 6.93E-03 6.46E-03 5.54E-03 1.00E-19 1.00E-07 4.60E-06 5.59E-04 3.15E-03 4.06E-03 3.97E-03 4.18E-03 3.90E-03 3.83E-03 3.75E-03 3.11E-03

7.58E-07 4.22E-05 2.42E-04 1.00E-03 1.93E-03 2.31E-03 2.43E-03 2.54E-03 2.58E-03 2.48E-03 2.43E-03 2.13E-03 1.00E-06 6.51E-05 5.13E-04 2.90E-03 5.12E-03 3.56E-03 2.24E-03 1.73E-03 1.63E-03 1.56E-03 1.52E-03 $1.37 E-03$ 4.76E-06 6.39E-04 2.39E-03 3.63E-03 2.63E-03 1.96E-03 2.08E-03 2.35E-03 2.36E-03 2.30E-03 2.21E-03 1.91E-03 1.00E-19 1.00E-09 3.81E-07 1.90E-04 1.23E-03 1.90E-03 1.94E-03 2.24E-03 2.26E-03 2.22E-03 2.21E-03 1.84E-03

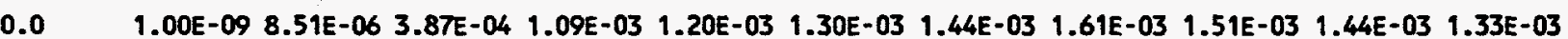
$0.0 \quad 5.93 E-07$ 2.73E-06 2.34E-05 9.78E-05 $1.35 E-04 \quad 2.09 E-04 \quad 3.49 E-04 \quad 3.90 E-04 \quad 4.92 E-04 \quad 4.64 E-04 \quad 4.70 E-04$ 1.00E-13 1.00E-08 5.00E-06 5.43E-04 2.55E-03 3.28E-03 3.29E-03 3.51E-03 3.62E-03 3.53E-03 3.28E-03 2.86E-03 1.26E-07 2.12E-05 8.07E-04 8.35E-03 1.67E-02 1.44E-02 1.22E-02 1.12E-02 1.03E-02 9.51E-03 9.01E-03 7.46E-03 6.40E-06 1.11E-04 8.39E-04 1.45E-02 3.27E-02 2.27E-02 $1.39 E-02$ 9.98E-03 9.01E-03 8.05E-03 7.90E-03 6.52E-03 1.65E-07 2.37E-05 8.03E-04 7.53E-03 1.46E-02 1.32E-02 1.19E-02 1.13E-02 1.05E-02 9.69E-03 9.17E-03 7.60E-03 1.92E-04 B.72E-03 2.56E-02 3.64E-02 2.72E-02 $1.85 E-02 \quad 1.72 E-02 \quad 1.70 E-02 \quad 1.57 E-02 \quad 1.47 E-02 \quad 1.36 E-02 \quad 1.13 E-02$ 2.47E-03 6.25E-03 8.70E-03 8.59E-03 5.99E-03 4.28E-03 4.13E-03 4.23E-03 4.08E-03 3.86E-03 3.64E-03 3.10E-03 
Table A.4 (cont'd). At Nine Months Gestation: Specific Absorbed Fraction of Photon Energy (kg').

SOURCE $=$ GI TRACT: UPPER LARGE INTESTINE WAU

Energy (MeV)

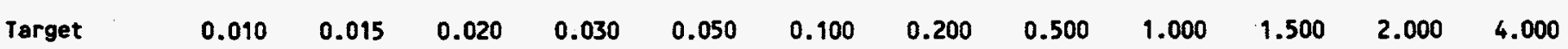

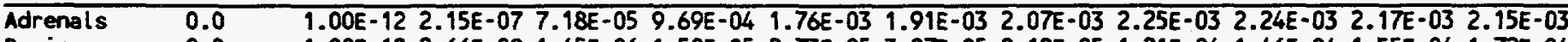

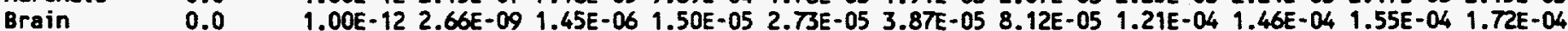
Breasts $\quad 7.46 E-06 \quad 1.36 E-04 \quad 1.06 E-03$ 3.61E-03 3.70E-03 2.82E-03 2.84E-03 3.10E-03 3.01E-03 2.86E-03 2.54E-03 2.21E-03 Gall Bl Wall 5.45E-09 5.23E-07 1.34E-05 1.28E-03 5.59E-03 6.67E-03 6.22E-03 5.99E-03 5.68E-03 5.37E-03 5.15E-03 4.44E-03 Gl tract:

Stomach Hall 1.39E-05 1.45E-04 7.61E-04 3.40E-03 6.52E-03 6.37E-03 5.85E-03 5.83E-03 5.55E-03 5.33E-03 5.06E-03 4.46E-03 SI Wall $\quad 0.0 \quad 3.33 E-09$ 5.10E-04 3.20E-03 7.64E-03 7.71E-03 7.18E-03 6.89E-03 6.60E-03 6.22E-03 5.95E-03 5.10E-03 ULI Wall 4.02E+00 2.64E+00 1.74E+00 8.37E-01 3.39E-01 2.02E-01 2.06E-01 2.18E-01 1.99E-01 $1.80 E-01$ 1.68E-01 $1.34 E-01$ LLI Wall 1.00E-08 5.30E-06 2.16E-04 1.26E-03 2.42E-03 2.66E-03 2.72E-03 3.04E-03 3.07E-03 3.13E-03 2.88E-03 2.54E-03

Heart Hall $\quad 0.0 \quad 1.00 E-09 \quad 1.37 E-05 \quad 3.97 E-04 \quad 1.53 E-03 \quad 1.97 E-03 \quad 2.00 E-03 \quad 2.21 E-03 \quad 2.29 E-03 \quad 2.17 E-03 \quad 2.22 E-03 \quad 1.99 E-03$ Kidneys Liver

Lungs

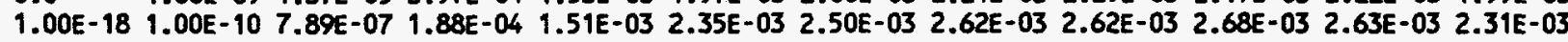
2.34E-07 1.21E-05 2.00E-04 2.38E-03 5.88E-03 5.89E-03 5.59E-03 5.71E-03 5.52E-03 5.27E-03 5.07E-03 4.38E-03 0.0 1.00E-11 3.83E-06 $1.77 \mathrm{E}-04 \quad 8.03 E-04 \quad 1.05 E-03 \quad 1.15 E-03 \quad 1.36 E-03 \quad 1.43 E-03 \quad 1.45 E-03 \quad 1.35 E-031.33 E-03$

Muscle

Ovaries

4.61E-03 8.81E-03 1.04E-02 8.98E-03 5.68E-03 4.03E-03 4.03E-03 4.23E-03 4.08E-03 3.87E-03 3.65E-03 3.09E-03

Ovaries

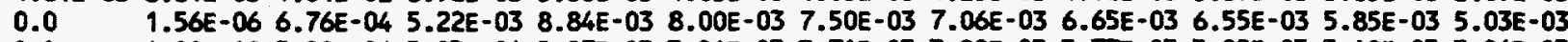

0.0

1.00E-10 5.28E-06 5.03E-04 2.87E-03 3.81E-03 3.71E-03 3.88E-03 3.77E-03 3.82E-03 3.40E-03 3.06E-03

Skeletal Tiss:

Act Marrow 9.16E-06 1.07E-04 3.27E-04 9.56E-04 1.73E-03 2.09E-03 2.21E-03 2.35E-03 2.35E-03 2.35E-03 2.25E-03 2.00E-03 Endosteum 1.22E-05 1.61E-04 6.60E-04 2.69E-03 4.55E-03 3.24E-03 2.06E-03 $1.60 E-03 \quad 1.51 E-03 \quad 1.46 E-03 \quad 1.43 E-03 \quad 1.29 E-03$ skin

Spleen 6.21E-05 1.05E-03 2.89E-03 3.83E-03 2.65E-03 1.95E-03 2.13E-03 2.35E-03 2.37E-03 2.26E-03 2.18E-03 1.88E-03 1.00E-12 1.00E-08 2.31E-06 1.82E-04 1.16E-03 1.77E-03 1.85E-03 2.00E-03 2.15E-03 2.24E-03 1.99E-03 1.84E-03

Thymus $\quad 0.0 \quad 1.00 E-07 \quad 1.13 E-05 \quad 3.61 E-04 \quad 1.02 E-03 \quad 1.09 E-03 \quad 1.18 E-03 \quad 1.46 E-03 \quad 1.55 E-03 \quad 1.52 E-03 \quad 1.34 E-03 \quad 1.30 E-03$ Thyroid $\quad 0.0 \quad 0.0 \quad 2.56 E-06$ 2.32E-05 $1.02 E-04$ 1.51E-04 2.15E-04 2.82E-04 4.23E-04 4.41E-04 5.31E-04 5.24E-04 Urin 8L Wall 1.00E-13 1.00E-08 6.51E-06 5.52E-04 2.52E-03 3.28E-03 3.24E-03 3.51E-03 3.58E-03 3.43E-03 3.40E-03 3.01E-03 Uterine Cont:

Fetus

4.10E-07 4.09E-05 1.07E-03 9.30E-03 1.75E-02 1.48E-02 1.25E-02 1.16E-02 1.06E-02 9.92E-03 9.36E-03 7.81E-03

Skeleton 9.19E-06 1.43E-04 1.01E-03 1.57E-02 3.34E-02 2.29E-02 1.40E-02 1.03E-02 9.09E-03 8.41E-03 7.75E-03 6.63E-03

Soft Tissue 5.45E-07 4.62E-05 1.08E-03 8.45E-03 1.54E-02 $1.37 E-02$ 1.23E-02 $1.17 \mathrm{E}-02 \quad 1.08 \mathrm{E}-02$ 1.01E-02 $9.56 \mathrm{E}-03$ 7.98E-03 Uterine Hall 1.87E-03 2.04E-02 3.93E-02 4.42E-02 2.99E-02 2.00E-02 1.89E-02 1.87E-02 1.76E-02 1.62E-02 $1.51 E-02$ 1.23E-02 Body Tissues 1.56E-02 1.42E-02 1.29E-02 9.79E-03 6.14E-03 4.34E-03 4.26E-03 4.41E-03 4.24E-03 4.00E-03 3.78E-03 3.19E-03

SOURCE $=$ GI TRACT: LOWER LARGE INTESTINE CONTENTS

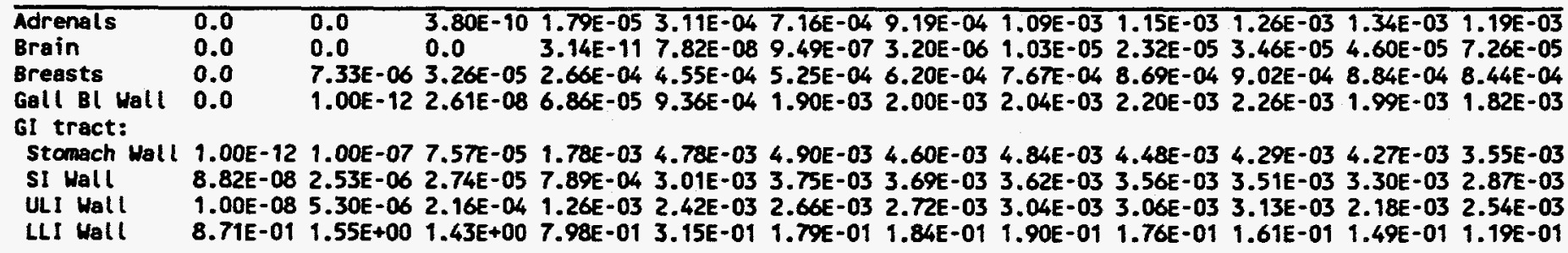

Heart Wall $0.0 \quad 1.00 E-17$ 1.00E-10 9.61E-06 1.50E-04 3.29E-04 4.29E-04 5.50E-04 6.47E-04 7.58E-04 $7.74 E-04 \quad 7.42 E-04$ Kidheys $\quad 1.00 E-15$ 1.00E-10 $1.00 E-07$ 7.78E-05 $7.86 E-04 \quad 1.46 E-03 \quad 1.67 E-03 \quad 1.85 E-03 \quad 1.96 E-03 \quad 1.88 E-03 \quad 1.86 E-03 \quad 1.72 E-03$

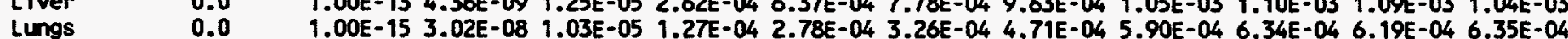

Muscle $\quad 3.77 E-04$ 3.65E-03 7.70E-03 1.00E-02 7.86E-03 5.72E-03 5.48E-03 5.52E-03 5.24E-03 4.92E-03 4.64E-03 3.91E-03 Ovaries $\quad 1.06 E-06$ 1.53E-04 5.20E-03 2.31E-02 2.67E-02 2.02E-02 $1.81 E-02 \quad 1.72 E-02 \quad 1.55 E-02 \quad 1.45 E-02 \quad 1.33 E-02 \quad 1.09 E-02$ Pancreas 1.00E-28 1.00E-10 4.50E-07 1.70E-04 1.42E-03 2.28E-03 2.24E-03 2.59E-03 2.55E-03 2.51E-03 2.32E-03 2.28E-03 Skeletal Tiss:

Act Marrow 2.43E-06 1.67E-04 1.12E-03 3.62E-03 5.23E-03 5.14E-03 5.01E-03 4.96E-03 4.72E-03 4.45E-03 4.26E-03 3.56E-03 Endosteum 3.01E-06 2.46E-04 $1.84 E-03$ 7.30E-03 1.11E-02 7.05E-03 4.13E-03 3.00E-03 2.69E-03 2.50E-03 $2.39 E-03$ 2.08E-03

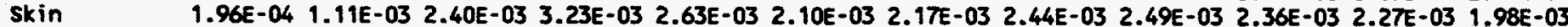

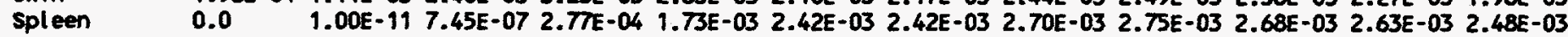

Thymus $\quad 0.0 \quad 0.0 \quad 1.00 E-12 \quad 1.00 E-07 \quad 3.87 E-05 \quad 1.13 E-04 \quad 1.42 E-04 \quad 2.42 E-04 \quad 3.50 E-04 \quad 3.49 E-04 \quad 4.12 E-04 \quad 4.27 E-04$

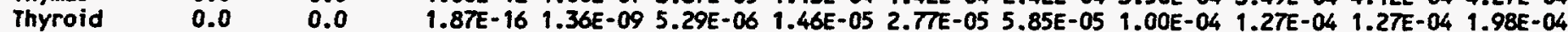
Urin Bl Wall $0.0 \quad 1.73 E-08$ 6.34E-04 8.83E-03 $1.63 E-02 \quad 1.35 E-02 \quad 1.24 E-02 \quad 1.21 E-02 \quad 1.13 E-02 \quad 1.01 E-02$ 9.94E-03 8.02E-03 Uterine Cont:

Fetus

5.00E-09 1.00E-05 6.26E-04 7.80E-03 1.65E-02 1.42E-02 1.18E-02 1.07E-02 9.73E-03 9.11E-03 8.52E-03 7.12E-03 Skeleton $\quad 2.10 E-07$ 4.83E-05 2.29E-03 2.33E-02 4.40E-02 2.91E-02 $1.74 E-02 \quad 1.27 E-02 \quad 1.09 E-02 \quad 1.00 E-02 \quad 9.48 E-03$ 7.79E-03 Soft Tissue 9.07E-09 4.75E-06 4.04E-04 5.73E-03 1.28E-02 1.22E-02 1.11E-02 1.04E-02 9.58E-03 8.98E-03 8.39E-03 7.03E-03 Uterine Hall 5.33E-05 3.99E-03 1.48E-02 2.54E-02 2.13E-02 $1.53 E-02$ 1.42E-02 $1.40 E-02 \quad 1.27 E-02$ 1.20E-02 1.11 E-02 $9.20 E-03$ Body Tissues 2.23E-03 6.20E-03 9.20E-03 1.01E-02 7.80E-03 5.55E-03 5.15E-03 5.13E-03 4.86E-03 4.57E-03 4.31E-03 3.63E-03 
Table A.4 (cont'd). At Nine Months Gestation: Specific Absorbed Fraction of Photon Energy (kg').

SOURCE $=$ GI TRACT: LOWER LARGE INTESTINE WALL

Energy (HeV)

\begin{tabular}{|c|c|c|c|c|c|c|c|c|c|c|c|c|}
\hline Target & 0.010 & 0.015 & 0.020 & 0.030 & 0.050 & 0.100 & 0.200 & 0.500 & 1.000 & 1.500 & 2.000 & 4.000 \\
\hline $\begin{array}{l}\text { Adrenals } \\
\text { Brain } \\
\text { Breasts } \\
\text { Gall Bl Wall } \\
\text { GI tract: } \\
\text { Stomech Wall } \\
\text { sI Wall } \\
\text { ULI Wall } \\
\text { LLI Hall }\end{array}$ & $\begin{array}{l}0.0 \\
0.0 \\
0.0 \\
0.0 \\
1.00 E-15 \\
8.15 E-08 \\
1.00 E-08 \\
5.03 E+00\end{array}$ & $\begin{array}{l}0.0 \\
0.0 \\
1.06 E-05 \\
1.00 E-11\end{array}$ & $\begin{array}{l}3.83 E-10 \\
0.0 \\
3.63 E-05 \\
1.00 E-07\end{array}$ & $\begin{array}{l}1.79 E-05 \\
2.35 E-11 \\
2.05 E-04 \\
4.82 E-05 \\
1.36 E-03 \\
6.18 E-04 \\
1.26 E-03 \\
9.94 E-01\end{array}$ & $\begin{array}{l}3.11 E-04 \\
6.01 E-08 \\
3.47 E-04 \\
7.88 E-04 \\
3.61 E-03 \\
2.38 E-03 \\
2.42 E-03 \\
3.87 E-09\end{array}$ & $\begin{array}{l}7.16 E-04 \\
7.53 E-07 \\
4.03 E-04 \\
1.59 E-03 \\
3.79 E-03 \\
3.06 E-03 \\
2.66 E-03 \\
2.30 E-01\end{array}$ & $\begin{array}{l}9.19 E-04 \\
2.60 E-06 \\
4.65 E-04 \\
1.71 E-03 \\
3.69 E-03 \\
3.11 E-03 \\
2.72 E-03 \\
2.35 E-01\end{array}$ & $\begin{array}{l}1.09 E-03 \\
8.68 E-06 \\
6.21 E-04 \\
1.89 E-03 \\
3.92 E-03 \\
3.11 E-03 \\
3.04 E-03 \\
2.50 E-01\end{array}$ & $\begin{array}{l}1.15 E-03 \\
2.01 E-05 \\
7.59 E-04 \\
1.81 E-03\end{array}$ & $\begin{array}{l}1.26 E-03 \\
3.04 E-05 \\
7.25 E-04 \\
1.86 E-03\end{array}$ & $\begin{array}{l}1.34 E-03 \\
4.07 E-05 \\
7.43 E-04 \\
2.01 E-03\end{array}$ & $\begin{array}{l}1.19 E-03 \\
7.73 E-05 \\
7.08 E-04 \\
1.75 E-03 \\
3.03 E-03 \\
2.54 E-03 \\
2.54 E-03 \\
1.57 E-01\end{array}$ \\
\hline
\end{tabular}

Heart Wall $\quad 0.0 \quad 8.10 E-16 \quad 3.74 E-09$ 9.61E-06 $1.50 E-04 \quad 3.29 E-04 \quad 4.30 E-04 \quad 5.50 E-04 \quad 6.47 E-04 \quad 7.58 E-04 \quad 7.74 E-04 \quad 7.42 E-04$

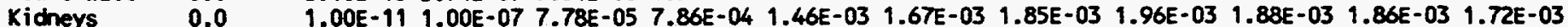

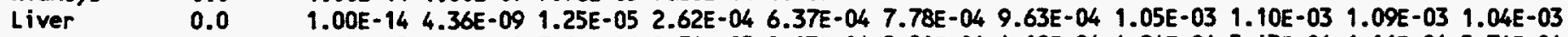
Lungs $\quad 0.0 \quad 2.13 E-15$ 4.43E-09 7.64E-06 9.74E-05 2.13E-04 2.81E-04 4.18E-04 4.94E-04 5.43E-04 6.16E-04 5.74E-04

Muscle $\quad 3.48 E-03 \quad 7.86 E-03 \quad 1.09 E-02 \quad 1.20 E-02 \quad 9.17 E-03 \quad 6.62 E-03 \quad 6.31 E-03 \quad 6.29 E-03 \quad 5.94 E-03 \quad 5.58 E-03$ 5.24E-03 4.39E-03 Ovaries $\quad 1.26 E-06$ 1.61E-04 5.04E-03 2.17E-02 2.62E-02 2.06E-02 $1.83 E-02 \quad 1.69 E-02 \quad 1.56 E-02 \quad 1.43 E-02$ 1.37E-02 $1.09 E-02$

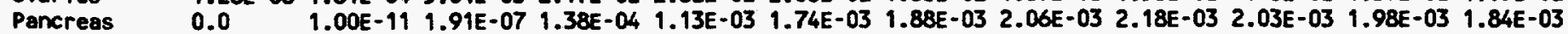
Skeletal Tiss:

Act Marrow 1.78E-05 3.61E-04 1.65E-03 4.71E-03 6.37E-03 6.12E-03 5.97E-03 5.85E-03 5.46E-03 5.17E-03 4.86E-03 4.05E-03 Endosteum 2.35E-05 5.33E-04 2.76E-03 9.66E-03 1.40E-02 8.67E-03 5.02E-03 3.60E-03 3.17E-03 2.96E-03 2.81E-03 2.41E-03 skin

Spleen 3.43E-04 1.53E-03 2.55E-03 3.18E-03 2.74E-03 2.19E-03 2.29E-03 2.52E-03 2.54E-03 2.39E-03 2.38E-03 $2.04 E-03$ 1.00E-16 1.00E-10 2.18E-06 2.15E-04 1.35E-03 1.97E-03 2.05E-03 2.27E-03 2.28E-03 2.26E-03 2.25E-03 2.08E-03

Thymus $\quad 0.0 \quad 0.0 \quad 2.70 E-12 \quad 8.82 E-07 \quad 3.00 E-05 \quad 7.89 E-05 \quad 1.36 E-04 \quad 2.22 E-04 \quad 2.88 E-04 \quad 3.22 E-04 \quad 3.57 E-04 \quad 3.88 E-04$

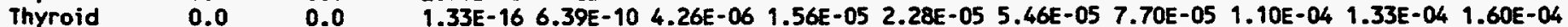
Urin Bl Wall $0.0 \quad 3.51 E-079.18 E-04 \quad 1.16 E-02 \quad 2.04 E-02 \quad 1.67 E-02 \quad 1.49 E-02 \quad 1.43 E-02 \quad 1.28 E-02 \quad 1.23 E-02 \quad 1.13 E-02 \quad 9.38 E-03$

Uterine Cont:

Fetus

3.83E-08 1.17E-05 6.81E-04 7.64E-03 1.58E-02 1.36E-02 1.13E-02 1.01E-02 9.31E-03 8.63E-03 8.12E-03 6.78E-03 skeleton $\quad 4.53 E-07$ 7.53E-05 2.84E-03 2.53E-02 $4.54 E-02 \quad 2.96 E-02 \quad 1.78 E-02 \quad 1.30 E-02 \quad 1.12 E-02 \quad 1.02 E-02 \quad 9.43 E-03 \quad 7.95 E-03$ Soft Tissue 1.12E-08 5.12E-06 3.93E-04 5.28E-03 1.19E-02 1.14E-02 1.04E-02 9.74E-03 9.04E-03 8.42E-03 7.93E-03 6.60E-03 Uterine Wall 3.99E-04 5.36E-03 1.40E-02 2.23E-02 1.92E-02 1.41 E-02 $1.30 E-02 \quad 1.26 E-02 \quad 1.18 E-02 \quad 1.09 E-02$ 1.02E-02 $8.43 E-03$ Body Tissues 1.60E-02 1.44E-02 1.39E-02 1.24E-02 9.10E-03 6.41E-03 5.93E-03 5.85E-03 5.52E-03 5.17E-03 4.87E-03 4.08E-03

SOURCE $=$ HEART CONTENTS

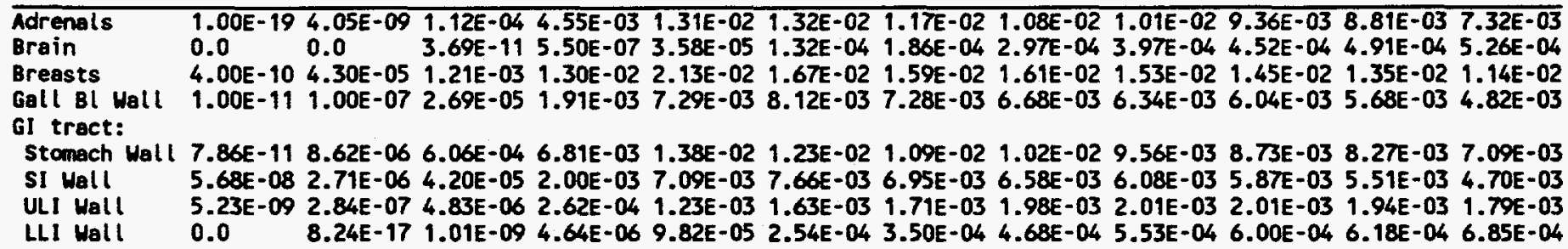

Heart Wall 2.48E-01 5.48E-01 6.86E-01 5.69E-01 2.93E-01 1.68E-01 1.60E-01 1.57E-01 1.45E-01 1.32E-01 1.22E-01 9.85E-02 Kidneys $\quad 1.00 E-17$ 1.00E-10 $1.40 E-06 \quad 4.03 E-04 \quad 2.63 E-03 \quad 3.69 E-03 \quad 3.71 E-03 \quad 3.74 E-03 \quad 3.60 E-03 \quad 3.66 E-03 \quad 3.45 E-03 \quad 2.96 E-03$ Liver $\quad 1.88 E-06$ 1.46E-04 2.26E-03 1.31E-02 $1.97 E-02$ 1.57E-02 $1.37 E-02 \quad 1.27 E-02 \quad 1.16 E-02 \quad 1.09 E-02$ 1.00E-02 8.2BE-03 Lungs 2.61E-05 3.10E-03 2.03E-02 5.51E-02 5.14E-02 3.27E-02 2.80E-02 2.59E-02 2.36E-02 2.13E-02 1.99E-02 1.62E-02

Muscle $\quad 8.61 E-05$ 1.19E-03 3.59E-03 7.48E-03 7.69E-03 5.75E-03 5.26E-03 5.10E-03 4.78E-03 4.47E-03 4.22E-03 3.53E-03

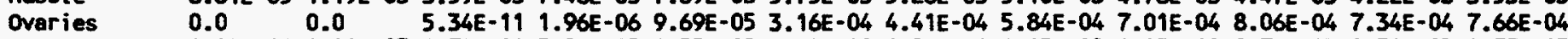
Pancreas 1.00E-16 1.00E-07 1.78E-04 5.80E-03 $1.55 E-02 \quad 1.45 E-02 \quad 1.26 E-02 \quad 1.15 E-02 \quad 1.05 E-02$ 9.79E-03 9.30E-03 7.77E-03 Skeletal Tiss:

Act Marrow 2.22E-07 4.46E-05 4.27E-04 2.52E-03 5.22E-03 5.66E-03 5.56E-03 5.51E-03 5.23E-03 4.93E-03 4.70E-03 4.00E-03 Endosteum 6.68E-07 1.45E-04 1.47E-03 9.39E-03 1.80E-02 1.15E-02 6.38E-03 4.49E-03 3.93E-03 3.62E-03 3.42E-03 2.95E-03

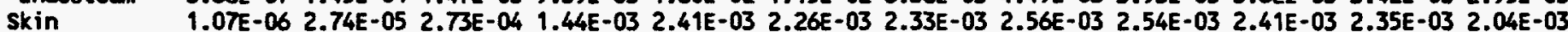
Spleen $\quad 1.00 E-23$ 1.00E-09 2.46E-05 1.69E-03 6.54E-03 7.16E-03 6.47E-03 6.20E-03 5.83E-03 5.61E-03 5.27E-03 4.49E-03

Thymus $\quad 4.32 E-05$ 4.99E-03 2.91E-02 7.94E-02 7.43E-02 4.81E-02 4.29E-02 4.08E-02 3.77E-02 3.44E-02 3.19E-02 2.59E-02 Thyroid $\quad 0.0 \quad 1.00 E-11$ 1.13E-06 $4.98 E-04$ 3.05E-03 3.80E-03 3.69E-03 3.79E-03 3.89E-03 3.96E-03 $3.69 E-03$ 3.34E-03 Urin Bl wall $0.0 \quad 0.0 \quad 3.69 E-13$ 1.61E-07 1.92E-05 $1.19 E-04$ 1.83E-04 2.65E-04 3.48E-04 4.00E-04 4.12E-04 4.13E-04

Uterine Cont: Fetus 0.0 Skeleton 0.0 Soft Tissue 0.0 Uterine Hall 0.0 $\begin{array}{llllllllllllllll}0.0 & 1.00 E-07 & 3.16 E-05 & 5.18 E-04 & 1.08 E-03 & 1.14 E-03 & 1.25 E-03 & 1.32 E-03 & 1.35 E-03 & 1.35 E-03 & 1.23 E-03\end{array}$ 0.0 2.56E-07 4.78E-05 9.34E-04 $1.79 \mathrm{E}-03$ 1.46E-03 $1.27 \mathrm{E}-03$ 1.19E-03 $1.17 \mathrm{E}-03$ 1.20E-03 $1.08 \mathrm{E}-03$ 0.04 4.86E-08 3.02E-05 $4.64 E-04 \quad 9.83 E-04 \quad 1.09 E-03 \quad 1.25 E-03 \quad 1.34 E-03 \quad 1.37 E-03 \quad 1.37 E-03 \quad 1.26 E-03$ 1.00E-11 3.95E-07 1.17E-04 8.64E-04 1.31E-03 1.38E-03 1.48E-03 1.60E-03 1.54E-03 1.57E-03 1.42E-03 Body Tissues 1.7TE-02 1.7TE-02 1.76E-02 1.66E-02 1.27E-02 8.64E-03 7.74E-03 7.48E-03 6.94E-03 6.44E-03 6.04E-03 5.00E-03 
Table A.4 (cont'd). At Nine Months Gestation: Specific Absorbed Fraction of Photon Energy ( $\left.\mathrm{kg}^{-1}\right)$.

SOURCE = HEART WALL

Energy (MeV)

\begin{tabular}{|c|c|c|c|c|c|c|c|c|c|c|c|c|}
\hline rget & .010 & 015 & .020 & .030 & .050 & 100 & 200 & .500 & .000 & 1.500 & 2.000 & 4.000 \\
\hline $\begin{array}{l}\text { Adrenals } \\
\text { Brain } \\
\text { Breasts } \\
\text { Gall Bl Wall } \\
\text { G1 tract: }\end{array}$ & $\begin{array}{l}1.00 E-17 \\
0.0 \\
2.38 E-06 \\
1.00 E-10\end{array}$ & $\begin{array}{l}0.0 \\
1.52 E-04 \\
5.00 E-07\end{array}$ & $\begin{array}{l}2.34 E-04 \\
3.08 E-11 \\
2.91 E-03 \\
7.07 E-05\end{array}$ & $\begin{array}{l}6.21 \\
4.71 \\
1.82 \\
2.68\end{array}$ & $\begin{array}{l}3.1 \\
2.4 \\
8.6\end{array}$ & $\begin{array}{l}1.44 \\
1.12 \\
1.87 \\
9.08\end{array}$ & $\begin{array}{l}1.6 \\
1.7 \\
8.0\end{array}$ & $\begin{array}{l}2.8 \\
1.8 \\
7.8\end{array}$ & $\begin{array}{l}3.6 \\
1.7\end{array}$ & $\frac{14}{12}$ & 4 & 1. \\
\hline $\begin{array}{l}\text { Stomach Hall } \\
\text { SI Wall } \\
\text { ULI Wall } \\
\text { LLI Wall }\end{array}$ & $\begin{array}{l}1.00 E-05 \\
4.71 E-07 \\
0.0 \\
0.0\end{array}$ & $\begin{array}{l}3.158 \\
1.25 \\
1.008 \\
8.10\end{array}$ & $\begin{array}{l}4 . \\
1 . \\
1 . \\
3 .\end{array}$ & $\begin{array}{l}1 . \\
3 . \\
3 . \\
9 .\end{array}$ & $\begin{array}{l}2 . \\
9 . \\
1 . \\
1 .\end{array}$ & & & $\begin{array}{l}1.56 E-02 \\
7.96 E-03 \\
2.21 E-03 \\
5.50 E-04\end{array}$ & & & & \\
\hline
\end{tabular}

Heart Wall 3.49E+00 2.54E+00 1.79E+00 9.29E-01 4.02E-01 2.35E-01 2.36E-01 2.46E-01 2.25E-01 2.03E-01 1.87E-01 1.52E-01 Kicheys 2.00E-26 2.00E-10 2.80E-06 5.59E-04 3.18E-03 4.32E-03 4.10E-03 4.13E-03 4.00E-03 3.93E-03 3.80E-03 3.35E-03 1.17E-05 1.08E-03 5.75E-03 $1.75 E-02$ 2.14E-02 $1.64 E-02$ 1.46E-02 $1.37 E-02$ 1.26E-02 $1.16 E-02$ 1.08E-02 9.07E-03 Liver

Lungs 2.10E-04 7.13E-03 2.72E-02 5.56E-02 4.83E-02 3.06E-02 2.63E-02 2.48E-02 2.24E-02 2.05E-02 1.90E-02 1.54E-02

Muscle

Ovaries

Pancreas

Skeletal Tiss:

Act Marrow

Endosteum

1.83E-03 4.51E-03 6.83E-03 9.10E-03 8.07E-03 5.91E-03 5.47E-03 5.36E-03 5.04E-03 4.70E-03 4-42E-03 3.71E-03 $0.0 \quad 1.00 E-19$ 1.00E-10 2.43E-06 1.23E-04 4.06E-04 5.29E-04 6.65E-04 8.27E-04 7.88E-04 8.72E-04 7.90E-04 1.00E-14 2.92E-08 6.42E-04 1.09E-02 2.21E-02 1.87E-02 1.60E-02 1.48E-02 1.36E-02 1.25E-02 1.17E-02 9.58E-03

skin

4.11E-06 1.44E-04 7.16E-04 2.77E-03 5.07E-03 5.36E-03 5.29E-03 5.30E-03 5.05E-03 4.81E-03 4.56E-03 3.90E-03 1.24E-05 4.76E-04 2.51E-03 1.05E-02 1.75E-02 1.09E-02 6.07E-03 4.33E-03 3.82E-03 3.55E-03 3.34E-03 2.87E-03

Spleen 1.00E-05 9.63E-05 4.99E-04 1.80E-03 2.58E-03 2.33E-03 2.42E-03 2.68E-03 2.62E-03 2.51E-03 2.44E-03 2.10E-03 1.00E-20 1.00E-07 8.10E-05 3.21E-03 9.64E-03 9.38E-03 8.40E-03 7.94E-03 7.29E-03 6.85E-03 6.42E-03 5.51E-03

Thyous

Thyroid

4.56E-04 9.39E-03 3.11E-02 6.71E-02 6.12E-02 4.03E-02 3.66E-02 3.48E-02 3.19E-02 2.96E-02 2.72E-02 2.25E-02

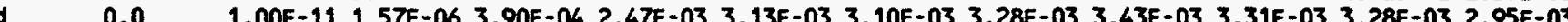

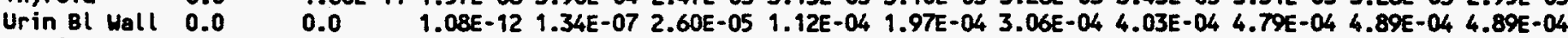
Uterine Cont:

Fetus $\quad 0.0$

Skeleton 0.0

Soft Tissue 0.0

Uterine Mall 0.0

1.00E- $11 \quad 1.18 E-07 \quad 5.53 E-05 \quad 6.95 E-04 \quad 1.32 E-03 \quad 1.37 E-03 \quad 1.45 E-03 \quad 1.51 E-03 \quad 1.55 E-03 \quad 1.48 E-03 \quad 1.35 E-03$ $\begin{array}{llllllllllll}0.0 & 0.0 & 8.27 E-05 & 1.26 E-03 & 2.11 E-03 & 1.75 E-03 & 1.47 E-03 & 1.27 E-03 & 1.35 E-03 & 1.30 E-03 & 1.20 E-03\end{array}$

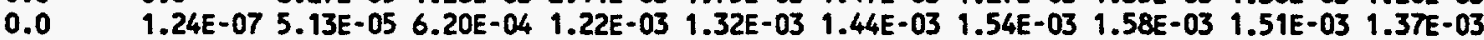
1.00E-10 $1.23 E-06 \quad 1.94 E-04$ 1.14E-03 $1.62 E-03 \quad 1.67 E-03$ 1.74E-03 $1.79 E-03 \quad 1.78 E-03 \quad 1.72 E-03 \quad 1.64 E-03$ Body Tissues 1.77E-02 1.77E-02 1.76E-02 1.64E-02 1.23E-02 8.38E-03 7.54E-03 7.31E-03 6.82E-03 6.32E-03 5.93E-03 4.93E-03

SOURCE $=$ KIDNEYS

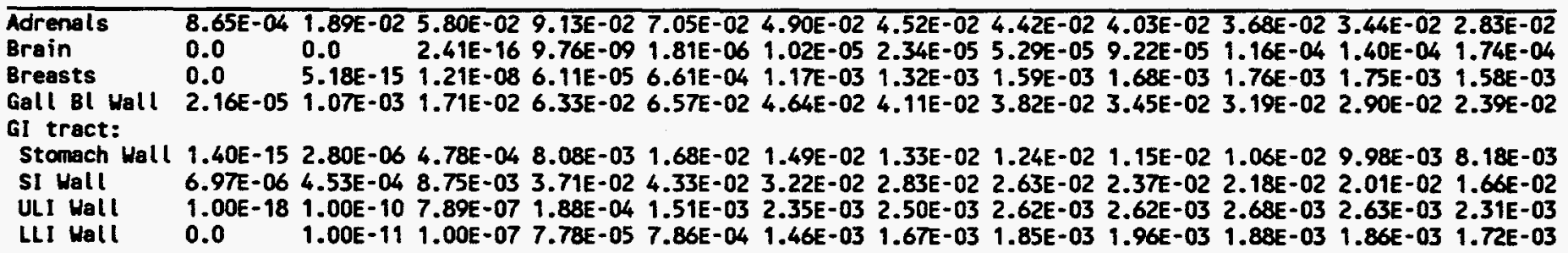

Heart Wall

Kicheys

Liver

2.00E-26 2.00E-10 2.80E-06 5.59E-04 3.18E-03 4.32E-03 4.10E-03 4.13E-03 4.00E-03 3.93E-03 3.80E-03 3.35E-03

Lungs $3.42 E+00$ 2.88E+00 2.15E+00 $1.09 E+00$ 4.27E-01 2.48E-01 2.59E-01 2.67E-01 2.46E-01 2.27E-01 2.11E-01 $1.72 E-01$ 1.10E-05 2.86E-04 2.88E-03 1.39E-02 2.01E-02 1.66 E-02 $1.48 E-02 \quad 1.39 E-02 \quad 1.30 E-02 \quad 1.20 E-02 \quad 1.14 E-02 \quad 9.35 E-03$ 1.00E-11 1.00E-07 4.59E-05 1.34E-03 3.83E-03 4.09E-03 3.91E-03 3.96E-03 3.93E-03 3.75E-03 3.59E-03 3.06E-03

Muscle

Ovaries

Pancreas

1.62E-03 4.99E-03 8.40E-03 9.79E-03 7.36E-03 5.51E-03 5.33E-03 5.37E-03 5.13E-03 4.82E-03 4.54E-03 3.83E-03 1.00E-27 1.00E-10 4.68E-06 5.74E-04 3.11E-03 4.39E-03 4.40E-03 4.49E-03 4.43E-03 4.16E-03 3.90E-03 3.61E-03 Skeletal Tiss:

Act Marrow

Endosteum

Skin

Spleen

4.09E-12 6.31E-05 4.00E-03 3.00E-02 4.17E-02 3.19E-02 2.77E-02 2.63E-02 2.39E-02 2.21E-02 2.05E-02 1.66E-02

4.36E-06 3.43E-04 1.88E-03 6.33E-03 9.91E-03 9.94E-03 9.95E-03 9.87E-03 9.29E-03 8.72E-03 8.22E-03 6.94E-03 8.52E-06 7.72E-04 4.62E-03 1.63E-02 2.25E-02 1.25E-02 7.18E-03 5.34E-03 4.77E-03 4.41E-03 4.17E-03 3.59E-03 1.61E-05 2.12E-04 1.32E-03 2.86E-03 2.54E-03 2.09E-03 2.24E-03 2.51E-03 2.55E-03 2.40E-03 2.39E-03 2.07E-03

Thymus $\quad 0.0 \quad 1.00 E-16 \quad 8.34 E-10 \quad 2.31 E-05 \quad 4.38 E-04 \quad 8.78 E-04 \quad 9.88 E-04 \quad 1.21 E-03 \quad 1.39 E-03 \quad 1.38 E-03 \quad 1.46 E-03 \quad 1.32 E-03$ Thyroid $\quad 0.0 \quad 0.0 \quad 9.85 E-13$ 2.06E-06 $6.63 E-05 \quad 1.92 E-04 \quad 2.58 E-04 \quad 3.20 E-04 \quad 4.67 E-04 \quad 5.29 E-04$ 5.53E-04 $5.56 E-04$ Urin Bl Wall $0.0 \quad 1.00 E-16 \quad 9.49 E-10 \quad 7.71 E-06 \quad 3.04 E-04 \quad 8.11 E-04 \quad 1.03 E-03 \quad 1.32 E-03 \quad 1.34 E-03 \quad 1.33 E-03 \quad 1.37 E-03 \quad 1.40 E-03$

Uterine Cont:

Fetus $\quad 0.0$

Skeleton $\quad 0.0$

Soft Tissue 0.0

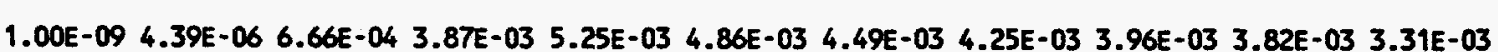
1.00E-09 1.01E-05 1.66E-03 9.23E-03 1.02E-02 7.13E-03 5.07E-03 4.38E-03 4.05E-03 3.97E-03 3.35E-03

1.00E-10 3.75E-06 5.34E-04 3.15E-03 4.59E-03 4.54E-03 4.40E-03 4.22E-03 3.94E-03 3.79E-03 3.29E-03 Uterine wall 1.00E-09 1.00E-06 1.13E-04 2.05E-03 5.16E-03 5.55E-03 5.18E-03 5.00E-03 4.80E-03 4.50E-03 4.24E-03 3.68E-03 Body Tissues 1.77E-02 1.76E-02 1.72E-02 1.49E-02 1.06E-02 7.36E-03 6.77E-03 6.68E-03 6.31E-03 5.89E-03 5.53E-03 4.63E-03 
Table A.4 (cont'd). At Nine Months Gestation: Specific Absorbed Fraction of Photon Energy (kg-1).

SOURCE = LIVER $\quad$ Energy (MeV)

Target $\quad$\begin{tabular}{lllllllllllll}
\hline 0.010 & 0.015 & 0.020 & 0.030 & 0.050 & 0.100 & 0.200 & 0.500 & 1.000 & 1.500 & 2.000 & 4.000
\end{tabular}

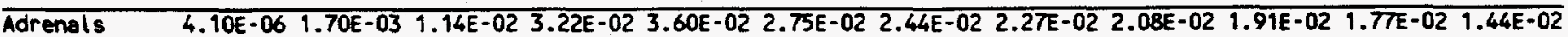

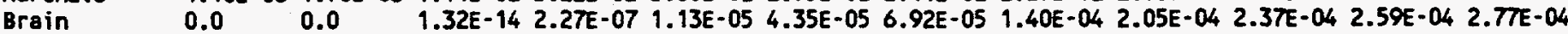
Breasts $\quad 1.00 E-09$ 1.00E-06 1.16E-04 2.34E-03 5.63E-03 5.33E-03 5.13E-03 5.43E-03 5.28E-03 5.07E-03 4.86E-03 4.14E-03 Gall Bl Wall 4.11E-06 3.19E-03 2.32E-02 7.077-02 7.25E-02 4.92E-02 4.24E-02 3.88E-02 3.51E-02 3.20E-02 2.96E-02 2.43E-02 GI tract:

Stomech Hall $1.78 E-06 \quad 7.76 E-05$ 1.13E-03 7.06E-03 1.28E-02 $1.14 E-02 \quad 1.02 E-02 \quad 9.67 E-03 \quad 8.90 E-03 \quad 8.26 E-03$ 7.78E-03 6.53E-03 s1 Wall 6.01E-03 1.83E-02 3.47E-02 5.49E-02 4.91E-02 3.42E-02 3.02E-02 2.81E-02 2.54E-02 2.32E-02 2.16E-02 1.77E-02 ULI Mall 2.34E-07 1.21E-05 2.00E-04 2.38E-03 5.8BE-03 5.89E-03 5.59E-03 5.71E-03 5.52E-03 5.27E-03 5.07E-03 4.38E-03 LLI Wall $\quad 0.0 \quad 1.00 E-14 \quad 4.36 E-09$ 1.25E-05 2.62E-04 6.37E-04 7.78E-04 9.63E-04 $1.05 E-03 \quad 1.10 E-03 \quad 1.09 E-03 \quad 1.04 E-03$

Heart Mall 1.17E-05 1.08E-03 5.75E-03 1.75E-02 2.14E-02 1.64E-02 1.46E-02 1.37E-02 1.26E-02 1.16E-02 1.08E-02 9.07E-03

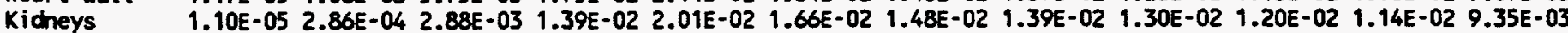
Liver 6.85E-01 6.32E-01 5.43E-01 3.64E-01 1.85E-01 1.11E-01 1.06E-01 1.07E-01 9.83E-02 B.96E-02 8.29E-02 6.80E-02

Lungs 2.63E-04 5.45E-03 1.53E-02 2.52E-02 2.14E-02 1.45E-02 1.27E-02 1.19E-02 1.09E-02 1.00E-02 9.41E-03 7.62E-03

Muscle

Ovaries

Pancreas

Skeletal Tiss:

Act Marrow

Endosteum

skin

6.33E-04 1.88E-03 3.46E-03 5.56E-03 5.59E-03 4.45E-03 4.19E-03 4.18E-03 3.98E-03 3.76E-03 3.56E-03 3.01E-03

Spleen

1.00E-16 1.00E-10 8.57E-07 1.83E-04 1.40E-03 2.30E-03 2.37E-03 2.45E-03 2.53E-03 2.36E-03 2.35E-03 2.12E-03 3.92E-06 2.35E-04 4.29E-03 2.32E-02 3.22E-02 2.48E-02 2.15E-02 1.97E-02 1.77E-02 $1.63 E-02 \quad 1.50 E-02 \quad 1.25 E-02$

4.55E-05 3.14E-04 1.03E-03 2.72E-03 4.41E-03 4.81E-03 4.84E-03 4.89E-03 4.70E-03 4.46E-03 4.22E-03 3.64E-03 1.36E-04 $1.09 E-03$ 4.11E-03 1.24E-02 $1.69 E-02$ 9.81E-03 5.59E-03 4.10E-03 3.66E-03 3.40E-03 $3.18 E-03$ 2.77E-03

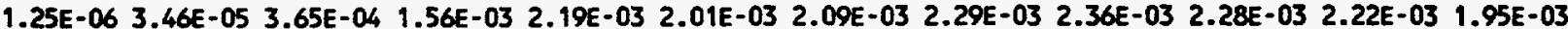
1.00E-22 1.00E-09 9.44E-06 9.31E-04 4.44E-03 5.56E-03 5.21E-03 5.07E-03 4.89E-03 4.59E-03 4.37E-03 3.81E-03

Thyons $\quad 2.96 E-15 \quad 6.96 E-10 \quad 7.20 E-06 \quad 7.92 E-04 \quad 3.44 E-03 \quad 3.97 E-03 \quad 3.74 E-03 \quad 3.80 E-03 \quad 3.78 E-03 \quad 3.62 E-03 \quad 3.44 E-03 \quad 2.99 E-03$ Thyroid $\quad 0.0 \quad 1.00 E-13 \quad 3.55 E-08$ 3.75E-05 $4.02 E-04 \quad 7.13 E-04$ 8.05E-04 $9.72 E-04 \quad 1.08 E-03 \quad 1.11 E-03 \quad 1.11 E-03 \quad 1.00 E-03$ Urin Bl Wall $0.0 \quad 1.00 E-16 \quad 1.00 E-09$ 6.56E-06 2.25E-04 6.28E-04 7.74E-04 9.29E-04 1.06E-03 1.07E-03 1.13E-03 1.04E-03 Uterine Cont:

Fetus

skeleton

Soft Tissue 0.0 Uterine Hall 6.64E-04 1.93E-03 3.53E-03 6.37E-03 7.88E-03 6.86E-03 6.30E-03 6.01E-03 5.78E-03 5.33E-03 5.10E-03 4.34E-03 Body Tissues 1.76E-02 1.76E-02 1.73E-02 1.57E-02 1.15E-02 7.88E-03 7.17E-03 7.00E-03 6.54E-03 6.09E-03 5.70E-03 4.74E-03

SOURCE $=$ LUNGS

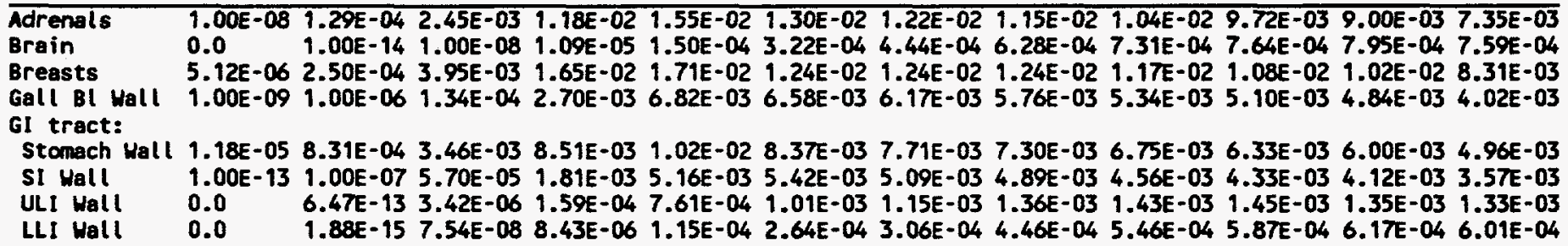

Heart Mall

Kichers

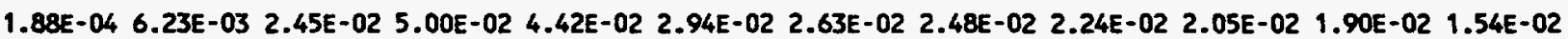
1.00E-10 1.00E-07 4.10E-05 1.24E-03 3.50E-03 3.91E-03 3.91E-03 3.96E-03 3.93E-03 3.75E-03 3.59E-03 3.06E-03 Liver 2.36E-04 4.85E-03 1.37E-02 2.27E-02 1.97E-02 1.39E-02 $1.27 E-02 \quad 1.19 E-02$ 1.09E-02 $1.00 E-02$ 9.41E-03 $7.62 E-03$

Lungs $1.38 E+00$ 1.07E+00 7.29E-01 3.36E-01 1.26E-01 6.93E-02 6.87E-02 6.93E-02 6.28E-02 5.74E-02 5.28E-02 4.19E-02

Muscle

Ovaries

Pancreas

Skeletal Tiss:

Act Marrow

Endosteum

2.56E-03 7.27E-03 1.14E-02 1.25E-02 8.71E-03 5.93E-03 5.60E-03 5.51E-03 5.12E-03 4.76E-03 4.45E-03 3.68E-03 $0.0 \quad 1.00 E-191.00 E-10$ 2.92E-06 9.27E-05 2.87E-04 3.96E-04 5.12E-04 6.40E-04 7.23E-04 6.75E-04 6.87E-04 5.00E-08 1.00E-05 5.67E-04 6.87E-03 1.24E-02 1.06E-02 9.56E-03 8.74E-03 7.99E-03 7.41E-03 6.98E-03 5.80E-03

Skin

2.40E-06 2.12E-04 1.42E-03 4.89E-03 6.76E-03 6.37E-03 6.27E-03 6.25E-03 5.92E-03 5.49E-03 5.21E-03 4.38E-03 7.17E-06 7.14E-04 5.27E-03 2.03E-02 2.52E-02 1.29E-02 7.16E-03 5.40E-03 4.81E-03 4.42E-03 4.15E-03 3.50E-03 Spleen 1.05E-06 4.04E-05 5.37E-04 2.34E-03 2.89E-03 2.48E-03 2.65E-03 2.90E-03 2.87E-03 2.72E-03 2.57E-03 2.19E-03 5.16E-06 1.82E-04 2.28E-03 9.27E-03 1.14E-02 8.98E-03 8.32E-03 7.84E-03 7.33E-03 6.83E-03 6.26E-03 5.29E-03

Thymus

Thyroid

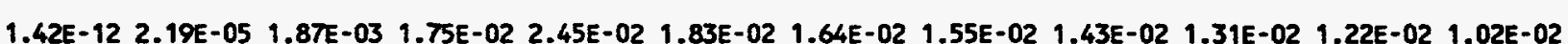
Uterine Cont:

Fetus 0.0

Skeleton 0.0

Soft Tissue 0.0

Uterine Mall 0.0

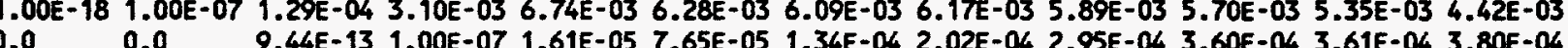

Body Tissues 1.77

1.30E-16 6.50E-09 2.75E-05 3.87E-04 7.87E-04 8.62E-04 9.79E-04 1.05E-03 1.06E-03 1.05E-03 9.75E-04 0.0 4.55E-08 $4.12 E-05$ 7.30E-04 1.31E-03 $1.10 E-03$ 9.12E-04 9.34E-04 9.63E-04 9.40E-04 8.73E-04 0.0 4.55E-08 2.57E-05 3.41E-04 7.17E-04 8.28E-04 9.67E-04 1.06E-03 1.07E-03 1.07E-03 9.71E-04 1.00E-11 5.71E-07 9.71E-05 6.15E-04 9.19E-04 9.87E-04 1.10E-03 1.18E-03 1.23E-03 1.21E-03 $1.10 E-03$ 
Table A.4 (cont'd). At Nine Months Gestation: Specific Absorbed Fraction of Photon Energy (kg').

SOURCE $=$ MUSCLE

Energy (MeV)

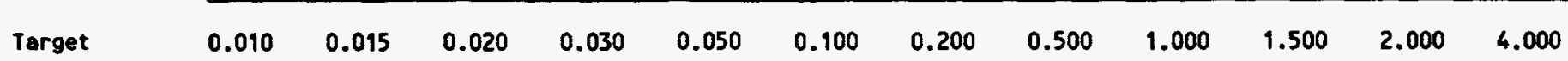

Adrenals $\quad 5.26 E-031.20 E-02 \quad 1.48 E-02$ 1.29E-02 8.78E-03 6.58E-03 6.38E-03 6.36E-03 5.99E-03 5.61E-03 5.25E-03 4.39E-03

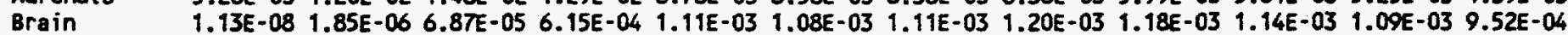

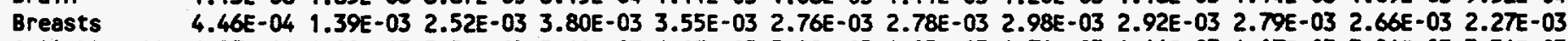

Gall BI Wall 6.27E-04 1.98E-03 3.54E-03 5.60E-03 6.17E-03 5.22E-03 4.85E-03 4.71E-03 4.44E-03 4.17E-03 3.94E-03 3.34E-03

Gl tract:

Stomach Wall 1.71E-03 4.29E-03 6.43E-03 8.47E-03 7.63E-03 5.78E-03 5.37E-03 5.27E-03 4.96E-03 4.64E-03 4.38E-03 3.68E-03

S1 Wall 7.39E-04 2.10E-03 3.70E-03 6.01E-03 6.47E-03 5.31E-03 4.93E-03 4.78E-03 4.51E-03 4.24E-03 4.01E-03 3.39E-03

ULI Wall 4.61E-03 8.81E-03 $1.04 E-02 \quad 8.98 E-03 \quad 5.68 E-03 \quad 4.03 E-03 \quad 4.03 E-03 \quad 4.23 E-03 \quad 4.08 E-03 \quad 3.87 E-03 \quad 3.65 E-03 \quad 3.09 E-03$

LLI Wall 3.48E-03 7.86E-03 1.09E-02 1.20E-02 9.17E-03 6.62E-03 6.31E-03 6.29E-03 5.94E-03 5.58E-03 5.24E-03 4.39E-03

Heart Wall 1.83E-03 4.51E-03 6.83E-03 9.10E-03 8.07E-03 5.91E-03 5.47E-03 5.36E-03 5.04E-03 4.70E-03 4.42E-03 3.71E-03

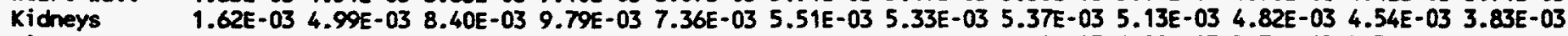
Liver $\quad 6.33 E-04 \quad 1.88 E-03 \quad 3.46 E-03 \quad 5.56 E-03 \quad 5.59 E-03 \quad 4.45 E-03 \quad 4.19 E-03 \quad 4.18 E-03 \quad 3.98 E-03 \quad 3.76 E-03 \quad 3.56 E-03 \quad 3.01 E-03$ Lungs $\quad 2.82 E-03$ 7.96E-03 $1.26 E-02 \quad 1.39 E-02$ 9.56E-03 6.12E-03 5.60E-03 5.51E-03 5.12E-03 4.76E-03 4.45E-03 3.68E-03

Muscle $\quad 2.41 E-02$ 2.22E-02 1.94E-02 1.40E-02 8.35E-03 5.83E-03 5.81E-03 6.02E-03 5.71E-03 5.34E-03 5.01E-03 4.17E-03 Ovaries $\quad 4.14 E-03$ 9.98E-03 $1.28 E-02$ 1.23E-02 9.83E-03 7.62E-03 7.22E-03 7.04E-03 6.58E-03 6.14E-03 5.7TE-03 4.81E-03 Pancreas $\quad 1.31 E-03$ 3.67E-03 6.12E-03 8.52E-03 7.98E-03 6.19E-03 5.74E-03 5.55E-03 5.19E-03 4.86E-03 4.57E-03 3.84E-03 Skeletal Tiss:

Act Harrow 6.19E-04 1.57E-03 2.92E-03 4.95E-03 5.74E-03 5.36E-03 5.26E-03 5.34E-03 5.07E-03 4.76E-03 4.49E-03 3.77E-03 Endosteum 2.11E-03 6.63E-03 1.33E-02 2.45E-02 2.55E-02 1.26E-02 7.33E-03 5.82E-03 5.29E-03 4.87E-03 4.60E-03 3.87E-03 Skin $\quad 5.35 E-03$ 8.16E-03 8.62E-03 7.07E-03 4.41E-03 3.22E-03 3.41E-03 3.73E-03 3.68E-03 3.50E-03 3.31E-03 2.80E-03 Spleen $\quad 1.42 E-03$ 4.38E-03 7.92E-03 1.08E-02 8.65E-03 6.14E-03 5.78E-03 5.75E-03 5.41E-03 5.06E-03 4.76E-03 3.98E-03

Thymus $\quad 2.71 E-03 \quad 7.85 E-03$ 1.21E-02 1.33E-02 9.52E-03 6.44E-03 6.10E-03 6.11E-03 5.76E-03 5.36E-03 5.01E-03 4.18E-03 Thyroid $\quad 4.32 E-03$ 1.03E-02 1.34E-02 1.26E-02 8.21E-03 5.56E-03 5.47E-03 5.62E-03 5.34E-03 4.96E-03 4.66E-03 3.86E-03 Urin Bl Mall 5.4TE-03 9.38E-03 1.12E-02 1.08E-02 7.93E-03 5.85E-03 5.66E-03 5.75E-03 5.49E-03 5.15E-03 4.86E-03 4.09E-03 Uterine Cont:

fetus

Skeleton 6.225

1.61E-06 3.17E-04 2.06E-03 8.23E-03 1.35E-02 9.40E-03 5.95E-03 4.45E-03 3.89E-03 3.68E-03 $3.50 E-032.98 E-03$ Soft Tissue 5.86E-07 2.72E-05 2.97E-04 1.86E-03 3.74E-03 3.77E-03 3.56E-03 3.48E-03 3.32E-03 3.16E-03 3.02E-03 2.60E-03 Uterine Wall 2.01E-03 4.93E-03 6.70E-03 7.24E-03 5.94E-03 4.64E-03 4.46E-03 4.47E-03 4.27E-03 4.02E-03 3.81E-03 3.23E-03 Body Tissues 1.76E-02 1.70E-02 1.59E-02 1.32E-02 8.92E-03 5.89E-03 5.47E-03 5.52E-03 5.21E-03 4.89E-03 4.59E-03 3.83E-03

\section{SOURCE = OVARIES}

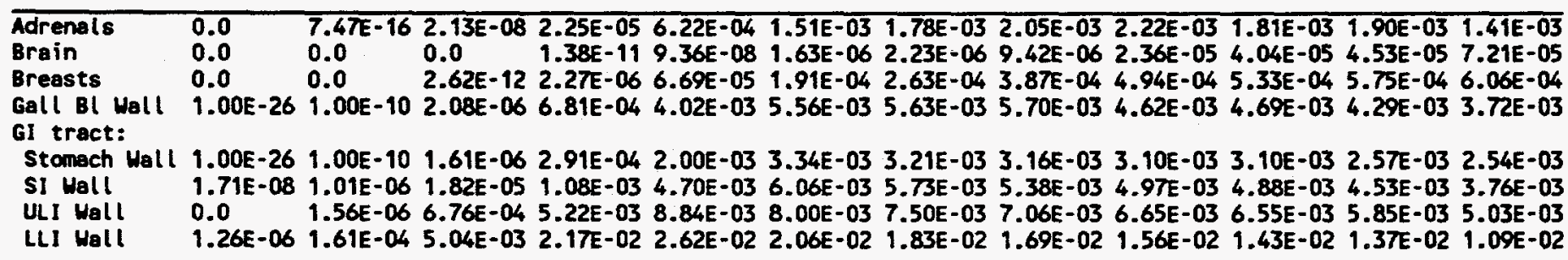

Heart Wall $0.0 \quad 1.00 E-19$ 1.00E-10 2.43E-06 1.23E-04 4.06E-04 5.29E-04 6.65E-04 8.27E-04 7.88E-04 8.72E-04 7.90E-04 Kidneys $\quad 1.00 E-27$ 1.00E-10 4.68E-06 5.74E-04 3.11E-03 $4.39 E-03 \quad 4.40 E-03 \quad 4.49 E-03 \quad 4.43 E-03 \quad 4.16 E-03$ 3.90E-03 $3.61 E-03$

Liver

Lungs

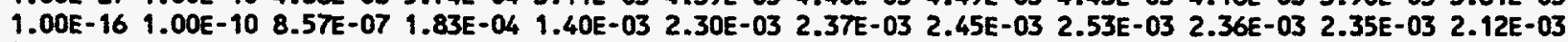

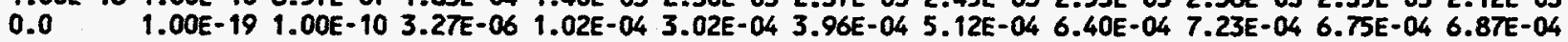

Muscle $\quad 4.14 E-03 \quad 9.98 E-03 \quad 1.28 E-02 \quad 1.23 E-02 \quad 9.83 E-03 \quad 7.62 E-03 \quad 7.22 E-03 \quad 7.04 E-03 \quad 6.58 E-03 \quad 6.14 E-03 \quad 5.77 E-03 \quad 4.81 E-03$ Ovaries $\quad 7.02 E+01 \quad 4.52 E+01 \quad 2.61 E+019.68 E+00 \quad 3.09 E+00 \quad 1.82 E+00 \quad 1.97 E+00 \quad 2.16 E+00 \quad 2.07 E+00 \quad 1.88 E+00 \quad 1.76 E+00 \quad 1.41 E+00$

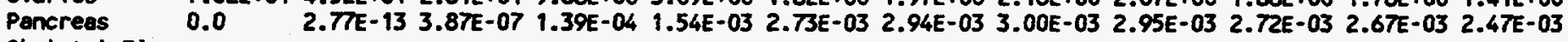
Skeletal Tiss:

$\begin{array}{lllllllllll}\text { Act Marrow 1.73E-05 4.46E-04 4.48E-03 } 1.49 E-02 & 1.78 E-02 & 1.53 E-02 & 1.44 E-02 & 1.37 E-02 & 1.26 E-02 & 1.17 E-02 & 1.09 E-02 & 9.00 E-03\end{array}$ Endosteum 2.29E-05 6.52E-04 7.01E-03 2.51E-02 2.78E-02 $1.42 E-02$ 7.90E-03 5.61E-03 $4.86 E-03$ 4.49E-03 4.20E-03 3.57E-03 Skin

Spleen

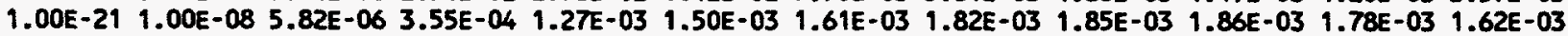

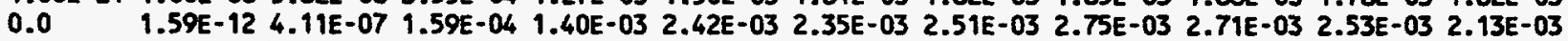

Thymus $\quad 0.0 \quad 0.0 \quad 1.00 E-14$ 1.31E-07 1.95E-05 7.79E-05 1.56E-04 2.71E-04 3.02E-04 3.61E-04 4.00E-04 4.15E-04

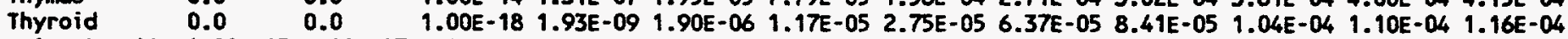
Urin Bl Wall 1.00E-15 1.00E-07 8.11E-05 4.04E-03 1.35E-02 1.38E-02 1.28E-02 1.16E-02 1.06E-02 1.00E-02 9.67E-03 7.84E-03 Uterine Cont:

Fetus

2.95E-06 1.85E-03 1.24E-02 3.78E-02 4.39E-02 3.08E-02 2.48E-02 2.19E-02 1.96E-02 $1.79 E-02 \quad 1.67 E-02 \quad 1.36 E-02$ Skeleton $1.41 E-05$ 1.11E-02 6.60E-02 $1.54 E-01$ 1.45E-01 7.21E-02 4.34E-02 3.28E-02 2.85E-02 $2.57 E-02$ 2.42E-02 $1.94 E-02$ Soft Tissue 1.46E-06 6.12E-04 5.23E-03 2.22E-02 3.04E-02 2.53E-02 2.23E-02 2.05E-02 $1.84 E-02 \quad 1.69 E-02$ 1.57E-02 $1.28 E-02$ Uterine Wall 5.65E-03 3.59E-02 5.95E-02 5.81E-02 3.82E-02 2.64E-02 2.46E-02 2.36E-02 2.15E-02 1.98E-02 1.84E-02 $1.48 E-02$ Body Tissues 1.77E-02 1.74E-02 1.64E-02 1.42E-02 1.09E-02 7.82E-03 7.05E-03 $6.78 E-03 \quad 6.32 E-03 \quad 5.89 E-03$ 5.53E-03 $4.61 E-03$ 
Table A.4 (cont'd). At Nine Months Gestation: Specific Absorbed Fraction of Photon Energy (kg').

SOURCE = PANCREAS

Energy (HeV)

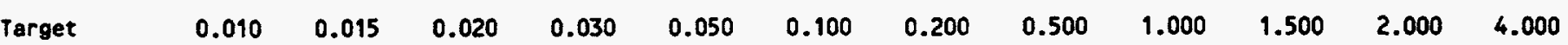

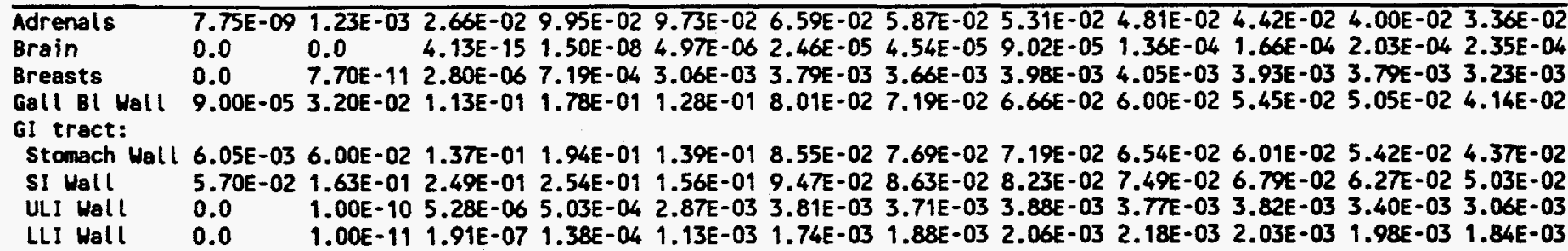

Heart Hall $1.00 E-14 \quad 2.92 E-08 \quad 6.42 E-04 \quad 1.09 E-02 \quad 2.21 E-02 \quad 1.87 E-02 \quad 1.60 E-02 \quad 1.48 E-02 \quad 1.36 E-02 \quad 1.25 E-02$ 1.17E-02 9.58E-03

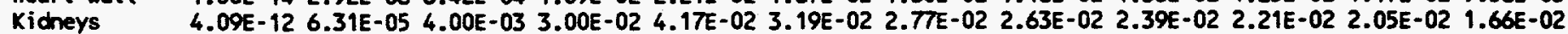
Liver $\quad 3.92 E-06$ 2.35E-04 4.29E-03 2.32E-02 3.22E-02 2.48E-02 2.15E-02 $1.97 E-02 \quad 1.77$ E-02 $1.63 E-02$ 1.50E-02 $1.25 E-02$ Lungs $\quad 0.0 \quad 4.00 E-06$ 6.35E-04 7.38E-03 $1.33 E-02$ 1.10E-02 9.56E-03 8.74E-03 7.99E-03 7.41E-03 6.98E-03 5.80E-03

Muscle $\quad 1.31 E-03 \quad 3.67 E-03 \quad 6.12 E-03 \quad 8.52 E-03 \quad 7.98 E-03 \quad 6.19 E-03 \quad 5.74 E-03$ 5.55E-03 5.19E-03 4.86E-03 4.57E-03 3.84E-03

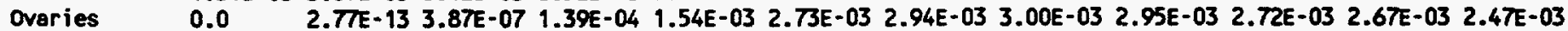
Pancreas 1.07E+01 8.31E+00 5.78E+00 2.66E+00 9.77E-01 5.63E-01 5.96E-01 6.31E-01 5.91E-01 5.35E-01 4.88E-01 3.84E-01 Skeletal Tiss:

Act Marrow 7.99E-07 3.35E-05 4.74E-04 3.14E-03 6.76E-03 7.61E-03 7.36E-03 7.30E-03 6.75E-03 6.30E-03 5.98E-03 5.16E-03 Endosteum 1.75E-06 8.80E-05 $1.42 E-03 \quad 1.02 E-02 \quad 1.96 E-02 \quad 1.27 E-02 \quad 7.09 E-03 \quad 4.86 E-03 \quad 4.25 E-03 \quad 3.93 E-03 \quad 3.71 E-03 \quad 3.23 E-03$ Skin $\quad 1.00 E-101.00 E-07 \quad 1.53 E-05 \quad 4.57 E-04 \quad 1.37 E-03 \quad 1.58 E-03 \quad 1.68 E-03 \quad 1.85 E-03 \quad 1.90 E-03 \quad 1.88 E-03 \quad 1.81 E-03 \quad 1.62 E-03$ Spleen $\quad 1.38 E-03$ 4.09E-02 1.27E-01 1.86E-01 1.31E-01 8.10E-02 7.20E-02 6.81E-02 6.42E-02 5.84E-02 5.35E-02 4.24E-02

Thymus $\quad 0.0 \quad 1.00 E-12 \quad 8.36 E-08 \quad 2.40 E-04 \quad 2.09 E-03 \quad 3.07 E-03 \quad 2.99 E-03 \quad 2.95 E-03 \quad 2.94 E-03 \quad 3.17 E-03 \quad 3.23 E-03 \quad 2.71 E-03$ Thyroid $\quad 0.0 \quad 0.0 \quad 3.91 E-114.79 E-06 \quad 1.69 E-04 \quad 4.67 E-04$ 5.55E-04 6.99E-04 8.81E-04 8.92E-04 8.54E-04 8.31E-04 Urin Bl Wall $0.0 \quad 1.00 E-18$ 2.40E-10 4.09E-06 2.46E-04 7.36E-04 9.69E-04 $1.19 E-03 \quad 1.16 E-03 \quad 1.08 E-03 \quad 1.27 E-03 \quad 1.04 E-03$ Uterine Cont:

Fetus $\quad 0.0$

Soft Uterine Wall 1.00E-08 1.00E-05 8.40E-04 5.33E-03 9.02E-03 8.17E-03 7.28E-03 6.77E-03 6.22E-03 5.90E-03 5.51E-03 4.63E-03 Body Tissues 1.74E-02 1.67E-02 1.59E-02 1.49E-02 1.22E-02 8.81E-03 7.84E-03 7.45E-03 6.90E-03 6.41E-03 5.99E-03 4.98E-03

SOURCE $=$ SKELETAL TISSUES: ACTIVE MARROW

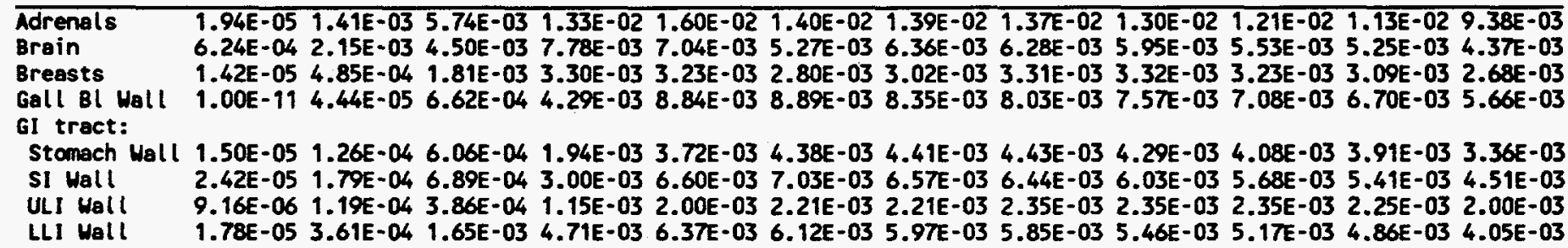

Heart Mall

Kidneys

Liver

Lungs

4.11E-06 1.61E-04 8.43E-04 3.22E-03 5.85E-03 5.67E-03 5.29E-03 5.30E-03 5.05E-03 4.81E-03 4.56E-03 3.90E-03 4.36E-06 3.81E-04 2.21E-03 7.44E-03 1.10E-02 1.03E-02 9.95E-03 9.87E-03 9.29E-03 8.72E-03 8.22E-03 6.94E-03 4.55E-05 3.50E-04 1.20E-03 3.17E-03 5.06E-03 5.13E-03 4.84E-03 4.89E-03 4.70E-03 4.46E-03 4.22E-03 3.64E-03 2.69E-06 2.65E-04 1.85E-03 6.31E-03 8.22E-03 6.78E-03 6.27E-03 6.25E-03 5.92E-03 5.49E-03 5.21E-03 4.38E-03

Muscle

Ovaries

Pancreas

Skeletal Tiss:

Act Marrow

Endosteum

Skin

Spleen

6.19E-04 1.80E-03 3.32E-03 5.53E-03 6.06E-03 5.29E-03 5.26E-03 5.34E-03 5.07E-03 4.76E-03 4.49E-03 3.77E-03 1.77E-05 4.96E-04 5.27E-03 1.73E-02 1.92E-02 1.60E-02 1.44E-02 1.37E-02 1.26E-02 1.17E-02 1.09E-02 9.00E-03 8.08E-07 3.54E-05 5.20E-04 3.38E-03 7.49E-03 7.88E-03 7.44E-03 7.30E-03 6.75E-03 6.30E-03 5.98E-03 5.16E-03

7.41E-02 6.48E-02 5.66E-02 4.31E-02 2.97E-02 2.39E-02 2.49E-02 2.59E-02 2.42E-02 2.25E-02 2.07E-02 1.71E-02 1.65E-01 1.59E-01 1.50E-01 1.23E-01 7.26E-02 2.78E-02 1.72E-02 $1.50 E-02$ 1.37E-02 $1.25 E-02$ 1.16E-02 9.62E-03 3.48E-04 1.16E-03 1.94E-03 2.65E-03 2.72E-03 2.54E-03 2.87E-03 3.16E-03 3.18E-03 3.01E-03 2.89E-03 2.52E-03 1.57E-06 1.39E-04 9.30E-04 3.06E-03 5.46E-03 5.48E-03 5.17E-03 5.20E-03 5.05E-03 4.80E-03 4.58E-03 3.96E-03

Thymus

Thyroid

9.43E-07 2.97E-04 1.76E-03 3.96E-03 4.93E-03 4.53E-03 4.36E-03 4.53E-03 4.40E-03 $4.23 E-03 \quad 4.03 E-03$ 3.41E-03 3.80E-06 7.61E-05 6.47E-04 2.70E-03 4.26E-03 4.62E-03 4.48E-03 4.65E-03 4.58E-03 4.36E-03 4.15E-03 3.57E-03 Urin Bl Wall

Fetus 1.00E-18 1.00E-09 3.55E-06 3.22E-04 1.76E-03 2.64E-03 2.76E-03 2.79E-03 2.73E-03 2.61E-03 2.56E-03 2.20E-03

Skeleton

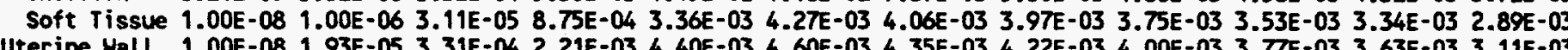

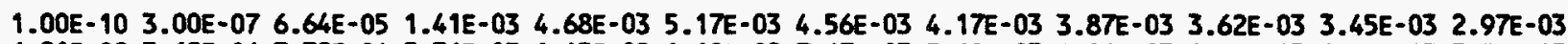
6.21E-09 3.62E-06 3.32E-04 5.36E-03 1.45E-02 $1.18 E-02 \quad 7.67 E-03$ 5.60E-03 $4.86 E-03 \quad 4.58 E-03 \quad 4.32 E-03 \quad 3.72 E-03$ Body Tissues 1.77E-02 1.76E-02 1.72E-02 1.57E-02 1.13E-02 7.08E-03 6.28E-03 6.23E-03 5.88E-03 5.49E-03 5.16E-03 4.33E-03 
Table A.4 (cont'd). At Nine Months Gestation: Specific Absorbed Fraction of Photon Energy (kg-1).

SOURCE = SKELETAL TISSUES: SKELETON

Energy (MeV)

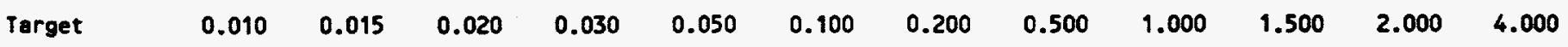

Adrenals $\quad 1.10 E-05$ 7.14E-04 2.87E-03 6.81E-03 8.04E-03 6.73E-03 6.32E-03 6.77E-03 6.50E-03 5.99E-03 5.63E-03 4.73E-03

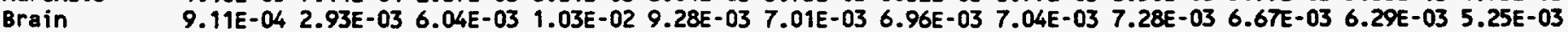
Breasts $\quad 9.12 E-06$ 2.82E-04 $1.05 E-03$ 2.03E-03 2.10E-03 $1.87 E-03 \quad 1.94 E-03 \quad 2.15 E-03 \quad 2.25 E-03 \quad 2.19 E-03 \quad 2.10 E-03 \quad 1.82 E-03$ Gall Bl Moll 4.68E-07 9.93E-05 2.69E-04 9.71E-03 3.96E-03 4.06E-03 3.75E-03 3.68E-03 3.50E-03 3.24E-03 3.09E-03 2.64E-03

GI trect:

Stomach Hall 5.81E-07 8.19E-05 4.13E-04 1.42E-03 2.57E-03 2.74E-03 2.61E-03 2.59E-03 2.51E-03 2.38E-03 2.27E-03 1.98E-03 SI Hall $\quad 1.57 E-05$ 9.35E-05 3.32E-04 1.33E-03 3.01E-03 3.23E-03 3.04E-03 3.05E-03 2.89E-03 2.72E-03 2.60E-03 2.22E-03 ULI Hall 2.65E-06 3.16E-05 $1.26 E-04$ 5.22E-04 9.37E-04 $1.04 E-03 \quad 1.09 E-03 \quad 1.21 E-03 \quad 1.24 E-03 \quad 1.22 E-03 \quad 1.20 E-03 \quad 1.10 E-03$ LLI Wall 5.12E-06 1.04E-04 5.46E-04 1.79E-03 2.83E-03 2.76E-03 2.68E-03 2.74E-03 2.63E-03 2.50E-03 2.37E-03 2.05E-03

Heart Wall 2.71E-06 9.31E-05 4.93E-04 1.96E-03 3.62E-03 3.53E-03 3.37E-03 3.35E-03 3.17E-03 2.99E-03 2.83E-03 2.43E-03 Kidneys $\quad 1.85 E-06$ 1.51E-04 8.86E-04 3.00E-03 4.53E-03 4.32E-03 4.11E-03 4.26E-03 3.98E-03 3.72E-03 3.54E-03 3.04E-03 liver $\quad 3.06 E-05$ 2.15E-04 7.92E-04 2.26E-03 3.44E-03 3.29E-03 3.18E-03 3.19E-03 3.05E-03 2.87E-03 2.70E-03 2.36E-03 lungs $\quad 1.03 E-05$ 1.62E-04 1.13E-03 4.09E-03 5.54E-03 4.59E-03 4.29E-03 4.30E-03 4.01E-03 3.73E-03 3.52E-03 2.97E-03

Muscle $\quad 4.76 E-04 \quad 1.33 E-03 \quad 2.43 E-03 \quad 4.22 E-03 \quad 4.85 E-03 \quad 4.32 E-03 \quad 4.38 E-03 \quad 4.52 E-03 \quad 4.32 E-03 \quad 4.08 E-03 \quad 3.84 E-03$ 3.24E-03 Ovaries $\quad 4.63 E-06$ 1.27E-04 1.34E-03 4.39E-03 5.64E-03 4.71E-03 4.28E-03 4.31E-03 $4.04 E-03 \quad 3.78 E-03$ 3.55E-03 $3.02 E-03$ Pancreas 3.94E-07 1.72E-05 2.55E-04 1.70E-03 3.89E-03 4.17E-03 3.91E-03 3.86E-03 3.53E-03 3.29E-03 3.13E-03 2.73E-03 Skeletal Tiss:

Act Marrow 3.24E-02 2.84E-02 2.49E-02 1.95E-02 1.42E-02 1.17 E-02 $1.21 E-02 \quad 1.26 E-02 \quad 1.15 E-02 \quad 1.08 E-02$ 9.92E-03 8.25E-03 Endosteum 1.66E-01 1.62E-01 1.56E-01 1.32E-01 7.94E-02 3.00E-02 1.86E-02 $1.65 E-02 \quad 1.50 E-02 \quad 1.38 E-02 \quad 1.27 E-02$ 1.05E-02 skin $\quad 4.63 E-041.36 E-03$ 2.16E-03 3.08E-03 3.24E-03 2.97E-03 3.26E-03 3.61E-03 3.52E-03 3.31E-03 3.17E-03 2.73E-03 Spleen $\quad 9.82 E-07$ 8.17E-05 5.31E-04 2.08E-03 3.44E-03 3.35E-03 3.15E-03 3.18E-03 3.09E-03 2.91E-03 2.79E-03 2.41E-03

Thymus $\quad 6.17 E-07$ 1.73E-04 1.01E-03 2.44E-03 3.19E-03 2.97E-03 2.82E-03 3.02E-03 2.96E-03 2.83E-03 2.70E-03 2.30E-03 Thyroid $\quad 3.52 E-10$ 1.07E-04 8.48E-04 3.28E-03 4.68E-03 4.39E-03 4.20E-03 4.67E-03 4.60E-03 4.34E-03 4.10E-03 3.49E-03 Urin BL Wall 1.00E-18 1.00E-09 $1.71 E-06 \quad 1.58 E-04 \quad 1.00 E-03 \quad 1.52 E-03 \quad 1.55 E-03 \quad 1.60 E-03 \quad 1.58 E-03 \quad 1.52 E-03 \quad 1.48 E-03 \quad 1.34 E-03$ Uterine Cont:

Fetus

Skeleton

Soft Tissue 1.67E-10 9.76E-08 8.95E-06 2.59E-04 1.13E-03 $1.61 E-03$ 1.66E-03 $1.61 E-03 \quad 1.61 E-03 \quad 1.54 E-03$ 1.48E-03 $1.34 E-03$ Uterine Wall 1.45E-07 6.67E-06 1.01E-04 6.78E-04 1.55E-03 $1.77 \mathrm{E}-03$ 1.73E-03 $1.75 E-03 \quad 1.73 E-03 \quad 1.66 E-03$ 1.60E-03 $1.42 E-03$ Body Tissues 1.7TE-02 1.76E-02 1.72E-02 1.55E-02 1.09E-02 6.43E-03 5.65E-03 5.65E-03 5.36E-03 5.02E-03 4.70E-03 3.95E-03

SOURCE $=$ SKIN

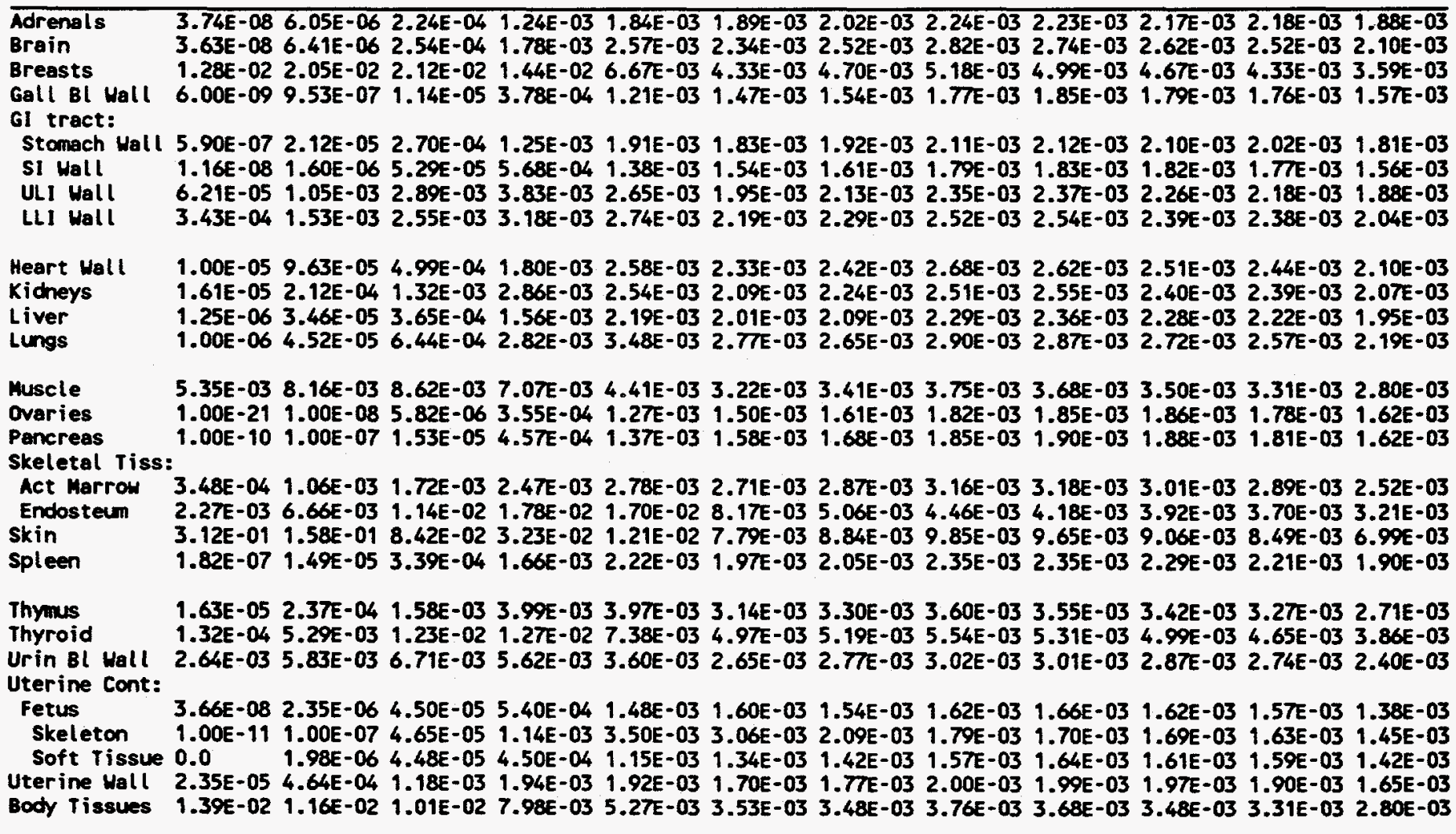


Table A.4 (cont'd). At Nine Months Gestation: Specific Absorbed Fraction of Photon Energy (kg').

SOURCE $=$ SPLEEN

Energy (MeV)

\begin{tabular}{|c|c|c|c|c|c|c|c|c|c|c|c|c|}
\hline Target & 0.010 & 0.015 & 0.020 & 0.030 & 0.050 & 0.100 & 0.200 & 0.500 & 1.000 & 1.500 & 2.000 & 4.000 \\
\hline $\begin{array}{l}\text { Adrenals } \\
\text { Brain } \\
\text { Breasts } \\
\text { Gall BL Wall } \\
\text { GI tract: } \\
\text { Stomach Wall } \\
\text { SI Wall } \\
\text { ULI Wall } \\
\text { ULI Wall }\end{array}$ & $\begin{array}{l}1.06 E-10 \\
0.0 \\
0.0 \\
1.00 E-23 \\
2.16 E-08 \\
3.22 E-03 \\
1.00 E-12 \\
1.00 E-16\end{array}$ & $\begin{array}{l}1.77 E-04 \\
0.0 \\
3.49 E-11 \\
1.00 E-08 \\
7.72 E-04 \\
1.29 E-02 \\
1.00 E-08 \\
1.00 E-10\end{array}$ & $\begin{array}{l}7.61 E-03 \\
3.72 E-15 \\
2.97 E-06 \\
7.87 E-05 \\
\\
1.48 E-02 \\
2.91 E-02 \\
2.31 E-06 \\
2.18 E-06\end{array}$ & $\begin{array}{l}3.98 E-02 \\
8.33 E-08 \\
4.48 E-04 \\
3.99 E-03 \\
\\
6.45 E-02 \\
5.07 E-02 \\
1.82 E-04 \\
2.15 E-04\end{array}$ & $\begin{array}{l}4.62 E-02 \\
9.44 E-06 \\
2.08 E-03 \\
1.25 E-02 \\
7.12 E-02 \\
4.69 E-02 \\
1.16 E-03 \\
1.35 E-03\end{array}$ & $\begin{array}{l}3.40 E-02 \\
3.47 E-05 \\
2.58 E-03 \\
1.30 E-02 \\
4.84 E-02 \\
3.29 E-02 \\
1.77 E-03 \\
1.97 E-03\end{array}$ & $\begin{array}{l}3.00 E-02 \\
6.15 E-05 \\
2.57 E-03 \\
1.16 E-02 \\
4.13 E-02 \\
2.89 E-02 \\
1.85 E-03 \\
2.05 E-03\end{array}$ & $\begin{array}{l}2.78 E-02 \\
1.27 E-04 \\
2.86 E-03 \\
1.04 E-02\end{array}$ & $\begin{array}{l}2.57 E-02 \\
1.93 E-04 \\
2.92 E-03 \\
9.77 E-03 \\
3.45 E-02 \\
2.43 E-02 \\
2.15 E-03 \\
2.28 E-03\end{array}$ & $\begin{array}{l}2.35 E-02 \\
2.32 E-04 \\
2.93 E-03 \\
9.24 E-03\end{array}$ & $\begin{array}{l}2.14 E-02 \\
2.55 E-04 \\
2.82 E-03 \\
8.45 E-03\end{array}$ & $\begin{array}{l}1.78 E-02 \\
2.74 E-04 \\
2.53 E-03 \\
7.35 E-03 \\
2.37 E-02 \\
1.71 E-02 \\
1.84 E-03 \\
2.08 E-03\end{array}$ \\
\hline
\end{tabular}

Heart Wall 1.00E-20 1.00E-07 8.10E-05 3.21E-03 9.64E-03 9.38E-03 8.40E-03 7.94E-03 7.29E-03 6.85E-03 6.42E-03 5.51E-03

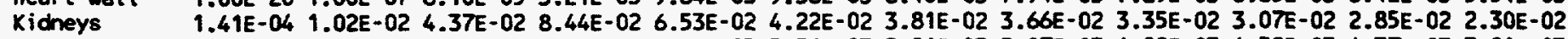

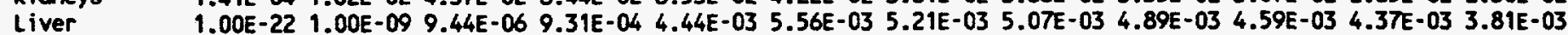
Lungs $\quad 5.74 E-06$ 2.04E-04 2.57E-03 1.02E-02 $1.23 E-02 \quad 9.22 E-03$ 8.32E-03 7.84E-03 $7.33 E-03$ 6.83E-03 6.26E-03 5.29E-03

Muscle $\quad 1.42 E-03$ 4.38E-03 7.92E-03 1.08E-02 8.65E-03 6.14E-03 5.78E-03 5.75E-03 5.41E-03 5.06E-03 4.76E-03 3.98E-03

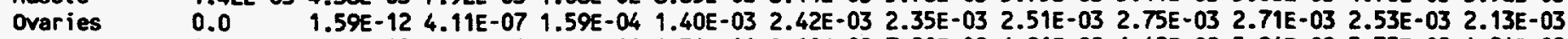
Pancreas $\quad 1.38 E-03$ 4.09E-02 1.27E-01 1.86E-01 1.31E-01 8.10E-02 7.20E-02 6.81E-02 6.42E-02 5.84E-02 5.35E-02 4.24E-02 Skeletal Tiss:

Act Marrow 1.57E-06 1.26E-04 7.56E-04 2.64E-03 4.69E-03 5.16E-03 5.17E-03 5.20E-03 5.05E-03 4.80E-03 4.58E-03 3.96E-03 Endosteum 4.51E-06 4.18E-04 2.79E-03 1.12E-02 1.70E-02 1.00E-02 5.71E-03 4.15E-03 3.71E-03 3.45E-03 3.29E-03 2.86E-03 Skin

Spleen 1.82E-07 1.49E-05 3.39E-04 1.66E-03 2.22E-03 1.97E-03 2.05E-03 2.35E-03 2.35E-03 2.29E-03 2.21E-03 1.90E-03 6.31E+00 5.40E+00 4.14E+00 2.13E+00 8.28E-01 4.76E-01 4.91E-01 5.16E-01 4.78E-01 4.39E-01 4.06E-01 3.21E-01

Thymus $\quad 0.0 \quad 5.50 E-15 \quad 5.00 E-08 \quad 1.24 E-04 \quad 1.26 E-03 \quad 1.94 E-03 \quad 1.97 E-03 \quad 2.02 E-03 \quad 2.15 E-03 \quad 2.13 E-03 \quad 2.05 E-03 \quad 1.78 E-03$ Thyroid $\quad 0.0 \quad 0.0 \quad 1.79 E-11$ 1.41E-05 2.53E-04 4.85E-04 5.87E-04 7.74E-04 8.42E-04 8.96E-04 9.87E-04 8.51E-04 Urin BI Wall $0.0 \quad 0.0 \quad 1.00 E-11$ 2.81E-06 1.72E-04 5.33E-04 6.75E-04 8.78E-04 1.02E-03 9.41E-04 9.30E-04 9.42E-04

Uterine Cont:

Fetus 0.0

Skeleton $\quad 0.0$

Soft Tissue 0.0

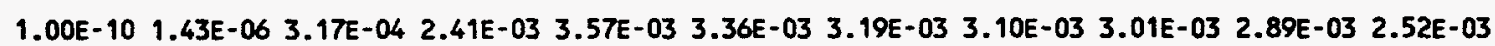
1.35E-08 1.16E-06 6.14E-04 5.07E-03 6.48E-03 4.69E-03 3.42E-03 2.95E-03 2.88E-03 2.72E-03 2.36E-03 3.49E-08 1.45E-06 2.77E-04 2.05E-03 3.18E-03 3.19E-03 3.16E-03 3.11E-03 3.02E-03 2.91E-03 2.54E-03 Uterine Wall 2.00E-07 4.90E-06 4.95E-05 4.29E-03 3.80E-03 4.18E-03 3.90E-03 3.84E-03 3.63E-03 3.48E-03 3.39E-03 2.94E-03 Body Tissues 1.77E-02 1.76E-02 1.74E-02 1.56E-02 1.12E-02 7.62E-03 6.96E-03 6.84E-03 6.39E-03 5.96E-03 5.59E-03 4.65E-03

SOURCE $=$ THYMUS

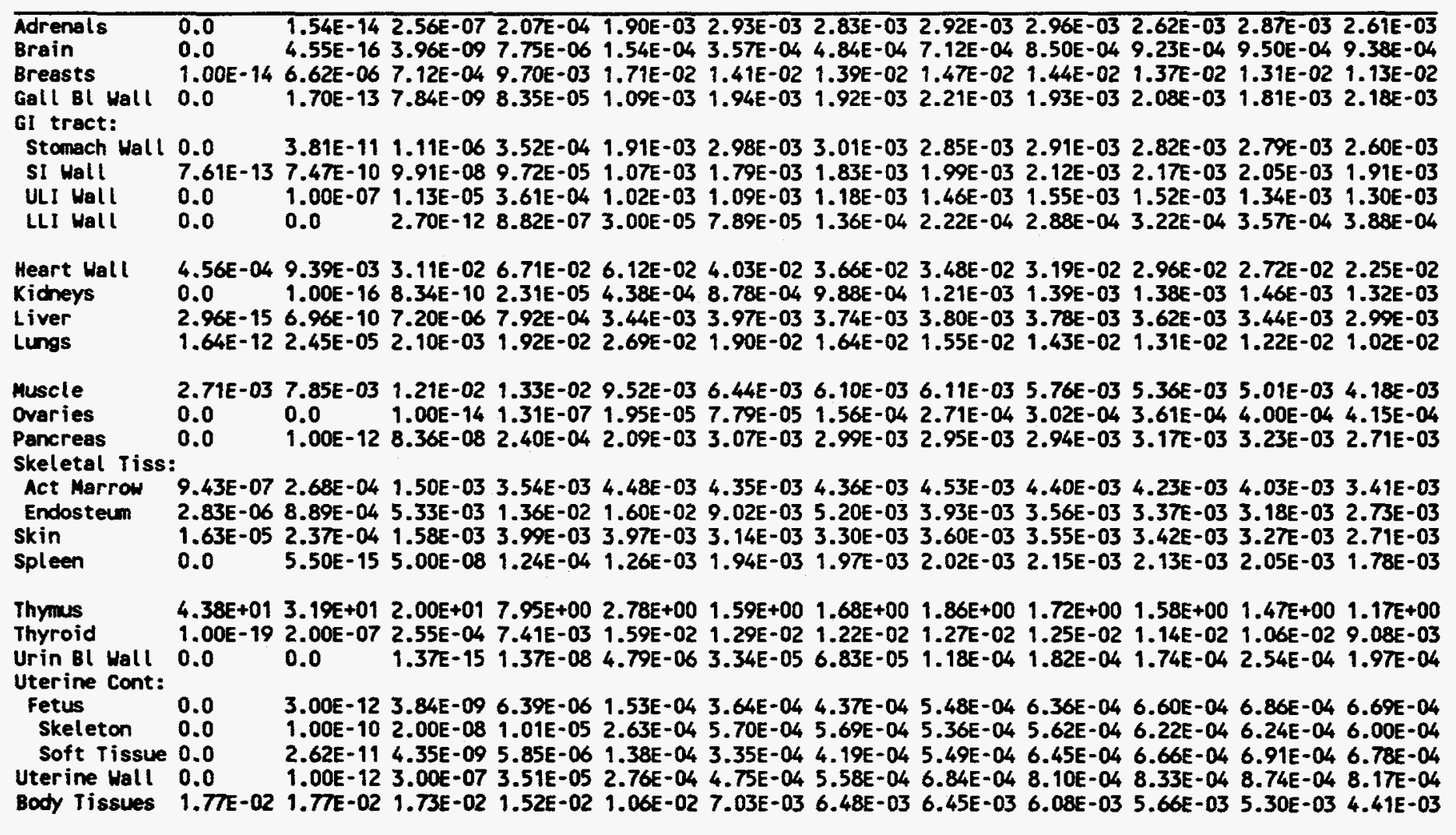


Table A.4 (cont'd). At Nine Months Gestation: Specific Absorbed Fraction of Photon Energy (kg-1).

SOURCE $=$ THYROID

Energy ( $\mathrm{HeV}$ )

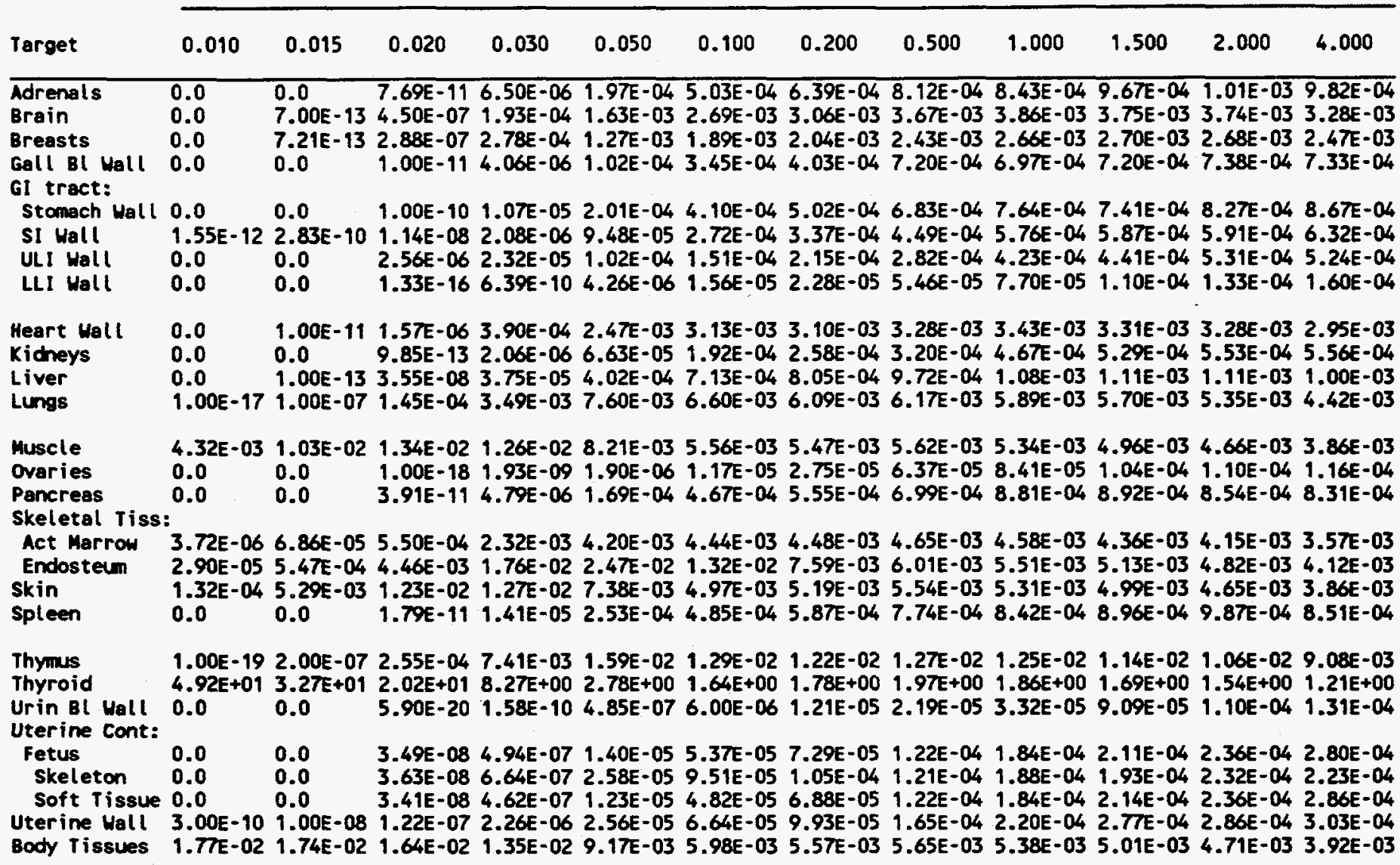

SOURCE = URINARY BLADDER CONTENTS

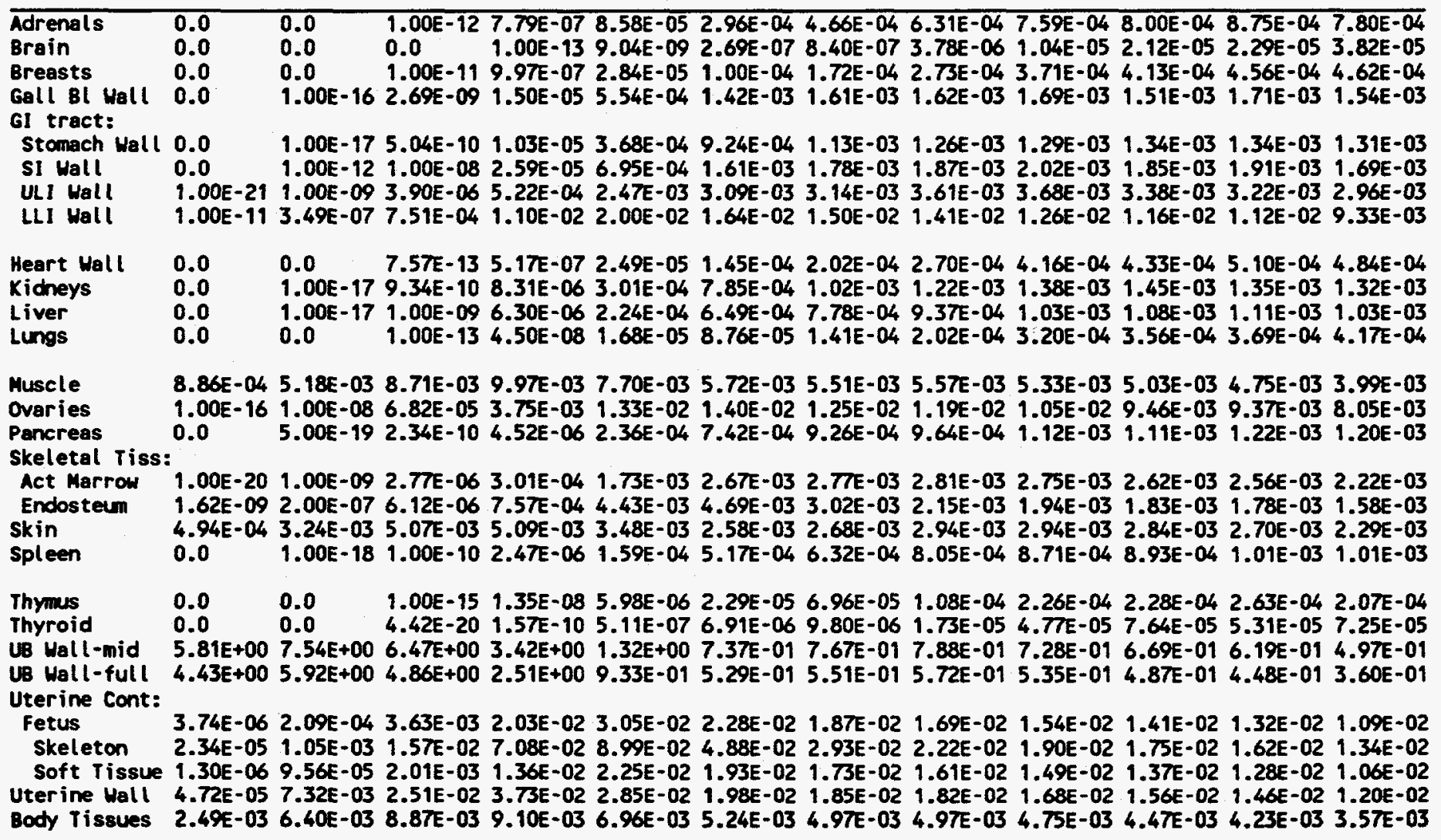


Table A.4 (cont'd). At Nine Months Gestation: Specific Absorbed Fraction of Photon Energy ( $\left.\mathrm{kg}^{-1}\right)$.

SOURCE = URINARY BLADDER WALL

Energy (MeV)

$\begin{array}{lllllllllllll}\text { Target } & 0.010 & 0.015 & 0.020 & 0.030 & 0.050 & 0.100 & 0.200 & 0.500 & 1.000 & 1.500 & 2.000 & 4.000\end{array}$

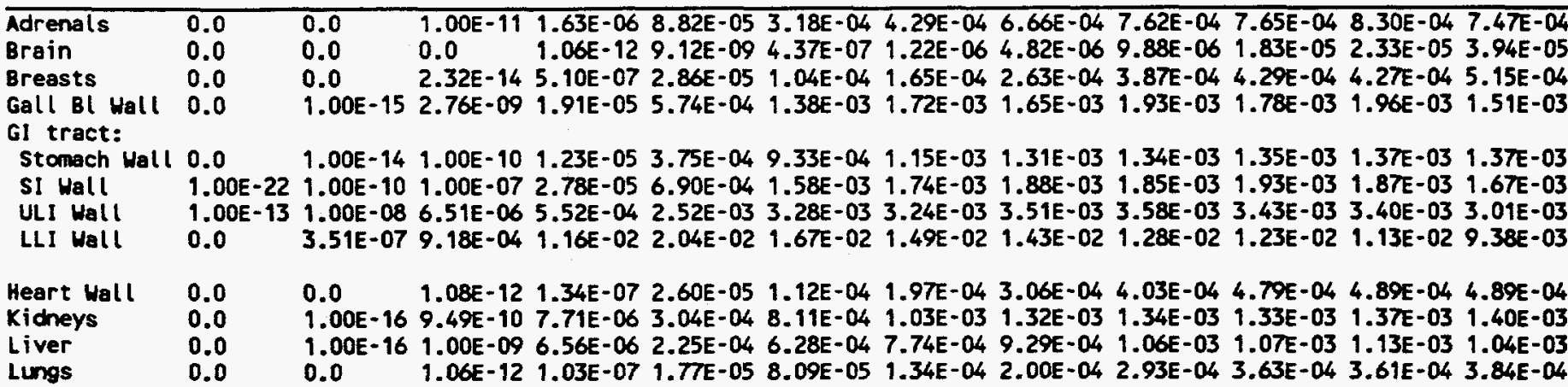

Muscle $\quad 5.47 E-03 \quad 9.38 E-03 \quad 1.12 E-02$ 1.08E-02 7.93E-03 5.85E-03 5.66E-03 5.75E-03 5.49E-03 5.15E-03 4.86E-03 4.09E-03 Ovaries $\quad 1.00 E-15$ 1.00E-07 8.11E-05 4.04E-03 $1.35 E-02 \quad 1.38 E-02 \quad 1.28 E-02 \quad 1.16 E-02 \quad 1.06 E-02 \quad 1.00 E-02$ 9.67E-03 $7.84 E-03$ $\begin{array}{lllllllllllllll}\text { Pancreas } & 0.0 & 1.00 E-18 & 2.40 E-10 & 4.09 E-06 & 2.46 E-04 & 7.36 E-04 & 9.69 E-04 & 1.19 E-03 & 1.16 E-03 & 1.08 E-03 & 1.27 E-03 & 1.04 E-03\end{array}$ Skeletal Tiss:

Act Marrow 1.00E-18 1.00E-09 3.55E-06 3.22E-04 1.76E-03 2.64E-03 2.76E-03 2.79E-03 2.75E-03 2.61E-03 2.56E-03 2.20E-03

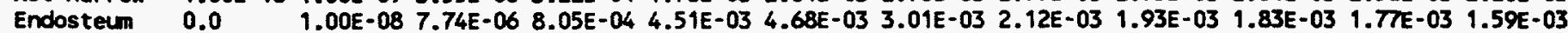
Skin

Spleen 2.64E-03 5.83E-03 6.71E-03 5.62E-03 3.60E-03 2.65E-03 2.77E-03 3.02E-03 3.01E-03 2.87E-03 2.74E-03 2.40E-03

Thymus $\quad 0.0 \quad 0.0 \quad 1.37 E-15 \quad 1.37 E-08 \quad 4.79 E-06 \quad 3.34 E-05 \quad 6.83 E-05 \quad 1.18 E-04 \quad 1.82 E-04 \quad 1.74 E-04 \quad 2.54 E-04 \quad 1.97 E-04$

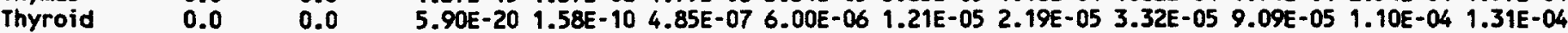
Ur BI Wall 2.44E+01 1.32E+01 7.76E+00 3.26E+00 1.13E+00 6.52E-01 7.00E-01 7.45E-01 6.95E-01 6.33E-01 5.91E-01 4.64E-01 Uterine cont:

Fetus

skeleton

7.64E-06 2.95E-04 3.94E-03 2.06E-02 3.03E-02 2.25E-02 1.86E-02 1.69E-02 1.54E-02 1.41E-02 1.32E-02 1.09E-02

Soft Tissue 2.90E-06 $1.45 E-03$ 1.73E-02 $7.21 E-02$ 8.95E-02 $4.85 E-02$ 2.93E-02 2.20E-02 $1.92 E-02$ 1.76E-02 $1.60 E-02$ 1.34E-02 Uterine Wall 2.55E-04 9.94E-03 2.78E-02 3.82E-02 2.85E-02 $1.98 E-02 \quad 1.85 E-02 \quad 1.82 E-02 \quad 1.69 E-02 \quad 1.57 E-02 \quad 1.47 E-02 \quad 1.21 E-02$ Body Tissues 1.43E-02 1.26E-02 1.20E-02 1.01E-02 7.22E-03 5.39E-03 5.14E-03 5.17E-03 4.93E-03 4.63E-03 4.37E-03 3.69E-03

SOURCE $=$ UTERINE CONTENTS: FETUS

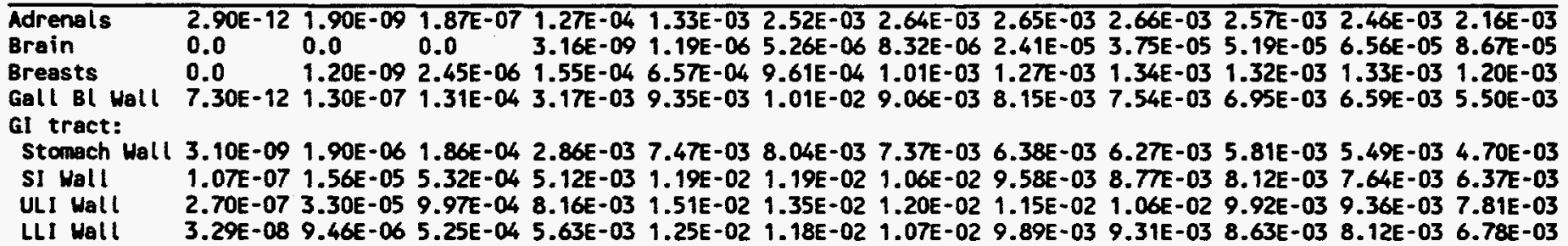

Heart Wall $\quad 0.0 \quad 7.70 E-12 \quad 7.70 E-08 \quad 4.51 E-05 \quad 6.09 E-04 \quad 1.20 E-03 \quad 1.28 E-03 \quad 1.37 E-03 \quad 1.51 E-03 \quad 1.55 E-03 \quad 1.48 E-03 \quad 1.35 E-03$ Liver $\quad 7.70 E-16 \quad 7.70 E-08$ 8.72E-05 1.20E-03 4.01E-03 4.85E-03 4.54E-03 $4.35 E-03 \quad 4.18 E-03 \quad 3.98 E-03$ 3.80E-03 $3.31 E-03$ Lungs $\quad 0.0 \quad 1.00 E-16 \quad 5.00 E-09$ 2.53E-05 3.47E-04 7.18E-04 8.06E-04 9.34E-04 $1.05 E-03 \quad 1.06 E-03 \quad 1.05 E-03$ 9.75E-04

Muscle $\quad 5.36 E-07$ 3.57E-05 3.32E-04 1.93E-03 3.83E-03 3.84E-03 3.63E-03 3.54E-03 3.40E-03 3.22E-03 3.06E-03 2.63E-03 Ovaries $\quad 2.54 E-06$ 1.49E-03 6.84E-03 2.46E-02 3.23E-02 2.65E-02 2.25E-02 2.15E-02 1.96E-02 1.79E-02 1.67E-02 1.36E-02

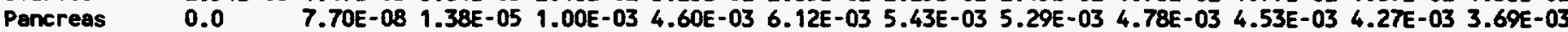
Skeletal Tiss:

Act Marrow 5.70E- 11 1.70E-07 3.35E-05 8.03E-04 3.05E-03 4.21E-03 4.14E-03 4.06E-03 3.87E-03 3.62E-03 3.45E-03 2.97E-03

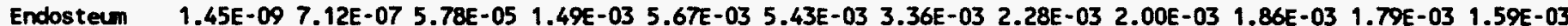
Skin Spleen 2.52E-08 1.89E-06 4.05E-05 4.33E-04 $1.13 E-03 \quad 1.32 E-03 \quad 1.39 E-03 \quad 1.56 E-03 \quad 1.66 E-03 \quad 1.62 E-03 \quad 1.57 E-03 \quad 1.38 E-03$ 0.0 7.70E-11 1.11E-06 2.56E-04 2.10E-03 3.25E-03 3.09E-03 3.17E-03 3.10E-03 3.01E-03 2.89E-03 2.52E-03

Thyous $\quad 0.0 \quad 2.30 E-12 \quad 3.84 E-09 \quad 6.39 E-06 \quad 1.53 E-04 \quad 3.64 E-04 \quad 4.37 E-04 \quad 5.48 E-04 \quad 6.36 E-04 \quad 6.60 E-04 \quad 6.86 E-04 \quad 6.69 E-04$

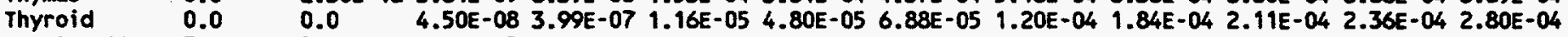
Ur BI Wall 5.90E-06 2.37E-04 2.75E-03 1.49E-02 2.34E-02 1.99E-02 $1.82 E-02 \quad 1.71 E-02 \quad 1.54 E-02 \quad 1.41 E-02 \quad 1.32 E-02 \quad 1.09 E-02$ Uterine Cont:

Ferus

3.31E-01 3.17E-01 2.94E-01 2.32E-01 1.39E-01 8.22E-02 7.29E-02 6.92E-02 6.25E-02 5.72E-02 5.31E-02 4.25E-02 Skeleton 3.39E-01 4.28E-01 5.06E-01 4.93E-01 2.98E-01 1.24E-01 7.94E-02 6.55E-02 5.93E-02 5.39E-02 5.00E-02 4.03E-02

Soft Tissue 3.30E-01 3.02E-01 2.66E-01 1.97E-01 1.17E-01 7.66E-02 7.20E-02 6.97E-02 6.31E-02 5.77E-02 5.35E-02 4.29E-02 Uterine Wall 2.62E-04 2.95E-03 1.01E-02 2.61E-02 3.11E-02 2.43E-02 2.18E-02 2.04E-02 $1.85 E-02 \quad 1.70 E-02$ 1.59E-02 1.31 E-02 Body Tissues 4.28E-06 8.30E-05 4.50E-04 2.15E-03 4.18E-03 4.09E-03 3.71E-03 3.53E-03 3.36E-03 3.18E-03 3.02E-03 2.60E-03 
Table A.4 (cont'd). At Nine Months Gestation: Specific Absorbed Fraction of Photon Energy (kg-1).

SOURCE = UTERINE CONTENTS: FETAL SKELETON

Energy (MeV)

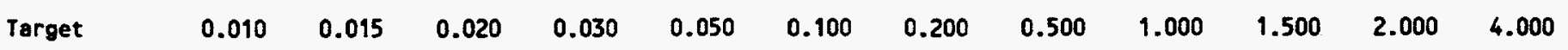

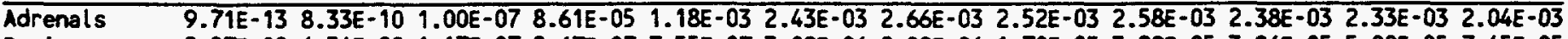
Brain $\quad 2.87 E-08$ 6.54E-08 $1.17 E-07$ 2.67E-07 7.55E-07 3.09E-06 8.08E-06 1.70E-05 3.29E-05 3.86E-05 5.00E-05 7.45E-05 Breasts $\quad 3.31 E-103.18 E-08$ 8.11E-07 7.78E-05 4.37E-04 7.01E-04 8.14E-04 1.01E-03 1.09E-03 $1.13 E-03 \quad 1.14 E-03 \quad 9.88 E-04$ Gall Bl Wall 1.36E-07 4.78E-06 5.97E-05 2.09E-03 7.78E-03 9.34E-03 8.36E-03 7.42E-03 7.19E-03 6.39E-03 5.93E-03 5.19E-03 GI tract:

Stonach Wall 1.38E-07 4.27E-06 4.87E-05 1.51E-03 5.48E-03 6.47E-03 5.93E-03 5.62E-03 5.47E-03 5.05E-03 4.64E-03 4.09E-03 SI Hall $\quad 5.98 E-07$ 1.40E-05 1.31E-04 3.08E-03 9.40E-03 1.03E-02 9.17E-03 8.28E-03 7.65E-03 7.03E-03 6.65E-03 5.56E-03 ULI Hall 2.87E-06 4.48E-05 3.15E-04 4.91E-03 $1.15 E-02 \quad 1.10 E-02 \quad 1.01 E-02 \quad 9.78 E-03 \quad 9.09 E-03$ 8.41E-03 $7.75 E-03$ 6.63E-03 LLI Wall 1.48E-07 2.43E-05 9.04E-04 7.86E-03 1.56E-02 1.43E-02 1.30E-02 1.18E-02 1.12E-02 1.02E-02 9.43E-03 7.95E-03

Heart Mall 5.87E-08 5.49E-07 2.68E-06 2.51E-05 4.19E-04 9.98E-04 1.04E-03 1.29E-03 1.27E-03 1.35E-03 1.30E-03 1.20E-03 Kidneys $\quad 5.11 E-10 \quad 8.44 E-08$ 3.16E-06 5.21E-04 3.09E-03 $4.50 E-03 \quad 4.53 E-03 \quad 4.39 E-03 \quad 4.38 E-03 \quad 4.05 E-03 \quad 3.97 E-03 \quad 3.35 E-03$

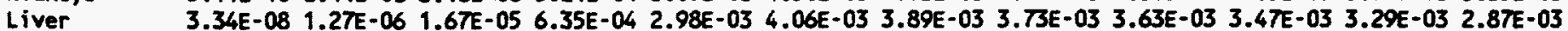
Lungs $\quad 2.64 E-08$ 2.67E-07 1.38E-06 1.40E-05 2.58E-04 5.78E-04 6.96E-04 7.84E-04 9.34E-04 9.63E-04 9.40E-04 8.73E-04

Muscle $\quad 5.74 E-07$ 1.02E-04 6.50E-04 2.57E-03 4.58E-03 4.48E-03 4.22E-03 4.09E-03 3.89E-03 3.68E-03 3.50E-03 2.98E-03

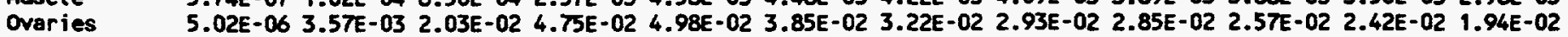
Pancreas 8.96E-10 1.29E-07 4.38E-06 6.29E-04 3.63E-03 5.32E-03 4.62E-03 4.22E-03 4.32E-03 4.07E-03 3.74E-03 3.43E-03 Skeletal Tiss:

Act Marrow 2.04E-09 1.05E-06 8.82E-05 1.39E-03 4.32E-03 5.44E-03 5.38E-03 5.14E-03 4.86E-03 4.58E-03 4.32E-03 3.72E-03 Endosteum 5.05E-09 2.02E-06 1.41E-04 2.45E-03 7.66E-03 6.63E-03 4.04E-03 2.73E-03 2.37E-03 2.22E-03 2.11E-03 1.86E-03 skin

Spleen 6.54E-08 1.54E-06 1.45E-05 3.43E-04 1.10E-03 $1.35 E-03 \quad 1.44 E-03 \quad 1.63 E-03 \quad 1.70 E-03 \quad 1.69 E-03 \quad 1.63 E-03 \quad 1.45 E-03$ 8.43E-12 4.32E-09 3.62E-07 1.86E-04 1.78E-03 2.94E-03 2.93E-03 3.03E-03 2.95E-03 2.88E-03 2.72E-03 2.36E-03

Thymus Thyroid $\quad 5.38 E-11$ 1.12E-09 $9.66 E-09$ 2.01E-07 9.23E-06 $4.55 E-05$ 7.12E-05 $1.08 E-04$ 1.88E-04 $1.93 E-04$ 2.32E-04 $2.23 E-04$

1.99E-09 2.99E-08 2.04E-07 3.07E-06 9.31E-05 2.73E-04 3.84E-04 4.78E-04 5.62E-04 6.22E-04 6.24E-04 6.00E-04 Uterine Cont:

Fetus

skeleton

Soft Tissue

Uterine Hall

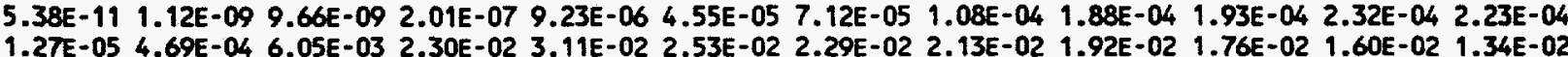

3.36E-01 3.25E-01 3.02E-01 2.38E-01 1.40E-01 7.92E-02 6.91E-02 6.55E-02 5.93E-02 5.39E-02 5.00E-02 4.03E-02 $2.65 E+00$ 2.22E+00 1.71E+00 1.04E+00 4.71E-01 1.79E-01 1.27E-01 1.16E-01 1.06E-01 9.56E-02 8.90E-02 7.23E-02 Body Tissues 1.10E-05 2.35E-04 8.42E-04 2.75E-03 4.87E-03 4.64E-03 4.20E-03 3.98E-03 3.76E-03 3.56E-03 3.37E-03 2.88E-03

\section{SOURCE = UTERINE CONTENTS: FETAL SOFT TISSUE}

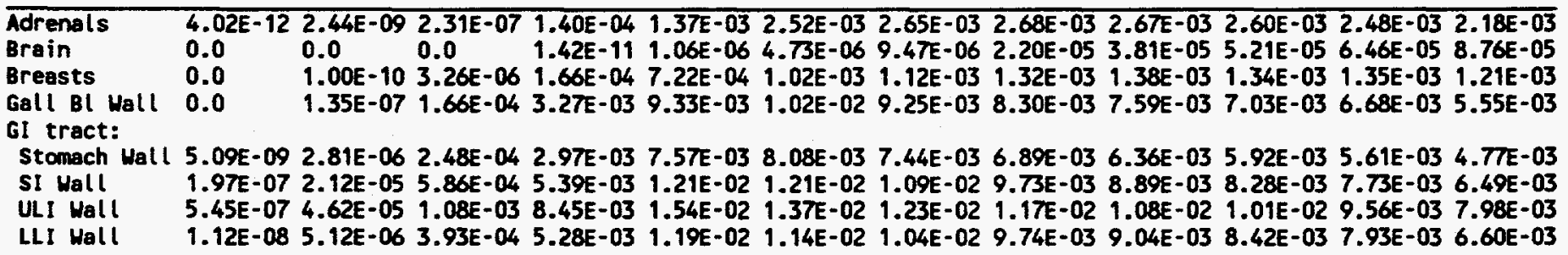

Heart Hall $\quad 0.0 \quad 0.0 \quad 1.24 E-07 \quad 5.13 E-05 \quad 6.20 E-04 \quad 1.22 E-03 \quad 1.32 E-03 \quad 1.44 E-03 \quad 1.54 E-03 \quad 1.58 E-03 \quad 1.51 E-03 \quad 1.37 E-03$ Kidneys $\quad 0.0 \quad 1.00 E-103.75 E-06$ 5.34E-04 3.15E-03 4.59E-03 4.54E-03 4.40E-03 4.22E-03 3.94E-03 3.79E-03 3.29E-03

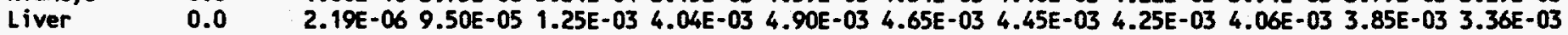
Lungs 1.16E-07 8.84E-07 3.73E-06 2.83E-05 3.65E-04 7.29E-04 8.28E-04 9.67E-04 1.06E-03 1.07E-03 1.07E-03 9.71E-04

Muscle Ovaries Pancreas Skeletal Tiss: Act Marrow Endosteum Skin Spleen

5.86E-07 2.72E-05 2.97E-04 1.86E-03 3.74E-03 3.77E-03 3.56E-03 3.48E-03 3.32E-03 3.16E-03 3.02E-03 2.60E-03 1.46E-06 6.12E-04 5.23E-03 2.22E-02 3.04E-02 2.53E-02 2.23E-02 2.05E-02 1.84E-02 1.69E-02 1.57E-02 1.28E-02

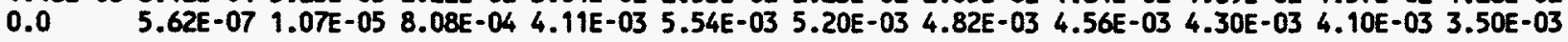

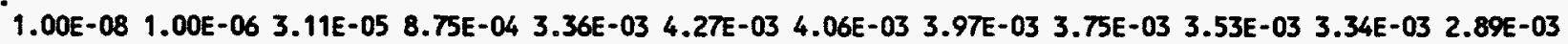
8.29E-10 5.00E-07 4.69E-05 $1.37 E-03 \quad 5.44 E-03 \quad 5.29 E-03 \quad 3.29 E-03 \quad 2.23 E-03 \quad 1.96 E-03 \quad 1.83 E-03 \quad 1.74 E-03 \quad 1.57 E-03$

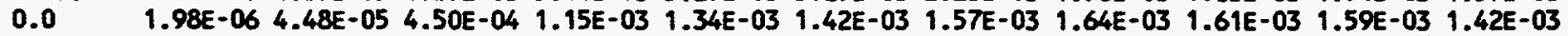

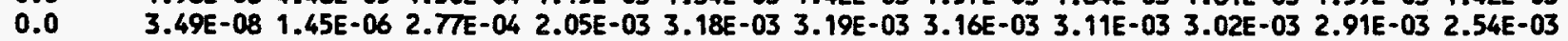

Thymus $\quad 0.0 \quad 2.62 E-11 \quad 4.35 E-09 \quad 5.85 E-06 \quad 1.38 E-04 \quad 3.35 E-04 \quad 4.19 E-04 \quad 5.49 E-04 \quad 6.45 E-04 \quad 6.66 E-04 \quad 6.91 E-04 \quad 6.78 E-04$

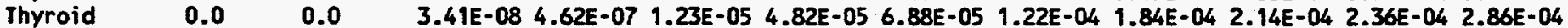
Ur Bl Wall 2.90E-06 1.38E-04 2.15E-03 1.36E-02 $2.24 E-02 \quad 1.91 E-02 \quad 1.72 E-02 \quad 1.62 E-02 \quad 1.49 E-02 \quad 1.36 E-02 \quad 1.28 E-02 \quad 1.05 E-02$ Uterine Cont:

Fetus

Skeleton

7.55E-02 2.24E-01 3.69E-01 4.30E-01 2.78E-01 1.18E-01 7.34E-02 5.96E-02 5.32E-02 4.84E-02 4.48E-02 3.61E-02 Soft Tissue 3.65E-01 3.29E-01 2.84E-01 2.05E-01 1.20E-01 7.81E-02 7.30E-02 7.09E-02 6.45E-02 5.89E-02 5.45E-02 4.37E-02 Uterine Wall 1.51E-04 2.30E-03 9.15E-03 2.55E-02 3.07E-02 2.42E-02 2.16E-02 2.01E-02 $1.83 E-02$ 1.68E-02 $1.57 E-02 \quad 1.29 E-02$ Body Tissues 3.34E-06 6.54E-05 4.07E-04 2.09E-03 4.10E-03 4.02E-03 3.65E-D3 3.47E-03 3.30E-03 3.14E-03 2.98E-03 2.58E-03 
Table A.4 (cont'd). At Nine Months Gestation: Specific Absorbed Fraction of Photon Energy (kg').

SOURCE = UTERINE CONTENTS: PLACENTA

Energy (MeV)

$\begin{array}{lllllllllllll}\text { Target } & 0.010 & 0.015 & 0.020 & 0.030 & 0.050 & 0.100 & 0.200 & 0.500 & 1.000 & 1.500 & 2.000 & 4.000\end{array}$

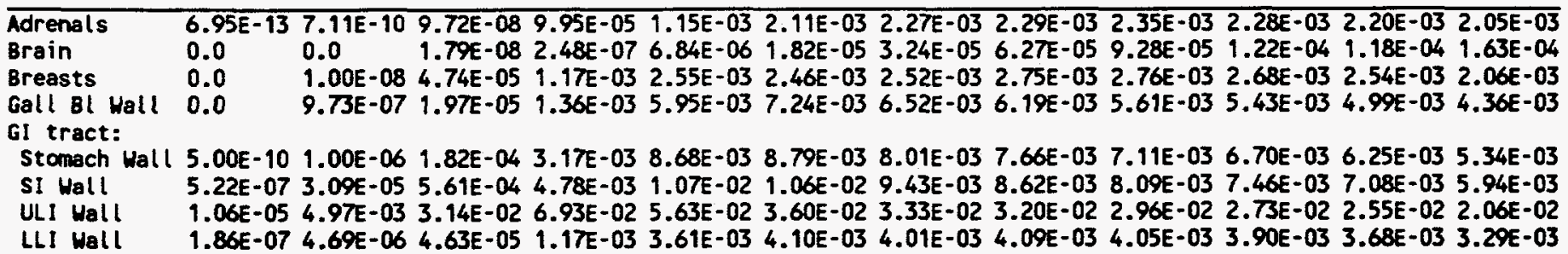

Heart Wall $\quad 0.0 \quad 0.0 \quad 8.98 E-07$ 1.60E-04 1.34E-03 2.01E-03 2.07E-03 2.15E-03 2.24E-03 2.31E-03 2.14E-03 2.04E-03

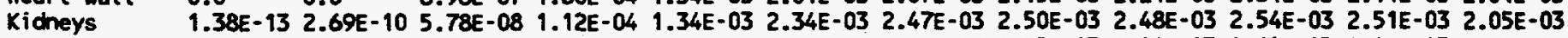
Liver $\quad 1.11 E-08$ 2.64E-06 1.06E-04 1.61E-03 4.94E-03 5.51E-03 5.16E-03 4.95E-03 4.81E-03 4.69E-03 4.44E-03 3.74E-03 Lungs $\quad 4.17 E-07$ 2.64E-06 9.80E-06 6.21E-05 6.36E-04 1.05E-03 1.08E-03 $1.23 E-03 \quad 1.32 E-03 \quad 1.38 E-03 \quad 1.41 E-03 \quad 1.26 E-03$

Muscle $\quad 1.87 E-06 \quad 2.87 E-04 \quad 1.36 E-03 \quad 3.10 E-03 \quad 3.28 E-03 \quad 2.76 E-03 \quad 2.68 E-03 \quad 2.76 E-03 \quad 2.71 E-03 \quad 2.61 E-03 \quad 2.50 E-03 \quad 2.17 E-03$ Ovaries $\quad 5.17 E-13 \quad 6.55 E-10 \quad 1.04 E-07$ 1.32E-04 1.84E-03 3.37E-03 3.44E-03 3.33E-03 3.35E-03 3.08E-03 3.11E-03 2.61E-03 Pancreas 3.58E-09 3.48E-07 8.94E-06 8.68E-04 4.36E-03 5.59E-03 5.13E-03 5.07E-03 4.66E-03 4.49E-03 4.41E-03 3.64E-03 Skeletal Tiss:

Act Marrow 8.79E-09 5.71E-07 1.10E-05 1.23E-04 5.96E-04 1.17E-03 1.35E-03 1.48E-03 1.56E-03 1.57E-03 1.56E-03 1.42E-03

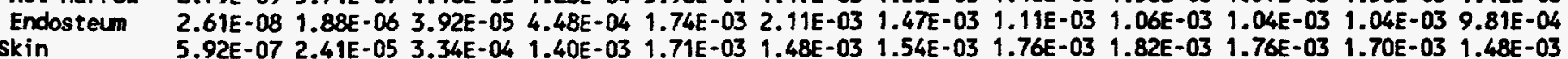
Skin Spleen $\quad 0.0 \quad 1.75 E-09$ 2.01E-07 1.62E-04 1.52E-03 2.47E-03 2.55E-03 2.62E-03 2.66E-03 2.58E-03 2.44E-03 2.31E-03

Thymus $\quad 0.0 \quad 6.33 E-093.09 E-07 \quad 7.39 E-05 \quad 5.91 E-04 \quad 8.75 E-04 \quad 9.96 E-04 \quad 1.22 E-03 \quad 1.29 E-03 \quad 1.38 E-03 \quad 1.36 E-03 \quad 1.19 E-03$

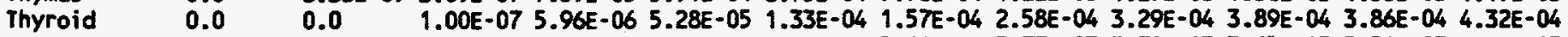
Ur Bl Hall 1.61E-11 7.55E-09 5.93E-07 2.78E-04 2.34E-03 3.55E-03 3.64E-03 3.77E-03 3.78E-03 3.63E-03 3.54E-03 3.18E-03 Uterine Cont:

Fetus Soft Tissue 8.T7E-03 2.57E-02 4.48E-02 6.22E-02 5.28E-02 3.71E-02 3.33E-02 3.14E-02 2.87E-02 2.60E-02 2.43E-02 $1.98 E-02$ Uterine Hall 2.54E-03 2.84E-02 5.51E-02 6.31E-02 4.35E-02 2.89E-02 2.67E-02 2.60E-02 2.40E-02 2.20E-02 2.06E-02 1.68E-02 Body Tissues 5.02E-05 7.67E-04 2.14E-03 3.79E-03 3.82E-03 3.17E-03 3.00E-03 3.02E-03 2.94E-03 2.82E-03 2.69E-03 2.31E-03

\section{SOURCE = UTERINE CONTENTS: OTHER UTERINE CONTENTS}

\begin{tabular}{llllllllllllll}
\hline Adrenals & $1.85 E-09$ & $1.96 E-07$ & $5.34 E-06$ & $5.65 E-04$ & $2.68 E-03$ & $3.66 E-03$ & $3.55 E-03$ & $3.44 E-03$ & $3.30 E-03$ & $3.15 E-03$ & $3.03 E-03$ & $2.64 E-03$ \\
Brain & 0.0 & 0.0 & 0.0 & $1.67 E-11$ & $1.22 E-06$ & $5.32 E-06$ & $1.05 E-05$ & $2.64 E-05$ & $4.54 E-05$ & $6.19 E-05$ & $7.02 E-05$ & $1.02 E-04$ \\
Breasts & 0.0 & $1.00 E-10$ & $8.19 E-06$ & $2.35 E-04$ & $8.19 E-04$ & $1.07 E-03$ & $1.14 E-03$ & $1.34 E-03$ & $1.41 E-03$ & $1.43 E-03$ & $1.37 E-03$ & $1.24 E-03$ \\
Gall Bl Wall & $2.25 E-05$ & $1.24 E-03$ & $6.22 E-03$ & $1.76 E-02$ & $2.07 E-02$ & $1.64 E-02$ & $1.44 E-02$ & $1.31 E-02$ & $1.19 E-02$ & $1.10 E-02$ & $1.02 E-02$ & $8.42 E-03$ \\
GI tract: & & & & & & & & & & & & & \\
Stomach Wall & $2.66 E-05$ & $1.14 E-03$ & $4.92 E-03$ & $1.26 E-02$ & $1.51 E-02$ & $1.22 E-02$ & $1.09 E-02$ & $1.01 E-02$ & $9.30 E-03$ & $8.65 E-03$ & $8.11 E-03$ & $6.70 E-03$ \\
SI Wall & $6.76 E-05$ & $2.69 E-03$ & $1.06 E-02$ & $2.38 E-02$ & $2.48 E-02$ & $1.88 E-02$ & $1.66 E-02$ & $1.52 E-02$ & $1.38 E-02$ & $1.28 E-02$ & $1.19 E-02$ & $9.79 E-03$ \\
ULI Wall & $1.93 E-05$ & $2.16 E-03$ & $9.30 E-03$ & $2.05 E-02$ & $2.08 E-02$ & $1.56 E-02$ & $1.43 E-02$ & $1.38 E-02$ & $1.29 E-02$ & $1.19 E-02$ & $1.12 E-02$ & $9.27 E-03$ \\
LLI Wall & $4.30 E-06$ & $8.85 E-04$ & $5.50 E-03$ & $1.51 E-02$ & $1.62 E-02$ & $1.27 E-02$ & $1.16 E-02$ & $1.13 E-02$ & $1.04 E-02$ & $9.72 E-03$ & $9.04 E-03$ & $7.54 E-03$
\end{tabular}

Heart Wall $\quad 0.0 \quad 0.0 \quad 5.02 E-07 \quad 1.27 E-04 \quad 9.78 E-04 \quad 1.56 E-03 \quad 1.62 E-03 \quad 1.68 E-03 \quad 1.77 E-03 \quad 1.74 E-03 \quad 1.73 E-03 \quad 1.56 E-03$ Kidneys $\quad 1.92 E-07$ 5.76E-06 6.42E-05 1.92E-03 5.65E-03 6.22E-03 5.74E-03 5.48E-03 5.18E-03 4.81E-03 4.62E-03 3.93E-03 Liver $\quad 4.42 E-06 \quad 2.48 E-04$ 1.29E-03 4.69E-03 7.63E-03 7.05E-03 6.41E-03 6.06E-03 5.71E-03 5.27E-03 5.04E-03 4.29E-03 lungs $\quad 3.10 E-11$ 7.09E-09 3.34E-07 7.64E-05 5.91E-04 9.43E-04 1.03E-03 $1.14 E-03 \quad 1.20 E-03 \quad 1.23 E-03 \quad 1.20 E-03 \quad 1.13 E-03$

Muscle $\quad 1.55 E-05 \quad 5.52 E-04$ 1.84E-03 3.91E-03 4.56E-03 3.95E-03 3.75E-03 3.72E-03 3.57E-03 3.37E-03 3.22E-03 2.75E-03 Ovaries $\quad 5.92 E-05$ 3.89E-03 1.31E-02 2.29E-02 2.34E-02 $1.89 E-02$ 1.69E-02 $1.57 E-02 \quad 1.43 E-02 \quad 1.32 E-02 \quad 1.23 E-02 \quad 1.02 E-02$ Pancreas 1.04E-08 4.98E-06 3.97E-04 4.47E-03 9.27E-03 8.89E-03 7.87E-03 7.17E-03 6.65E-03 6.22E-03 5.85E-03 4.88E-03 Skeletal Tiss:

Act Marrow 1.99E-08 2.73E-06 9.06E-05 9.91E-04 2.83E-03 3.67E-03 3.72E-03 3.63E-03 3.48E-03 3.33E-03 3.16E-03 2.77E-03 Endosteum 3.04E-08 4.64E-06 1.64E-04 2.02E-03 5.79E-03 5.01E-03 3.08E-03 2.15E-03 1.92E-03 1.82E-03 1.71E-03 1.57E-03

Skin

Spleen 5.29E-06 6.62E-05 3.98E-04 1.21E-03 $1.59 E-03 \quad 1.52 E-03 \quad 1.61 E-03 \quad 1.7 B E-03 \quad 1.81 E-03 \quad 1.79 E-03 \quad 1.73 E-03 \quad 1.53 E-03$ $0.0 \quad 1.91 E-06 \quad 2.72 E-05 \quad 1.15 E-03 \quad 4.04 E-03 \quad 4.62 E-03 \quad 4.32 E-03 \quad 4.12 E-03 \quad 3.95 E-03 \quad 3.73 E-03 \quad 3.63 E-03 \quad 3.10 E-03$

Thymus $\quad 0.0 \quad 4.74 E-103.02 E-08$ 1.05E-05 $1.76 E-04 \quad 3.90 E-04 \quad 4.80 E-04 \quad 5.83 E-04 \quad 7.05 E-04 \quad 7.29 E-04 \quad 7.87 E-04 \quad 7.58 E-04$

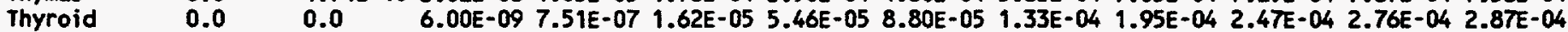
Ur BI Wall 2.22E-06 1.75E-03 1.04E-02 2.50E-02 2.46E-02 1.84E-02 1.69E-02 $1.64 E-02 \quad 1.52 E-02 \quad 1.41 E-02 \quad 1.32 E-02 \quad 1.08 E-02$ Uterine Cont:

Fetus

6.42E-03 1.94E-02 3.64E-02 5.85E-02 5.59E-02 3.80E-02 3.20E-02 2.89E-02 2.61E-02 2.40E-02 2.22E-02 1.81E-02 Skeleton $\quad 5.96 E-03$ 2.69E-02 6.28E-02 1.23E-01 1.21E-01 6.17E-02 3.72E-02 2.80E-02 2.43E-02 2.24E-02 2.07E-02 1.69E-02 Soft Tissue 6.52E-03 1.85E-02 3.29E-02 5.00E-02 4.74E-02 3.50E-02 3.13E-02 2.90E-02 2.63E-02 2.41E-02 2.23E-02 1.83E-02 Uterine Hall 3.01E-02 6.63E-02 8.09E-02 7.15E-02 4.56E-02 3.05E-02 2.85E-02 2.78E-02 2.56E-02 2.35E-02 2.18E-02 1.77E-02 Body Tissues 5.95E-04 1.72E-03 3.08E-03 4.85E-03 5.27E-03 4.40E-03 4.03E-03 3.92E-03 3.73E-03 3.51E-03 3.34E-03 2.85E-03 
Table A.4 (cont'd). At Nine Months Gestation: Specific Absorbed Fraction of Photon Energy ( $\left.\mathrm{kg}^{-1}\right)$.

SOURCE = UTERINE WALL

Energy (MeV)

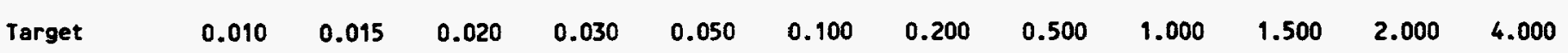

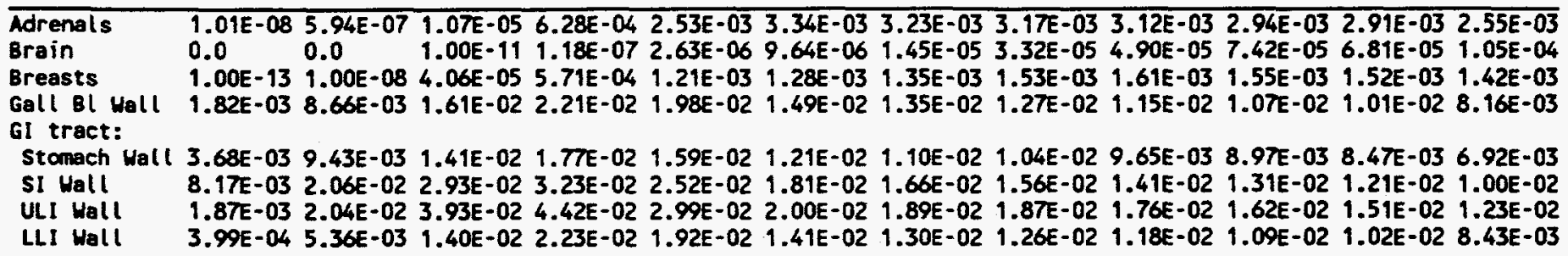

Heart Wall $\quad 0.0 \quad 1.00 E-10 \quad 1.23 E-06 \quad 1.94 E-04 \quad 1.14 E-03 \quad 1.62 E-03 \quad 1.67 E-03 \quad 1.74 E-03 \quad 1.79 E-03 \quad 1.78 E-03 \quad 1.72 E-03 \quad 1.64 E-03$ Kidneys $\quad 1.00 E-09$ 1.00E-06 $1.13 E-04$ 2.05E-03 5.16E-03 $5.55 E-03$ 5.18E-03 5.00E-03 $4.80 E-03 \quad 4.50 E-03$ 4.24E-03 $3.68 E-03$ liver $\quad 6.64 E-04 \quad 1.93 E-03$ 3.53E-03 6.37E-03 7.88E-03 6.86E-03 6.30E-03 6.01E-03 5.78E-03 5.33E-03 5.10E-03 4.34E-03 Lungs $\quad 0.0 \quad 1.12 E-11 \quad 6.40 E-07$ 1.06E-04 6.53E-04 9.48E-04 9.87E-04 $1.10 E-03 \quad 1.18 E-03 \quad 1.23 E-03 \quad 1.21 E-03 \quad 1.10 E-03$

Muscle $\quad 2.01 E-03 \quad 4.93 E-03 \quad 6.70 E-03 \quad 7.24 E-03 \quad 5.94 E-03 \quad 4.64 E-03 \quad 4.46 E-03 \quad 4.47 E-03 \quad 4.27 E-03 \quad 4.02 E-03 \quad 3.81 E-03 \quad 3.23 E-03$ Ovaries $\quad 5.65 E-03$ 3.59E-02 5.95E-02 5.81E-02 3.82E-02 2.64E-02 2.46E-02 2.36E-02 2.15E-02 1.98E-02 1.84E-02 1.48E-02 Pancreas 1.00E-08 1.00E-05 8.40E-04 5.33E-03 9.02E-03 8.17E-03 7.28E-03 6.7TE-03 6.22E-03 5.90E-03 5.51E-03 4.63E-03 Skeletal Tiss:

Act Merrow 1.00E-08 1.93E-05 3.31E-04 2.21E-03 4.40E-03 4.60E-03 4.35E-03 4.22E-03 4.00E-03 3.77E-03 3.63E-03 3.11E-03 $\begin{array}{lllllllll}\text { Endosteum 7.40E-07 3.38E-05 5.09E-04 3.64E-03 } & 7.45 E-03 & 5.53 E-03 & 3.36 E-03 & 2.34 E-03 & 2.09 E-03 & 1.97 E-03 & 1.89 E-03 & 1.68 E-03\end{array}$ Skin $\quad 2.35 E-05 \quad 4.64 E-041.18 E-031.94 E-03 \quad 1.92 E-03 \quad 1.70 E-03 \quad 1.77 E-03 \quad 2.00 E-03 \quad 1.99 E-03 \quad 1.97 E-03 \quad 1.90 E-03 \quad 1.65 E-03$ Spleen $\quad 2.00 E-07$ 4.90E-06 4.95E-05 1.29E-03 3.80E-03 4.18E-03 3.90E-03 3.84E-03 3.63E-03 3.48E-03 3.39E-03 2.94E-03

Thyomus $\quad 0.0 \quad 1.00 E-12 \quad 3.00 E-07 \quad 3.51 E-05 \quad 2.76 E-04 \quad 4.75 E-04 \quad 5.58 E-04 \quad 6.84 E-04 \quad 8.10 E-04 \quad 8.33 E-04$ 8.74E-04 8.17E-04 Thyroid $\quad 3.00 E-101.00 E-08$ 1.22E-07 2.26E-06 $2.56 E-05$ 6.64E-05 9.93E-05 $1.65 E-04 \quad 2.20$ - 04 2.77E-04 $2.86 E-04$ 3.03E-04 Ur Bl Wall 2.55E-04 9.94E-03 2.78E-02 3.82E-02 2.85E-02 $1.98 E-02$ 1.85E-02 $1.82 E-02 \quad 1.69 E-02 \quad 1.57 E-02$ 1.47E-02 $1.21 E-02$ Uterine Cont:

Fetus

Skeleton

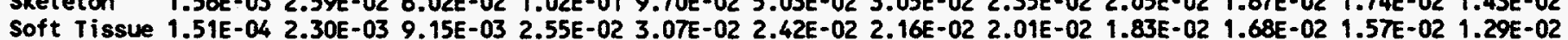
Uterine Hall 7.59E-01 5.19E-01 3.36E-01 1.62E-01 7.03E-02 4.43E-02 4.47E-02 4.60E-02 4.27E-02 3.90E-02 3.61E-02 2.92E-02 Body Tissues 1.62E-02 1.39E-02 $1.19 E-02$ 9.36E-03 6.93E-03 5.22E-03 4.90E-03 4.85E-03 4.60E-03 4.32E-03 4.08E-03 3.44E-03

SOURCE $=$ BODY TISSUES

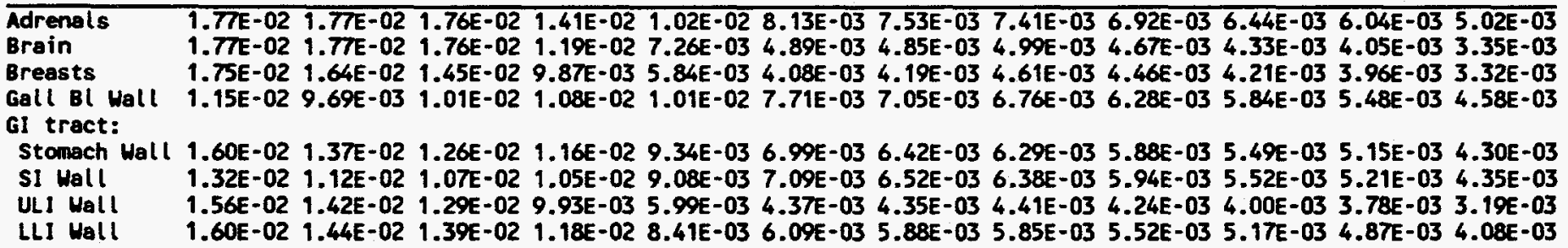

Heart Wall

Kidneys

1.77E-02 1.77E-02 1.76E-02 1.56E-02 1.13E-02 7.87E-03 7.38E-03 7.31E-03 6.82E-03 6.32E-03 5.93E-03 4.93E-03 Liver

Lungs 1.TTE-02 $1.76 E-02$ 1.72E-02 1.38E-02 9.28E-03 6.85E-03 6.72E-03 6.68E-03 6.31E-03 5.89E-03 5.53E-03 4.63E-03 1.76E-02 1.76E-02 1.73E-02 1.49E-02 1.06E-02 7.59E-03 7.09E-03 7.00E-03 6.54E-03 6.09E-03 5.70E-03 4.74E-03 1.88E-02 1.88E-02 1.82E-02 1.58E-02 1.05E-02 6.92E-03 6.33E-03 6.15E-03 5.73E-03 5.31E-03 4.96E-03 4.09E-03

Muscle

Ovaries

Pancreas

Skeletal Tiss:

Act Marrow

Endosteum

Skin

Spleen

1.72E-02 1.63E-02 $1.48 E-02 \quad 1.14 E-02 \quad 7.33 E-03 \quad 5.30 E-03 \quad 5.27 E-03 \quad 5.45 E-03 \quad 5.21 E-03 \quad 4.89 E-03 \quad 4.59 E-03 \quad 3.83 E-03$ 1.77E-02 1.74E-02 1.64E-02 1.25E-02 9.22E-03 7.38E-03 6.92E-03 6.78E-03 6.32E-03 5.89E-03 5.53E-03 4.61E-03 1.74E-02 1.67E-02 1.59E-02 1.41E-02 1.13E-02 8.43E-03 7.86E-03 7.45E-03 6.90E-03 6.41E-03 5.99E-03 4.98E-03

4.99E-03 5.25E-03 5.90E-03 6.91E-03 6.93E-03 6.29E-03 6.35E-03 6.42E-03 5.88E-03 5.49E-03 5.16E-03 4.33E-03 2.53E-02 2.86E-02 3.32E-02 3.94E-02 3.26E-02 1.48E-02 8.79E-03 7.21E-03 6.52E-03 6.05E-03 5.60E-03 4.75E-03 1.40E-02 1.13E-02 9.47E-03 6.79E-03 4.23E-03 3.18E-03 3.41E-03 3.82E-03 3.68E-03 3.48E-03 3.31E-03 2.80E-03 1.77E-02 1.76E-02 1.74E-02 1.48E-02 1.02E-02 7.31E-03 7.08E-03 6.84E-03 6.39E-03 5.96E-03 5.59E-03 4.65E-03

Thymus

Thyroid

1.7TE-02 1.77E-02 1.73E-02 1.45E-02 9.73E-03 6.63E-03 6.35E-03 6.45E-03 6.08E-03 5.66E-03 5.30E-03 4.41E-03 Ur Bl Vall Uterine Cont:

Fetus 1.77E-02 1.74E-02 1.64E-02 1.25E-02 7.71E-03 5.65E-03 5.46E-03 5.65E-03 5.37E-03 5.01E-03 4.71E-03 3.93E-03 1.43E-02 1.26E-02 1.20E-02 1.01E-02 6.88E-03 5.08E-03 5.03E-03 5.17E-03 4.93E-03 4.63E-03 4.37E-03 3.69E-03

Skeleton

5.57E-06 1.43E-04 6.75E-04 2.75E-03 4.86E-03 4.41E-03 3.88E-03 3.60E-03 3.36E-03 3.18E-03 3.02E-03 2.60E-03 Soft Tissue 3.30E-06 6.54E-05 4.07E-04 1.99E-03 3.78E-03 3.80E-03 3.62E-03 3.48E-03 3.30E-03 3.14E-03 2.98E-03 2.58E-03 Uterine Wall 1.62E-02 1.39E-02 1.19E-02 9.12E-03 6.52E-03 4.98E-03 4.87E-03 $4.85 E-03 \quad 4.60 E-03 \quad 4.32 E-03 \quad 4.08 E-03 \quad 3.44 E-03$ Body Tissues 1.74E-02 1.68E-02 1.59E-02 1.34E-02 9.20E-03 5.98E-03 5.49E-03 5.53E-03 5.23E-03 4.90E-03 4.60E-03 3.84E-03 


\section{INTERNAL DISTRIBUTION}

1. B. A. Berven

2. J. S. Bogard

3-7. M. Cristy

8-12. K. F. Eckerman

13. C. E. Easterly

14. G. D. Kerr

15. D. C. Kocher

16. R. W. Leggett

17. D. A. McLaughlin

18. G. L. Murphy

19. R. R Rawl

20. D. E. Reichle

21. P. S. Rohwer
22.

23.

24.

25.

26.

27.

28.

29.

30.

31.

32.
J. C. Ryman
A. L. Sjoreen
R. E. Swaja
C. C. Travis
J. E. Turner
R. C. Ward
Central Research Library
ORNL Y-12 Research Library
Document Reference Section
Laboratory Records Department
Laboratory Records, ORNL (RC)
ORNL Patent Office

\section{EXTERNAL DISTRIBUTION}

33. Assistant Manager of Energy Research and Development, U. S. Department of Energy, Oak Ridge Operations Office, P. O. Box 2001, Oak Ridge, TN 37831-8600

34. S. J. Adelstein, Harvard Medical School, 25 Shattuck Street, Boston MA 02115

35. H. L. Atkins, Medical Department, Brookhaven National Laboratory, Upton, NY 11973

36. M. R. Bailey, Head, Dose Assessments Department, NRPB, Chilton, Didcot, Oxfordshire, OX11 ORQ, United Kingdom

37. V. Berkovski, Ukraine Scientific Centre for Radiological Medicine 53, Melnikova Street, 252050, Kiev, Ukraine

38. R. E. Bigler, The New York Hospital/Cornell Medical Center, 525 East 68th Street, New York, NY 10021

39. A. Birchall, NRPB, Chilton, Didcot, Oxon, OX11 ORQ, United Kingdom

40. B. B. Boecker, Inhalation Toxicology Research Institute, Lovelace Biomedical and Environmental Research Institute, P. O. Box 5890, Albuquerque, NM 87185

41. W. E. Bolch, 205 Nuclear Science Center, University of Florida, Gainesville, FL 32611

42. L. Bologna, ENEA-DISP, Direzione Centrale Sicurezza, Nucleare E. Protezione Sanitaria, Via V. Brancati 48, 00144 Roma, Italy 
43. A. Bouville, National Cancer Institute, Radiation Effects Branch, Executive Plaza North, Suite 530, Bethesda, MD 20892

44. E. M. Brackett, 4815 Wilkshire Drive, Knoxville, TN 37921

45. A. B. Brill, Department of Nuclear Medicine, University of Massachusetts Medical Center, 55 Lake Avenue North, Worchester, MA 06155

46. A. Brodsky, 2765 Ocean Pines, Berlin, MD 21811-9127

47. T. F. Budinger, Center for Functional Imaging, Lawrence Berkeley Laboratory, University of California, Berkeley, CA 94720

48. J. T. Bushberg, Department of Diagnostic Radiology, Section of Nuclear Medicine, Sacramento Medical Ctr., University of California, Davis, 2315 Stockton Boulevard, Sacramento, CA 95817

49. R. S. Caswell, U. S. Department of Commerce, NIST, Bldg. 245/C-229, Gaithersburg, MD 20899

50. R. Chatterjee, Atomic Energy Control, P. O. Box 1046, 270 Albert Street, Ottawa, Ontario K1P 5S9, Canada

51. R. J. Cloutier, 168 Cumberland View Drive, Oak Ridge, TN 37830

52. D. Cole, Medical Applications and Biophysical Research Division, ER-73, Office of Health and Environmental Research, Office of Energy Research, Department of Energy, Washington, D.C. 20585

53. D. Cool, Radiation Protection and Health Effects Branch, U. S. Nuclear Regulatory Commission, MS NLS-130, Washington, DC 20555

54. F. T. Cross, Battelle Pacific Northwest Laboratories, P. O. Box 999, K4-13, Richland, WA 99352

55. L. T. Dillman, 184 W. Lincoln Ave., Delaware, OH 43015

56. K. Ennow, National Board of Health, National Institute for Radiation Hygiene, Frederikssundsvej 378, DK-2700 Bronshoj, Denmark

57. R. J. Farkas, Division of Medical Imaging, Surgical and Dental Drug Products, (HFD-160), Office of Drug Evaluation I, Center for Drug Evaluation and Research, Food and Drug Administration, 5600 Fishers Lane, Rockville, MD 20857

58. L. E. Feinendeigen, Institute of Medicine, P. O. Box 1913, 517-Juelich, Germany

59. F. A. Fry, National Radiological Protection Board, Chilton, Didcot, Oxon, OX11 ORQ, United Kingdom 
60. R. V. Griffith, IAEA, Wagramerstrasse 5, P.O. Box 200, A-1400, Vienna, Austria. AA008.

61. R. Guilmette, Inhalation \& Toxicology Research Institute, Lovelace Biomedical \& Environmental Research Institute, P. O. Box 5890, Albuquerque, NM 87185

62. A. Hach, Institut f. Nukleaarmedizin Medizinische Univ. - Klinik, Langenbeckstra6e 1, D-6500 Mainz, Germany

63. J. D. Harrison, National Radiological Protection Board, Chilton, Didcot, Oxon, OX11 ORQ, United Kingdom

64. M. T. Hays, VA Medical Center 151, 3801 Miranda Avenue, Palo Alto, CA 94304

65. K. Henrichs, Siemens, 2PL1 VWS3, D-81730 München, Germany

66. W. Hladik, The University of New Mexico College of Pharmacy, Albuquerque, NM 87131

67. J. S. Hughes, National Radiological Protection Board, Chilton, Didcot, Oxon, OX11 ORQ, United Kingdom

68. T. E. Hui, Pacific Northwest Laboratories, P. O. Box 999 K3-51, Richland, WA 99352

69. D. B. Howe, Nuclear Regulatory Commission, Mail Stop TWFN 8-F-5 OWFN, Washington, DC 20555

70. L. A. Ilyin, Institute of Biophysics, Ministry of Health, Zhivopisnaya ul. 46, 123182 Moscow, Russia

71. J. Inaba, National Institute of Radiological Sciences, 9-1 Anagawa-4-Chome, Chiba-shi, 260, Japan

72. A. C. James, Pacific Northwest Laboratory, P. O. Box 999, Richland WA 99352

73. L. Johansson, Department of Radiation Physics, Umea University Hospital, S-901 85 Umea, Sweden

74. E. Jones, Division of Medical Imaging, Surgical and Dental Drug Products, (HFD-160), Office of Drug Evaluation I, Center for Drug Evaluation and Research, 5600 Fishers Lane, Rockville, MD 20857

75. H. Kawamura, Laboratory for Radioecology National Institute of Radiological Sciences, 3609 Isozaki, Nakaminato-shi, Ibaraki, 311-12, Japan

76. K. Kearfott, Nuclear Engineering, University of Michigan, 108 Cooley Lab, North Campus, Ann Arbor, MI 48109-2104

77. G. M. Kendall, National Radiological Protection Board, Chilton, Didcot, Oxon OX11 ORQ, United Kingdom 
78. L. Koblinger, KFKI Atomic Energy Research Institute, P. O. Box 49, H-1525 Budapest, Hungary

79. E. Lamothe, AECL Research, Chalk River Nuclear Laboratories, Chalk River, Ontario, Canada KOJ 1 JO

80. I. A. Likhtarev, Ukraine Scientific Centre for Radiation Medicine, Melnikova Street 53, 252050 Kiev-50, Ukraine

81. J. Liniecki, Department of Nuclear Medicine, Medical Academy of Lodz, Czechoslowacka 8/10, 92-216 Lodz, Poland

82. J. L. Lipsztein, Rua Itajuru 132, Rio De Janeiro, RJ, CEKD2641, Brazil

83. C. S. Marcus, Nuclear Medicine Outpatient Clinic, Building A-13, UCLA School of Medicine, Harbor-UCLA Medical Center, 1000 Carson Street, Torrance, CA 90509

84. D. R. Melo, Comissao Nacional de Energia Nuclear, Instituto de Radioprotecao e Dosimetria, Av.

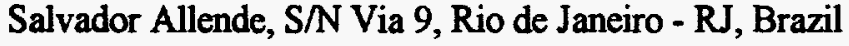

85. J. Melogranna, Office of Drug Research \& Review, Division of Radiopharmaceutical Drug Products, Center for Drug Evaluation and Research, Food and Drug Administration, 18BOB HFD 160, 5600 Fishers Lane, Rockville, MD 20857

86. H. Menzel, DGXII, Commission of the European Communities, Joint Research Centre, Rue de la Loi 200, B-1049 Brussels, Belgium

87. H. Metivier, CEA/ISPN, Direction des Racherches en Securite Nucleaire, BP No. 6-92265 Fontenay-aux-Roses, France

88. F. Mettler, Department of Radiology, The School of Medicine, University of New Mexico, Albuquerque, NM 87131

89. G. Mills, Center for Biologics, Department of Clinical Trials, Food and Drug Administration, Woodmont Building, 208C, II, 1401 Rockville Pike, Rockville, MD 20852

90. N. Mocanu, Str. Giuseppe Garibaldi, Nr. 4, Sc.D., Ap. 50, Sect. 2, 71441 Bucharest, Romania

91. P. J. Mountford, Department of Nuclear Medicine, Kent \& Canterbury Hospital, Ethelbert Road, Canterbury, Kent CT13NG, UK

92. T. Nakano, National Institute of Radiological Sciences, 4-9-1, Anagawa, Inage-ku, Chiba-shi, 263, Japan

93. C. B. Nelson, U. S. EPA Office of Radiation Programs, 401 M. Street, S. W., Mail Code 6602J, Washington, DC 20460

94. J. C. Nenot, Institut de Protection et de Surete Nucleaire, Commissariat a l'Energie Atomique, B P No 6, 92265 Fontenay-aux-Roses, Cedex, France 
95. D. Nosske, Bundesamt für Strahlenschutz, Institut für Strahlenhygiene, Ingolstädter Landstrasse 1, D-8042 Neuherberg, Germany

96. B. Nosslin, Knut Wickells, Väg 19, S-22466, Lung, Sweden

97. R. O'Brien, Australian Radiation Laboratory, Lower Plenty Road, Yallambie, Victoria 3085, Australia

98. J. Piechowski, Institut de Protection et de Surete Nucleaire, Departement de Protection Sanitaire, Centre d'Etudes de Fontenay-aux-Roses, France

99. J. W. Poston, Department of Nuclear Engineering, College of Engineering, Texas A\&M University, College Station, TX 77843

100. A. K. Poznanski, Department of Radiology, The Children's Memorial Hospital, 2300 Children's Plaza, Chicago, IL 60614

101. P. V. Ramzaev, Institute of Radiation Hygiene, 8 Mira ul, St. Petersburg 197101, Russia

102. A. C. B. Richardson, Deputy Director for Federal Guidance Criteria and Standards Division, Office of Air and Radiation, United States Environmental Protection Agency, Washington, DC 20460

103. P. J. Roberts, Medical Physics \& Medical Engineering, Southampton General Hospital, Tremona Road, Southampton, Hants S09 4XY, United Kingdom

104. J. S. Robertson, 18909 Tributary Lane, Gaithersburg, MD 20879-3409

105. G. S. Roessler, Route 1, Box 139H, Elysian, MN 56028

106. J. C. Rosen, GSPH, Department RADHEALTH, University of Pittsburg, Pittsburg, PA 15261

107. M. Rosenstein, Office of Health Physics (HFZ-60), Center for Devices and Radiological Health, 2094 Gaither Road, Rockville, MD 20850

108. D. van Rotterdam, Medical Biological Laboratory, TNO-Health Research, P. O. Box 5815, 2288 HV Rijswijk, The Netherlands

109. M. Roy, Commissariat Energie Atomique, Institut de Protection et Surete Nucleaire, BP No 6, F992265 Fontenay-aux-Roses Cedex, France

110. G. Runkle, U. S. Department of Energy, Albuquerque Operations, Environmental Safety and Health Division, Albuquerque, NM 87115

111. E. L. Saenger, Eugene L. Saenger Radioisotope Laboratory, University of Cincinnati Hospital, Cincinnati General Division, 234 Goodman Street, Cincinnati, OH 45267 
112. Y. Sasaki, Department of Radiology, Faculty of Medicine, The University of Tokyo, 7-3-1 Hongo, Bunkyou-ku, Tokyo 113, Japan

113. J. A. Siegel, Cooper Hospital, Department of Radiation Oncology, University Medical Center, 1 Cooper Plaza, Camden, NJ 08103

114. M. R. Sikov, Biology \& Chemistry Department, P7-53, Battelle, Pacific Northwest Laboratory, O. Box 999, Richland, WA 99352

115. K. Skulina, Lawrence Livermore National Laboratory, P. O. Box 808, Livermore, CA 94550

116. L. A. Slaback, NIST, Bldg. 245/C125, Gaithersburg, MD 20899

117. E. B. Sokole, Department of Nuclear Medicine, Academic Medical Center, Meibergdreef 9, 1105 AZ Amsterdam ZO, The Netherlands

118. M. G. Stabin, Radiopharmaceutical Internal Dose Center, Oak Ridge Associated Universities, MERT, P.O. Box 117, Oak Ridge, TN 37831

119. J. W. Stather, National Radiological Protection Board, Chilton, Didcot, Oxon, OX11 ORQ, United Kingdom

120. S. E. Strand, Radiation Physics Department, Lund University, Lasarettet, S-221 85 Lund, Sweden

121. J. B. Stubbs, Oak Ridge Associated University, MERT Division, Building 2714-G, Room W-17, P. O. Box 117, Oak Ridge, TN 37830

122. C. Sun, Radiological Sciences Division, Department of Nuclear Energy, Brookhaven National Laboratory, Building 703M, Upton, NY 11973

123. D. Swanson, School of Pharmacy, University of Pittsburgh, Pittsburgh, PA 15261

124. C. Taliaferro, Radiology Department, Norfolk General Hospital, 600 Gresham Drive, Norfolk, VA 23507

125. G. Tanaka, Asian Center for Reference Man Studies, 4-20-3 Ogikubo, Syuginami-ku, Tokyo 167, Japan

126. J. E. Tanner, Battelle, P.O. Box 999, Richland, WA 99352

127. D. M. Taylor, University of Wales, Cardiff School of Chemistry and Applied Chemistry, P. O. Box 912, Cardiff CF1 3TB, United Kingdom

128. K. Thomas, Nuclear Medicine Service (115), V. A. Medical Center, Gainesville, FL 32602

129. S. R. Thomas, Division of Medical Physics, University of Cincinnati, Medical Sciences Bldg. E560, Cincinnati, OH 45267-0579 
131. S. Treves, Division of Nuclear Medicine, Childrens Hospital Medical Center, 300 Longwood Avenue, Boston, MA 02115

132. E. A. Tupin, 518 Meadow Hall Drive, Rockville, MD 20851

133. B. W. Wachholz, Low Level Radiation Effects Branch, National Cancer Institute, Landow Building 8C-09, 9000 Rockville Pike, Bethesda, MD 20205

134. H. N. Wagner, Jr., Johns Hopkins Medical Institutions, 615 North Wolfe Street, Room 1102, Baltimore, MD 21205

135. M. E. Wangler, Director, Transportation \& Packaging Safety Division, U. S. Department of Energy, Office of Safety \& Quality Assurance, EH-33.2, 270 CC, Washington, DC 20585

136. E. E. Watson, Radiopharmaceutical Internal Dose Center, Oak Ridge Associated Universities, MERT, P. O. Box 117, Oak Ridge, TN 37831

137. D. A. Weber, Brookhaven National Laboratory, Medical Department, Building 490, Upton, NY $11973-5000$

138. A. V. Wegst, 5420 Pawnee, Fairway, KS 66205

139. B. W. Wessels, Department of Radiology, George Washington Univ Med Center, 901 Twentythird St., NW, Washington, DC 20037

140. W. Whicker, Professor, Department of Radiology and Health Sciences, Colorado State University, Fort Collins, CO 80523

141. L. E. Williams, Radiology Division, City of Hope, 1500 East Duarte Rd., Duarte, CA 91010

142. L. R. Williams, Indiana University at South Bend, Mathematics Department, 1700 Mishawaka Avenue, South Bend, IN 46634

143. B. C. Winkler, Private Bag X256, Pretoria, 0001, South Africa

144. D. F. Wong, Nuclear Medicine/Tower Basement, The Johns Hopkins Hospital, 600 North Wolfe Street, Baltimore, MD 21205

145. R. W. Wood, Physical and Technology Research Division, ER-74, Office of Health and Environmental Research, U. S. Department of Energy (GTN), Washington, DC 20545

146. M. E. Wrenn, Environmental Radiation \& Toxicology Laboratory School of Medicine, 1771 So., 900 W., Suite 10, Salt Lake City, Utah 84104

147. H. Yamaguchi, National Institute of Radiological Sciences, Division of Physics, 9-1, Anagawa-4chome, Chiba-shi 260, Japan 
148. S. Yaniv, Health Effects Branch - MS 1130-SS, Division of Health, Siting and Waste Management, RES, U. S. Nuclear Regulatory Commission, Washington, DC 20555

149. P. B. Zanzonico, Division of Nuclear Medicine, New York Hospital/Cornell Medical Center, 525 East 68th Street, New York, NY 10021

150-152.Office of Scientific and Technical Information, P. O. Box 62, Oak Ridge, TN 37831 